NBS

Publi-

cations

\title{
TABULATION OF DATA ON MICROWAVE TUBES
}

Handbook 104

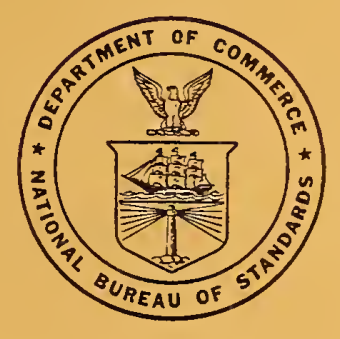

United States Department of Commerce National Bureau of Standards 

UNITED STATES DEPARTMENT OF COMMERCE - Alexander B. Trowbridge, Secretary NATIONAL BUREAU OF STANDARDS - A. V. Astin, Director

\title{
Tabulation of Data on Microwave Tubes
}

\author{
J. K. Moffitt
}

The National Bureau of Standards

Electron Devices Data Service

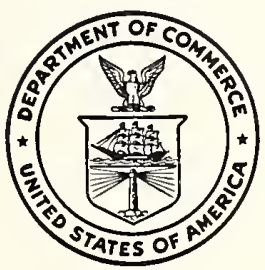

National Bureau of Standards Handbook 104

Issued September 29, 1967

Supersedes Handbook 70

For sale by the Superintendent of Documents, U.S. Government Printing Office, Washington, D.C. 20402 Price $\$ 1.25$ 
Library of Congress Catalog Card Number : 67-62080 


\section{Foreword}

This tabulation of data on microwave tubes currently in use has been prepared as part of the National Bureau of Standards Electron Devices Data Service. Established in 1948 to provide technical data on radio tubes to members of the Bureau Staff, the service has since been cxtended to all scientists and engineers in government and industry. In the course of the program, a large volume of information on domestic and foreign devices was accumulated on punched cards from which it could be automatically printed. It was felt desirable to make these data available in a single reference sourcc as an aid to circuit designers in selecting tube types for particular uses.

The engineer should find this manual useful in narrowing down the choicc of tubes to one or a few types. However, it is not practical to give all possible operating conditions or to provide the characteristic curves for each tube in a tabulation such as this. It will still be necessary to consult the tube manufacturer's literature for such detailed information.

All information appearing in this publication was taken from manufacturer's published specifications, and every effort has been made to ensure accuracy and completeness. However, the Bureau cannot assume responsibility for omissions nor for results obtained with these data.

A tabulation of data on receiving tubes has been issued previously as NBS Handbook 103. Additional tabulations for o ther electron devices are being developed and will be issued as rapidly as they are completed. Also, revisions of this and other tabulations will be issued as deemed necessary to keep them up to date.

A. V. Astix, Director. 


\section{Contents}

Foreword Page

Foreword

1. Introduction

2. Organization of the tabulation $\ldots \ldots \ldots$

3. Sorting and terminology of the tabulation $\ldots \ldots$

3.1. Sorting methods $\ldots \ldots \ldots \ldots$

3.2. Terminology $\ldots \ldots \ldots \ldots$

Definitions

3.3. Unit symbols

4. Numerical listing of tube types

5. Characteristic listing of tube types 


\title{
Tabulation of Data on Microwave Tubes
}

\author{
J. K. Moffitt
}

\begin{abstract}
A tabulation of microwave electron tubes with characteristies of each type has been arranged in the form of two major listings, a Numerical Listing in which the tubes are arranged by type number, and a Characteristic Listing in which the tubes are arranged by the kind of tube, and further ordered on the basis of minimum frequeney and power output.
\end{abstract}

\section{Introduction}

The Electron Devices Section of the National Bureau of Standards has developed over the past decade an Electron Devices Data Service. This service collects and maintains a file of data on all electron devices, i.e., tubes, transistors, diodes, etc., manufactured in the United States and other countries. In an effort to make this service more readily available to engineers applying electronics in the laboratories throughout the country, it was decided to develop a method of tabulating the essential information pertaining to tube characteristics in handbook form for ready reference. For this publication on Microwave 'Tubes, an easily decipherable code and format of the tube characteristics which was adaptable to punched cards was developed. Both accuracy and flexibility are incorporated into the system. The sources of information were the manufacturers' published handbooks and data sheets. The accuracy of the printed information is reasonably assured by verifying tabulations by various sortings and cross checking with manufacturers' publications. This tabulation includes only the information normally furnished by the manufacturers in their handbooks or data sheets. All data in our files up to April 1, 1967, is included in this tabulation.

This tabulation includes only those tube types of the general class known in the field of electronics as microwave tubes, i.e., tubes employed as oscillators and power amplifiers at frequencies above approximately $1,000 \mathrm{MHz}$ and which are "velocity controlled" with transit times in excess of 0.3 radian. Some developmental types have been included.

\section{Organization of the Tabulation}

The Microwave Tabulation comprises two principal sections as follows:
1. Numerical Listing. In this, the tubes are arranged by type number in the numerical-alphabetical sequence which is standard in the industry.

2. Characteristics Listing. Here the tubes are sorted into the basic groups and then are arranged by increasing value of one or more pertinent characteristics.

\section{Sorting and Terminology of the Tabulation}

'To assist the user in understanding and applying the tabulation, the method of sorting and the definition of terms and code used, are explained in this section.

\subsection{Sorting Methods}

The Numerical Listing is arranged in the numerical-alphabetical sequence by tube type number which is explained under the heading "Type Number" in section 3.2.

In the Characteristic Listing, the tube types are arranged into basic groups or kinds, e.g., klystron amplifier, klystron oscillators, magnetrons, etc., and then sorted primarily by minimum frequency and secondarily by power output. The basic groups are listed inder the heading "Kind" in section 3.2, Terminology. This method of sorting permits the user to find the type numbers which most completely satisfy his requirements.

\subsection{Terminology}

The Numerical and Characteristic Listings are in tabular form containing 24 columns. The headings of these columns and their meanings. are given below.

A blank in any column indicates that the characteristic designated by the column is not applicable to the type number in question or that no value was given in the available data. 


\section{Definitions}

Type Number. This column lists the numericalalphabetical designation assigned by the manufacturer. The Numerical Listing on page 7 has been arranged according to the industry standard, i.e., primarily by arranging the initial numerical portion in sequence, secondarily by arranging the alphabetical prefix in sequence and finally by arranging the alphabetical suffix in order e.g.

2V315
2W401
HA3
HK3
W3MQ/1F
3K2500SG
3K3000LQ
3KM300LA

Symbols. An asterisk (*) is used to designate tube types found in the Military Preferred list issued by the Department of Defense as "Military Standard Electron Tubes, Selection and use of" MIL-STD-200H, 17 January, 1966.

An equal sign $(=)$ designates a reliable or premium type as indicated by the manufacturer.

Kind. A three-letter code is used to describe the generic group of tubes. This is used as the primary sorting means for the Characteristic Listing.

$\begin{array}{ll}\text { AMA } & \text { Amplitron Amplifier or Platinotron } \\ \text { BWA } & \text { Backward Wave Amplifier } \\ \text { BWO } & \text { Backward Wave Oscillator } \\ \text { CAR } & \text { Carcinotron } \\ \text { CEA } & \text { Crossed-Field Amplifier } \\ \text { KLA } & \text { Klystron Amplifier } \\ \text { KLM } & \text { Klystron Multiplier } \\ \text { KLO } & \text { Klystron Oscillator } \\ \text { MAG } & \text { Magnetron } \\ \text { TWA } & \text { Traveling Wave Amplifier } \\ \text { TWM } & \text { Traveling Wave Multiplier }\end{array}$

Frequency. Both minimum and maximum frequency of operation are listed in gigahertz $\left(10^{9} \mathrm{~Hz}\right)$ with the exception that the "letterband" designation (i.e., S-band, X-band) is used when more specific information is unavailable. Fixed-tuned tubes are available at any frequency within the tabulated range. In the case of frequency multiplier tubes, output frequency is tabulated.

Duty Cycle. The duty cycle for pulsed operation is often specified as a percentage, although it may also appear as the product of the pulseduration time $\left(t_{p}\right)$ and the pulse-repetition rate $(\mathrm{prr})$. The tabulated value is the product of the pulse-duration time and the pulse-repetition rate times $10^{4}$. The following table illustrates both methods of specifying Duty Cycle and the corresponding tabulated value.

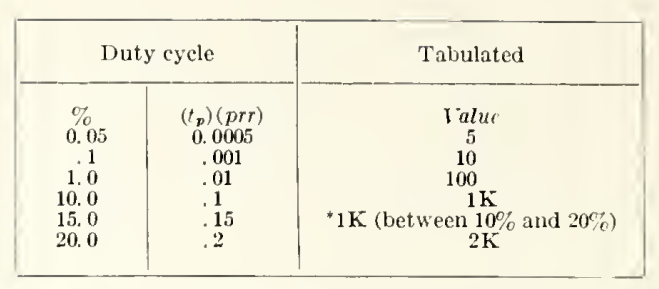

Tuning. The method by which the tube is frequency-tuned is shown by the following alphabetical code.

$\begin{array}{ll}\text { FX } & \text { Fixed tuned } \\ \text { HY } & \text { Hydraulically tuned } \\ \text { MEC } & \text { Mechanically tuned } \\ \text { TH } & \text { Thermally tuned } \\ \text { V'T } & \text { Voltage tuned }\end{array}$

Manufacturer. 'The alphabetical code refers to either the company originally registering the tube type with the Electronic Industries Association or the manufacturer of the type. In the case of tubes produced by more than one manufacturer, this column has been left blank.

AM Amperex Electronic Corp.

BE Bendix Aviation Corp.

BL Bomac Div. of Varian Assoc.

BM Beam Tube Corporation

CS Compagnie General de T.S.F.

CV Canadian Varian

EC Microwave Electronics Corp.

Palo Alto

EE English Electric Valve Co.

EI Eimac Div. of Varian

EM EMI Electronics Ltd.

FE Ferranti Electric Co.

GC General Electric Co. Ltd.

GE General Electric Co.

HI Hitachi, Ltd.

HP Hughes Microwave Tube Div.

$\mathrm{HU}$ Huggins Laboratories, Inc.

I'T International Telephone \& 'Telegraph Corp.

KK Kobe Kogyo

LI Litton Industries

LT Lignes Telegraphiques et 'Telephoniques

MA Microwave Associates, Inc.

ME Metcom

MR Mictron, Inc.

MU Mullard Ltd.

NC Aksjeselskapot Nera

NE Nippon Electric Co.

OK OKI Electric Industries Co.

$\mathrm{PH}$ Philips Laboratories

PO Polarad Electric Corp.

RA Raytheon Co.

RC Radio Corporation of America

SD SFD Laboratories

SE Stewart, Div. of Watkins-Johnson

SH Siemens America, Inc. 
SP Sperry Electronic Tube Division

S'T Standard Telephones \& Cables Ltd.

TE Telefunken G.M.B.H.

TO 'Tokyo Shibaura

TV Thomson-Varian

VA Varian Associates

VV Valvo G.M.B.H.

WH Westinghouse Electric Co.

WJ Watkins-Johnson Co.

W'T Warnecke Electron 'Tube Co.

Operation. This is an alphabetical code to indicate the type or operation, as shown,

C Continuous Wave Operation

$P$ Pulsed operation (If a manufacturer has specified different operating parameters (i.e. for both pulsed and CW), the tube is tabulated twice to include both sets of conditions.

$\mathbf{E}_{1}$. The nominal heater or filament voltage in volts.

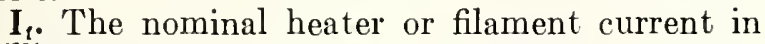
milliamperes.

Beam or Anode Volts. The maximum anode, beam or resonator voltage. All roltages and currents given in this and the following columns are maximum values (peak values in the case of pulsed tubes) as these will aid in determining the power supplies needed to operate the tube over its complete range.

$\mathbf{I}_{\mathrm{k}}$. The maximum cathode or plate current in milliamperes.

$\mathbf{P}_{0}$. The maximum power output.

Collector, Reflector, etc. Volts. The maximum collector, reflector, line, etc. voltage whichever is most applicable.

Control Volts $\mathbf{E}_{\mathrm{g} 1}$. The maximum control, focusing or grid roltage.

Helix Voltage. The maximum helix or delayline roltage of traveling wave tubes.

Gain. The maximum, small-signal gain in decibels or, in the case of frequency multiplier tubes, the multiplication factor.

NF. The typical noise factor in decibels. hertz.

Pulling Factor. The pulling factor in mega-
Bandwidth. 'The frequency difference between half-power points for amplifier tubes or the electronic tuning range for oscillator tubes.

Magnetic Field. Numerical figures indicate the magnetic field strength in millitesla $(0.1 \mathrm{~m}$ T =Gauss); or the alphabetical code "IM" indicates an integral permanent magnet.

Cavity. The number and type of cavities characteristic of the tube is indicated by the code.

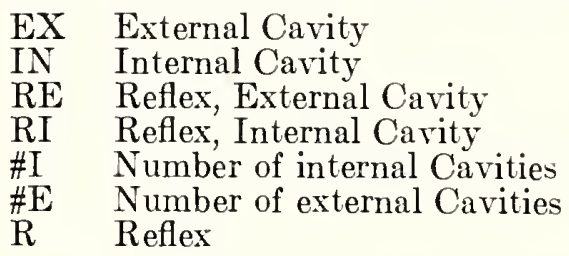

Coupling. 'The type of output coupling of the tube is coded as follows:

$\begin{array}{ll}\text { CO } & \text { Coaxial } \\ \text { SP } & \text { Special } \\ \text { WG } & \text { Waveguide } \\ \text { WC } & \text { Waveguide or Coaxial }\end{array}$

\subsection{Unit Symbols}

While the normally used electrical unit is printed at the top of each column, it will be noted that letter symbols are used following some numbers to indicate a change of unit.

\begin{tabular}{|c|c|c|}
\hline Symbol & Heading & Unit \\
\hline $\begin{array}{l}\mathrm{H} \\
\mathrm{A} \\
\mathrm{K} \\
\mathrm{A} \\
\mathrm{U} \\
\mathrm{K} \\
\mathrm{M} \\
\mathrm{U} \\
\mathbf{W} \\
\mathrm{K} \\
\mathrm{K} \\
\mathrm{K} \\
\mathrm{K}\end{array}$ & 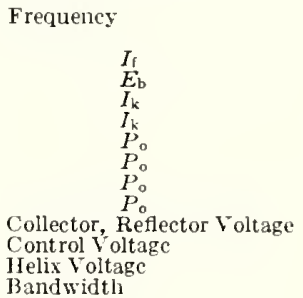 & $\begin{array}{l}100 \text { Gigahertz } \\
\text { (1011 Hz) } \\
\text { Amperes } \\
\text { Kilovolts } \\
\text { Amperes } \\
\text { Microamperes } \\
\text { Kilowatts } \\
\text { Megawatts } \\
\text { Microwatts } \\
\text { Watts } \\
\text { Kilovolts } \\
\text { Kilovolts } \\
\text { Kilovolts } \\
\text { Kilohertz }\end{array}$ \\
\hline
\end{tabular}




\section{Numerical Listing of Tube Types}




\begin{tabular}{|c|c|c|c|c|c|c|c|c|c|c|c|}
\hline 9 9MI TnCO & 8 & $\begin{array}{ll} & 8\end{array}$ & $8 \% \frac{5}{3}$ & 8 ४ & 이요 & 언웅 & 언 옹어 & 웅 & O & 엉엉으 & 엉엉ㅇ \\
\hline ALAPS & & & & & $\vec{\alpha}$ & $\bar{\alpha}$ & & & & & \\
\hline $0131 \mathrm{~s}$ & $\Sigma$ & & $\Sigma$ & $\Sigma$ & $\Sigma$ & & & & & & \\
\hline HLOMarro & 퐆요 & & 음 & & & $\stackrel{\circ}{m}$ & & & & & \\
\hline 2*1าרกs & $\stackrel{n}{\sim}$ & $\stackrel{n}{\sim}$ & & $\stackrel{n}{\sim}$ & $\simeq \cong$ & $\stackrel{\simeq}{\sim}$ & & & & & \\
\hline $\begin{array}{l}8013 \times 3 \\
3510 \%\end{array}$ & $:$ & & & & & & & & & & \\
\hline hivg & : & & & & & & & & & & \\
\hline $\begin{array}{l}\text { S170h } \\
\times 17734\end{array}$ & & & 芯 $\stackrel{\sim}{\sim}$ & $\stackrel{N}{N}$ & 弚 & & & & & & \\
\hline $\begin{array}{c}\text { S170 } \\
7081403\end{array}$ & $\rightarrow$ & & : & $\stackrel{\circ}{\sim}$ & \& & 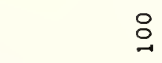 & 욤 & $\stackrel{\circ}{N}$ & $\stackrel{\text { ㅇ }}{ }$ & $\stackrel{\circ}{D} \stackrel{\circ}{D}$ & 웅 욤 \\
\hline 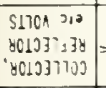 & $>\int_{0}^{\infty}$ & & & & $\stackrel{\circ}{\circ}$ & $\stackrel{\circ}{\infty}$ & 엄 & $\stackrel{\circ}{\sim}$ & & $\stackrel{\circ}{\stackrel{\circ}{N}}$ & 옹 \\
\hline $0^{\circ}$ & E No & 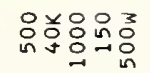 & 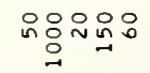 & "nz잉요임 & "n & 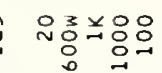 & 品品品品怘 & 总品品管 & so & 음용요 & 总总品品品 \\
\hline 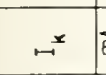 & Em & 욤욤음 & 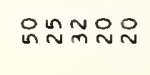 & 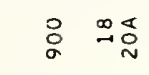 & $\stackrel{\infty}{\circ} \underset{\exists}{0}$ & m品䠐 N & $m$ in in in & in in in & $\stackrel{0}{-1}$ & 으음으으으 & $m m m O m$ \\
\hline 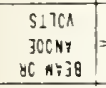 & 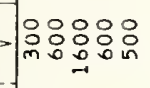 & 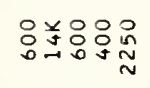 & : & 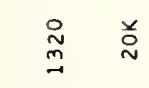 & 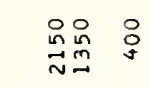 & 总品品 品 & 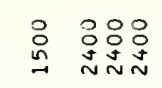 & 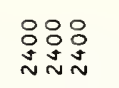 & : & 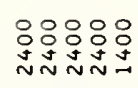 & 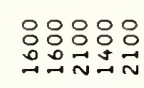 \\
\hline+ & El & 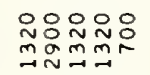 & 요=윰유. & : : & 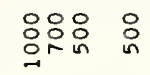 & 용유 & : $:$ : & : & : & 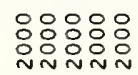 & 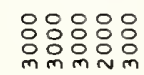 \\
\hline$\omega^{-}$ & : & 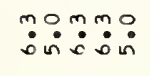 & 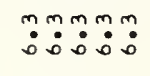 & in & $\ddot{0} \dot{0}: \stackrel{m}{0}$ & $\dot{m}: \dot{0}: 0$ & $\dot{m} \dot{m} \dot{m} \dot{m} \dot{m}$ & $\ddot{m} \dot{0} \dot{0} \dot{m}$ & $\dot{m}$ & $\dot{m} \dot{m} \dot{m} \dot{m} \dot{m} \dot{m}$ & $\ddot{m} \dot{m} \dot{0} \dot{m} \dot{m} \dot{m}$ \\
\hline $\mathrm{NO} 11 \mathrm{Hy}] \mathrm{JC}$ & טua u & Ua U & טuטu & ט ט ט & ua u & uauv & טיטu & טuט & טג & טنטu & טuט \\
\hline$\overline{y \cdot n}$ & ळ & $\vec{\omega} \omega \vec{\omega} \omega \vec{\omega}$ & 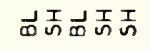 & こロ & ๙ & जิळ & $\frac{\alpha}{\Sigma} \circlearrowleft \frac{\alpha}{\Sigma} \frac{\alpha}{\Sigma} \frac{\alpha}{\Sigma}$ & $\frac{\alpha}{\Sigma} \frac{\alpha}{\Sigma} \frac{\alpha}{\Sigma} \mathcal{U}$ & $\frac{\alpha}{\Sigma}$ & $\frac{\alpha}{\Sigma} \frac{\alpha}{\Sigma} \frac{\alpha}{\Sigma} \frac{\alpha}{\Sigma} \frac{\alpha}{\Sigma}$ & $\frac{\alpha}{\Sigma} \frac{\alpha}{\Sigma} \frac{\alpha}{\Sigma} \frac{\alpha}{\Sigma} \frac{\alpha}{\Sigma}$ \\
\hline $3 \mathrm{~N} \cap 1$ & $\stackrel{u}{\mathrm{u}}$ & $\begin{array}{ll}\times & \breve{w} \\
\end{array}$ & $\stackrel{\breve{山}}{\Sigma} 5$ & $\stackrel{\breve{山}}{\Sigma} 5$ & $\stackrel{\breve{w}}{\lessgtr}$ & 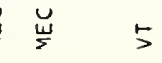 & ๖ トレ゙ & ららら & 5 & トレ5ケら & トレレレ5 \\
\hline $0: 18 y$ aIs & $\stackrel{\circ}{\sim}$ & $\stackrel{i}{i}$ & & $\stackrel{\sim}{\sim}$ & 옷 & 웅 & & & & & \\
\hline 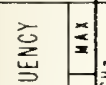 & $\approx$ & 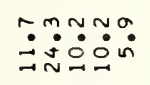 & 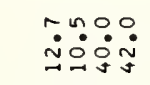 & mañoㅇำ & 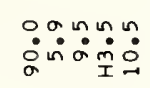 & 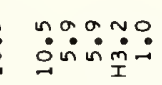 & 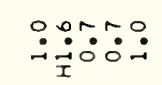 & $\because \because$ & $\therefore$ & 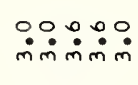 & 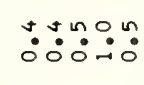 \\
\hline \begin{tabular}{|l|l|}
$\underset{\tilde{u}}{\tilde{u}}$ & $\frac{z}{x}$ \\
\end{tabular} & ॥ & : & 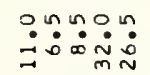 & 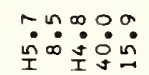 & 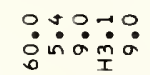 & 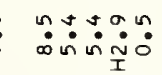 & mm : & $\ddot{0}: \because 0$ & $:$ & $\because \because \because ニ ュ$ & $\because \because \because \because \because 0$ \\
\hline ONIXX & 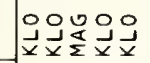 & 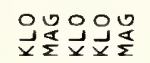 & 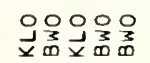 & 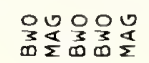 & 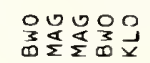 & 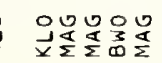 & 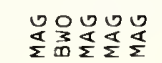 & 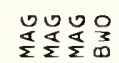 & $\frac{0}{2}$ & 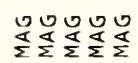 & 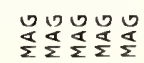 \\
\hline 709 W LS & " & & & & & & & & & & \\
\hline 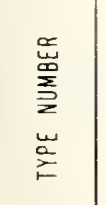 & 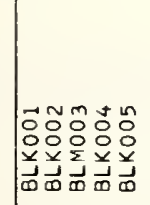 & 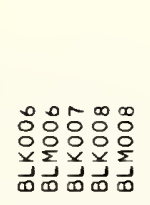 & 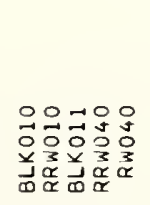 & 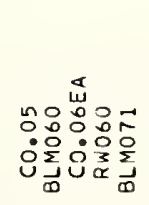 & 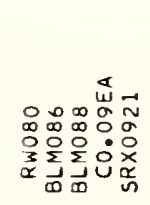 & 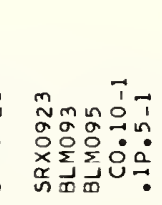 & 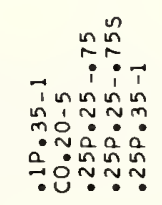 & 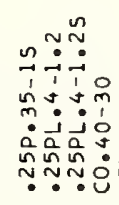 & à & 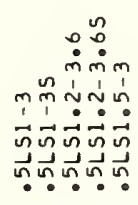 & 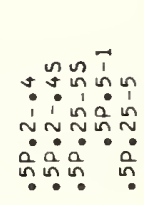 \\
\hline
\end{tabular}




\begin{tabular}{|c|c|c|c|c|c|c|c|c|c|c|}
\hline 9MITdnOS & 웅옹 & 운워눈 & 号 & 80 & 운옹 & 웅요 & 8요논 & 눈원 & 운운오 & 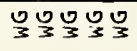 \\
\hline AlIAYJ & & $\vec{\alpha}$ & $\vec{\alpha} w \vec{\alpha} \vec{\alpha} \vec{\alpha} \vec{\alpha}$ & $\vec{\alpha} \vec{\alpha} \underset{\alpha}{w} \vec{\alpha} \vec{\alpha}$ & $\vec{x}$ & & $\vec{\alpha}$ & $\vec{\alpha}$ & & \\
\hline 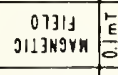 & $\Sigma$ & 음욤 & & & & $\Sigma$ & $\Sigma \sum \Sigma_{0}$ & 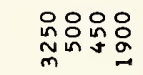 & 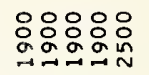 & ミ 品别 \\
\hline H101MONYA $\simeq$ & & in & 임요묘의 & 임용ㅇㅁ & 웅 & & in & 요 웅 & 악영연 & : \\
\hline 9Mורח & & a & & & & & & $\stackrel{\Perp n}{\sim}$ & 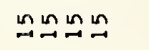 & $\cong \stackrel{n}{2} \pi$ \\
\hline $\begin{array}{c}\text { YoIJYJ } \\
\text { JSION }\end{array}$ & & & & & & & 욤 & & & \\
\hline \begin{tabular}{l|l} 
NiYg & : \\
\end{tabular} & & $\stackrel{n}{m}$ & & & & $\stackrel{\infty}{m}$ & 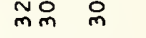 & 웅요 & & \\
\hline $\begin{array}{l}51701 \\
\times 1134 \\
X\end{array}$ & & $\stackrel{n}{\sim}$ & & & & 䛔 & $\stackrel{y}{\sim} \stackrel{x}{\sim}$ & 丷ㅠㅅㅛ & & \\
\hline $\begin{array}{c}\text { S170A } \\
\text { 1001 NOJ }\end{array}$ & : & & & & 总总 总 & 윰이 & $\stackrel{\circ}{\circ} \stackrel{\circ}{\circ}$ & 弚 品吕 & & \\
\hline 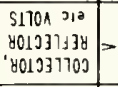 & : & 吕 & 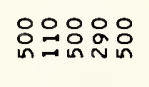 & 웅융요 & 怘 $\stackrel{\circ}{\sim}$ & 品 总隽 & 品品品品 & 品 总品 & & \\
\hline $0^{\circ}$ & 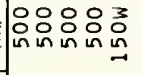 & 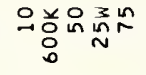 & 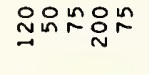 & 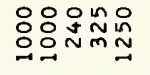 & 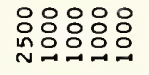 & 응응ㅇㅇㅇㅁㅇ & 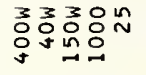 & 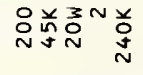 & 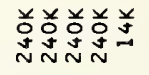 & 面吕兑兑兑 \\
\hline$\curvearrowleft$ & 루으으으욨 & 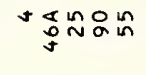 & 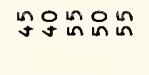 & 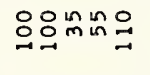 & $\varrho^{\circ} \ln ^{\ln \infty} \infty$ & $\infty \infty \underset{\sim}{\infty}$ 융 & 욤용쇼요 & 잉ㅇㅇㅁㅇㅇㅁㅇㅁㅇ & ర్లేర్లేర్లీర్లీన్ & 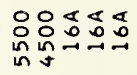 \\
\hline 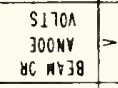 & 施吕: & 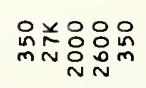 & 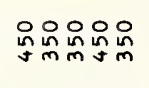 & 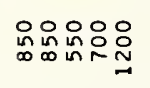 & 욤유ㅁㅠㅠ & 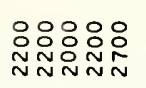 & 응용응 & 品前 员并 & 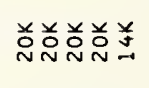 & 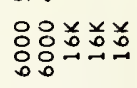 \\
\hline+ & 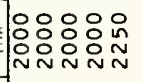 & 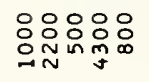 & 웅용ㅇㅇㅇㅛ & 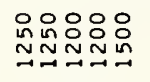 & 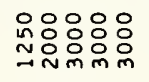 & 음음유 & 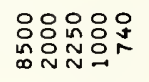 & 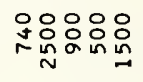 & 응ㅇㅇㅇㅇㅇㅇㅛ & 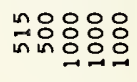 \\
\hline w" & & 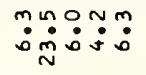 & 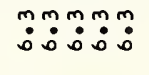 & 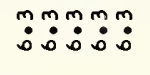 & 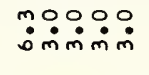 & ஸिmin்: & 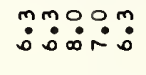 & 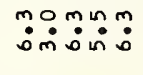 & m & 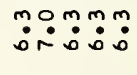 \\
\hline NO1_HYy 30 & & vauuv & טuטu & טuטu & טuטu & טuטu & טบטบ & vauva & 00000 & $0, a 00$ \\
\hline$y=$ & $\frac{\alpha}{\Sigma} \frac{\alpha}{\Sigma} \frac{\alpha}{\Sigma} \frac{\alpha}{\Sigma} U$ & 고로ऽ늠 & 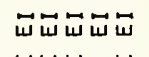 & 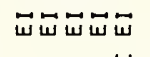 & $\vec{w} \frac{\alpha}{\Sigma} \frac{\alpha}{\Sigma} \frac{\alpha}{\Sigma} \frac{\alpha}{\Sigma}$ & $\frac{\alpha}{\Sigma} \frac{\alpha}{\Sigma} \frac{\alpha}{\Sigma} \frac{\alpha}{\Sigma} \frac{\omega}{Z}$ & 욦어ㅁㅗㅗ & 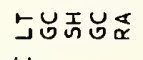 & $\underset{\alpha \propto \alpha}{\alpha \propto \alpha} \underset{\alpha}{\alpha}$ & \\
\hline \begin{tabular}{|l|}
$3 \times \cap 1$ \\
\end{tabular} & ららレ5 & 岁 & 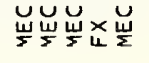 & 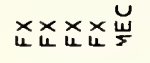 & 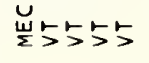 & 5555 & xs & 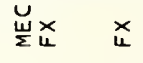 & 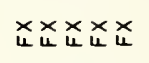 & 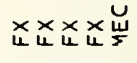 \\
\hline $0116 y<x i n 0$ & & $\therefore$ & & & & & & $\stackrel{\circ}{-}$ & 윽으우응요 & $\stackrel{\sim}{\sim} \cap \cong \simeq ニ$ \\
\hline 总 & & 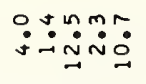 & 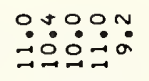 & 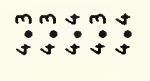 & 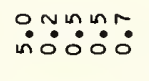 & $\because \because \because \because \circ$ & 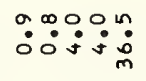 & 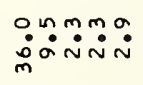 & 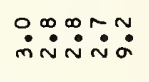 & 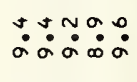 \\
\hline 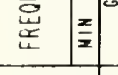 & 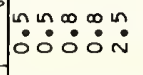 & 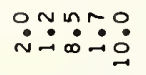 & 我 & 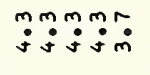 & 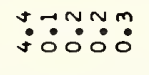 & min & $\because \because 0 \because \ddot{\sim} \dot{\sim}$ & 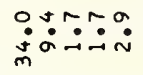 & 悉: & 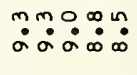 \\
\hline ONIX & 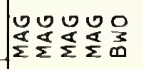 & 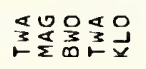 & 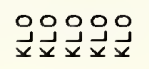 & 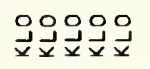 & 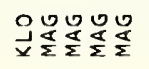 & 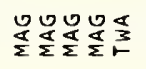 & 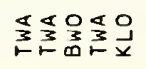 & 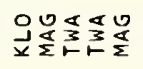 & 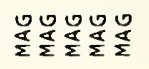 & 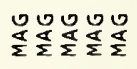 \\
\hline 108W/S & & & " " " " " & " " " " " & & & & & & $"$ \\
\hline 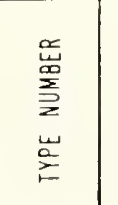 & 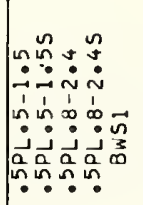 & 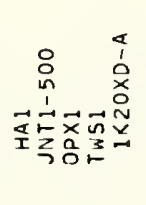 & 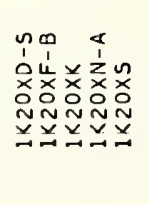 & 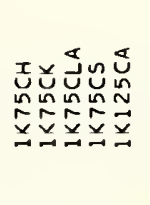 & 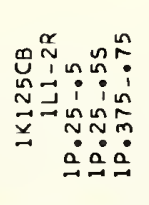 & 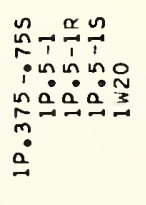 & 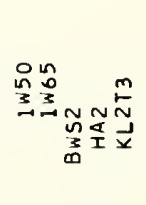 & 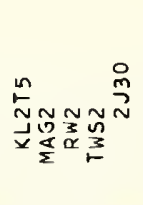 & 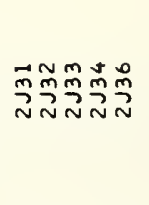 & 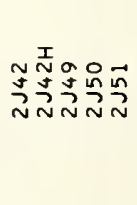 \\
\hline
\end{tabular}




\begin{tabular}{|c|c|c|c|c|c|c|c|c|c|c|}
\hline 9NI?dกOS & W & 8 & צ̧יO ૬ & $\frac{0}{3}$ & 운용 & 웅ㅇㅇ & 웅워용 & 웡어 & 웅웡 & 웅ㅇㅇ \\
\hline hlars & & & $\vec{\alpha} \vec{\alpha} \vec{\alpha} \times \vec{\alpha}$ & $\vec{\alpha} \vec{\alpha} \vec{\alpha} \vec{\alpha} \vec{\alpha}$ & $\vec{\alpha} \vec{\alpha} \vec{\alpha} \vec{\alpha} \vec{\alpha}$ & $\vec{\alpha} z$ & & & $\vec{\alpha} \vec{\alpha} \vec{\alpha} \vec{\alpha}$ & $\vec{\alpha} \vec{\alpha} \vec{\alpha}$ \\
\hline ग1719 & 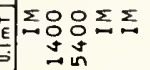 & 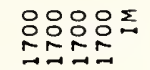 & & & & & $\sum \sum \sum_{n}$ & & & $\Sigma$ \\
\hline H10) & & & $\tilde{m} \tilde{m} \quad n$ & 守出 은 & $\stackrel{n}{\sim} \underset{\sim}{n}$ & 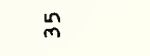 & & & ำกำ & $\stackrel{0}{ }$ \\
\hline Sחרורואי & $=\infty$ & $\stackrel{m}{-}$ & & & & & & & & \\
\hline $\begin{array}{l}\text { yoljws } \\
\text { 3sion }\end{array}$ & 7 & & & & & & & & & \\
\hline NiYg & : & & & & & & & & & 욤 \\
\hline $\begin{array}{l}51701 \\
\times 1173 H \\
\end{array}$ & & & & & & & & & & 㝹 \\
\hline $\begin{array}{c}51701 \\
\text { 10811003 }\end{array}=$ & & & & $\stackrel{\circ}{\circ}$ & & $\stackrel{\circ}{\circ}$ & & 品 & & $\stackrel{i}{N}$ \\
\hline 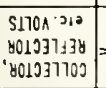 & & & 点品品品品品 & 읏욤욧요 & 웃음음음 & 品 & 品 & 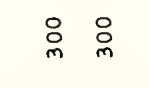 & 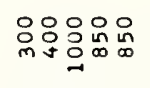 & 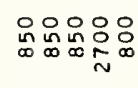 \\
\hline $0^{\circ}$ & 莌总总总总 & 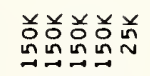 & 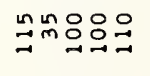 & 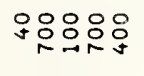 & 웅욤욤요 & 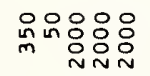 & 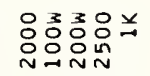 & 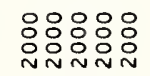 & 品品品品 & 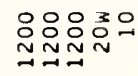 \\
\hline$\ldots$ & 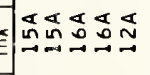 & 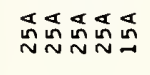 & 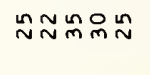 & " 品织品品 & $\mathcal{O}_{\sim}^{\infty} \stackrel{\infty}{\sim} \underset{\sim}{\sim} \underset{\sim}{\sim} \underset{\sim}{N}$ & 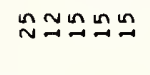 & 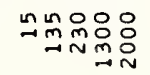 & 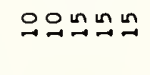 & 뜼요 & 으름요 \\
\hline $\begin{array}{c}5170 \mathrm{~A} \\
300 \mathrm{NY} \\
8 \mathrm{Ar} \rightarrow \mathrm{Bg}\end{array}$ & 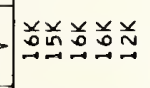 & 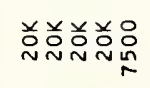 & 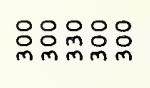 & 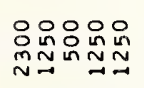 & 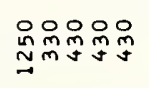 & 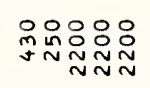 & 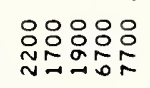 & 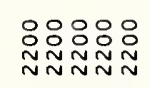 & 윴임용ㅇㅁ & 영영옹요 \\
\hline 5 & 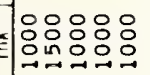 & 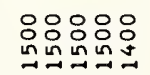 & 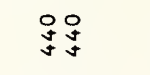 & 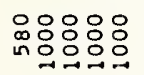 & 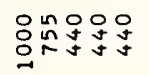 & 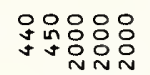 & 㗊总品品 & 용유윰윰유 & 品等品品品 & 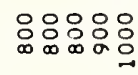 \\
\hline ш" & 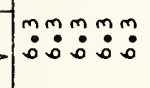 & mmmm & $\ddot{m}: \ddot{m}: \stackrel{m}{0}$ & 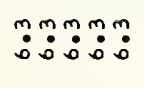 & 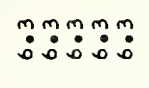 & 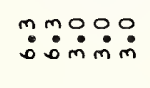 & 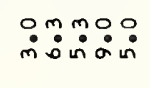 & 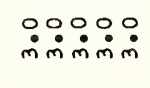 & $\dot{m} \dot{m}: \dot{m}: \dot{m}: 0$ & $\ddot{0}: \ddot{m}: \dot{m}: \dot{m}$ \\
\hline N011Fy3dO & 00200 & 00000 & טưu & yuuve & ưuu & טuטu & טuטu & טuטu & טuטu & טuטu \\
\hline$y+N$ & & $\underset{\alpha \propto \alpha \underset{\alpha}{\alpha} \underset{\alpha}{\alpha} \underset{\alpha}{\alpha}}{\alpha}$ & $\underset{\propto}{\propto} \quad \underset{x}{\alpha} \underset{\alpha}{\alpha}$ & ब旅的的的 & in $\underset{x}{ } \ddot{x} \ddot{x}$ & 爻宸品 $\frac{\alpha}{\Sigma} \frac{\alpha}{\Sigma}$ & 똗온온오 & $\frac{\alpha}{\Sigma} \frac{\alpha}{\Sigma} \frac{\alpha}{\Sigma} \frac{\alpha}{\Sigma} \frac{\alpha}{\Sigma}$ & 뚤은온유안 & 암안안오고 \\
\hline JNח1 & Uü & Uự & UUU⿺乚一匕 & 岁 & 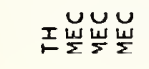 & 岀岕ら55 & 5 $5 \times x \times x$ & ら555 & 々岂岂岕岕 & 岀山ّ山 \\
\hline $011 y 4$ anno & $\exists=\because N \cong ㅇ ㅡ ㅇ ㅡ$ & 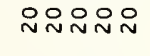 & & & & & & & & \\
\hline 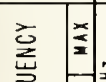 & 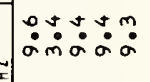 & 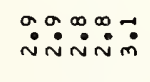 & 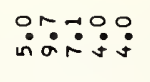 & 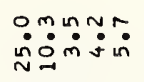 & 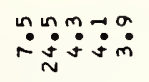 & 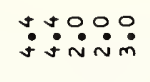 & $\dot{m} \dot{\sim} \dot{N} \dot{N} \dot{N}$ & 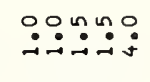 & 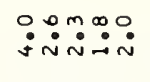 & 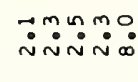 \\
\hline 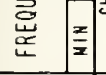 & 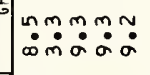 & $\begin{array}{l}\infty \sim \sim \sim 0 \\
\dot{N} \dot{N} \dot{m}\end{array}$ & 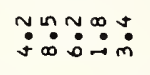 & $\dot{\sim} \underset{\sim}{\dot{N} \dot{\sim} \dot{m}}$ & $\ddot{n} \ddot{\sim} \dot{\sim} \dot{j} \dot{m} \dot{m}$ & บ: & $\ddot{\sim} \dot{\sim} \dot{\sim} \dot{N} \dot{N}$ & 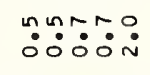 & 讪: & 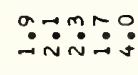 \\
\hline ONIY & 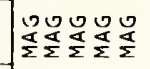 & 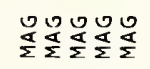 & 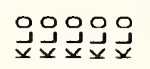 & 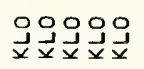 & 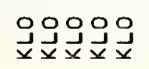 & 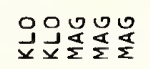 & 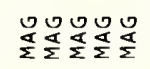 & 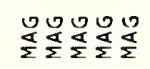 & 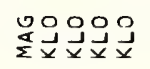 & 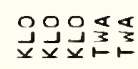 \\
\hline T08WLS & & & & & & & & & & \\
\hline 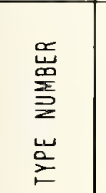 & 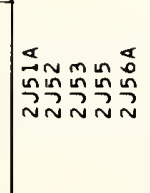 & 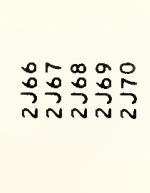 & $\begin{array}{l}\underset{N}{\sim} \underset{\sim}{\sim} \underset{\sim}{\sim} \underset{\sim}{\sim} \underset{\sim}{\sim} \\
\underset{\sim}{\sim} \underset{\sim}{\sim} \underset{\sim}{\sim}\end{array}$ & 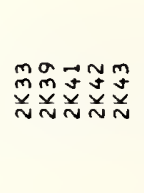 & 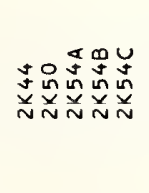 & 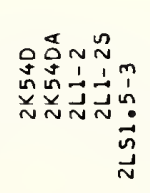 & 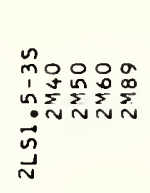 & 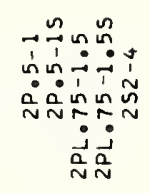 & 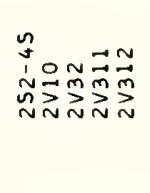 & 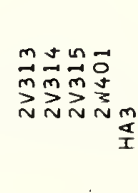 \\
\hline
\end{tabular}




\begin{tabular}{|c|c|c|c|c|c|c|c|c|c|c|}
\hline 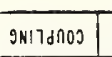 & 무유용 & 풍엉 & 엉ㅇㅇㅇ & 엉워 & OOOOOO & 엉어오 & OOOOOY & 엉ㅇㅇ & 엉ㅁㅇ & पण \\
\hline LL1AYS & $\vec{\sigma} \vec{\alpha}$ & $\vec{m} m \vec{m} m$ & 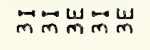 & 萹菂 & & $\vec{\alpha}$ & & $\vec{\alpha}$ & & \\
\hline $\begin{array}{c}0731 \pm \\
\text { J13\%9YK }\end{array}$ & $\Sigma \Sigma$ & $\Sigma$ & & $\Sigma \quad \Sigma$ & $\sum \sum \sum \sum$ & 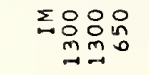 & 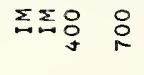 & 蕰 & 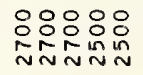 & 品: \\
\hline H10IMONYP & $\stackrel{-}{-}$ & & & & & $\stackrel{\text { n }}{\sim}$ & & & & \\
\hline 9M17ากd & 풀 & & & & & & & $\cong$ & 뜸ำ음 & 으으으오 \\
\hline $\begin{array}{l}801983 \\
35104\end{array}$ & 으 & $\stackrel{0}{-1}$ & & & & 0 & $\stackrel{m}{\sim} \stackrel{n}{\sim}$ & & & \\
\hline mited & $m i n$ & 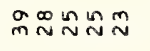 & $\stackrel{m}{\sim}$ & $m i$ & & 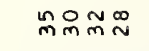 & $\stackrel{\infty}{m}$ 욤요 & 25 & & \\
\hline $\begin{array}{l}1701 \\
\times 173 \mathrm{H}\end{array}$ & $\underset{\forall}{\forall}$ & $\stackrel{x}{\rightarrow}$ & & & & 并芯品品 & $\underset{m}{ } \underset{m}{ }$ & $\underset{m}{\because}$ & & \\
\hline $\begin{array}{c}51701 \\
1081403=\end{array}=$ & 并 & & $\stackrel{x}{\Perp}$ & 음윰 & $\stackrel{\circ}{\circ}$ & $\stackrel{i}{\sim}$ & 요 오욨 & m & & \\
\hline 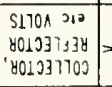 & : & $\stackrel{\circ}{\cong}$ & & & & 용요 & 욤ㅇㅇㅛ & 용욤ㅇㅁㅇ & & \\
\hline$\alpha^{\circ}$ & 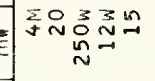 & $\stackrel{\sim}{\sim} \underset{\exists}{x} \underset{\sim}{\mathrm{m}} \underset{\mathrm{N}}{\mathrm{N}}$ & 芯前㒸若兑 & 前品品点 & 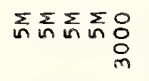 & 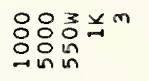 & 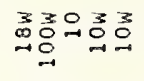 & 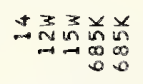 & 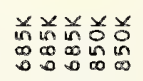 & 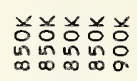 \\
\hline$\omega^{*}$ & Ex & 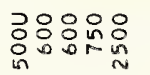 & 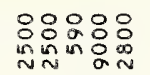 & 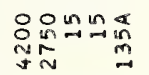 & 点出芯吕n & 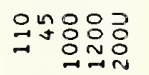 & 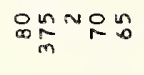 & mo암ㅇㅁㅇㅁㅇ & 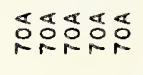 & 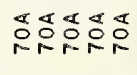 \\
\hline 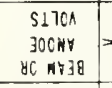 & 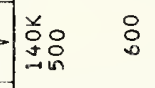 & 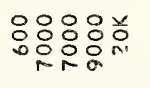 & 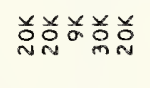 & 总前哭照 & 并前总并品 & 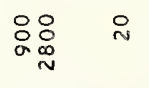 & 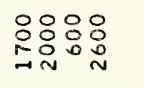 & 品怘 喕喃 & 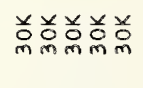 & 僱总总品 \\
\hline 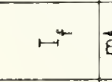 & 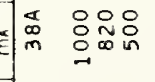 & 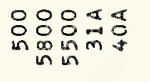 & 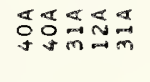 & 品乔品品哭 & 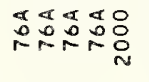 & 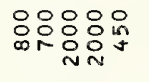 & 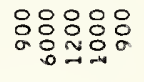 & 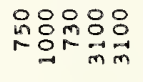 & $\begin{array}{l}88: 80 \\
\text { mलmmm }\end{array}$ & 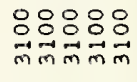 \\
\hline$w^{ \pm}$ & 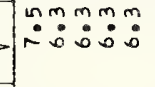 & Muño: & $\because: 0: 0: 0$ & $\ddot{\sim} \ddot{n} \dot{m} \dot{0}$ & 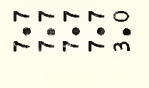 & mmmmo & mmmm & 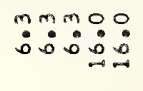 & ن & $\because 0: 0$ \\
\hline $\mathrm{NO} \perp \angle 84730$ & auvuu & yบuบy & yuuva & auvua. & aaau & uvau & บบuบ & Uuva & $a a a a$ & \\
\hline$y+N$ & 吃う占占 & 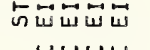 & 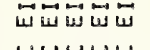 & யี山ี & 안안안안 $\frac{\alpha}{x}$ & 은온안안류 & 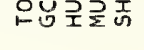 & 岗㟧らðð & 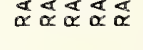 & $\underset{\alpha x}{\mathscr{x}}$ \\
\hline JNก1 & 崖岇5 & 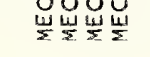 & 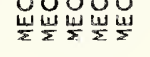 & 岀岕ら5岀 & 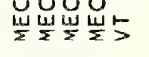 & $\stackrel{\breve{w}}{\Sigma}$ & 55 & 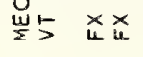 & 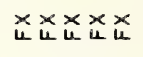 & $\begin{array}{l}x \times x \\
u k u\end{array}$ \\
\hline $018 \forall+\times 100=$ & 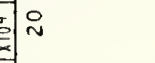 & & : & $\therefore$ & 오음어음 & $ㅇ ㅜ$ & & 어윽 & 으응ㅇㅇㅇㄱㅇㅡ & \\
\hline 咅 & 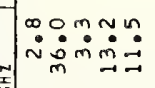 & 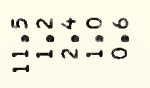 & $\because \because \because 000$ & 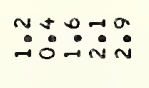 & 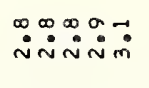 & 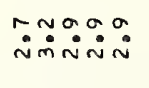 & 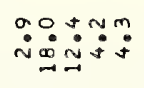 & 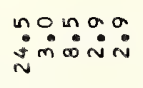 & $\begin{array}{l}\infty \\
\dot{\sim} \dot{\sim} \dot{\sim} \dot{m}\end{array}$ & ஜோஷ் \\
\hline 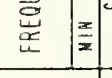 & $\ddot{\sim} \dot{\sim} \dot{\sim} \dot{0} \dot{\sim}$ & 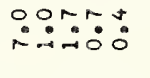 & $\because \because: m: 0$ & 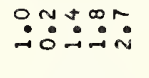 & 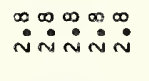 & 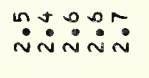 & marm & 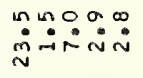 & 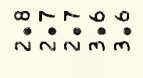 & $\ddot{m} \dot{m} \dot{m} \dot{m}$ \\
\hline ONIX & 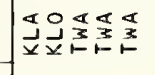 & 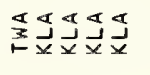 & & 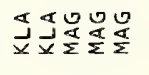 & 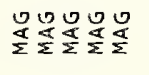 & 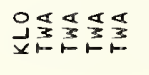 & 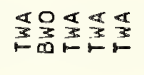 & 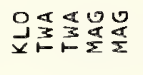 & 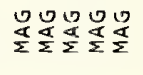 & $\frac{d}{2} \frac{1}{2}$ \\
\hline $708 \mathrm{~W} / \mathrm{SS}$ & & & & & & & & & & \\
\hline 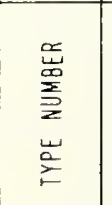 & 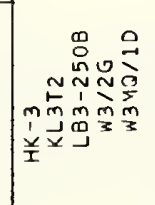 & 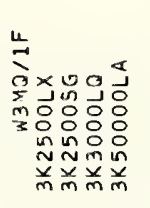 & 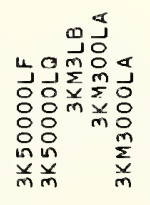 & 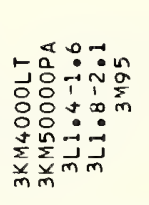 & 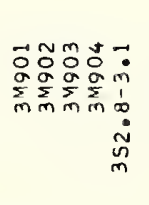 & 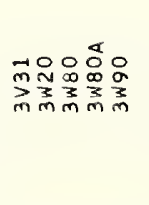 & 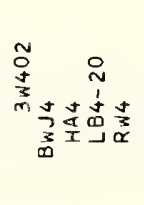 & 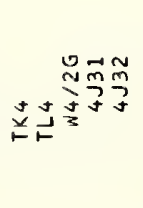 & 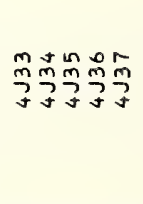 & 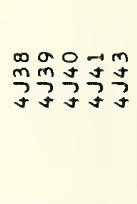 \\
\hline
\end{tabular}




\begin{tabular}{|c|c|c|c|c|c|c|c|c|c|c|}
\hline 9พ17d & QOु & 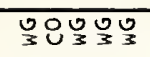 & 우엉어 & O요요 & יভ엉 & 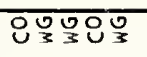 & ייㅇㅇㅇ & יㅓㅇ엉 & ত্ৰওיত & ত্তৃত্য \\
\hline 1114rs & & & & $F \overrightarrow{5}$ & テすデす & 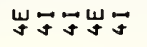 & $\underset{\mho}{U 山}$ & 山す山岁岁 & 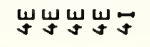 & 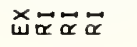 \\
\hline $\begin{array}{c}01314 \\
\text { ग13k9YM }\end{array}$ & 国 & $\sum_{\underset{N}{n}} \underset{n}{n} \Sigma \Sigma \Sigma \Sigma$ & $\sum \Sigma \Sigma \Sigma \Sigma$ & $\Sigma \Sigma \sum \Sigma$ & $\sum \sum \sum$ & & & & & \\
\hline H1010Now日 & & & & $m r \infty$ & 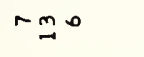 & $m \simeq \approx \infty N$ & $\infty \infty r r$ & $\ln v \min r$ & 으 & $\stackrel{\circ}{m}$ \\
\hline 9 9אורוา & $\simeq \cong$ & 요 & 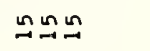 & $\cong$ & q & & q & & 웅ㅇㅇㅇ & \\
\hline 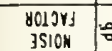 & & & & & & & & & & \\
\hline NiYg & : & & & nngs & 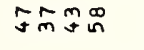 & m웡ㅇㅇㅁㅇㅢ & 일 일요 & 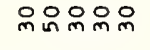 & fn & $\stackrel{n}{m}$ \\
\hline $\begin{array}{l}51701 \\
\times 173 H \\
\end{array}$ & & & & & & & & & & 并 \\
\hline $\begin{array}{c}51701 \\
1081400\end{array}=$ & & & & & 总 & & 웅 & 음음음 & 号 & \\
\hline 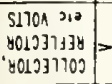 & & & & & & & & & & 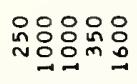 \\
\hline$a^{\circ}$ & 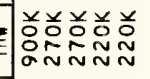 & 居总总总总 & 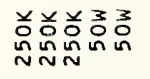 & 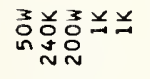 & 证并兰 & 뭉ㅁㅁ웜ㅇ & 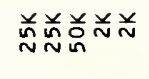 & 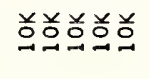 & 并总总总兑 & 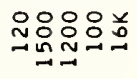 \\
\hline$=$ & 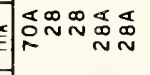 & 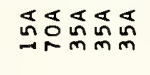 & 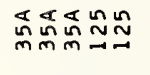 & 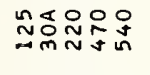 & 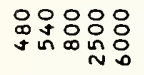 & 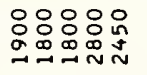 & 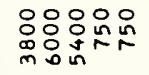 & 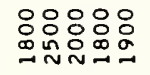 & 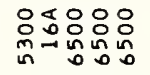 & 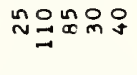 \\
\hline 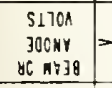 & 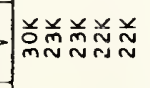 & 苛兑芯芯芯 & 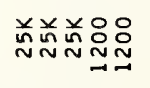 & 总㒸品品: & 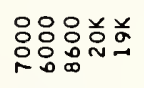 & 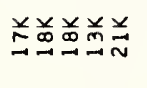 & 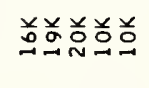 & 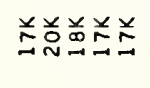 & 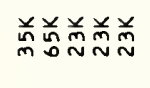 & 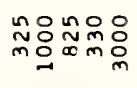 \\
\hline- & | & 总总总点员员品 & 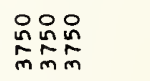 & 品品品品 & 总品品顿 & 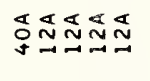 & 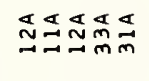 & 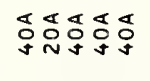 & 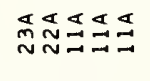 & 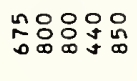 \\
\hline " & 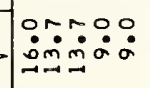 & 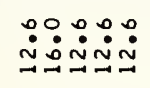 & & $\ddot{m}$ & : & 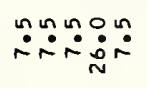 & $\begin{array}{l}: \\
\dot{0} \\
\sim\end{array}$ & 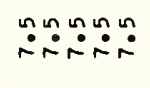 & 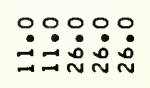 & m: \\
\hline $\mathrm{NO} 117 \mathrm{Y} 3 \mathrm{dO}$ & aana & 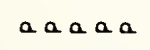 & anauv & vauuv & טuטu & טưư & טuטu & טuטu & vauuv & ưư \\
\hline yנn & 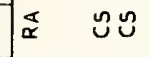 & 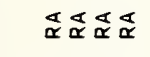 & $\underset{\alpha \propto \alpha \underset{\alpha}{\alpha} \underset{\alpha}{\alpha} \underset{\alpha}{\alpha}}{\alpha}$ & 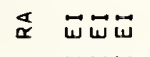 & யேயயேயら & $\vec{w} \tilde{w} \bar{w} \tilde{w} \vec{w}$ & ひらでで & 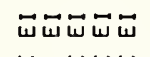 & ゴてらららら & 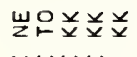 \\
\hline 3Nח1 & $\begin{array}{l}x \times x \\
\text { Lutu. }\end{array}$ & x $x \times x \times x$ & 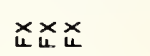 & 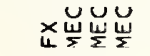 & 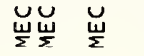 & 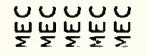 & 岀 $\frac{w}{\Sigma} \frac{w}{\Sigma} \frac{w}{\Sigma}$ & 岀 岀岀岕 & 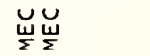 & 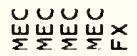 \\
\hline $0118 y+1 \cap 0=$ & 웅ㅇㅁ워으으 & 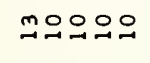 & 우움으 & $\stackrel{\circ}{N}$ & & & & & 요 & \\
\hline 咅 & 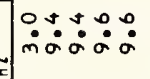 & 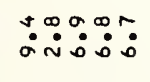 & 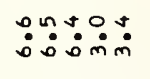 & 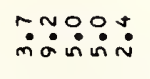 & 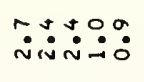 & 足: & 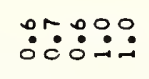 & $\ddot{0}: \ddot{0}: \circ:$ & $\because: 0: 0:$ & 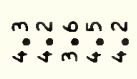 \\
\hline 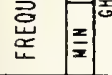 & 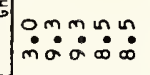 & 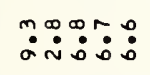 & 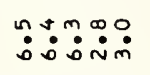 & mo: & 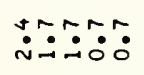 & ப̊: & 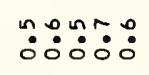 & $\dot{0}: 0: 00$ & 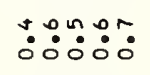 & $\dot{m} \dot{m} \dot{m} \dot{m} \dot{m} \dot{m}$ \\
\hline ONIX & 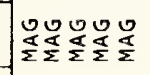 & 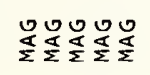 & 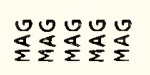 & 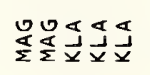 & 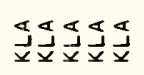 & 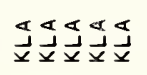 & 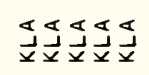 & 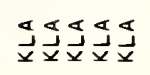 & 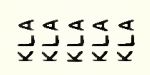 & 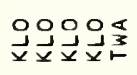 \\
\hline 708W/S & & " " " & " " " & " " " & & " " & & & & \\
\hline 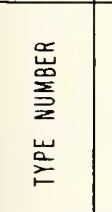 & 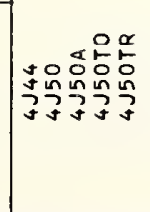 & 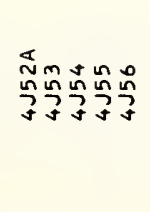 & 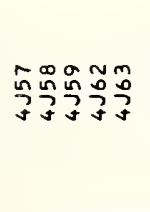 & 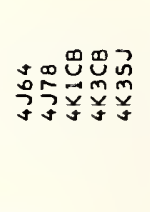 & 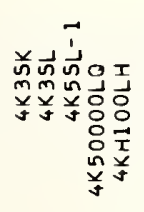 & 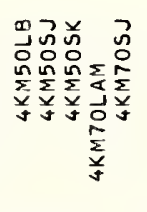 & 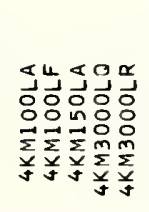 & 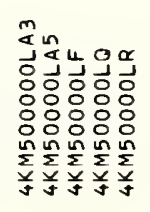 & 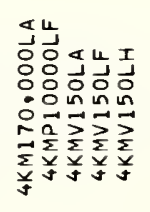 & 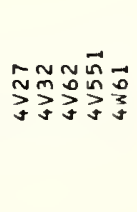 \\
\hline
\end{tabular}




\begin{tabular}{|c|c|c|c|c|c|c|c|c|c|c|}
\hline 9NITdNOS & 윯원요 & UO & 욱ㅇㅇㅇㅇ & 엉원운 & OO & 언언언 & 엉웡ㅇㅇ & 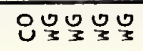 & OY & 언언엉 \\
\hline dilars & & & & ถึ心 & 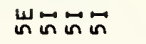 & & $\vec{\alpha} \tilde{\alpha}$ & $\vec{\alpha} \vec{\alpha} \vec{\alpha} \vec{\alpha}$ & & $\vec{\alpha}$ \\
\hline \begin{tabular}{c|c|c|}
01311 \\
ग13N9\%
\end{tabular} & $\sum \sum \sum_{j} \sum_{-1}$ & $\Sigma$ & $\sum \sum \Sigma$ & : & $\Sigma$ & & & & $\Sigma$ & $\Sigma \quad$ in \\
\hline H101monrg & $\approx$ & & & $\stackrel{\text { S }}{\sim}$ & 욱우옥 & & & & & 品 \\
\hline - & $\cong$ & $\cong$ & $\cong$ & & & & & & & \\
\hline $\begin{array}{l}\text { Holjof } \\
\text { JSION }\end{array}$ & $=$ & 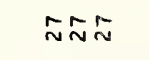 & 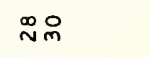 & & & & & & 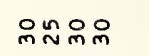 & 요 \\
\hline$\frac{130 \mathrm{~N}}{\mathrm{NIYO}}=$ & : $\ln _{m}$ 品 & ำกำ的品 & $\underset{f}{q}$ & 요 & 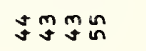 & & & 요 & 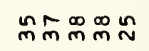 & $\prod_{m}^{\infty}$ \\
\hline $\begin{array}{l}5170 \Lambda \\
\times 173 \mathrm{H}\end{array}=$ & $\Rightarrow \rightarrow$ m & 弚羊羊前 & 并鬲 & & & & & 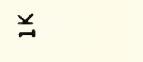 & 并前前羊兰 & $\ddot{\sim}$ \\
\hline $\begin{array}{c}51701 \\
7041 \mathrm{NOS}\end{array}=$ & in & 음임 & 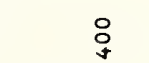 & 方 & & : & 잉요 & & 일 & $\stackrel{0}{n}$ \\
\hline 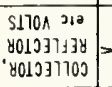 & 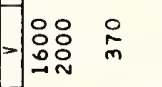 & 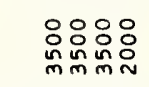 & 욤요 & 웅 & & 品 & 용 范 & 吠品品品品 & 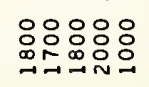 & 음윰윰 \\
\hline$a^{\circ}$ & Ex & 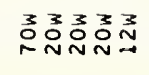 & 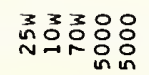 & 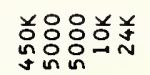 & 点弟兑兑总 & 응용요 & 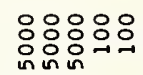 & 융윰윰유유 & 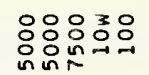 & 용요 \\
\hline$\mapsto$ & E⿱ & 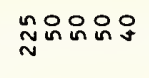 & 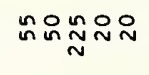 & ్ㅠㅅㅠ: & 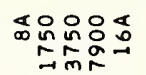 & 움요 & N N유욤요 & 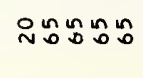 & 웅웡ㅇㅇㅇㅛ & 요운ㅉำ \\
\hline $\begin{array}{c}51700 \\
300 \mathrm{NY} \\
86 \mathrm{NY} \rightarrow 3 \mathrm{~B}\end{array}$ & $>$ > & 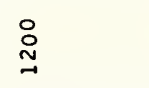 & 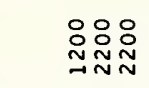 & 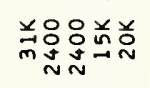 & 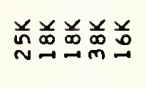 & 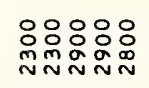 & 吠品品品 & 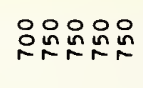 & 응융유 & 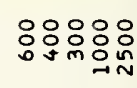 \\
\hline$H^{*}$ & 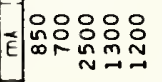 & 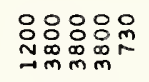 & 옷윰윰유 & 윰요 & 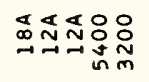 & 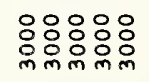 & 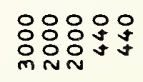 & 임이 & 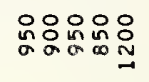 & 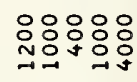 \\
\hline L5 & - & m:o: & 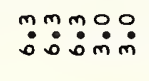 & 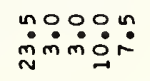 & 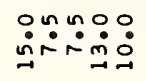 & 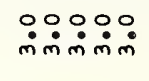 & $\ddot{m} \dot{m} \dot{m} \dot{m} \dot{m} \dot{m}$ & 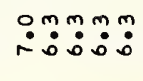 & 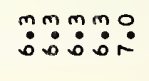 & $\ddot{n}$ \\
\hline 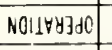 & auטa & auuvu & טuם & auvuv & םטuט & טuטu & บบบบy & טuטu & טuטu & auvuu \\
\hline$\overline{y j n}$ & × & 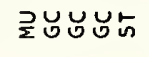 & ららつつ & $\stackrel{I}{a} \frac{\alpha}{\Sigma} \frac{\alpha}{\Sigma} \mathfrak{w} \vec{w}$ & 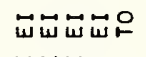 & $\frac{\alpha}{\Sigma} \frac{\alpha}{\Sigma} \frac{\alpha}{\Sigma} \frac{\alpha}{\Sigma} \frac{\alpha}{\Sigma}$ & $\frac{\alpha}{\Sigma} \frac{\alpha}{\Sigma} \frac{\alpha}{\Sigma} \not{x} \underline{x}$ & 고글로롤로 & 골로롤로고 & 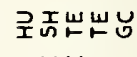 \\
\hline$\frac{3 \mathrm{~N} \cap 1}{0118 \mathrm{Y} \text { AIro) }}$ & 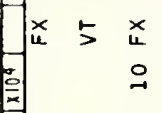 & $\begin{array}{l}x \\
u \\
0\end{array}$ & 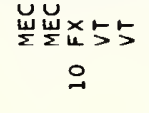 & 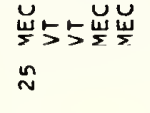 & 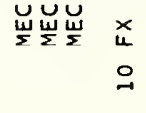 & ケらケケケ & ケケケ岕岕 & 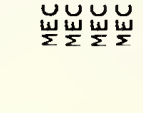 & 5555 & 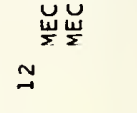 \\
\hline 离 & $\mid \begin{array}{l}0 \\
\dot{n}\end{array}$ & 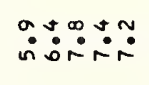 & 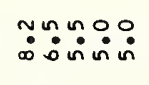 & 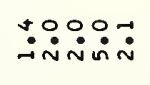 & 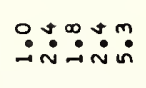 & 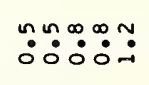 & 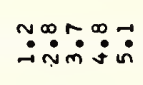 & 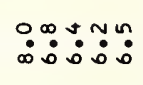 & 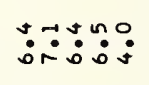 & 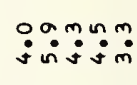 \\
\hline 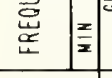 & 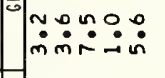 & 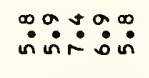 & 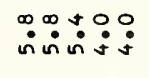 & ペ: & 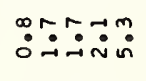 & $\ddot{0}: \because: 00$ & $\ddot{\circ} \dot{\sim} \dot{\sim} \dot{q}: \dot{q}$ & 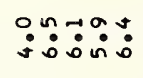 & 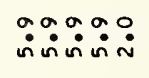 & ஸ்: \\
\hline ONIX & 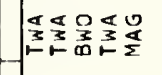 & 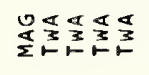 & 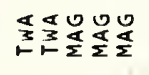 & 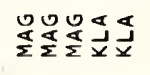 & 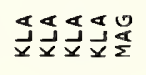 & 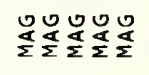 & 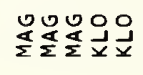 & 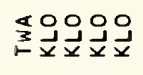 & 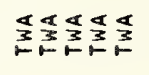 & 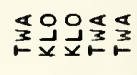 \\
\hline 70gwis & & & & & & & & & & \\
\hline 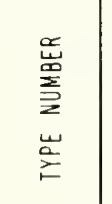 & 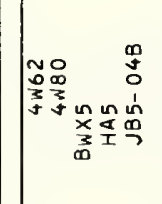 & 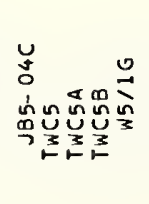 & 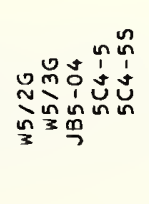 & 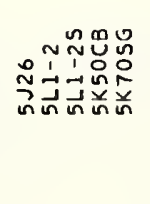 & 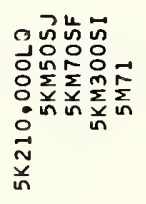 & 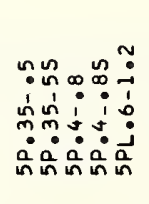 & 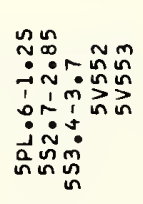 & 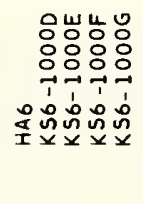 & 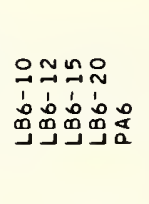 & 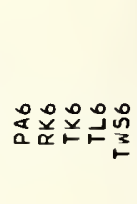 \\
\hline
\end{tabular}




\begin{tabular}{|c|c|c|c|c|c|c|c|c|c|c|}
\hline 9N17dnOS & $\frac{O N}{3}$ & 엉ํㅛ & 묵요 & OOW & OOW & Wणु & 엉요 & 뭎ㅇㅇ & 언모 & 엉요 \\
\hline 1L1AY3 & 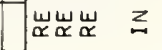 & ż心证 & z즈즌 & zzzzz心 & $\vec{\sim} \vec{\alpha} \vec{\alpha} \vec{\alpha} \vec{\alpha}$ & $\vec{\alpha} \vec{\alpha}$ & $\vec{\alpha} \vec{\alpha} \vec{\alpha}$ & $\vec{\alpha} \vec{\alpha} \quad \vec{\alpha} \vec{\alpha}$ & $\vec{\alpha} \vec{\alpha}$ & $\vec{\forall} \vec{\alpha} z z$ \\
\hline $\begin{array}{c}01311 \\
\text { J113M9YK }\end{array}$ & 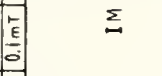 & & & & & ㅍㅇㅇㅇㅠ & 응 & & ${ }_{i n}=$ & $\Sigma$ \\
\hline RLOIMONYB & In & 证品品品 & 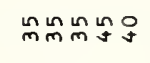 & 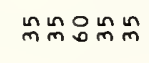 & 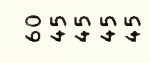 & $\mathfrak{s}\{$ & & 요 & 유 & $\stackrel{n}{m} \tilde{n}$ \\
\hline 9אורח & & & & & & & & & & \\
\hline $\begin{array}{c}8019 y+1 \\
\text { Jsion }\end{array}$ & is & & & & & & 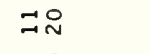 & i & $\stackrel{\circ}{m}$ & \\
\hline nirg & 圈 & $\stackrel{\circ}{n}$ & & & & 욨 & $\stackrel{n}{\sim}$ & $\stackrel{m}{m}$ & 욤 & $\stackrel{9}{9}$ \\
\hline $\begin{array}{l}5170 A \\
\times 1734 \\
\end{array}$ & & $\stackrel{N}{N}$ & & & & 弟兰 & 욤 & 并 & 芯首前 & 苾 \\
\hline $\begin{array}{c}51701 \\
1081402\end{array}$ & & & & & & o in & in & 品 趷 & 品品 并并 & 品 \\
\hline 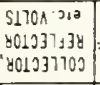 & Nㅗㅇㅇㅛ & 숬욤요 & 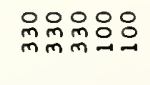 & 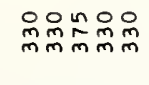 & 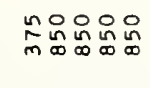 & 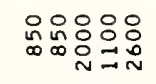 & 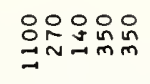 & 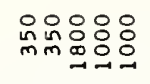 & 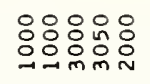 & 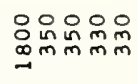 \\
\hline $0^{\circ}$ & Honn & 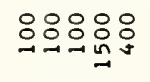 & 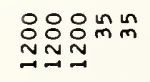 & 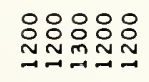 & 양요 & 용용유 & No욤요 & 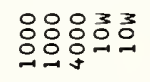 & 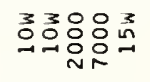 & 윰엄요 \\
\hline$\rightarrow$ & 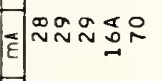 & 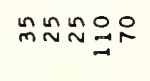 & 웃유N & 우순담오 & 늨읔읔 & 읔웍요 & 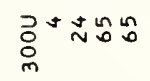 & 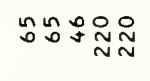 & 윳요 & 을음웃 \\
\hline $\begin{array}{c}51701 \\
300 \mathrm{HY} \\
80 \mathrm{Ki}: 38\end{array}$ & 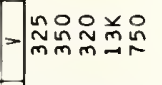 & 있윰윰요 & 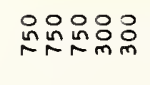 & 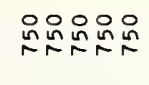 & 읏응영ㅇㅇㅇ & 음옹요요 & 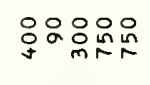 & 웃용요 & 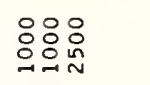 & 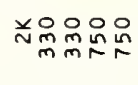 \\
\hline$\leftarrow$ & E| & 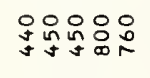 & 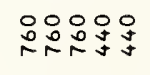 & 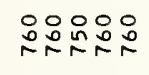 & 숫요 용요 & 양용요 & 잉유. & 잉용유유 & 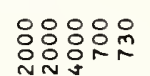 & 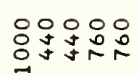 \\
\hline แั & mm:0" & : & 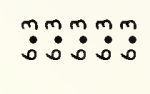 & 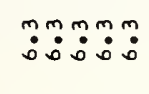 & 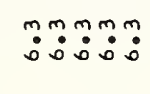 & : & : & : & 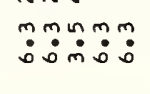 & 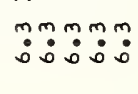 \\
\hline $\mathrm{N} 011 \forall \mathrm{y}] \mathrm{dO}$ & $\operatorname{aga} a$ & 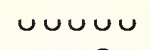 & טuטu & טレンט & uบuบu & טuטu & 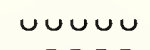 & טบטu & 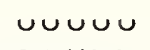 & טu৩u \\
\hline$y \mathrm{y}$ & 운 & 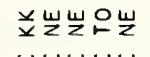 & 岂岀岀崖宸 & 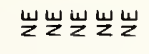 & 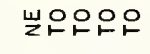 & 안안르로포 & 고고롤로를 & 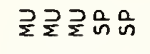 & 於的占 & ら立立岂岩 \\
\hline JNก1 & Hư & 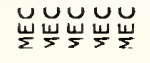 & UU⿺乚一匕U & 岀岀UU⿺乚一匕 & U⿺辶ّ山ّ & 岀岁 & 岀岀嵌 & 岀岕っ岀岀 & 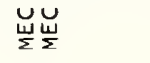 & 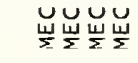 \\
\hline Ol15y AIro & $\stackrel{\circ}{\circ}$ & & & & & & & & & \\
\hline 恖 & 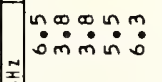 & فํ: & 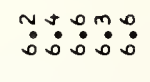 & : & : & 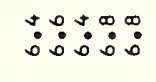 & 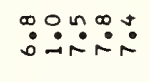 & $\ddot{\therefore} \therefore$ & 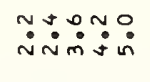 & $\because \sim \infty: a$ \\
\hline \begin{tabular}{|l|l|}
$\underset{\underline{x}}{\overrightarrow{\underline{x}}}$ & $\frac{z}{x}$ \\
\end{tabular} & $\ddot{0}: 00$ : & 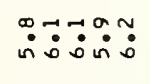 & ڤُ & 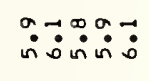 & 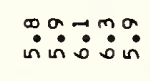 & 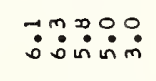 & $\ddot{n}$ & 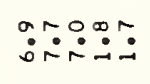 & $\ddot{\dot{N}} \dot{\sim} \dot{\sim} \dot{m} \dot{m}$ & 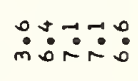 \\
\hline ONIX & $\mid \begin{array}{l}0 \\
\vec{x} \\
\vec{x} \\
\end{array}$ & 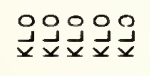 & 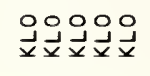 & 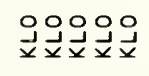 & 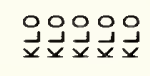 & 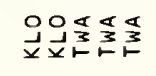 & 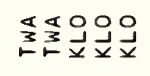 & 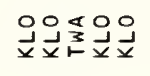 & 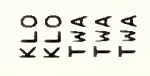 & 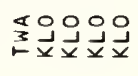 \\
\hline $708 \mathrm{~N} / \mathrm{S}$ & & & & & & & & & & \\
\hline 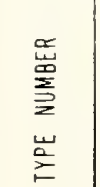 & 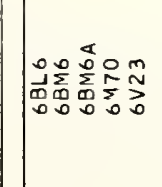 & 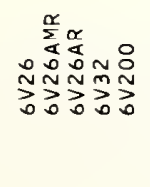 & 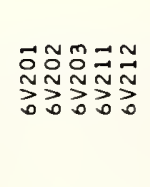 & 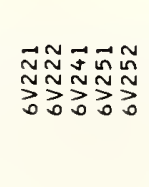 & 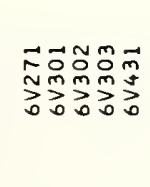 & 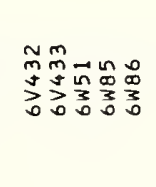 & 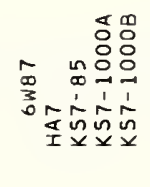 & 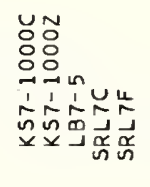 & 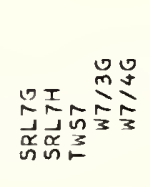 & 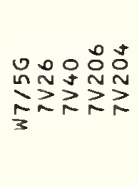 \\
\hline
\end{tabular}




\begin{tabular}{|c|c|c|c|c|c|c|c|c|c|c|}
\hline 2HITd d OOS & U్३ & 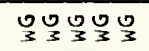 & Wִ & Wִ & 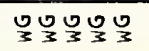 & 옹్ㅀ & WO & ডড়엉요 & 언워놇 & Wִ \\
\hline LIIAYS & $\vec{\alpha} \vec{\alpha} \geq z z$ & $z z z z z$ & 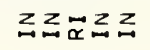 & $z z \vec{\alpha} \ddot{\alpha} \vec{\alpha}$ & $\vec{\alpha} \vec{\alpha} \vec{\alpha}$ & & & $\ddot{\alpha}$ & 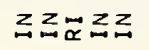 & $z \geq z \geq z$ \\
\hline \begin{tabular}{l|l|}
01311 \\
J113\%9VM
\end{tabular} & & & & & $\Sigma$ & 足沙莡沙 & $\sum \sum \sum \sum \sum \sum$ & $\sum \stackrel{0}{0}$ & & \\
\hline HLOIMOHYY $\tilde{\underline{\mathbf{x}}}$ & ingog & gognnm nn & ñ & 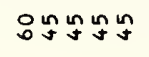 & ใู่ & & $\tilde{m}$ & $\tilde{N}$ & 우 윳 & 임요묘 \\
\hline 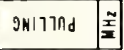 & & & & & & $\simeq \cong$ & 요요 & $\stackrel{\sim}{N}$ & & \\
\hline 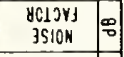 & & & & & & & & 욤 & & \\
\hline Mirs $:$ & & & & & $\stackrel{\sim}{\sim} \underset{m}{\infty}$ & 롱요 & & $\stackrel{\infty}{m}$ & & \\
\hline $\begin{array}{r}51701 \\
\times 1173 \mathrm{H} \\
\end{array}$ & & & & & 吕前 & 希莳品 & & $\stackrel{*}{*}$ & & \\
\hline $\begin{array}{c}51701 \\
108 \mathrm{H} \text { HOS }\end{array}$ & & & & & $\stackrel{\sim}{N}$ & 유윰 & & $\stackrel{\circ}{\sim}$ & & \\
\hline 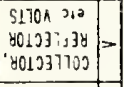 & 음임요 & 음있일요 & 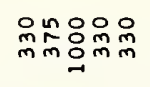 & 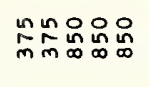 & 品员品路品 & 送品 & & 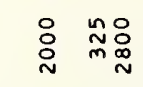 & 욤요 & 울율욜 \\
\hline$a^{\circ}=$ & 음ㅇㅇㅇㅇㅁ & 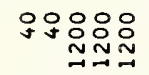 & 옹임욤윰워 & 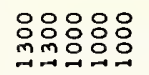 & 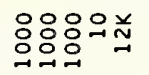 & 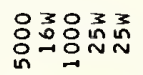 & 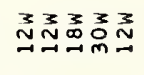 & 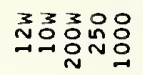 & ஃ:음을 & 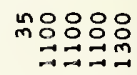 \\
\hline$\omega^{x} \mid \overrightarrow{\mathrm{E}}$ & 몽NNN & mariR우 & 움슴웅 & 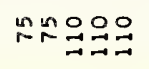 & 윽육요 & 음은용욤요 & 음요욤요 & 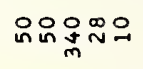 & Nㅡㅁ우우 & mo우움 \\
\hline $\begin{array}{c}S 170 \mathrm{~N} \\
300 \mathrm{NY} \\
86 \mathrm{Ar} 3 \mathrm{BO}\end{array}$ & 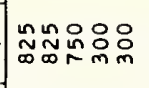 & 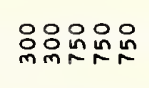 & 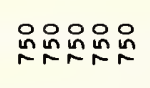 & 은윰응ㅇㅇㅇㅇㅇ & 응응용융ㅁㅇ & 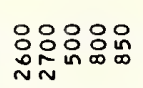 & 영ㅇㅇㅇㅇㅇㅇ & 염욤요 & 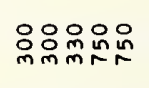 & 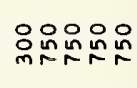 \\
\hline$F$ & 员员品昌卞 & 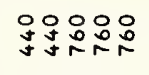 & 음용요 & 욧용용요 & 임윰요 & 음욤윰워 & 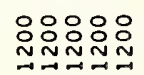 & 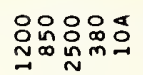 & 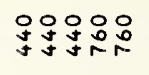 & 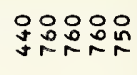 \\
\hline$w^{t}$ & mamm: & 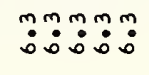 & 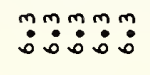 & : & 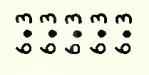 & : & 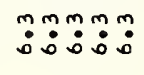 & 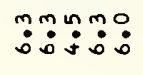 & $\ddot{m}: \mathfrak{m}: m \mathfrak{m}: m$ & 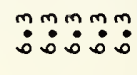 \\
\hline NOIIVHJdO & טטuט| & טuטu & טטטu & טיטu & טטuט & טưa & טaםu & טטuט & טיטטי & טuטu \\
\hline$\forall \mathrm{HN}$ & 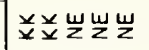 & 岂岂岂岂岂 & 岁 $\quad \frac{w}{z}$ & 뜰얀요 & 옴옹ㅇㅁㅈㅏ & 롱ㅇㄱ고롤골 & 굴굴골굴골 & 골곪ㄸㄴㅂㅓ & 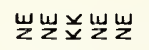 & 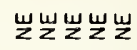 \\
\hline \begin{tabular}{l|l} 
JNกI \\
\end{tabular} & 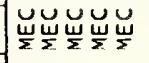 & 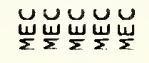 & 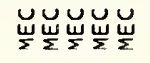 & 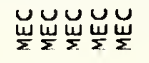 & 岀岕岕 $\underset{u}{ }$ & $x_{4}^{x}$ & 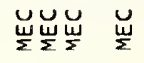 & 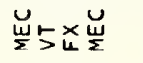 & 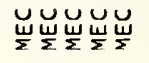 & 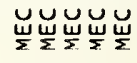 \\
\hline $011 y+4$ ano & & & & & & $N \tilde{N}$ & 옹 & & & \\
\hline 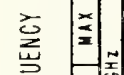 & $\ddot{\because} \because \because \therefore \dot{0}$ & 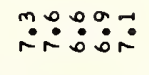 & 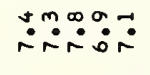 & 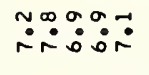 & 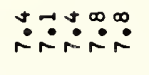 & 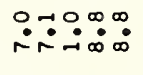 & $\because \because \infty: 0$ : & 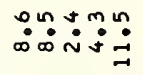 & 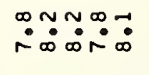 & 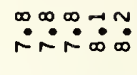 \\
\hline 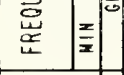 & 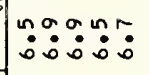 & $\ddot{\sim} \stackrel{m}{\sim}: \stackrel{0}{0}$ & 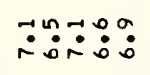 & 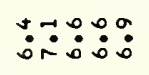 & 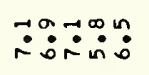 & 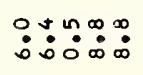 & 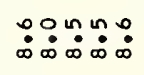 & 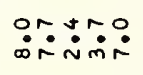 & 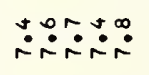 & 華芒芒 \\
\hline ONIX & 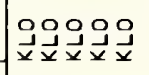 & 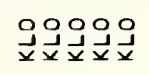 & & 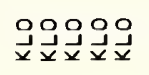 & 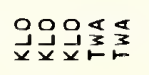 & 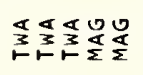 & 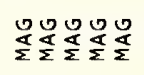 & 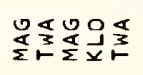 & 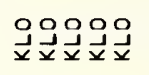 & لُح \\
\hline 109w/S & & & & & & & & & & \\
\hline 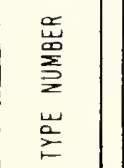 & 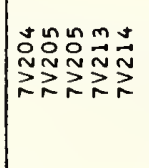 & 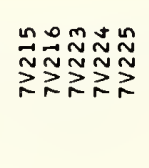 & 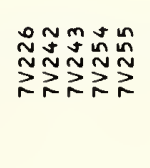 & 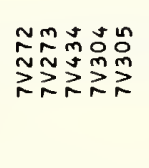 & 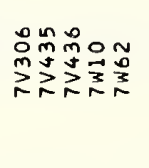 & 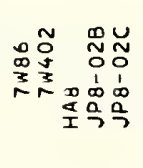 & 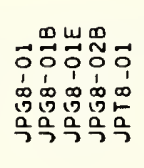 & 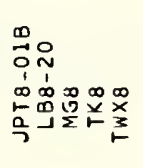 & 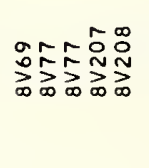 & 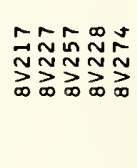 \\
\hline
\end{tabular}




\begin{tabular}{|c|c|c|c|c|c|c|c|c|c|c|}
\hline 9KITd חOS & OOOWO & 야요 & 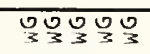 & OWO & WO & 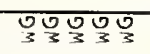 & WO & $\frac{0}{3} 3 \frac{O}{3}$ & TSOS:O & 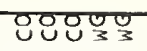 \\
\hline 111nes & $\vec{\alpha} \vec{\alpha} \vec{\alpha}$ & & & & & & & & $\vec{\alpha}$ & $\vec{\alpha} \vec{\alpha} \vec{\alpha} \vec{\alpha} \vec{\alpha}$ \\
\hline $\begin{array}{c}07314 \\
\text { ग13н9ук }\end{array}$ & ह & 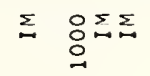 & $\Sigma \Sigma \Sigma \Sigma \Sigma$ & $\sum \sum \sum \sum \sum \sum$ & $\sum \sum \sum \sum \sum$ & $\sum \sum \sum \sum \sum$ & 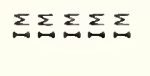 & 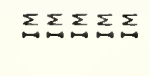 & $\sum \sum \sum \sum$ & \\
\hline HLOMOHYP & 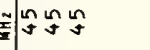 & & & & & & & $\tilde{m}$ & 웅 & 웡웧워 \\
\hline פ & $\underline{\mathbf{s}}$ & $m \sim$ & $\stackrel{\infty}{\sim} \underset{\sim}{ } \stackrel{\infty}{\sim} \underset{\sim}{\sim}$ & 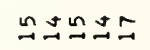 & 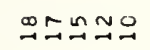 & $\stackrel{\sim}{\sim} \simeq \underset{\sim}{ }$ & さささささ & $\stackrel{\circ}{\sim} \stackrel{\sim}{\sim} \stackrel{0}{N}$ & 음오 & \\
\hline $\begin{array}{c}\text { yelov } \\
\text { JSION }\end{array}$ & & & & & & & & & & \\
\hline Hivo & $q^{m}$ & g $m$ & & & & & & & & \\
\hline $\begin{array}{l}51700 \\
\times 113 \mathrm{H}\end{array}$ & 并 & $\stackrel{*}{*}$ & & & & & & & & \\
\hline $\begin{array}{r}51701 \\
7081400\end{array}=$ & 잉요 & 욤 & & & & & & & & \\
\hline 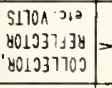 & 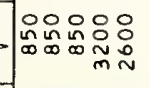 & 욤요 & & & & & & & $\stackrel{0}{0}$ & 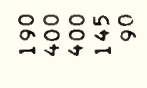 \\
\hline $0^{\circ}$ & 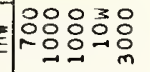 & 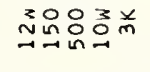 & 弟并并并酋 & 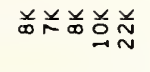 & 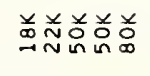 & 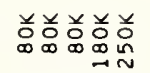 & 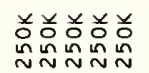 & 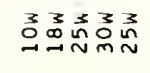 & 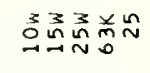 & ํำ $\sim \sim n$ \\
\hline$-x$ & 읔으웄 & ํำn요용 & 응융요윰 & 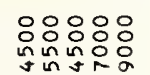 & 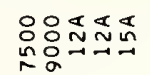 & 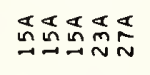 & 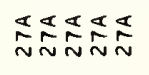 & 용웜워 & 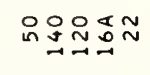 & 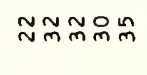 \\
\hline 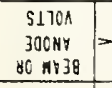 & 믐유슈 & 㧵品品品品 & 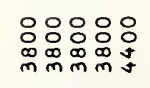 & 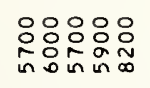 & 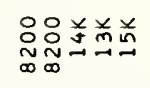 & 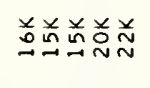 & $\underset{\sim}{\sim} \underset{\sim}{\sim} \underset{N}{N} \stackrel{\times}{N}$ & 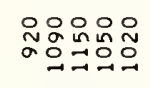 & 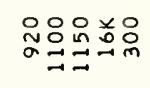 & 임있욤요 \\
\hline-5 & 징요 & 음윰욤요 & 品品品品品 & 응영요 & 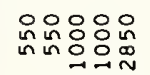 & 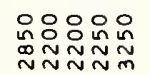 & 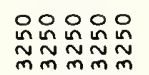 & 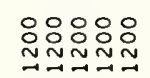 & 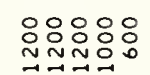 & 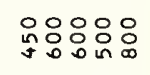 \\
\hline " & 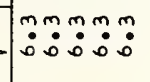 & 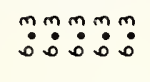 & 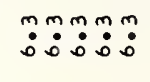 & 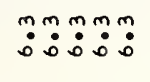 & 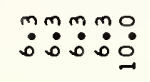 & : & 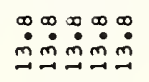 & 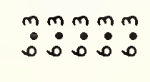 & 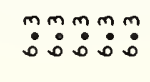 & 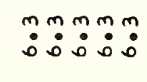 \\
\hline Nol 1 *y? $\mathrm{dO}$ & uטu & 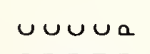 & acaas & anaa & anaag & a a a a & a a a a & vuaaa & vaaau & טuט \\
\hline$y+N$ & 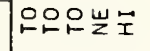 & 은고로 & 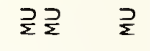 & 고ㄹㅗㅡㅁ골로 & そ २ & ว & 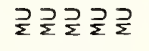 & 当灵专导导 & 골돔글 $\frac{\partial}{\Sigma} \frac{\partial}{\Sigma}$ & 골동몸고 \\
\hline $3 \mathrm{~N} \cap 1$ & 慰岕 & $5 \underset{4}{>}$ & 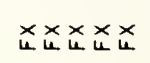 & 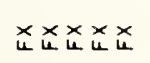 & $\begin{array}{l}x \times x \times x \\
4\end{array}$ & $\begin{array}{l}\times \times \times \times x \\
u\end{array}$ & 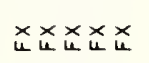 & 岕岕岕岕 & 岀剀比 & 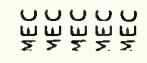 \\
\hline oilyy 사요 & & & NNNNS & 은요 & 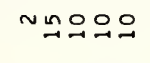 & 으으움으 & 으으으오 & ng음요 & $\mathbb{N}^{n} \Xi$ & \\
\hline 产 & 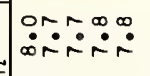 & 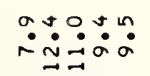 & åå & 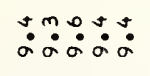 & 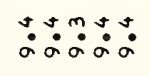 & $\dot{\sigma} \dot{\alpha} \dot{\alpha} \dot{\alpha} \dot{\alpha}$ & $\because \because \therefore: \infty \cdot$ & 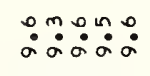 & 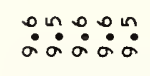 & 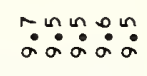 \\
\hline 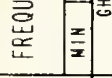 & 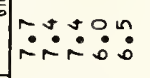 & 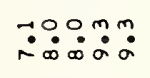 & 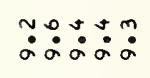 & 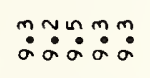 & 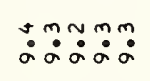 & 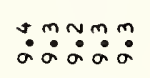 & 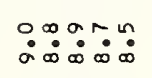 & 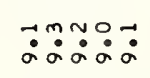 & 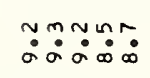 & 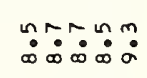 \\
\hline ONIX & 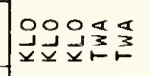 & 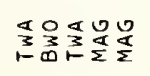 & 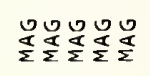 & 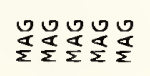 & 造造造造造 & 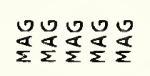 & 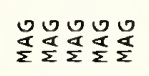 & 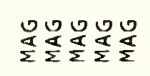 & 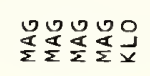 & 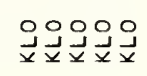 \\
\hline $708 \mathrm{w}$ /S & & & & & & & & & & \\
\hline 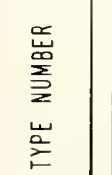 & 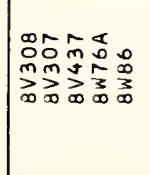 & 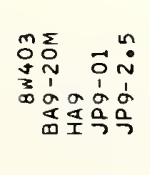 & 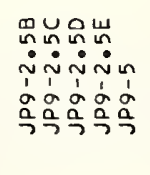 & 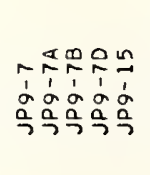 & 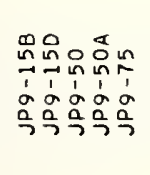 & 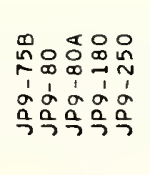 & 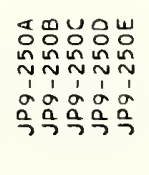 & 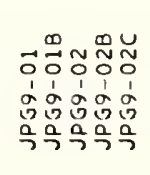 & 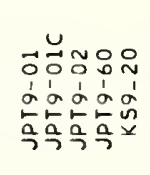 & 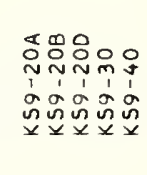 \\
\hline
\end{tabular}




\begin{tabular}{|c|c|c|c|c|c|c|c|c|c|c|}
\hline 9NITdnos & 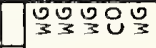 & 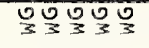 & 38095 & 8800 & 88888 & 88988 & 88980 & $8989 \%$ & 98889 & 3898 \\
\hline 111AY3 & $\vec{\alpha}$ & $\ddot{\alpha} \boldsymbol{\alpha}$ & & & & & & $\Xi$ & $z$ & $\ddot{\sim} z$ \\
\hline 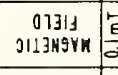 & [- & $\sum_{i=1} \sum_{n=1}$ & 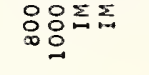 & 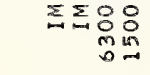 & $\Sigma$ & & & & & \\
\hline H101100HFA & $\sqrt{\bar{m}}=\frac{9}{4}$ & 융ㅇㅁ & & & & & & $\stackrel{6}{0}$ & s & \\
\hline 9Miา7nd & $\stackrel{n}{\sim}$ & $\stackrel{\text { In }}{\rightarrow}$ & m & $\cong$ & & & & & & 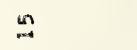 \\
\hline $\begin{array}{c}\text { yolivy } \\
\text { ISIOH }\end{array}$ & 옹 & & 0 & m & & & & & $\cong$ & \\
\hline Mirg & a & & $\tilde{N}$ & 尊 & * & & & & $\stackrel{\sim}{\sim}$ & 。 \\
\hline $\begin{array}{r}5.70 \mathrm{~A} \\
\times 1773 \mathrm{H} \\
\end{array}$ & in & & 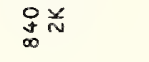 & $\because$ 并 & m & & & & $\stackrel{n}{\sim}$ & \\
\hline $\begin{array}{c}51701 \\
7081403\end{array}=$ & $\stackrel{\circ}{\circ}$ & & in & $\rightarrow \quad 0$ & 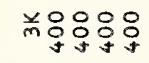 & 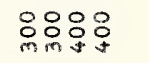 & 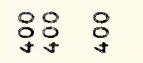 & \begin{tabular}{ll}
80 \\
8 \\
\hdashline \\
9
\end{tabular} & 웅영 & \\
\hline 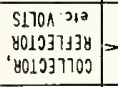 & $=\mid \begin{array}{ll}\infty & 0 \\
\infty & 0 \\
0\end{array}$ & 옹옹 & 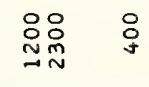 & 응 & : & $\stackrel{8}{9}$ & : & : 8 & $\stackrel{\circ}{\stackrel{n}{a}}$ & \\
\hline $0^{\circ}$ & E & 并兑銪号 & m品爻욤 & 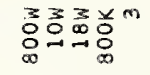 & 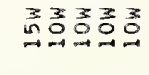 & 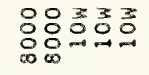 & 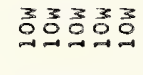 & 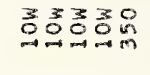 & 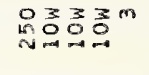 & 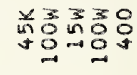 \\
\hline$\varpi^{x}$ & Ex & 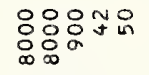 & 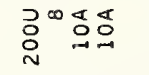 & 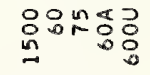 & 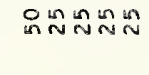 & 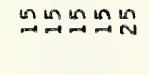 & 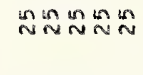 & 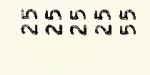 & 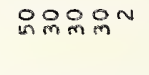 & 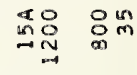 \\
\hline 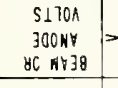 & 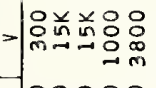 & 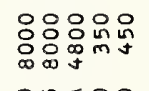 & 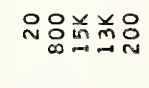 & 옹요 & & 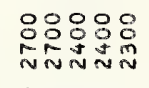 & 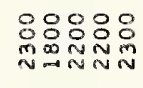 & 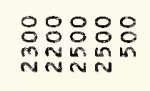 & 음ㅇㅇㅇㅛ & 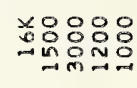 \\
\hline$H^{-}$ & É & 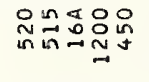 & 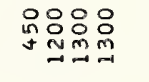 & 总 品 & 요요 & $\begin{array}{l}8: \\
8: 0 \\
0 \% n\end{array}$ & 응요 & 욤요 & 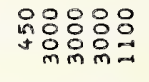 & 总 \\
\hline$w^{5}$ & 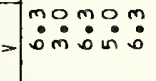 & 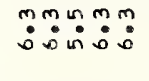 & $\stackrel{0}{\circ}: m m$ & 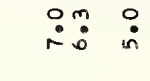 & ஸ:0: & 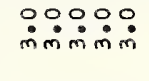 & ஸ் & 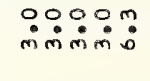 & mo: & $\dot{\sim}$ \\
\hline NO1_by $3 d 0$ & Ua a u & a auv & uva u & a auau & & & & uuuvu & yuuv & a a uau \\
\hline y」N & 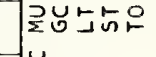 & 은쁠일 & 岂공이 & 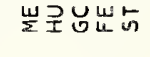 & $\hbar \frac{\hbar}{2} \frac{\alpha}{2} \frac{\alpha}{\Sigma} \frac{\alpha}{\Sigma} \frac{\alpha}{\Sigma}$ & $\frac{\alpha}{\Sigma} \frac{\alpha}{\Sigma} \frac{\alpha}{\Sigma} \frac{\alpha}{\Sigma} \frac{\alpha}{\Sigma}$ & $\frac{\alpha}{\Sigma} \frac{\alpha}{\Sigma} \frac{\alpha}{\Sigma} \frac{\alpha}{\Sigma} \frac{\alpha}{\Sigma}$ & $\frac{\alpha}{\Sigma} \frac{\alpha}{\Sigma} \frac{\alpha}{\Sigma} \frac{\alpha}{\Sigma} \underset{\Sigma}{\Sigma}$ & 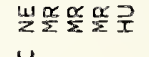 & 犯崖崖崖品 \\
\hline \begin{tabular}{|l|l}
$3 \mathrm{~N} \cap 1$ \\
\end{tabular} & 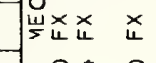 & 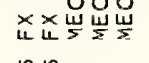 & 肴希 & $\begin{array}{ll}x & x \\
u & \mathbb{u}\end{array}$ & 5555 & らララら5 & 5555 & 5555 & 岀555 & 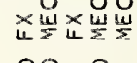 \\
\hline $0118 y$ a Irio $=$ & $\circ 0^{\circ}$ & $\stackrel{n}{N} \mathfrak{n}$ & in m & $\cong \infty$ & & & & & & 요 $\stackrel{i}{2}$ \\
\hline 总 & 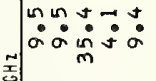 & 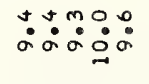 & 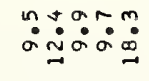 & 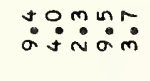 & $\because ّ$ & $\because \because \because \because: 0$ & & & & 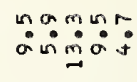 \\
\hline 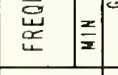 & 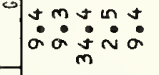 & 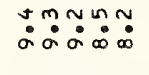 & 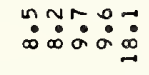 & 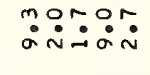 & 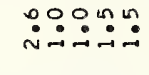 & 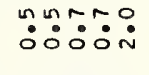 & 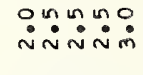 & 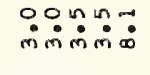 & 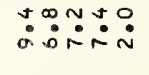 & 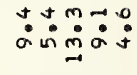 \\
\hline ONIX & 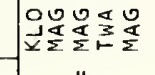 & 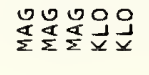 & 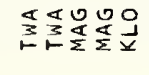 & 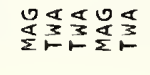 & 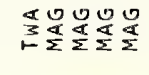 & 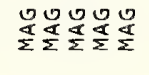 & 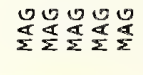 & 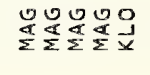 & 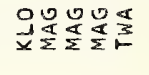 & 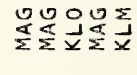 \\
\hline $708 \mathrm{~W} \times \mathrm{S}$ & & & & & & & & & & \\
\hline 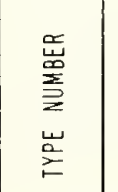 & 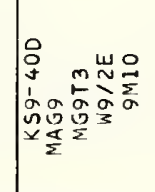 & 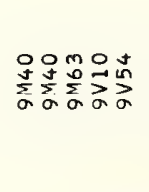 & 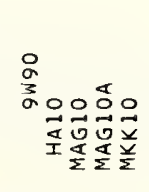 & 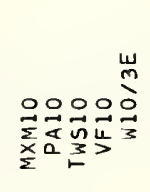 & 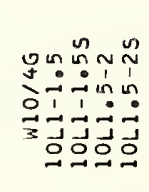 & 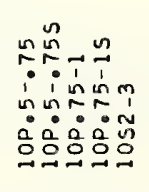 & 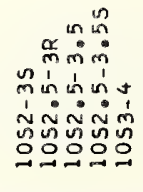 & 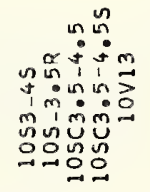 & 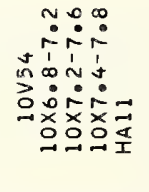 & 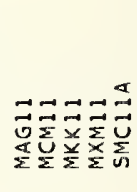 \\
\hline
\end{tabular}




\begin{tabular}{|c|c|c|c|c|c|c|c|c|c|c|}
\hline 9NITdnos & 언언엉 & 언엉은 & Q오오 & ৩ソ & ৩৩ & 옹ํㅛ & 운? & OOS & 号号 & OY \\
\hline L1/AY3 & $\leq z \leq z \leq$ & $z z z z z$ & $z \quad z Z \vec{\alpha}$ & $\vec{\alpha} z \vec{\alpha} z z$ & $z z$ & & $\vec{\sim} \vec{\alpha} \vec{\alpha}$ & $\vec{\alpha} \vec{\alpha} \vec{\alpha} z$ & $\vec{\alpha}$ & \\
\hline \begin{tabular}{|c|c|}
01311 & $\frac{E}{E}$ \\
\end{tabular} & & & $\begin{array}{l}\stackrel{\circ}{0} \\
0 \\
0\end{array}$ & & $\sum \sum \Sigma$ & $\Sigma \Sigma$ & & & $\begin{array}{l}\stackrel{0}{\circ} \\
\vdots \\
0\end{array}$ & $\sum \Sigma \Sigma \Sigma$ \\
\hline HLOMONY8 & & & $\stackrel{n}{\sim} \stackrel{n}{5}$ & in in & in $\operatorname{nn}$ & & 虫 in & ㅇํำ年 & & \\
\hline 9אוากด & & & & & & $\stackrel{n}{\sim} \underset{-1}{n}$ & & & $\stackrel{\sim}{\sim}$ & $\stackrel{m}{\rightarrow}$ \\
\hline 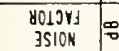 & & & & & & & $\stackrel{p}{r}$ & $\hat{\sim}$ & 옥 & $\stackrel{\infty}{\infty} \underset{\sim}{\infty} \sim$ \\
\hline Hirg & 图 & 00000 & $\circ$ & & 일요 & 绝 & $m$ & ? & $\stackrel{\Perp}{\sim}$ & 웅ㅇㅇㅇ \\
\hline $\begin{array}{l}S_{X 1704} \\
\times 1134\end{array}$ & & & & & 并鬲 & 并羊 & $\stackrel{\sim}{N}$ & 当 & $\stackrel{\circ}{\sim}$ & 并并并 \\
\hline $\begin{array}{c}51701 \\
1041 \mathrm{k03}\end{array}=$ & & & & & 옹요 & 只 & & : & & \\
\hline 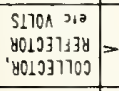 & & & 용요 & 음용요 & 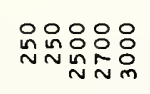 & 옹 & 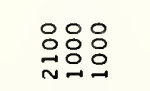 & 웃음욤 & 品 总 & 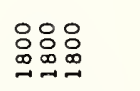 \\
\hline $0^{\circ}$ & |l & 영영영영 & 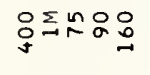 & 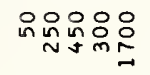 & 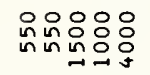 & $\begin{array}{l}0 \\
\text { in } \\
y\end{array}$ & 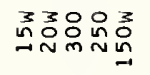 & 㝋员ㅇㅇㅛ & 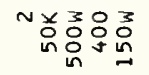 & 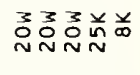 \\
\hline$\curvearrowleft$ & Eే & 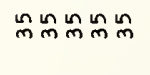 & $\underset{n}{n} \underset{\sim}{n} \stackrel{0}{\sim} \sim \stackrel{\infty}{\sim}$ & 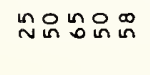 & 옹오오 유 & 옹ㅇㅇㅇㅇㅁㅇㅛ & 숭ㅇㅇㅇㅇ & 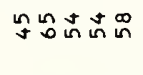 & N哭음 & 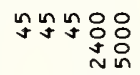 \\
\hline 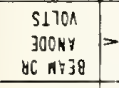 & 음음음 & 음음윰 & 总兽总尔 & 唰品品品品 & 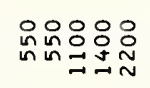 & 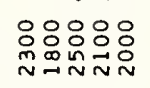 & 品品品品 & 음요요요 & 只兰咨品品 & 용 \\
\hline$=$ & 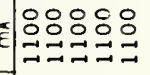 & 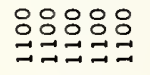 & 음 & 品品品品品 & 总总员员品 & 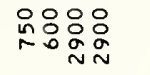 & 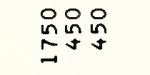 & 品员品品品 & 욤 & 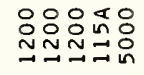 \\
\hline$\omega^{\omega}$ & 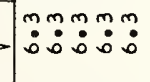 & 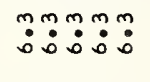 & $\stackrel{m}{:}: \ddot{m}:$ & $\ddot{m}: m \stackrel{m}{m}:$ & $\ddot{m}: m: m:$ & : & $\ddot{0}: \ddot{m}$ & $\stackrel{m}{m}: m m: ?$ & $\ddot{m}:$ & $\ddot{0} \ddot{0} \ddot{0}: \dot{0} \dot{0}$ \\
\hline N011YY] & טuטu & טuטu & טuטu & บuบuט & טuטu & $a \cup a a a$ & טuט & טuטu & Uuava & טuט \\
\hline$y, x$ & 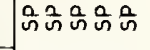 & 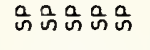 & 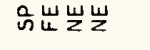 & 岂 岂岂 & 岂岂岸욤요 & 屰岁鸟岂 & 山Uऽㅁㅁㅣ & பூひタチ岂 & 곯⼭ㅣㄹ & Uூֵ \\
\hline 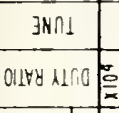 & $\mid$ & 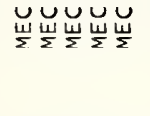 & 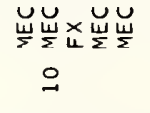 & 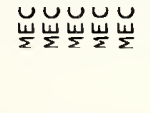 & 岀岀 & 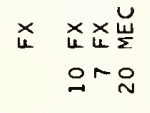 & 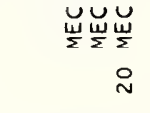 & 岕岕嵌嵌 & 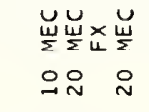 & 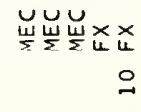 \\
\hline 竞 & 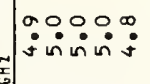 & 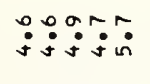 & 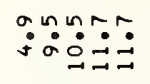 & 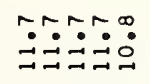 & 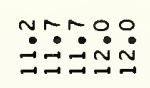 & $\because \because \because 000$ & 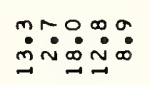 & 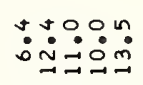 & 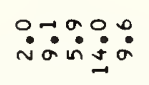 & 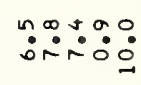 \\
\hline 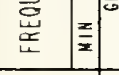 & 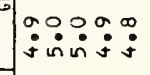 & 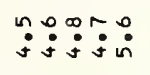 & 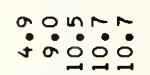 & $\because \because \because \dot{0} \dot{0}$ & 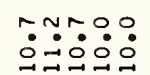 & $\because \because 000$ : & 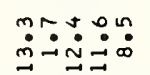 & 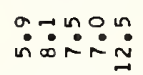 & 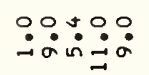 & ம்: \\
\hline ONIX & 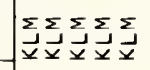 & 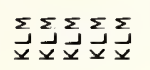 & 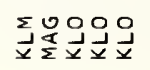 & 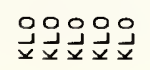 & 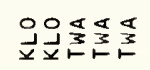 & 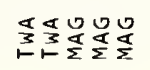 & 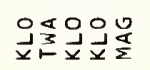 & 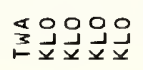 & 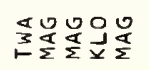 & 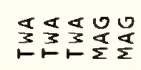 \\
\hline $108 W \times S$ & & & & & & & & & $"$ & \\
\hline 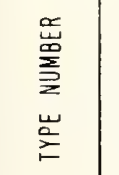 & 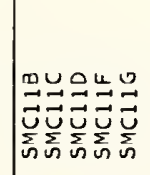 & 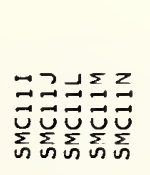 & 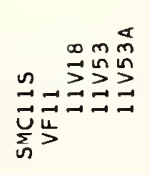 & 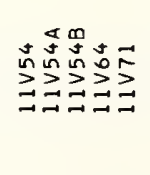 & 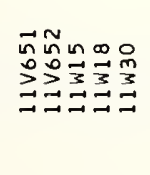 & 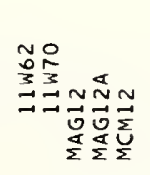 & 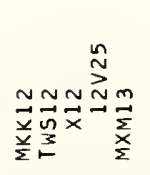 & 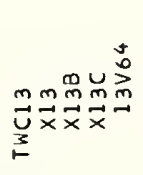 & 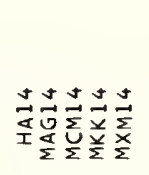 & 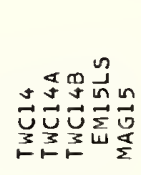 \\
\hline
\end{tabular}




\begin{tabular}{|c|c|c|c|c|c|c|c|c|c|c|}
\hline TNI TnOSO & O & 8Y & 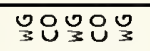 & 언 언 & 8ㅇ & 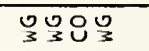 & 언 인 & 웅 & OYOYত & 야요 \\
\hline L1/nes & ZZZ & $\vec{\sim}$ & $\stackrel{\alpha}{\sim} \vec{\alpha}$ & & $\underset{\sim}{\sim}$ & & $\ddot{\sim} \vec{\sim}$ & $z$ & Z品 & z \\
\hline $\begin{array}{c}01711 \\
\text { ग113N9YK }\end{array}$ & है & $: \Sigma$ & $\stackrel{\circ}{\circ}$ & 음 & 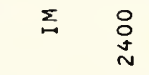 & $\underset{\substack{0 \\
\text { 足 }}}{\Sigma}$ & 음요 & $\stackrel{\circ}{\circ}$ & 号 & \\
\hline HLOIMON日P & in & & 品 on & & $\begin{array}{l}8 \\
0 \\
0\end{array}$ & $\stackrel{\text { ก }}{+}$ & & 9 & $\stackrel{m}{N}$ & 응요용 \\
\hline 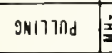 & 空 & $\stackrel{\sim}{\sim}$ & & & & $\stackrel{n}{\sim}$ & & & & \\
\hline $\begin{array}{c}\text { Yolors } \\
\text { ISION }\end{array}$ & $\infty$ & & $\stackrel{n}{-1}$ & $\stackrel{n}{\sim}$ & & & & $\stackrel{\circ}{\circ}$ & & \\
\hline niYs $=$ & 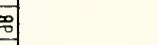 & in & $9 \stackrel{n}{N}$ & $\stackrel{m}{m} \stackrel{n}{\sim}$ & 요 & $\stackrel{m}{m}$ & 욤 & $\stackrel{\sim}{\sim}$ & $\stackrel{n}{\sim} \quad$ in & \\
\hline $\begin{array}{l}S 1700 \\
x 113 H\end{array}$ & & 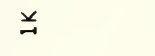 & ్ㅠㅇㅇ & 怘 & 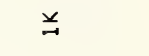 & $\stackrel{\sim}{N}$ & 并品 & 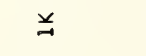 & $\ddot{\sim}$ & \\
\hline $\begin{array}{c}\text { S170A } \\
\text { 1082NOS }\end{array}$ & $\stackrel{\circ}{n}$ & & 윰ำ & & & $\stackrel{\circ}{\circ}$ & $\stackrel{\circ}{m}$ & $\stackrel{\circ}{\circ}$ & in & 윰요 \\
\hline 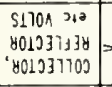 & - 윰욤ํำ & $\stackrel{\circ}{\stackrel{ }{二}}$ & 并品 品品 & 요 & 욤 & 品号욤 & 品品 & 윰욤욤 & $\underset{\sim}{\stackrel{ }{9}}$ & 음욤요 \\
\hline $0^{\circ}$ & 풍 & 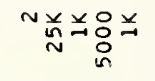 & 㒸 N品品品 & 总㐓: ${ }^{m}{ }^{m}$ & 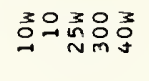 & 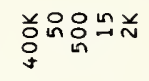 & 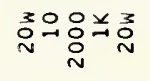 & 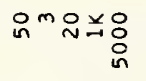 & 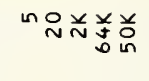 & 品品识品琶 \\
\hline$\omega$ & Eิ & 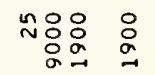 & in & n: N & № $\underset{\sim}{\stackrel{N}{=}}$ & 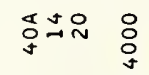 & 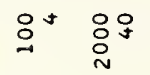 & $\pm \sim \quad \underset{N}{\stackrel{0}{\sim} \underset{N}{\sim}}$ & $\begin{array}{l}\sim \\
\text { 윴융유 }\end{array}$ & 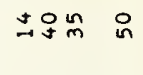 \\
\hline 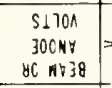 & 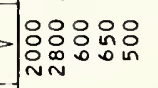 & 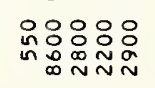 & 옥유 & 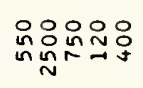 & 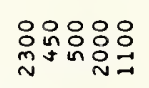 & 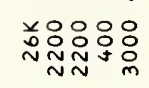 & 용요 & 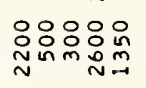 & 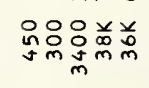 & 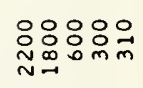 \\
\hline$\mapsto^{-}$ & 트 & 总品 & 品品 总品 & $\begin{array}{ll}\circ & 0 \\
& 0\end{array}$ & : & $\begin{array}{l}\text { 요욤 } \\
\text { ind }\end{array}$ & 응 & 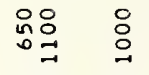 & $\begin{array}{l}\stackrel{\circ}{\stackrel{O}{N}} \\
\underset{\sim}{*}\end{array}$ & 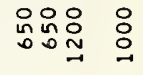 \\
\hline$w^{t}$ & 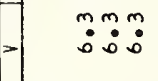 & $\stackrel{m}{i} \dot{0}$ & $\ddot{r}: \stackrel{m}{0}: \dot{0}$ & $\stackrel{m}{\dot{0}}$ & 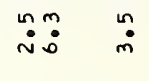 & $\ddot{m} \dot{0}$ & $\ddot{m}: \dot{m}$ & $\ddot{m} \quad \stackrel{m}{0}$ & $\stackrel{m}{\ddot{0}} \quad \ddot{0}:$ & $\ddot{m}: \ddot{m}$ \\
\hline N01.6939O & aauvu & Ua a u & auuvu & vauvu & uvauv & a Uu & u u & uvuau & vuaaa & טuטu \\
\hline yis & 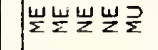 & 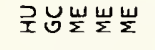 & 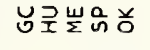 & 고뽀ㄹㅣㅗ롤 & Ф고플 & 빤호로필 & 台고를ㄴ & 응로롱 & 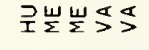 & 吕并岂岂杰 \\
\hline JNก1 & 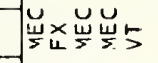 & 苨岕 & 岕岮 & 岀ら 5 & $5 \stackrel{\breve{J}}{\lessgtr} \underset{4}{x}$ & 嵌 $5 x$ & $5 x$ & 岀 嵌岀 & 岀 & 岕岕岕岕岕 \\
\hline $0118 y$ alro 0 & 을 & 유 웃 & $\therefore$ & $\stackrel{\circ}{\sim}$ & $\stackrel{2}{N}$ & $\stackrel{i}{2}$ & $\stackrel{\sim}{N}$ & $\stackrel{\sim}{N}$ & i & \\
\hline 离 & 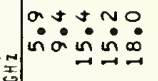 & 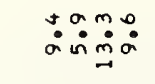 & 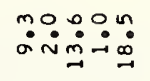 & 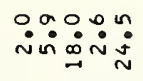 & 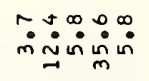 & 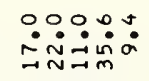 & 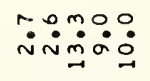 & 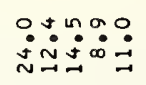 & 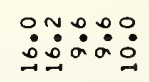 & :ำ: \\
\hline 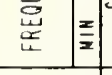 & 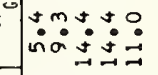 & 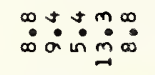 & 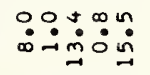 & 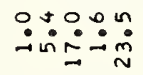 & 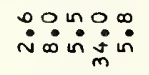 & $\because \because 00$. & 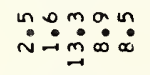 & 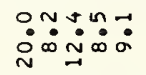 & 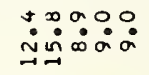 & 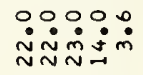 \\
\hline ONIX & 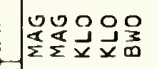 & 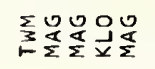 & 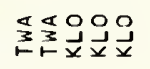 & 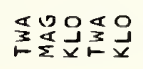 & 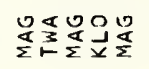 & 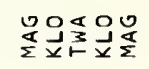 & 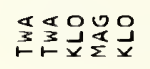 & 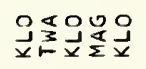 & 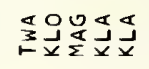 & 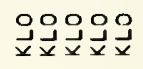 \\
\hline 708W/S & & & & & & & & & & \\
\hline 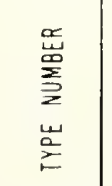 & 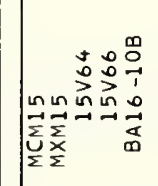 & 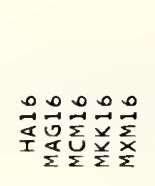 & 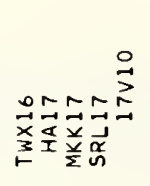 & 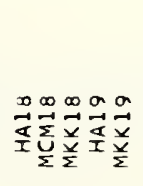 & 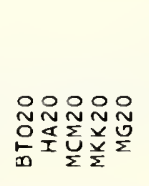 & 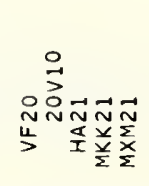 & 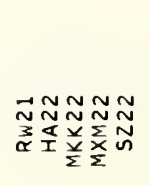 & 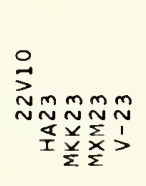 & 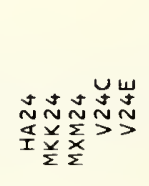 & 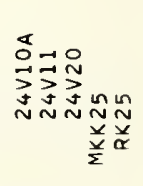 \\
\hline
\end{tabular}




\begin{tabular}{|c|c|c|c|c|c|c|c|c|c|c|}
\hline 9k17dחOS & 웅 엉 & 8OY & 옹 & 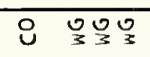 & 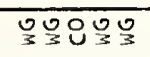 & 언 号号 & 언 눙언 & 은은 & 뭉요 & पО अणु \\
\hline LIAN & & $z$ & $\vec{m}$ & & & $\vec{\sim}$ & $\vec{m}$ & $\vec{\alpha}$ & $\vec{\alpha}$ & $z$ \\
\hline \begin{tabular}{c|c}
$07\} 11$ \\
ग1139VKK
\end{tabular} & $\sum \sum \begin{array}{l}0 \\
0\end{array}$ & $\sum$ & $\sum \sum$ & : & $\Sigma$ & $\Sigma \Sigma$ & $\stackrel{\Sigma}{\vdots}$ & in $\Sigma$ & $\Sigma$ & \\
\hline H101Monry $=$ & & & & 오 & 잉 & & & $\stackrel{\circ}{\sim}$ & $:$ & 요 \\
\hline 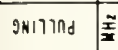 & $\stackrel{\sim}{\sim} \sim$ & & & & & 웅 & & & & \\
\hline \begin{tabular}{c|c}
$8013 Y\}$ \\
3S10N
\end{tabular} & & & & & & & $\stackrel{n}{\sim}$ & & & \\
\hline \begin{tabular}{l|l} 
Nirg \\
Nito
\end{tabular} & $\stackrel{\circ}{m}$ & 임요 & $\stackrel{\infty}{N} \stackrel{n}{m}$ & & 을 & & $\stackrel{n}{\sim} \sim$ & & 임 & \\
\hline $\begin{array}{l}5170 \mathrm{~A} \\
\times 173 \mathrm{H}\end{array}=$ & $\stackrel{\infty}{\circ}$ & 응 & $\underset{\sim n}{\sim} \stackrel{\sim}{\sim}$ & & $\stackrel{\circ}{N}$ & & $\stackrel{2}{\stackrel{2}{2}}$ & in & $\stackrel{\sim}{N}$ & \\
\hline $\begin{array}{c}51701 \\
7081603\end{array}=$ & & 웅 & $\stackrel{\circ}{N}$ & : & 욤 : & & & 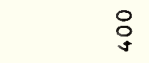 & $\stackrel{\circ}{\circ}$ & 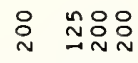 \\
\hline 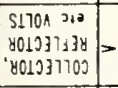 & : & 总 & $\stackrel{n}{\sim} \underset{0}{\circ}$ & :웅 & 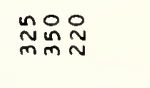 & & $\stackrel{\circ}{\circ}$ & 怘 & 임욤 & 品品证品品 \\
\hline $0^{\circ}$ & 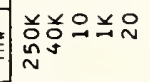 & 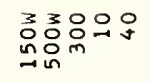 & ․్ㅁㅇㅛ & 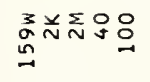 & 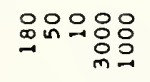 & 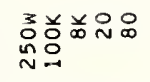 & 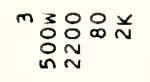 & 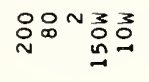 & 요요요 & Nㅗㅇㅛ \\
\hline 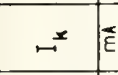 & 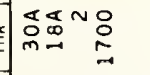 & 음ㅇN & 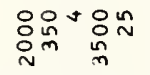 & 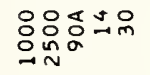 & 욤ㅇㅛㅛ & 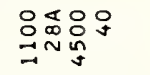 & No: & 욤요 & 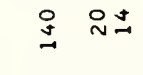 & 욜 $\tilde{n} \sim \tilde{n}$ \\
\hline $\begin{array}{c}S 1701 \\
300 N Y \\
\forall C A Y 38\end{array} \mid>$ & 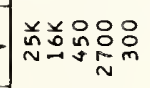 & 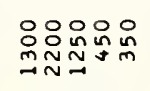 & 娟品品品 & 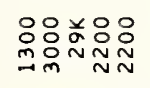 & 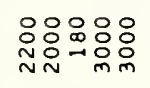 & 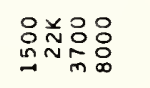 & 品品品 & 유 욧윰유 & ::우 & :::유 \\
\hline$F^{-} \bar{E}$ & 总: & 웅요 & ¿̊융 & 웃요 & 号 & $\Xi$ & $\begin{array}{l}\circ \\
\vdots\end{array}$ & $\begin{array}{l}\circ \\
:\end{array}$ & 品 总员 & 总足 \\
\hline$\omega^{4}$ & $\ddot{n}:$ & $\ddot{m}_{0}^{m}:$ & $\stackrel{n}{i}: \stackrel{0}{i}$ & $\ddot{m}: m$ & $\ddot{0} \dot{m}: \dot{m}: \dot{m}$ & $\ddot{m}$ & $\dot{n}: \dot{m}$ & $\ddot{0} \dot{0}$ & $\ddot{0}: \ddot{0}$ & 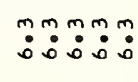 \\
\hline $\mathrm{NO1178300}$ & anuau & aguuu & auuau & $a a g u v$ & uvuuu & $a a a u v$ & vauva & ưu & טưu & טưuบ \\
\hline$\forall נ n$ & 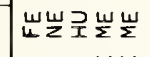 & 崖岂吕豆崖 & 崖品고르ㄹㅗㅗ & 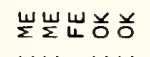 & 등고등 & 㞫ち岂山。 & 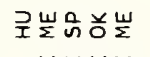 & 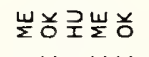 & 吕吕岂全长 & 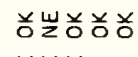 \\
\hline \begin{tabular}{|l|l}
$3 N \cap 1$ \\
\end{tabular} & 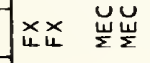 & wüw & 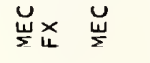 & 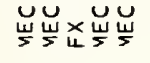 & 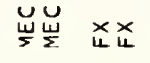 & 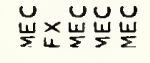 & 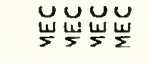 & ヶÜ & 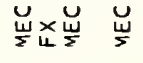 & 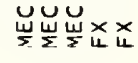 \\
\hline Outry AnO & $\stackrel{n}{\sim} \stackrel{\circ}{N}$ & 요요 & 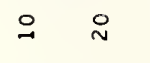 & 오음 & & 오응ㅇ & $\stackrel{\circ}{\sim}$ & $\stackrel{i}{N}$ & & \\
\hline 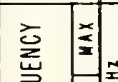 & 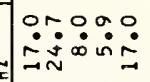 & 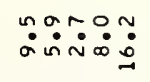 & 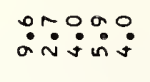 & 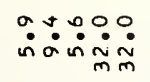 & 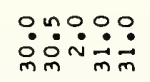 & ம் & 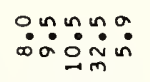 & 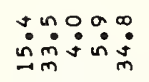 & 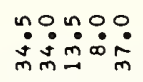 & 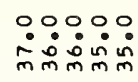 \\
\hline 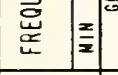 & 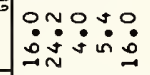 & 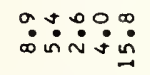 & 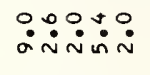 & 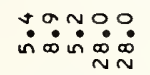 & مّ & 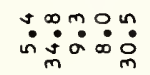 & 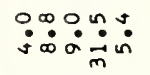 & 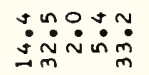 & 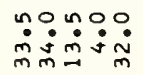 & 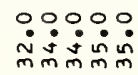 \\
\hline ONIX & 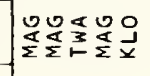 & 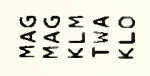 & 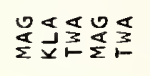 & 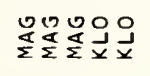 & 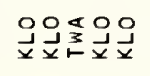 & 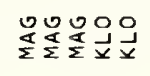 & 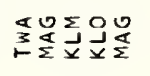 & 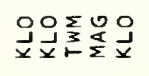 & 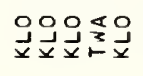 & 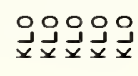 \\
\hline $108 \mathrm{~W} / \mathrm{S}$ & & & & & & & & & & \\
\hline 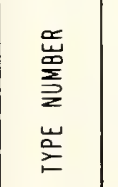 & 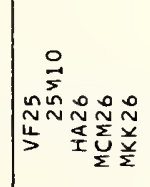 & 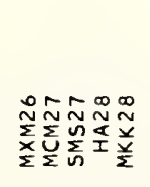 & 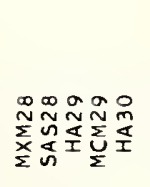 & 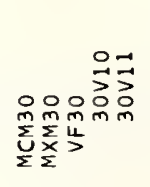 & 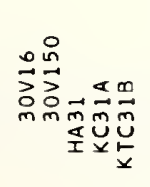 & 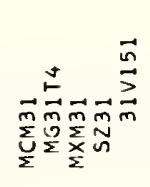 & 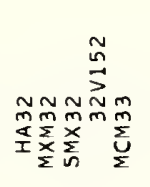 & 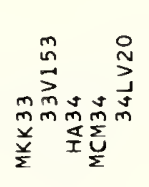 & 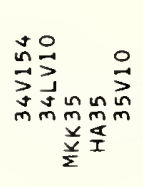 & 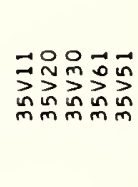 \\
\hline
\end{tabular}




\begin{tabular}{|c|c|c|c|c|c|c|c|c|c|c|}
\hline 9.1 ר d d & 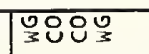 & ত̧०0 & 웡ㅇㅇㅇㅛ & 언원의 & 언 인 & 억언 & 엉워워 & 엉워워 & 언엉요 & 웡워 \\
\hline A1IAYS & & $\vec{N}$ & & $\underset{\alpha}{\underline{\alpha}} \quad \stackrel{\vec{\alpha}}{\underline{\alpha}}$ & జx & $F$ & & wz & 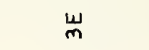 & 峁. \\
\hline 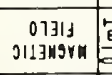 & $\Sigma$ & & 品 & $\underset{\infty}{\stackrel{\infty}{\infty}}$ & & 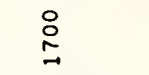 & : & $\Sigma$ & $\sum \Sigma$ & \\
\hline H10140Hrg: & $\mid \begin{array}{l}\infty \\
\infty\end{array}$ & & & N & $\simeq \simeq$ & $\stackrel{-}{\sim}$ & & n & $\stackrel{n}{n}$ & 는을 \\
\hline 94ו17าก & & & & & & & : & & in & \\
\hline $\begin{array}{c}8019 \mathrm{rj} \\
\mathrm{JS10N}\end{array}$ & & $\therefore$ & & $\cong$ & & & $\cong 0$ & & & \\
\hline Hivg & $\stackrel{2}{2}$ & $\stackrel{n}{N}$ & 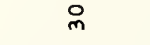 & $\stackrel{n}{N}$ & & $\stackrel{n}{\sim} \stackrel{\infty}{m}$ & $\stackrel{n}{\sim}$ & $\stackrel{m}{m}$ & $\stackrel{m}{m}$ & $\stackrel{n}{\sim}$ \\
\hline $\begin{array}{l}51700 \\
\times 1173 H\end{array}$ & 品 & $\stackrel{n}{\xi}$ & $\ddot{\forall}$ & $\stackrel{\circ}{\underset{7}{~}}$ & & $\stackrel{x}{N}$ & $\cong \stackrel{\circ}{=}$ & & 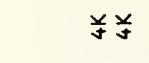 & $\stackrel{\circ}{\circ}$ \\
\hline $\begin{array}{c}5170 \mathrm{~N} \\
1081400\end{array}$ & . & & & & 总虽 & $\stackrel{\circ}{N}$ & & ㅋㅇㅇㅁㅇㅁ & 음요 & $\stackrel{\circ}{N}$ \\
\hline 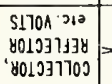 & 용요 & 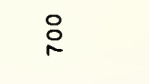 & $\stackrel{i}{\underline{n}}$ & 용요 욤 & 웅 & 吕 & 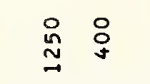 & $\stackrel{\circ}{\circ}$ in & $\stackrel{\text { 足 }}{N}$ & $\stackrel{\circ}{\stackrel{n}{\infty} \underset{N}{N}}$ \\
\hline$a^{\circ}$ & 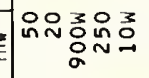 & : & 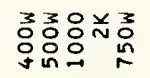 & 윰종용여 & 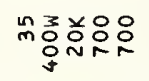 & 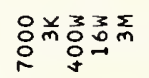 & $\begin{array}{l}z \rightarrow 0 \times m \\
\vdots \\
0\end{array}$ & 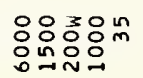 & 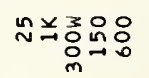 & 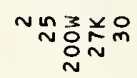 \\
\hline- & $=1+\infty$ & No : & 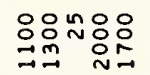 & 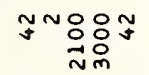 & 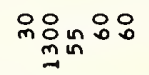 & 웅움욤ㅆ & \begin{tabular}{l} 
\& \\
\hdashline \\
\hdashline
\end{tabular} & 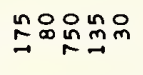 & 옹융용 & N 总品品 \\
\hline $\begin{array}{c}51701 \\
300 \mathrm{HY} \\
80 \mathrm{KH}=38\end{array}$ & $\mid \begin{array}{l}a n \\
\mathbb{N}_{N}^{n}\end{array}$ & 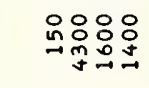 & 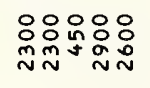 & 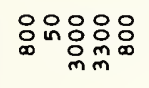 & 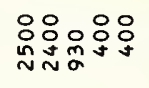 & 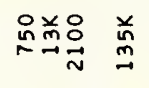 & 路品兑识品 & 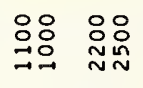 & ㅇㅇㅇㅇㅇㅇㅇㅇㅇㅛ & 웃윰윰유 \\
\hline- & $=$ & $\stackrel{\circ}{\circ}$ & $\stackrel{\circ}{\stackrel{D}{\sim}}$ & 윰 & $\stackrel{m}{m}$ & $\stackrel{\circ}{\circ}$ & 음융ㅇํ & 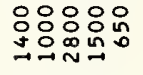 & 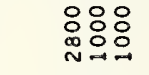 & 品 \\
\hline " & 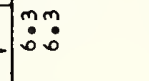 & i. & $\stackrel{\circ}{i}$ & 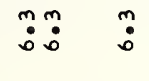 & $\ddot{m} \quad \ddot{j}_{\dot{j}}^{\infty}$ & $\stackrel{n}{:} \quad \stackrel{m}{0}$ & $\ddot{m} \ddot{m}: \stackrel{m}{0}$ & m:m:mm: & 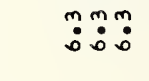 & $\ddot{m}: m$ \\
\hline Nol1Yy3do & Uบ & 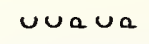 & a auoa & uva u & vauuv & vuaua & auaua & טuטus & vauuv & vuauv \\
\hline$\forall J M$ & 하ㅁㅣㅡㄹ & 응고 $\frac{\omega}{\Sigma} \frac{\omega}{\Sigma} \frac{\omega}{\Sigma}$ & 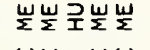 & 顿岂崖】 & 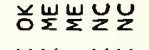 & 跸岂忈品 & 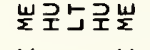 & 部占。 & 㞤岂的芫。 & 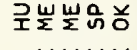 \\
\hline $3 \times \cap 1$ & 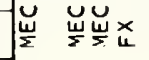 & 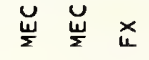 & 岀岕 岕岕 & 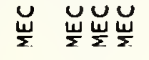 & 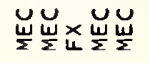 & ラ訔㞱 & $\begin{array}{l}\breve{w} \Sigma \\
\frac{\omega}{\Sigma}\end{array}$ & 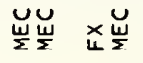 & 巡岕岕ら5 & 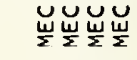 \\
\hline OLYY «InO & $\stackrel{i}{n}$ & $\therefore$ 용 & 오 & 윳ำ & i & $\stackrel{\text { q }}{\sim} \stackrel{\text { N }}{2}$ & $\stackrel{\sim}{\sim} \stackrel{\circ}{\sim}$ & & 오요 & 욤 \\
\hline 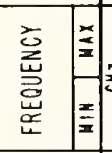 & 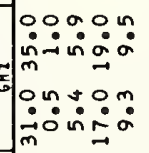 & 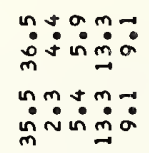 & 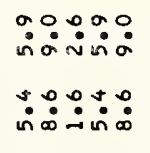 & 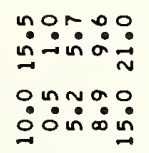 & 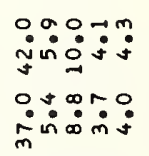 & 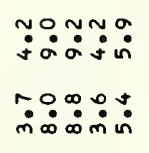 & 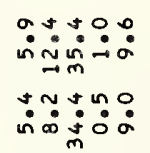 & 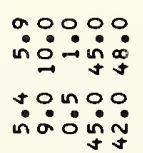 & 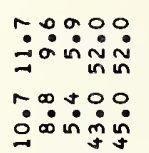 & 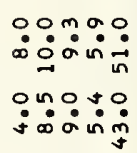 \\
\hline ONIY & 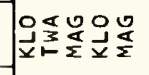 & 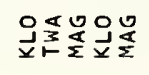 & 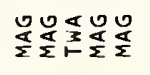 & 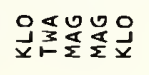 & 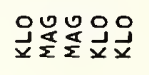 & 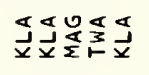 & 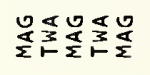 & 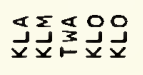 & 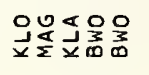 & 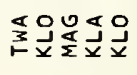 \\
\hline 70gW/S & & & & & & & & & & \\
\hline 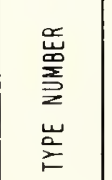 & 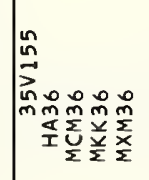 & 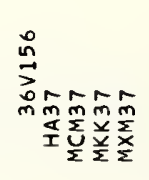 & 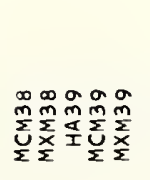 & 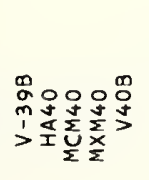 & 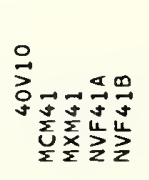 & 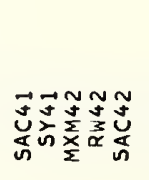 & 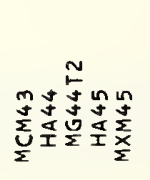 & 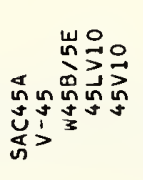 & 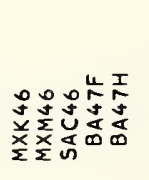 & 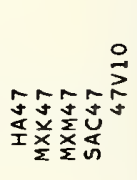 \\
\hline
\end{tabular}




\begin{tabular}{|c|c|c|c|c|c|c|c|c|c|c|}
\hline 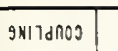 & :Оত্ত & 엉 & 언원오 & 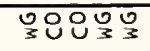 & पू O OU & 을엉 & 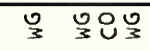 & 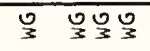 & 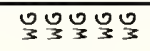 & 언 \\
\hline LL1Ars & & $\vec{\alpha}$ & $\underset{\propto}{\sim}$ & $\vec{w} \vec{x}$ & $\vec{\alpha}$ & $\vec{\alpha} \vec{\alpha}$ & & $\vec{\alpha}$ & $\vec{\alpha} \vec{\alpha}$ & \\
\hline 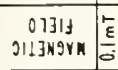 & $\Sigma \quad \Sigma$ & $\sum \Sigma$ & $\Sigma$ & $\Sigma$ & $\Sigma$ & $\Sigma \Sigma$ & $\sum \Sigma$ & & 음 & $\Sigma$ \\
\hline H101MOHYA & $\tilde{\mathbf{I}} \cong$ & $\cong$ & 昌昌 & $\simeq \infty$ & : & 우 & & s & 品品 & $\stackrel{2}{\sigma}$ \\
\hline 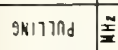 & 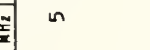 & n & & & & n & & & n & \\
\hline 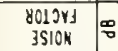 & & $\stackrel{2}{N}$ & & & & $\simeq$ & $\stackrel{\circ}{N}$ & & & $\stackrel{n}{\sim}$ \\
\hline Nirg & 目 & 요 & & $\stackrel{\circ}{N}$ & $\stackrel{m}{m}$ & 요 & m & & & $\stackrel{m}{m}$ \\
\hline $\begin{array}{l}51701 \\
\times 173 H\end{array}$ & 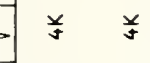 & 감용 & $\stackrel{\varpi}{\exists}$ & $\stackrel{i}{\sim}$ & $\underline{\Xi}$ & ㅇㅇㅛ & 羊兰 & & & $\stackrel{\ddot{N}}{\sim}$ \\
\hline $\begin{array}{r}51701 \\
7001803\end{array}=$ & $\mid \begin{array}{ll}8 & \circ \\
i & 0\end{array}$ & 品 & 용요 & 웅 品 & 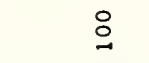 & 임 & 욤 & & $\stackrel{\sim}{\sim}$ & $\stackrel{i}{i}$ \\
\hline 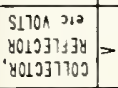 & ho & 品品 总 & 음요 & 웄잉요요 & 욤 & 品 윰 & 옹 & 号 & 응 & 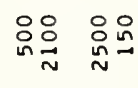 \\
\hline $0^{\circ}$ & 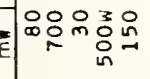 & 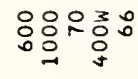 & 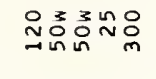 & 음욤요 & 으음윰ㅇㅇํ & 움웅요 & : & 品爻舟怘怘 & 육용ㅇㅁㅇㅁㅇ & 염중융유 \\
\hline$\mapsto$ & 탕욤 & :임 & 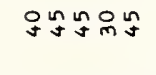 & 윰으 옹 & $0 \underset{\substack{0 \\
0}}{0}$ & 总 & 윳용요 & $\stackrel{D}{n}_{\substack{n \\
m}}$ & 우수윰요 & 윢웄욤요 \\
\hline $\begin{array}{c}5170 \mathrm{~s} \\
300 \mathrm{kr} \\
80 \mathrm{hr} 3 \mathrm{~g}\end{array}$ & 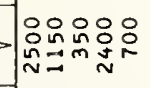 & 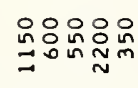 & 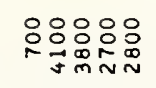 & 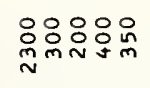 & 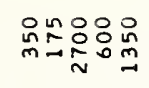 & 总品品品品 & 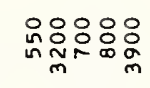 & 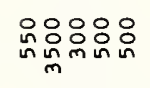 & 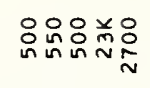 & 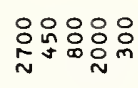 \\
\hline$\leftarrow$ & 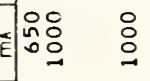 & 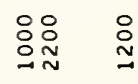 & 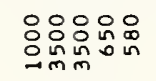 & 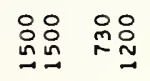 & $\stackrel{\circ}{\stackrel{\circ}{ }}$ & 음임ㅇㅇㅇ응 & 웅음 & 员吕品 & 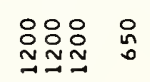 & 응요 \\
\hline$\omega^{ \pm}$ & $m ?$ & $\ddot{0}: 0$ & :̊ํ: & 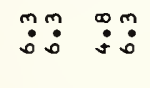 & $\stackrel{m}{:} \stackrel{0}{i}$ & $\ddot{0}: \ddot{:}: \dot{m}$ & $\ddot{0} \dot{0}: 0$ & $\ddot{m} \ddot{0}: \dot{m}$ & $\ddot{0}: \ddot{m}: m:$ & $\stackrel{m}{\ddot{0}} \stackrel{0}{:}$ \\
\hline N011Yy $7 \mathrm{dO}$ & Uu & ư & บบบบบ & טưu & U & \&บบบบ & UaUu & $\cup a \cup \cup \cup$ & uU $\cup \cup$ & uveuv \\
\hline$y d m$ & 各吕岂岂首 & 差预崖崖山 & 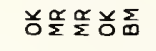 & 히롤uu & 嵌岂㞫兲崖 & 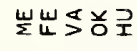 & 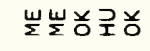 & 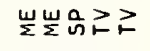 & 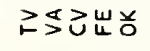 & 흥고리로필 \\
\hline 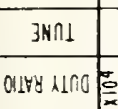 & 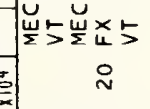 & 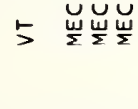 & 555 & 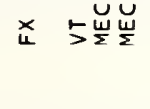 & 岀っ & 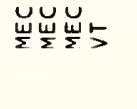 & เ㞱ヶ & 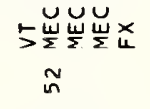 & 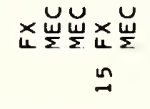 & 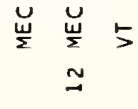 \\
\hline 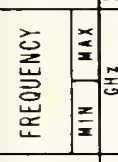 & 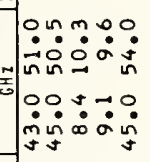 & 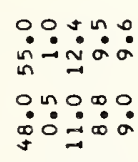 & 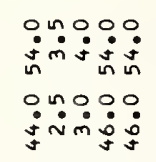 & 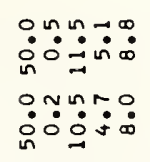 & 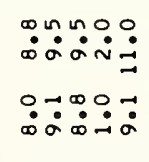 & 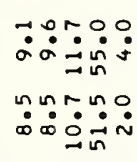 & 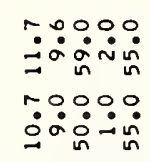 & 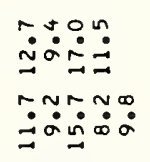 & 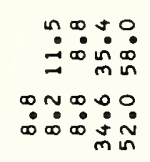 & 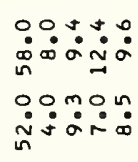 \\
\hline ONIX & 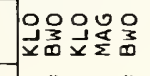 & 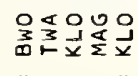 & 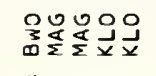 & 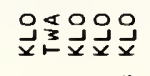 & 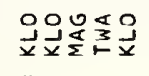 & 울울 & 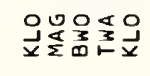 & 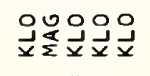 & 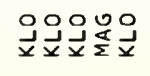 & 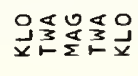 \\
\hline $708 w / S$ & " & $"$ & $"$ & & $"$ & " " & " & " & & \\
\hline 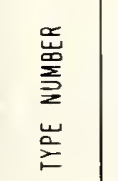 & 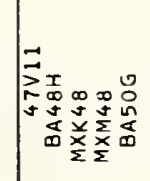 & 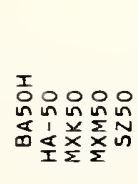 & 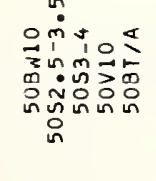 & 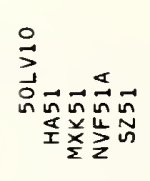 & 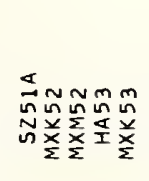 & 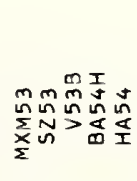 & 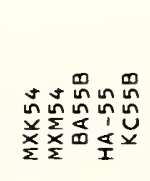 & 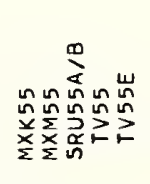 & 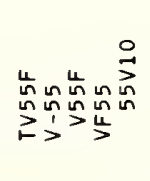 & 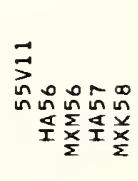 \\
\hline
\end{tabular}




\begin{tabular}{|c|c|c|c|c|c|c|c|c|c|c|}
\hline 9พ1าdก0ง & 9088 & 30980 & 53008 & 엉ㅇㄴ? & $\frac{9}{3}$ & 8800 & OOYOOO & 8808 & $89 \div 85$ & 뭉ㅇㅇㅇ \\
\hline Al|AVs & $\operatorname{us}_{\propto \alpha} \quad \vec{\alpha}$ & $\overrightarrow{m m}$ & $\vec{\sim} \quad \vec{m}$ & $\vec{\alpha} \quad \vec{\alpha}$ & $\vec{\alpha}$ & $\ddot{\alpha} \underset{\alpha}{u}$ & $\underset{\alpha}{u}$ & $\vec{\alpha} \quad \vec{\alpha}$ & $\overrightarrow{a x}$ & $\ddot{*}$ \\
\hline \begin{tabular}{|c|c}
01319 \\
ग11399VK
\end{tabular} & $\Sigma$ & $\Sigma \Sigma$ & $\stackrel{\circ}{\circ}$ & & & $\Sigma \quad \Sigma$ & $\sum_{\substack{\infty \\
\infty}}$ & $\stackrel{8}{\stackrel{8}{N}}$ & $\sum \begin{array}{l}0 \\
0 \\
0\end{array}$ & $\sum \Sigma \Sigma \Sigma$ \\
\hline H101monva & $:$ & $\infty$ & $\stackrel{0}{ \pm}$ & : & $\stackrel{n}{m}$ & $9 \stackrel{9}{9}$ & 요묘 & 02 & m & 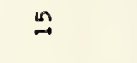 \\
\hline 9M17าnd $\bar{x}$ & 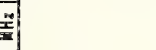 & n & & & & & ' & & & \\
\hline 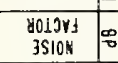 & $\stackrel{N}{N}$ & $\stackrel{\Delta}{\rightarrow}$ & $\stackrel{\varpi}{\sim}$ & $\stackrel{\circ}{\circ}$ & & $=$ & $\infty$ & $\infty$ & $\stackrel{\ln }{N}$ & \pm \pm \\
\hline Hirg & $\stackrel{n}{m}$ & $\stackrel{n}{N} \stackrel{n}{\sim}$ & $\stackrel{\text { nn }}{\sim} \stackrel{\circ}{m}$ & $\stackrel{\leftrightarrow}{\sim}$ & & 品 & $\stackrel{N}{N}$ & $\stackrel{i s}{\sim}$ & 용ㅇ & 䔞织品出兄 \\
\hline $\begin{array}{l}91701 \\
\times 1739\end{array}$ & 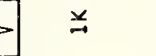 & $\underset{z}{*}$ & $\underset{x}{*}$ & 品 & & $\stackrel{i}{\mathrm{~N}}$ & జO & $\stackrel{8}{N}$ & $x:$ & 希 总品品 \\
\hline $\begin{array}{c}51700 \\
10811400\end{array}=$ & & 욤 & $\stackrel{\vdots}{\sim}$ & & & $\therefore$ & 웍윰요 & & is : & 요 \\
\hline 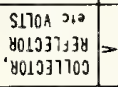 & $\begin{array}{l}800 \\
88 \\
80\end{array}$ & 욤용 & 总品品 & 品吕总 品 & 品品品品品 & 음 总号 & 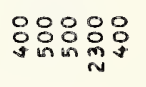 & 品品: & 踶品怘品 & 음 \\
\hline$a^{\circ}$ & 용요 & 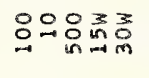 & 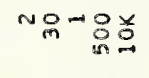 & 品以只品品 & 욤요요 & 品总点品品 & 멤요 & 象品总品 & 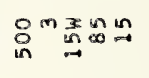 & $\underset{N}{3} \underset{N}{2}=0$ in $N$ \\
\hline$\approx$ & $\stackrel{\sim}{\sim}$ & 㽞 品 & nON & $\stackrel{\infty}{N}$ & $\therefore$ & $\therefore \quad$ 照 & 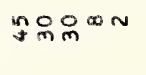 & Nọg & NO & 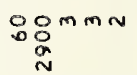 \\
\hline $\begin{array}{c}\text { S1700 } \\
300 \mathrm{ny} \\
80 \mathrm{nb39}\end{array}=$ & 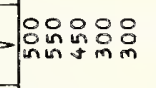 & 움욤요 & 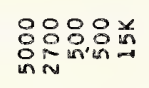 & 음욤욤요 & 용ㅇㅇㅇㅇㅛ & 品品 号 & 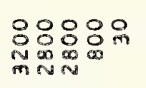 & 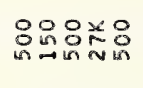 & 品 品品 & 요욤요 \\
\hline 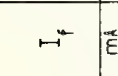 & S: & 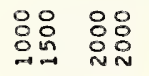 & 용요 & 윰 & : & $\begin{array}{l}8 \\
8 \\
8\end{array}$ & 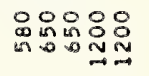 & 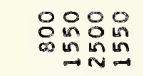 & 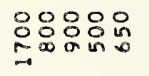 & 웅욤요 \\
\hline w" & :० & $\ddot{0}: 03$ & $\ddot{:}: \stackrel{m}{:}$ & $\ddot{m}:$ & 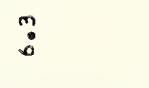 & $\ddot{m} \quad \because \because:$ & $\ddot{0}: \ddot{0}: \ddot{\circ}$ & 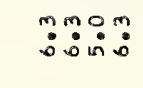 & 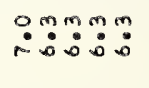 & 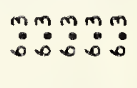 \\
\hline NOLIYYJ]dO & & uuua & บบưa. & yuuyu & yuune & yuuvu & yuuve & uuau & טuบu & vauuv \\
\hline$y+k$ & 我골씨 & 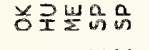 & 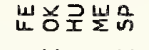 & 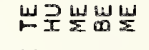 & 息改崖崖崖 & 坴崖出括音 & 흠응로로 & 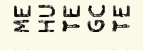 & 로로됨ㅇㅇ & 존로로고 \\
\hline $3 \mathrm{~K} \cap \perp$ & 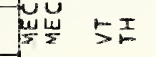 & 5 ร岀㟧 & 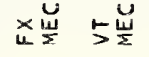 & 岀 政 & 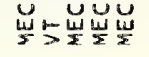 & 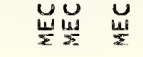 & 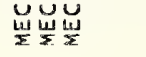 & 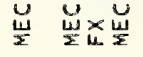 & 㟧岂 & 希 \\
\hline $01148 \times 110=$ & & $\underset{N}{N}$ & 9 & & & & & 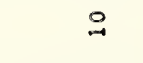 & & in \\
\hline 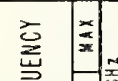 & $\because \because \because: 0$ & $\begin{array}{l}\because \because m a \\
\dot{0}=0 \dot{0}\end{array}$ & 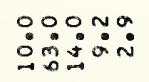 & $\because \because \because 0:$ & 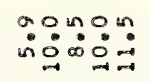 & 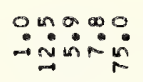 & $\begin{array}{l}00: \div 0 \\
\ln \end{array}$ & 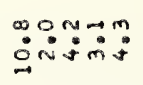 & 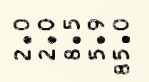 & $\because \because 0: 0$ \\
\hline 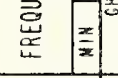 & $\ln _{0}$ & 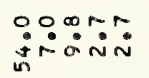 & $\because 0: 0$. & 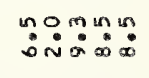 & 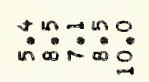 & 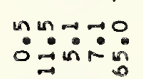 & $\because \because 0:$ & $\dot{0}$ & \begin{tabular}{l}
0000 \\
\hdashline-10
\end{tabular} & 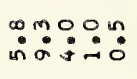 \\
\hline ONIX & 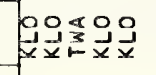 & 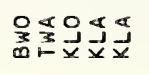 & 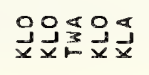 & 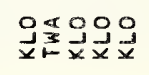 & 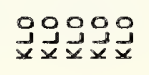 & 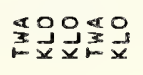 & 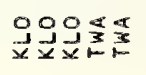 & 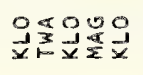 & 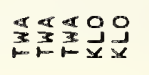 & 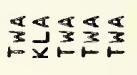 \\
\hline 708W/S & & $"$ & & & & & & & & \\
\hline 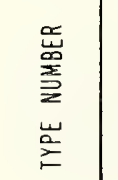 & 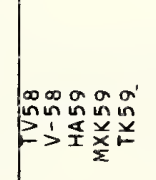 & 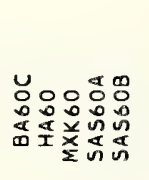 & 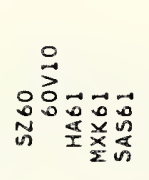 & 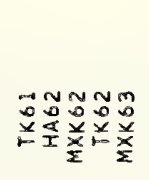 & 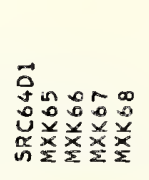 & 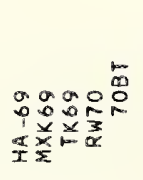 & 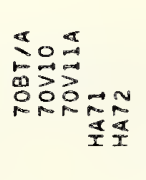 & 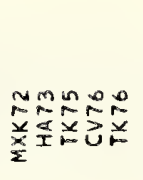 & 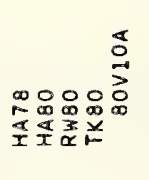 & 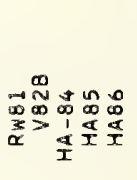 \\
\hline
\end{tabular}




\begin{tabular}{|c|c|c|c|c|c|c|c|c|c|c|}
\hline 9w17dnos & 8 : & 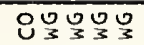 & 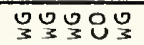 & 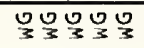 & ช88 & ים & 언언원 & Зू० & 언엉어 & ים \\
\hline d1וAY & $\ddot{m} \tilde{N} \vec{\jmath}$ & $\vec{m} \vec{\alpha} \vec{\alpha} \vec{\alpha} \vec{\alpha}$ & $\underset{\alpha}{\vec{\alpha}} \underset{\alpha}{\vec{\alpha}} \vec{\alpha} \underset{\alpha}{\sim}$ & $\vec{\alpha} \underset{\alpha}{\vec{\alpha}} \vec{\alpha}$ & $\vec{\alpha}$ & $\vec{\alpha}$ & $\vec{\alpha} \quad \vec{\alpha}$ & $\vec{\alpha} \quad \vec{\alpha}$ & $\vec{\alpha} \quad \vec{\alpha}$ & $\ddot{\alpha}$ \\
\hline 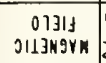 & & & & & $\Sigma$ & $\Sigma$ & $\Sigma \Sigma \Sigma$ & $\Sigma \Sigma$ & $\Sigma \Sigma$ & $\sum \quad \sum \Sigma$ \\
\hline H101monro & $\tilde{y} 00 \mathrm{~m} m$ & 요 욤 & 용 & 阅品泪品品 & & $\stackrel{\circ}{\sim}$ in & $\therefore$ & $\therefore \quad:$ & $\therefore \rightarrow$ & No \\
\hline dחהרוואפ & 풀 & & & & $\simeq$ & & & $\stackrel{\circ}{N}$ & & \\
\hline $\begin{array}{l}8019 \mathrm{YH} \\
35104\end{array}$ & $\infty$ & & & & $m$ & $\stackrel{\circ}{N}$ & 욜 & $\stackrel{n}{n}$ & $\stackrel{m}{=}$ & $\stackrel{\sim}{\sim} \quad \stackrel{\sim}{N}$ \\
\hline Alrg & :용 & $\stackrel{\circ}{m}$ & in & & in & 욤 & $\stackrel{n}{m} m m$ & 요 & $m$ 욜 & 있 \\
\hline $\begin{array}{l}51701 \\
\times 113 H\end{array}$ & on & & & & 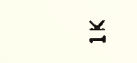 & $\underline{\sim}$ & $\underline{\sim}$ & 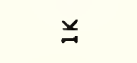 & 弚 品 & $\ddot{\sim} \underset{\infty}{\infty}$ \\
\hline $\begin{array}{c}\text { s170n } \\
\text { 70y11003 }\end{array}$ & 0 & 品 & & 於 & $\begin{array}{l}0 \\
0 \\
0\end{array}$ & 首 & & & & $\stackrel{\circ}{N}$ \\
\hline 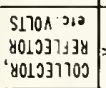 & 品 & 음욤어 & 응용 & 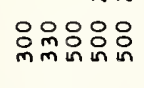 & : $:^{-1}$ & 욤욤 & 윰윰윰요 & 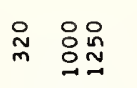 & $\begin{array}{ll}0 & 0 \\
0 \\
0 \\
\infty\end{array}$ & 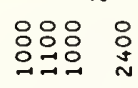 \\
\hline $0^{\circ}$ & ] & 㒸品望品品 & 윰유욤우 & 욱으=요 & 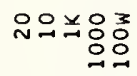 & 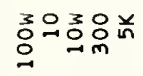 & 强品的员员 & 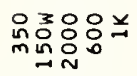 & ㅇㅇㅇㅇㅇㅇ & 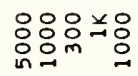 \\
\hline$-*$ & 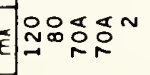 & 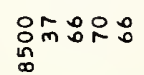 & mog o & 约웝요 & 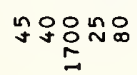 & 유음 용 & 응용옹우 & 윴욤유 & $\stackrel{n}{\sim}: \stackrel{0}{0}$ & 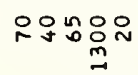 \\
\hline 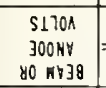 & 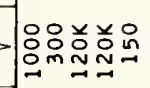 & 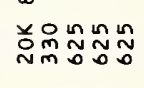 & 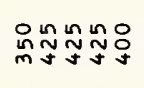 & 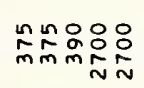 & 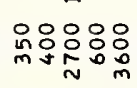 & 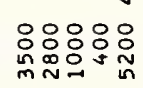 & 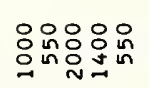 & 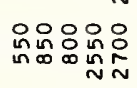 & 음욤요요 & 잉용요잉 \\
\hline$\mapsto^{+}$ & 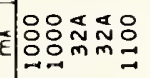 & 중용ㅇㅇㅇㅛ & 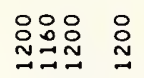 & 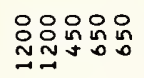 & 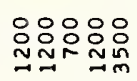 & 路: & 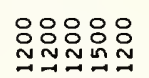 & 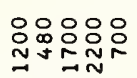 & $\begin{array}{l}0 \\
: \\
\vdots \\
0\end{array}$ & 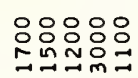 \\
\hline$w^{*}$ & 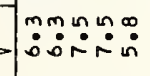 & 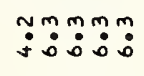 & $\ddot{m}: \ddot{m}: \stackrel{m}{0}$ & 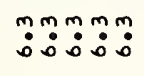 & 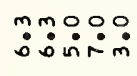 & $\ddot{m} \dot{m}: \dot{m}:$ & 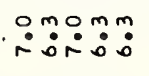 & 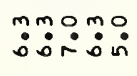 & $\stackrel{\circ}{\circ} \stackrel{m}{i}$ & 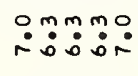 \\
\hline NOIIVG & Juanu & auvuu & บบบuั & טuטu & טuט & uบưa & טuטu & טauบa & טuטư & uUuau \\
\hline$\forall \mathrm{JW}$ & 는ை로 & 佂路 & 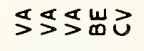 & びン゚゙す & 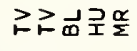 & 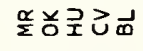 & 고ㄹㅗㅗㄴ & 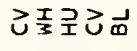 & 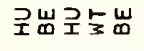 & 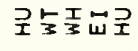 \\
\hline 3Nก1 & 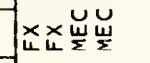 & 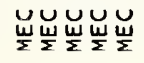 & 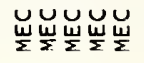 & Uu & 崖岮 5 & ヶ岕 & $\stackrel{U}{\stackrel{U}{\Sigma}} \quad \stackrel{U}{\Sigma}$ & 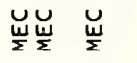 & I $\quad$ 崖 & $\underset{w}{\breve{w}}$ \\
\hline $0.16 y<190$ & 음우 & $\cong$ & & & & n & & $\stackrel{\circ}{\stackrel{2}{N}}$ & & $\stackrel{P}{\sim}$ \\
\hline 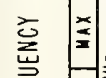 & ำ & 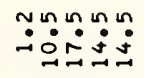 & & 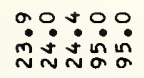 & & & & 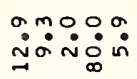 & :゚̊:゚ロ & $\because \because \because \because \vdots$ \\
\hline 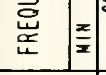 & 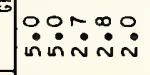 & 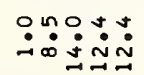 & : : : & 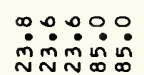 & OOサ̊n & & 무요 & 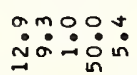 & 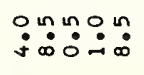 & 品華是 \\
\hline ONIX & 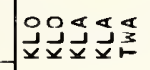 & 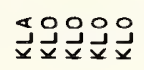 & طُ & & 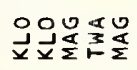 & 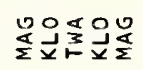 & 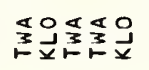 & 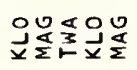 & 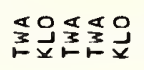 & 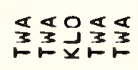 \\
\hline 708W/S & " " " & $"$ & " " " " & " " & & & & $"$ & & \\
\hline 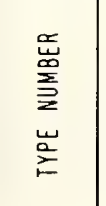 & 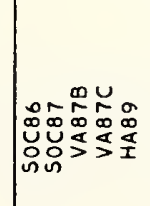 & 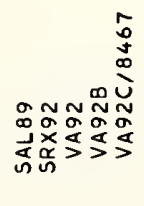 & 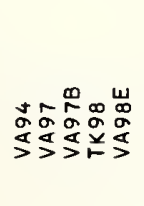 & 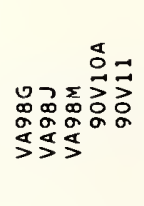 & 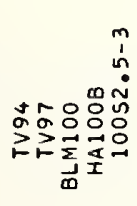 & 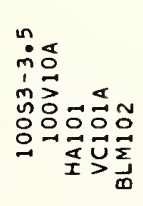 & 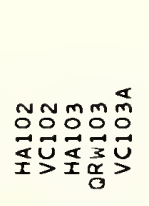 & $\begin{array}{l}\text { mom } \\
0\end{array}$ & 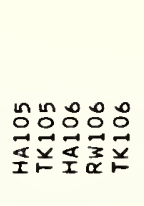 & 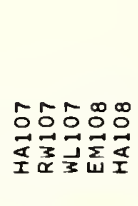 \\
\hline
\end{tabular}




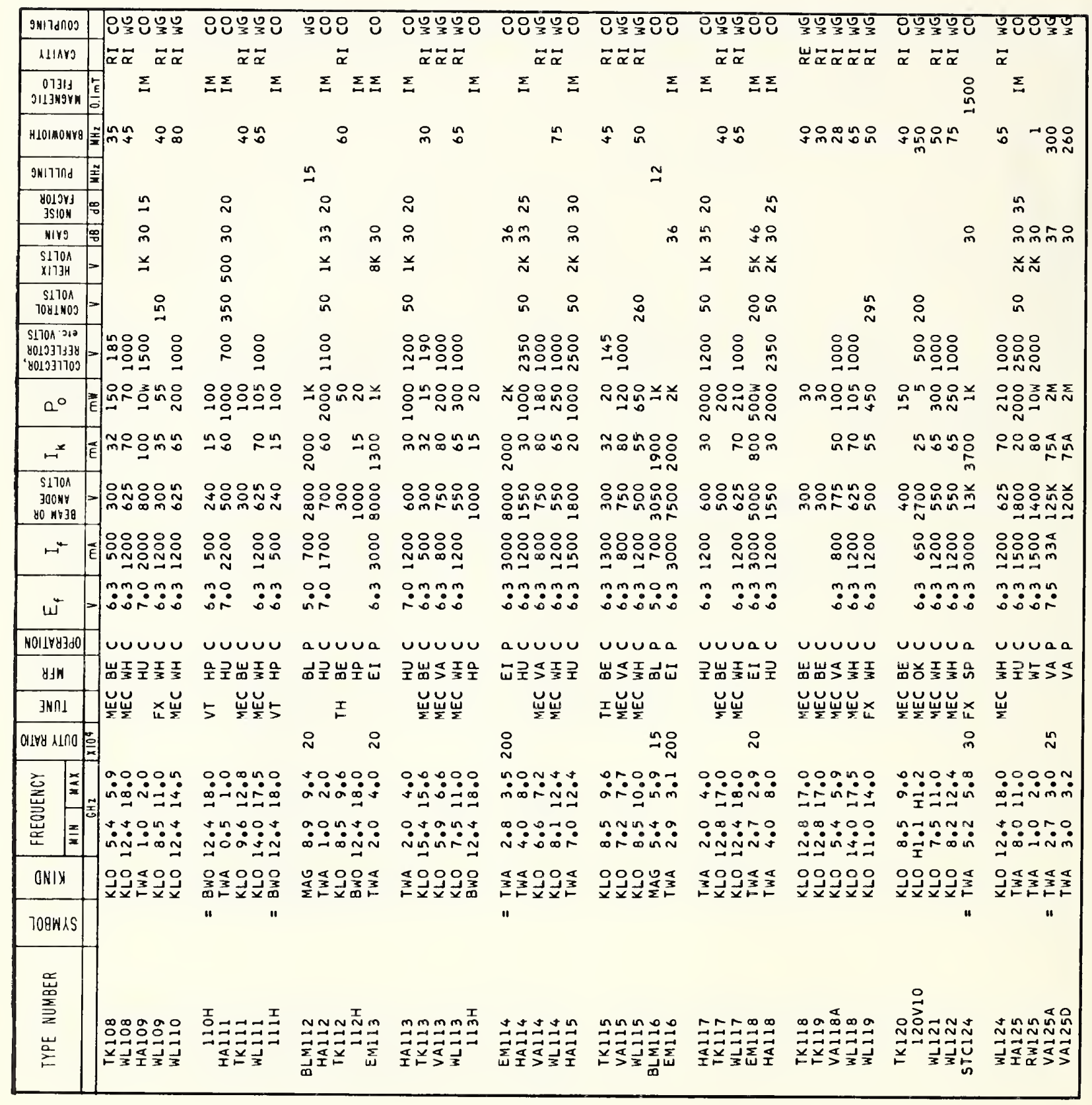




\begin{tabular}{|c|c|c|c|c|c|c|c|c|c|c|}
\hline 9MITdnos & 언오엉 & 논언언 & 언언언 & 언언옳 & 원어엉 & 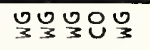 & 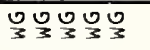 & Оुणुण & 우융어 & 8000 \\
\hline d11Aro & $\vec{\alpha}$ & $\vec{\alpha}$ & $\vec{\alpha}$ & $\vec{\alpha} \quad \vec{\alpha}$ & $\vec{\alpha}$ & $\vec{\alpha} \vec{\alpha} \vec{\alpha}$ & & $\vec{\alpha} \vec{\alpha}$ & $\vec{\alpha} \vec{\alpha} \bar{\alpha}$ & $\vec{\alpha} \quad \vec{\alpha}$ \\
\hline 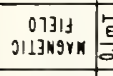 & 品 & $\Sigma \Sigma$ & $\sum \sum \Sigma \Sigma$ & $\Sigma$ & $\Sigma \Sigma$ & $\stackrel{\Xi}{\Xi}$ & $\Sigma$ & & 品 & \\
\hline H1OMONYG $=$ & $\sim$ & $\stackrel{n}{n}-\longrightarrow$ & 암윰욤요 & 욤 $\quad$ 용 & 兽壂 总 & 은 앙요 & $\stackrel{F}{F}$ & mogñn & 둥요 & $\sim$ \\
\hline 9M:17nd & $\cong$ & & & $\cong$ & $\cong$ & & 유N유 ㅇ & & $\stackrel{n}{N}$ & $N \cong$ \\
\hline $\begin{array}{l}\text { 8013Yy } \\
\text { JSION }\end{array}$ & & & $\stackrel{n}{m}$ & & & & & & & \\
\hline Hirg & $m N$ & 윰욤ํํํำ & Fิำ & 요 & mo & in $\stackrel{\circ}{N}$ & $\stackrel{\infty}{m}$ & gm & $\stackrel{\infty}{\sim}$ & \\
\hline \begin{tabular}{l|}
$5170 n$ \\
$\times 173 H$
\end{tabular}$=$ & & $\stackrel{D}{ } \underset{N}{ }$ & & & & & & & & \\
\hline $\begin{array}{c}51701 \\
1081100\end{array}=$ & $\stackrel{\circ}{n}$ & & 옹 욤 & 욤 & 只只 & 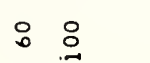 & & & $\stackrel{\Perp n}{\sim}$ & \\
\hline 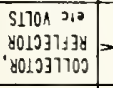 & : & 음윰유 & & & & 음 & & 욤 & 욤요 & 욤 $\stackrel{\circ}{\circ}$ \\
\hline $0^{\circ}$ & 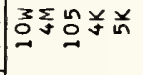 & 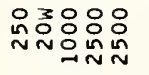 & 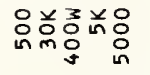 & 씅으믕ㅇㅇㅇ & 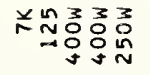 & 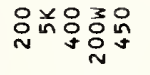 & 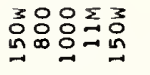 & 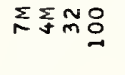 & 品品楒趾弚 & 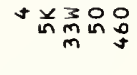 \\
\hline$-x$ & 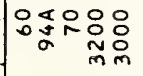 & n̊ng & 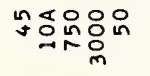 & 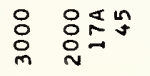 & 용용음음 & 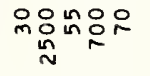 & 品造品 & 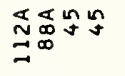 & 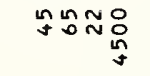 & 용 웅요 \\
\hline $\begin{array}{r}5170 \mathrm{~A} \\
300 \mathrm{WY} \\
y \in \mathrm{ABP} \\
\end{array}$ & 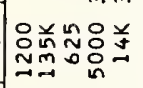 & 융요윰ㅇㅁㅇ & 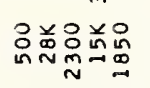 & 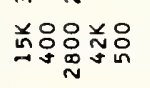 & 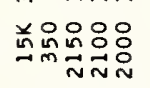 & 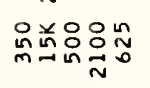 & 品 总品 & 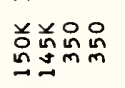 & 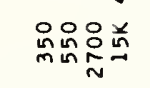 & 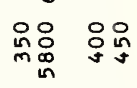 \\
\hline$F^{4}$ & 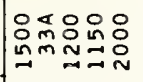 & 总品品 品 & 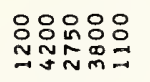 & 品 品柋品 & 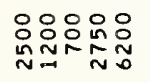 & 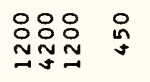 & 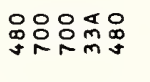 & 总售品品 & 只品品品品 & 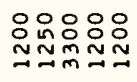 \\
\hline$\omega^{ \pm}$ & 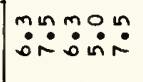 & $\ddot{0}: \stackrel{m}{:}:$ & 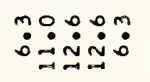 & : & 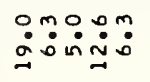 & $\stackrel{m}{:}: m: m$ & 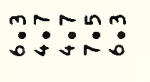 & 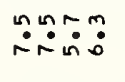 & 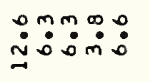 & 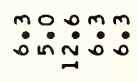 \\
\hline NOLI I8y3do & vavaa & טuvuru & vauu & auagu & auauv & vauuv & agao & a auu & avuva & va uv \\
\hline 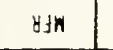 & 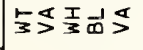 & 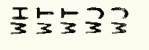 & 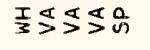 & 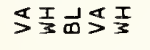 & 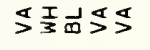 & $\frac{1}{3} \ \frac{T}{3} \ \frac{T}{3}$ & 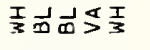 & $\triangle S \frac{T}{3} \frac{T}{3}$ & 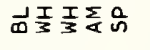 & 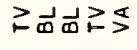 \\
\hline \begin{tabular}{|l|} 
INnI \\
\end{tabular} & 㞭 & $\stackrel{u}{\Sigma}$ & 岁 & $\begin{array}{ll}\times & \breve{u} \\
\end{array}$ & 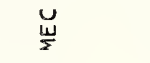 & 岀 岀 荘 & 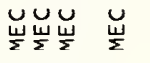 & $\underset{4}{x}$ & ×x崖岮 & 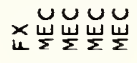 \\
\hline $0118 y$ anco & $\stackrel{i}{O} \stackrel{\circ}{N}$ & & 용 & 용 $\cong$ & $\stackrel{\circ}{\sim}$ & $\stackrel{\circ}{\sim}$ & 옷ㅇㅁㅇㅁㅇㅛ & $\pm \stackrel{N}{N}$ & in $\quad \stackrel{\sim}{N}$ & ㅇm \\
\hline 总 & 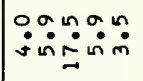 & 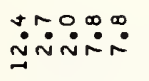 & 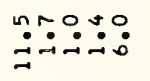 & 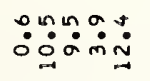 & 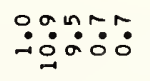 & 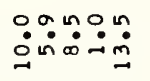 & 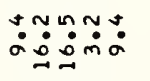 & : & 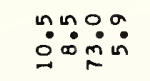 & $\stackrel{\sim}{\sim} \underset{\sim}{\sim} \stackrel{\sim}{\sim}$ \\
\hline 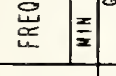 & 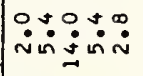 & 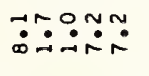 & $\ddot{0} \because \because 0 \%$ & $\ddot{0}$ & 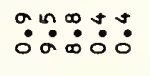 & 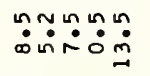 & mo:0め & : & 近品品品 & 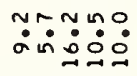 \\
\hline ONIX & 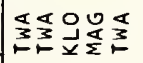 & 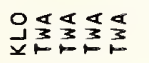 & 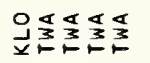 & 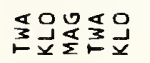 & 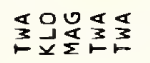 & 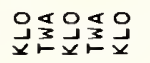 & 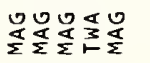 & 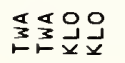 & 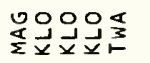 & 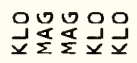 \\
\hline 700NAS & & & " " & " & $"$ & $"$ & $"$ & " " & $"$ & " " \\
\hline 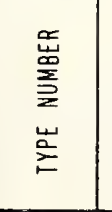 & 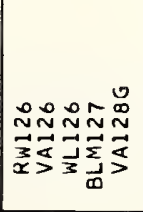 & 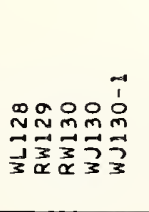 & 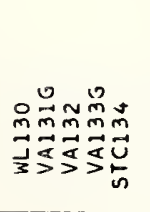 & 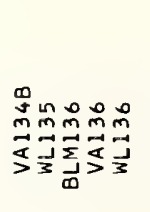 & 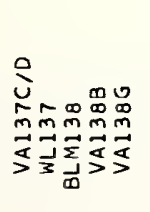 & 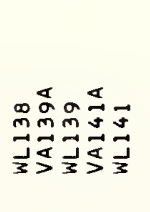 & 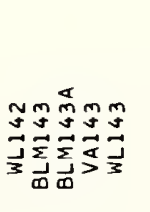 & 落 & 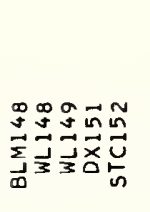 & 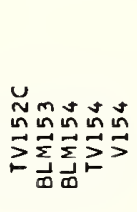 \\
\hline
\end{tabular}




\begin{tabular}{|c|c|c|c|c|c|c|c|c|c|c|}
\hline 9אוาdก0ว & 엉웡 & 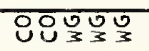 & তুতু & 웡으 & 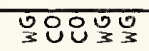 & 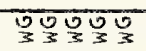 & 80505 & 80ㅇㅇㅇ & 웅연요 & 이요 \\
\hline A1AAY & 虫 & & & 亗 & & $\ddot{\alpha}$ & & $\vec{\alpha} \alpha \vec{\alpha}$ & $\mathbb{F}_{\alpha}$ & \\
\hline $\begin{array}{c}01314 \\
\text { ग1369VK }\end{array}$ & 品 & 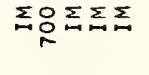 & 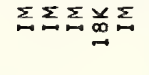 & 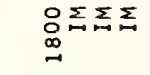 & $\sum \Sigma \Sigma \Sigma \Sigma$ & $\Sigma \Sigma \sum \Sigma$ & $\sum \sum \sum$ & $\Sigma \quad \stackrel{O}{N}$ & $\Sigma \quad \Sigma \Sigma$ & $\Sigma \Sigma \Sigma \Sigma \Sigma$ \\
\hline H.101mowre & $\stackrel{\sim}{N}$ & & & 욤어 & & $\stackrel{n}{5}$ & : & ning & 象品 & $\infty$ \\
\hline גחרורואפ & $\approx$ & & & & & & & & & $\cong$ \\
\hline${ }_{3510 \mathrm{~N}}^{\text {8010 }}=$ & $=$ & & $\circ$ & & & & & & & \\
\hline NiYg $\frac{9}{8}$ & in & $\stackrel{9}{9}$ & & $\mathfrak{m}$ & 임 & & & & $\stackrel{m}{9}$ & \\
\hline $\begin{array}{l}5170 \mathrm{~A} \\
\times 173 \mathrm{H} \\
\end{array}$ & 品 & 옳 品员品 & 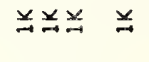 & $\ddot{\Xi}$ & $\cong \quad \cong=$ & $\underline{\Xi}$ & $\ddot{z} \quad \underline{\sim}$ & $\cong$ & $\stackrel{x}{\rightarrow}$ & 兰兰 吕 \\
\hline $\begin{array}{c}51701 \\
1081 N 00\end{array}=$ & & & $\stackrel{-1}{\circ}$ & & $\stackrel{\circ}{\circ}$ & $\frac{n}{0} \frac{n}{m}$ & $\stackrel{\circ}{\circ}$ & 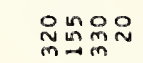 & $\stackrel{:}{N}$ & \\
\hline 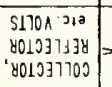 & & & & & & & 品 & $\stackrel{8}{9}$ & & \\
\hline$a^{\circ}$ & E & 웅응유요 in & 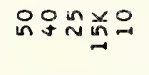 & 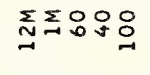 & 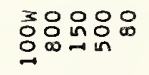 & 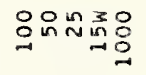 & 으증음용유 & 욤욤유 & 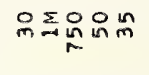 & 웅요맘요 \\
\hline 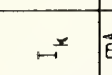 & 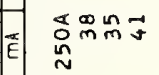 & 궁요 & กニニฐ & 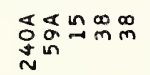 & minn & mr n & 욱 & 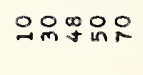 & 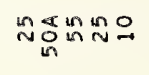 & $\pm \quad \underset{N}{\mathbb{N}}$ \\
\hline $\begin{array}{c}51700 \\
300 N y \\
80 \% 638\end{array} \mid=$ & 兑品 & 前品 : & 怘 & 㒸首号 & 응용요 & 品品总志 & : & 영용요 & 品品品品 & $\stackrel{\circ}{\stackrel{\circ}{\alpha}} \stackrel{\circ}{\sim}$ \\
\hline- & E & 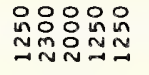 & 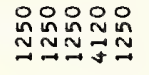 & 倠 员员 & 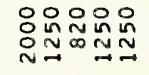 & 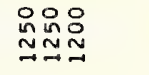 & $\begin{array}{ll}0 & 0 \\
0 \\
0\end{array}$ & 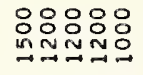 & 总品 品 & 员员尔品 \\
\hline w" & $=\operatorname{lom}$ & $\ddot{m}: \ddot{m}: m \stackrel{m}{0}:$ & $\ddot{m} \ddot{0}: \dot{m}: \dot{m}$ & $\ddot{n}: \ddot{m}$ & 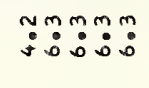 & $\ddot{m}: m$ & $\ddot{m}: \stackrel{m}{0}:$ & ח: & $\ddot{n} \ddot{0}: \stackrel{m}{0}$ & חִ: \\
\hline Nol1 $\forall y 3 d 0$ & a. auu & vouvu & uvuau & a. auu & טบטu & טuטu & טuบ & טuטu & vauuv & uบ u \\
\hline yun & $\mid \cos s \leq s$ & 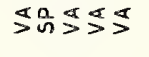 & 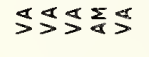 & 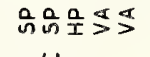 & aㅗ $>$ aㅗ & 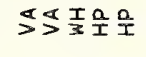 & 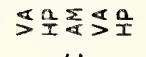 & 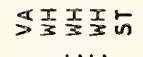 & 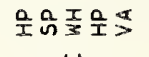 & 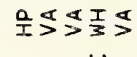 \\
\hline 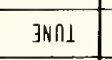 & 崖宏 & 55 & $5 \underset{4}{x}$ & xü & 㚲5 & $5 x$ & $5 \frac{u}{2}$ & 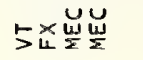 & $x_{1} \frac{u}{x} 5$ & 岁 \\
\hline 01184 xino & in & $\overrightarrow{0}$ & $\sim$ & $\underset{f}{\infty}$ & & & & & $\approx$ & $\stackrel{m}{\rightarrow}$ \\
\hline 总 & 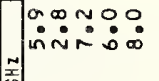 & 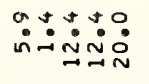 & 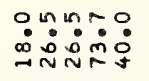 & ๓ams & 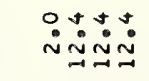 & $\begin{array}{l}+\circ 0 \circ 0 \\
\sim \\
\sim \\
\sim\end{array}$ & 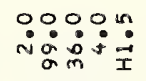 & $\because \because 00$ & 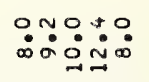 & 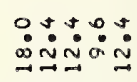 \\
\hline 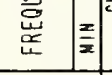 & 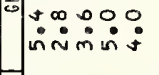 & 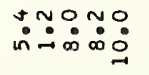 & 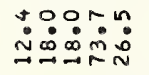 & 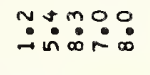 & $\begin{array}{r}\because \sim 0 \\
\times \therefore \infty \\
\times \square \infty\end{array}$ & 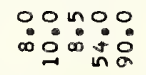 & 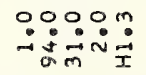 & 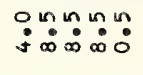 & 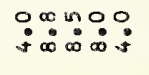 & 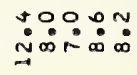 \\
\hline ONIX & 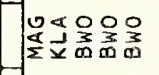 & 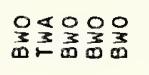 & 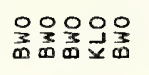 & 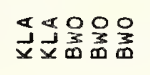 & 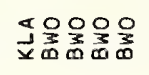 & 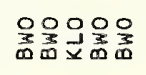 & 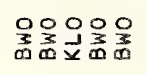 & 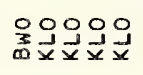 & 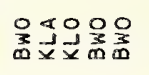 & 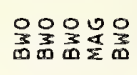 \\
\hline $709 W / S$ & & & & & & & & & & " \\
\hline 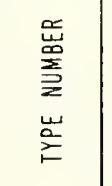 & 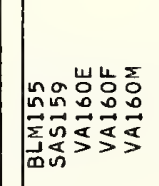 & 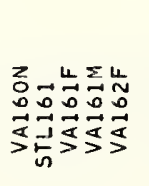 & 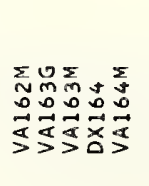 & 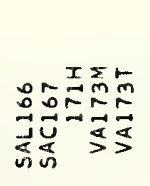 & 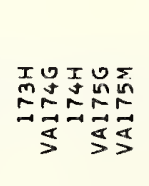 & 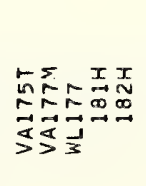 & 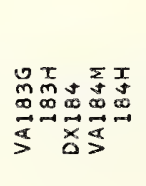 & 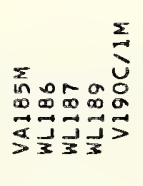 & 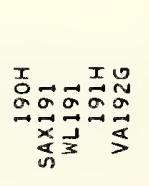 & 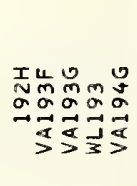 \\
\hline
\end{tabular}




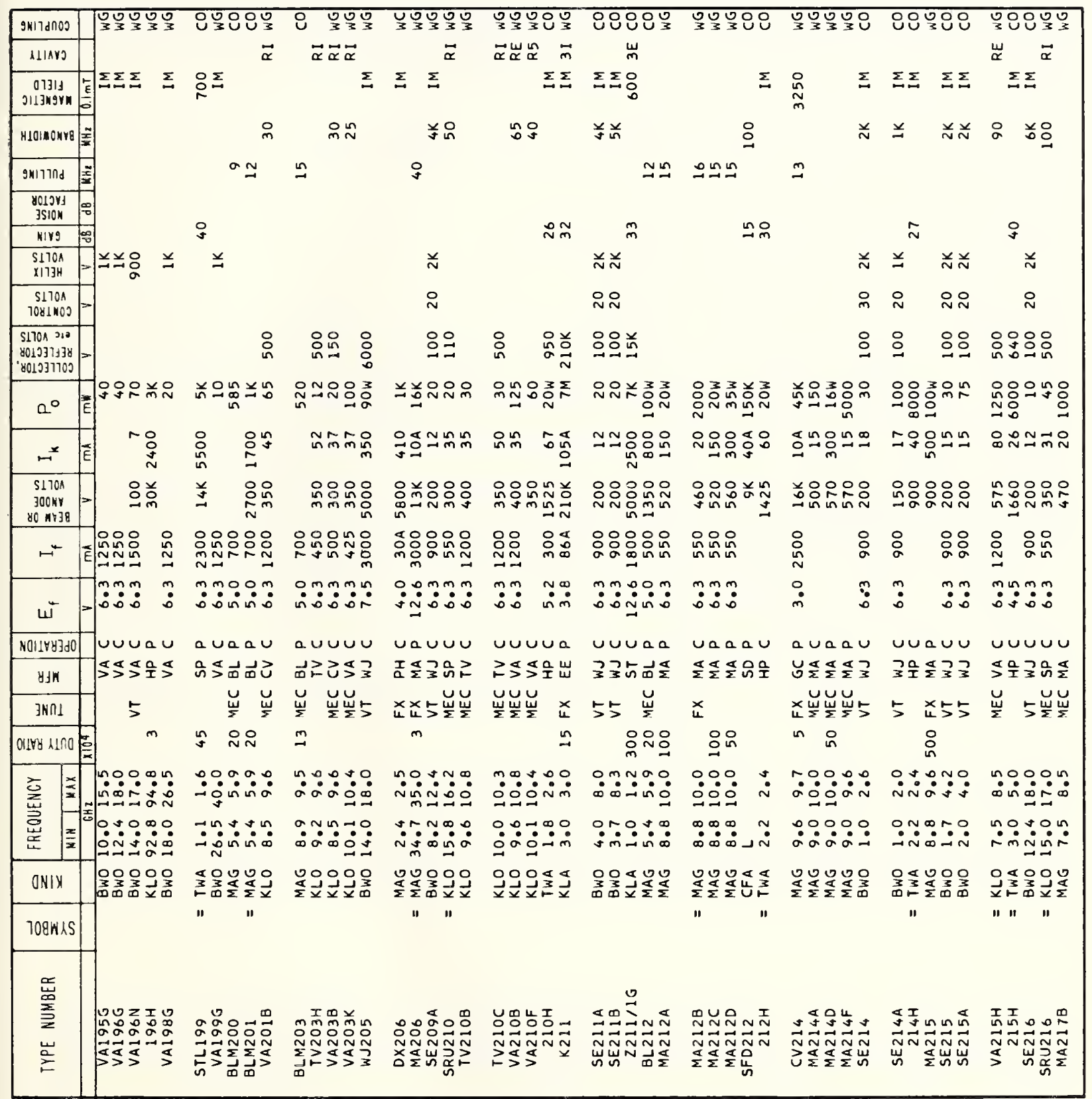




\begin{tabular}{|c|c|c|c|c|c|c|c|c|c|c|}
\hline 9HIT TnOS & گ్ర & 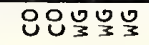 & 뭉요 & 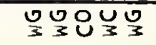 & 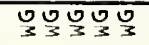 & 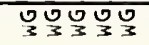 & 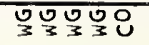 & & 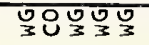 & 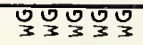 \\
\hline sliars & $\vec{\alpha} \ddot{\alpha}$ & & & $\vec{m}$ & & $\vec{\alpha} \vec{\alpha} \vec{\alpha} \underset{\alpha}{w} \boldsymbol{\alpha}$ & 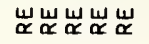 & & $\vec{\alpha} \vec{\alpha} \vec{\alpha}$ & $\vec{\alpha} \vec{\alpha} \vec{\alpha} \vec{\alpha} \vec{\alpha}$ \\
\hline $\begin{array}{l}01319 \\
\text { ग113N9\% } \\
\end{array}$ & $\Sigma \Sigma$ & $\sum \Sigma \Sigma \Sigma$ & $\Sigma \Sigma$ & $\sum \Sigma$ & & & & $\Sigma$ & $\Sigma$ & \\
\hline 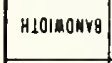 & 品 & 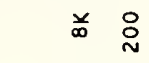 & 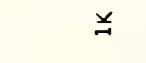 & 并 & & $\stackrel{n}{\sim} \approx$ & $\stackrel{\infty}{\sim} \stackrel{\infty}{\sim} \sim_{\sim}^{\infty}$ & & $\stackrel{\sim}{\sim}$ & \\
\hline פ פחרורח & $\underline{\underline{z}}$ & & & & & & & 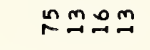 & $\stackrel{m}{\rightarrow}$ & \\
\hline $\begin{array}{l}\text { yolors } \\
\text { 3Sion }\end{array}$ & 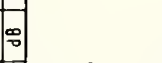 & & & & & & & & & \\
\hline Nirg & $\stackrel{\infty}{\rightarrow}$ & 前 $\stackrel{0}{-1}$ & $\stackrel{2}{\sim}$ & $\approx$ N & & & & $\stackrel{n}{\sim}$ & & \\
\hline $\begin{array}{l}51701 \\
\times 113 H \\
\end{array}$ & 品 & $\stackrel{\sim}{N}$ & $\dddot{=}$ & N & & & & & $\stackrel{x}{N}$ & \\
\hline $\begin{array}{r}5170 \mathrm{~A} \\
70 y+1 \mathrm{NOS}\end{array}$ & $\stackrel{i}{\sim}$ & $\stackrel{\circ}{\sim}$ & $\stackrel{i}{N}$ & $\stackrel{p}{N}^{N}$ & & & $\stackrel{\circ}{n}$ & & $\stackrel{\circ}{\sim}$ & \\
\hline 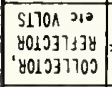 & 음 웅 & 옹요음 & 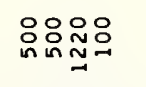 & 움 & & ㅇㅇㅇㅇㅁㅇㅁㅇ & 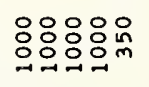 & $\stackrel{0}{r}$ & $\stackrel{8}{\circ}$ & 음 \\
\hline $\mathrm{Q}^{\circ}$ & F & 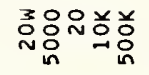 & 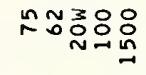 & 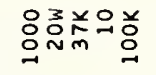 & 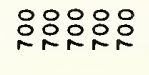 & 웃움융ㅇㅁ & 음윰요 & 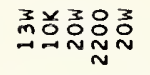 & 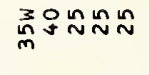 & 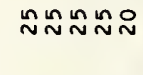 \\
\hline 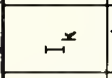 & 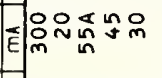 & 유쇼 & $\tilde{m} \tilde{m} \tilde{n}=0$ & 임요 & : ০০০০) & : & 잉요 & 융ㅇㅁ욤욤요 & 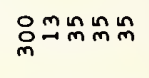 & 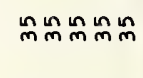 \\
\hline $\begin{array}{c}\text { S170N } \\
300 \mathrm{NY} \\
80 \mathrm{NHzB}\end{array}$ & 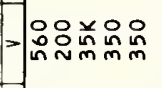 & 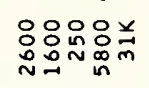 & 品品员品号 & 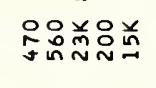 & 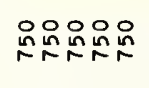 & 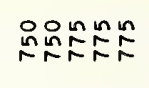 & 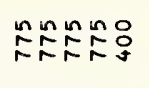 & 号 品品品 & 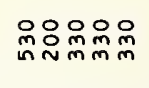 & 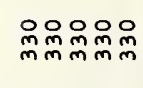 \\
\hline- & $|=| \begin{array}{l}: \\
\end{array}$ & 음음음 & 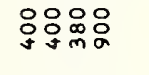 & 송요 & : & 용용요 & 음용요 & 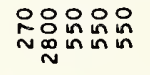 & 융ㅇㅇㅇㅇㅇㅇ & 웅ㅇㅇㅇㅇㅇㅇㅇ \\
\hline$\omega^{5}$ & $:_{0}^{m}: \ddot{0}_{0}^{m}$ & 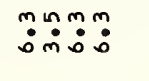 & :mm & $\because \dddot{m}$ & 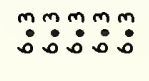 & mamm & 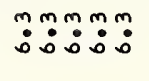 & 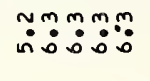 & 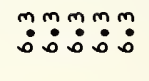 & 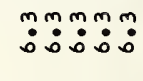 \\
\hline 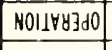 & auau & บuvaa & טuטu & U०口 U & บบบบบ & טบบบ & טบบบ & voםoa & auuuu & yuบuบ \\
\hline 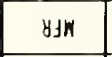 & 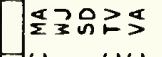 & 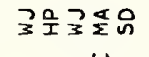 & 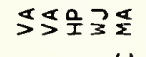 & 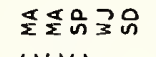 & 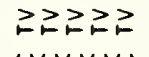 & 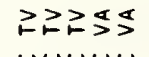 & 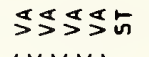 & 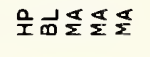 & 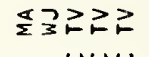 & ZZZZZZ \\
\hline 3Kก1 & 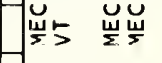 & ร㟔. & 炭 5 岂 & 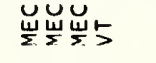 & 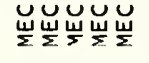 & 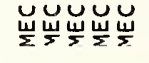 & 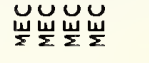 & $\underset{u}{x \times x} \times x$ & 圠岕岕岀 & U⿺辶巛ّ \\
\hline 01145 k1ก0 & in & 오 & & 요욧 & & & & no용 & in & \\
\hline 畜 & 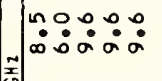 & 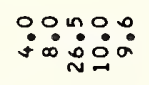 & 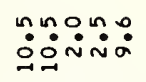 & 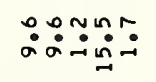 & 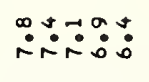 & 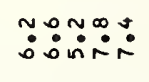 & $\because \because \because \because \because \dot{\sim}$ & 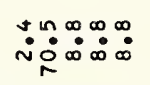 & 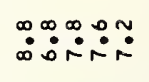 & 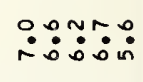 \\
\hline 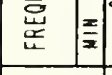 & 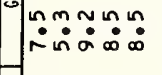 & $\because \because: \dot{\infty}$ & ڤ̊ & 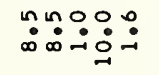 & 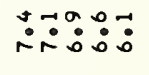 & 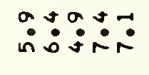 & 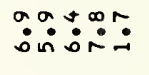 & ஸon & ஜோ:ペ: & 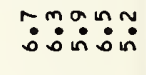 \\
\hline ONIY & 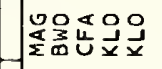 & 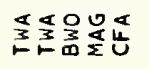 & 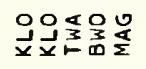 & 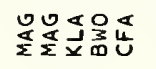 & 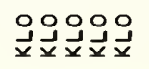 & جُ & 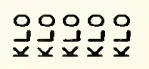 & 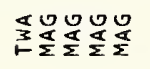 & 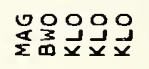 & 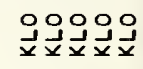 \\
\hline $708 \mathrm{~W} / \mathrm{S}$ & & " " " " & " " & " & & & & " " " " & $"$ & \\
\hline 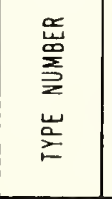 & 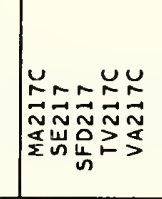 & 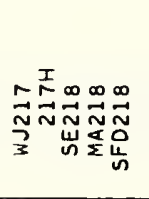 & 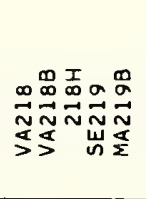 & 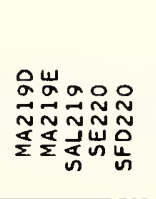 & 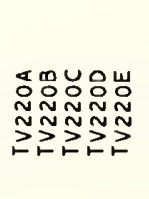 & 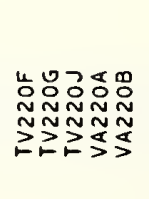 & 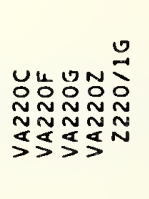 & 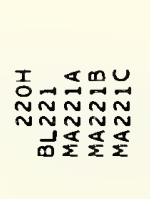 & 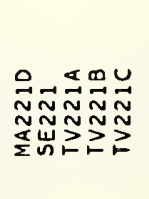 & 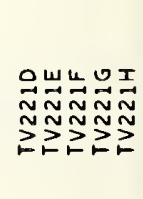 \\
\hline
\end{tabular}




\begin{tabular}{|c|c|c|c|c|c|c|c|c|c|c|}
\hline 9N17dnos & 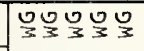 & 次 & 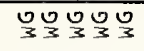 & 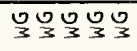 & 웅요 & ํㅜㅇㅛ & OQY3 & 언ㅁำ & 눙요 & 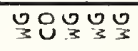 \\
\hline kl1Av3 & 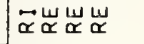 & $\vec{\alpha} \vec{\alpha} \vec{\alpha} \vec{\alpha} \vec{\alpha}$ & $\vec{\alpha} \vec{\alpha} \vec{\alpha} \vec{\alpha} \vec{\alpha}$ & $\vec{\alpha} \vec{\alpha} \vec{\alpha} \vec{\alpha} \vec{\alpha}$ & 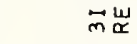 & $\stackrel{w}{\propto}$ & 爻 & $\vec{x}$ & $\underset{\alpha}{u} \quad \underset{w}{x}$ & 虍 \\
\hline 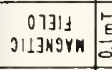 & $\Sigma$ & & & & $\sum \sum$ & $\sum \sum \sum \Sigma$ & $\stackrel{\circ}{\cong}$ & $\sum \sum$ & $\stackrel{8}{\stackrel{2}{2} \Sigma}$ & $\stackrel{\circ}{\stackrel{0}{\cong}}$ \\
\hline HLOMONAg & 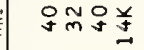 & & $\stackrel{\infty}{\sim} \underset{\sim}{\sim} \stackrel{\infty}{N}$ & $\stackrel{\infty}{\sim} \stackrel{\infty}{\sim} \underset{\sim}{\sim} \stackrel{\infty}{\sim} \underset{\sim}{\sim}$ & $\stackrel{n}{\sim}$ & $\stackrel{n}{N}$ & 즈욤 & N & $g N$ & $\sim$ \\
\hline פח & & & & & $\stackrel{n}{=}$ & & $m m$ & $m m$ & $\stackrel{n}{n}$ & $\stackrel{n}{r} \stackrel{n}{r} \underset{r}{n}$ \\
\hline $\begin{array}{l}\text { YolJYS } \\
\text { JSION }\end{array}$ & & & & & & $\stackrel{n}{m}$ & & 욤 & 운 & \\
\hline Nirg & & & & & in $\stackrel{n}{n}$ & in $\stackrel{n}{n}$ & & $\overrightarrow{+}$ & In & \\
\hline $\begin{array}{l}5170 \% \\
\times 173 H\end{array}$ & $\stackrel{x}{N}$ & & & & $\stackrel{\sim}{\sim}$ & 픈 $\underset{\sim}{\sim}$ & 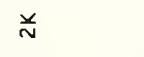 & 首并 & & \\
\hline $\begin{array}{r}51701 \\
1081403 \\
\end{array}$ & $\stackrel{i}{N}$ & & & & $\stackrel{i}{N}$ & $\stackrel{i}{n}$ & $\stackrel{N}{N}$ & & 옷N & q \\
\hline 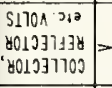 & 음욤요 & 응ㅇㅁㅇㅁㅇㅁㅇ & ㅇ::ㅇ: & ㅇ:ㅇㅇㅕ & $\stackrel{\circ}{\circ}$ & $\begin{array}{l}\circ \\
\vdots\end{array}$ & $\stackrel{\circ}{-}$ & 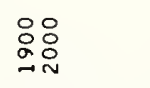 & 品 : & 草 \\
\hline $0^{\circ}$ & 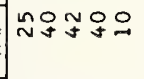 & 음옴윰요 & 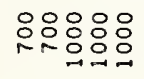 & 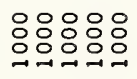 & 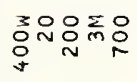 & 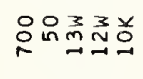 & 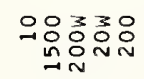 & 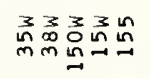 & 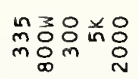 & 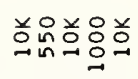 \\
\hline$\mapsto^{-x}$ & 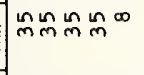 & 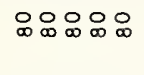 & : & 임ㅇㅇㅇㅇㅇㅇㅇ & 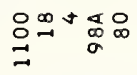 & 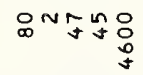 & 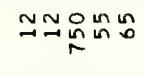 & 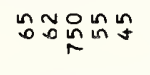 & 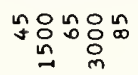 & 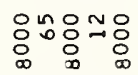 \\
\hline 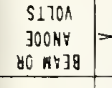 & 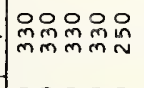 & 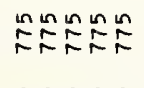 & 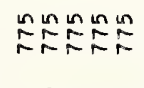 & 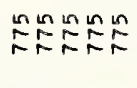 & 品品总 & 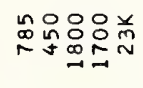 & 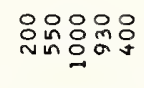 & 盛员品品占 & 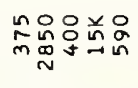 & 并品总品总 \\
\hline$F^{-}$ & 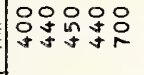 & : & 웅용요 & 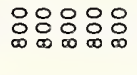 & 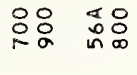 & : & 음욤욤욤요 & 음욤임 & 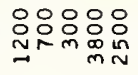 & 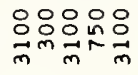 \\
\hline ш" & $m m m m$ & 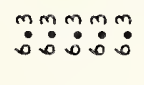 & $\ddot{n}$ & 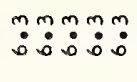 & 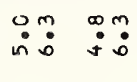 & 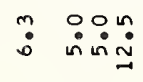 & 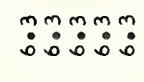 & 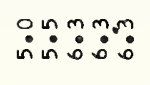 & mom & m \\
\hline NOI IYYy $\mathrm{dO}$ & טuטu & טuטu & טuט & טuטu & auuu & טuטua & ư u & ưau & vauv & $a \cup a \cup a$ \\
\hline 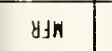 & $\not 1 \leq \leq \leq 3$ & ロロマセロ & 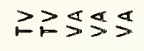 & 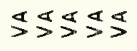 & 两踶员的 & 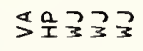 & 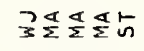 & 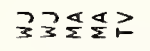 & 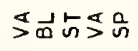 & 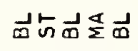 \\
\hline 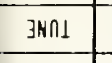 & 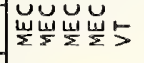 & 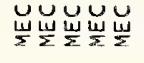 & 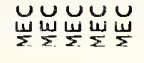 & UuWüu & 岁々 & 岁 & $5 \times x \times$ & $\underset{u}{\times} \times \frac{u}{\Sigma}$ & & $\begin{array}{lll}x & x & x \\
u & x\end{array}$ \\
\hline 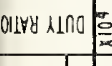 & & & & & & $\stackrel{0}{\infty}$ & 웅 & 웅 & $\stackrel{n}{\sim} 8$ & $\simeq \simeq \approx$ \\
\hline 总 & ำ & $\ddot{\sim} \because \ddot{\sim} \dot{\sim}: \dot{0}$ & 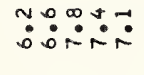 & $\ddot{\square} \ddot{0} \ddot{0}: \overrightarrow{0}$ & 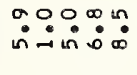 & 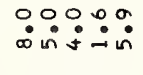 & 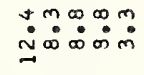 & 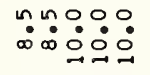 & 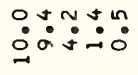 & \\
\hline 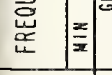 & 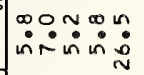 & 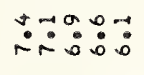 & 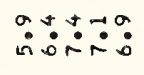 & 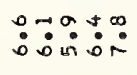 & 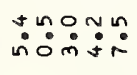 & 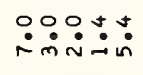 & 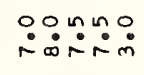 & $\dot{\sim} \dot{\sim} \dot{\infty} \infty \dot{\infty} \infty \dot{\infty}$ & 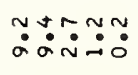 & $\begin{array}{l}0 \\
\dot{A n} \\
\dot{n}\end{array}$ \\
\hline CNIY & 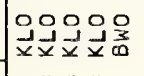 & & 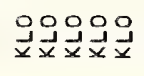 & 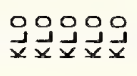 & 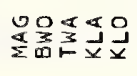 & 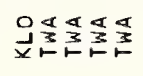 & 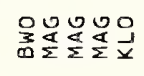 & 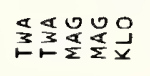 & 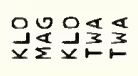 & 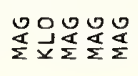 \\
\hline TogkLS & " " " & & & & & " & " " " & " " " & & \\
\hline 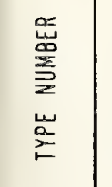 & 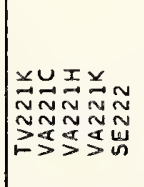 & 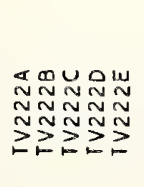 & 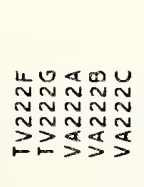 & 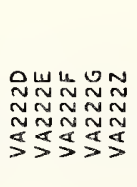 & 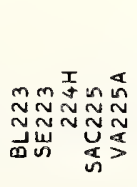 & 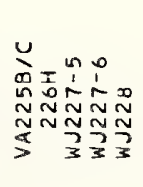 & 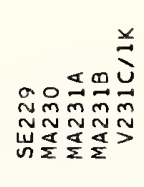 & 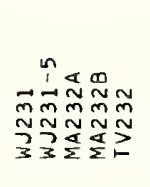 & 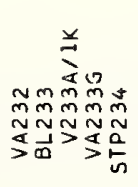 & 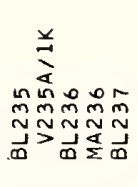 \\
\hline
\end{tabular}




\begin{tabular}{|c|c|c|c|c|c|c|c|c|c|c|}
\hline 9M1 Tdnos & $\leqslant 35$ & 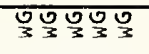 & 어원요 & 엉워요 & प్ & 영요은 & WO & 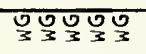 & 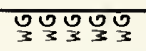 & 언웍 \\
\hline Alavg & 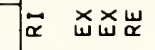 & 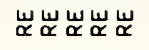 & $\stackrel{\breve{\alpha}}{\ddot{w}} \ddot{\propto}$ & 蔍 & సֻx & & $\vec{m} \vec{x} \vec{x} \vec{\alpha}$ & $\vec{\alpha} \widetilde{\alpha} \vec{\alpha} \vec{\alpha}$ & $\vec{\alpha} \underset{w}{x} \vec{\alpha}$ & $\vec{\alpha} \boldsymbol{w} \vec{\alpha} \widetilde{\alpha} \vec{\alpha}$ \\
\hline $\begin{array}{c}07315 \\
\text { ग13\%9Yพ }\end{array}$ & 旨 $\quad \underset{N}{\stackrel{O}{O}}$ & & 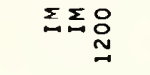 & 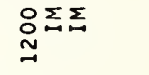 & 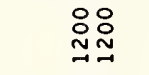 & $\Sigma$ & $\Sigma$ & & 임ำ & 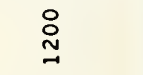 \\
\hline HLOIMONY8, & 푀요 & 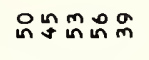 & ก & $\sim$ & Nr $=\underset{N}{N}$ & m & 응요요 & ০০০০০ & 品=品 & in \\
\hline 9 & 폰 & & & 유 & $\stackrel{\circ}{N}$ & $\approx N$ & $\stackrel{\circ}{\sim}$ & & & \\
\hline $\begin{array}{l}\text { 8019YJ } \\
\text { 3SION }\end{array}$ & & & $\stackrel{n}{m}$ & & & & & & & \\
\hline NiYg & & & 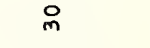 & $\tilde{N} N$ & & $\stackrel{\sim}{\sim}$ & 욤 & & & \\
\hline $\begin{array}{l}S 1701 \\
\times 1173 H\end{array}$ & & & 栾呙 & N & & 弚 & & & & \\
\hline $\begin{array}{c}51701 \\
7081400\end{array}$ & $=$ li & & o & o & 웅 & & & & 앙우욤 & q \\
\hline 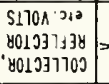 & = & 응ㅇㅇㅇㅇㅁ & 品品品品品 & 웅영 & 잉용ㅁㅁ & 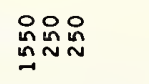 & 응요 & 임요 & 잉용ㅇㅇㅇㅇㅇㅇㅇ & 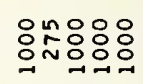 \\
\hline$\alpha^{\circ}$ & & 욤웍워 & 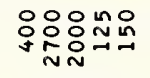 & 응중종중정 & 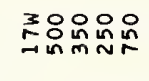 & 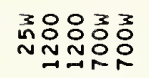 & 并部总品品 & 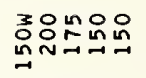 & 음욨요웅요 & 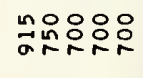 \\
\hline$-*$ & Fn요요요 & 암영여 & 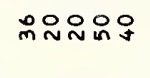 & 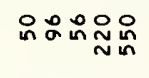 & 옹요용ㅇㅇ & 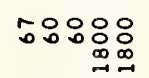 & 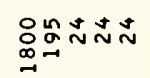 & 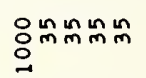 & 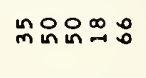 & 능용요 \\
\hline $\begin{array}{c}5170 \mathrm{~N} \\
30 \mathrm{NH} \\
80 \mathrm{Mr} 38\end{array}$ & $\mid \begin{array}{l}\text { Oon } \\
0 \\
0\end{array}$ & : & 양용용요 & 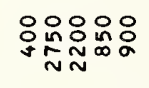 & 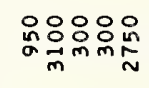 & 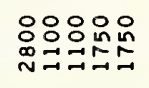 & 웃융ㅇㅇㅇㅇㅇ & 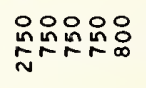 & 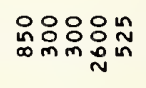 & 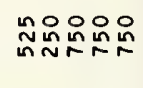 \\
\hline$\leftarrow$ & E & 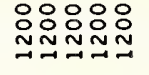 & 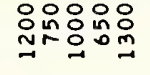 & 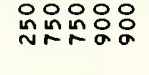 & 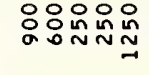 & 양응ㅇㅁㅇㅇㅇ & 응용ㅇㅇㅇㅇㅇㅇ & 总品品品 & 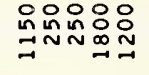 & 육윰요 \\
\hline$\omega^{ \pm}$ & 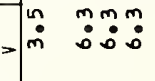 & 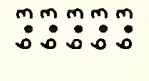 & :0: & & "מ: & mmmm & 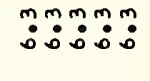 & m:m: & & 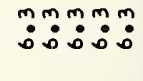 \\
\hline NOII $\forall y \exists] \mathrm{dO}$ & טuטu & uบuvu & טuטu & uUua & uuvur & บUบaa & auUuu & aus & บบบบบ & טบบบ \\
\hline$y+n$ & 高ららう & ダダタ & 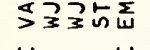 & 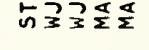 & 高䏠ららな & そららミミ文 & 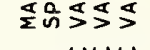 & 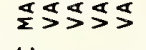 & アらららミ & アロロロロる \\
\hline \begin{tabular}{c|}
$3 \mathrm{~N} \cap 1$ \\
$011 \forall \forall \times 1 \cap 0$
\end{tabular} & $w \underline{w} \quad \underline{w}$ & w w w w w w & W $\quad$ W & $\begin{array}{l}\times x \\
\mathbb{L} \\
\mathbb{N} \\
\mathbb{N}\end{array}$ & $x_{u}^{x} \underset{z}{\bar{w}}$ & 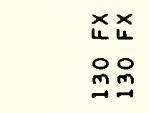 & 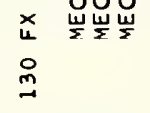 & 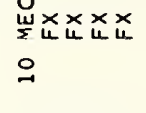 & 妾 & x $\frac{w}{\Sigma} w_{\Sigma}$ \\
\hline 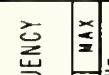 & 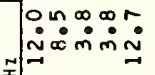 & 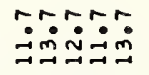 & 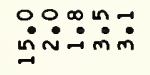 & $\ddot{s} \dot{q}: \dot{m}: \dot{m}$ & 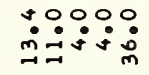 & 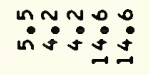 & 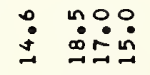 & :O:O:O: & 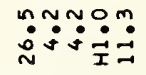 & 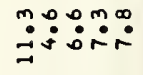 \\
\hline 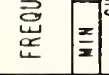 & & ํㅜㅇㅇㅛ & 过 & mºmmo & $\ddot{m} \dot{m} 0 \dot{m}^{\infty} \dot{m} \dot{m}$ & 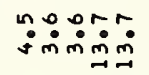 & 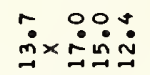 & $\overrightarrow{0}: 0000$ & 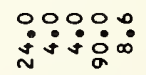 & 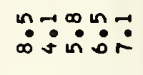 \\
\hline ONIX & 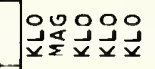 & 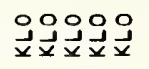 & 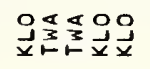 & 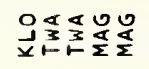 & 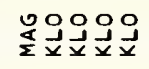 & 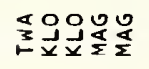 & 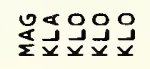 & 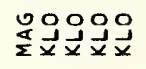 & & 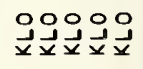 \\
\hline 70gW/S & & & & & & & " " & & & \\
\hline 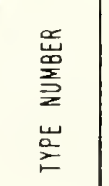 & 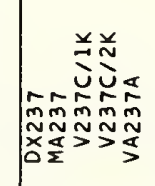 & 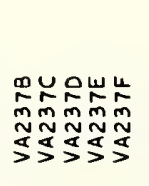 & 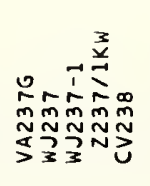 & 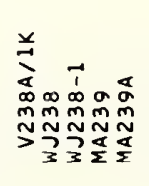 & 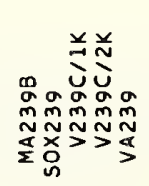 & 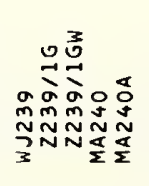 & 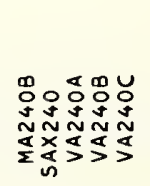 & 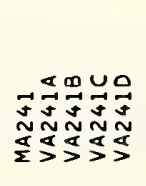 & 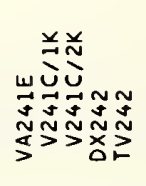 & 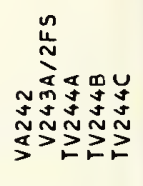 \\
\hline
\end{tabular}




\begin{tabular}{|c|c|c|c|c|c|c|c|c|c|c|}
\hline 9M17dnOS & ऊणु३ & З & 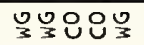 & 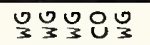 & 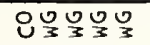 & 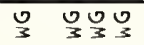 & 뭉요 & 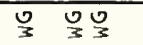 & 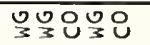 & ב \\
\hline dilavo & $\vec{\alpha} \vec{\alpha} \vec{\alpha} \vec{\alpha} \vec{\alpha}$ & $\vec{\alpha} \vec{\alpha} \vec{\alpha} \vec{\alpha} \vec{\alpha}$ & $\vec{\alpha} \vec{\alpha}$ & $\stackrel{x}{\omega} \quad \vec{m} \vec{x}$ & 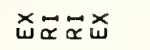 & $\vec{\alpha} \quad \underset{w}{\alpha} \vec{\alpha}$ & $\vec{x}$ & $\vec{\alpha} \vec{\alpha}$ & $\underset{\alpha}{u} \quad \vec{\alpha}$ & $\vec{m} \vec{\alpha} \vec{\alpha}$ \\
\hline 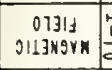 & & & $\Sigma$ & & $\stackrel{\circ}{\stackrel{一}{二}}$ & 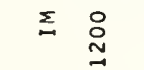 & $\sum \Sigma$ & & $\Sigma \Sigma$ & $\Sigma$ \\
\hline H10 Mowro: & n & 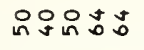 & 苛 & $\infty$ & ñ: $0^{\infty}$ & in $\quad \infty \stackrel{\infty}{m}$ & 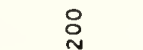 & $\because$ in in & 용 & in \\
\hline 9พוากา & & & $\approx 0$ & no & & & $\stackrel{\circ}{-}$ & 으 & $\stackrel{n}{\sim}$ & \\
\hline 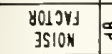 & & & & & & & & & q & \\
\hline Nirs $=$ & & & $\stackrel{s}{m}$ & $\stackrel{\text { m }}{2}$ & & in & 盀 & & $\tilde{m}$ & $\stackrel{m}{m}$ \\
\hline $\begin{array}{l}\text { S170A } \\
\text { XIIJH }\end{array}$ & & & $\underline{\exists}$ & & & $\stackrel{\sim}{\sim}$ & 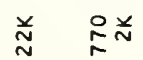 & & $\ddot{*}$ & \\
\hline $\begin{array}{c}S 1701 \\
70 y+1103 \\
\end{array}$ & & & & q & 웅 & o & & & $\stackrel{\sim}{\sim}$ & $\underline{\exists}$ \\
\hline 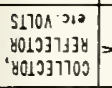 & $=\left(\begin{array}{ll}\circ & \stackrel{n}{*} \\
0 & \multirow{\sigma}{*}{}\end{array}\right.$ & 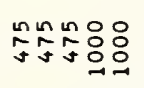 & 客品宫 & $\stackrel{\circ}{\circ}$ & 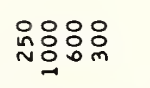 & 品 菂品 & 克资足 & 웅요 & 임 & \\
\hline$a^{\circ}$ & 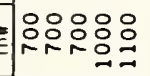 & 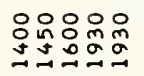 & 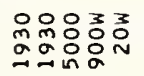 & 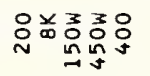 & 앳웡유요 & 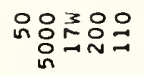 & 品前克品品 & 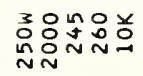 & : & 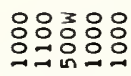 \\
\hline$\mapsto$ & 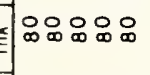 & 잉잉ㅇㅇㅇㅛ & 임욤요 & 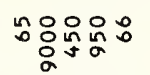 & 웅요 용요 & 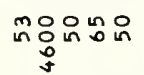 & 萹品 & 융요묘 $\underset{\sim}{n} \underset{n}{n}$ & 임욤요 & 유솟ำ \\
\hline 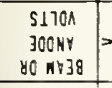 & 굿용용요 & 잉잉잉 & 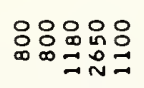 & 品 潧品品 & 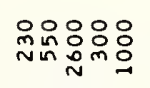 & 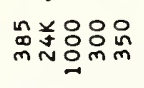 & 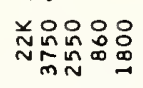 & 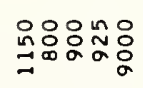 & 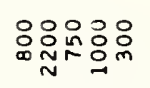 & 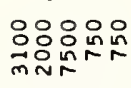 \\
\hline$\varpi^{-}$ & = & 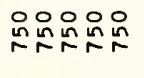 & 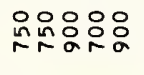 & 윰요 & 郘总品品 & 品 总品 & 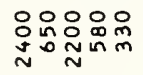 & 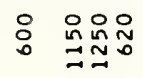 & 웃옹욤욤요 & 옷윰욧요 \\
\hline$\omega^{ \pm}$ & 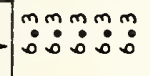 & 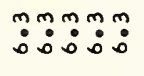 & 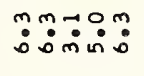 & : & $\dot{m}: \dot{m}: m$ & 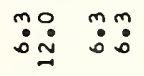 & 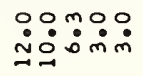 & 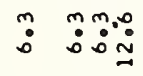 & mo: & 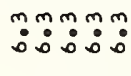 \\
\hline N01178380 & טuטu & טuטu & ם טים & Ua & טuטu & טauUu & Q a Uu & a & טu ט & Ua \\
\hline $84 \mathrm{~m}$ & ママママタ & 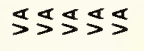 & 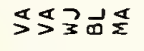 & 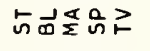 & らンエロ文 & 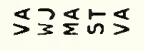 & 锌的? & $\left.\frac{\pi}{\Sigma} \frac{\pi}{\Sigma}\right\rangle \frac{\pi}{\Sigma}$ & 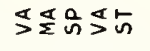 & 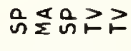 \\
\hline JNก1 & 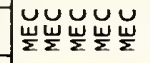 & 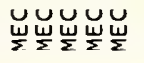 & 岀耑 $\quad x$ & 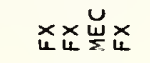 & 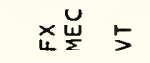 & 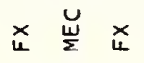 & $\frac{x}{u}$ & 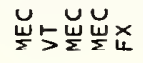 & 岀区 崫 & 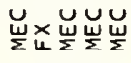 \\
\hline 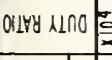 & & & 요ํ & n요요 & & $\stackrel{2}{\sim}$ & iํ & in & i & 유요 \\
\hline 产 & 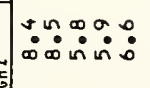 & 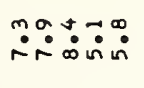 & mana: & 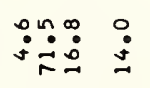 & 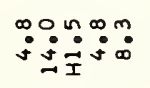 & 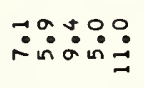 & $\because 0: 00$ & 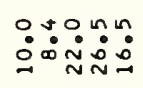 & & 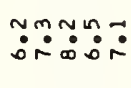 \\
\hline \begin{tabular}{l|l}
$\underline{x}$ & $\frac{x}{2}$ \\
\end{tabular} & 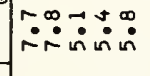 & 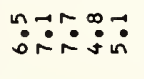 & 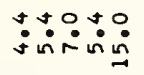 & ホon & 寸゚ง:웅 & 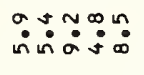 & 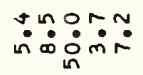 & Oㅇㅇㅇ & 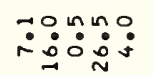 & Noำ \\
\hline ONIX & 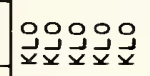 & 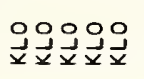 & 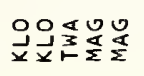 & 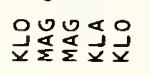 & 울울월 & 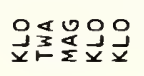 & 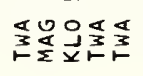 & 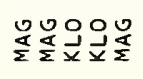 & 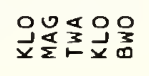 & 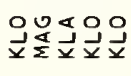 \\
\hline 708W $1 \mathrm{SS}$ & & & & & & & " " & & $"$ & " \\
\hline 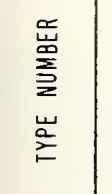 & 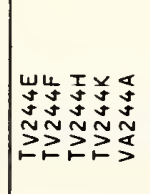 & 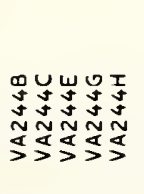 & 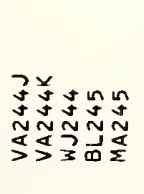 & 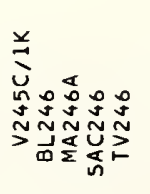 & 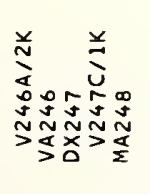 & 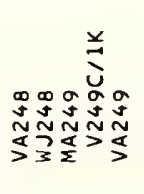 & 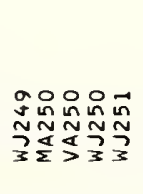 & 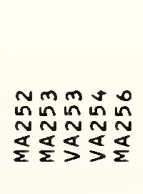 & 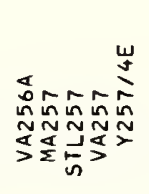 & 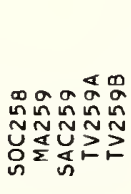 \\
\hline
\end{tabular}




\begin{tabular}{|c|c|c|c|c|c|c|c|c|c|c|}
\hline 9MIId dnos & 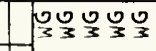 & 원요 & 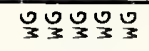 & 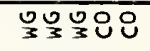 & 우웅요 & 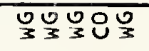 & 운워원 & 원 요 & Oㅇ & 웡ㅇㅁ \\
\hline RLAAY & $\vec{\alpha} \vec{\alpha} \vec{\alpha} \vec{\alpha} \vec{\alpha}$ & $\underset{w}{x}$ & $\vec{\alpha} \tilde{\alpha} \tilde{\alpha} \underset{\widetilde{\alpha}}{\boldsymbol{\alpha}}$ & $\vec{\alpha} \times \vec{u}$ & 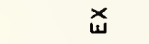 & జ゙n $\quad$ u & $\underset{\alpha}{\tilde{\alpha}} \quad \ddot{\alpha}$ & $\ddot{\alpha}$ & $\ddot{x}$ & $\ddot{\alpha}$ \\
\hline $\begin{array}{c}011319 \\
\text { ग113n9v" }\end{array}$ & & $\Sigma$ & $\Sigma$ & $\sum \sum \sum$ & $\sum \sum g_{n} \sum_{n}$ & $\Sigma \Sigma$ & $\Sigma \Sigma$ & $\sum \Sigma \Sigma \Sigma$ & $\Sigma$ & $\Sigma \sum \Sigma$ \\
\hline HLOMOHYG & ถู & $\tilde{n}$ & 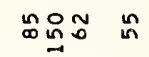 & $\underset{\infty}{\infty}$ & $\infty$ & nñ & 요 ${ }_{m}^{m}$ & & in & $\stackrel{\circ}{\circ}$ \\
\hline גחרוואפ & 솦 & & & & & & & & & \\
\hline $\begin{array}{l}0012 Y \mathrm{~S} \\
\text { 3SIOH } \\
\end{array}$ & $\approx$ & & & no & O D & $\approx$ & $\stackrel{n}{m} 0$ & $\operatorname{lin}_{n} n_{n}$ & $\infty$ & \\
\hline nirg & $\nabla$ & $: 8 m$ & & $\stackrel{n}{N}$ & $\stackrel{n}{\sim} \cong \stackrel{n}{\sim}$ & $\stackrel{p}{m}$ & $\stackrel{m}{n} \stackrel{n}{\sim}$ & $\tilde{m} \tilde{n} \stackrel{m}{m} \tilde{m}$ & $\stackrel{n}{m} \quad \stackrel{\infty}{N}$ & in \\
\hline $\begin{array}{l}51701 \\
\times 11734 \\
\end{array}$ & & & & & & $\stackrel{x}{N}$ & & & $\underset{\sim}{\stackrel{䒑}{\sim}}$ & 原 \\
\hline $\begin{array}{c}5170 \mathrm{~A} \\
1081102\end{array}$ & & $\stackrel{s}{\infty}$ & $\stackrel{\circ}{n}$ & in & in & in & $\hat{m}$ & $\stackrel{\circ}{\stackrel{0}{N}}$ & & in \\
\hline 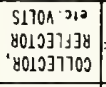 & 응음요 & 음 욤욤 & 음윰엄 & 움용ㅇㅁ & in & 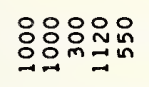 & 응 : & $:$ & $:$ & 응용요 \\
\hline $0^{\circ}$ & = & 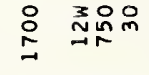 & 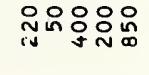 & 임요 & 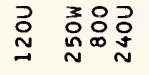 & 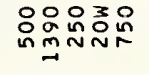 & 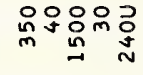 & 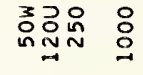 & 윰조옹요 & 吠录录兑 \\
\hline 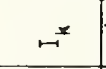 & E & 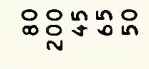 & Nㅗ요요 & min & 용 & ํำ & 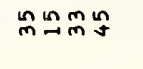 & $\stackrel{\text { D }}{\circ}$ & moginn & 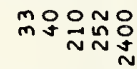 \\
\hline $\begin{array}{c}5170 \mathrm{~A} \\
30 \mathrm{NH} \\
80 \mathrm{Ag3g} \\
\end{array}$ & 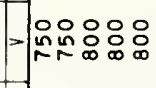 & :용유 & 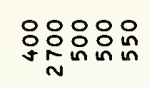 & 윰용 & 융응 & 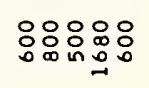 & 品品品品 & 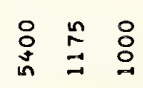 & 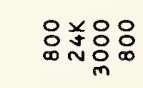 & 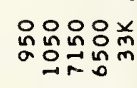 \\
\hline$\varpi$ & 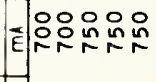 & 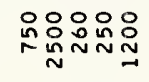 & 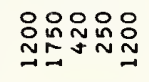 & ํํํํํํำ & 윰 & 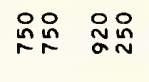 & 足员品品 & 只 : & 용용요 & 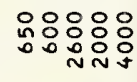 \\
\hline$\omega^{4}$ & 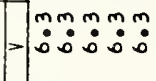 & $\ddot{m}: m: m$ & 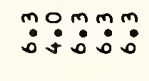 & $\ddot{m}: m$ & $\stackrel{0}{\stackrel{0}{*}}$ & $\ddot{m} \ddot{m}: \ddot{m}$ & $\stackrel{m}{m}: m m$ & $\stackrel{m}{\ddot{0}} \stackrel{0}{\vdots} \cdot$ & : & :m:m \\
\hline NO11 $19 y] d 0$ & טu טu & uauuv & uบuบu & vouvu & טuบuบ & טuטu & uטuンu & auvu & טuטu & บบบบa \\
\hline$y=$ & 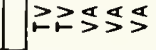 & 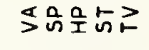 & 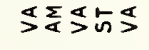 & 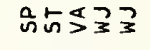 & ろろ路? & 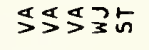 & 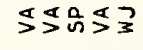 & 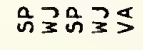 & ろォろニム & 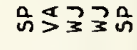 \\
\hline $3 \mathrm{~N} \cap 1$ & 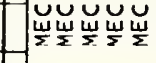 & $\stackrel{\breve{w}}{\stackrel{u}{\Sigma}}$ & 岀岀岕 岀 & 岀 & & $\underset{w}{u} x_{u}$ & 岁岕 × & x. & $\underset{4}{x}$ & 岀 \\
\hline Ollyy uIroo & & $\stackrel{n}{r}$ & & & & & & & & $\simeq$ \\
\hline 总 & 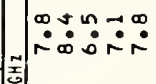 & 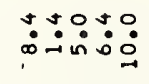 & 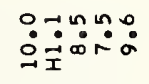 & 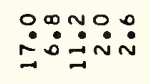 & 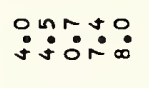 & 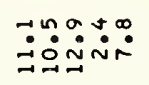 & 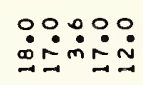 & 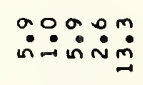 & 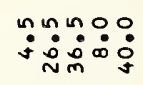 & 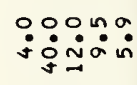 \\
\hline \begin{tabular}{l|l}
$\substack{\tilde{\alpha} \\
世}$ & $\frac{x}{2}$ \\
\end{tabular} & 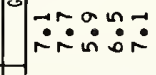 & 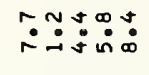 & 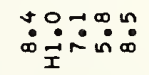 & ஜேஃ゚ロ & i்: & 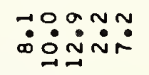 & 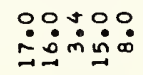 & 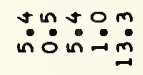 & 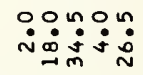 & 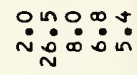 \\
\hline ONIX & |ج & 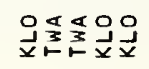 & 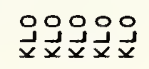 & 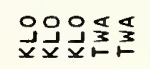 & 造乐乐是乐 & 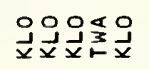 & 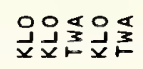 & 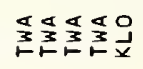 & 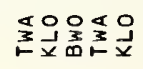 & 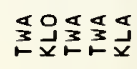 \\
\hline 70gW/S & & " & & & & " " & & & & " \\
\hline 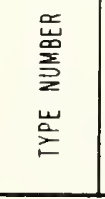 & 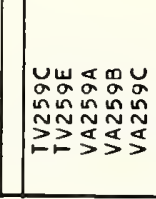 & 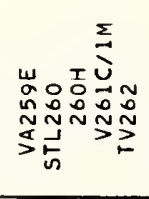 & 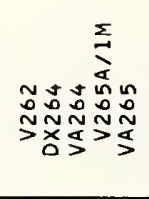 & 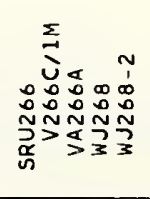 & 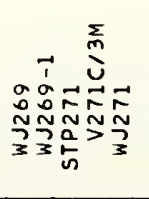 & 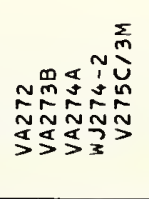 & 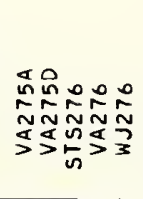 & 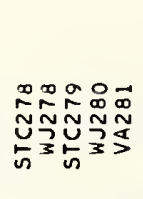 & 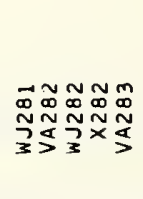 & 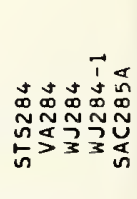 \\
\hline
\end{tabular}




\begin{tabular}{|c|c|c|c|c|c|c|c|c|c|c|}
\hline 9N17dחOO & 언연운 & 언워 & 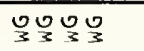 & 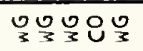 & 꾼언온은 & \ִ & 언원요 & U్ & 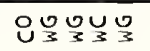 & ЗО \\
\hline AlAAY & $\vec{\alpha} \quad \underset{\alpha}{\sim} \underset{\alpha}{\sim} \underset{\alpha}{\alpha}$ & $\underset{\alpha}{u} \quad \vec{\alpha} \vec{\alpha}$ & $\vec{\alpha} \vec{\alpha} \vec{\alpha} \vec{\alpha} \vec{N}$ & $\vec{\alpha} \vec{\alpha} \ddot{\alpha} \quad \vec{\alpha}$ & $\underset{\propto}{\breve{~}}$ & 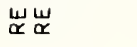 & $\vec{\alpha} \ddot{\alpha} \quad \vec{\alpha}$ & $\vec{\alpha}$ & $\vec{\alpha} \vec{\alpha}$ & $\vec{\alpha} \quad \vec{\alpha}$ \\
\hline 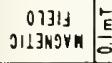 & 会 & $\Sigma \Sigma$ & & $\Sigma$ & $\sum \Sigma \sum$ & & $\Sigma \Sigma$ & $\Sigma \sum \Sigma \Sigma$ & $\Sigma \Sigma \Sigma$ & $\Sigma \sum \Sigma$ \\
\hline HLOIMONY & 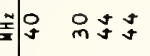 & a & 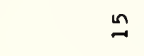 & n & 요 & Nํㅇㅇㅝ & 品㫌 & 䠰羊 & F $\stackrel{n}{n} \underset{\sim}{n} \stackrel{n}{m}$ & 影品 并 \\
\hline 9אורח & 妾 & & & & & & $\stackrel{i}{\sim}$ & $\circ$ & t & \\
\hline $\begin{array}{l}\text { yoloys } \\
\text { Jsion }\end{array}$ & $\infty$ & Oㄹ & & $\infty$ & $\infty a$ ? & & & & & \\
\hline NIY & $\stackrel{n}{m}$ & min & & $\stackrel{n}{N}$ & $\tilde{N} \stackrel{n}{N}$ & & & & & $\underset{f}{q}$ \\
\hline $\begin{array}{l}\text { S170N } \\
\times 173 \mathrm{H}\end{array}$ & & & & & & & $\stackrel{\mathrm{N}}{\mathrm{N}}$ & $\stackrel{x}{N}$ & $\stackrel{\Perp}{N}$ & $\dddot{\exists}$ \\
\hline $\begin{array}{c}51700 \\
7001403 \\
\end{array}$ & & $\stackrel{\circ}{\sim}$ & 品品品品 & $\stackrel{\circ}{\stackrel{0}{N}}$ & & & $\stackrel{\circ}{N}$ & $\stackrel{\sim}{\sim}$ & $\stackrel{\circ}{\sim}$ & 윰 $\stackrel{\circ}{N}$ \\
\hline 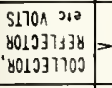 & $\mid$\begin{tabular}{lll}
0 & 0 & 0 \\
0 & 0 & 0 \\
0 & 0 & 0 \\
\hdashline & 0 & 0
\end{tabular} & 总品 & 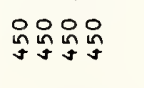 & 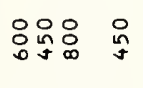 & 음 & 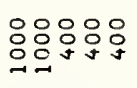 & 음요 & 음음엄 & 옥 윽욤 & 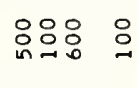 \\
\hline $0^{\circ}$ & Ell & 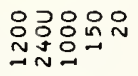 & 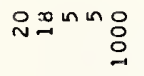 & 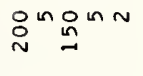 & 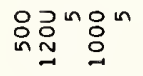 & :::요 & 임음ㅇㅇ임요 & 음유 & $\underset{\sim}{0} \underset{\sim}{0} \stackrel{n}{\sim} \stackrel{n}{\sim} \underset{N}{0}$ & 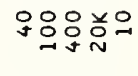 \\
\hline 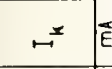 & 各走去 & * ำำ & 뜨유요 & miñ & $\therefore \quad$ & $\mathfrak{n}_{\infty}^{n} \stackrel{\sim}{n} \vec{\infty} \vec{\infty} \vec{\infty}$ & 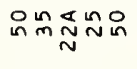 & N & ㄸ్ల & an \\
\hline 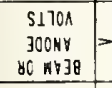 & 总足足 & 웃 总品号品 & 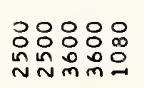 & 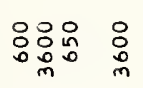 & $\stackrel{\circ}{n}$ & 응요ㅇㅛㅛ쇼쇼 & 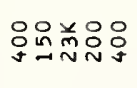 & 品品尊鬲 & 옷ㅇㅇㅇㅇㅇㅇㅇ & 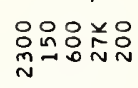 \\
\hline$\rightarrow$ & 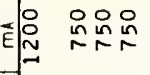 & 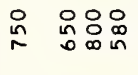 & $\begin{array}{l}O \\
0\end{array}$ & 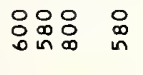 & 号 & 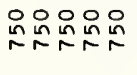 & 응ㅇㅇㅇ영 & 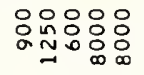 & $\begin{array}{l}\circ \vdots 0 \\
0 \pm 0\end{array}$ & 品品 : \\
\hline$\omega^{\star}$ & 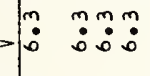 & 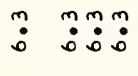 & 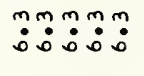 & 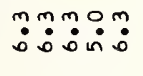 & 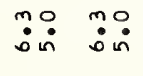 & 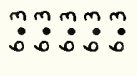 & 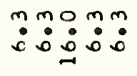 & mmm & 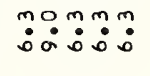 & 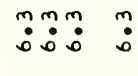 \\
\hline NO1_Hy\}dO & & บบบบ & บบบบบ & บบบบบ & บบบบบ & บบบuv & ưau & uuva & vauuv & uUuau \\
\hline$\forall A M$ & \つฺタ & ১プロ & 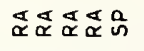 & 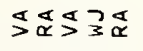 & 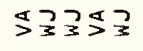 & 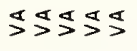 & 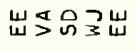 & 岕出岕に & 岕灾岕ऽ & ๔出〉足虫 \\
\hline 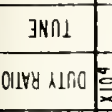 & $\left.\right|^{u}$ & 荘 岀嵌 & 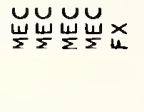 & 嵌岀 岁 & $\begin{array}{ll}x & \breve{w} \\
\end{array}$ & 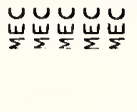 & 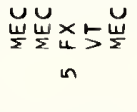 & $\begin{array}{r}5 \times 5 \times x \\
000 \\
00\end{array}$ & 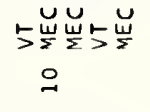 & 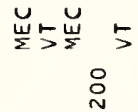 \\
\hline 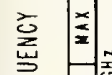 & 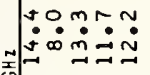 & 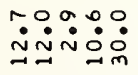 & 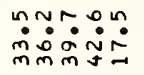 & ¿: & $\because \because 0 \div 0$ & 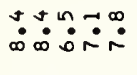 & 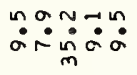 & 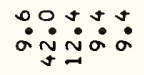 & 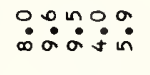 & 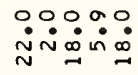 \\
\hline 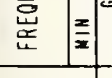 & 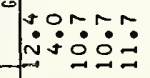 & 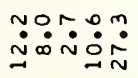 & 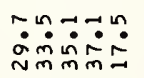 & 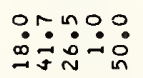 & 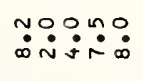 & 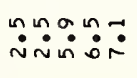 & 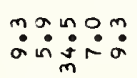 & 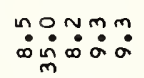 & 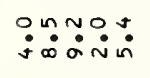 & \\
\hline ONIX & 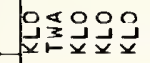 & 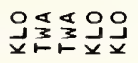 & 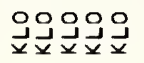 & 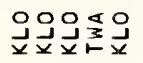 & 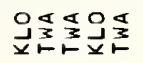 & 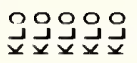 & 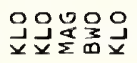 & 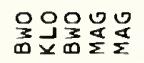 & 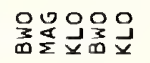 & 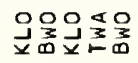 \\
\hline 70gW/S & & & & & & & & " & & \\
\hline 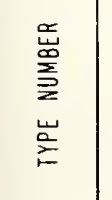 & 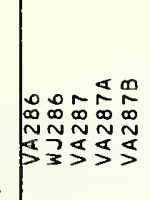 & 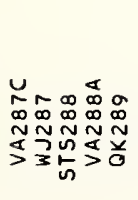 & 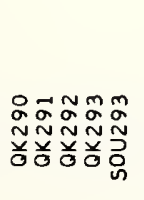 & 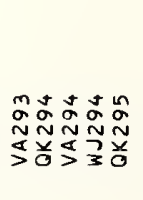 & 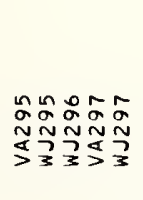 & 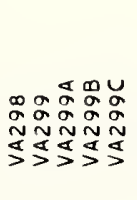 & 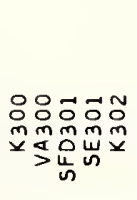 & 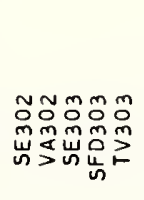 & 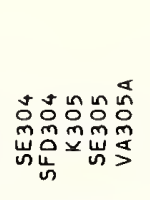 & 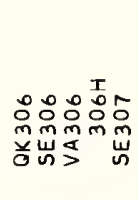 \\
\hline
\end{tabular}




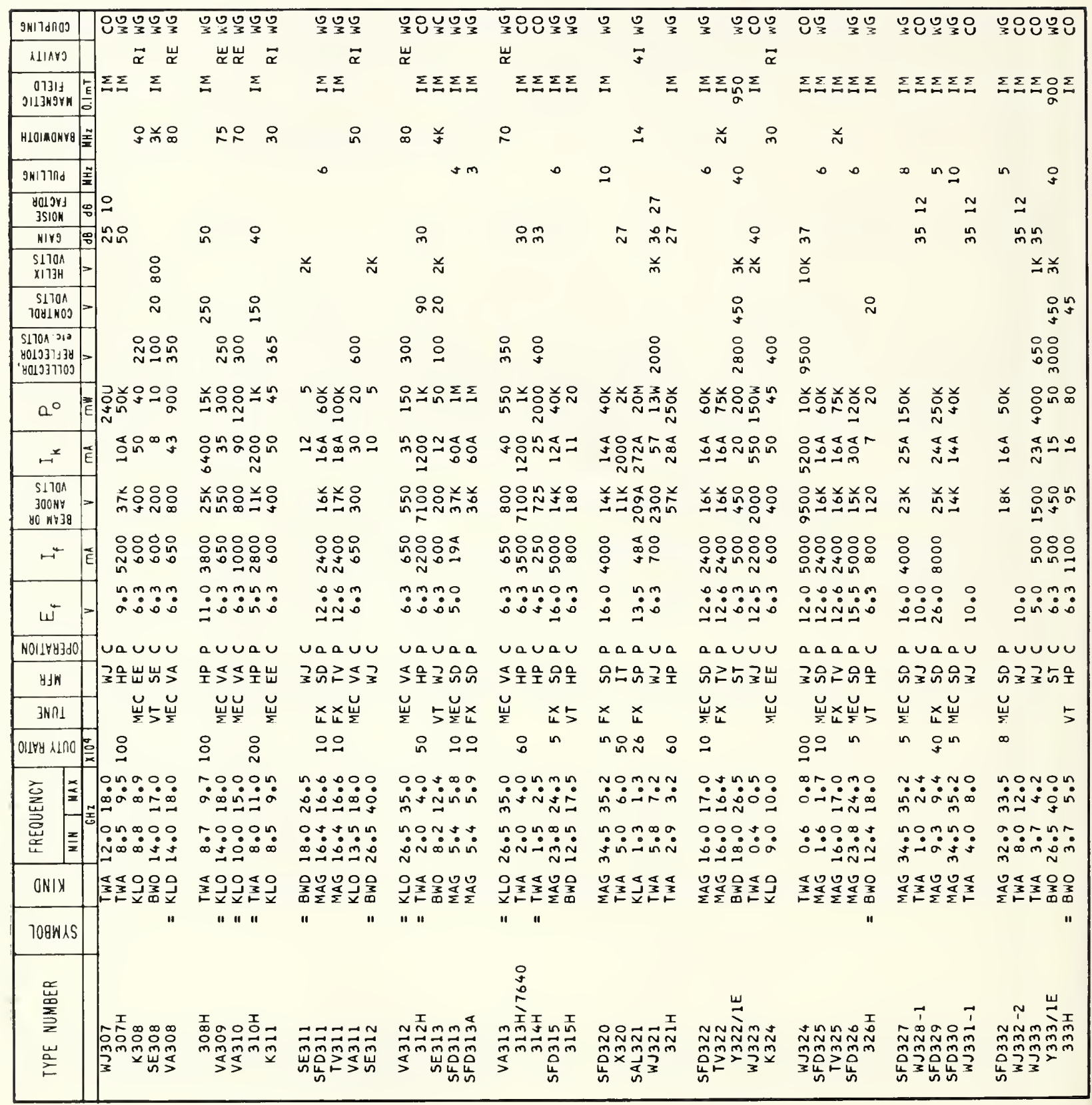




\begin{tabular}{|c|c|c|c|c|c|c|c|c|c|c|}
\hline 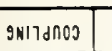 & & 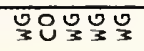 & \$883 & $4=3$ & 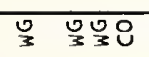 & UU & :0880 & 어 & 엉어 & 3085 \\
\hline Aliars & $\vec{\alpha}$ & $\vec{\alpha}$ & $\vec{\alpha}$ & $\vec{\alpha}$ & $\vec{\alpha}$ & $\ddot{\alpha} \vec{m}$ & & $\vec{\sim} \quad \vec{\alpha} \bar{m}$ & $\vec{m} \quad \vec{\alpha}$ & \\
\hline \begin{tabular}{c|c}
01311 \\
ग113\%94x
\end{tabular} & 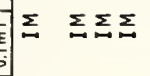 & $\Sigma \Sigma \Sigma \Sigma$ & $\sum_{-} \underset{\sim}{N} \sum_{n=\Sigma}$ & $\Sigma \Sigma_{n} \Sigma_{n}$ & $\sum_{n=0}$ & $\sum \sum$ 品 & $\sum \Sigma \Sigma \Sigma \Sigma$ & $\Sigma$ & $\Sigma \Sigma$ & $\Sigma$ \\
\hline HLOMONFA & 욤 & $\stackrel{ \pm}{N}$ & q & 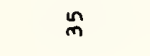 & ○ & $\stackrel{\stackrel{I}{I}}{=}$ & & $\approx$ i $\tilde{y}$ & $:$ & \\
\hline \begin{tabular}{l|l} 
9וIרח & $\frac{x}{x}$
\end{tabular} & $\infty$ & $\because \quad$ & 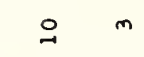 & 0 & mo & $m$ & $\checkmark$ & & s & s \\
\hline 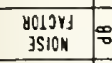 & & & & $\Rightarrow$ & r & $\infty$ & $\ln s$ & in & 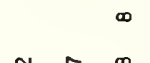 & \\
\hline Mirg & in & & 윰요 & $\circ \stackrel{m}{m}$ & $\approx$ & $\tilde{N} \stackrel{m}{n}$ & NOPON & Sᄋ요 & $\cong \tilde{m} \stackrel{\Phi}{\sim}$ & $m m$ \\
\hline $\begin{array}{l}\text { S170A } \\
\times 1173 \mathrm{H}\end{array}$ & in & & & & & & & & $\stackrel{N}{N}$ & $\underline{N}$ \\
\hline $\begin{array}{c}51701 \\
7011 \times 03\end{array}=$ & $\stackrel{\text { in }}{+}$ & $\stackrel{n}{f} \stackrel{n}{f}$ & $\stackrel{\circ}{N}$ & 응 & & & & 용 & & \\
\hline 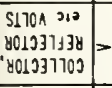 & $\begin{array}{ll}0 & 0 \\
\infty & 0\end{array}$ & 品 & $\stackrel{n}{\simeq}$ & 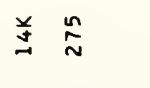 & $\stackrel{\circ}{m}$ & 品 & $\stackrel{0}{q}$ & $\stackrel{\circ}{\circ}$ & 兰 品: & $\begin{array}{l}8 \\
0 \\
\substack{++}\end{array}$ \\
\hline $0^{\circ}$ & Nㅜㅇㅇㅛ & 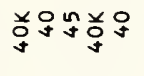 & mif & 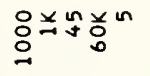 & 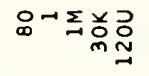 & 证总 & 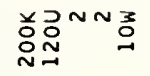 & $\underset{\sim}{\stackrel{D}{\sim}} \cong n \Sigma$ & 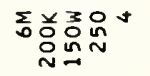 & 항ㅎㅁ웜엄 \\
\hline 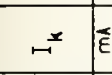 & 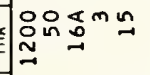 & 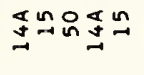 & 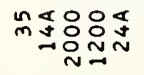 & 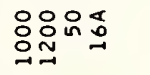 & i & 웡ㅁㅇ & $\stackrel{\infty}{N} \quad$ in & 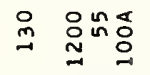 & 우웡ㅇㅇㅇㅁ & $\begin{array}{l}\alpha \sim n O \\
\sim \\
\sim\end{array}$ \\
\hline $\begin{array}{c}\text { S1100 } \\
300 \mathrm{NY} \\
80 \mathrm{AHBg}\end{array} \mid>$ & 음요요 & 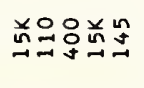 & 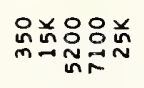 & 美品号并 & 哻 㒸芯 & 웅퓨 & $\stackrel{\sim}{\sim}$ & 品 总品首 & 㒸总品品 & 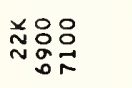 \\
\hline $5^{-}$ & : & 宓品 & 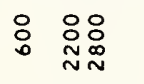 & 요윰유 & 웅 & 品芯 & 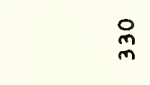 & : & 용 & 요 \\
\hline$\omega^{\prime \prime}$ & $\ddot{m} \ddot{m}: \stackrel{m}{m}:$ & $\ddot{m}: \stackrel{m}{0}:$ & $\ddot{m}: \ddot{n}:$ & 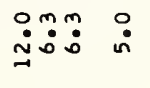 & : & mo: & $\dot{n}$ & 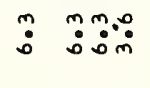 & $\stackrel{0}{\dot{m}} \ddot{0}:$ & $\ddot{n}$ \\
\hline NOIIYYIdO & vauv & auvau & voa & vavau & unau & Jaa & u & vuaua & aauuu & $a u$ \\
\hline YIJ & 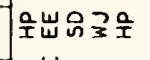 & 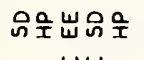 & 㟧品구오응 & 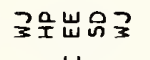 & 岀 & 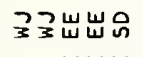 & ติว?ำำ & 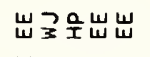 & ㄸํำำำ & 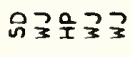 \\
\hline $3 \mathrm{~N} \cap 1$ & 岀宏 5 & ×ら岀岕5 & 岀岕 & 岀区 & 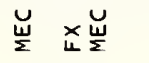 & 崖岮岮 & 岕 & 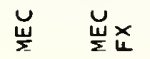 & 㛶崖 & 岁 \\
\hline $0.18 y<1 n 0 \frac{2}{2}$ & io in & in in & 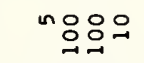 & $\stackrel{\circ}{\circ}$ & $ㅇ ㅡ$ & Ni & $\therefore$ & $\stackrel{2}{\simeq}$ & $\stackrel{n}{\sim} 0$ & $\stackrel{\circ}{\circ}$ \\
\hline 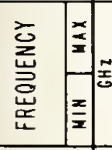 & 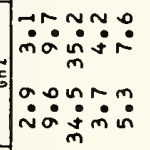 & 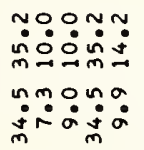 & 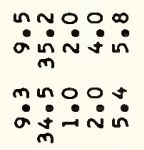 & 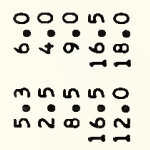 & 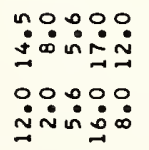 & 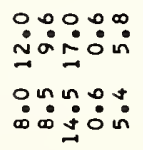 & 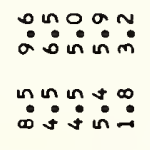 & 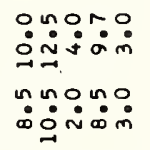 & 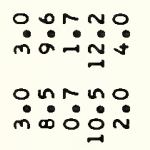 & 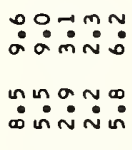 \\
\hline ONIX & 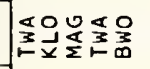 & 울울 & 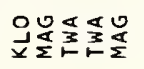 & 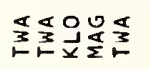 & 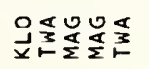 & 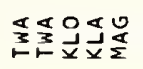 & 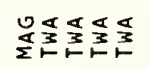 & 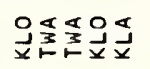 & 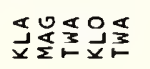 & 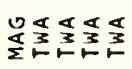 \\
\hline 708w KS & $"$ & " " " & " " & " " & " & & " & " " & " & $"$ \\
\hline 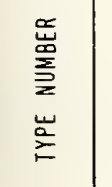 & 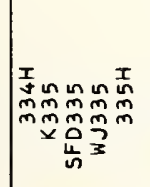 & 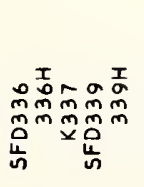 & 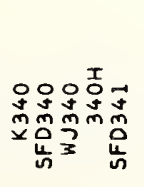 & 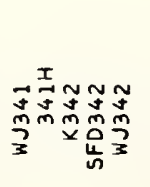 & 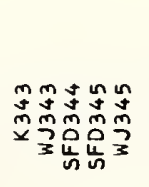 & 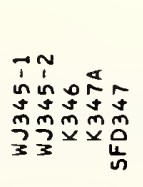 & 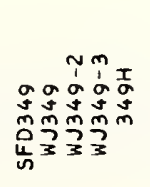 & 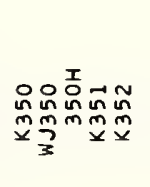 & 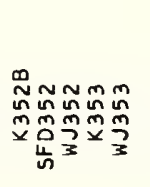 & 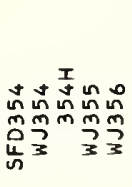 \\
\hline
\end{tabular}




\begin{tabular}{|c|c|c|c|c|c|c|c|c|c|c|}
\hline 9k17dnos & | & 뭉요 & 문응 & 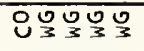 & ОणОु & OOW & Wण & 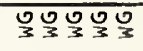 & 엉원오 & 웜ㅇㅇ \\
\hline LIIAVJ & $\vec{\alpha} \vec{\alpha} \vec{\alpha} \vec{\alpha}$ & & $\vec{\alpha}$ & 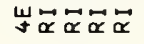 & $\vec{\alpha} \vec{\alpha} \vec{\alpha} \vec{\alpha} \vec{\alpha}$ & $\vec{\alpha} \vec{\alpha} \vec{\alpha} \vec{\alpha} \vec{\alpha}$ & $\vec{\alpha} \vec{\alpha} \vec{\alpha} \vec{\alpha} \vec{\alpha}$ & $\vec{\alpha} \vec{\alpha} \widetilde{\alpha} \vec{\alpha} \vec{\alpha}$ & 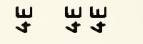 & $\vec{\xi} \underset{\jmath}{\tilde{q}}$ \\
\hline $\begin{array}{c}01314 \\
\text { ग11399YM }\end{array}$ & $\underline{E}$ & $\Sigma \Sigma \Sigma$ & $\sum \Sigma \Sigma \Sigma($ & & & & & & $\Sigma$ & $\Sigma$ \\
\hline H1OMOMUP & ㅇํำำ & & q & omnnm & 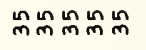 & 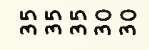 & 있욜요 & 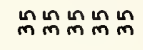 & & $\infty \infty$ \\
\hline 9*17าก & 폴 & $\infty m$ & & & & & & & & \\
\hline $\begin{array}{l}\text { yoljory } \\
\text { JSIOH }\end{array}$ & 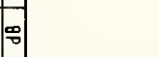 & 웅 & & & & & & & + & \\
\hline nivg & $:$ & 웅ㅇㅇㅛ & $\stackrel{m}{\sim m} m \stackrel{m}{N}$ & 옹 & & & & & 웅우웅ㅇ & $\underset{v}{*} \stackrel{0}{\sim}$ \\
\hline $\begin{array}{l}5170 A \\
\times 1173 H \\
\end{array}$ & 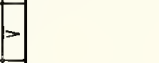 & & 并 & & & & & & & $\ddot{\sim}$ \\
\hline $\begin{array}{c}51701 \\
\text { TOY1003 }\end{array}$ & in & $\stackrel{n}{m}$ & & : & & & & & $\stackrel{n}{5}$ & \\
\hline 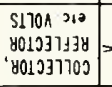 & 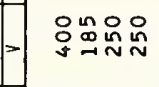 & & 융요 & 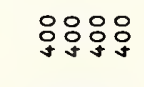 & 웅웅응응이 & 응용요 & 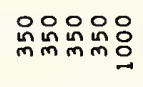 & 응ㅇㅇㅇ음 & 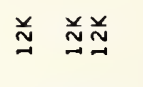 & $\stackrel{\circ}{\circ}$ \\
\hline $0^{\circ}$ & E & 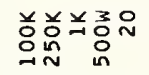 & 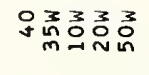 & 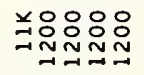 & 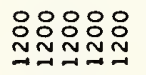 & 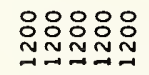 & 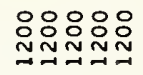 & 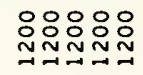 & 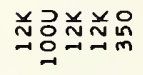 & 㒸总路品 \\
\hline$\mapsto$ & & 总芯 品 & s & 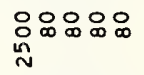 & 잉요 & 임요ㅁㅠㅛ & กำ & 잉용ㅇ & : & : \\
\hline $\begin{array}{c}51701 \\
300 \mathrm{AH} \\
80 \mathrm{KH}\} \mathrm{Gg}\end{array}$ & $=$ N & 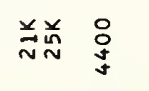 & 怘 & 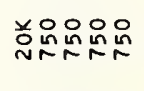 & 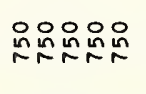 & 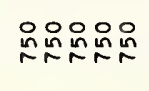 & 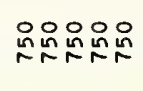 & 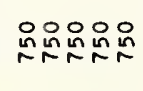 & 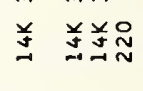 & 훙웅유 \\
\hline- & E & $\stackrel{i}{\sim}$ & $:$ & కீ: & :용요요 & :용요 & 융용요 & : & 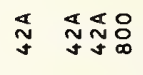 & $\underset{\exists}{\mathbb{N}} \underset{N}{\mathbb{N}}$ \\
\hline$\omega^{ \pm}$ & $\Rightarrow$ & $\ddot{m}$ & $\ddot{:}$ & 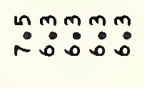 & mmmm & 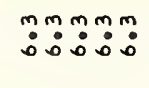 & mmmm & 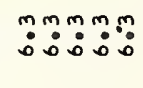 & 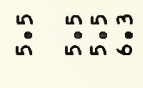 & 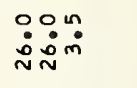 \\
\hline $\mathrm{NO} \mid \angle \forall \mathrm{GJO}$ & |uบuvu & $a a a a u$ & yบบบบ & yuบuท & ưưu & yuบu & yบuบ & yuบuy & บบบบบ & บบบบบ \\
\hline$\forall \pm N$ & 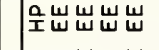 & 응ำำ? & 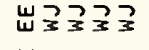 & سّ山سّ山س & 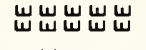 & 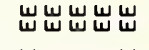 & س山ّ山س & 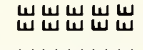 & 뽏ㅃㅃㅃ뭄 & 岀出这匹? \\
\hline 3NกI & క & $\underline{\underline{w}}$ & 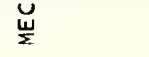 & 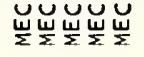 & 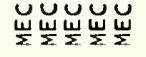 & 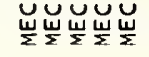 & 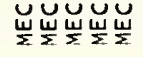 & 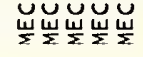 & 岀 & 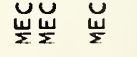 \\
\hline 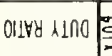 & & 앙용요 & & & & & & & & \\
\hline 惫 & 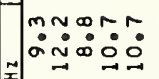 & 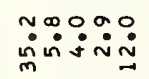 & 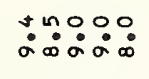 & 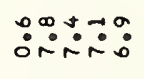 & 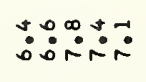 & 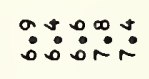 & $\ddot{\sim} \ddot{0}: \stackrel{0}{0}: 0$ & 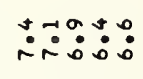 & $\because m \sim: 00$ & 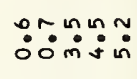 \\
\hline 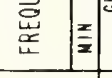 & : & 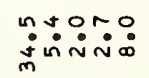 & m: & ษัง & 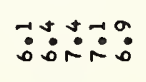 & 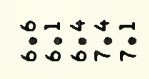 & 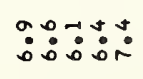 & $\because \because: \because::$ & 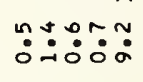 & $\ddot{\circ}: \ddot{0} \dot{m} \dot{\sim} \dot{\sim}$ \\
\hline ONIX & 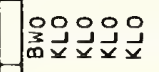 & 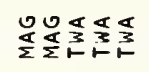 & 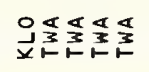 & 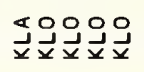 & 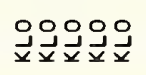 & 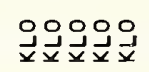 & 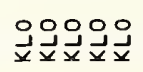 & 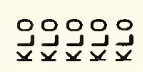 & 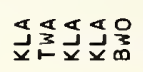 & 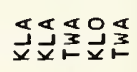 \\
\hline 70gw/S & $"$ & & & & & & & & $"$ & " $"$ \\
\hline 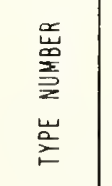 & 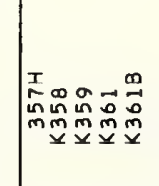 & 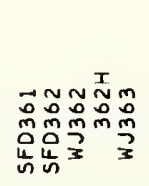 & 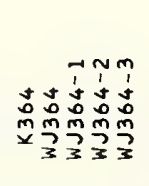 & 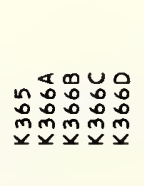 & 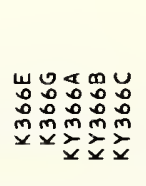 & 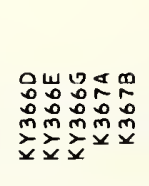 & 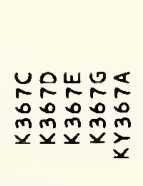 & 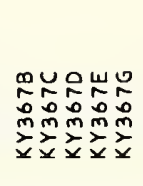 & 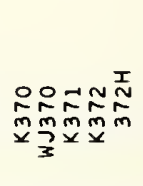 & 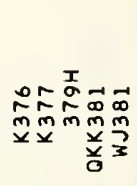 \\
\hline
\end{tabular}




\begin{tabular}{|c|c|c|c|c|c|c|c|c|c|c|}
\hline 9N17dnos & 웅으 & 엉워논 & 웅요 & 언운옹 & $\frac{0}{3}$ & 월 & 운은 & OO & $\frac{0}{3}$ & 옹요 \\
\hline Rl1AY & $\underset{\varpi}{\varpi}$ & 岁ひす & & $\vec{\sim} \vec{\alpha}$ & mี゙ே & デัテ & $\vec{\alpha} \quad \vec{\alpha}$ & $\vec{\alpha} \tilde{N} \vec{v}$ & $\vec{\alpha} \vec{\alpha} \vec{\sim}$ & $\ddot{\alpha}$ \\
\hline 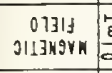 & 熟 $\sum \sum$ & $\Sigma \quad \Sigma$ & $\Sigma \Sigma \Sigma$ & $\Sigma \quad \Sigma$ & & i & $\Sigma \Sigma \Sigma$ & & & $\sum \Sigma$ \\
\hline HLOMONY8 $=$ & & & 앙우 & $\circ \stackrel{\circ}{\sim}$ & $\stackrel{\infty}{\sim} \simeq$ & 욤 $\infty$ & $\stackrel{n}{m}$ & $\stackrel{\infty}{\rightarrow}$ 요 & 요 & 8 \\
\hline 9או17רח & & & & & & & & & & \\
\hline 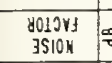 & $\approx$ & & $m$ & $\infty \exists$ & & & & & & \\
\hline NIV9 & ํำ & 용워 & $\stackrel{m}{m} \underset{m}{\infty}$ & $\stackrel{\sim}{\sim} \stackrel{\infty}{\sim}$ & $\stackrel{\sim}{\sim} \stackrel{n}{\sim} \stackrel{n}{N}$ & min & $\stackrel{+}{\sim}$ & 용요 & & $\stackrel{n}{N}$ \\
\hline 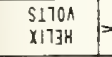 & & & & & & & & & & $\underline{\sim}$ \\
\hline $\begin{array}{c}51701 \\
7081 \times 00\end{array}=$ & & & $\stackrel{n}{\xi}$ & & & & & & & \\
\hline 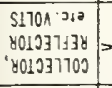 & :응 & 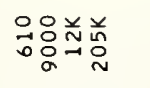 & 응 & 只 & & & $\stackrel{n}{N}$ & & 客 & $\stackrel{n}{\sim}$ \\
\hline $0^{\circ}$ & E & 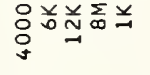 & 9:0요이 & $\begin{array}{l}\text { OOONO } \\
\text { OOON }\end{array}$ & 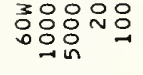 & 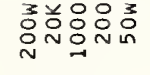 & 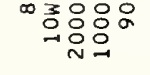 & 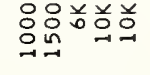 & 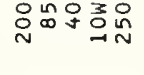 & 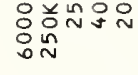 \\
\hline 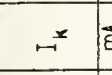 & Eิ & 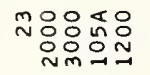 & ํํ용ㅇㅇ & $\stackrel{\text { 웅 }}{n}$ & 윴용요 & 路品品 & $\stackrel{\sim}{\sim} \mathcal{O}_{\sim}^{\infty} \underset{\sim}{\mathbb{N}} \stackrel{\sim}{\sim}$ & 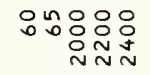 & $: \hat{m}$ in & 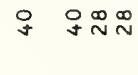 \\
\hline 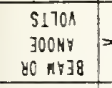 & 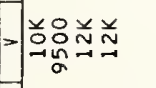 & 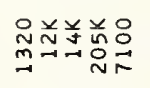 & 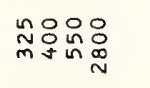 & 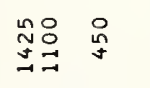 & 욤요 & 总总品品 & 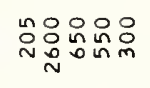 & 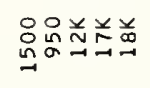 & 다요 & : \\
\hline 5 & $\underset{J}{\stackrel{J}{N}}$ & 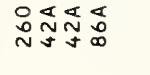 & $\begin{array}{ll}: & 0 \\
0 & \stackrel{0}{N}\end{array}$ & 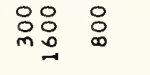 & 品品: & 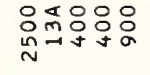 & 웅 & 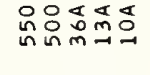 & $\begin{array}{lll}0 & 0 \\
n & 0 & 0 \\
\text { in } & +\end{array}$ & 옹요 \\
\hline wँ & 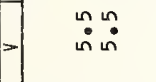 & 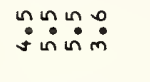 & $\stackrel{m}{m} \stackrel{m}{m}$ & $\stackrel{n}{\sim} \stackrel{m}{:}$ & 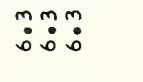 & 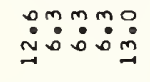 & 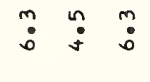 & 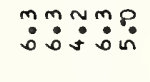 & 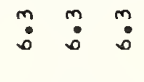 & $\ddot{m}: \dot{m}$ \\
\hline NOLIVEJ & Jauvu & บบบaa & טu৩au & טuטu & u u & טuט & ט & U & 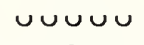 & $\cup \propto \cup \cup \cup$ \\
\hline yง & 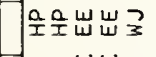 & 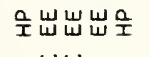 & 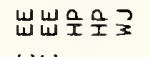 & 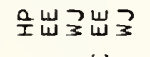 & 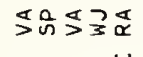 & 的的的文 & 的路呈品 & 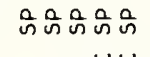 & 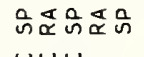 & 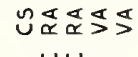 \\
\hline $\mathrm{JN} \cap 1$ & 岀 & 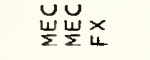 & Wu & $\times \underset{u}{\breve{w}}$ & $\underset{x \times x}{x}$ & 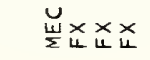 & $\begin{array}{ll}\breve{w} & \breve{w}\end{array}$ & 希 岀崖 & 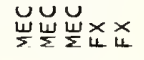 & 岂㞱 \\
\hline $0118 y+1100$ & 옹 & $\stackrel{n}{\sim} \underset{N}{(1)}$ & & & & & & $\stackrel{i}{\sim}$ & & 우 \\
\hline 曾 & 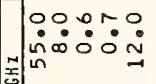 & $\ddot{n} \because \because \because \dot{0}$ & 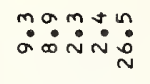 & 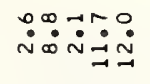 & ن. & 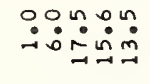 & 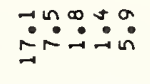 & 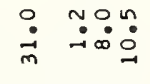 & 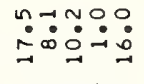 & ن் \\
\hline \begin{tabular}{l|l}
$\stackrel{ㅍ}{\dddot{x}}$ \\
\end{tabular} & $\ddot{\ddot{n}} \because \because 00$ & $\ddot{m} \dot{0} 0 \dot{m} \dot{m}$ & \begin{tabular}{c}
$N \infty$ \\
\hdashline \\
\hdashline
\end{tabular} & 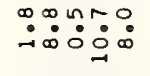 & 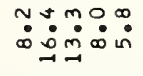 & 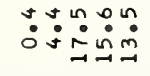 & 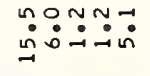 & 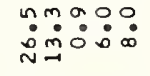 & 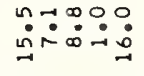 & 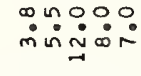 \\
\hline ONIX & 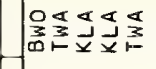 & 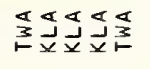 & 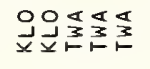 & 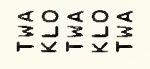 & 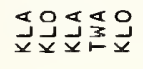 & 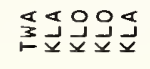 & 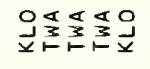 & 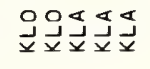 & 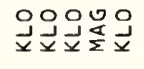 & 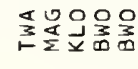 \\
\hline 7ognis & $"$ & $"$ & " " " & " " " & " $11 "$ & & & $"$ & & \\
\hline 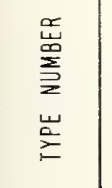 & 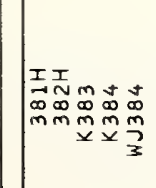 & 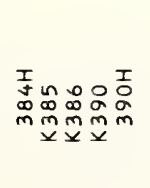 & 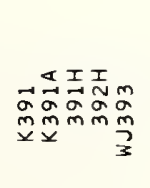 & 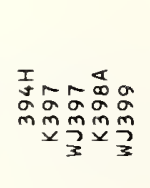 & 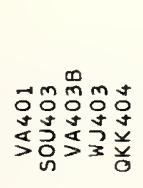 & 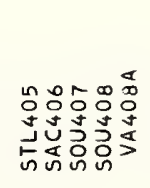 & 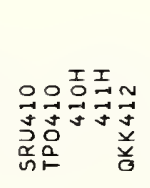 & 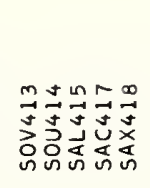 & 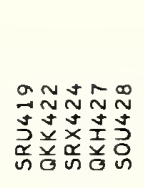 & 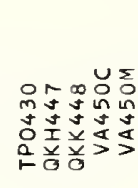 \\
\hline
\end{tabular}




\begin{tabular}{|c|c|c|c|c|c|c|c|c|c|c|}
\hline 9M17dก03 & 엉요 & Uত⿱ & पू & 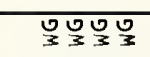 & 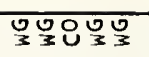 & 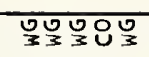 & OU్ర & ত্ৰ엉이 & 엉ㅇ & 엉ㅇㅇㅇ \\
\hline A1/AY3 & & $\vec{\alpha} \quad \vec{\alpha}$ & & $\vec{N} \tilde{N} \vec{N}$ & $\vec{\sim}$ & $\vec{\sim} \tilde{N} \vec{N}$ & $\vec{\sim} \vec{N}$ & $\vec{N}$ & $\vec{N} \vec{N} \vec{N}$ & $\vec{N} \vec{N}$ \\
\hline 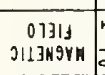 & 甸 & $\Sigma \Sigma \sum$ & $\Sigma \Sigma$ & $\Sigma$ & $\underset{n}{\Sigma 0 \Sigma \Sigma}$ & & $\sum \Sigma \Sigma$ & $\Sigma \Sigma$ & $\Sigma$ & $\underset{\sim}{\stackrel{p}{\sim}} \Sigma \Sigma$ \\
\hline H101MOMY8 & $\approx$ & o & & & $\stackrel{\circ}{\circ}$ & & in & in & ก̊ & \\
\hline 9 & : & & & $\stackrel{\text { n }}{\sim}$ & $\cong$ & & $\cong$ & $\stackrel{\infty}{\rightarrow}$ & & \\
\hline \begin{tabular}{l|}
$y 013 Y 1$ \\
JSIOH
\end{tabular} & 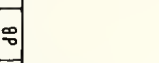 & & & & $\stackrel{n}{\sim}$ & & & & & \\
\hline \begin{tabular}{l|l} 
M1Y9 & $=$ \\
\end{tabular} & 圈 & & & & r & $\stackrel{0}{\sim}$ & $\stackrel{m}{m}$ & 品 & & $\stackrel{m}{m} \stackrel{\infty}{N}$ \\
\hline $\begin{array}{l}5170 \mathrm{~A} \\
\times 173 \mathrm{H} \\
\end{array}$ & $=\mid$\begin{tabular}{ll}
$O$ \\
\hdashline
\end{tabular} & 융요 물 & $\stackrel{\circ}{\circ} \cong \pm$ & 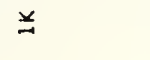 & 弟 & & & & $\stackrel{x}{N}$ & \\
\hline $\begin{array}{c}\text { S1701 } \\
1041003\end{array}$ & & & & & in & & & & $\therefore$ & in \\
\hline 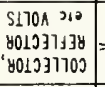 & & $\stackrel{\circ}{\vec{N}} \stackrel{0}{m}$ & & & : & & & & & \\
\hline $0^{\circ}$ & 踏品关只 & 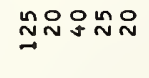 & 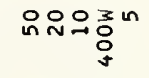 & 只酋品品录 & 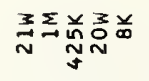 & 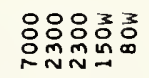 & 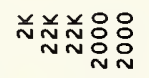 & 落: & 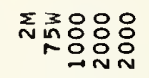 & 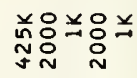 \\
\hline- & $\stackrel{\infty}{\sim} \underset{\sim}{[\infty}$ & $\stackrel{\sim}{\sim} \underset{\sim}{\sim} \sim \infty$ & $\stackrel{\infty}{\rightarrow}$ & 范 N & 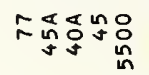 & 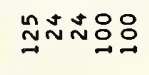 & 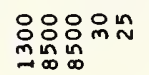 & 옹윯ㅇ్ㅇㅇㅁ & 영으우임요 & 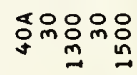 \\
\hline $\begin{array}{c}S 170 \mathrm{~A} \\
300 \mathrm{HY} \\
80 \mathrm{Hr} 3 \mathrm{~B}\end{array}$ & \% & $\stackrel{\circ}{\circ} \stackrel{\circ}{\circ}$ & & 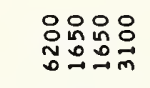 & : & 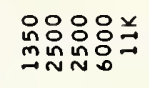 & 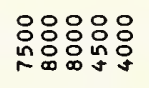 & 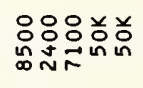 & 关兰员怘品品 & 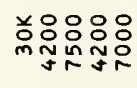 \\
\hline$\mapsto$ & 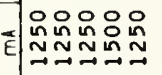 & 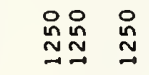 & 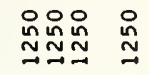 & 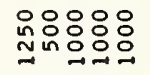 & : O용 & 융용요 & 윰용요 & 융요 & 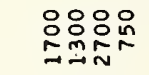 & 品 \\
\hline$\omega^{ \pm}$ & mamm & m:mm & $\ddot{m}: m \stackrel{m}{m}:$ & 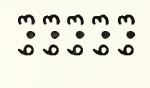 & $\ddot{m}: \ddot{n}: m$ & $\ddot{m}: \dot{m}: \dot{m}$ & mmm & $\ddot{m}:$ & $\ddot{m}: m m{ }_{0}^{m}$ & $\ddot{n} \ddot{0}: \ddot{m}$ \\
\hline$N 011 \forall \forall\}] d 0$ & yuaru & טuטu & uvuau & vauuv & vana & טuטu & $a a a u b$ & avaan & auuuu & $a \cup a \cup a$ \\
\hline$\forall y W$ & 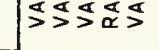 & 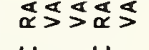 & $\stackrel{\Delta}{>}>\Delta \alpha^{\alpha}>$ & 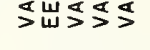 & \出出出 & 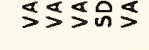 & 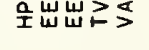 & 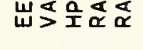 & 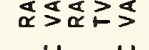 & 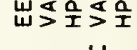 \\
\hline 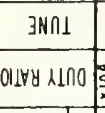 & $\begin{array}{l}x \\
4 \\
0\end{array}$ & $\frac{W}{\Sigma} \quad \frac{W}{\Sigma}$ & $\frac{\mathrm{w}}{\Sigma}$ & $\begin{array}{l}x_{4} \times x_{4} \times \mathbb{u} \\
\stackrel{n}{N}\end{array}$ & $\begin{array}{rl}x \times x & x \\
4 & 4 \\
00 & \stackrel{n}{N}\end{array}$ & $\begin{array}{lll}x \times 4 & x \\
4 & x\end{array}$ & 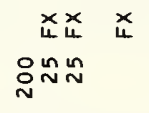 & 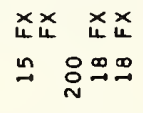 & 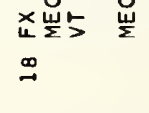 & 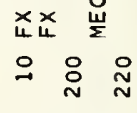 \\
\hline 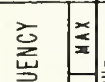 & 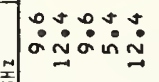 & 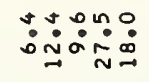 & 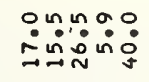 & 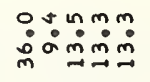 & 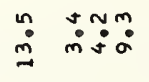 & 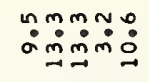 & ن் & 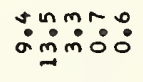 & :0: & \\
\hline 总 & 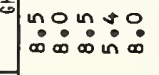 & 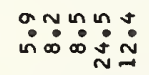 & :00 & :minmm & 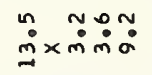 & 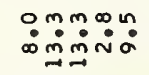 & 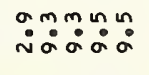 & 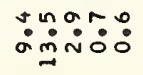 & 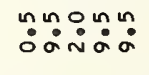 & $\begin{array}{l}\dot{n} \\
\dot{m} \\
\dot{m}\end{array}$ \\
\hline ONIY & 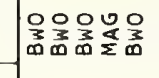 & 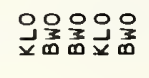 & 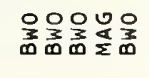 & 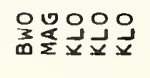 & 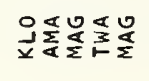 & 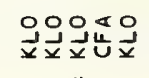 & 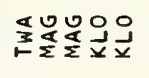 & 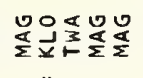 & 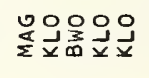 & 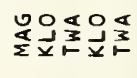 \\
\hline 708W/S & & & & & & & & & & \\
\hline 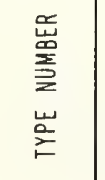 & 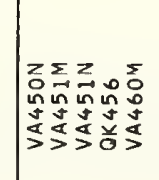 & 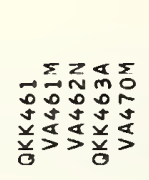 & 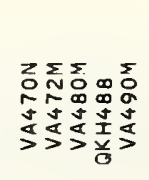 & 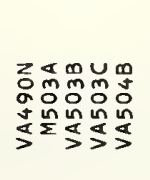 & 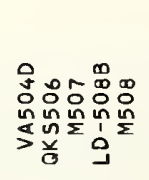 & 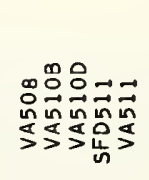 & 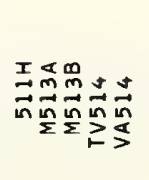 & 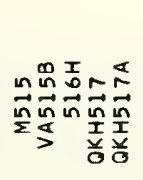 & 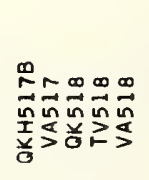 & 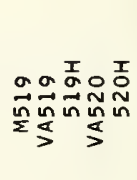 \\
\hline
\end{tabular}




\begin{tabular}{|c|c|c|c|c|c|c|c|c|c|c|}
\hline 9k17dnos & ॠ & Oי & 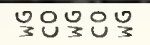 & 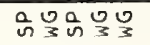 & 엉 & \్ & 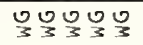 & טִ & 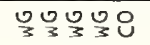 & 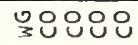 \\
\hline 111ArJ & $\vec{\sim} \vec{\sim}$ & $\vec{\sim} \vec{\sim}$ & $\vec{\sim} \quad \vec{\alpha}$ & $\vec{N} \vec{\propto} \stackrel{\sim}{\sim} \vec{N}$ & $\vec{\sim}$ & & $\vec{\alpha}$ & & & \\
\hline 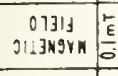 & $\underset{m}{\stackrel{\circ}{N}} \Sigma$ & $\sum_{-1}$ & $\sum \Sigma \Sigma$ & & $\Sigma \Sigma \Sigma \Sigma$ & $\Sigma \sum \Sigma \Sigma$ & 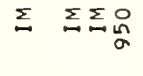 & 品哭哭 & 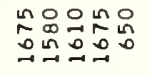 & 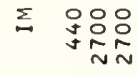 \\
\hline 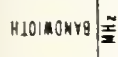 & & i & $\stackrel{n}{N}$ & 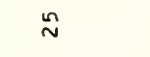 & $\rightarrow$ & & $\stackrel{n}{\sim}$ & $\therefore \quad-$ & & \\
\hline 9אור & $\cong \cong$ & $r$ & $\cong$ & & $\cong \bumpeq$ & $\simeq \approx$ & $\cong \quad+$ & $\circ r$ & arra & $\cong \cong$ \\
\hline 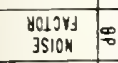 & & & & & & & $\stackrel{\sim}{N} \stackrel{\infty}{N}$ & & & \\
\hline Mirg $\$$ & & 을 & 요 & & $\stackrel{\sim}{\sim}$ & $\tilde{m}$ & mo & & $\stackrel{\circ}{\circ}$ & $\stackrel{\sim}{\sim}$ \\
\hline $\begin{array}{l}{ }_{S}^{5} 170 N \\
\times 173 H\end{array}$ & & & $\stackrel{x}{N}$ & & & & 弟弚 & & & \\
\hline $\begin{array}{c}\text { S1701 } \\
70 y 1 \text { NOS }\end{array}=$ & & & $\stackrel{\circ}{\circ}$ & & $\cong$ & : & 웅 & & & \\
\hline 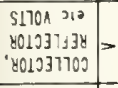 & & $\stackrel{x}{\Xi}$ & $\underset{\infty}{\infty}$ & 品 & & 品 & 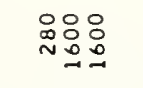 & $\stackrel{\sim}{\sim}$ & & \\
\hline$a^{\circ}$ & 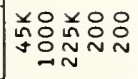 & 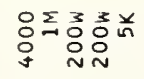 & 胥员兑总莡 & 욤융응ㅇ & 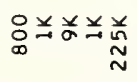 & 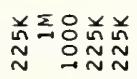 & 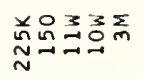 & O O & $\sum_{N} \sum_{N} \sum_{N} \sum m$ & 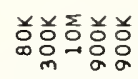 \\
\hline 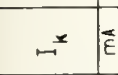 & 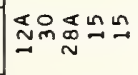 & 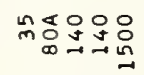 & 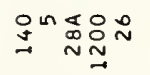 & 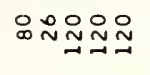 & 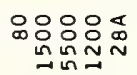 & 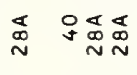 & 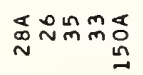 & 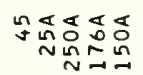 & 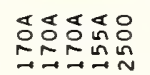 & 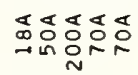 \\
\hline 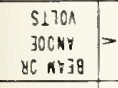 & 용요 & 总弟恶兽总 & 弟品㒸品品 & 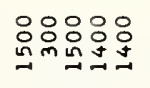 & 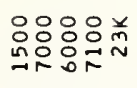 & 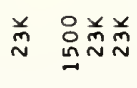 & 并楶品品怘 & 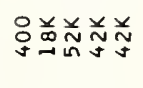 & 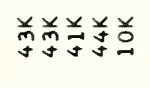 & 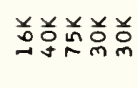 \\
\hline$\mapsto$ & 品品品 & 영윰용요 & $\begin{array}{l}\text { 용유 } \\
\text { 윰에 }\end{array}$ & 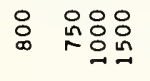 & 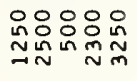 & 怘 总怘 & 怘 & 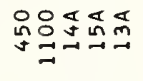 & 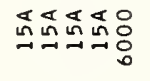 & 品 造品品 \\
\hline$\omega^{ \pm}$ & 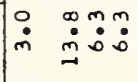 & ஸ் & 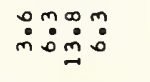 & 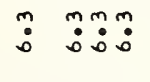 & 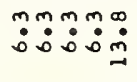 & 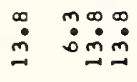 & 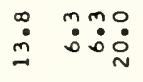 & M:O: & 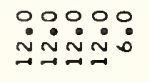 & $\begin{array}{lll}0 & \vdots \\
\vdots & \vdots\end{array}$ \\
\hline Nol $1 Y y\} d 0$ & auauv & vauuv & uvaau & טuบ & vacaa & $a a v a a$ & a uvua & vaaa & $a a a a$ & $a, a a a$ \\
\hline y. & 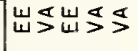 & 呈山\くU & \৫山出呈む & 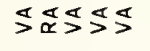 & 积呈出呈出 & 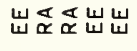 & 岀郧岂岂 & 㞱山岀岀こ & 岀出岀岕๔ & 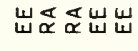 \\
\hline J $\mathrm{x} \cap 1$ & 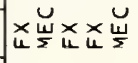 & ×岀岁 & x & $\underset{\Sigma}{u} x_{u}$ & $\begin{array}{lll}x & x & x \\
u\end{array}$ & $\begin{array}{ll}x \times \\
u\end{array}$ & 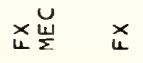 & 岀始始 & 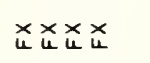 & $\begin{array}{ll}x & x \\
u\end{array}$ \\
\hline 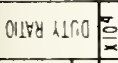 & $\div 0$ & $\simeq$ & 용여 & & 유쇼요음 & $\therefore \quad ㅇ ㅡ$ & $\stackrel{n}{=}$ & $\stackrel{O}{\sim} \stackrel{\sim}{\sim} \sim \sim$ & 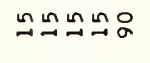 & 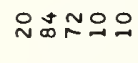 \\
\hline 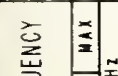 & 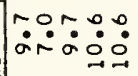 & $\dot{\sigma} \dot{\sim} \dot{\alpha} 000$ & 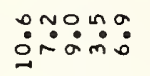 & 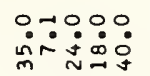 & 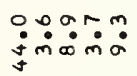 & 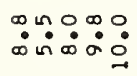 & 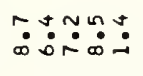 & $\dot{\sim} \dot{\sim} \dot{m} \dot{\sim} \dot{\sim}$ & $\ddot{m} \dot{m} \dot{m} \dot{m} \dot{0}$ & $\dot{0}: 00: \dot{m}$ \\
\hline 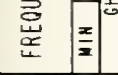 & 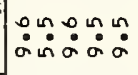 & 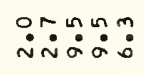 & 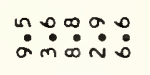 & $\ddot{\sim} \dot{\sim} \dot{0} \dot{0} \dot{\sim} \dot{\sim} \dot{m}$ & $\ddot{g} \dot{\sim} \dot{\sim} \dot{m} \dot{m}$ & 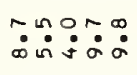 & 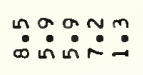 & 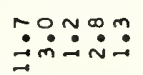 & $\ddot{\sim} \dot{\sim} \dot{\sim} \dot{\sim} \dot{m} \dot{0}$ & $\dot{m} \dot{0}: \dot{0} \dot{m} \dot{m}$ \\
\hline ONIX & 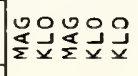 & 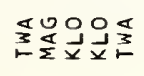 & 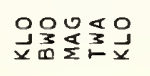 & 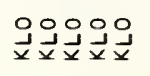 & 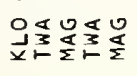 & 过造造造造 & 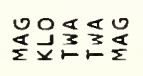 & 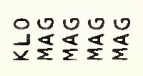 & 员造造造造 & 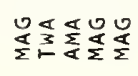 \\
\hline 708w/S & & & & & & & & & & \\
\hline 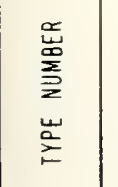 & 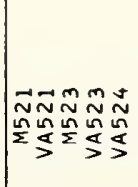 & 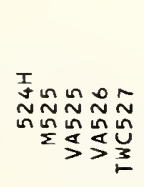 & 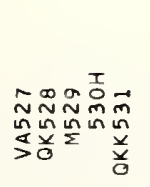 & 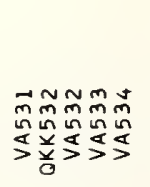 & 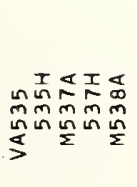 & 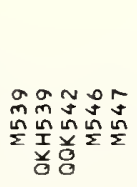 & 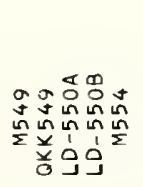 & 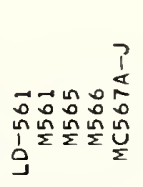 & 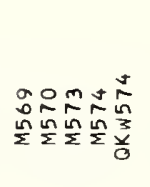 & 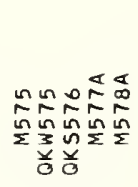 \\
\hline
\end{tabular}




\begin{tabular}{|c|c|c|c|c|c|c|c|c|c|c|}
\hline 9MITdnOS & 야엃요 & ৩৩ฺฺ & 은언 운 & OUß워 & 언언 언언 & 언언언언 & 엉원이 & 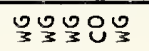 & 언언언 & 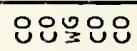 \\
\hline hlarg & 3 & & $\underset{\exists}{\Psi}$ & $\vec{\alpha}$ & & & & $\vec{\alpha}$ & $z$ & 崫 \\
\hline \begin{tabular}{l|l|l}
01319 \\
J113K9VK
\end{tabular} & 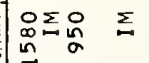 & $\sum \Sigma \Sigma \sum$ & $\sum \sum$ & $\sum \sum$ & $\Sigma \Sigma \Sigma \Sigma \Sigma$ & $\sum \Sigma \sum \Sigma \Sigma$ & $\sum \sum \sum \sum \sum$ & $\Sigma \Sigma$ & $\Sigma \Sigma \Sigma \Sigma$ & $\Sigma \Sigma \Sigma$ \\
\hline HLOMONHA & in & 응ㅇ & & in $\stackrel{\text { N }}{\text { N }}$ & & & & $\stackrel{n}{\sim}$ & 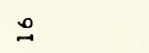 & \\
\hline פח & $\cong \sim$ & $m \stackrel{n}{\sim} \approx$ & $\stackrel{\infty}{-}$ & & & & & & & \\
\hline $\begin{array}{l}8019 y \mathrm{~s} \\
3510 \mathrm{~N}\end{array}$ & & $\stackrel{n}{\sim}$ & $\stackrel{n}{m}$ & $\stackrel{n}{\sim}$ & 있욤요 & 있 욤 & 있임요 & & $\stackrel{n}{m} \stackrel{n}{m}$ & $\stackrel{n}{m} \stackrel{n}{m} m$ \\
\hline NIYS & & $\stackrel{n}{m} \stackrel{m}{m}$ & $\underset{s}{n} m$ & 워 $\quad \stackrel{n}{\sim}$ & 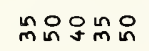 & 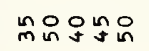 & 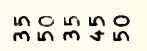 & ro 욤요 & 있 웄요 & 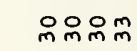 \\
\hline $\begin{array}{l}5170 \AA \\
\times 173 H\end{array}$ & & 当 & 弟 & $\ddot{m} \stackrel{x}{\sim}$ & $\stackrel{x}{\sim}$ & 并 & $\stackrel{Ð}{\exists}$ & & 芯㒸并 & 羊并兑 \\
\hline 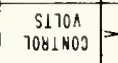 & & $\stackrel{n}{m} \stackrel{\circ}{n}$ & $\ddot{*}$ & $\stackrel{n}{m} \stackrel{0}{n}$ & & & 号 & & & 웅 \\
\hline 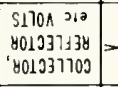 & $\stackrel{\circ}{\circ}$ & $\stackrel{\circ}{:}$ & $\begin{array}{l}0 \\
0 \\
\text { m }\end{array}$ & : & $\stackrel{\circ}{\circ}$ & & $\stackrel{i}{i n}$ & $\stackrel{\circ}{\infty}$ & 嗢 & \\
\hline$a^{\circ}$ & 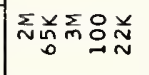 & 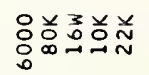 & 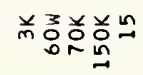 & 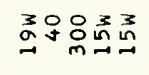 & 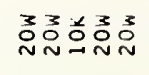 & 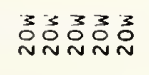 & 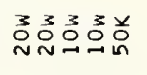 & 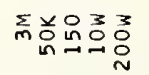 & 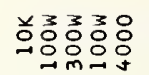 & 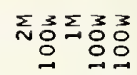 \\
\hline 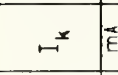 & 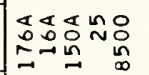 & N氐的品品 & 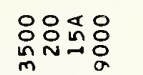 & 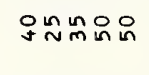 & 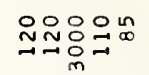 & 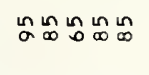 & 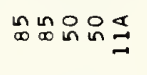 & 迁㗄品品 & 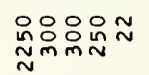 & 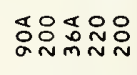 \\
\hline 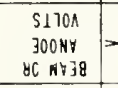 & 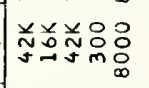 & O. & 总总总 & 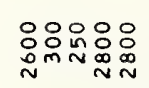 & $\begin{array}{l}: \\
:\end{array}$ & 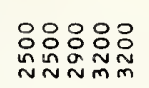 & 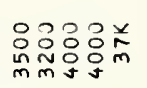 & 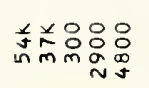 & 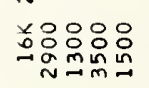 & 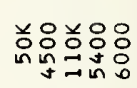 \\
\hline$\mapsto^{-}$ & 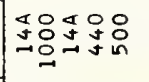 & 응유욤요 & 욤욤요 & 品 总品品 & 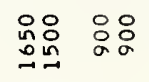 & 응 & 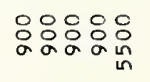 & $\begin{array}{ll}\circ & 0 \\
0 & 0 \\
n & n \\
n\end{array}$ & 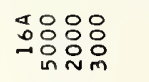 & 요요요 \\
\hline$\omega^{\leftarrow}$ & 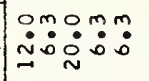 & m: & 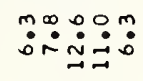 & 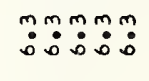 & 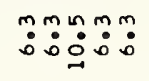 & $\stackrel{m m}{\ddot{m}} \stackrel{m}{m}:$ & $\ddot{0}: \ddot{m} \ddot{m}: \dot{0}$ & $\stackrel{n}{\ddot{\sigma}} \ddot{\dot{b}}$ & $\ddot{m}: \because: 0:$ & : \\
\hline $\mathrm{NO} \perp 18 \mathrm{YG}+\mathrm{O}$ & anaua & טanan & ט a a & טuบ & עטu & טיטט & מטט טים & aguuv & טنטن & a a \\
\hline 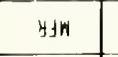 & 岀岀岀崖崖 & 岂岀㞾岀 & 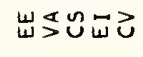 & 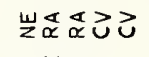 & 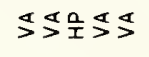 & ダ呈ダ & 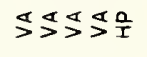 & 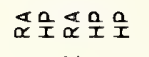 & 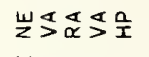 & 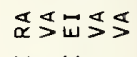 \\
\hline $3 \mathrm{~N} \cap 1$ & 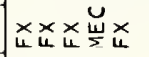 & x $x \underset{u}{x}$ & $x$ & 岁 & & & & 岁 & $\stackrel{\breve{w}}{5} 5$ & \\
\hline $018 y+$ al: 0 & $\stackrel{2}{\sim} \cong \stackrel{n}{\sim}$ & 요 $\stackrel{n}{N}$ & 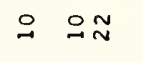 & & & & 요 & $\stackrel{\circ}{\sim}$ & & $\stackrel{\infty}{-}:$ \\
\hline 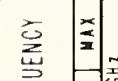 & 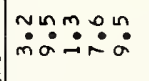 & 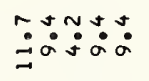 & 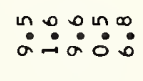 & $\ddot{\sim} \dot{\alpha} \doteq \dot{\sim} \dot{\sim}$ & $\dot{\sim} \dot{\sim} \dot{0}: 00$ & 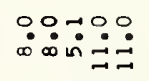 & 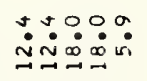 & 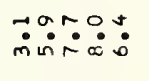 & 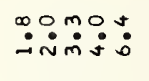 & 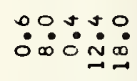 \\
\hline 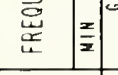 & 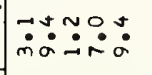 & $\ddot{0} \dot{0} \dot{m} \dot{0}$ & 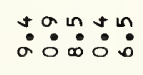 & 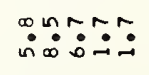 & $\because \because 000$ & O̊min & $\ddot{r} \dot{\sim} \dot{\cong} \dot{\sim} \dot{\sim}$ & 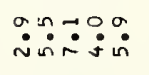 & 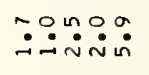 & 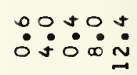 \\
\hline ONIX & $\begin{array}{l}001000 \\
\frac{\pi}{2} \frac{1}{2} \frac{\alpha}{2} \frac{\alpha}{2}\end{array}$ & 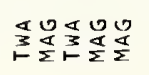 & 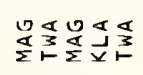 & 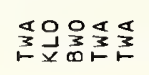 & 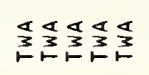 & 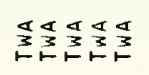 & 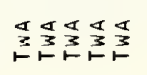 & 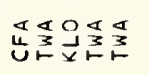 & 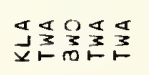 & 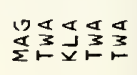 \\
\hline $708 \mathrm{~W} \times \mathrm{S}$ & & & $"$ & & $"$ & & & & & \\
\hline 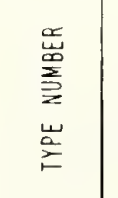 & 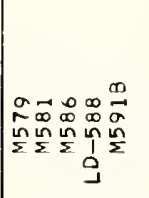 & 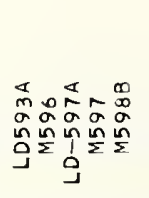 & 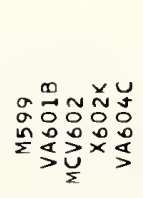 & 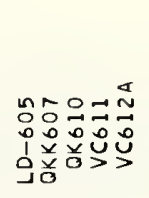 & 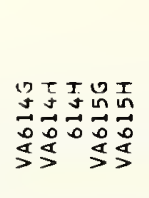 & 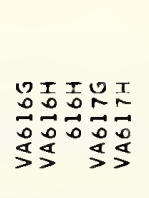 & 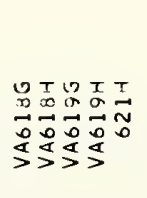 & 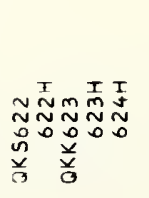 & 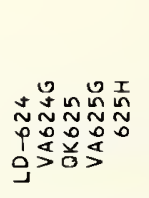 & 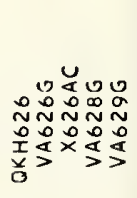 \\
\hline
\end{tabular}




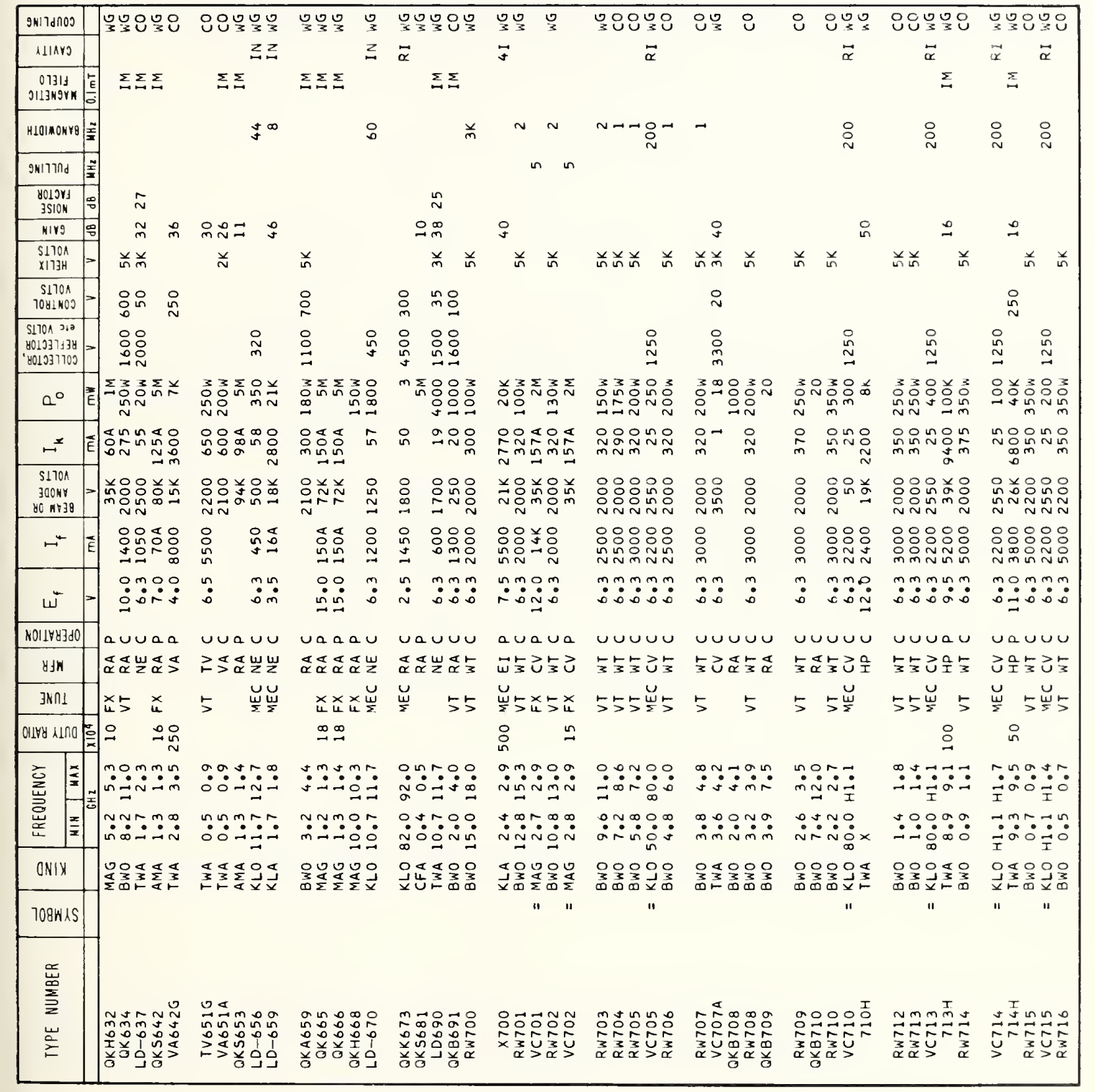




\begin{tabular}{|c|c|c|c|c|c|c|c|c|c|c|}
\hline 9NITdก03 & 8008\% & प0 & 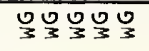 & पण & O్ß & O० & WO & 엉जㅜ엉 & $8 \%$ & ํํํํํㅇㅇㅇ \\
\hline AlARS & $\vec{\alpha} \quad \vec{\alpha} \vec{\alpha}$ & $\vec{\alpha} \vec{\alpha} \vec{\alpha}$ & $\vec{\alpha} \vec{\alpha} \vec{\alpha} \vec{\alpha} \vec{\alpha}$ & $\vec{\alpha} \vec{\alpha} \vec{\alpha} \vec{\alpha}$ & $\vec{\alpha} \vec{\alpha} \vec{\alpha} \vec{\alpha} \vec{\alpha}$ & $\vec{\alpha} \vec{\alpha} \vec{\alpha} \vec{\alpha}$ & $\vec{\alpha} \vec{\alpha} \vec{x} \vec{x}$ & $\vec{\alpha}$ & $\vec{\alpha}$ & \\
\hline $\begin{array}{l}0131 \mathrm{f} \\
\text { J113HOVK }\end{array}$ & & $\sum \sum$ & & & & & & $8 \Sigma$ & $\Sigma$ & $\sum \sum \sum \sum \sum$ \\
\hline HLOIMONYA & 응요 & $: 0: 0$ & 웅ㅇㅇㅇ & 品品品品 & 욤욤요 & 영용ㅇㅇ & :ㅇ::용 & o & 욤 & \\
\hline 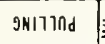 & 출 & 욱유오 & 윽우워우우 & & & & & $\cong$ & & \\
\hline $\begin{array}{l}\text { Bolyy- } \\
\text { 3SIOON }\end{array}$ & & & & & & & & & & $\stackrel{n}{\sim}$ \\
\hline Nirg & & $\ln _{\mathfrak{q}}$ & & & & & ? & $\begin{array}{l}9 \\
9\end{array}$ & $e^{9}$ & $\underset{\sim m}{\sim}$ \\
\hline $\begin{array}{l}\text { S1770 } \\
X 113 H\end{array}$ & $\frac{x}{r}$ & & & 品 & & & & & & 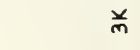 \\
\hline $\begin{array}{c}\text { S1701 } \\
\text { 7081400 }\end{array}$ & & $\stackrel{\circ}{\sim}$ & & 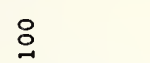 & & & & & $\stackrel{\circ}{\sim}$ & o \\
\hline 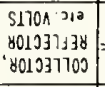 & $\stackrel{\text { N }}{\underset{N}{N}} \underset{\sim}{\stackrel{N}{*}}$ & 응이 & 응ㅇㅇㅇㅁㅇ & 总品品品 & 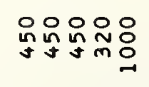 & ㅇㅇㅇㅇㅇㅛ & 웅ำ & \& & 号 & $\stackrel{\circ}{\circ}$ \\
\hline $0^{\circ}$ & E & 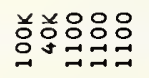 & 응응응음음 & 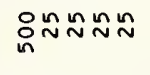 & 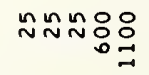 & 응음음ㅇ & 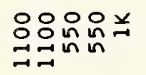 & 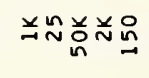 & 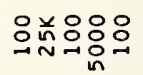 & 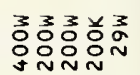 \\
\hline$\mapsto$ & noror & 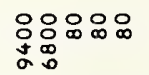 & 잉용요 & 으우음으 & 으으음ㅇㅇㅇ & 임잉요 & 잉웅유 & 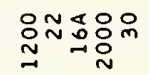 & nn: & 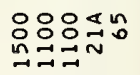 \\
\hline 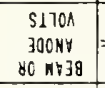 & > 음욤욤요 & 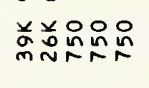 & 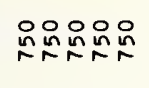 & 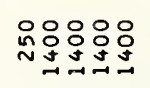 & 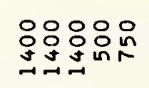 & 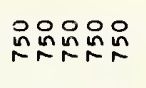 & 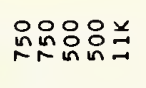 & 임욤요 & 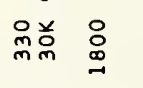 & 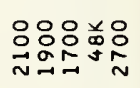 \\
\hline$\mapsto$ & E & 옹용ㅇㅇㅇㅇㅇㅇ & 응응응응응 & 송ㅇㅇㅇㅇㅇㅇㅛ & 용ㅇㅇㅇㅕ & 응응응ㅇㅇㅇㅇ & 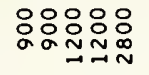 & 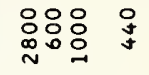 & 家是 & : \\
\hline " & & 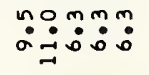 & $\ddot{2}: m: m$ & 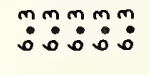 & 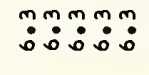 & 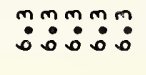 & 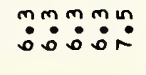 & $\ddot{n}$ & $\ddot{\square}:$ & $\ddot{m}$ \\
\hline N0IIyyjdO & טuטu & a auu & טنטu & טuטu & טuטu & טuטu & טuטu & $a v a a u$ & טuט & 00000 \\
\hline ענW & 水らさひ & 오모ココ & コココココ & 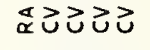 & ひさむひコ & コココゴ & ココさび & 呈＼cjkstart赶 & 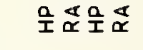 & 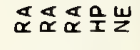 \\
\hline JNחI & ケ岁ら岂岂 & 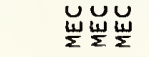 & 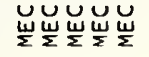 & 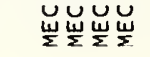 & 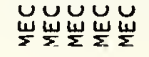 & U⿺辶ِّ & 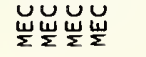 & 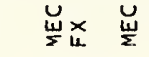 & $\frac{u}{\Sigma}$ & 适岕岕 \\
\hline OIEY $\times 1 \cap 0$ & & in in & & & & & & N 응요 & in & 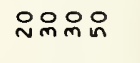 \\
\hline 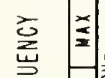 & 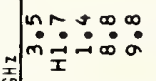 & 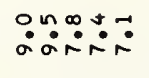 & 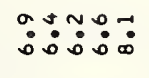 & 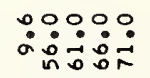 & $\ddot{0}: 0 \infty \dot{\infty}$ & 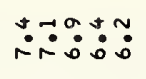 & 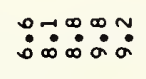 & 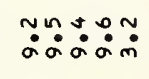 & 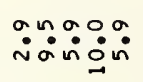 & 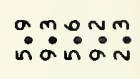 \\
\hline 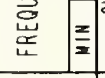 & 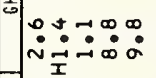 & 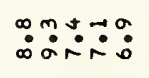 & 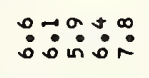 & ๓:0웅 & 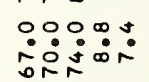 & 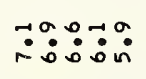 & 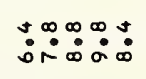 & $\begin{array}{l}+\sim \dot{m} \backsim \dot{a} \\
\dot{\infty} \dot{\infty} \dot{\infty} \dot{\infty}\end{array}$ & i̊ñ & 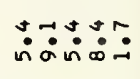 \\
\hline ONIX & 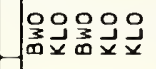 & 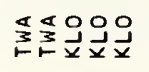 & 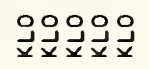 & 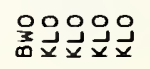 & 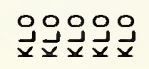 & 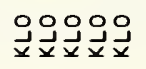 & 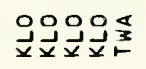 & 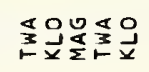 & 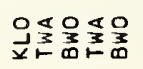 & 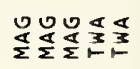 \\
\hline $708 w<S$ & & & & " " " " & " " " & & $"$ & & & \\
\hline 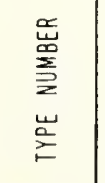 & 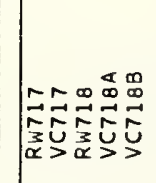 & 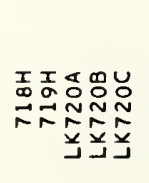 & 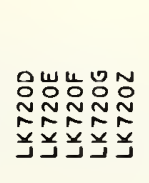 & 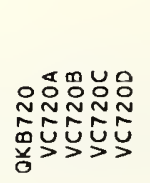 & 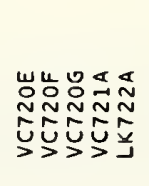 & 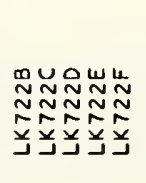 & 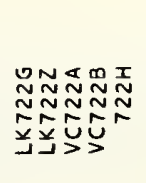 & 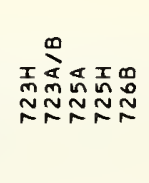 & 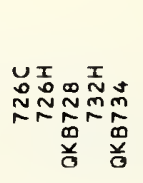 & 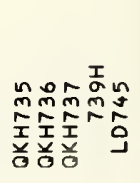 \\
\hline
\end{tabular}




\begin{tabular}{|c|c|c|c|c|c|c|c|c|c|c|}
\hline 9אוTdnOS & 언 욱 & 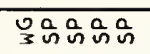 & 언의 & 엉웅어 & 엉요 & তত্যOי & 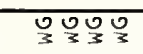 & ততুహত্ & ত్ऊणु్ & 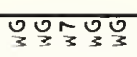 \\
\hline HLAYS & $\vec{x}$ & $\vec{\alpha} \vec{\alpha} \vec{\alpha} \vec{\alpha} \vec{x}$ & $\bar{\alpha}$ & $\vec{s}$ & & テすテすす & $F \overline{7}$ & テすテすす & テすテテす & 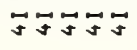 \\
\hline 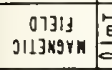 & $\sum \Sigma$ & & $\Sigma \Sigma \Sigma$ & $\sum \Sigma \sum \Sigma$ & $\Sigma \Sigma \Sigma$ & $\Sigma \Sigma$ & $\Sigma \Sigma$ & & & \\
\hline H101M0Kr8 & $\stackrel{n}{\sim}$ & ํㅗㅇㅇㅝ & s & & & $\infty \simeq$ & $\infty 0$ & & & \\
\hline 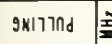 & 푼. & & & & & & & & & \\
\hline $\begin{array}{l}\text { Yo19ys } \\
\text { 3S10x }\end{array}$ & $\tilde{N}$ & & $\stackrel{ }{m}$ & min & & & & & & \\
\hline xirg & 司守 & & & :용 & & 웅워 & ogo요요 & 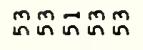 & 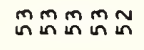 & กำ \\
\hline $\begin{array}{l}51701 \\
\times 11334 \\
\end{array}$ & 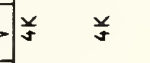 & & 并夈 & 并前 & & & & & & \\
\hline $\begin{array}{c}51701 \\
1001 \times 03\end{array}$ & In & & 음웅음 & 욱 & & & $\stackrel{n}{N} \underset{n}{n} \stackrel{n}{n}$ & & & \\
\hline 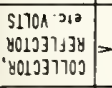 & $=$ & 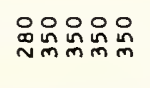 & 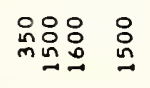 & 品 品 & 응욤요 & & $\stackrel{n}{2}$ & & & \\
\hline$Q^{\circ}$ & $\leq=$ & 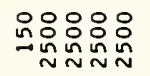 & 윰웅욤ㅇㅇㅁ & 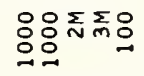 & 윰유 & 兰兰兰兰兰 & 㒸兰并 & 弚弚兰弚 & 弚弚并并 & $\underset{N}{ } \underset{N}{ } \underset{\sim}{ } \stackrel{x}{N}$ \\
\hline 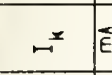 & 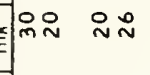 & N & mino $\begin{array}{l}n \\
0\end{array}$ & 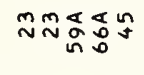 & $\begin{array}{ll}\circ & \circ \\
0 & 0 \\
0\end{array}$ & 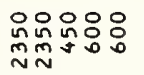 & n응ㅇㅇㅇㅇㅛ & 잉용용 & 잉용요 & :০০০চ⿻心: \\
\hline 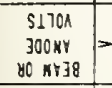 & $=\mid$\begin{tabular}{ll}
$\circ$ \\
\hdashline \\
\hdashline
\end{tabular} & 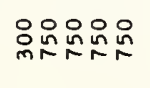 & 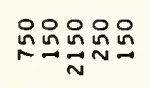 & 吠总恙怘 & : & 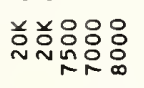 & 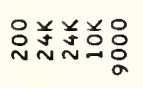 & 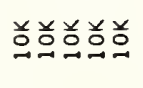 & 局弟兰㒸 & 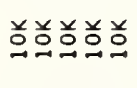 \\
\hline- & $: \begin{array}{ll}0 \\
00 & 0 \\
0 \infty & 0\end{array}$ & & 옹 $\stackrel{n}{\sim}$ & ::ㅇㅇㅇ & & 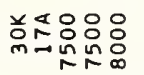 & 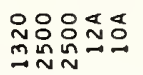 & 우유유류 & 유ㅇㅠㅠㅇㅠ & :유유유 \\
\hline$\omega^{ \pm}$ & $\stackrel{n}{m}: \stackrel{m}{:}$ & & $\ddot{n}: \ddot{n}_{0}^{\infty}: \dot{0}$ & $\ddot{0}: \ddot{0}: 0$ & $\ddot{m}: \ddot{m}$ & 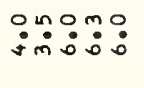 & : & $\because \because 0000$ & $\because \because 00:$ & $\because \because 00: 0$ \\
\hline NOIIFYG & טuט & טuטu & טuטuン & Uua & $a \cup \cup \cup a$ & טuטu & ua & טuטu & טuט & טu \\
\hline$\forall \mathrm{JW}$ & 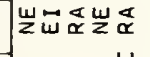 & 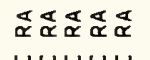 & 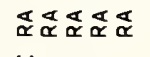 & 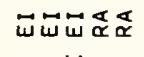 & $\underset{\alpha \underset{\alpha}{\alpha} \underset{\alpha}{\alpha} \underset{\alpha}{\alpha}}{\alpha}$ & \メマメ゙ & 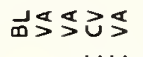 & 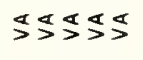 & 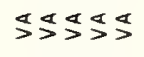 & タタタタை \\
\hline 3Nก1 & $\vdash \quad \stackrel{u}{\Sigma}$ & 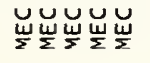 & 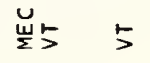 & $\stackrel{\breve{w}}{\Sigma}\llcorner$ & 岕レレレ゙ & U⿺乚一匕 & $\underset{\Sigma}{\underline{\Sigma}} \frac{u}{\Sigma}$ & $x_{u}^{\times x \times x}$ & 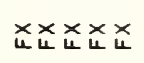 & 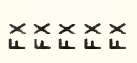 \\
\hline Oalyy dino & & & & 응요 & & & 응음 & & & \\
\hline 䇋 & $\because \because \sin ^{\infty}$ & 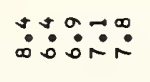 & 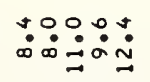 & $\because \because 00$ & 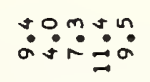 & iñن் & 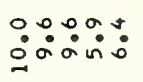 & 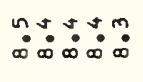 & 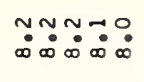 & 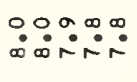 \\
\hline 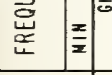 & $\because \because \because \because$ & 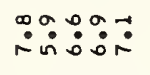 & 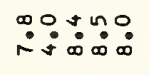 & 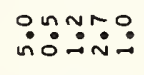 & 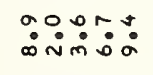 & $\ddot{\sim} \because \dot{\sim} \therefore \dot{\sim}$ & 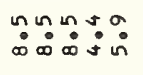 & $\begin{array}{l}\checkmark \\
\infty \\
\infty \\
\infty\end{array}$ & $\because \because \because \because \therefore \circ$ & 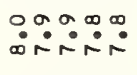 \\
\hline ONIX & 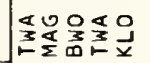 & ئُ & 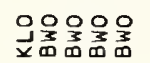 & 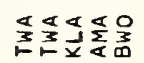 & 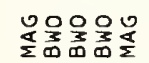 & 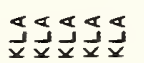 & 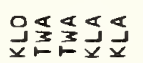 & 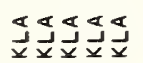 & 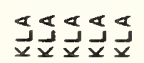 & 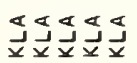 \\
\hline Togh & $"$ & & " " & & & $" \quad "$ & " " " & " " " " " & " " " " & " " " " \\
\hline 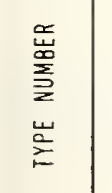 & 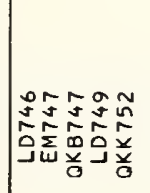 & 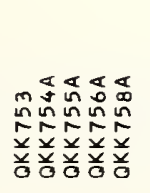 & 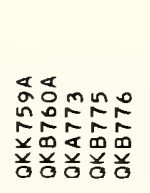 & 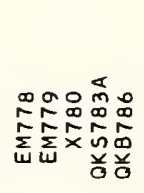 & 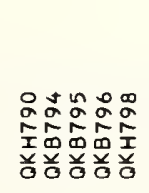 & 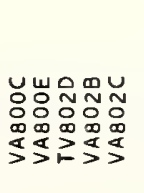 & 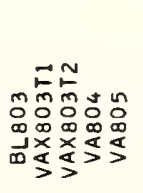 & 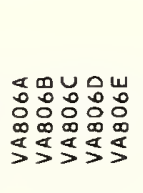 & 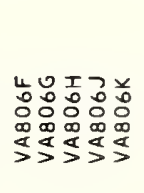 & 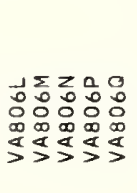 \\
\hline
\end{tabular}




\begin{tabular}{|c|c|c|c|c|c|c|c|c|c|c|}
\hline 9.17dnos & गण & उОणु & 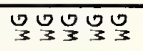 & 웡ㅇ & 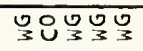 & 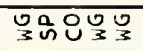 & पुऊ & 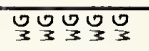 & ত़ु & WO \\
\hline Allaro & ษすテษษ & $F$ & nn & $\ddot{\alpha} \quad \vec{n}$ & テす心 & $\ddot{\alpha} 5$ & テすすテす & テテ & テすテすテ & デすテす \\
\hline 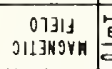 & & $\sum \sum \sum$ & $\Sigma \quad \sum$ & $\Sigma \Sigma$ & $\Sigma$ & $\sum \sum \sum \sum$ & $\sum \sum \sum \sum \sum \sum$ & $\sum \sum$ & & \\
\hline H1OMONY9 & & $\stackrel{m}{=}$ & 용 & $\stackrel{n}{n}$ & 용 & ำก웅ㅇ & 유윰유 & 율N & $\mathbb{N} N \mathbb{N} N$ & $\approx N N \mathbb{N}$ \\
\hline 9חורורו & त्) & & & & & & & & & \\
\hline $\begin{array}{r}8013 y+1 \\
35 \text { Siok } \\
\end{array}$ & & $\stackrel{n}{m}$ & & 욤 & & & & & & \\
\hline Nivg & 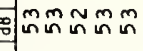 & in & $\stackrel{\sim}{\simeq}$ 品 & $\stackrel{\sim}{\sim}$ & 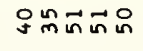 & $\tilde{N}$ in & กี $N$ ก $N$ N $N$ N & Nㅗำ & $\mathfrak{q} \mathfrak{q} \mathfrak{q} q \mathfrak{q}$ & 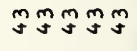 \\
\hline $\begin{array}{l}51701 \\
\times 173 H \\
\end{array}$ & & $\stackrel{\sim}{N}$ & & & & & & & & \\
\hline $\begin{array}{c}S 1701 \\
7081 * 03\end{array}$ & & $\stackrel{\circ}{n}$ & & $\stackrel{\circ}{\circ}$ & & & & & & \\
\hline 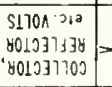 & & $\stackrel{n}{\sim}$ & & 品 品品 & & 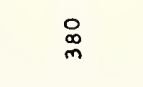 & & & & \\
\hline $0^{\circ}$ & 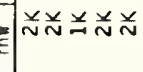 & 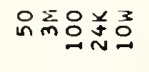 & 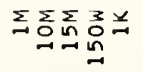 & 음윰유수 & 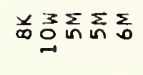 & 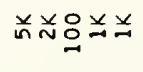 & 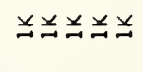 & ㅡㅡ임ㅇ & 丷。。ㅡㅁ음 & 吕吕 \\
\hline 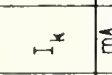 & 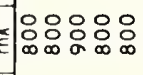 & 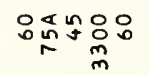 & 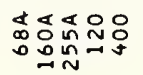 & 요의 & 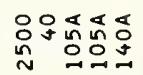 & 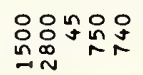 & 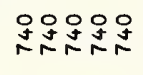 & 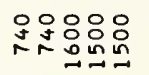 & 웅용요 & 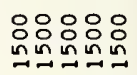 \\
\hline $\begin{array}{c}5170 \mathrm{~A} \\
300 \mathrm{NY} \\
80 \mathrm{KH} 3 \mathrm{~B}\end{array}$ & 뭉ㅁㅁㅇㅁㅇㅁㅇ & 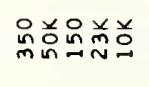 & 詞总总总总 & 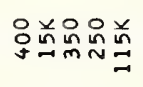 & 总品总总点㽞 & 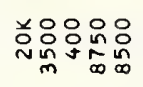 & 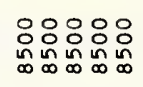 & 总总总总总 & 恙总芯并总 & 总酋总酋总 \\
\hline$\mapsto$ & | & 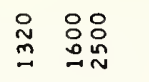 & 过 & $\underset{\sim}{\stackrel{O}{m}} \underset{\sim}{\stackrel{i}{N}}$ & 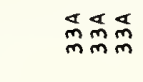 & 웅요 & $\begin{array}{l}\circ \circ \\
\circ \circ \\
\circ 0\end{array}$ & 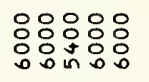 & 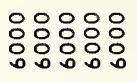 & $\begin{array}{l}\circ: 0 \\
: 00: 0 \\
\circ 0 \\
00000\end{array}$ \\
\hline w" & $\because \because \because 000$ & $\stackrel{m}{:}: m$ & ño & $\ddot{m}: m \ddot{m}:$ & 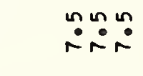 & $\ddot{2}: 00$ & $\because: 00: 0$ & $\because: \because: \because: 0$ & $\because \because: 0:$ & $\because \because: 00$ \\
\hline NO1_t8y $3 \mathrm{dO}$ & טuטu & ט & a a au & aטuט & Uuaaa & טuu & טu & טu & ט ט & טบט \\
\hline yJw & 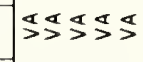 & $\vec{\omega}_{\alpha}^{\alpha} \underset{\alpha}{\alpha} \mathbf{a}$ & 乐乐呈呈 & 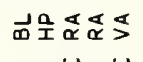 & 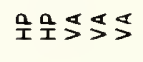 & $a_{\alpha}^{a} \propto \alpha \Delta$ & 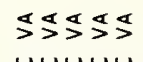 & \ロダタ & 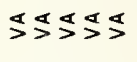 & 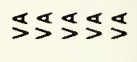 \\
\hline 3NחL & 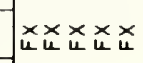 & $5 x$ & xxx & 㭊ヶ岕 & 宏原原 & 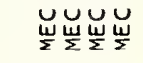 & 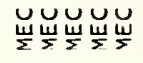 & 岕出 $\times x$ & 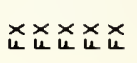 & 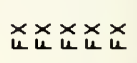 \\
\hline Ollty $x$ LIro & & $=$ : & 웅유 & q & ํํำ & $\stackrel{\circ}{\circ}$ & & & & \\
\hline 总 & $\mid \begin{array}{l}\infty \\
\check{\sim} \\
\ddot{\sim}\end{array}$ & \begin{tabular}{l}
0 \\
$\dot{0} \sim 0$ \\
\hdashline
\end{tabular} & 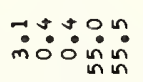 & 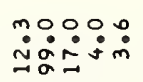 & 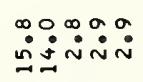 & 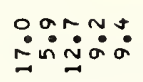 & : & 品品: & 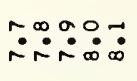 & 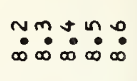 \\
\hline 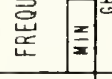 & 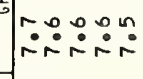 & 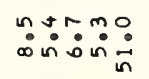 & OJ & †ㅇㅇㅁㅇㅛ & 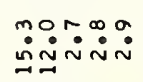 & 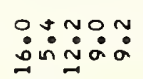 & 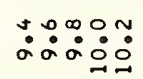 & 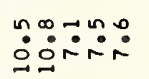 & 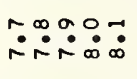 & 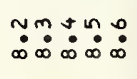 \\
\hline ONIX & 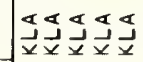 & 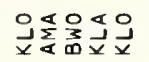 & 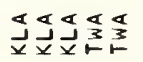 & 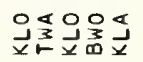 & 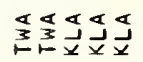 & 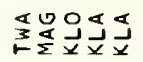 & 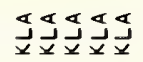 & 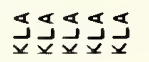 & 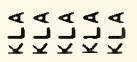 & 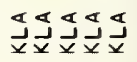 \\
\hline 70gW AS & " " " " " & " " & & " " & " " & " " & " " " " & " " " & & \\
\hline 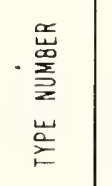 & 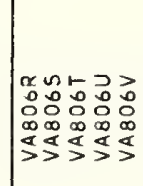 & 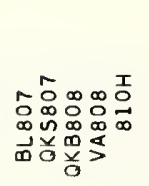 & 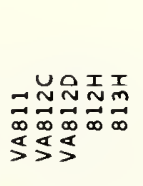 & 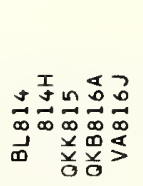 & 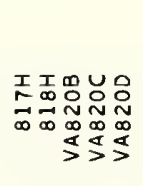 & 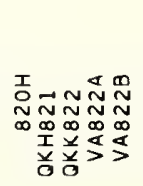 & 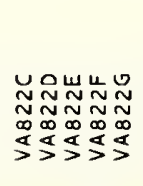 & 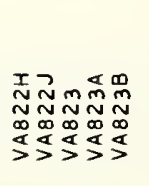 & 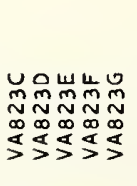 & 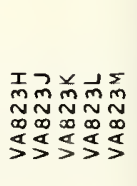 \\
\hline
\end{tabular}




\begin{tabular}{|c|c|c|c|c|c|c|c|c|c|c|}
\hline 9NITdחOS & 00000 & 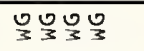 & 인 & 온언은 & OU. & ํํำ & Uण & 은영 & OUல & WO \\
\hline ALAYJ & けすすすす & テすデ & $\vec{x}$ & $\vec{\alpha} \bar{\alpha} \vec{\alpha} \vec{\alpha}$ & ひすすすす & $\vec{\alpha} \bar{\alpha} \tilde{\sim} \vec{\alpha}$ & $\vec{\alpha} \vec{\alpha} \vec{\varpi}$ & ฉேる゙ & $\vec{\square} \vec{\jmath}$ & テすコすす \\
\hline $\begin{array}{c}01311 \\
\text { ग113N9VM }\end{array}$ & & & $\Sigma$ & & $\Sigma \Sigma$ & $\Sigma$ & $\Sigma$ & & $\Sigma$ & \\
\hline hLOMONYB & $\tilde{I} \sim \sim \sim N \sim N$ & $\tilde{N} N$ P & ming & ㅇmㅇ오음 & ベニさ里 & $\stackrel{n}{\sim} \underset{f}{m}$ & 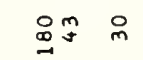 & mong on & $\stackrel{\infty}{2} \quad \infty \infty$ & $\infty \infty \infty \infty \infty$ \\
\hline פחרדורו & $\underline{x}$ & & & & & & & & & \\
\hline 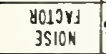 & & & $\stackrel{n}{\sim}$ & & & & & & & \\
\hline Mirg & 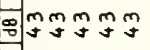 & $\underset{\sim}{m} \underset{\sim}{m} \underset{v}{m}$ & $\stackrel{n}{m}$ & q & 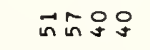 & in & $\stackrel{\sigma}{m} \stackrel{\rho}{s}$ & mo & nn $\operatorname{rnn}_{n} n$ & 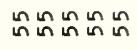 \\
\hline $\begin{array}{l}51701 \\
\times 1134\end{array}$ & & & & & & & & & & \\
\hline $\begin{array}{c}\text { S170n } \\
\text { 1081N03 }\end{array}$ & & & : & & & 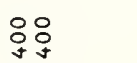 & & & & \\
\hline 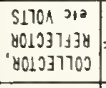 & & 号 & 윴용요 & 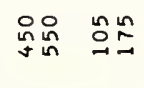 & $\stackrel{\circ}{\sim}$ & 字: & $\stackrel{\circ}{\circ}$ & $\stackrel{n}{m}$ & $\stackrel{\circ}{n}$ & \\
\hline$a^{\circ}$ & 关关关首 & 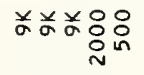 & 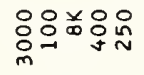 & 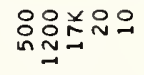 & 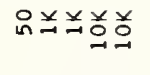 & 四望总总总 & 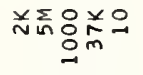 & 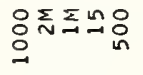 & 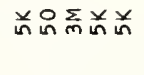 & 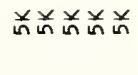 \\
\hline 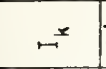 & Eᄐ & 웅용요 & 암욤욤 & nñ & 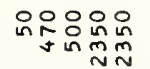 & ơ & 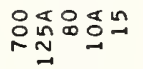 & \&芯品 & 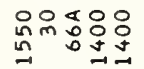 & $\begin{array}{l}\circ \circ 800 \\
09 \\
\end{array}$ \\
\hline 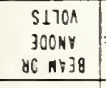 & 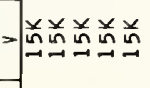 & 芯芯酋踶 & 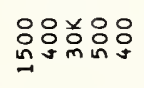 & 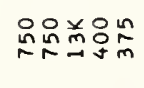 & 路哭总酋 & 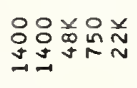 & 表兑员总品 & 虽㒸美的品 & 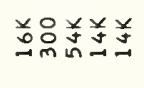 & 弟姜总姜总 \\
\hline 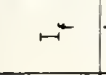 & Ê & 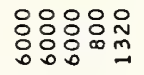 & 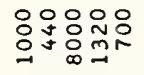 & $\frac{n}{5} \leq 0$ & 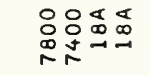 & 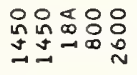 & : & : & : & 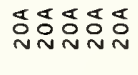 \\
\hline$\omega^{\leftarrow}$ & $>\quad \because \because \because \because \because$ & $\because \because: 0 m:$ & $\ddot{m}:{ }^{m}:{ }^{n}: m m:$ & $\stackrel{m}{m}: \ddot{m}: m$ & 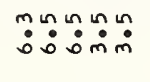 & $\begin{array}{l}n \sim 0 m m \\
\dot{\sim} \sim \dot{\sim}\end{array}$ & 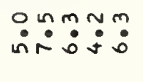 & mo:m & $\ddot{n} \ddot{m} \dot{m} \dot{m}: \dot{m}$ & $\dot{m} \dot{m} \dot{m} \dot{m} \dot{m}$ \\
\hline NOIIYyJdO & טuטu & บบบuั & บบบบy & บบบuy & บบบบa & vบava & $\cup a \cup a U$ & va au & auauv & บบบบบ \\
\hline ydu & 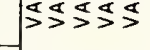 & 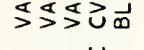 & 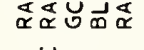 & 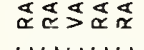 & 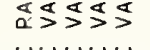 & ๔๘ょコ & 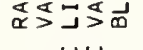 & $コ$ & ১\৫ひU & ひさひンさ \\
\hline $3 \mathrm{~N} \cap 1$ & 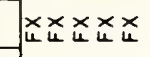 & 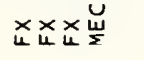 & $\underset{\Sigma}{w} \times 5$ & WuUw & Wư & 岀岕岕岕 & 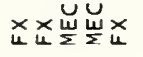 & 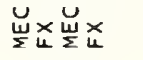 & 岀x & 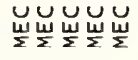 \\
\hline 0 olyy ungo & & & & & & ${ }_{9}$ in & 요 & 용요 & & \\
\hline 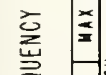 & 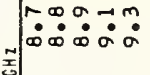 & 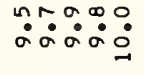 & 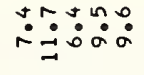 & 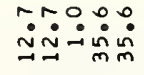 & 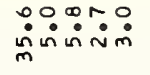 & 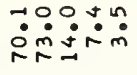 & 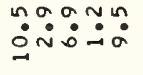 & 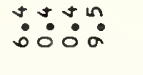 & 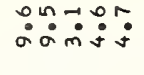 & 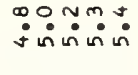 \\
\hline 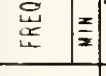 & 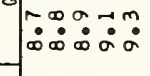 & 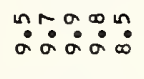 & 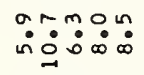 & ஸ̃:O: & 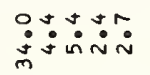 & 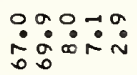 & 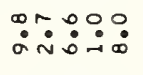 & $\ddot{0}: \dot{0}: \stackrel{m}{0} \dot{m}$ & 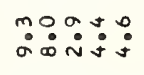 & 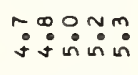 \\
\hline ONIX & 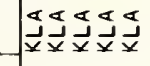 & 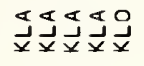 & 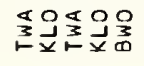 & 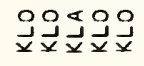 & 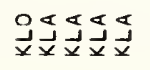 & 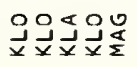 & 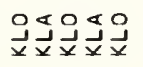 & 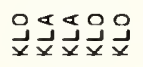 & 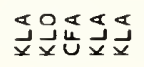 & 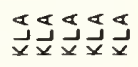 \\
\hline $708 \mathrm{~W} / \mathrm{S}$ & & & " & & & & " & " " & " " " " & " " " " " \\
\hline 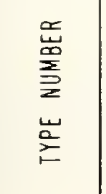 & 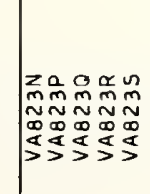 & 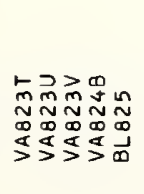 & 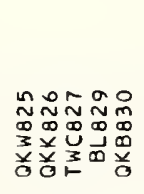 & 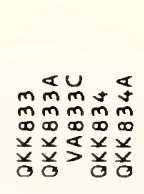 & 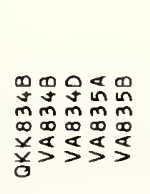 & 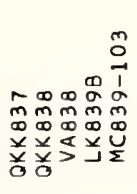 & 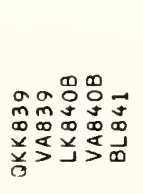 & 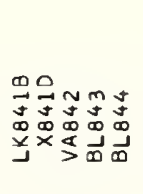 & 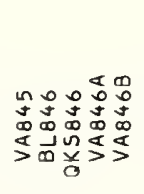 & 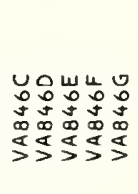 \\
\hline
\end{tabular}




\begin{tabular}{|c|c|c|c|c|c|c|c|c|c|c|}
\hline 9RITdחOS & O०ण & 芆 & पण & 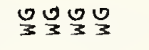 & O० & पण उण & 웡요 & 뭉옹요 & 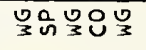 & 어 \\
\hline LIIAVS & งテすす & $F \overrightarrow{5}$ & ガルすすす & テすすデ & テデロ゙れ & $\vec{n} \vec{\alpha} \bar{\alpha} \quad \vec{\alpha}$ & 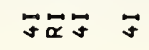 & 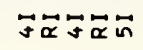 & $\vec{n} \tilde{\alpha}$ & $\vec{\alpha} \vec{\alpha} \quad \vec{\jmath}$ \\
\hline $\begin{array}{ll}01311 \\
\text { J119K9VK }\end{array}$ & & $\Sigma$ & $\Sigma$ & & $\Sigma$ & $\Sigma$ & $\Sigma \Sigma \Sigma$ & & $\Sigma \Sigma$ & $\Sigma$ \\
\hline HLOMONYA & $=\infty \infty \infty \infty$ & $\pm \pm \tilde{N}$ & $\sigma=z$ & 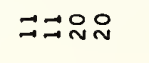 & $\tilde{m}=\tilde{m}$ & $\stackrel{\circ}{\sim}$ & $\because n$ & 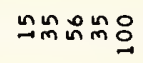 & $\stackrel{n}{n} \dot{m}$ & 욨n一요요 \\
\hline dחרדואי & & & & & & & & & & \\
\hline $\begin{array}{l}\text { yoljors } \\
\text { JSlon }\end{array}$ & & & & & & & $\stackrel{n}{m}$ & & & \\
\hline 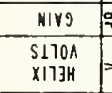 & 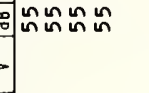 & 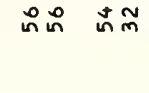 & No: & ํำถนก & No & in & $n$ in 2 & in in in & in in $\tilde{0}$ & s \\
\hline $\begin{array}{c}5170 \mathrm{~A} \\
10 \mathrm{OH} 1 \mathrm{NOS}\end{array}=$ & & $\stackrel{\circ}{\underset{\sim}{~}}$ & & 욤요 & 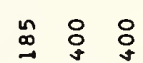 & 잉유 & $\stackrel{8}{\Omega}$ & & & $\stackrel{8}{\circ}$ \\
\hline 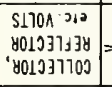 & $\stackrel{\sim}{\sim}$ & : & & & 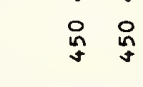 & 용요 & 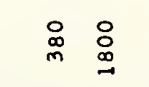 & 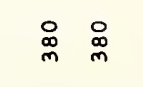 & 号 & 웅유 \\
\hline$a^{\circ}$ & 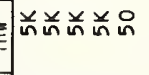 & 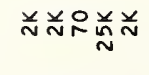 & 弟关次次 & 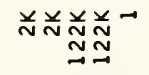 & $\underset{N}{N} \underset{\sim}{N} \underset{N}{\mathbb{N}}$ & 뭉 $\sim \sim ⿻ 上$ & 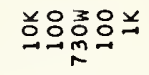 & 음ㅎㅇㅇ음ㅇㅁ & 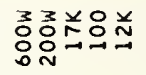 & 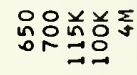 \\
\hline 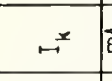 & 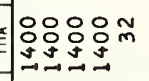 & 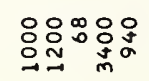 & 응융요 & 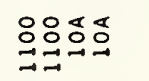 & 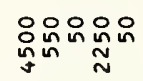 & 음요요요용 & 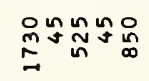 & ำㅇํㅇㅇㅇㅇㅇㅇ & 응요 & 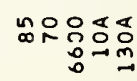 \\
\hline 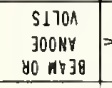 & 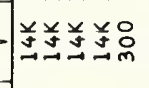 & 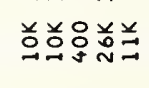 & 兰酋兰兰兰 & 兰兰鬲首 & 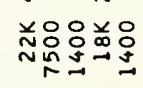 & 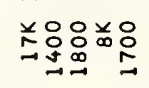 & 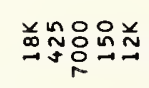 & 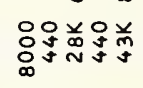 & 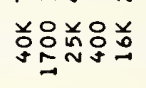 & 品员并㒸㒸 \\
\hline$H^{4}$ & 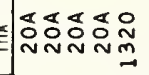 & 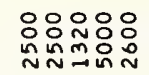 & 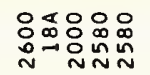 & 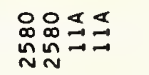 & 응용요 & 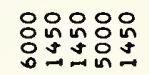 & 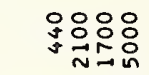 & 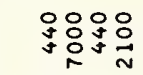 & $\begin{array}{ll}\circ & \circ \\
\vec{N} & 9\end{array}$ & $\begin{array}{l}0 \\
0 \\
0 \\
0\end{array}$ \\
\hline$\omega^{4}$ & 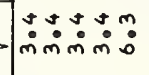 & 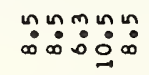 & 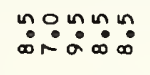 & $\begin{array}{l}n \sim 00 \\
\dot{\infty} \infty \\
\infty \\
\infty\end{array}$ & 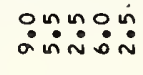 & 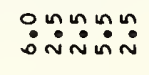 & mo:m: & $\ddot{m}: \stackrel{m}{m}: \dot{m}$ & 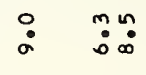 & 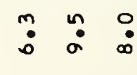 \\
\hline N0117y9d0 & טטuנ & טuטu & vaauv & ưưu & uטuv & טuטu & vuava & & a auvu & uบưa \\
\hline yנW & ひさむさ & マタガタ & 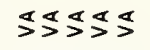 & 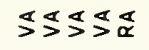 & 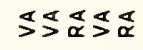 & 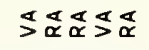 & 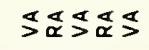 & 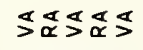 & 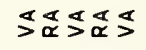 & 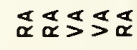 \\
\hline JNח1 & 䦽岕岕岕 & 岂岕 岂 & 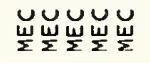 & 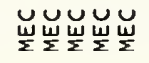 & 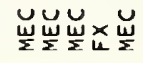 & 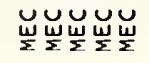 & 嵩岕岕 & 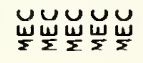 & 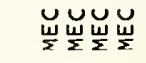 & 訔岕 \\
\hline Oll1yㅓ AInO & & & & & & & 용 & in & go & \\
\hline 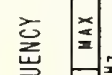 & 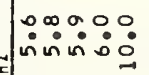 & 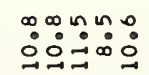 & 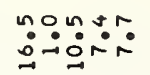 & 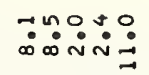 & 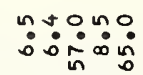 & 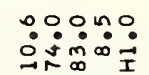 & งNNง: & & 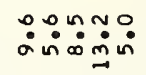 & $\because \because \because \ddot{\infty}$ \\
\hline 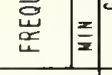 & 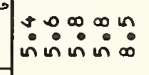 & 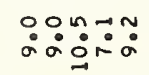 & 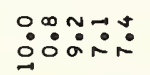 & 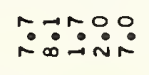 & 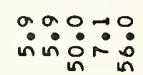 & 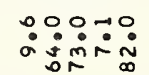 & 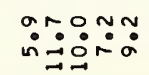 & ON & 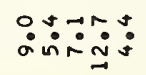 & $\because \because \because ே ゚$ \\
\hline ONIY & 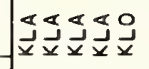 & 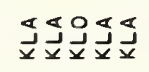 & 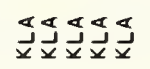 & 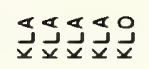 & 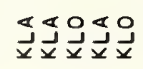 & & 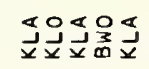 & 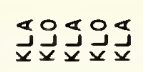 & 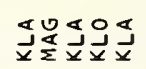 & 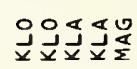 \\
\hline 708w 25 & " " " " " & & & & & & & & & \\
\hline 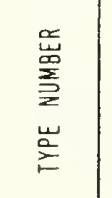 & 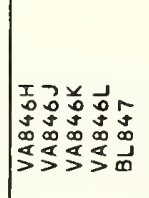 & 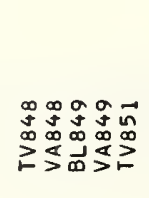 & 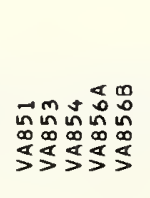 & 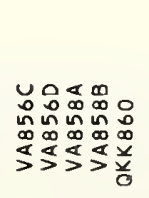 & 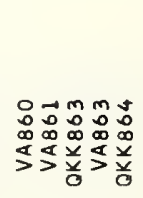 & 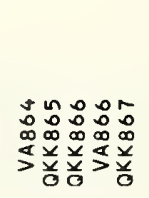 & 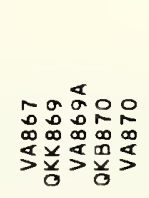 & 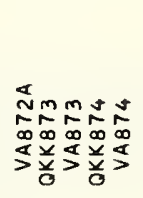 & 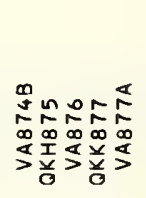 & 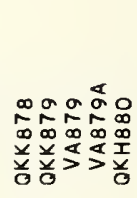 \\
\hline
\end{tabular}




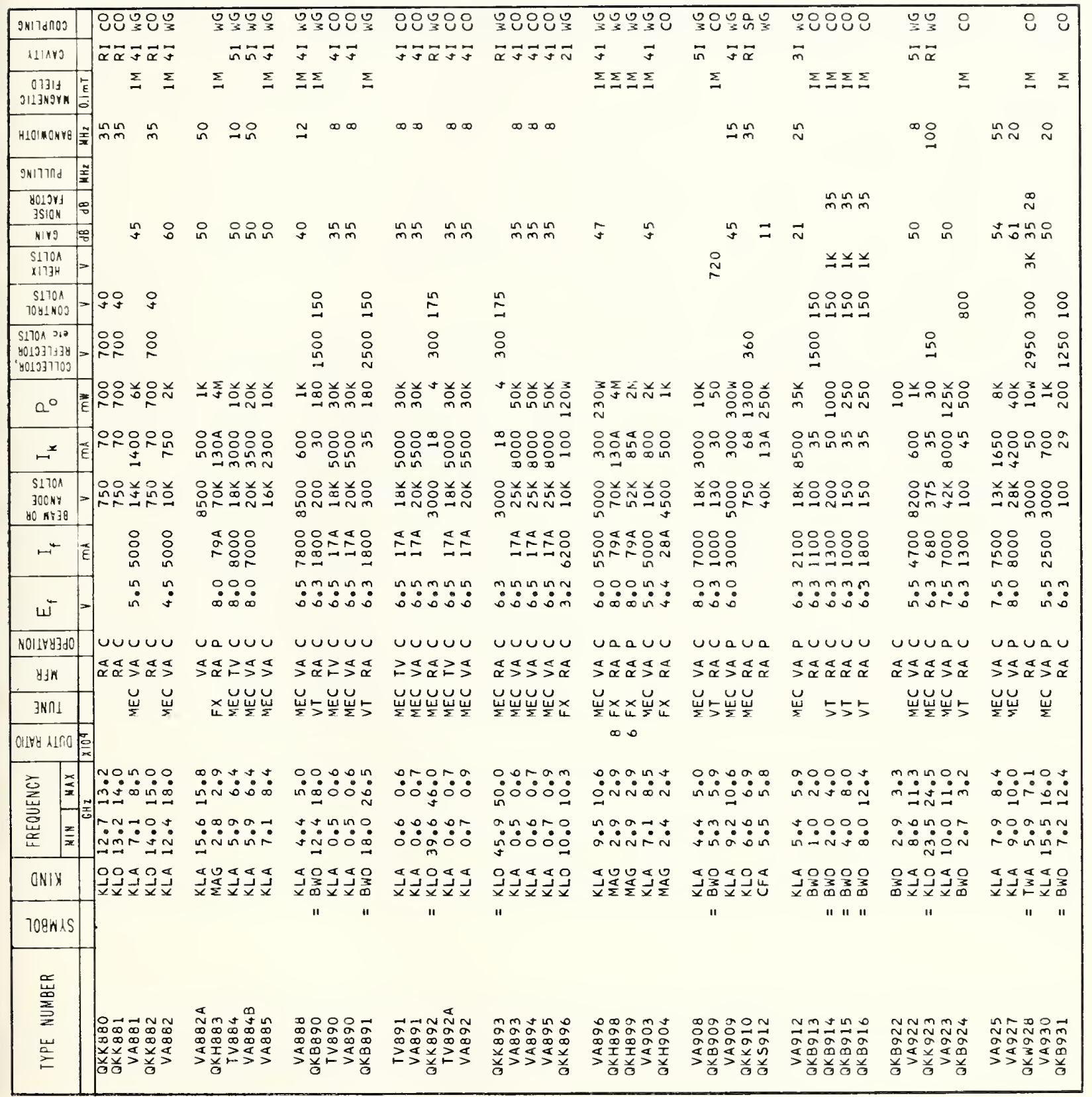




\begin{tabular}{|c|c|c|c|c|c|c|c|c|c|c|}
\hline 9N17d 103 & 웅요 & 운옹 & 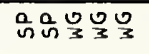 & \%우요 & 8000 & $8 \% \frac{0}{3}$ & \%० & $\begin{array}{lll}0 & 0 & 0 \\
3 & 3 & 3\end{array}$ & 엉엉요 & 엉ㅇㅇㄴ \\
\hline RHANO & こี & $\vec{x} \bar{x}$ & $\vec{\alpha} \vec{\alpha} \vec{x} \quad \vec{\sim}$ & $\vec{\alpha} \vec{\alpha} \quad \vec{\alpha}$ & & $\underset{\mho}{w}$ & $\begin{array}{ll}\varpi \\
\mho\end{array}$ & 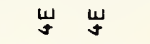 & $\underset{\propto}{\mathbf{\alpha}} \underset{\alpha}{\sim}$ & \\
\hline $\begin{array}{l}01314 \\
013 N 9 \times K\end{array}$ & $\Sigma \Sigma$ & $\sum \Sigma$ & & & $\Sigma \Sigma \Sigma$ & $\sum \stackrel{0}{0}$ & $\Sigma \Sigma$ & \begin{tabular}{lll}
$\circ$ & $\circ$ & 0 \\
0 & 0 & 0 \\
\hdashline & 0 & 0
\end{tabular} & 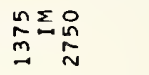 & $\sum \sum \Sigma \sum$ \\
\hline HLOMONYG & $\pm n^{n}$ & $\stackrel{n}{m} \tilde{m}$ & $\stackrel{n}{m} \stackrel{n}{m}$ & 요 & $N N$ & & $N N$ & & 0 & \\
\hline 9w17nd & 퐆 & & & & & $\cong r \underline{n}$ & & $r r$ & $\sim \stackrel{n}{2}$ & \pm \\
\hline $\begin{array}{l}012 \mathrm{HI} \\
3 \mathrm{~S} / 0 \mathrm{~K} \\
\end{array}$ & 0 & & & & & & & & $\stackrel{m}{m}$ & $\stackrel{s}{m}$ \\
\hline Mirg & 웝응요 i & & $a$ & $\sigma$ & $i_{m}^{\infty}$ & $\stackrel{\circ}{m}$ & $\stackrel{\circ}{\circ}$ & $\stackrel{\circ}{\circ}$ & 8 & : \\
\hline $\begin{array}{l}5170 \mathrm{~N} \\
\times 113 \mathrm{H}\end{array}$ & $\Rightarrow \quad \stackrel{i}{N}$ & & & & 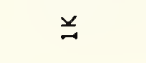 & 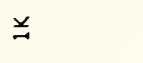 & & & $\cong$ & mNN \\
\hline $\begin{array}{c}5170 \mathrm{~s} \\
10 \mathrm{~N} 1 \mathrm{NOS}=\end{array}=$ & & & 임 & 品 & & & & & 요 욤 & 웅욤욤요 \\
\hline 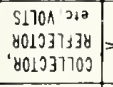 & & $\begin{array}{l}0 \\
\text { 品 } \\
\text { m }\end{array}$ & 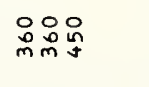 & 品吕 & 总 & $\stackrel{\circ}{\stackrel{\circ}{\infty}} \stackrel{-}{-}$ & $\stackrel{\sim}{\exists} \stackrel{\vee}{\square}$ & 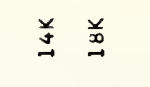 & 욜 욱요 & 总 \\
\hline $0^{\circ}$ & 兹品望 & 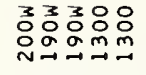 & 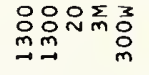 & 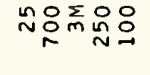 & 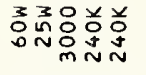 & 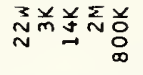 & 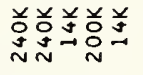 & 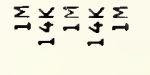 & 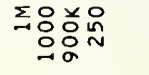 & 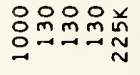 \\
\hline$\mapsto$ & E & 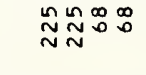 & 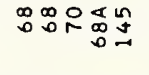 & o̊施 & 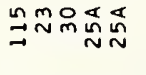 & 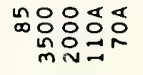 & 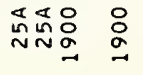 & 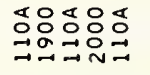 & 맘요묘 & 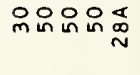 \\
\hline $\begin{array}{c}S 1701 \\
\text { J0oNy } \\
\text { HC N⿴囗十 }\end{array}$ & 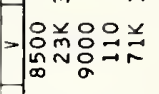 & 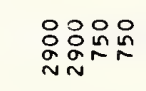 & 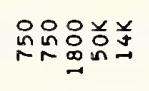 & 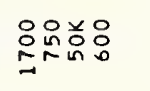 & 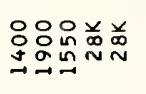 & 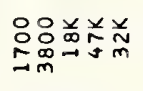 & 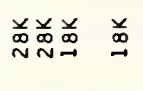 & 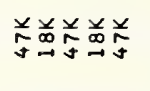 & 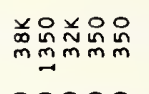 & 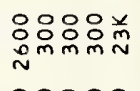 \\
\hline$H^{+}$ & E⿸ & & 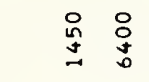 & $\begin{array}{l}0 \\
\text { in } \\
\text { I }\end{array}$ & 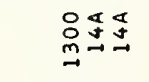 & 슈윯ำ & 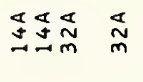 & :্̊సి: & :용요 & :윰윰유. \\
\hline$\omega^{ \pm}$ & $\stackrel{n}{n}$ & & $\tilde{\sim} \dot{m}$ & $\stackrel{n}{\sim} \quad \stackrel{m}{\dot{n}}$ & mmo: & 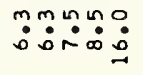 & 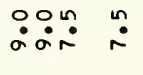 & 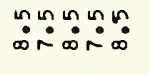 & 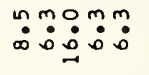 & 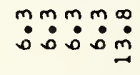 \\
\hline N011 Yy $3 d 0$ & 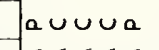 & auvuu & vưau & vuauv & บuvaa & vava & a auau & avaua & auaua & 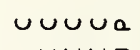 \\
\hline y」n & 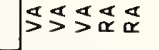 & 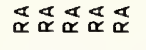 & 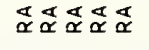 & 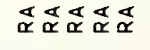 & 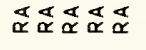 & แェㅗㄴ & 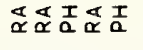 & UI & 山ே゙ヌ゚ㅇㅇㅇ & 岀岀岀 \\
\hline 3Nก1 & 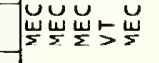 & 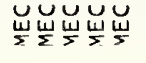 & 岀岀㟐 $\underset{u}{ }$ & 岀 岀 & $\underset{u}{x} \underset{\Sigma}{\breve{w}} \underset{\Sigma}{u}$ & 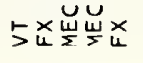 & 主主岂て岌 & 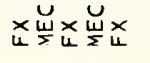 & 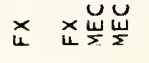 & ケらケメ \\
\hline $0116 y$ a difo & $\stackrel{\infty}{=}$ & & & & $\stackrel{m}{\rightarrow}$ & $\stackrel{\square}{\sim} \cong$ & $m m$ & $\cong \cong$ & $\cong \stackrel{\circ}{=}$ & $\therefore$ \\
\hline 曾 & 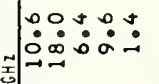 & 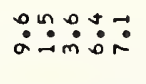 & 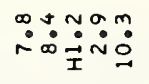 & 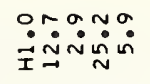 & 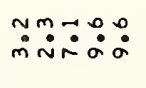 & $\dot{\sim} \dot{\sim} \dot{m}: \dot{m} \dot{m}$ & 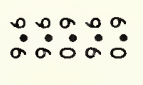 & 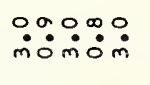 & $\because \because \dot{m} \dot{m}: \dot{0}$ & 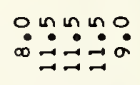 \\
\hline 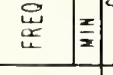 & 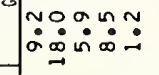 & 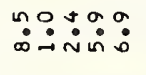 & 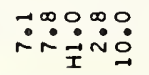 & 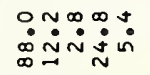 & 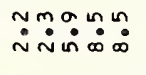 & $\because \because \because \because \dot{m}$ & 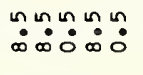 & $\ddot{m o n} \dot{m} \dot{m}: \dot{\sim}$ & 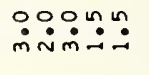 & 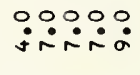 \\
\hline ONIY & 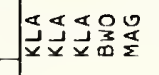 & 울 & 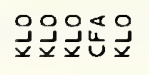 & 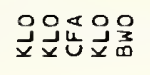 & 이일 & 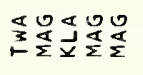 & 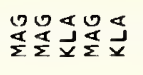 & 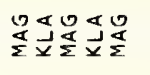 & 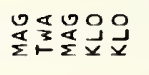 & 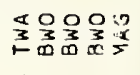 \\
\hline $708 \mathrm{w}$ is & " & & & " & " " " & & " " & & " & " \\
\hline 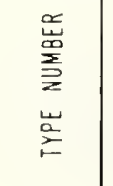 & 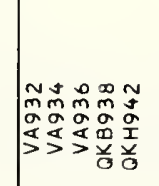 & 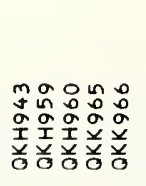 & 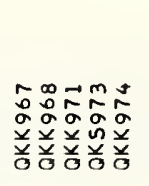 & 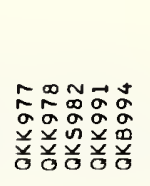 & 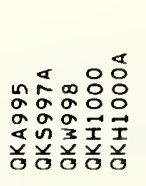 & 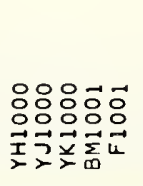 & 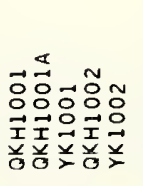 & 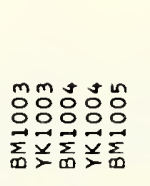 & 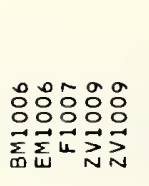 & 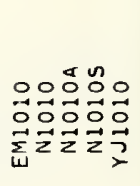 \\
\hline
\end{tabular}




\begin{tabular}{|c|c|c|c|c|c|c|c|c|c|c|}
\hline $9 \times 17$ dnos & 껀웡 & 엉요 & 엉ㅇㅇ & 엉ㅇㅇ웡 & 웅ㅇㅇ & 8080 & 엉연연ㅇ & 的 3 O & 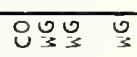 & \begin{tabular}{ll}
0 \\
\hdashline & 0 \\
3
\end{tabular} \\
\hline A11Ars & $\vec{\alpha} \underset{\alpha}{w}$ & 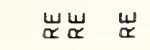 & $\underset{\alpha}{w}$ & & $\vec{\alpha} \quad \vec{\alpha}$ & $\vec{\alpha} \underset{\alpha}{u}$ & $\vec{\alpha} \widetilde{\alpha}$ & $\vec{\alpha}$ & & \\
\hline $\begin{array}{c}01314 \\
\text { ग1399VK }\end{array}$ & $\sum \Sigma$ & $\Sigma$ & 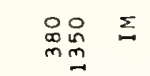 & 品페 & 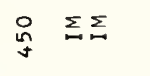 & $\sum \Sigma$ & $\sum \sum \Sigma$ & $\Sigma \quad \Sigma \Sigma$ & 品品 & $\begin{array}{l}0 \\
:\end{array}$ \\
\hline HLOMONY8 & 20 & $\stackrel{\circ}{\sim}$ & & & $\stackrel{\sim}{\sim}$ & $\stackrel{\sim}{\sim}$ & & $\stackrel{\circ}{\sim}$ & & \\
\hline פאורוา & $\tilde{\varepsilon}$ & $\stackrel{n}{\sim}$ & & & 요 & o & & & $\cong \cong$ & $\cong \cong$ \\
\hline 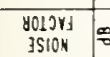 & $\stackrel{+}{m}$ & & $\stackrel{n}{m}$ & 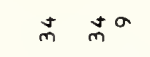 & 0 & in & & $\stackrel{5}{m}$ & $\vec{\sim}$ & \\
\hline \begin{tabular}{l|l} 
Nirg & D \\
\end{tabular} & 암암 & $m$ & 品 & 용 웅우 & $\stackrel{c}{\sim} \underset{n}{n}$ & o & & o & $\stackrel{m}{m}$ & m \\
\hline $\begin{array}{l}5170 \mathrm{~A} \\
\times 173 \mathrm{H} \\
\end{array}$ & 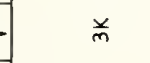 & & & 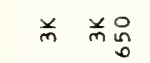 & $\stackrel{\circ}{\circ}$ & & & & $\stackrel{\circ}{\infty}$ & 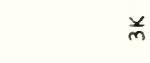 \\
\hline $\begin{aligned} 51701 \\
10011402\end{aligned}$ & 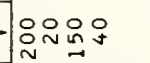 & 욤 & 品 & in in & & $\stackrel{i}{i}$ & 品 & 웅 & in & 号 \\
\hline \begin{tabular}{|l|}
$51704-312$ \\
8019374734 \\
8002237700
\end{tabular} & 음윰유 & 总品 움 & 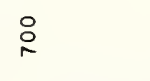 & 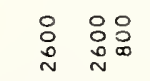 & 品品品 品 & 윰요 & 웅요 & $\stackrel{\circ}{0}$ & $\stackrel{\circ}{\circ}$ & : \\
\hline $0^{0}$ & 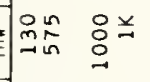 & 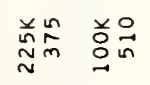 & 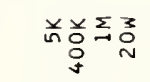 & 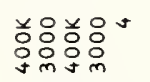 & N & 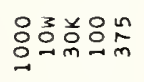 & 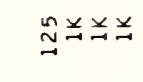 & 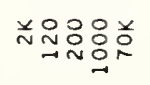 & 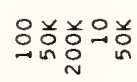 & 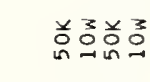 \\
\hline$\mapsto$ & Non min & 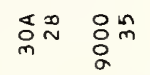 & 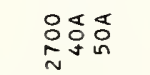 & 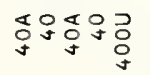 & 긋요쇼요 & 욤 & 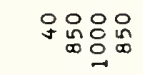 & 욱 $\stackrel{\text { N }}{\sim}$ & N芯芯 & $\stackrel{\substack{0 \\
n}}{n}$ \\
\hline 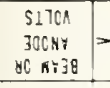 & 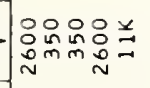 & 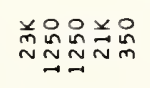 & 品单总总 & 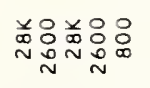 & 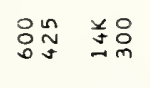 & 总孳品品 & 颔品品品品 & 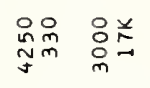 & 总晜总 & 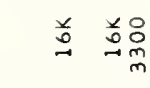 \\
\hline$-\vec{E}$ & 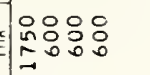 & 용요 & : : & 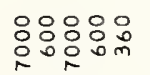 & 品品品品 & 品品品 & 용 & 范 & 品品品 : & : : \\
\hline$\omega^{\top}$ & 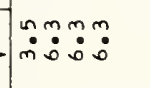 & $\ddot{m} \dot{m} \ddot{0}: \stackrel{m}{0}$ & $\begin{array}{l}n \\
:\end{array}$ & $\dot{0}: \dot{0}: \dot{0}: \dot{m}$ & 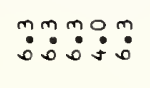 & $\ddot{0}: \ddot{0}: 0$ & 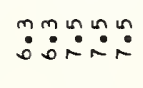 & 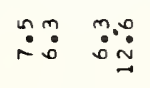 & $\ddot{n}$ & $\ddot{:} \ddot{0}: \ddot{0}$ \\
\hline Nol $1 F y=3$ & טuם & aUa u & $a a a v$ & טuau & טư & vuauv & auvuu & ם & U ט Q & Ua Ua \\
\hline yin & 동ㅇㅇㅁ음 $\approx$ & Iㅇㅇㅇㅇㅇㅇㅇ & 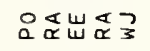 & 岀ே山゙山 & 岀䅹台屴 & ๔壮エ山。 & :@®ZZZ & 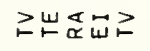 & 岀 & 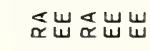 \\
\hline \begin{tabular}{l|l}
$3 N \cap \perp$ \\
\end{tabular} & 岀岕岀 & 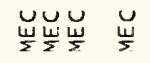 & 岀 岀 & & ヶ & xuّ & 岀岕屈屈屈 & 原 & 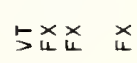 & $\underset{w}{x}$ \\
\hline 016 a dinco & i : & $=\stackrel{\sim}{\stackrel{\circ}{N}}$ & $\dot{m}_{\infty}^{\circ} \operatorname{con}^{n}=$ & in in & m & m & 일 & N & $\therefore ㅇ$ & $\circ \quad$ \\
\hline 昱 & 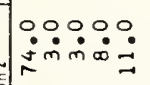 & 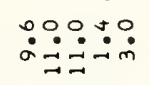 & 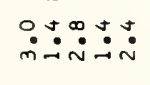 & 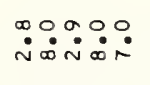 & 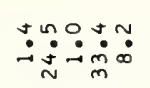 & ง: & Oån & 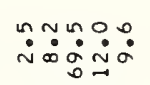 & 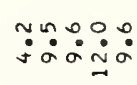 & $\because \because \dot{0} \dot{\sim}$ \\
\hline $\begin{array}{ll}\vec{g} \\
\underline{x}\end{array}$ & $\ddot{\dot{0}} \because \because 000$ & 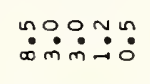 & $\ddot{\circ} \because \dot{\sim} \dot{\sim} \dot{\sim}$ & $\dot{\sim}: \dot{\sim} \dot{\sim} \dot{j}: \dot{j}$ & 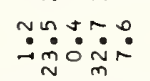 & 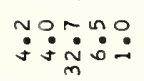 & $\ddot{\because} \dot{\sim} \dot{N} \dot{N} \dot{N}$ & 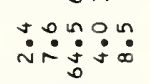 & 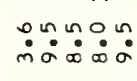 & 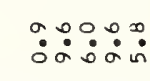 \\
\hline ONIX & 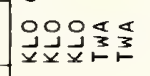 & 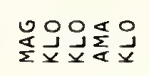 & 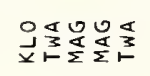 & 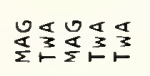 & 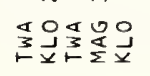 & 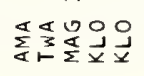 & 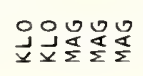 & 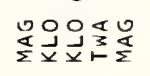 & 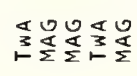 & 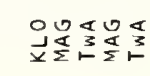 \\
\hline 70gkis & $n$ & & & $"$ & $"$ & $"$ & & " & & \\
\hline 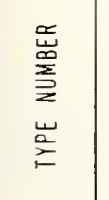 & 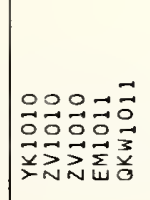 & 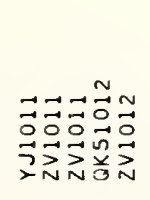 & 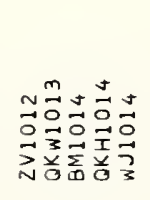 & 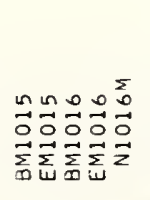 & 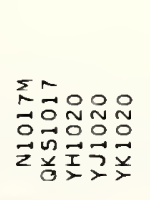 & 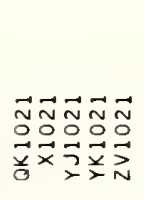 & 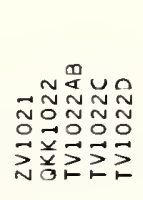 & 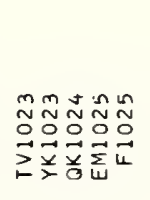 & 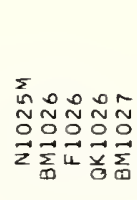 & 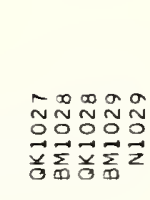 \\
\hline
\end{tabular}




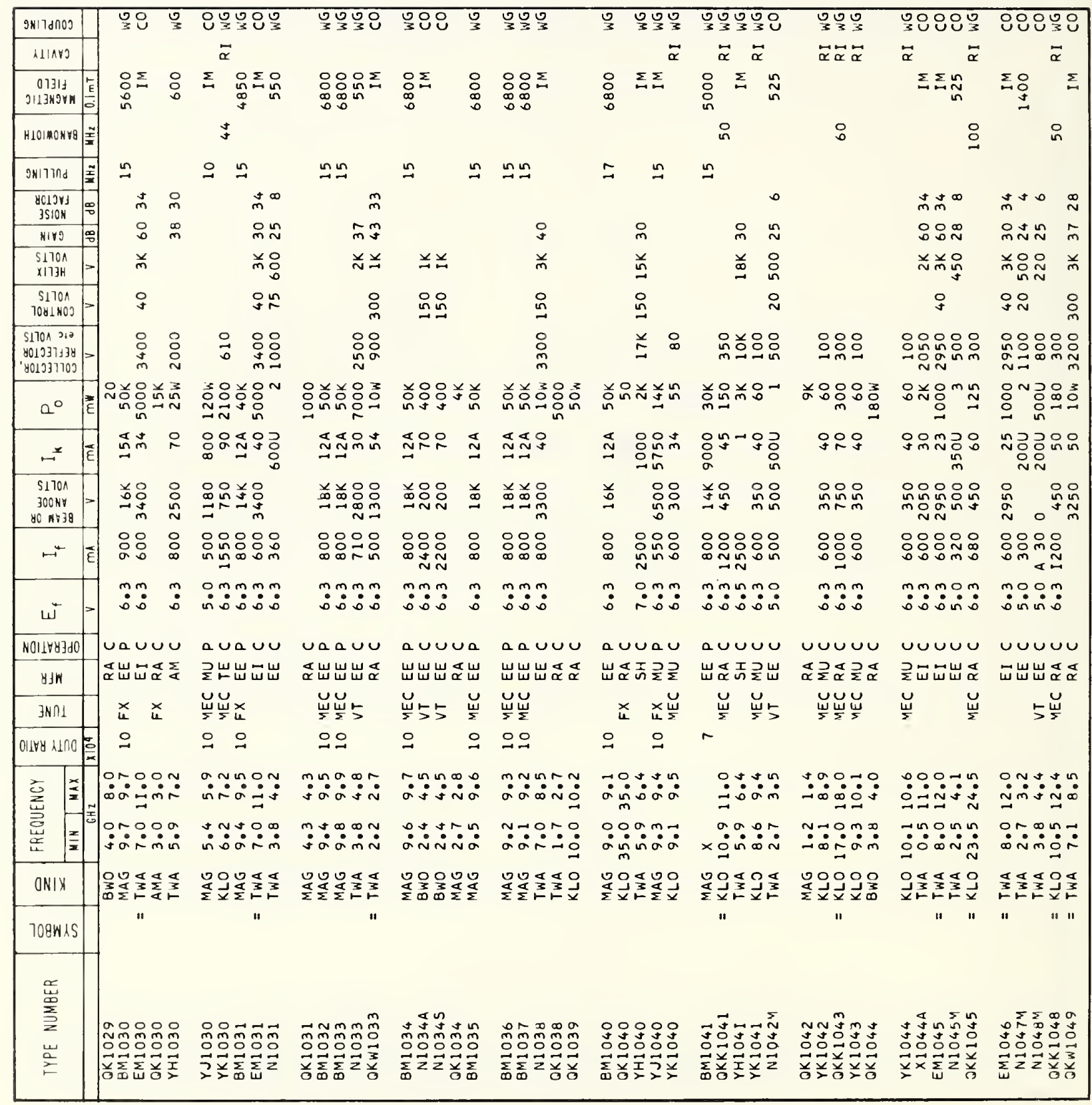




\begin{tabular}{|c|c|c|c|c|c|c|c|c|c|c|}
\hline 9N1 TdnOS & 원 & 언 & 8 & 엉 은 & 엉요 & & $\mathrm{S}_{3}^{\circ}$ & $\frac{0}{3} \overline{3}$ & 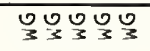 & ㅇß엉ㅇ \\
\hline delavs & $\vec{\alpha}$ & & & & & 山岁 & $\vec{x}$ & $\vec{\alpha} \quad \vec{\alpha}$ & $\vec{\alpha} \vec{\alpha} \vec{\alpha} \vec{\alpha} \vec{\alpha}$ & $\vec{\alpha} \vec{\alpha} \vec{\alpha}$ \\
\hline \begin{tabular}{c|c|c|}
01319 \\
$113 \times 94 M$
\end{tabular} & $\underline{E}$ & $\Sigma$ & $\Sigma$ & $\sum_{-\infty}$ & $\sum \sum \sum \Sigma$ & & $\Sigma$ & $\sum$ & & 응 \\
\hline HLOMONH : & o & & & & & & $\infty$ & $\stackrel{n}{\sim} \stackrel{\infty}{\sim}$ & $\stackrel{\infty}{\sim} \stackrel{\infty}{\sim} \stackrel{\infty}{\sim}$ & $\stackrel{\infty}{\sim} \stackrel{\infty}{N}$ \\
\hline dחורוומר & $\cong$ & & & & \pm & & & & & \\
\hline$\frac{8019 \mathrm{YH}]}{3 \mathrm{SION}}=$ & 间 & $\stackrel{+m}{m}$ & $\stackrel{p}{?}$ & $\hat{N}^{N} \hat{N}$ & $\stackrel{\infty}{N} \stackrel{+}{m}$ & & & & & $\infty \infty$ \\
\hline $\begin{array}{ll}\text { Nivg } \\
\text { Nivg }\end{array}$ & : 0 & 웅 & in & 웅요 웅 & $\hat{m} \infty_{m}^{\infty}$ & 있임요 & in & & & 융 \\
\hline $\begin{array}{l}S 170 \mathrm{~A} \\
\times 173 \mathrm{H}\end{array}$ & 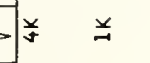 & 并 & 弟 & 兰怘 并 & 并并并 & & N & $\cong \cong$ & & 용 \\
\hline $\begin{array}{c}51701 \\
1081003\end{array}=$ & 0 & in & : & 只 只 & in $\cong 0$ & & $\stackrel{\circ}{-}$ & $\therefore$ 움 & : & : \\
\hline 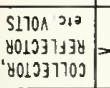 & 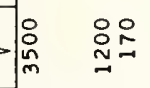 & : & $\stackrel{\circ}{\stackrel{\leftrightarrow}{\alpha}}$ & 品 : & 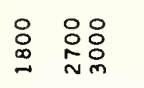 & 弟弟 & in & 品 品 & 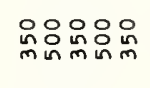 & 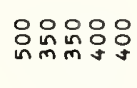 \\
\hline $0^{\circ}$ & 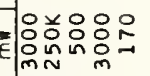 & 总录并兰 & $\Xi_{0} \Sigma \Sigma \Sigma \Sigma \Sigma$ & 竞忌 & 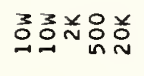 & 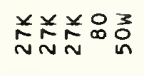 & 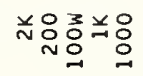 & 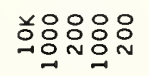 & 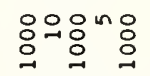 & 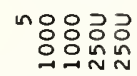 \\
\hline$\rightarrow$ & గొ & 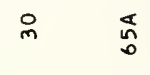 & ํำ & 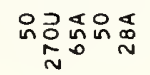 & 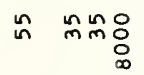 & 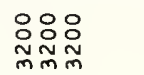 & 응 육욱 & 웅 웅 & 암암웃 & 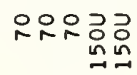 \\
\hline $\begin{array}{c}5170 \mathrm{~N} \\
300 \mathrm{NY} \\
80 \mathrm{H \mapsto yg}\end{array}$ & 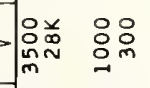 & 品 & 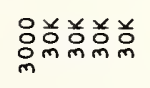 & 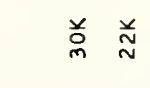 & 品 品品品 & $\underset{\sim}{\stackrel{䒑}{\sim}} \stackrel{\sim}{\sim}$ & 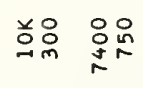 & 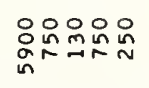 & 只希员范品 & 爻黾员 \\
\hline $5^{-}$ & 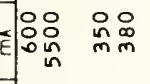 & $\stackrel{\circ}{i}$ & 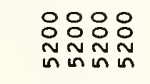 & 음윯요 & 음 : 욤 & 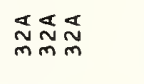 & 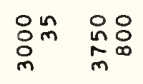 & 앵요임요 & 唡品品品 & 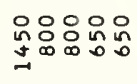 \\
\hline$\omega$ & 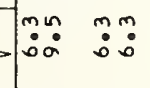 & $\stackrel{0}{\dot{0}}$ & 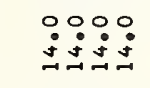 & mo: & $\ddot{0}: \ddot{0}: \stackrel{m}{0}$ & 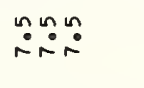 & 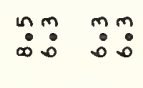 & mmmm & m: & 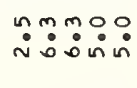 \\
\hline$N 0118430$ & Juavu & vuบua & vana & vuava & บบบบa & טuบu & uบuau & auบu & טบบ & טuטu \\
\hline$y$ y & 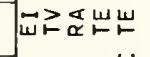 & 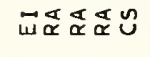 & ๕こひすと & 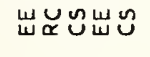 & 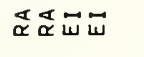 & II I I I & 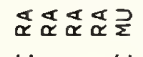 & 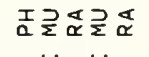 & 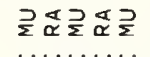 & 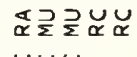 \\
\hline JNח1 & 宏 $5 \frac{u}{\Sigma}$ & & & & x & 岀岕岕 & $\begin{array}{ll}\breve{w} & \breve{w} \\
\end{array}$ & 嵌 & 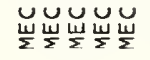 & 岀岀岀 \\
\hline $014 y$ aIno & $\cong$ & 우 & 엄어어오 & $\stackrel{n}{\prime}$ & $\therefore$ & & 옹 & $\stackrel{\circ}{N}$ & & \\
\hline 妾 & 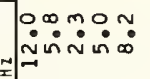 & 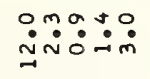 & 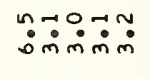 & 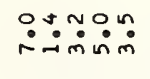 & :ே0்: & o:a: & $\because \because \underset{\sim}{0} \dot{0} \dot{0}$ & 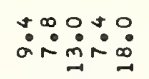 & $\ddot{\sim} \because \ddot{0}: 00$ & 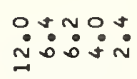 \\
\hline$\underset{\frac{3}{\tilde{w}}}{\vec{z}}$ & 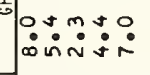 & 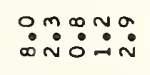 & 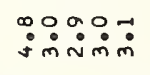 & $\ddot{\sim} \dot{\sim} \dot{m} \dot{m} \dot{\sim}$ & 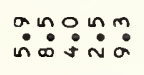 & 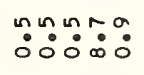 & 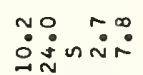 & 守送: & $\ddot{\circ}: \dot{0}: 0$ & 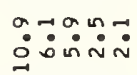 \\
\hline ONIY & 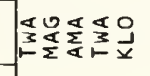 & 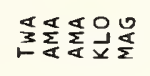 & $\begin{array}{l}\mathbb{4} 0000 \\
3 \frac{\pi}{2} \frac{\pi}{2} \frac{\pi}{2}\end{array}$ & 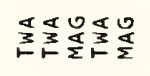 & 速乐乐造 & 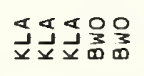 & 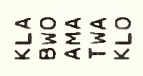 & 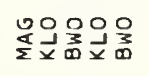 & 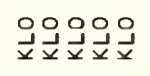 & 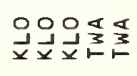 \\
\hline $708 \mathrm{WLS}$ & $"$ & $"$ & $"$ & & & & & & & \\
\hline 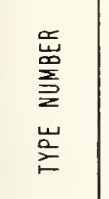 & 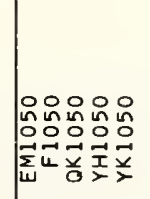 & 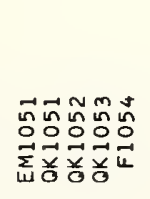 & 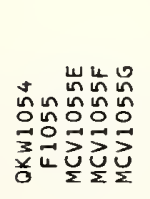 & 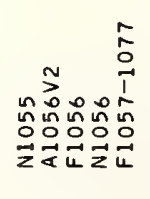 & 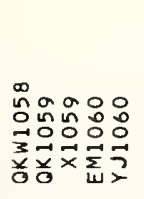 & 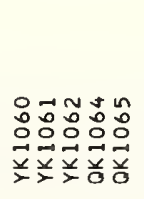 & 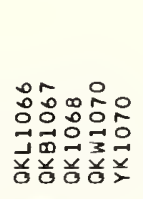 & 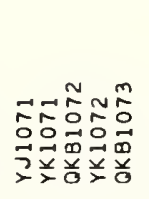 & 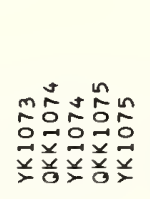 & 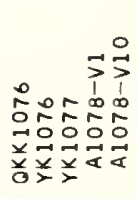 \\
\hline
\end{tabular}




\begin{tabular}{|c|c|c|c|c|c|c|c|c|c|c|}
\hline 9אורdกOS & 잉요 & 웅요 & 88 & $8 \%$ & 언 언언 & 88 & 8 & 엉 & 요요 & $\stackrel{0}{3}$ \\
\hline 11114Y & $\vec{\alpha} \vec{\alpha} \quad \vec{\alpha}$ & & & $\vec{\alpha}$ & $\vec{\alpha}$ & & $\underset{\boldsymbol{\alpha}}{w}$ & & $\vec{\alpha}$ & $\vec{\alpha} \vec{\sim} \vec{\alpha}$ \\
\hline \begin{tabular}{c|c|c|}
01311 \\
ग1399vk
\end{tabular} & & 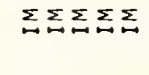 & $\Sigma \Sigma$ & $\Sigma$ & $\Sigma \Sigma \Sigma$ & $\sum \Sigma$ & $\Sigma$ & $\Sigma \Sigma \Sigma$ & $\Sigma \Sigma$ & \\
\hline HבOIMONY & & & & & & & $\therefore$ & & $\tilde{n}$ & $n$ nn \\
\hline 9אוा7רกd & E. & & & m & m & & & & $\cong \cong$ & \\
\hline $\begin{array}{l}\text { yolort } \\
\text { 3S10N }\end{array}$ & $\tilde{N}$ & $\tilde{m}$ & & $\stackrel{n}{\sim}$ & & & & & & \\
\hline Nirg & $\hat{m}$ & $\hat{m}$ & & o & & & m & & & \\
\hline $\begin{array}{l}S 170 \mathrm{~A} \\
\times 1173 \mathrm{H} \\
\end{array}$ & F & $\cong$ & & $\stackrel{\Perp}{N}$ & & & $\stackrel{丷}{\exists}$ & & & \\
\hline $\begin{array}{c}51701 \\
7081003\end{array}$ & 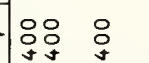 & $\stackrel{\circ}{0}$ & & & & & & & $\stackrel{\circ}{\circledR}$ & \\
\hline 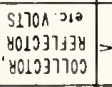 & 总品 品品 & $\stackrel{8}{\stackrel{8}{0}}$ & & 옹 & 品 & & $\stackrel{\Xi}{\Xi} \underset{N}{N}$ & & : & 웅 \\
\hline $0^{\circ}$ & 웁용요 & 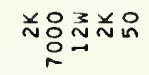 & 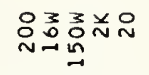 & 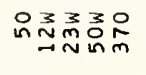 & 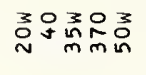 & 品品 & 兰品品웅 & 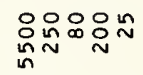 & 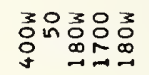 & 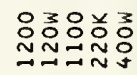 \\
\hline$\mapsto$ & 웅용ㅇㅇㅇ & 용용요 & 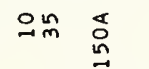 & 음 nूogo & 우 용요 & $\stackrel{n}{\sim}$ & 总品 吕 & 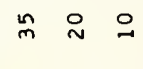 & 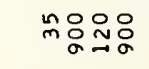 & nू임 \\
\hline $\begin{array}{c}51701 \\
300 \% 7 \\
80 \mathrm{Hryg}\end{array}$ & 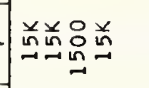 & 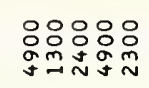 & 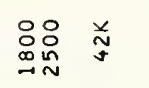 & $\begin{array}{ll}\stackrel{O}{O} & \stackrel{O}{0} \\
\stackrel{m}{\sim} & \stackrel{\infty}{=}\end{array}$ & 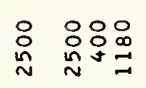 & $\begin{array}{l}\text { 요 } \\
\text { o: } \\
\text { on } \\
\text { n }\end{array}$ & $\cong 0$ & 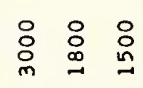 & 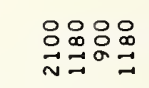 & 용ㅇㅁㅇㅛ \\
\hline- & 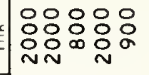 & 离 & 용 & 옹 ํㅐ욤유 & 응 & 总品 & 品 & 요 & 욤욤음 & 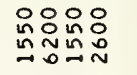 \\
\hline$\omega^{ \pm}$ & 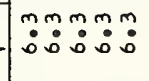 & 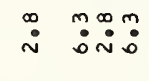 & $\ddot{m}: \stackrel{0}{0}$ & 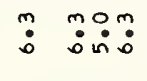 & $\ddot{m}: \ddot{m}: \dot{m}:$ & $\ddot{m} \quad \stackrel{m}{:}$ & $\ddot{m} \stackrel{m}{\dot{0}}$ & $\ddot{m} \quad \stackrel{m}{:}: \stackrel{m}{:}$ & 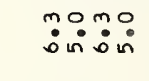 & $\ddot{m}: \stackrel{m}{0}: 00$ \\
\hline NOIIYYIdO & טưu & טuบu & uvuau & uvuau & טuva & טuטu & auvau & vuuu & UUA Ua & טיט \\
\hline$\forall \mathrm{d}$ & 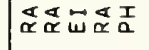 & 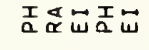 & 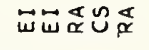 & 跣员号 & \㫣志 & 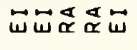 & 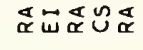 & 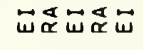 & 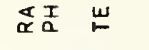 & แ๕山ひ凹 \\
\hline JNก1 & 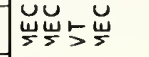 & 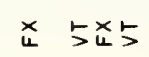 & らら & 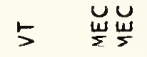 & $55 \breve{u}$ & 55 & $\stackrel{\breve{~}}{\Sigma}$ & 55 & ๖岕岕岂 & $\stackrel{\breve{U}}{\Sigma}$ \\
\hline Oiltey $\wedge 1 n 0$ & & & $\stackrel{n}{\sim}$ & $\stackrel{\circ}{\sim}$ & 운 & & $\stackrel{\circ}{\circ}$ & & 요 & $\stackrel{-}{-}$ \\
\hline 总 & ヘீำ: & 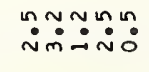 & 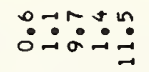 & 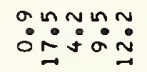 & ஸ்: & 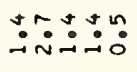 & 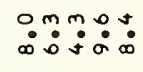 & 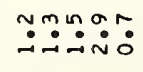 & 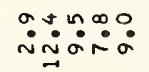 & 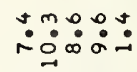 \\
\hline 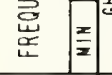 & 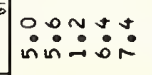 & $\dot{\sim} \dot{\sim} \dot{0}: \dot{0}$ & $\ddot{m}: \circ: m: 0$ & 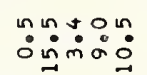 & 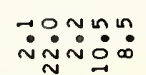 & 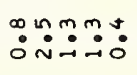 & 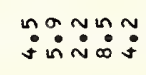 & 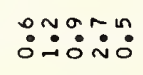 & 站 & 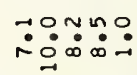 \\
\hline ONIX & 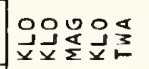 & 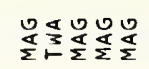 & 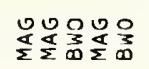 & 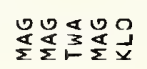 & 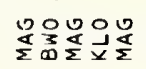 & 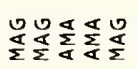 & 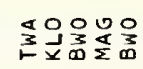 & 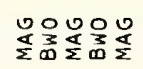 & 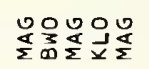 & 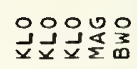 \\
\hline 708WhS & $"$ & " " " & " " & $"$ & $"$ & " " " & $"$ & " " " & & \\
\hline 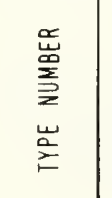 & 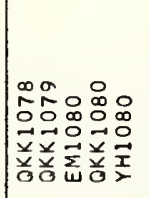 & 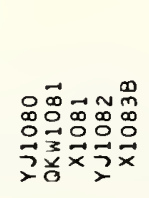 & 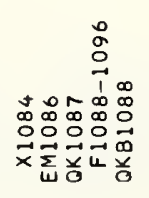 & 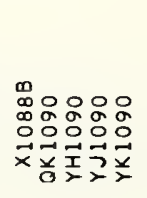 & 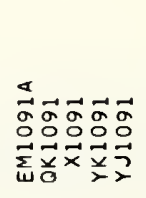 & 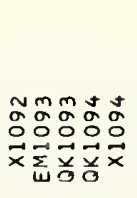 & 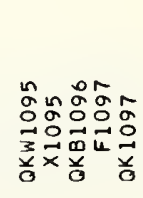 & 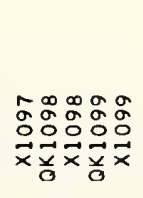 & 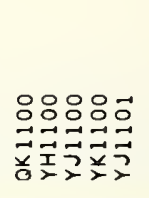 & 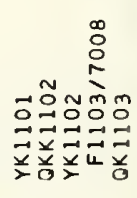 \\
\hline
\end{tabular}




\begin{tabular}{|c|c|c|c|c|c|c|c|c|c|c|}
\hline 9 & Y YOS & Y & एू़ & पूज़ण & जण & $\frac{0}{3} \frac{O}{3}{ }_{3}$ & पOOWO & $\begin{array}{l}\text { OO } \\
\end{array}$ & पू & $\frac{0}{3} \quad 0 \frac{9}{3}$ \\
\hline R11AvJ & $\approx$ & & $\vec{\alpha} \vec{\alpha} \vec{\alpha}$ & $\vec{\alpha} \vec{\alpha} \vec{\alpha} \vec{\alpha} \vec{\alpha}$ & $\vec{\alpha} \vec{\alpha}$ & $\vec{\alpha} \vec{\alpha} \vec{\alpha}$ & & $\vec{\alpha}$ & $\vec{\alpha}$ & \\
\hline 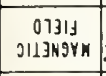 & $\sum_{\infty} \sum_{\infty}$ & $\Sigma$ & $\Sigma$ & & & & 嗢 & $\Sigma$ & $\Sigma$ & 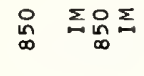 \\
\hline HLOIAONYB & $\frac{1}{ \pm}$ & & $\stackrel{n}{m} \approx \tilde{m}$ & 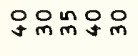 & of & 영영 & $\stackrel{n}{n} \tilde{n}$ & 욤 & q & \\
\hline פחי & 풀 & $\stackrel{\infty}{\sim}$ & & & & & $\stackrel{\infty}{=}$ & $\stackrel{\infty}{=}$ & & \\
\hline 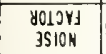 & 8 & & & & & & & & 品 & $\stackrel{\infty}{N}$ \\
\hline NiYg & $\simeq$ ? & 음 & o & & & & $\stackrel{n}{s}$ & & $\stackrel{n}{m} \approx$ & 영 영영 \\
\hline $\begin{array}{l}S 170 \mathrm{~A} \\
\times 117 \mathrm{H}\end{array}$ & 弟 & 웅 & 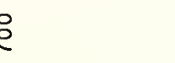 & & & & 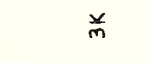 & & 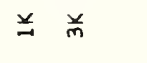 & 弟 兰首 \\
\hline $\begin{array}{c}51701 \\
7001 \mathrm{NOO} \\
\end{array}$ & d & & & & & & in & & 용 & \\
\hline 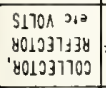 & 品 & $\stackrel{\circ}{i}$ & 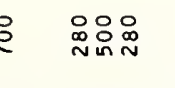 & 品品品品 & 잉 & 웅 융음 & 움욤움 & 品 & 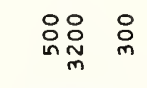 & 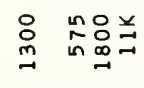 \\
\hline $0^{\circ}$ & E| & 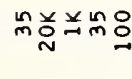 & 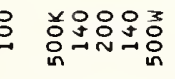 & 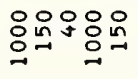 & 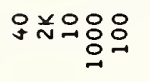 & 움용응음 & 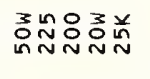 & 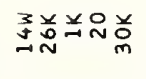 & 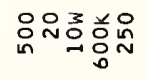 & 预强并录 \\
\hline$\mapsto^{x}$ & 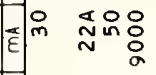 & 总品 & 夰触品 & ㅇㅇㅋ용용ㅇㅇ & 눙용ㅇㅇ & 两 & 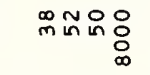 & 总品䜣兄 & ถูก员 กี & 임 \\
\hline $\begin{array}{c}5.770 \mathrm{~N} \\
30 \mathrm{NH} \\
80 \mathrm{kH} \rightarrow 3 \mathrm{~B}\end{array}$ & 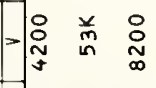 & 总品 & 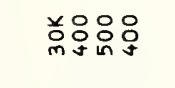 & 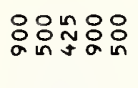 & 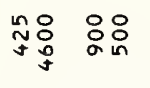 & $\stackrel{n}{\mathcal{N}}$ 品品 & 号吕 品 & 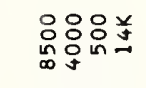 & 욧요 总 & $\stackrel{\circ}{\circ}$ \\
\hline$\rightarrow$ & 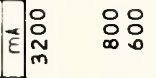 & 욤욤 & $\stackrel{\leftarrow}{\stackrel{0}{二}}$ & 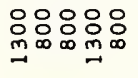 & 용요 & 음 & 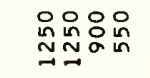 & : :욤요 & 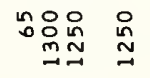 & 옹 品品品 \\
\hline$\omega^{ \pm}$ & 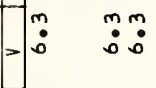 & $\stackrel{m}{\ddot{0}} \stackrel{m}{\stackrel{0}{0}}$ & $\stackrel{m}{\dot{\infty}}: \dot{m}$ & $\ddot{m}: m m m$ & $\dot{m}: \dot{m}: \dot{m}$ & $\ddot{0}: m \ddot{m}:$ & $\ddot{m}: m m:$ & m: & 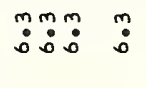 & 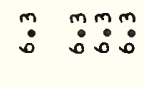 \\
\hline $\mathrm{N} 011 \forall \mathrm{YZ} 3 \mathrm{dO}$ & Uuaua & vauu & auuvu & 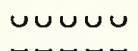 & บบบบบ & טuטu & 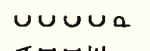 & บaUua & ưuau & บuUu \\
\hline$\forall y \mathrm{~W}$ & 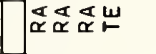 & 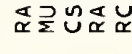 & 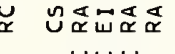 & ேேேே゙ & ேつチே゙ี & 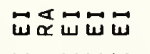 & 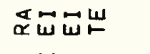 & 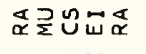 & 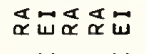 & 㟔乔山゙ \\
\hline JNก1 & $\underset{u}{x}$ & $u_{u}^{x}$ & 崖岮㟐 & 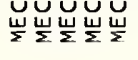 & 岂岂 & U⿺ & $\breve{w}_{z}^{\Psi_{u}} \underset{u}{x}$ & ×xư & 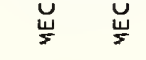 & \\
\hline 011 ty $A 1 \cap 0$ & $\stackrel{\circ}{\stackrel{n}{\sim}}$ & n & $\stackrel{2}{N}$ & & & & & & & \\
\hline 总 & 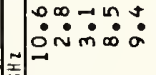 & $\ddot{\infty}$ & nُ & 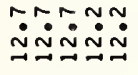 & 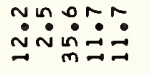 & 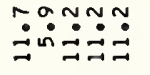 & 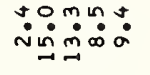 & 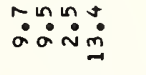 & 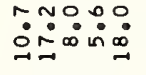 & 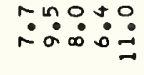 \\
\hline 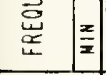 & 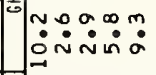 & 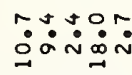 & mạ: & 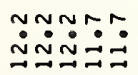 & 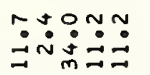 & 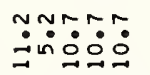 & 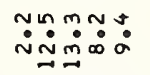 & 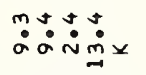 & 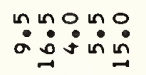 & 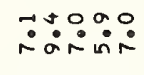 \\
\hline ONIX & 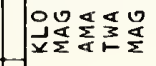 & 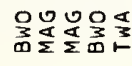 & 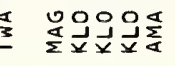 & 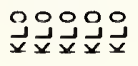 & 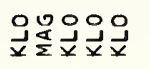 & 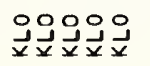 & 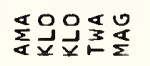 & 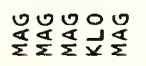 & 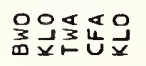 & 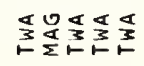 \\
\hline $708 \mathrm{WLS}$ & & & " & " " " " & " " " & " " " " & " " & & " " & \\
\hline 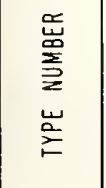 & 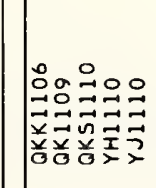 & 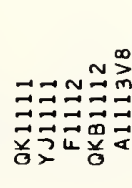 & 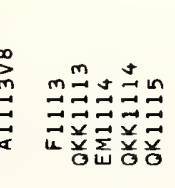 & 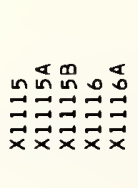 & 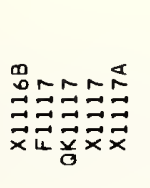 & 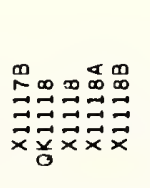 & 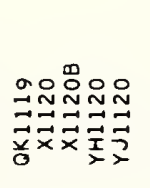 & 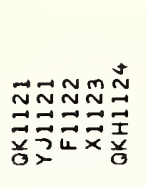 & 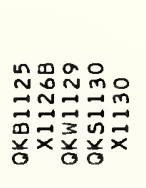 & 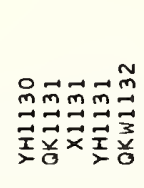 \\
\hline
\end{tabular}




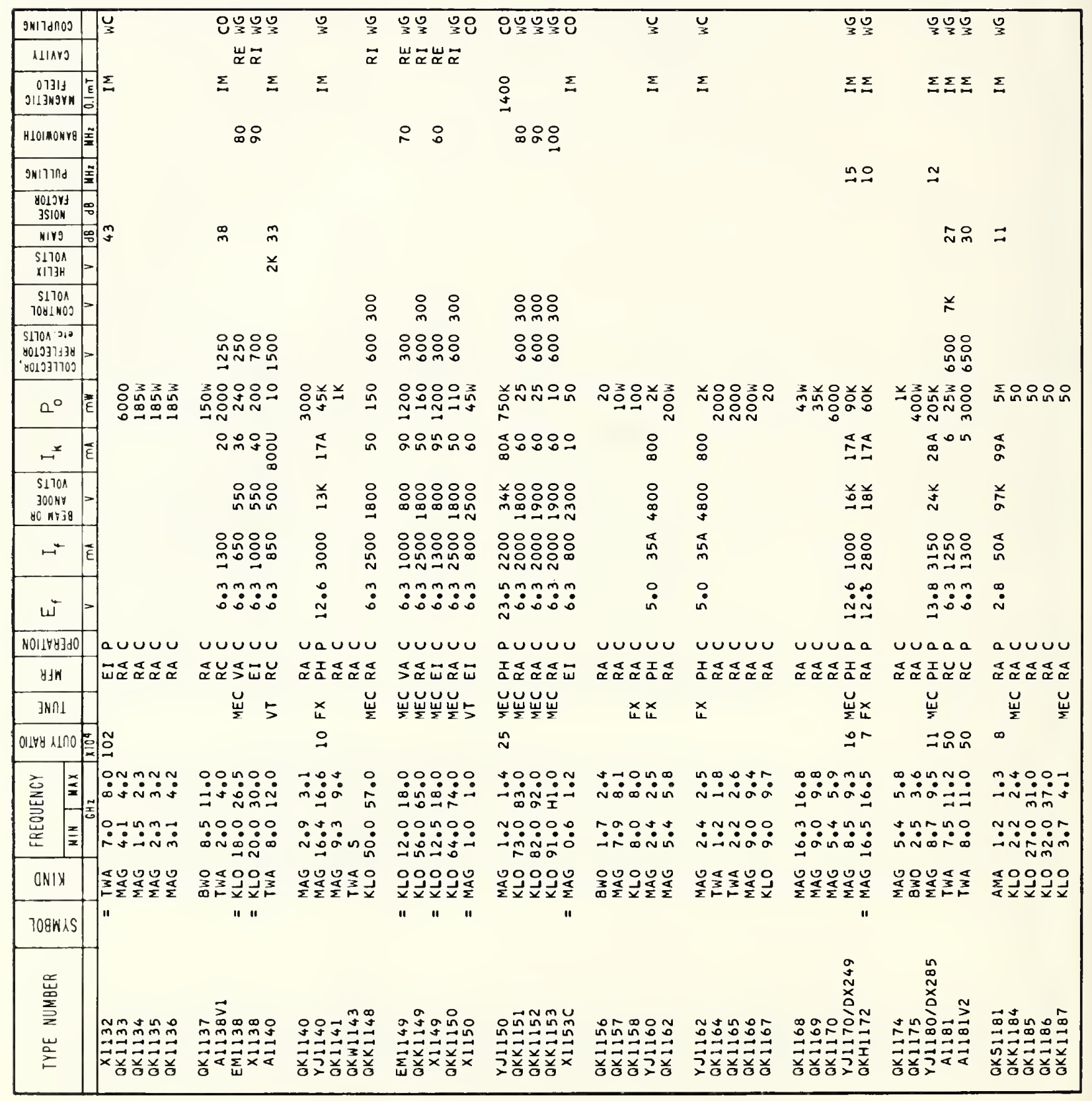




\begin{tabular}{|c|c|c|c|c|c|c|c|c|c|c|c|}
\hline 9k17dחOO & $\frac{0}{3} 88$ & そ๐゚ֻ & ים & יי요 & 8 \& & 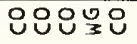 & $\stackrel{0}{3}$ & is 8 & एँ & & 언 문인 \\
\hline s1lard & $\ddot{\alpha}$ & & & & & & $\vec{\alpha}$ & $\vec{\alpha}$ & & & \\
\hline 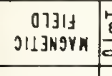 & $\Sigma \Sigma$ & $\Sigma$ & $\Sigma \Sigma$ & $\Sigma \Sigma_{-\infty}^{0}: 0$ & 怘 $\Sigma \Sigma$ & $\sum_{\infty} 0_{\infty} 0_{\infty}$ & & $\Sigma$ & $\Sigma \Sigma$ & 足 & 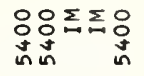 \\
\hline HLOMONYP & 푚ㅇㅇㅇ & & & & & $\therefore$ & $:$ & \& & & & \\
\hline 9אורור & 竧 & $\cong$ & & & & & & & & $\cong$ & $\stackrel{n}{\sim}$ \\
\hline $\begin{array}{l}8013 y+1 \\
\text { JSION }\end{array}$ & 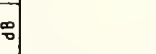 & & $\stackrel{\circ}{-}$ & in in & $n=$ & & & & & & \\
\hline Nirg & $\stackrel{m}{m}$ & 웅유 & $\vec{m}$ 움 & 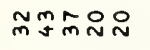 & $\stackrel{\sim}{N}$ & 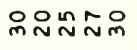 & & 品 & \pm & & \\
\hline $\begin{array}{l}S 170 \mathrm{~A} \\
\times 1173 \mathrm{H} \\
\end{array}$ & $\rightarrow$ & & & $\underset{\sim}{*} \underset{m}{n} \underset{m}{n}$ & 兰 & 임 & & $\because$ & & & 并首 \\
\hline $\begin{array}{c}51701 \\
1081100\end{array}=$ & $\stackrel{\sim}{N}$ & & & & & 요 & & & & & 兰兰 \\
\hline 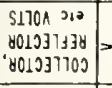 & 足 & & 吕 & 怘 : & $\begin{array}{ll}\circ & 0 \\
0 & 0 \\
0 & 0\end{array}$ & : & 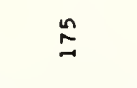 & 品 吕 & & & 品品 \\
\hline $0^{\circ}$ & 웃응요 & 并前兑总忐 & 뭉종ㅇㅇㅇㅇㅇㅇ & 웅윰- & -ㅉㅇㅇ으믐 & 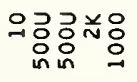 & 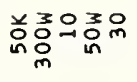 & 응ㅇㅇㅇㅇㅇㅇㅇ & 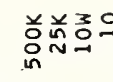 & 前 & 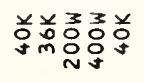 \\
\hline 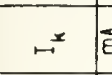 & Eิ & 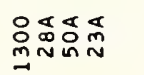 & $-O$ & 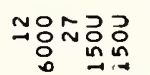 & $\begin{array}{l}\overrightarrow{0} \\
\stackrel{0}{n}\end{array}$ & m금음요 & in & $\stackrel{n}{\sim}$ 品 & $\begin{array}{l}\text { 죠 } \\
\text { No } \\
\text { N }\end{array}$ & $\stackrel{a}{n}$ & 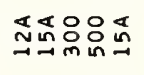 \\
\hline 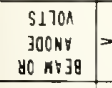 & $>$ - & 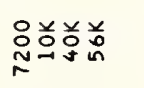 & & 弟 & 煎 & $\stackrel{\Xi}{\exists}$ & 字 & $\stackrel{i}{i n}$ & $\underset{m}{\stackrel{0}{0}}$ & 酋 & 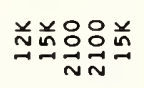 \\
\hline$\mapsto$ & Eᄐ & : & 品 & 品 & 융 : & 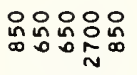 & & 足 & & : & 욤 \\
\hline w" & 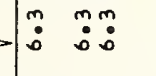 & $\ddot{n}$ & $\ddot{m}:$ & $\ddot{m}: \ddot{m}_{0}^{0}$ & $\stackrel{0}{0}:$ & 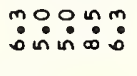 & & $\because$ & & $\stackrel{m}{:}$ & $\ddot{m}:{ }^{m}$ \\
\hline NOII $\forall y 3 \mathrm{dO}$ & u & vaan & טuט & vauuu & $u$ ua & & & auuv & $a a u b$ & $a$ & vau \\
\hline$y \mathrm{JN}$ & 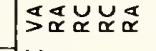 & I & 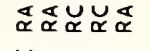 & 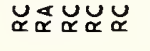 & 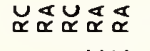 & $\mathfrak{x}_{\propto}^{u} \underset{\alpha}{\Psi_{\alpha}}$ & 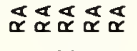 & 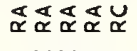 & 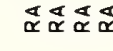 & $\geq$ & 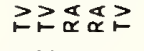 \\
\hline Jknı & 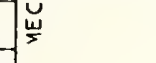 & $\underset{u}{x}$ & 岀 & & 岀㟧 & $\stackrel{\breve{u}}{\Sigma}$ & $\breve{y}_{y} x_{u}$ & 訔㞬 & & x & 宸宸 \\
\hline $0118 y$ asia & & 요 & & $\stackrel{\sim}{\sim}$ & 웅 & & & & $\pm \stackrel{\infty}{N}$ & 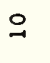 & 욱 \\
\hline 曾 & 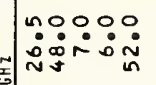 & 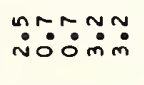 & 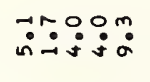 & 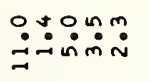 & 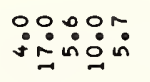 & & 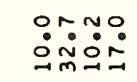 & $\because \cong \sim \because \dot{0}$ & คேㅜㅇ & $\approx$ & 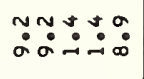 \\
\hline 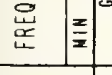 & :00:00 & 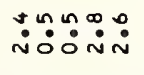 & 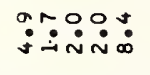 & 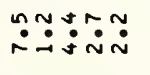 & 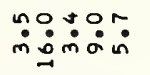 & 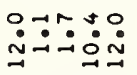 & 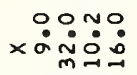 & 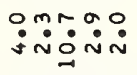 & $\begin{array}{l}n \text { no: } \\
\text { åjo: }\end{array}$ & $\because$ & ペ。: \\
\hline ONIX & 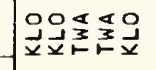 & 员乐乐事员 & 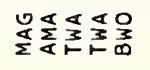 & 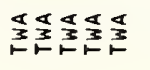 & 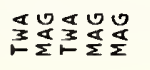 & 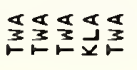 & 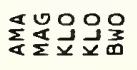 & 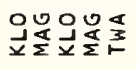 & 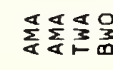 & 济 & 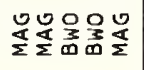 \\
\hline $100 \mathrm{w} / \mathrm{S}$ & $"$ & & & & & & & & & & \\
\hline 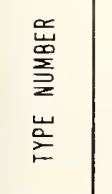 & 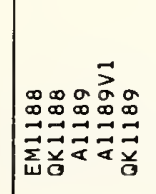 & 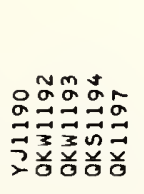 & 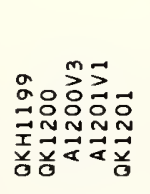 & 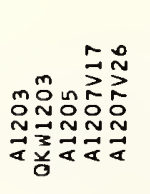 & 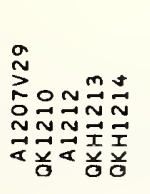 & 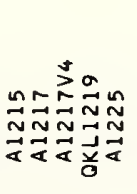 & 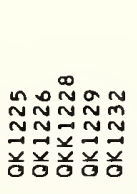 & 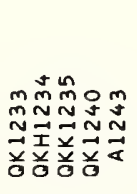 & 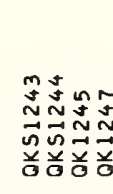 & & 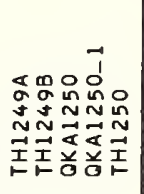 \\
\hline
\end{tabular}




\begin{tabular}{|c|c|c|c|c|c|c|c|c|c|c|}
\hline 9\%1/dnos & कणज़ण & 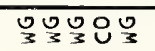 & 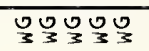 & 원엉 & 8워영 & 8888 & তО: & 웡ㅇㅇㅇ & 운 웅어 & 808 \\
\hline d1IAYs & & & & & $\vec{\alpha}$ & & & & $\ddot{\alpha}$ & \\
\hline $\begin{array}{c}01314 \\
\text { ग113\%9VM }\end{array}$ & 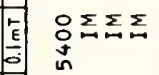 & $\sum \Sigma \sum \Sigma \Sigma$ & $\sum \sum \sum \sum \sum$ & $\sum \sum \Sigma$ & $\Sigma$ & $\sum \sum \sum$ & $\sum \sum$ & $\Sigma \Sigma \Sigma$ & $\Sigma \sum \sum$ & $\sum \sum \sum$ \\
\hline HLOIMOHYP & & & & & 앙우 & & & & $\stackrel{n}{\sim}$ & \\
\hline 9אוาרก & 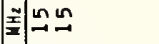 & & & & & & & & & \\
\hline $\begin{array}{l}\text { yolors } \\
\text { 3SION }\end{array}$ & 8 & & & & & $\simeq M$ & 음 & & & \\
\hline Hirg & 圈 & & & 요 & 900 & $\min \tilde{m}=$ & 욤 & $\stackrel{n}{m} \stackrel{n}{m} \stackrel{n}{m}$ & $\stackrel{\circ}{\circ} \stackrel{m}{m}$ & 蛋品 \\
\hline $\begin{array}{l}51101 \\
\times 1173 \mathrm{H}\end{array}$ & 并首前 & 芯芯兑 首 & 并首首前 & 兰兰兰 & $\stackrel{\Xi}{\exists}$ & $\stackrel{n}{\sim} \underset{n}{n}$ & $\stackrel{\mathfrak{N}}{\mathfrak{J}}$ & 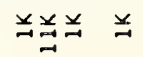 & 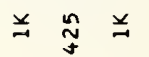 & 并 \\
\hline $\begin{array}{c}\text { S1701 } \\
10 \mathrm{HINOS}=\end{array}=$ & $\cong \cong$ & 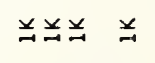 & 픙ㅇㅁㅇㅛ & 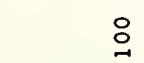 & & & & & & \\
\hline 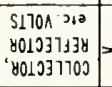 & 总品品 & 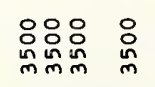 & 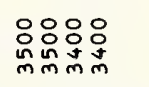 & 莫 & 品 $\quad$ 品品 & 음욤요 & 吕品 & 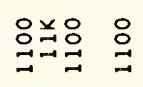 & 品 总品品 & 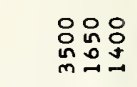 \\
\hline $0^{\circ}$ & E & 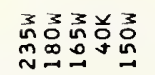 & 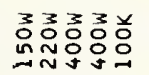 & 只兑吕芯品 & 次旅品 & 웅요 요 & 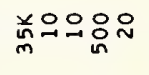 & 음욤욤욤 & 용요욤윰 & 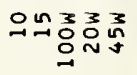 \\
\hline$\mapsto$ & 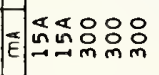 & 윴윰윰유 & 임윰욤요 & $\stackrel{0}{0}^{\circ}:$ & 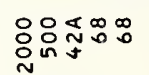 & nato & 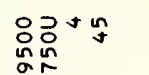 & 임윰요 & 品 J $\underset{N}{n}$ & 을 in \\
\hline 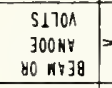 & 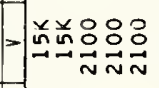 & 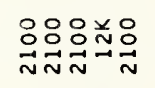 & 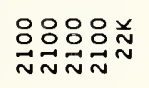 & 㒸 总虽 & 兰路慈品员 & $\stackrel{\circ}{\text { N }}$ & 品 & 芯 & 品 & \\
\hline$\mapsto$ & 충요 & 음 & & 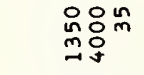 & 品 & ::윰 & 옴 & 品品品品品 & 品 $\stackrel{\circ}{\circ} \stackrel{\circ}{\circ}$ & 总总品品 \\
\hline$\omega^{*}$ & $\rightarrow \because \ddot{0}$ & $\stackrel{m}{:}$ & & $\ddot{m}: \stackrel{m}{0}$ & 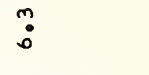 & 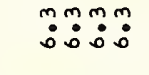 & $\ddot{m}: \stackrel{m}{0}$ & 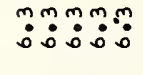 & $\stackrel{0}{0}: \stackrel{m}{0}$ & m:mo: \\
\hline NOI LFYZdO & aauuu & טuטu & טuטu & u vau & $a \cup a \cup u$ & טuטu & טuטa & ט ט ט & טuטu & טu \\
\hline$y j n$ & 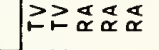 & 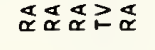 & $\underset{\alpha}{\alpha} \alpha \propto \alpha \alpha \alpha$ & 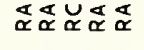 & 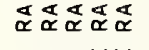 & 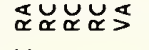 & ๔ữ & 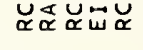 & 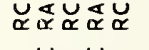 & 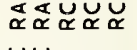 \\
\hline $3 \mathrm{~N} \cap 1$ & 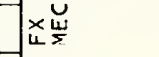 & $\underset{4}{x}$ & & & 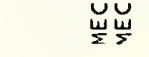 & $\stackrel{\breve{w}}{\Sigma} \quad 5$ & $\stackrel{\breve{w}}{\bar{w}} \quad \stackrel{\breve{U}}{\Sigma}$ & 5 & 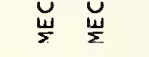 & 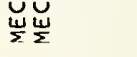 \\
\hline OA18t ALrio & 움요 & & & 웅 욤 & $\stackrel{\circ}{\sim}$ & & 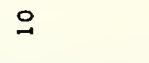 & $\stackrel{i}{\sim}$ & & $\simeq$ \\
\hline 离 & 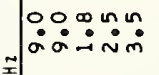 & 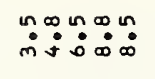 & 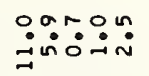 & 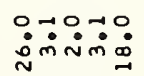 & 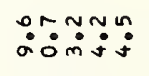 & 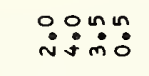 & 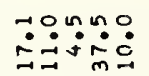 & $\because \because: 00:$ & 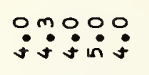 & 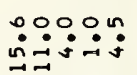 \\
\hline 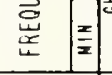 & 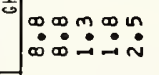 & 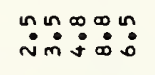 & 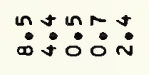 & $\begin{array}{l}00000 \\
\sim \sim N \\
\sim\end{array}$ & 舟品品品: & 象: & 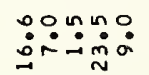 & 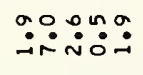 & $\ddot{\sim} \dot{\sim} \dot{\sim} \dot{\sim}: \dot{\dot{N}}$ & 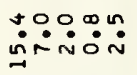 \\
\hline ONIX & 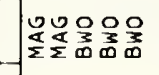 & 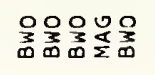 & 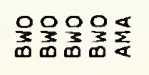 & 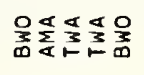 & 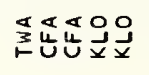 & 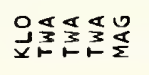 & 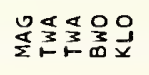 & 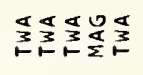 & 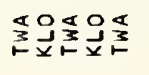 & 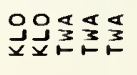 \\
\hline 708WAS & & & & & & & & & & \\
\hline 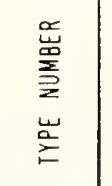 & 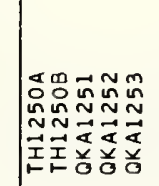 & 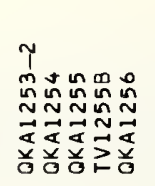 & 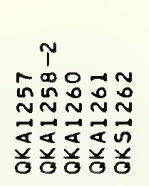 & 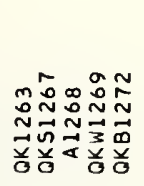 & 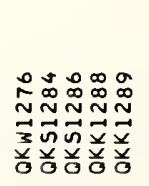 & 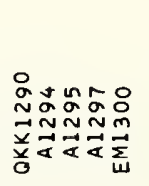 & 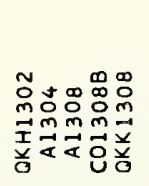 & 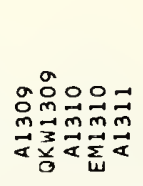 & 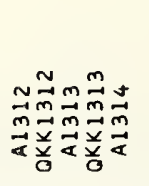 & 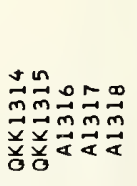 \\
\hline
\end{tabular}




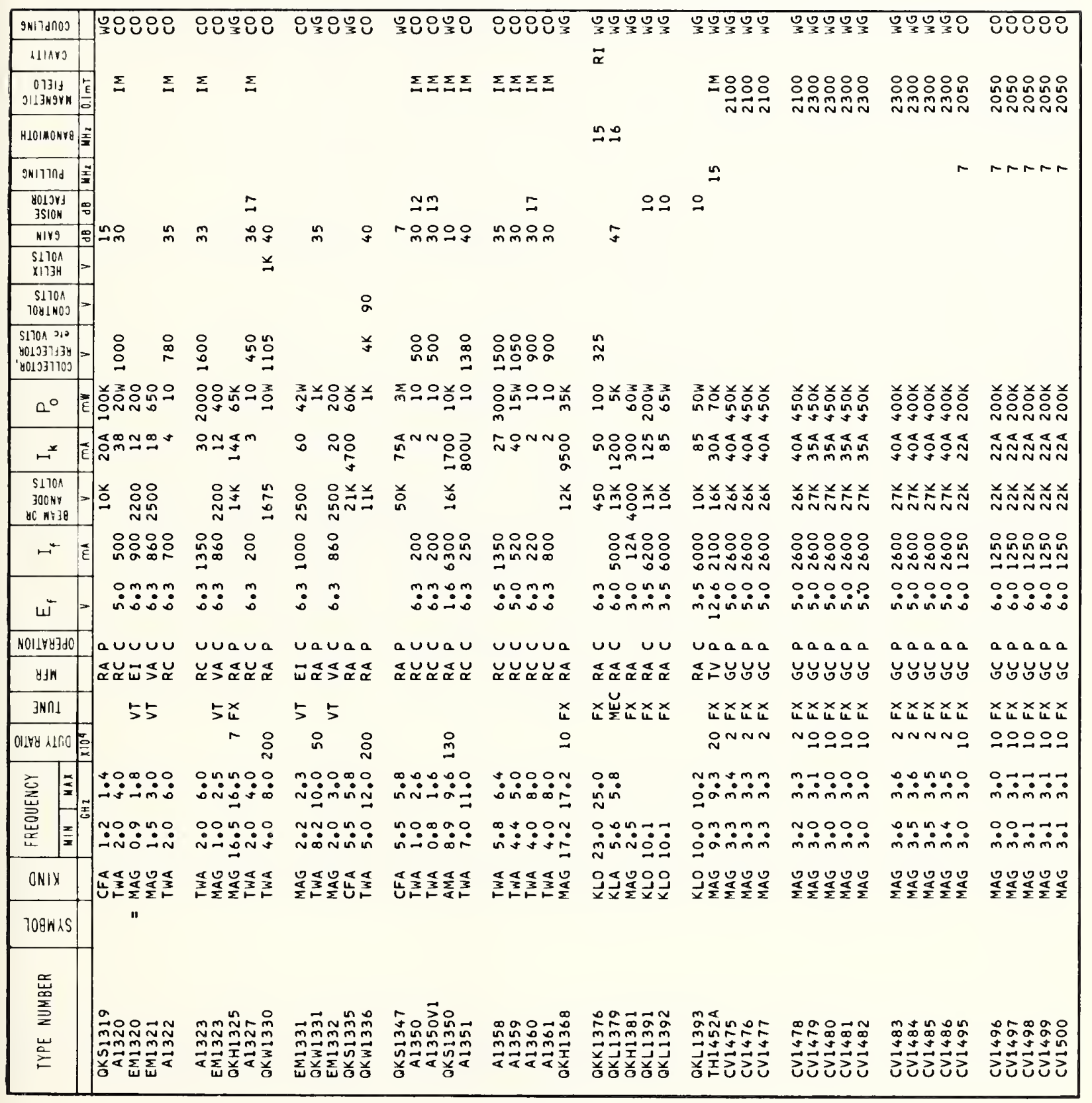




\begin{tabular}{|c|c|c|c|c|c|c|c|c|c|c|}
\hline 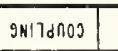 & in 8 & 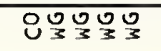 & 몽엉어 & UOO & (ত) & 88 & \begin{tabular}{ll}
0 \\
\multirow{3}{*}{$\frac{0}{3}$}
\end{tabular} & $\frac{0}{3}$ & पू़ & \% \\
\hline R11Ars & & & & & $F$ & & in & & in & in \\
\hline 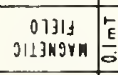 & 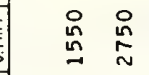 & 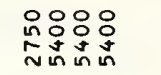 & $\sum_{n} \underset{\Xi}{0} \sum_{0}$ & $\sum \sum \sum$ & $\sum_{n=1}$ & & & $\Sigma$ & & $\Sigma \quad \Sigma$ \\
\hline HLOMONYVO & $\stackrel{\circ}{\sim}$ & 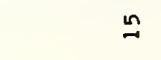 & 음 & $\stackrel{F}{\xi}$ & $\checkmark$ & & $\stackrel{n}{\sim}$ & $n$ & $:$ & 吕年 \\
\hline 9אוาרก & 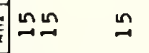 & $\stackrel{n}{n} \cong$ & $\cong$ & & & & & & & \\
\hline $\begin{array}{c}8019 \mathrm{ys} \\
\text { JSIOH }\end{array}$ & & & & $\stackrel{\infty}{\sim}$ & & & & & & \\
\hline nirg to & $\stackrel{a}{s}$ & & in & "ేำ & nn & $\stackrel{m}{m} \tilde{N} \stackrel{\infty}{N}$ & 읏 N品品 & Ninñ & 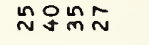 & $\stackrel{n}{N} \stackrel{n}{m}$ 욤 \\
\hline $\begin{array}{l}S 110 \mathrm{~A} \\
\times 113 \mathrm{H}\end{array}$ & & & $\stackrel{*}{N}$ & 并并并并品 & $\stackrel{\Perp}{N} \stackrel{⿱}{N}$ & 픈 & & N & $\underline{x}$ & $\stackrel{\varpi}{\sim}$ \\
\hline $\begin{array}{r}51700 \\
1041103\end{array}=$ & & & $\stackrel{\circ}{\sim}$ & $\stackrel{\sim}{\sim}$ & r & & & $r$ & & $\stackrel{\text { ㅇ }}{2}$ \\
\hline 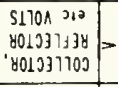 & & & $\stackrel{\circ}{\circ}$ & 옹욤엄 & & 品 & & : & 品 & 品 \\
\hline$a^{\circ}$ & 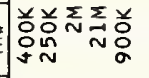 & 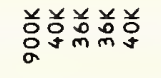 & 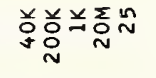 & 용요용요 & 웅요요워N & 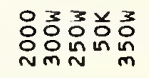 & 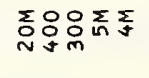 & 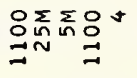 & 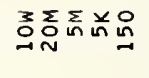 & 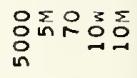 \\
\hline 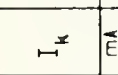 & mర్లో & 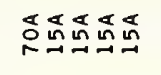 & 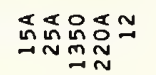 & 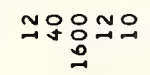 & ㄱㅇㅛ & n앵요요요 & 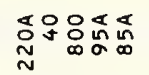 & 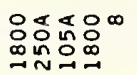 & 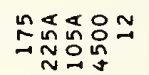 & 员芯士心员 \\
\hline 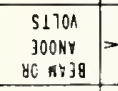 & 㒸酋兑总总 & 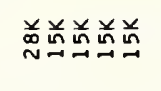 & 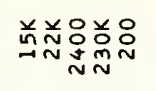 & 음욤유 & 옹 酋욤 & 옹 & 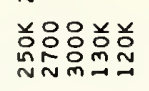 & 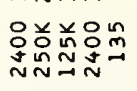 & 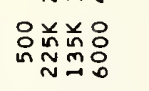 & 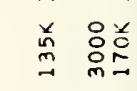 \\
\hline- & 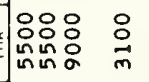 & 용요 & 品品品敌品 & 응융유 & 品 & 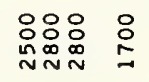 & 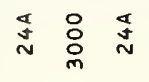 & 品 & 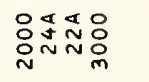 & : \\
\hline$\omega^{\leftarrow}$ & 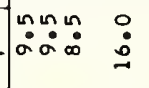 & $\ddot{0}: \stackrel{m}{0}:{ }^{m}:$ & 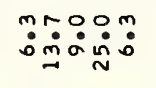 & 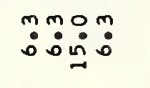 & $\stackrel{n}{:}$ & 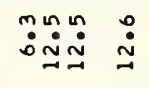 & $\stackrel{\circ}{\stackrel{\dot{n}}{\sim}} \stackrel{\circ}{\stackrel{n}{N}}$ & $\stackrel{\dot{0}}{\cong} \quad \stackrel{0}{\sim}:$ & 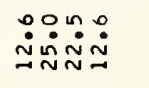 & 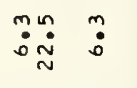 \\
\hline NOIIFYJ JO & a $a$ ua & 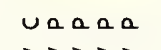 & auvau & טuטu & uUau & auvau & auvaa & va $a \cup v$ & vaauv & טロ \\
\hline$\forall \mathrm{JW}$ & ママーマダー & ママママZマ & 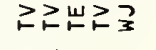 & $3 \frac{6}{2} \frac{\alpha}{2} 3$ & 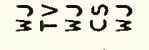 & $\sqsubseteq \frac{\alpha}{2} \frac{\alpha}{2} U \frac{\alpha}{2}$ & マびマママ & 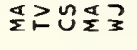 & 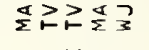 & こマアミュ \\
\hline \begin{tabular}{l|l}
$3 \times \cap 1$ \\
\end{tabular} & 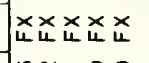 & 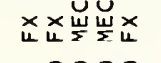 & 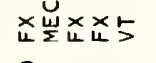 & 5 & 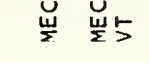 & x & $\underset{k}{x}$ & $\begin{array}{ll}x & 5\end{array}$ & $\stackrel{\breve{w}}{\Sigma}$ & 品 \\
\hline $0118 y$ 스요 & $\ln \approx 00$ & 오오오오 & $\stackrel{\circ}{\sim}$ & & & $\stackrel{9}{q}$ & $\stackrel{i}{\sim}$ & $\stackrel{\sim}{\sim}$ & & $\tilde{\jmath}$ \\
\hline 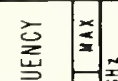 & 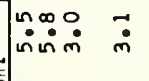 & 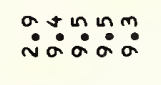 & 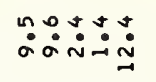 & 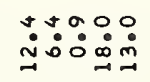 & 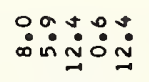 & 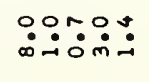 & $\ddot{m} \underset{\sim}{+} \dot{q} \dot{m}$ & 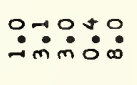 & $\therefore \quad \because \because ̊$ & 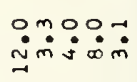 \\
\hline 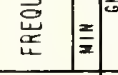 & $\ddot{\forall}$ & 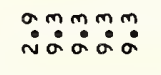 & 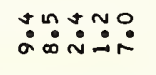 & 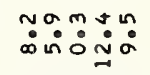 & 过: & 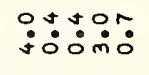 & 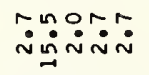 & 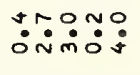 & 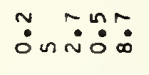 & : \\
\hline ONIX & $\mid \frac{0}{2} \frac{0}{2} \frac{\alpha}{2} \frac{0}{2}$ & 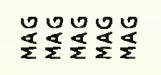 & 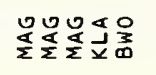 & 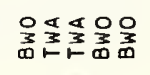 & 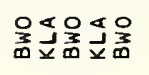 & 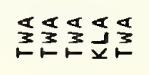 & 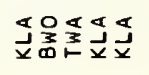 & 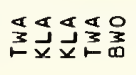 & 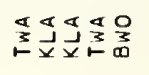 & 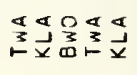 \\
\hline $108 \mathrm{~W} / \mathrm{S}$ & & & & " " & " " & " " & & $"$ & " & " " \\
\hline 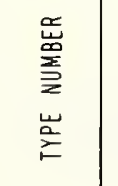 & 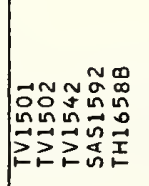 & 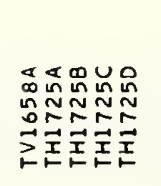 & 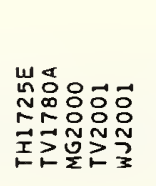 & 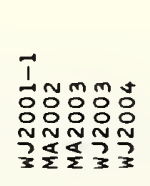 & 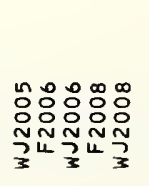 & 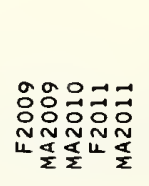 & 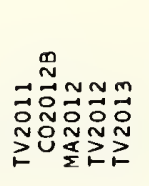 & 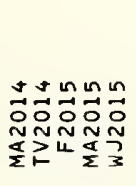 & 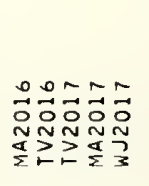 & 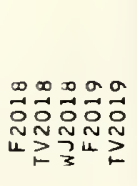 \\
\hline
\end{tabular}




\begin{tabular}{|c|c|c|c|c|c|c|c|c|c|c|}
\hline $9 \times 17$ वnOO & $\overline{3} \quad \overline{3}$ & शบ & 눈언언 & 언엉온오 & 은 옹 & $\frac{0}{3}$ & पू & 엉응 & 88 & 온오 \\
\hline LIIAY3 & $\vec{n} \vec{x}$ & $\vec{\alpha} \operatorname{n} \vec{\alpha} \vec{n} \vec{\alpha}$ & $\vec{n} \bar{\alpha} \bar{\alpha}$ & $\bar{\alpha} \bar{\alpha} \bar{n}$ & $\ddot{\alpha}$ & $\ddot{\alpha}$ & $\vec{\alpha}$ & 岗岁岁 㟧 & & \\
\hline 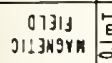 & & & $\Sigma \Sigma$ & $\Sigma \sum_{H}$ & $\Sigma \Sigma \Sigma$ & & & & $\sum$ & \\
\hline H1OMONYrg & $\stackrel{\circ}{\circ}$ & ํํํ요요 & $\stackrel{N}{\sim} \stackrel{n}{N}$ & $\stackrel{n}{\sim} \stackrel{0}{N}$ & 움 & $\stackrel{\circ}{-}$ & $\stackrel{n}{N}$ & & & \\
\hline 9אורור & & & in & & & & & & & \\
\hline 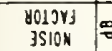 & & & & & & & & & & \\
\hline MiYg & $\stackrel{n}{m}$ & in in & 요 & in & $\stackrel{n}{n}$ & $\stackrel{\infty}{N}$ & 옹요요 & 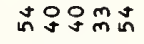 & 잉맘 & 푬욤요 \\
\hline $\begin{array}{l}51701 \\
\times 1173 k\end{array}$ & $\stackrel{\sim}{\sim} \stackrel{䒑}{\sim}$ & & $\ddot{\sim}$ & $\stackrel{x}{\sim}$ & 弚芯品 并 & 芯兰 & 兰 兰 & 差 & 弚 & \\
\hline $\begin{array}{c}51701 \\
70811003\end{array}$ & & & $r$ & $\circ r$ & $\circ \stackrel{\circ}{\circ}$ & & & 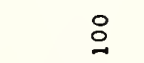 & $\stackrel{\sim}{\underset{N}{N}}$ & 음음 \\
\hline 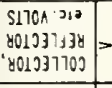 & 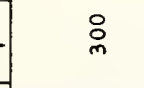 & \begin{tabular}{lll}
$\stackrel{n}{n}$ & 0 & 0 \\
\multirow{j}{*}{} & \multirow{1}{*}{} & 8
\end{tabular} & $\stackrel{\circ}{\circ} \stackrel{n}{9}$ & 용 & 용 & $\underset{\sim}{\stackrel{\text { 省 }}{n}}$ & 号 号 & 兰 & $\begin{array}{l}\circ \\
\circ: 0 \\
\infty \\
\infty\end{array}$ & ㅇ:ㅇㅇㅇㅣ \\
\hline $0^{\circ}$ & 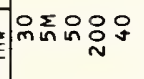 & 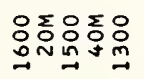 & 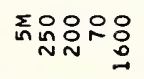 & 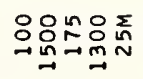 & 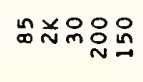 & : & 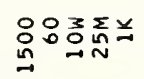 & 立京㒸兰京 & $=0 ㅇ ㅡ$ & 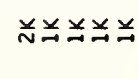 \\
\hline 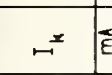 & $\underset{\sim}{N}$ & ธ。্N & 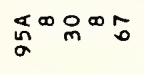 & $\infty 5^{\infty} \tilde{\sim}$ & 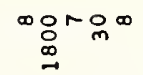 & 品さ资 & 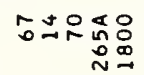 & 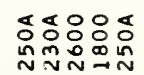 & :̊ & : \\
\hline 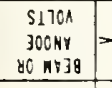 & 总 & 品弟品总总品 & 总第总品品 & 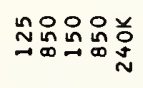 & 음 윰윰음 & 号染 & 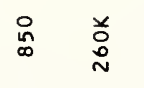 & 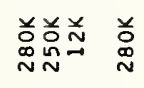 & 융 & 요 \\
\hline$F^{-}$ & $\stackrel{a}{ \pm} \stackrel{0}{\sim}$ & 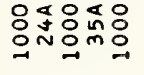 & 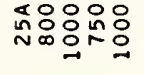 & 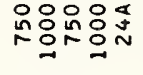 & 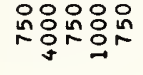 & 용 & 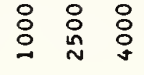 & 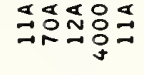 & 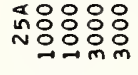 & ::ㅇํ \\
\hline W" & $\dot{n}:$ & : & 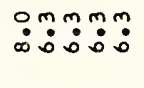 & :3:m: & 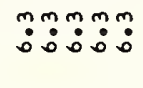 & $\ddot{:} \quad: \stackrel{m}{:}$ & $\stackrel{m}{a} \quad \stackrel{m}{0}:$ & 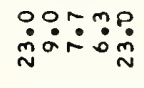 & $\ddot{\infty}: \ddot{:}: \dot{m}:$ & $\ddot{:}: m: m:$ \\
\hline NOIIFy3 30 & vauuv & vauau & auuuv & บบบบa & UaUu & ưu & uvua & aogao & vas & \\
\hline$\forall \mathrm{y} N$ & 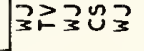 & 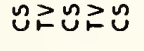 & 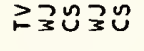 & 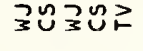 & วேろつア & さろアヒさ & 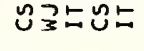 & びさざル & マロヒヒヒこ & $\sqsubseteq$ \\
\hline 3NกI & x & $\begin{array}{ll}x \\
4\end{array}$ & 㚲5 & $55 x$ & 555 & & & $\begin{array}{lll}x & x \\
4\end{array}$ & & \\
\hline $014 y+$ anno & & & & & in & in & $\cong$ & in & 윰 & :융요 \\
\hline 总 & 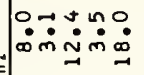 & 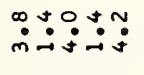 & 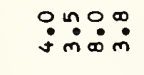 & 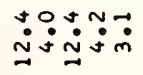 & 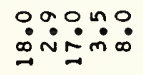 & 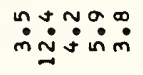 & 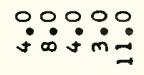 & 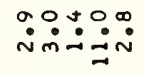 & $\dot{\sim}: \dot{0}: \dot{0}$ & $\dot{m} \dot{m}: \because: \dot{m}$ \\
\hline 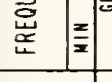 & نஷ & 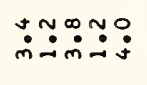 & 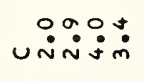 & 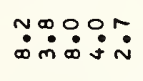 & 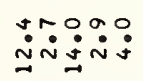 & $\ddot{\sim}: \vec{\sim} \dot{\sim} \dot{\sim} \dot{m}$ & 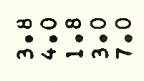 & $\dot{\sim} \dot{\sim} \dot{\sim} \dot{\sim} \dot{\sim}$ & 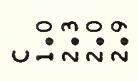 & 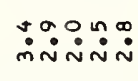 \\
\hline ONIX & 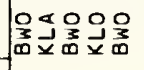 & 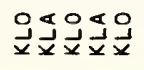 & 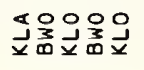 & 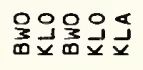 & 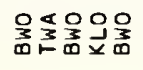 & 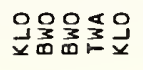 & 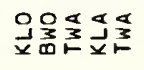 & 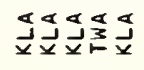 & 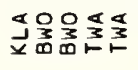 & 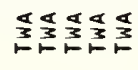 \\
\hline $708 \mathrm{k} / \mathrm{s}$ & " 11 & & & & & & " & & & \\
\hline 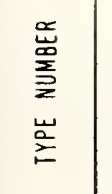 & 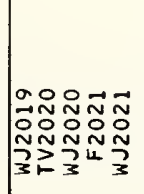 & 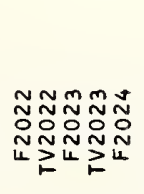 & 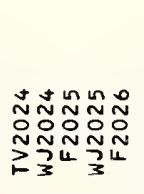 & 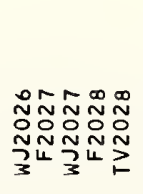 & 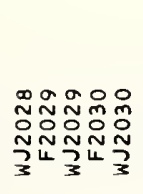 & 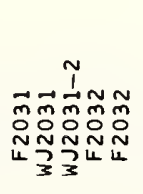 & 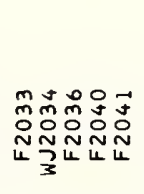 & 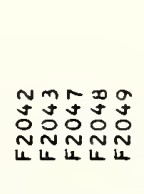 & 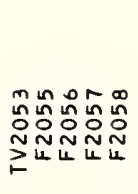 & 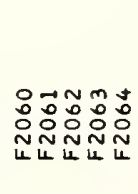 \\
\hline
\end{tabular}




\begin{tabular}{|c|c|c|c|c|c|c|c|c|c|c|}
\hline 9M: Tdก0S & 88 8 & 엉엉ㅇㅇㅇ & 웅웡ㅇㅇ & 엉ㅇㅇ & 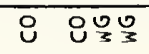 & 문요 & $\frac{0}{3} 80$ & 엉웡으 & 엉워 & 88888 \\
\hline Aliars & & & 5 & $\overrightarrow{0}$ & & $\vec{\alpha} \vec{J}$ & $\vec{\alpha} \vec{\alpha}$ & & $\vec{\sim}$ & \\
\hline $\begin{array}{c}07314 \\
\text { J11392n }\end{array}$ & $\frac{\varepsilon}{0}=\Sigma \Sigma \Sigma \Sigma \Sigma$ & $\sum \sum \sum \sum \sum$ & $\Sigma \Sigma \Sigma$ & $\Sigma \Sigma \Sigma \Sigma$ & 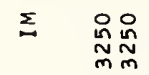 & 总员足 & 용여 & $\sum \Sigma d_{0}$ & 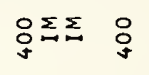 & 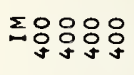 \\
\hline HLOMONY & & & & & $\stackrel{\circ}{N}$ & $\therefore$ & nf & & $\stackrel{\sim}{N}$ & \\
\hline 9אורורו & 풀 & & & & $m m$ & $m m$ & & & & \\
\hline 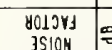 & a & 오 엉웡ㅇ & $m \stackrel{n}{m} \sim$ & 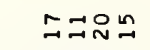 & i & $\stackrel{n}{N}$ & 임음 & 임임임으 & 임임요 & \\
\hline Mirg & 国 & 용ㅇㅇㅇㅇㅛ & 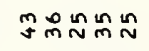 & 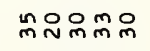 & 욨nn & i $\quad$ g & 요ำ욤 & 앝음임요 & ㅇm요 N & 요묘 \\
\hline $\begin{array}{l}S 170 \wedge \\
\times 1173 H \\
\end{array}$ & 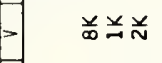 & 유 윰윰ㅇ & 옷 옷 & $\underline{a} \underline{\sim}$ & 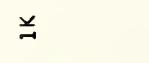 & 并 & 兰 & 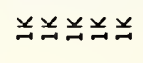 & ㅡㅡㅁㅇㅛ & 品兰品 \\
\hline $\begin{array}{c}51701 \\
1081 \mathrm{H} 03\end{array}$ & 㝏 min & 용잉요 & 웅응요 in & 응요요요 & 品 & 品 & & & & \\
\hline 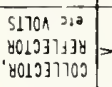 & | & : : & & $\stackrel{\circ}{\stackrel{ }{N}}$ & & in & 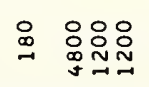 & $\stackrel{\circ}{\cong}$ & : & \\
\hline $0^{\circ}$ & E & 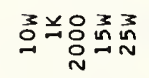 & 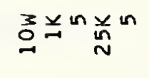 & 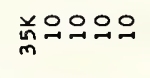 & 説总关关 & 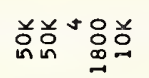 & 궁요 & 우웅으으음 & 옥으욧요음 & \\
\hline$\leftrightarrow$ & El & 암용요요 & 욤윰 & 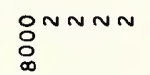 & 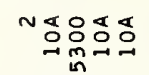 & 떰ㅇㅁㅇㅇㅠ & 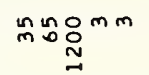 & mmmmm & $\mathrm{mmm} \mathrm{m}$ & $\mathrm{mmmmm}$ \\
\hline 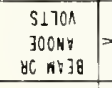 & 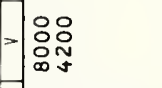 & 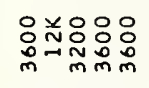 & 品兰呆总品 & 씃용요 & 品总吕声寺 & 弟弟只员希 & 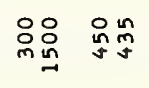 & 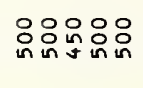 & 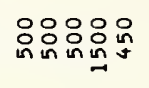 & 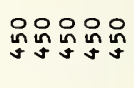 \\
\hline$F^{+}$ & | & 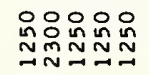 & 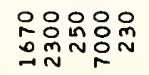 & 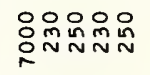 & 오N 总品品 & 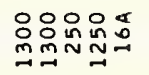 & 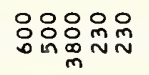 & 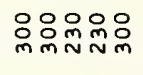 & 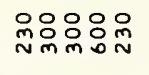 & \\
\hline w & 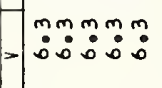 & 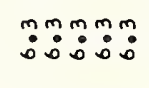 & $\ddot{m}: m: m: m$ & ma:mo: & m: man: & mmo: & 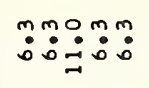 & $\ddot{m}: m \ddot{m}: \dot{m}:$ & $\ddot{m}: m \ddot{m}^{m}: \dot{m}$ & \\
\hline $\mathrm{NO} 1 \mathrm{YH}\} \mathrm{dC}$ & auauv & vauuv & $\cup a \cup a u$ & auuuv & uavaa & a auuu & טuטu & טuטu & ưuט & บบบบบ \\
\hline YJW & ヒニヒヒヒ & ヒこニこニ & ヒニU゙マU゙ & 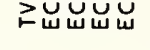 & யüZUু匕 & પூUু & 路出㫐 & 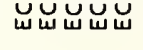 & 㟧岕出的㟧 & 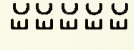 \\
\hline 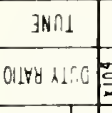 & 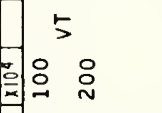 & 욤 & 5 & & $\begin{array}{l}x \times x \\
u \in u \\
n n\end{array}$ & 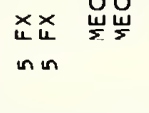 & $\stackrel{\breve{w}}{\Sigma}$ & & $x_{u}^{x}$ & \\
\hline 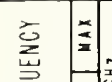 & 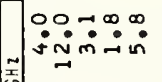 & 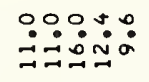 & $\because \because \dot{\sim} \quad:$ & \begin{tabular}{l}
$\because 0$ \\
\hdashline$\vdots$ \\
\hdashline
\end{tabular} & 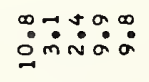 & & 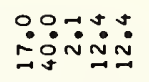 & 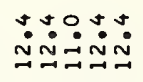 & 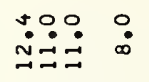 & 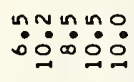 \\
\hline 总 & $\mid$ & ث̊ำ: & 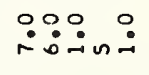 & 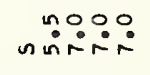 & 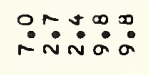 & 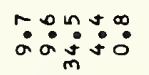 & $\because \because \because \therefore:$ & வீ: & ஊ̊? & 品華递 \\
\hline ONIY & $\mid \begin{array}{l}4 \\
3 \\
1 \\
1\end{array}$ & 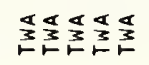 & 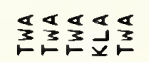 & 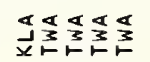 & 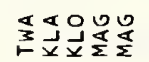 & 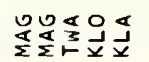 & 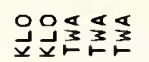 & 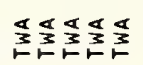 & 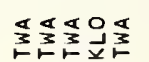 & 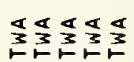 \\
\hline 708wis & & " " " " " & " " & " & & & " " " & " & " & \\
\hline 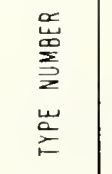 & 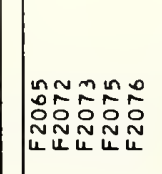 & 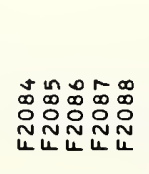 & 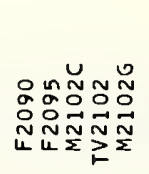 & 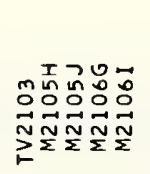 & 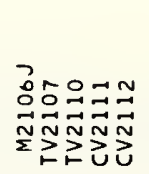 & 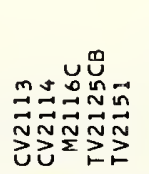 & 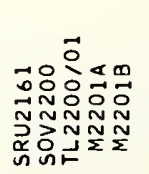 & 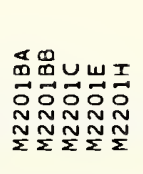 & 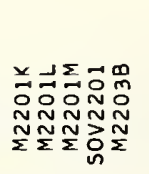 & 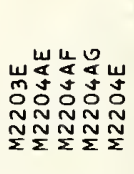 \\
\hline
\end{tabular}




\begin{tabular}{|c|c|c|c|c|c|c|c|c|c|c|}
\hline 9x:7dnos & \multirow[t]{2}{*}{ 엉요 } & 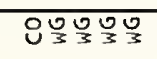 & \% & \multicolumn{2}{|c|}{ 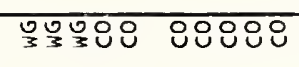 } & \multirow[t]{2}{*}{ 엉요 } & \multirow[t]{2}{*}{80808} & \multirow[t]{2}{*}{80808} & \multirow[t]{2}{*}{ Oण 305} & \multirow[t]{2}{*}{ जणूउ } \\
\hline 111ArJ & & $\tilde{\alpha} \tilde{\alpha}$ & $\vec{\alpha} \vec{\alpha} \vec{\alpha} \vec{\alpha}$ & $\ddot{\sim} \tilde{N}$ & & & & & & \\
\hline $\begin{array}{c}01311 \\
\text { ग13N9VK }\end{array}$ & 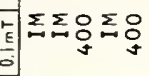 & $\sum \underset{j}{g}$ & $\Sigma$ & 표 & $\sum \sum \sum n \sum \sum$ & 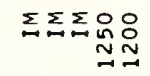 & 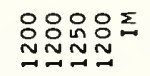 & 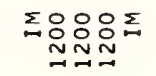 & 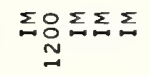 & 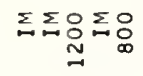 \\
\hline HLOMONYG & & & & & & & & & & \\
\hline 2W177nd & 害 & & & & & & & & & \\
\hline $\begin{array}{l}\text { yolovis } \\
\text { 3S10k }\end{array}$ & : No욤 & 윰융요 & $\tilde{m}$ & $\tilde{m} \tilde{m}$ & $\tilde{m} \tilde{m} \tilde{m} n \tilde{m}$ & mnnmmnn & nnnmmnn & $\tilde{m} \tilde{m} \tilde{m} n \tilde{m}$ & 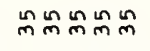 & 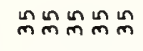 \\
\hline$\frac{19180}{4199}$ & 国舟品品品 & 을윰요 & in & 임 & 음임임요 & 잍윰윰요 & 잍윰윰요 & 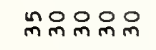 & 윰윰윰유 & 있임임요 \\
\hline $\begin{array}{l}5: 70 \mathrm{~A} \\
\mathrm{XI173H}\end{array}$ & 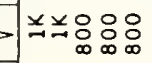 & $\stackrel{0}{O_{\infty}} \underset{-}{ }$ & & $\stackrel{\sim}{\sim}$ & 弚芯五 & 弚芯前羊杀 & 当羊杀杀古 & 羊羊羊羊羊 & 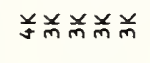 & 并并首前 \\
\hline $\begin{array}{c}S 1700 \\
7041 \text { NOS }\end{array}$ & & & 용 & 응요 & 욧요요요 & 읏요요요 & 음요요요요 & 용요요요 & 品品品品品 & 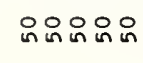 \\
\hline 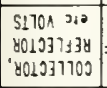 & : & 吕 & 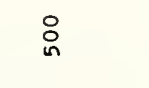 & & & & & & & \\
\hline $0^{\circ}$ & 어으으 & 욱어으욤욤 & 음윰욤유 & 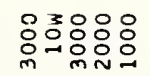 & 응융ㅇㅁㅇㅁㅇ & 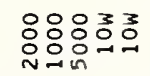 & 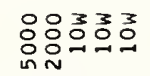 & 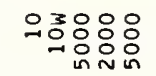 & 중임잉융ㅇㅁㅇㅇ & 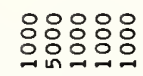 \\
\hline- & Emmmm & $m+t 0_{\infty} n$ & 욤ำ & 암아임윰요 & 임임임요 & 을용ㅇㅇ & 00000 & 옹요 & 일ming & 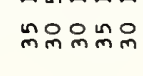 \\
\hline 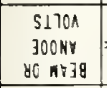 & 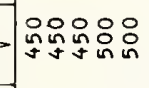 & 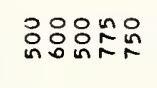 & 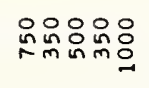 & 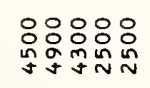 & :ㅇ: & :ㅇ: & 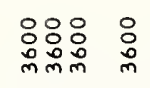 & 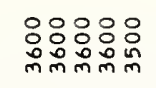 & 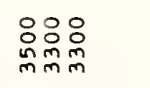 & 을음요 \\
\hline$\omega^{4}$ & 品怘怘 & 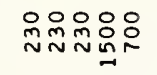 & 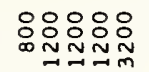 & 응용요 & 음윰용요 & 음욤요 & 음음요 & 음욤요 & 品品品品品 & 윰욤요 \\
\hline$\omega^{5}$ & $\ddot{m}: \stackrel{m}{0}$ & 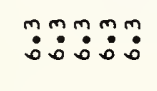 & 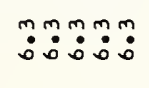 & 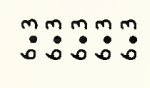 & "̊: & "̊: & m: & : & 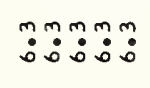 & : \\
\hline NOLIYYy] 00 & & טuンu & טuטu & ưuบu & & uบuบu & & & & \\
\hline 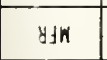 & 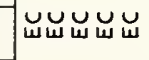 & 岕岲きる & 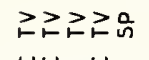 & 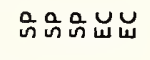 & 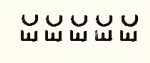 & س̆ & 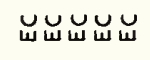 & 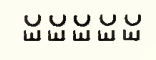 & 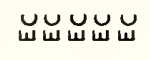 & 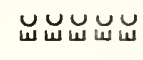 \\
\hline JNก1 & & $\frac{\breve{u}}{\Sigma}$ & 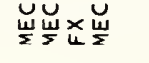 & $x_{4 \times 4}^{x \times x}$ & & & & & & \\
\hline Ol18y $x 110$ & & & & & & & & & & \\
\hline 意 & 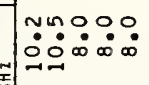 & $\because \because 00 n$ & 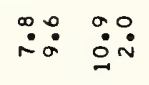 & 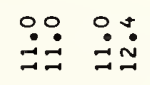 & O゚: & ث. & ○シ゚○゚ & 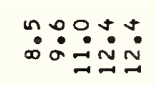 & 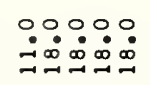 & $\because: 0000$ \\
\hline 总 & ONO::0: & 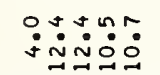 & 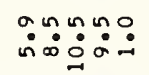 & 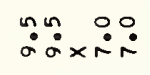 & 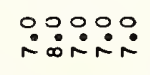 & 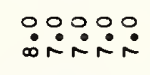 & 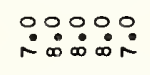 & $\because \because \because 000$ & 苾芯过 & : \\
\hline ONIX & 通乐乐我 & 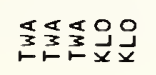 & 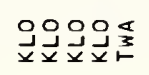 & 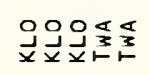 & 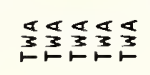 & 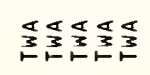 & 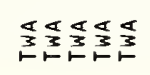 & 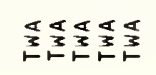 & 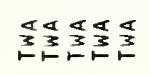 & 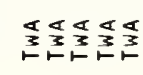 \\
\hline 708 W 15 & $"$ & $"$ & & & & & & & & \\
\hline 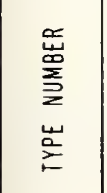 & 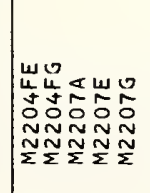 & 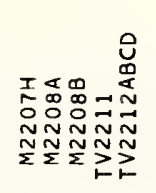 & 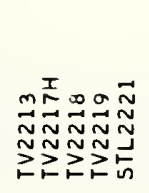 & 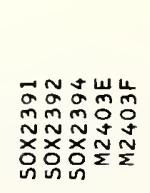 & 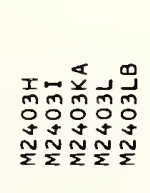 & 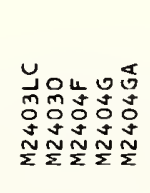 & 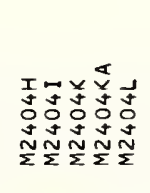 & 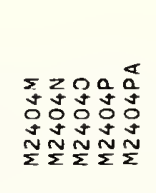 & 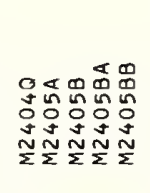 & 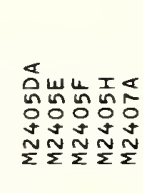 \\
\hline
\end{tabular}




\begin{tabular}{|c|c|c|c|c|c|c|c|c|c|c|}
\hline 9אור InOS & 엉ㅇㅇㅇㅇ & 엉어엉엉 & তㅜㅇㅇㅇ엉 & 엉어언언 & 어원엉 & ㅇㅇㅇㅇㅛ & రত্যত্য়ত্য & ত & 뭉 § & 엉엉어 \\
\hline AIIAYS & & & $\vec{\alpha}$ & & & & $\vec{\alpha} \vec{\alpha} \vec{\alpha}$ & $\vec{\alpha} \vec{\sim} \vec{\sim} \vec{\sim} \vec{N}$ & $\vec{\sim} \tilde{N}$ & $\vec{\alpha} \vec{m} \vec{\alpha} w$ \\
\hline $\begin{array}{c}01119 \\
\text { ग113KVVM }\end{array}$ & 甸 & $\Sigma \Sigma \Sigma \Sigma \Sigma$ & $\sum \quad \sum \Sigma$ & 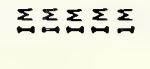 & $\sum_{n \rightarrow \infty} \Sigma_{-1} \Sigma \Sigma_{-1}$ & 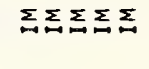 & $\Sigma$ & & $\sum_{i=1}$ & \\
\hline H101MOKY8 & & & & & & & nin & 윰 & q & ? \\
\hline 9אורาnd & 폰 & & & & & & & & & \\
\hline 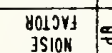 & 耳mnnnm & 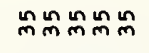 & $\stackrel{n}{m}$ & & & q & & & $\stackrel{\text { n }}{m}$ & \\
\hline NIYg & 임욤욤요 & 임윰윰욤 & 品 & & & ก & ? & & $\stackrel{m}{m}$ & $\stackrel{m}{\sim} \underset{m}{\infty} \stackrel{\infty}{m}$ \\
\hline $\begin{array}{l}\text { S170A } \\
\times 17 y H\end{array}$ & 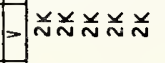 & 프N & 㒸 & & $\cong \cong$ & $\underset{\sim}{\sim}$ 品 & $\stackrel{\sim}{N}$ & & & \\
\hline $\begin{array}{c}\text { S170n } \\
\text { 1081 KOOO }\end{array}$ & 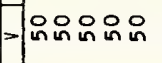 & 은은은은요 & 음음요 & 웄임윰요 & 임윰욤요 & 암윰임잉요 & 익 & $\stackrel{\circ}{\circ}$ & $\stackrel{\circ}{N}$ & in \\
\hline 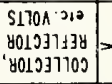 & & & 品品品品 & 盖品识品员 & 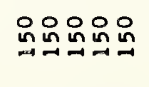 & 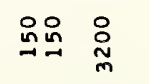 & 응응 & 吕 & 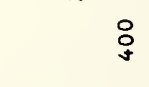 & 웅 \\
\hline$a^{\circ}$ & Ho & 응ㅇㅇㅇㅇㅇㅁㅇㅠ & 응욤융요 & 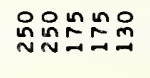 & 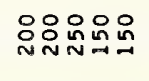 & 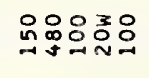 & 응응용ㅇㅁ & 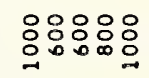 & 总品品员n & 욱용요 \\
\hline 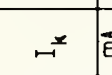 & 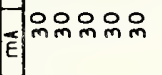 & 윰유있요 & 요ำn & 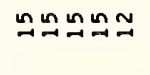 & $\cong \simeq$ & $\cong \cong \sim \sim ⿻ 上 丨$ & 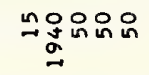 & 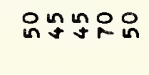 & ${ }^{\infty} 0^{\infty}{ }^{\infty}$ in & 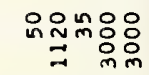 \\
\hline $\begin{array}{c}5170 \mathrm{~N} \\
300 \mathrm{NY} \\
80 \mathrm{AHBg} \\
\end{array}$ & > & 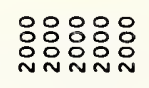 & 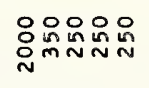 & 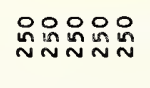 & 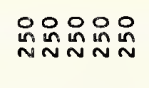 & 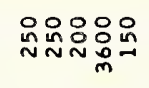 & 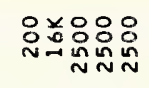 & 品品品品 & 品员品踶 & 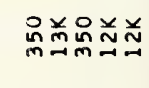 \\
\hline$\omega$ & 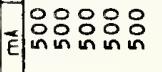 & 움욤요욤 & 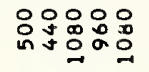 & 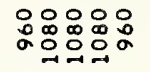 & 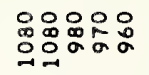 & 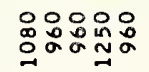 & 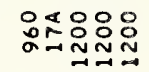 & 品品路品品 & :ㅇㅇㅇ융ㅇㅇ & 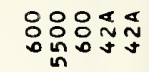 \\
\hline шँ & 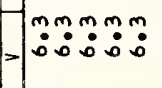 & 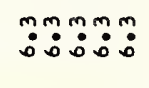 & : & 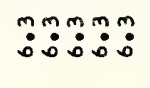 & 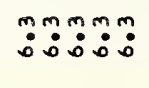 & : & m: & mo: & 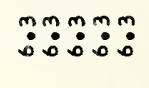 & 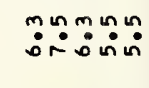 \\
\hline Nol1YY3dO & |ưuบu & บบบบบ & vouบ & & yưư & vưue & vauuv & טuטu & บบบư & vavuv \\
\hline$\forall j \mathrm{~W}$ & |ئسنس & سئ山ن & ய゙マニヒロ & こニヒヒニ & ヒニヒロヒ & ヒヒヒヒヒ & 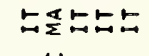 & こ命的号合 & 解的的岩 & 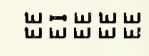 \\
\hline JNกL & & & แั山55ร & ケ55ร5 & ら5っらら & เ55 & 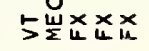 & $x_{4}^{x \times x \times}$ & $x_{u}^{x}$ & w \\
\hline 01184 a $1 \cap 0 \mathrm{E}$ & & & & & & & $\stackrel{0}{n}$ & & & 品 \\
\hline 离 & 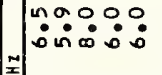 & $\because: 00^{\circ}: 0$. & 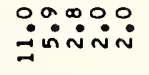 & 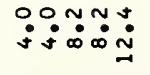 & 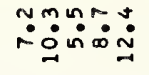 & $\begin{array}{l}+9: 00 \\
\dddot{\sim} \\
\sim\end{array}$ & $\because \because 0: 0:$ & 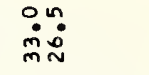 & :゚̊: & 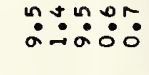 \\
\hline 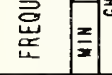 & 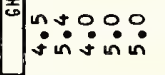 & m::0:0 & 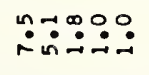 & 㝵: : : & 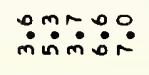 & 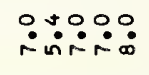 & 棺品品品品 & 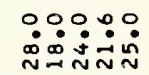 & 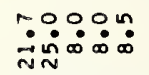 & 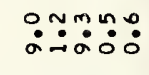 \\
\hline ONIX & 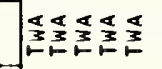 & 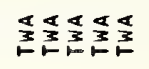 & 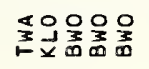 & 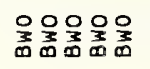 & 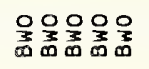 & 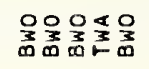 & 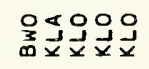 & 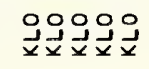 & 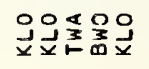 & 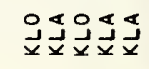 \\
\hline $708 W / S$ & & & & & " " & " " " & " " " " " & $"$ & & \\
\hline 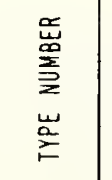 & 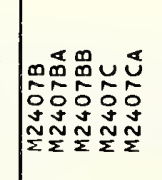 & 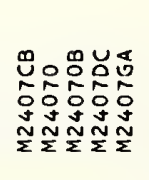 & 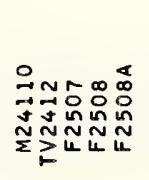 & 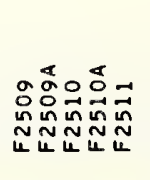 & 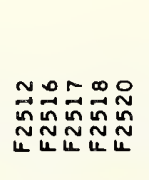 & 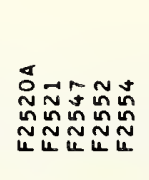 & 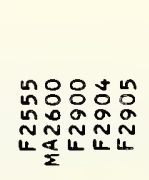 & 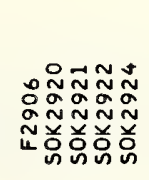 & 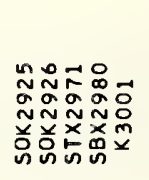 & 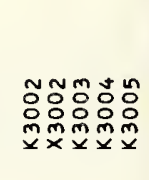 \\
\hline
\end{tabular}




\begin{tabular}{|c|c|c|c|c|c|c|c|c|c|c|}
\hline 9kild dnos & 웅ㅇㅇㅇ & 언우ㄴㅜㅗ & & 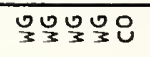 & 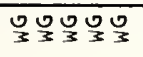 & 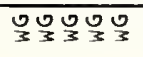 & 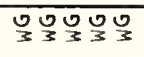 & पण & OOO & 엉원요 \\
\hline L11ars & 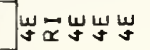 & $w \underset{\xi}{w} \underset{\jmath}{w}$ & & & $\vec{m}$ & & & $\approx \pi$ & 五政 & テデี \\
\hline 01311, & & $\Sigma$ & $\sum \sum \sum \sum \sum \sum$ & $\Sigma \Sigma \Sigma \Sigma$ & $\Sigma \Sigma \Sigma \Sigma \Sigma$ & $\Sigma \Sigma \Sigma \Sigma \Sigma$ & $\Sigma \Sigma \Sigma \Sigma \Sigma$ & $\Sigma \Sigma \Sigma$ & $\Sigma \Sigma \Sigma$ & $\Sigma \Sigma$ \\
\hline HLOMONry & $\sim$ & g & $\sim \sim N \sim N$ & $\mathrm{mmm}$ & $\sim \sim \sim N$ & $\sim N \sim N N$ & $\sim N N \sim N$ & NN & $d^{2} \operatorname{mn}$ & 유 \\
\hline 9אור & 훌 & & ㅇNㅇN유 & $\stackrel{\sim}{2} \cong \backsim$ & 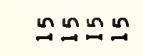 & 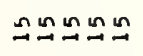 & 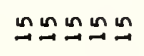 & $\stackrel{n \sim n}{\sim}$ & $\stackrel{i}{\sim}$ & $\stackrel{\infty}{\sim}$ \\
\hline 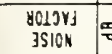 & & & & & & & & & & \\
\hline Mirg & 国 & 웡워 & & $\stackrel{n}{\sim}$ & $\stackrel{m}{m}$ & & & $\hat{m} \tilde{m}$ & 只告品 品 & 융요 \\
\hline $\begin{array}{l}S 1701 \\
\times 11] \mathrm{HH}\end{array}$ & & & & & & & & & & 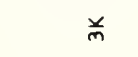 \\
\hline $\begin{array}{c}\text { S170n } \\
\text { TO81No3 }\end{array}$ & & & & & & & & & & \\
\hline 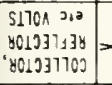 & i̊ & & & & & & & & & 品 \\
\hline$a^{\circ}$ & 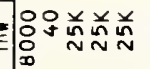 & 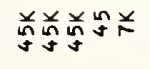 & 疍 & 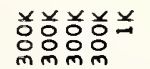 & 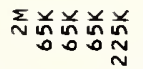 & 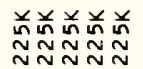 & 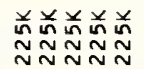 & 总总总京兰 & 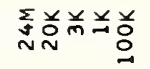 & 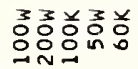 \\
\hline$-x$ & 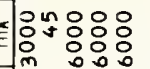 & 응윰유 & 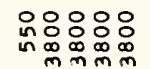 & 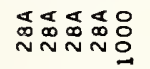 & 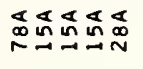 & 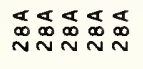 & 恶 & 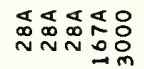 & 乐品品路品 & 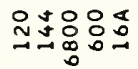 \\
\hline 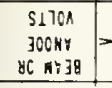 & 证㒸兑 & 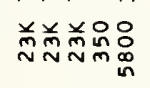 & 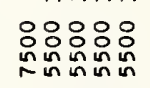 & 前㒸㒸㒸前 & 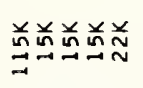 & 프NNNN N & $\underset{N}{\mathbb{N}} \underset{N}{\mathbb{N}} \underset{N}{N} \stackrel{N}{N}$ & 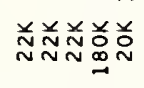 & 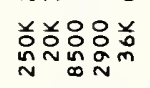 & 影品嗯 兰 \\
\hline$\leftarrow$ & 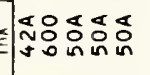 & 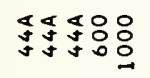 & 응ㅇㅇㅇㅇㅇㅇ응 & 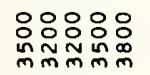 & 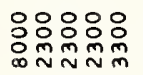 & 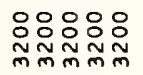 & 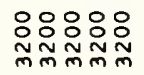 & 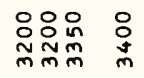 & 乐品品品品 & 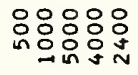 \\
\hline$\omega^{\leftarrow}$ & 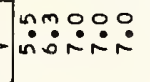 & 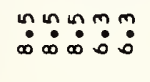 & 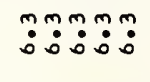 & 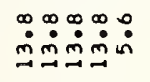 & 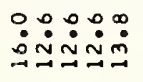 & 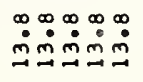 & 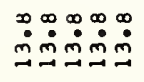 & 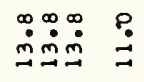 & 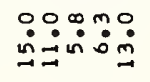 & 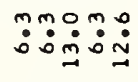 \\
\hline No:18G930 & טuטuט & aטuט & 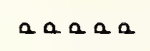 & $a a a a v$ & $a 0 a a$ & $a a a a a$ & $a, a, a$ & $a a a u v$ & auua.u & uuvua \\
\hline yJW & س山ّ山س & 岀岕岕 & ココココ & ココココび & コココココ & コココココ & コココココ & コココேี & ேีコேゴ & ேีயேี้ \\
\hline $3 \times \cap 1$ & 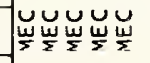 & 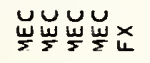 & 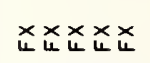 & 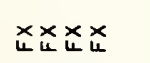 & 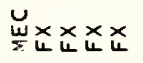 & $\begin{array}{l}x \times \times x \\
4\end{array}$ & $\begin{array}{l}x \times x \times x \\
4 u[u\end{array}$ & 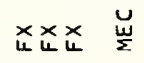 & 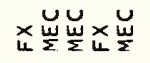 & 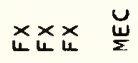 \\
\hline $0118+4$ in: 0 & & $\cong \quad 8$ & 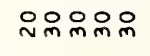 & 오오오오 & 앝오어오으 & 어오오어으 & 응ㅇㅇㅇㅇㅇㅇ & 오우음 & $=0$ & 웅 \\
\hline 产 & 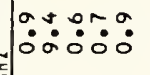 & 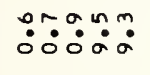 & 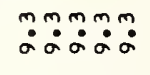 & 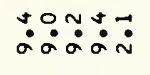 & 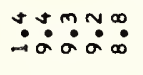 & 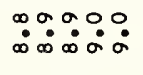 & 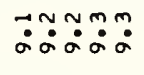 & $\dot{\sigma} \sigma_{\infty}^{+\infty}: \Delta$ & 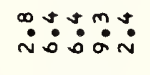 & 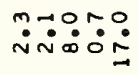 \\
\hline 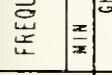 & 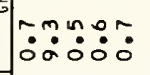 & $\ddot{0}: \mathfrak{0}: \dot{m}$ & 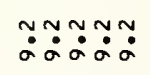 & 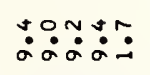 & 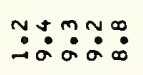 & 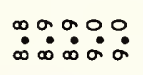 & 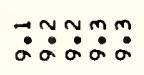 & 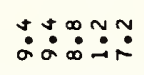 & 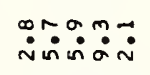 & $\dot{\sim} \sim 0: 0:$ \\
\hline ON!X & 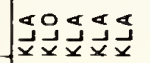 & 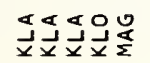 & 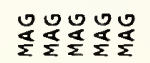 & 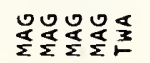 & 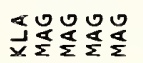 & 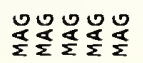 & 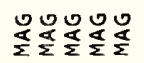 & 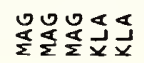 & 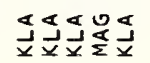 & 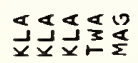 \\
\hline $709 \mathrm{kis}$ & & $"$ & & & " " " & & & " " & & " \\
\hline 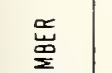 & & & & & & & & & & \\
\hline 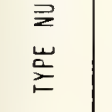 & 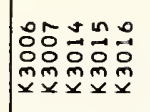 & 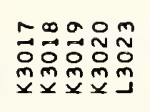 & 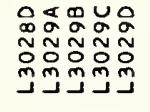 & 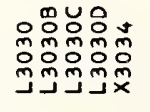 & 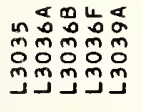 & 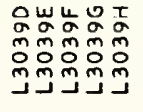 & 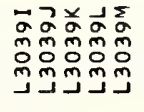 & 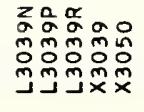 & 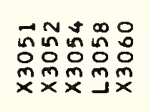 & 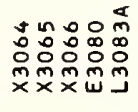 \\
\hline
\end{tabular}




\begin{tabular}{|c|c|c|c|c|c|c|c|c|c|c|}
\hline 9HITdOOS & पुणु & पूण & पण्ड & OOOY & W & पण्ड & 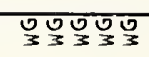 & 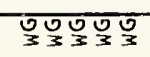 & তু & Wज़ \\
\hline 111980 & & & & & $\bar{m}$ & & & & & \\
\hline 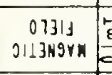 & $\varepsilon \Sigma \Sigma \Sigma \Sigma \Sigma$ & $\sum \sum \Sigma \Sigma \Sigma$ & $\Sigma \Sigma \Sigma$ & $\Sigma \Sigma \Sigma \Sigma \Sigma \Sigma$ & $\Sigma \Sigma \Sigma$ & $\Sigma \Sigma \Sigma \Sigma$ & $\Sigma \Sigma \Sigma \Sigma$ & $\sum \sum \sum \sum \sum$ & $\sum$ & $\sum \sum \sum$ \\
\hline HOOMOHY $\risingdotseq$ & $=m \backsim N N m$ & $N N N \infty$ & NNNm & $\sim N N m$ & $\sim m m$ & m: & 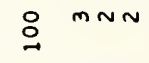 & NNNN & m & $m m N$ \\
\hline 9*17רnd & 풀유욤요 & 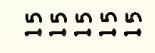 & $\cong$ 온 & 우유ํํํ & 우수 & $\stackrel{\sim}{\sim} \stackrel{\sim}{\sim}$ & $\stackrel{N}{N} \vec{N}$ & 오ำ요 & 유 & 유유 운 \\
\hline 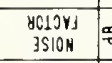 & & & & & & & & & & \\
\hline NIYO & & & & & $\stackrel{\circ}{\circ} \quad \stackrel{\circ}{m}$ & $\vec{m}$ & $\stackrel{\circ}{m}$ & & 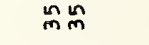 & \\
\hline $\begin{array}{l}51700 \\
\times 173 H\end{array}$ & & & & & & & & & & \\
\hline $\begin{array}{c}51701 \\
1001 \mathrm{NOS}\end{array}=$ & & & & & & & & & & \\
\hline $\begin{array}{l}\text { S170R 3.2 } \\
\text { 40103?134 }\end{array}$ & & & & & & & & & & \\
\hline 'y0120371703 & & & & & & & & & & \\
\hline$a^{\circ}$ & & 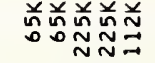 & 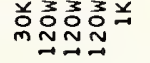 & 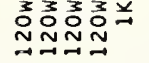 & 疍品品 & 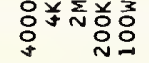 & 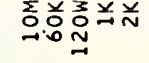 & 吕夈前首兰 & 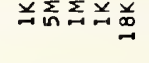 & 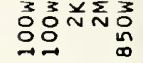 \\
\hline 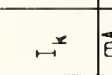 & 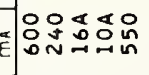 & 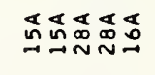 & 喝品品 & 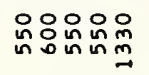 & 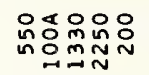 & 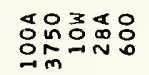 & 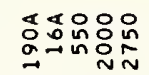 & 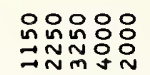 & 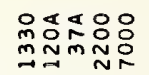 & 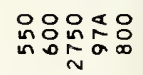 \\
\hline 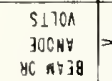 & 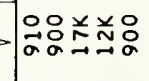 & 总芯恙并首 & 임요 & 잉잉잉요 & 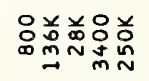 & 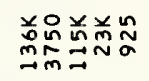 & 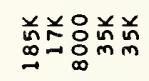 & 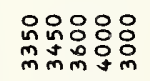 & 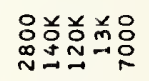 & 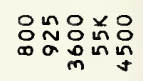 \\
\hline$\varpi^{-} \vec{E}$ & E| & 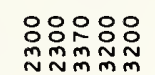 & 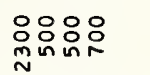 & 음윰욤욤요 & 윰용요 & 잉윰요 & 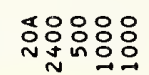 & 응양옹응영 & 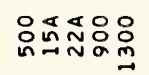 & 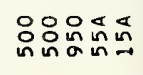 \\
\hline$\omega^{5}$ & & : & :mmm & $: m: m: m$ & mำ & : & 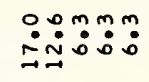 & 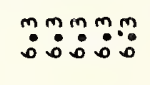 & m: & 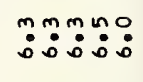 \\
\hline $\mathrm{NOLI} 1 \mathrm{YHZ} \mathrm{dO}$ & acana & acaaa & $a, a a u$ & anaba & anaa & anaa & aaca & aana & a a a a & \\
\hline$y+n$ & コココココ & コココココ & コココロ & تコココ & تココゴ & & コココココ & コココざ & コココココ & בココココ \\
\hline JNก1 & $\mid x \times$ & 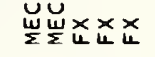 & $x_{4}^{x} \times x^{x} \times x$ & 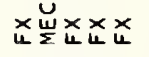 & ×5 & $\begin{array}{l}x \\
4\end{array}$ & 走宏 & 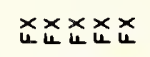 & 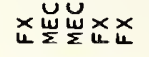 & 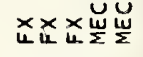 \\
\hline Oilty AIrio & 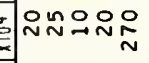 & 으으으엉ㅇ & 오유오 & 유숫윰ำ & 우수요묘 & m음요오 & 융어유으으 & 임웅으음요 & 묭용요 & 옹윰유 \\
\hline 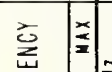 & 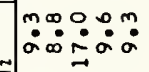 & 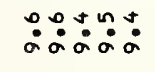 & 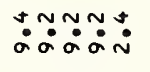 & 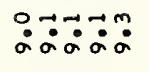 & 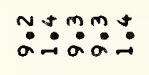 & 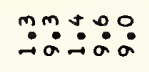 & 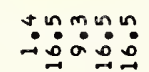 & 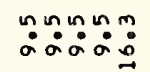 & 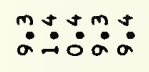 & 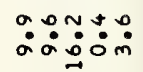 \\
\hline 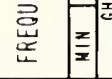 & 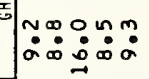 & 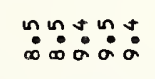 & 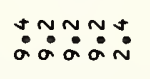 & 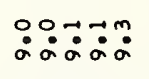 & 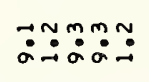 & 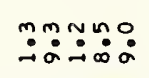 & 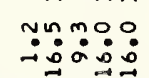 & 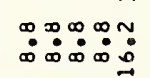 & 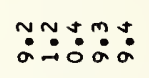 & 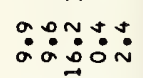 \\
\hline ONIX & $\mid \frac{0}{2} \frac{0}{2} \frac{0}{2} \frac{0}{2} \frac{\pi}{2}$ & 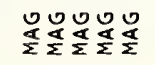 & 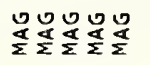 & 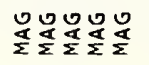 & 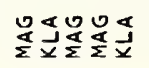 & 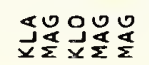 & 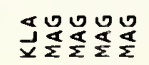 & 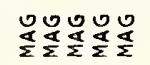 & 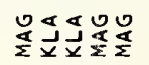 & 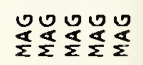 \\
\hline $708 \mathrm{~W} / \mathrm{S}$ & & " " " " & & $"$ & & & " " " & & & " " " \\
\hline $\begin{array}{l}\text { 虽 } \\
\text { 焉 }\end{array}$ & & & & & & & & & & \\
\hline 竞 & 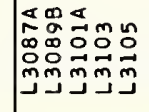 & 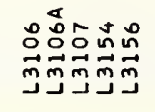 & 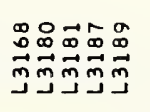 & 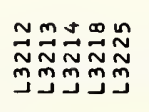 & 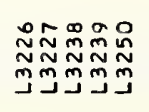 & 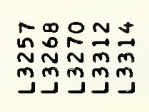 & 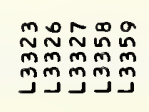 & 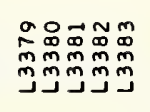 & 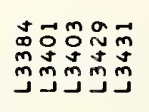 & 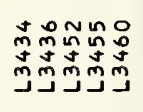 \\
\hline
\end{tabular}




\begin{tabular}{|c|c|c|c|c|c|c|c|c|c|c|}
\hline 9 9พ17 & \multirow[t]{2}{*}{ 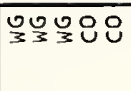 } & \multirow{2}{*}{ 웅어어 } & \multirow[t]{2}{*}{ 옹 } & \multirow[t]{2}{*}{ 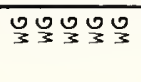 } & \multirow[t]{2}{*}{ 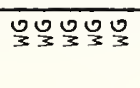 } & WO & \multirow[t]{2}{*}{ 88 8\% } & \multirow[t]{2}{*}{ 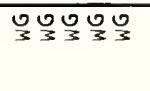 } & \multirow[t]{2}{*}{ ఇতৃ } & \multirow[t]{2}{*}{ OO $\frac{O}{3}$} \\
\hline Alars & & & & & & $\vec{J}$ & & & & \\
\hline \begin{tabular}{l|l}
01111 \\
J1139VYK
\end{tabular} & $\sum \Sigma \Sigma \Sigma \Sigma \Sigma$ & $\Sigma \Sigma \Sigma \Sigma \Sigma$ & $\Sigma \Sigma \Sigma \Sigma$ & $\sum \Sigma \sum \Sigma \Sigma$ & $\sum \Sigma \sum \Sigma \Sigma$ & $\Sigma \Sigma \Sigma$ & $\Sigma \Sigma$ & & $\Sigma$ & $\Sigma$ \\
\hline 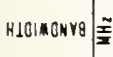 & $\sim \sim \sim \sim N$ & $\sim N \quad \underset{F}{F}$ & & & $\sim$ & $\sim N N$ & $\underset{3}{*}$ & & & $:$ \\
\hline 9M1717nd & 음ำ & $\stackrel{\sim}{\sim} N$ & & & 요 & 융ㅇ & $\stackrel{D}{\sim}$ & & & \\
\hline $\left.\begin{array}{l|l}8013 \gamma \mathrm{Y} \\
3 \mathrm{SSON}\end{array}\right)$ & & & & & & & & & & \\
\hline \begin{tabular}{l|l} 
Mirg & \%
\end{tabular} & & ํㅛ & 윰ำ & & & & 悉品 & o요 요 & & $\tilde{m} \tilde{N}$ \\
\hline $\begin{array}{l}51701 \\
\times 173 \mathrm{H}\end{array}$ & & & & & & & & & & \\
\hline $\begin{array}{c}51701 \\
1081400\end{array}=$ & & & & & & & & $\ddot{\sim} \ddot{\sim}$ & 品 & $\stackrel{\circ}{\circ}$ \\
\hline 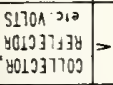 & & & & & & & & & & 品 尊 \\
\hline $0^{\circ}$ & 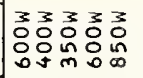 & 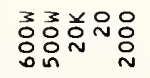 & 丞모N & 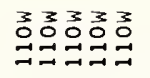 & 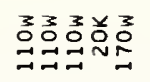 & 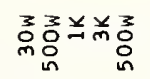 & 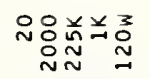 & 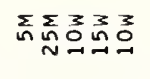 & 응엉ㅇㅁㅇㅇㅁㅇㅁㅇ & 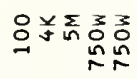 \\
\hline 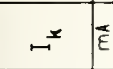 & 잉 & 㗊品 & 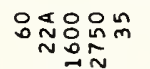 & 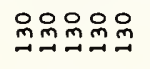 & 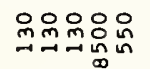 & 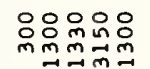 & 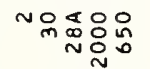 & 赵芯品品员 & 只品品品只 & 品员的品品 \\
\hline 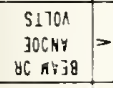 & 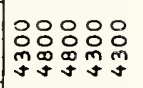 & 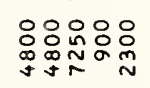 & : : & 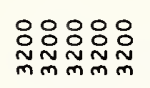 & 次: & 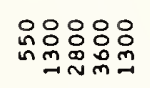 & 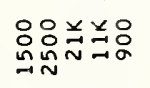 & 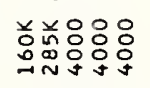 & 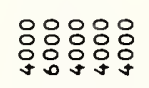 & 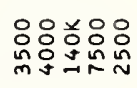 \\
\hline- & 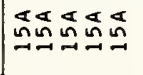 & 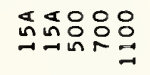 & 임윰욤욤 & ミミミミミ & 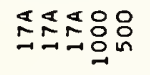 & 음음욤음 & 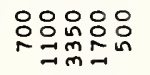 & 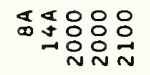 & 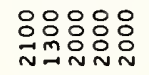 & 总员 \\
\hline$\omega^{-}$ & $\because \because: 0: 0$ & : & 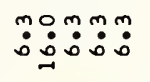 & 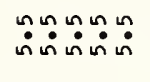 & 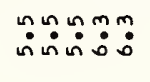 & 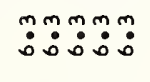 & 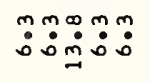 & 品: & 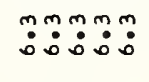 & $\ddot{m}: \ddot{m}: \ddot{m}$ \\
\hline Nol18y330 & טuטu & טUa & Uaaau & טuטu & Uưa & $a \triangle a \square a$ & Uuaa & a auuu & uauuu & $u a a a u$ \\
\hline$y \mathrm{JN}$ & ココココ & コココココ & コココココ & コココココ & コココココ & コココココ & コココココ & コココココ & コココココ & コココココ \\
\hline 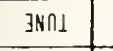 & Uu & 岀岀x & ระx & 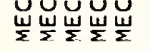 & 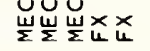 & 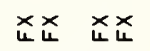 & 层 & 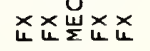 & 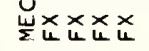 & 岁宏 \\
\hline oiley ansio & & $\stackrel{\circ}{\circ}$ & 옹용ㅇ & & 오 & 웃윰융ㅁㅇㅇㅁ & 웃 & 윰요 & $\stackrel{\circ}{\text { N }}$ & 음 \\
\hline 离 & 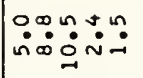 & 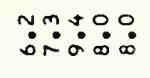 & 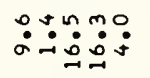 & 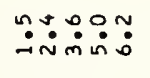 & 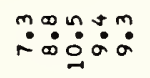 & 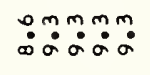 & ஊ̊:゚ロ & 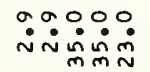 & 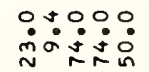 & 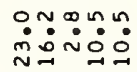 \\
\hline 总 & 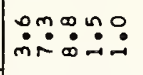 & 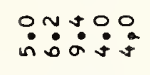 & : & 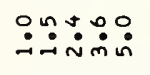 & 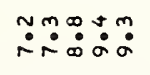 & 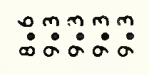 & 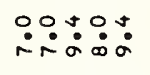 & $\begin{array}{l}\sigma \sigma: 0: 0 \\
\dot{\sim} \dot{\sim} \dot{m} \dot{m} \dot{\sim}\end{array}$ & 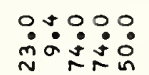 & 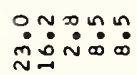 \\
\hline ONIX & 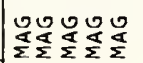 & 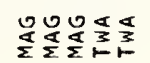 & 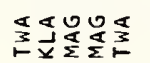 & 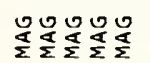 & 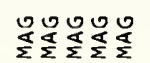 & 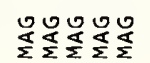 & 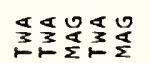 & 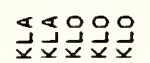 & 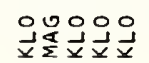 & 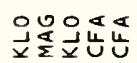 \\
\hline 70gh/S & & & " " " & & & " & $"$ & " " " & " " " " & " " \\
\hline 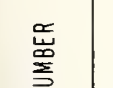 & & & & & & & & & & \\
\hline $\begin{array}{l}\vec{z} \\
\underline{w} \\
\underline{a}\end{array}$ & 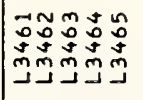 & 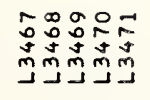 & 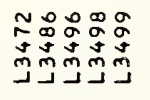 & 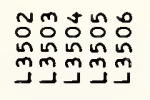 & 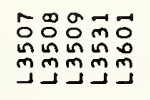 & 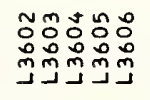 & 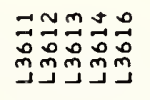 & 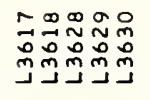 & 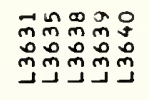 & 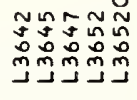 \\
\hline
\end{tabular}




\begin{tabular}{|c|c|c|c|c|c|c|c|c|c|c|}
\hline 9H17dก03 & $38 \%$ & 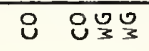 & 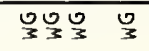 & 요 & 윽 언언 & 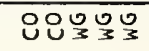 & $\frac{0}{3} \%$ & $\frac{00}{3}$ & 뭉ㅇ & 언 운 \\
\hline klars & & & $\vec{\alpha}$ & $\vec{\alpha}$ & & & $\vec{x}$ & & & \\
\hline 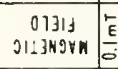 & $\underline{\underline{\epsilon}}$ & $\Sigma \Sigma$ & $\Sigma$ & & $\Sigma \Sigma$ & & $\Sigma$ & $\Sigma$ & $\Sigma \Sigma$ & $\Sigma \Sigma$ \\
\hline HLOMONYH & & $\cong$ & $:$ & $: 8:$ & N & & $\cong$ & & & $\therefore$ \\
\hline 9Milרח & $\underline{\underline{x}}$ & & $\cong$ & & $m$ & & & $\stackrel{n}{f}$ & fn $q$ & $\stackrel{\sim}{2}$ \\
\hline 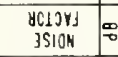 & 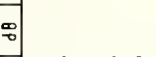 & & & & & & & & & \\
\hline Hivg \pm & $m m$ & mon & $\stackrel{n}{m}$ & $m m m ~ m$ & & & mํํ & 品 & $8=$ & $8 \mathrm{~m}$ \\
\hline $\begin{array}{l}\text { S170A } \\
\times 173 \mathrm{H}\end{array}$ & & & & & $\underset{f}{*}$ & 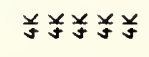 & $\stackrel{\Perp}{N}$ & & & \\
\hline $\begin{array}{c}51701 \\
7081402\end{array}=$ & $\cong$ & $\underline{二}$ & $\cong:$ & 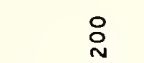 & 웅 & 옷웃ㅇㅇㅇㅠ & 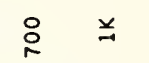 & & $\stackrel{n}{f}$ & \\
\hline 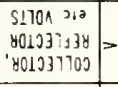 & & & $\stackrel{\circ}{\circ}$ & $\stackrel{\circ}{\sim}$ & & & & & & \\
\hline$a^{\circ}$ & 군 & 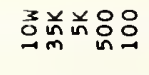 & 융욤ㅇㅁㅇ & 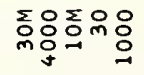 & 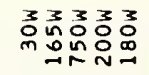 & 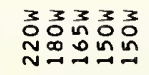 & 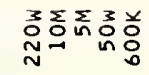 & 弟弚 & 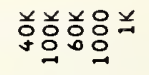 & 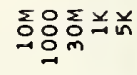 \\
\hline$\stackrel{x}{*}$ & E & 욕 욕 욕 & 品品品芯留 & 品 & 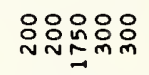 & 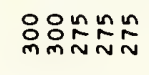 & 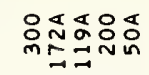 & 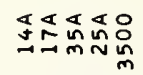 & 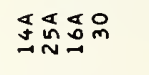 & 品 \\
\hline 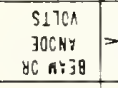 & 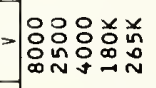 & 용 & 总品品总并 & 总 总品品 & 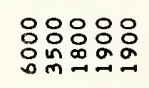 & 양임욤이 & 总兑总总㒸 & 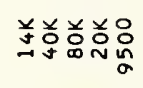 & 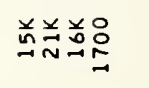 & 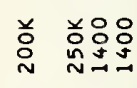 \\
\hline$m-\vec{E}$ & Eే & : & 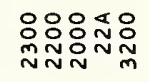 & 芯 芯品品 & 㳭品品 & : & $\begin{array}{l}\text { ON } \\
\stackrel{O}{N} \\
\text { N } \\
\text { N }\end{array}$ & 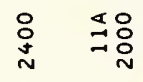 & 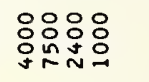 & 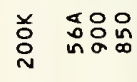 \\
\hline$\omega^{ \pm}$ & 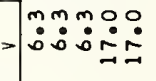 & $\stackrel{m}{:} \quad m$ & 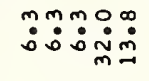 & $\dot{m}: \dot{m}: \dot{m}$ & m: & m: & $\dot{0}: \dot{\circ}$ & $\stackrel{m}{\cong}:$ & 草: & ¿ $\underset{m}{2}$ \\
\hline $\mathrm{N} 011 \mathrm{~B} \forall \mathrm{y} \mathrm{jC}$ & auvaa & u auv & טưaa & auauv & Uuaa & $a a a a a$ & vanua & $a, a, a$ & $a a a v u$ & avaan \\
\hline$y J M$ & コココここ & コココゴ & コココココ & コココココ & コココココ & コココココ & ココココ五 & ココゴ & ココゴ & ココココ \\
\hline $3 \mathrm{~N} \cap 1$ & 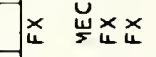 & x 岾㟧 & 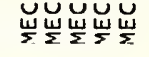 & 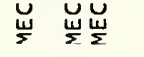 & 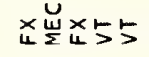 & ら5555 & ๖ & 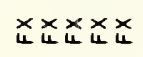 & $x_{4}^{x} \times$ & 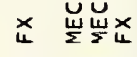 \\
\hline OLby alio & ton & : & 욱 & & : & & $\stackrel{m}{\sim}$ & $\therefore \quad ㅇ$ & ง & ㄱ \\
\hline 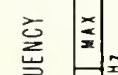 & 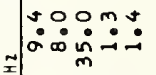 & 舟華薄: & :융유 & mo: & 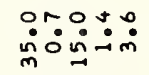 & 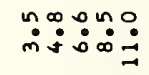 & 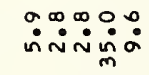 & 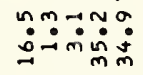 & 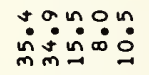 & 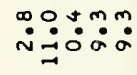 \\
\hline \begin{tabular}{|l|l|}
$\underline{X}$ & $\frac{x}{x}$ \\
\end{tabular} & ப요 & 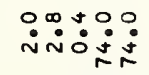 & :융유 & 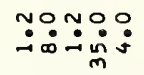 & 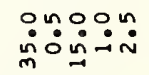 & 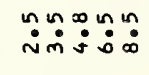 & 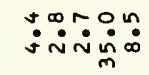 & 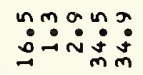 & $\begin{array}{l}+0 \\
\dot{q} \\
\dot{m}\end{array}$ & 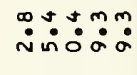 \\
\hline ONIX & 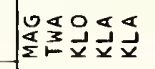 & 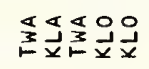 & 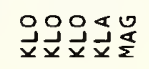 & 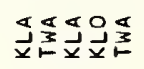 & 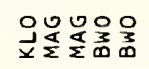 & 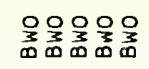 & 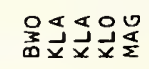 & 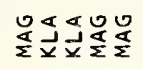 & 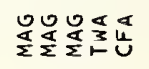 & 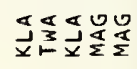 \\
\hline $708 \mathrm{~N} \times \mathrm{S}$ & " 1 & & " " " " & " " & " " " " & " " " " & & $"$ & " " " & " " \\
\hline 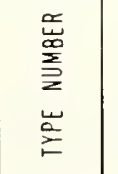 & 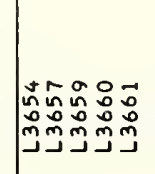 & 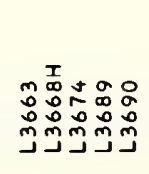 & 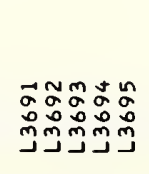 & 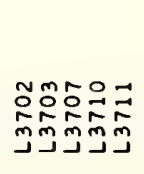 & m心̃ & 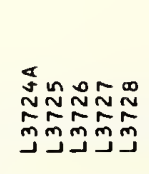 & 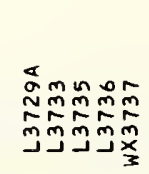 & 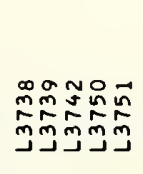 & 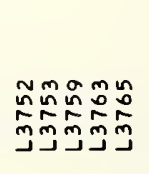 & 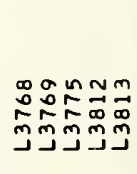 \\
\hline
\end{tabular}




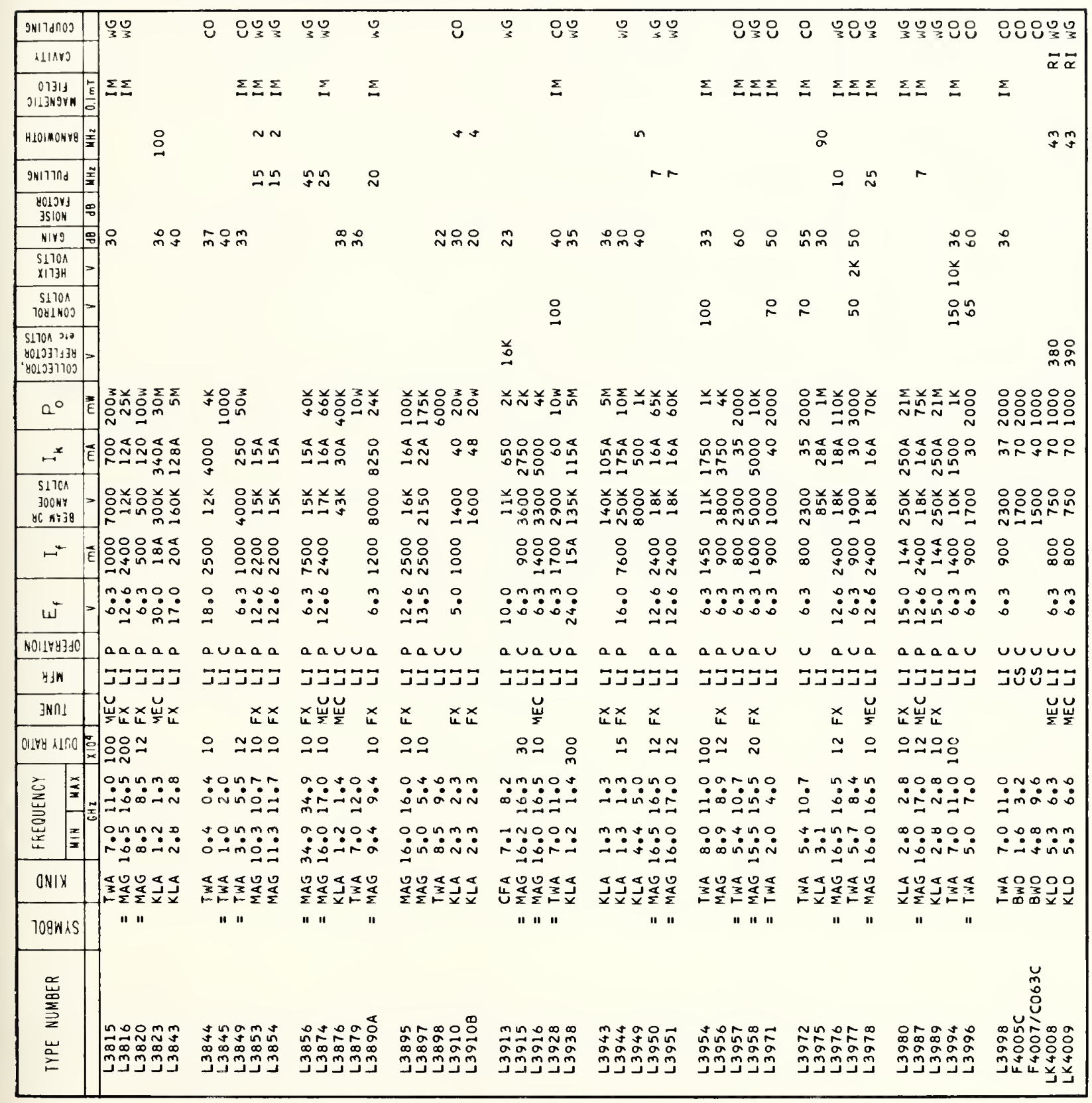




\begin{tabular}{|c|c|c|c|c|c|c|c|c|c|c|}
\hline 9*17dno3 & 원 & ৪ 오 & ㅇㅇ원 & ত & 응 8뭉 & 을 & 유용 & & 워요 & 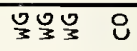 \\
\hline Alans & $\vec{\alpha} \alpha$ & & & $\vec{\sim} \vec{\sim}$ & & & & & $\underset{\xi}{\omega}$ & \\
\hline $\begin{array}{c}01311 \\
\text { ग113N9YM }\end{array}$ & $\sum \sum$ & $\sum \Sigma \Sigma \Sigma \Sigma$ & & $\Sigma$ & 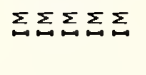 & $\sum_{-1} \sum_{n} \Sigma_{n}$ & & & 品 & $\Sigma$ \\
\hline H10:monYo & $\frac{7}{ \pm}=\frac{9}{9}$ & & & $\cong$ & & & & $\stackrel{\Perp}{N}$ & & \\
\hline 9*iา & 폴 & & & & & 品 & & & & \\
\hline $\begin{array}{l}\text { yoljors } \\
3510 \mathrm{H}\end{array}$ & $\stackrel{0}{\circ}$ & $\simeq \simeq \simeq$ & & & $\stackrel{m}{\sim}$ & $+\infty$ & & $\simeq \cong$ & \pm & $\simeq 0$ \\
\hline Nivg & 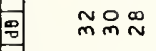 & $\hat{\sim} \approx \mathcal{y}$ & & $\tilde{m}$ & $\stackrel{n}{\sim} \underset{\sim}{N} \stackrel{\sim}{n} \tilde{m}$ & $\stackrel{n}{N} \mathfrak{N} \stackrel{n}{N}$ & $\stackrel{n}{N}$ & N N N & $\underset{\sim}{\sim} \stackrel{n}{m}$ & $\stackrel{n}{m} \vec{m}$ \\
\hline $\begin{array}{r}5170 \mathrm{~N} \\
\times 173 \mathrm{H} \\
\end{array}$ & $\stackrel{\Xi}{ }$ & 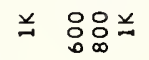 & & & $\stackrel{x}{\sim} \quad \underline{\sim}$ & & & & & \\
\hline $\begin{array}{c}\text { S170A } \\
1081 \mathrm{NOOS}\end{array}$ & 욤 & $\stackrel{-}{\circ}$ & & & $\stackrel{-}{\circ}$ & & & & $\stackrel{i}{N}$ & \\
\hline 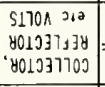 & 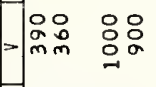 & 영 : & & 음 & 品品 : & & & & $\stackrel{\infty}{=}$ & \\
\hline$a^{\circ}$ & 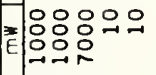 & 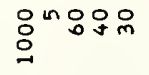 & 응용용요 & 응ㅇㅇㅇㅇㅇㅁㅇ & 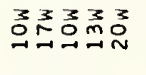 & 总羊总芯 & 용용응이 & 응응음음으 & 궁응ㅇㅁㅇㅇㅁㅇㅁㅇ & 응음에 \\
\hline 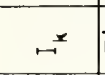 & $: \frac{d i n}{a n}$ & $\stackrel{N}{N} \mathbb{N}^{-1-}$ & 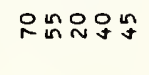 & 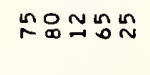 & 웃어요 m n & 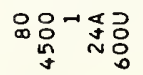 & 암용ㅇㅁ운 & 임임 & $\underset{N}{\stackrel{D}{N}} \underset{\sim}{n} \underset{n}{n}$ & 姵肙品 N \\
\hline 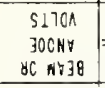 & 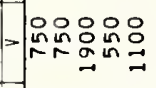 & 임윰욤요 & 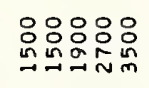 & 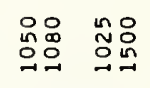 & 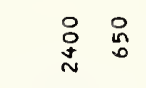 & 品芯品兰品 & 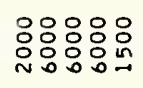 & 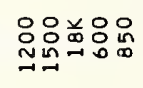 & 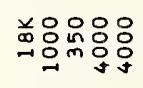 & 잉용요 \\
\hline$t^{-}$ & 데 & 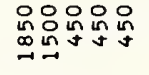 & & 응용요용 & 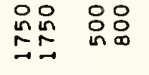 & 品 & & $\begin{array}{l}8 \\
: \\
0\end{array}$ & 孚 & 品 \\
\hline$\omega^{\leftarrow}$ & $\Rightarrow$ & ח: & & 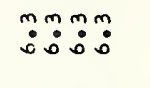 & $\ddot{m}: \stackrel{m}{0}: \stackrel{m}{0}$ & $\ddot{:}$ & & $\stackrel{\circ}{0}$ & $\stackrel{n}{:}:$ & $\stackrel{3}{0}$ \\
\hline $\mathrm{N} 011 \mathrm{ty} 3 \mathrm{3O}$ & บบบบบ & טบบบ & טレンט & 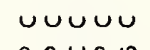 & บบบบบ & $a a \cup a u$ & 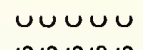 & บบบบบ & บบบบบ & 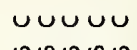 \\
\hline$\forall \cup N$ & 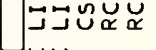 & บํษำ & 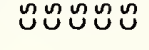 & 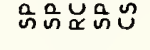 & 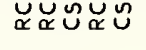 & ルேさコル゙ & 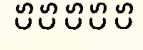 & コังேัง & 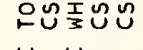 & 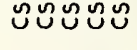 \\
\hline 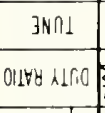 & 宸 & & & $\begin{array}{ll}x & x \\
\mathbf{u} & x\end{array}$ & & $\begin{array}{l}x \\
4 \\
\infty\end{array}$ & & $\stackrel{\breve{w}}{\Sigma}$ & $\stackrel{\breve{~}}{\Sigma}$ & \\
\hline 立 & 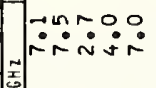 & 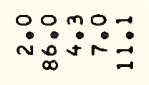 & 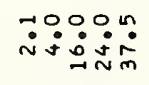 & 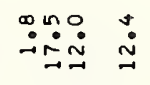 & 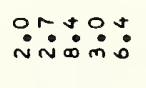 & 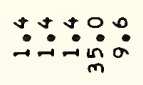 & 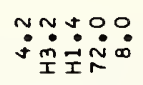 & $\dot{\sim} \because \because 000$ & 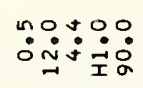 & 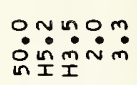 \\
\hline \begin{tabular}{|l|l|}
$\underline{u}$ & $z$ \\
$z$ & $\frac{z}{z}$ \\
\end{tabular} & 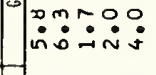 & 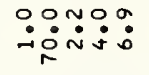 & 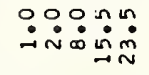 & mů: & 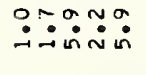 & 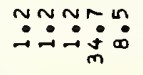 & 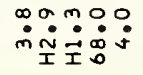 & $\ddot{\because} \dot{\sim} \dot{*} \dot{\sim}$ & $\because \because \because \because \because: 0$ & $\begin{array}{l}0 \\
\dot{m} \dot{m} \dot{m} \dot{m}\end{array}$ \\
\hline ONIX & 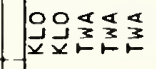 & 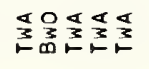 & 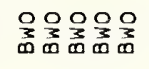 & 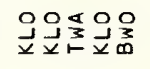 & 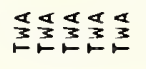 & $\begin{array}{l}0 \\
3 \\
1 \\
1\end{array}$ & 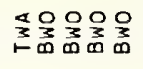 & 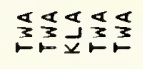 & 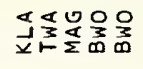 & 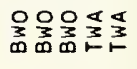 \\
\hline 708wis & & " " & & & & & & & & \\
\hline 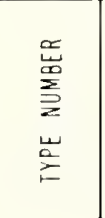 & 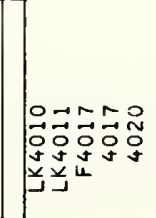 & 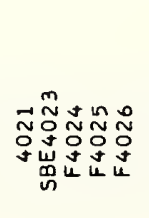 & 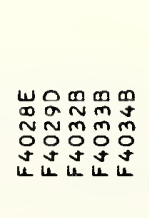 & 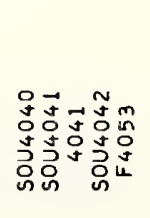 & 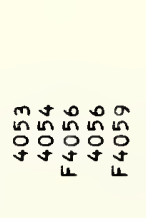 & 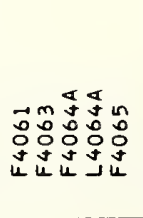 & 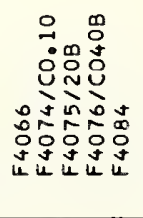 & 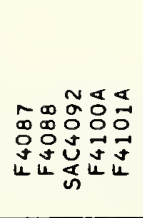 & 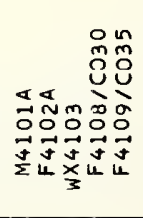 & 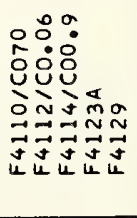 \\
\hline
\end{tabular}




\begin{tabular}{|c|c|c|c|c|c|c|c|c|c|c|}
\hline 9א17dnos & 웅 & OO & QYO & 욱 & $\stackrel{5}{3}$ & $\frac{0}{3}$ & पO & ত़ & OOYOY & ONO \\
\hline delaro & $\vec{\sim} \quad \vec{\sim}$ & & & & & $\vec{x}$ & $\vec{a}$ & $\vec{x}$ & $\vec{x} \vec{x}$ & $\vec{\alpha} \vec{\alpha} \vec{x}$ \\
\hline ग11311 & $\frac{1}{0} \sum_{n=1}$ & $\Sigma$ & $\sum \sum \sum \sum$ & $\sum \sum$ & $\Sigma$ & & $\sum \Sigma \Sigma \Sigma$ & $\Sigma$ & $\sum \sum \Sigma$ & $\sum \Sigma$ \\
\hline H10 MONY8 & $\stackrel{n}{\sim} \infty$ & & & $\stackrel{\ln }{q}$ & 요 & 웃욤유용 & $\stackrel{n}{\sigma}$ & 욤 & ñ & 았용요 \\
\hline 9M|17ก & 퐆 & & & $\simeq$ & & & 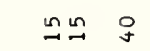 & $\stackrel{n}{\rightarrow}$ & $\simeq O^{\circ}$ & $\stackrel{m}{\rightarrow}$ \\
\hline 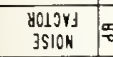 & 맘용 용요 & & & & & & & & & \\
\hline Nivg & 圆痛 & $\stackrel{m}{\sim}$ & & 요요 & 용 & 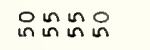 & & & & \\
\hline $\begin{array}{l}51701 \\
\times 173 H \\
\end{array}$ & $\rightarrow$ 品 & & & & & & & & & \\
\hline $\begin{array}{c}51701 \\
7081000\end{array}$ & & $\stackrel{\text { : }}{2}$ & & & & & & & & \\
\hline 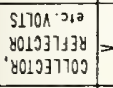 & 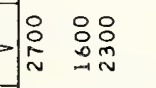 & & & & & 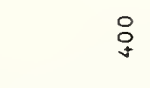 & 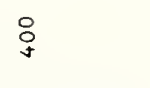 & & 음 & 음웃 \\
\hline$a^{\circ}$ & 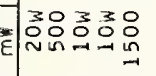 & 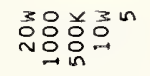 & 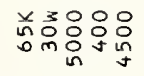 & 음음웜 & 뭉ㅇㅁ용ㅁㅇㅁㅇ & 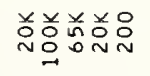 & 윰ㅇㅇㅇㅇㅁㅇㅛ & 吅的点总总 & 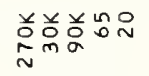 & 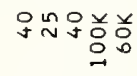 \\
\hline$\because$ & E & 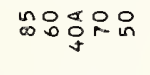 & 조N웅요요 & 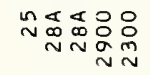 & 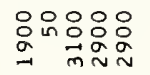 & 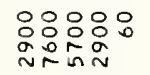 & 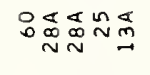 & 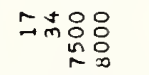 & 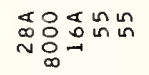 & 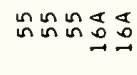 \\
\hline 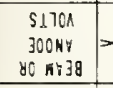 & 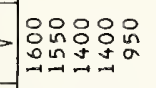 & 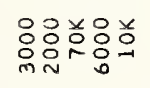 & 永品品品总导 & 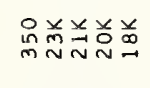 & 芯总兰兑兑 & 总前总总虽 & 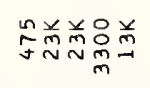 & 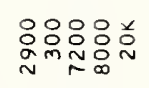 & 总总杀品号品 & 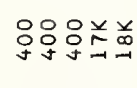 \\
\hline 5 & 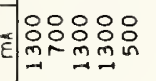 & 品 & $\stackrel{\circ}{\circ}$ & 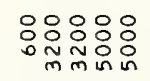 & $\begin{array}{l}\circ \\
\therefore\end{array}$ & 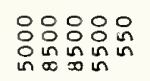 & 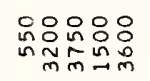 & 윽 & 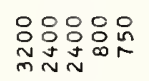 & 응요 \\
\hline$\omega^{\leftarrow}$ & 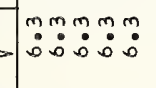 & $\dot{m}$ & $\dot{0} \quad \dot{0}$ & 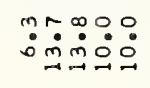 & 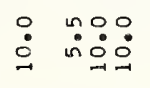 & : & 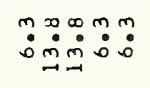 & 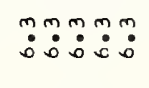 & ஸ் & 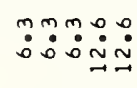 \\
\hline N011YYZdO & yuuv & Uu & a uuvu & vacuu & vuauv & vaauv & vacua & vuaaa & $a a a u v$ & vuva \\
\hline$\forall x$ & 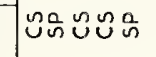 & 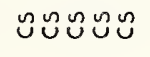 & コクタクコ & 辛ココ的的 & 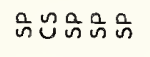 & की 疑的的 & 斿コ的 & 絡ココ & コココココ & コココココ \\
\hline \begin{tabular}{|c|c|}
$j \mathrm{~N} \cap 1$ \\
$0118 \mathrm{y}$ 스
\end{tabular} & $\begin{array}{ll}x & x \\
4 & 4\end{array}$ & & $\begin{array}{ll}x & \breve{w} \\
u & \stackrel{u}{\Sigma} \\
0 & \end{array}$ & 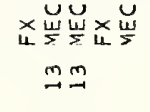 & 肴 & × $\quad \times \frac{u}{\Sigma}$ & 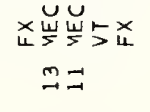 & 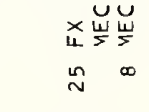 & 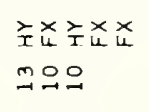 & 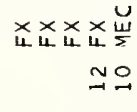 \\
\hline 产 & 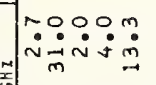 & 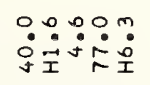 & 品踪品: & $\ddot{j} \ddot{0}: \stackrel{0}{0}$ & : & $\stackrel{\text { n }}{\check{7}}$ & 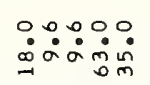 & 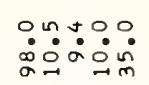 & 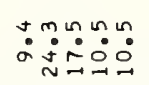 & 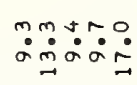 \\
\hline 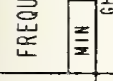 & 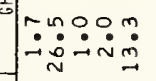 & 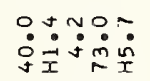 & 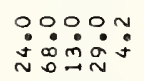 & 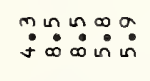 & 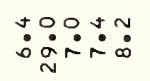 & 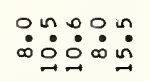 & 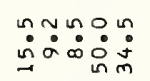 & 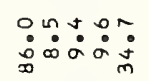 & 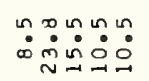 & 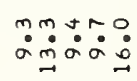 \\
\hline ONIX & 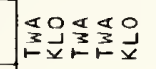 & 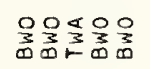 & 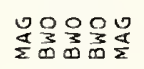 & 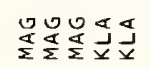 & 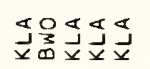 & 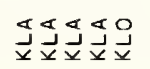 & 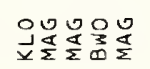 & 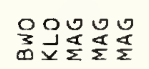 & 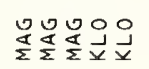 & 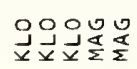 \\
\hline $70 \mathrm{gw} / \mathrm{S}^{-}$ & & & & $"$ & & & " " " & & & \\
\hline 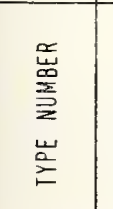 & 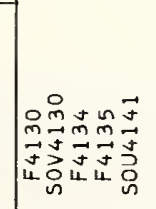 & 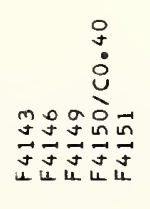 & 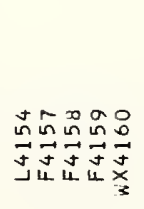 & 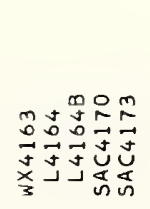 & 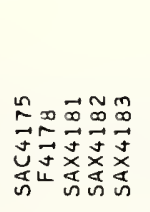 & 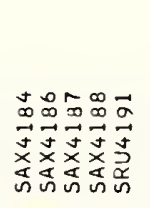 & 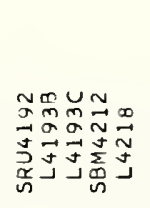 & 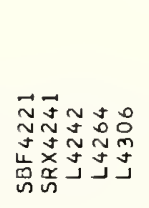 & 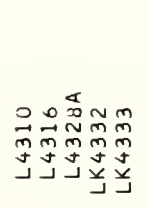 & 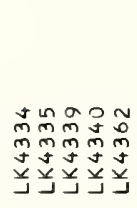 \\
\hline
\end{tabular}




\begin{tabular}{|c|c|c|c|c|c|c|c|c|c|c|}
\hline 9xitdnOS & $\begin{array}{|ll|}0 & 0 \\
3 & 0\end{array}$ & 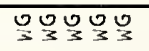 & पुणु & पु & पू & पू & 38 & 8 & তั & \begin{tabular}{ll}
0 \\
\hdashline
\end{tabular} \\
\hline Rliays & & $\vec{\alpha} \vec{\alpha} \vec{\alpha}$ & $\vec{\alpha} \widetilde{\alpha} \vec{\alpha} \vec{\alpha}$ & $\vec{\alpha} \vec{\alpha} \vec{\alpha} \vec{\alpha}$ & $\vec{\alpha} \ddot{\alpha}$ & $\vec{\alpha} \vec{\alpha} \vec{\alpha} \vec{\alpha}$ & $\ddot{\alpha}$ & $\vec{\alpha} \boldsymbol{\alpha} \widetilde{\alpha} \underset{v}{ }$ & $\vec{\sigma} \vec{\sigma} \quad \vec{\sigma} \vec{\alpha}$ & $\vec{\alpha} \vec{\alpha}$ \\
\hline 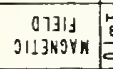 & $\underline{E} \leq \sum \sum \sum \Sigma$ & $\sum \Sigma$ & & $\Sigma$ & $\Sigma \Sigma_{-1} \sum_{-1}$ & $\sum$ & $\sum \sum \sum$ & in & 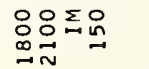 & $\Sigma$ \\
\hline HOOMONYO & & 一曲只品品 & 용요욤요 & 品品㶽 in & n & 윰요요 & 요 & ng용요 & $\stackrel{\circ}{\circ}$ & 웅 \\
\hline 9kורדת & : & 온 & & $\simeq$ & 00 & & $\simeq$ & & & $m$ \\
\hline $\begin{array}{l}y 013 y+1 \\
3510 k\end{array}$ & & & & & & & & & & \\
\hline 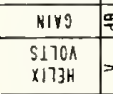 & & & $:$ & & in & & & in & 물용 & f \\
\hline $\begin{array}{r}51701 \\
7081003\end{array}$ & & & & & & & & $\stackrel{i}{\sim}$ & 웅 品 & \\
\hline 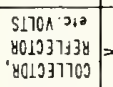 & & 品: & 유ำ몸 & 黾昌品。 & $\stackrel{\circ}{-}$ & 웄웄움움 & 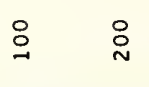 & 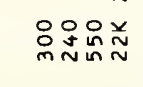 & 弟 总怘 & 요음 \\
\hline$a^{\circ}=$ & Elo & 着秥品品品 & 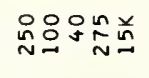 & 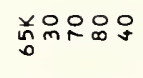 & 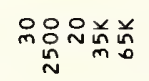 & 웅웅용ㅇㅇㅇㅇㅇ & 웃욧용요 & 응응용요 & 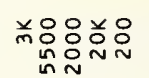 & 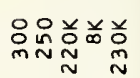 \\
\hline$\rightarrow$ & Ê & 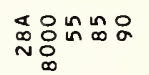 & 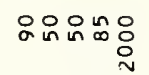 & 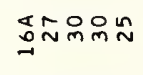 & 욜nᅭm & 品尔等肙总 & $\stackrel{\infty}{\sim} \underset{n}{\mathbb{n}} \quad \stackrel{m}{\sim}$ & 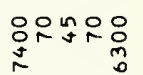 & 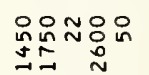 & 은 \\
\hline 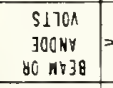 & - & 弟品윰윰우 & 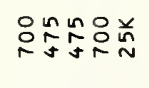 & 음임욤요 & 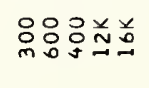 & 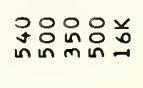 & 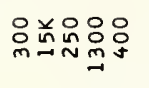 & 㒸品品品总 & 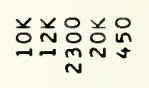 & 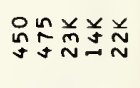 \\
\hline 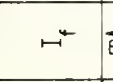 & El & 을윰요 & 옹윰유 & 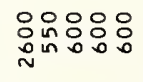 & 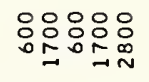 & 응응용요 & 음윰용 & 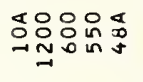 & 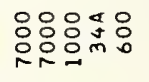 & 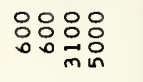 \\
\hline " & 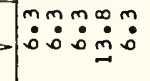 & $\ddot{m} \ddot{g}: \ddot{m}: m$ & 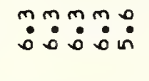 & 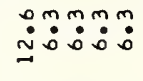 & 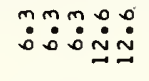 & 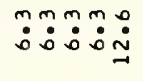 & : & :m: & 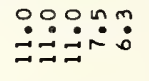 & 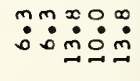 \\
\hline N011 14380 & a & a auU & هuט & טuטa & טua & םטuט & טuטu & טuטu & טuט & טUロ \\
\hline$y, W$ & ココ的コこ & $\Xi コ$ 的的品 & 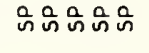 & 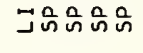 & 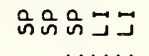 & 路路 & 距青的的 & 으응ํㅇำ & 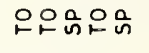 & 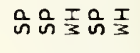 \\
\hline 3Nก1 & $\int_{u x}^{x}$ 岀x & 岀希 & & 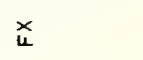 & 岀岀岀 & $\stackrel{\breve{~}}{\Sigma}$ & 状出 & $\stackrel{\breve{~}}{5}$ & 密 & 岀岀岁 \\
\hline Ollye $x 1 n 0$ & $\therefore \quad m 0$ & mo & $\simeq$ & & $\therefore$ & & $m$ & & & 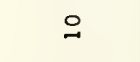 \\
\hline 离 & mingos & $\ddot{\circ}:$ & $\overrightarrow{\dot{m}}$ & 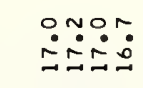 & 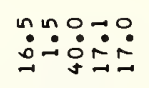 & $\stackrel{\circ}{\check{I}}$ & 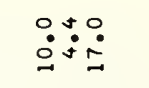 & $\dot{\dot{n}}$ & 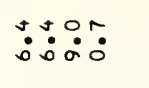 & 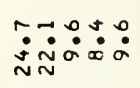 \\
\hline 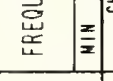 & 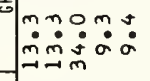 & 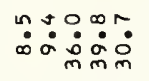 & 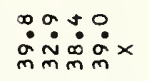 & 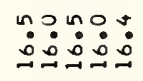 & $\ddot{n}$ & مُ & 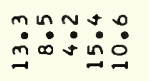 & 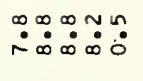 & 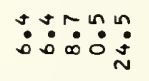 & 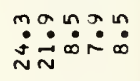 \\
\hline ONIX & 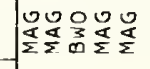 & 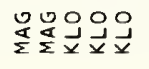 & 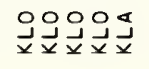 & 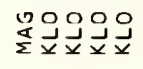 & 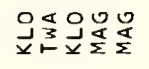 & 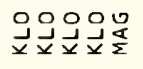 & 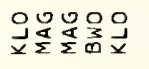 & 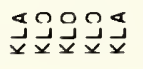 & 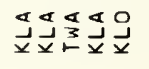 & 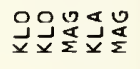 \\
\hline $708 \mathrm{~W} / \mathrm{S}$ & & & & " " & & 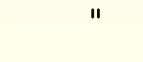 & & & & \\
\hline 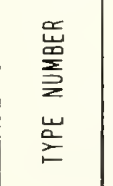 & 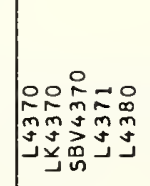 & 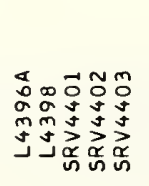 & 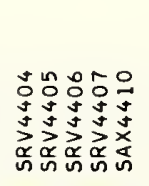 & 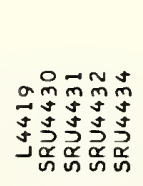 & 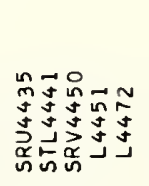 & 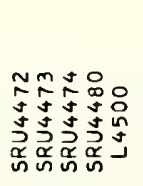 & 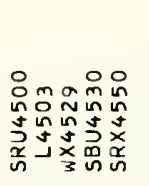 & 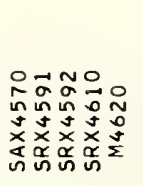 & 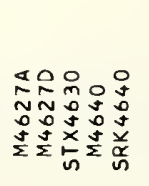 & 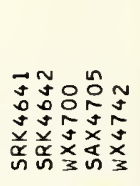 \\
\hline
\end{tabular}




\begin{tabular}{|c|c|c|c|c|c|c|c|c|c|c|}
\hline 9.17d ก03 & $\frac{0}{3}$ & WO & $\frac{5}{3}$ & 웡요 & 웅요 & 8눈요 & 8888 & O & 언 & 8000 \\
\hline 11114r3 & $\vec{\alpha} \quad \vec{\alpha} \vec{\alpha}$ & $\vec{\alpha} \boldsymbol{\alpha} \sim \vec{N}$ & $\ddot{\alpha}$ & & & $\vec{m}$ & & & & \\
\hline \begin{tabular}{c|c}
07311 \\
ग11399VK
\end{tabular} & $\Sigma$ & & $\Sigma$ & $\Sigma \Sigma \Sigma \Sigma$ & 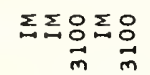 & $\Sigma \Sigma D_{n} \sum_{n=}$ & $\Sigma \Sigma \Sigma \Sigma$ & 品 & $\Sigma \Sigma$ & $\sum \Sigma \Sigma_{m} \Sigma_{n}$ \\
\hline HIOIMONYG & 용 & 음요 & 总识品品 & & -- & & & & & $n$ \\
\hline 9w17nd & & & & $m \cong$ & $\cong 00$ & $\stackrel{n}{\sim} n$ & & $\cong$ & & n \\
\hline $\begin{array}{l}8013 \% 1 \\
3510 \mathrm{~W}\end{array}$ & $\stackrel{a}{\sim}$ & & & & & & & & & $\stackrel{n}{m} m$ \\
\hline hivg & $\hat{m}$ & $\stackrel{n}{f}$ & 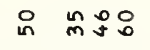 & $m \stackrel{m}{m} \stackrel{0}{n}$ & $\stackrel{\circ}{m}$ 只 & $\stackrel{m}{m} \stackrel{i}{m}$ & 일일임ㅇ & $\stackrel{D}{N}$ & ㅇmㅆ & 음요 \\
\hline $\begin{array}{l}51701 \\
\times 173 H \\
\end{array}$ & & & N & & & & 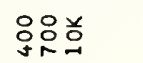 & N & 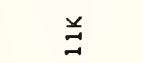 & 羊 \\
\hline $\begin{array}{c}51701 \\
7041103\end{array}=$ & & & $:$ & & o in & $\stackrel{\circ}{\circ}$ & $\stackrel{\circ}{n}$ & & $\stackrel{0}{\circ}$ & oin in \\
\hline 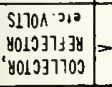 & 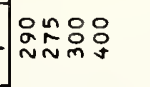 & 임 & $\stackrel{8}{\circ}$ & & & & & : & & \\
\hline $0^{\circ}$ & 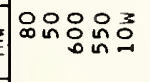 & 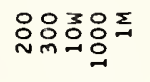 & 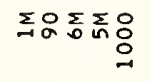 & 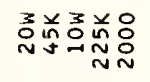 & 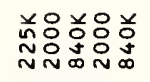 & 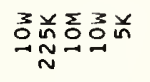 & 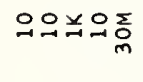 & 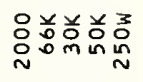 & 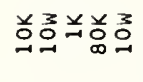 & 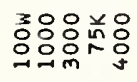 \\
\hline$\rightarrow$ & 국용ㅇㅇ & 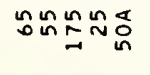 & 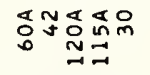 & 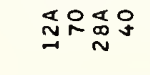 & 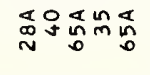 & 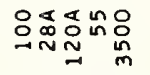 & mv总占 & 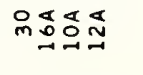 & 용ㅇㅇㅇㅇ & 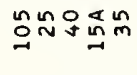 \\
\hline 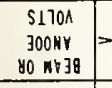 & 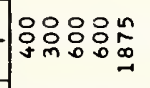 & 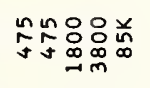 & 总品总会品 & 弟品㒸品 & 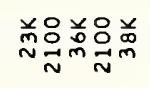 & 总前总品品品 & 郘品总品兑 & 虽并总羊 & 吕 兰并品 & 路品前品 \\
\hline$F^{+}$ & 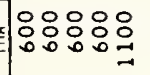 & 웅ㅇㅇㅇㅇㅇ & $\stackrel{\circ}{\stackrel{\circ}{0}}$ & 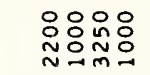 & 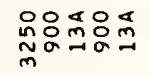 & 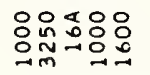 & 品品品虽 & 웅요 & $\begin{array}{l}\circ \\
\circ \\
\circ\end{array}$ & 응요욤요 \\
\hline ш" & $m m m m$ & $\ddot{m}: m m$ & $\stackrel{n}{0} \quad \stackrel{m}{0}$ & 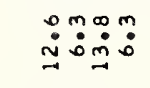 & 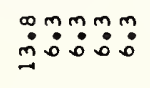 & 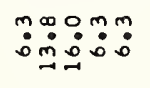 & $\dot{m}: \dot{m}^{m}:{ }^{m}: \dot{0}$ & 象: & 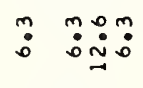 & $\ddot{0}: \ddot{m}: \stackrel{m}{0}:$ \\
\hline NOI1YGJ 30 & טuטuט & 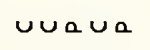 & $a \cup a a u$ & vauav & auaua & vacua & vuava & Ua.au & $a \cup a a v$ & vuau \\
\hline $8+n$ & 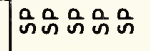 & 的品的品品 & 蛙的品 & コ岀コ岕さ & س山ّ山岀コ岀 & コ岀岕ココ & コココココ & コココビ & コココゴ & エゴ゙ّ \\
\hline \begin{tabular}{l|l}
$3 \times \cap 1$ \\
\end{tabular} & & xxx & 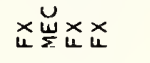 & $\underset{u}{x} \quad \underset{u}{x}$ & $\begin{array}{lll}\underset{u}{x} & \underset{u}{x} & \underset{u}{x}\end{array}$ & xü $\underset{u}{u}$ & 嵌 & 密岕 & 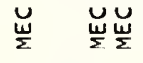 & 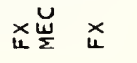 \\
\hline Oivy Anno & & $\stackrel{m}{r}$ & 오 으오 & $\therefore \quad$ & $\cong \cong \cong$ & $\therefore \quad \therefore$ & $\stackrel{\circ}{\circ}$ & 어융으 & 웅 :웅 & $\simeq$ \\
\hline 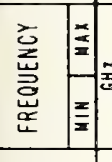 & 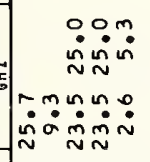 & 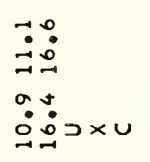 & 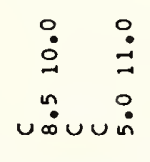 & 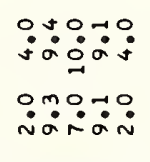 & 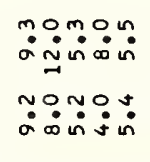 & 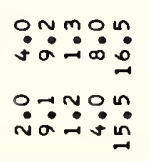 & 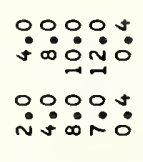 & 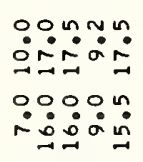 & 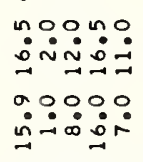 & 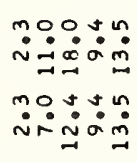 \\
\hline ONIX & | & 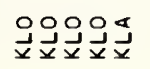 & 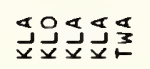 & 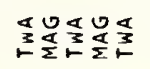 & 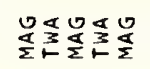 & 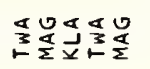 & 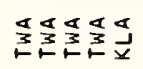 & 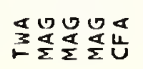 & 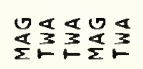 & 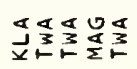 \\
\hline $708 \mathrm{WSS}$ & & & & $"$ & 11 & & " & & " " & \\
\hline 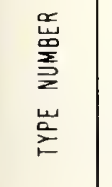 & 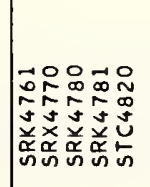 & 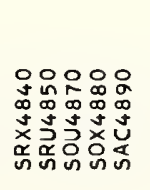 & 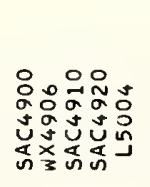 & 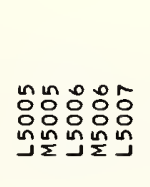 & 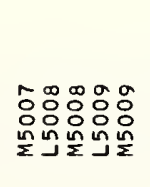 & 次ㅇํำ & 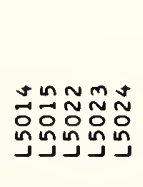 & 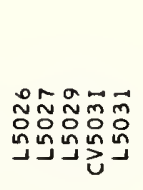 & 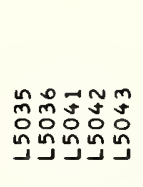 & 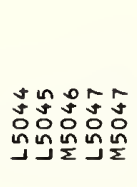 \\
\hline
\end{tabular}




\begin{tabular}{|c|c|c|c|c|c|c|c|c|c|c|}
\hline 9M17dnos & 농요 & ৪৪৪৪ & \ত & 엉원 & 옹ㅇㅇㅇ & ๖יㅇㅇㅛ & 웅ㅇㄴ논 & 옹요 & & SOY \\
\hline LIIAVS & & & $\vec{\alpha}$ & & & & & $\bar{\alpha}$ & & \\
\hline 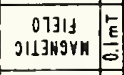 & $\sum \sum$ & $\Sigma \Sigma \Sigma$ & $\Sigma \quad \Sigma \Sigma$ & $\Sigma \Sigma \Sigma \Sigma \Sigma$ & $\Sigma \Sigma \Sigma \Sigma \Sigma$ & $\Sigma \Sigma \Sigma \Sigma \Sigma$ & 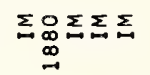 & $\Sigma \Sigma \Sigma \Sigma$ & $\Sigma \Sigma \Sigma \Sigma \Sigma$ & $\Sigma \Sigma \Sigma \Sigma \Sigma$ \\
\hline H.01monv $\tilde{\text { I }}$ & & & ᄋ & 并 & & & & : & & \\
\hline 9н177nd & $\infty \infty$ & & $\cong$ & & & & $r$ & & & \\
\hline \begin{tabular}{c|c} 
Holjyy \\
3Slon
\end{tabular} & 욤욤요 & 용 & 욕 & $\cong$ 요 & $\cong$ กニュュ & 오ำ & $\cong \stackrel{\sim}{\sim} \stackrel{\sim}{\sim}$ & 동요 & & 웅요 $\underset{\sim}{\infty} \stackrel{m}{\sim}$ \\
\hline \begin{tabular}{l|l} 
HIY & : \\
\end{tabular} & 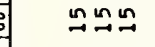 & モ용용요 & 乐星品 & 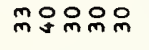 & 윰윰윰요 & 융요윰요 & 융요요 & 웅유소 & & 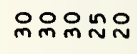 \\
\hline $\begin{array}{l}51701 \\
\times 173 \mathrm{H} \\
\end{array}$ & 응음 & $\underline{\sim}$ & & 음응 & 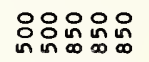 & 品员品兰兰 & $\ddot{\Rightarrow} \underset{\sim}{\longrightarrow} \underset{\sim}{ }$ & ㅡㅇㅛ & 决资品前 & 总品并并并 \\
\hline $\begin{array}{c}51701 \\
7081 \mathrm{NOS}\end{array}$ & & 요ํnn & : & 융ํ요요 & 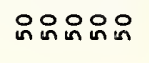 & 으으요요 & 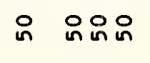 & 부요요요 & & 음요요으요 \\
\hline 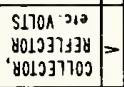 & & & 嘼 & & & & & 욤 & & \\
\hline \begin{tabular}{l|l}
$a^{\circ}$ & $E$
\end{tabular} & $\because \approx 00 n$ 品 & n:ㅇㅇㅇ융ㅁㅇ & 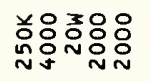 & nะn & 옹ㅇㅁ응ㅇ & 픙으으으 & 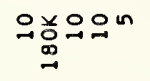 & $\stackrel{ }{N}^{\text {in }} \operatorname{lin}^{n}$ & 오욤옹 & $\ln u \sin s$ in \\
\hline 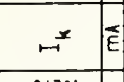 & $\int_{0}^{2}$ & oinn & 윰요 웅 & N & NNNNN & $\begin{array}{l}\Xi_{m} N N N N \\
\vec{n}\end{array}$ & $\sim \underset{N}{N} N N N$ & ดัN & ㅇN유슈 & $\sim \sim N \sim N$ \\
\hline $\begin{array}{r}5170 \mathrm{~A} \\
300 \mathrm{HY} \\
80 \mathrm{HYBO}\end{array}$ & 最盖品品品 & 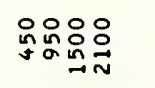 & 弚嗢 品怘 & 品兰品守品 & 영응용 & 응ㅇㅇㅇㅇㅇㅇ & 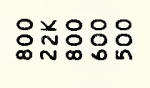 & 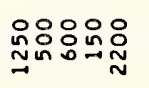 & 옴유옹 & 웅요욤ำ \\
\hline$\mapsto \vec{E}$ & 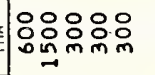 & 잉ㅇㅇㅇㅇㅛ & 응 융요 & 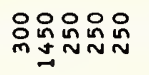 & 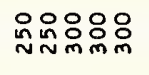 & 임윰요 & 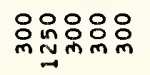 & 욤욤윰요 & 임윰욤요 & 员品品品怘 \\
\hline$w^{ \pm}$ & 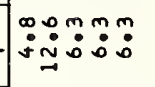 & 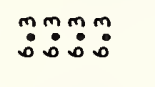 & $\ddot{n}: \stackrel{m}{0}: \stackrel{m}{0}$ & mm: & 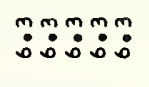 & mmm & 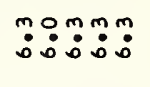 & 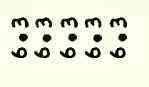 & 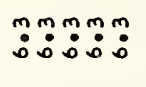 & 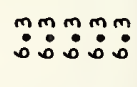 \\
\hline NO118Y3dO & anuuv & טuטu & auvuv & vauuv & uUưu & a uuvu & vauuu & ưư & vưư & טuטu \\
\hline HJW & تَّưu & エコさココ & コ命コココ & 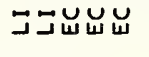 & 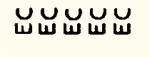 & 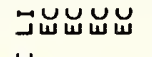 & سِّنّ & 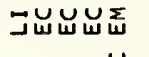 & 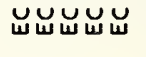 & نيّنّ \\
\hline JNก1 & $\mid \underline{\breve{w}}$ & & 岀崖 $\quad x$ & $x_{k}^{x}$ & & 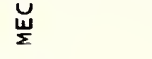 & x & $\stackrel{\breve{w}}{\Sigma}$ & & \\
\hline OIIty AInO & ino & & $\cong$ & 욤 & & 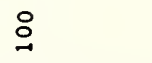 & $\circ$ & & & \\
\hline 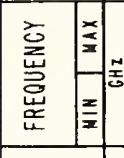 & 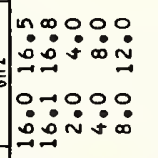 & 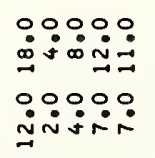 & 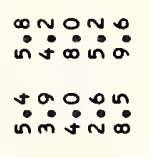 & 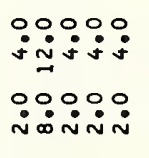 & 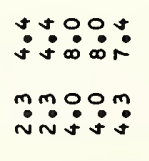 & 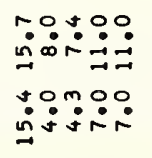 & 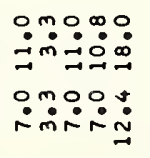 & 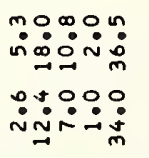 & 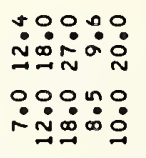 & 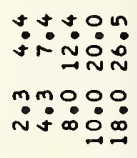 \\
\hline ONIX & 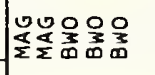 & 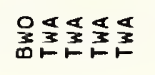 & 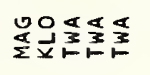 & 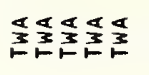 & 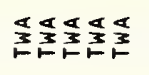 & 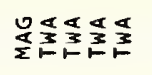 & 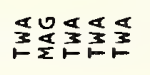 & 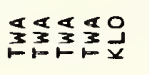 & 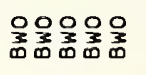 & 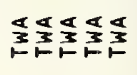 \\
\hline 708W/S & & " " " & " " " & & & $"$ & & & " " " & \\
\hline 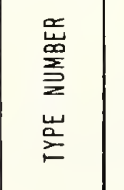 & 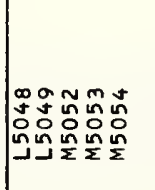 & 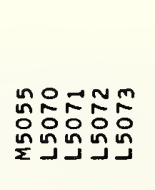 & 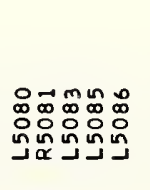 & 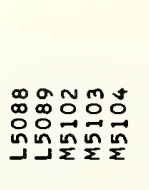 & 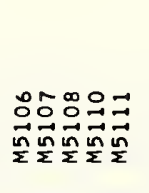 & 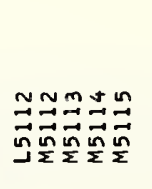 & 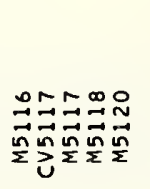 & 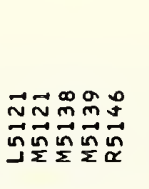 & 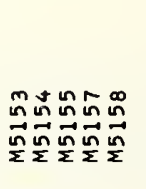 & 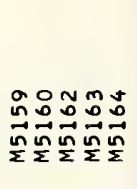 \\
\hline
\end{tabular}




\begin{tabular}{|c|c|c|c|c|c|c|c|c|c|c|}
\hline 9พ1/1ก00 & $\begin{array}{ll}0 & 0 \\
3 & 0\end{array}$ & $\mho_{3}$ & 엉ㅇㅇㅇ & 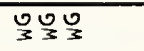 & ㅇํㄴ운 & OOOYOO & 언웡 & 언 옹 & 우을 & 워온 \\
\hline 11/AYS & & $\underset{\alpha}{\omega}$ & $\underset{w}{\times} \underset{\sim}{w}$ & $\underset{\boldsymbol{\alpha}}{\breve{\varpi}}$ & $\vec{\alpha} \vec{\alpha} \quad \vec{\alpha} \vec{\alpha}$ & & $\vec{\alpha}$ & $\underset{\alpha \vec{\alpha} \vec{\alpha}}{w}$ & w $\vec{\alpha}$ & \\
\hline \begin{tabular}{|l|l}
01311 \\
J11399vM
\end{tabular} & $\sum \sum \sum \sum$ & $\Sigma$ & 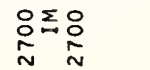 & $\Sigma \Sigma \Sigma$ & $\Sigma$ & $\Sigma \Sigma \Sigma \Sigma \Sigma$ & $\Sigma \Sigma \Sigma$ & & $\Sigma \Sigma \Sigma$ & $\Sigma \Sigma \Sigma$ \\
\hline HLOIMONYP & $\approx$ & 앙요 음 & $\infty$ & $\cong \infty \infty$ & in & & $\stackrel{9}{q}$ & $\stackrel{n}{s}$ & $\cong \cong$ & \\
\hline 9w17nd & & n & r & $\stackrel{\operatorname{nn} n}{\sim} \operatorname{n} 0$ & $\cong$ & $\stackrel{\sim}{\sim} 0$ & & & $q^{5}$ & \\
\hline 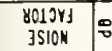 & $\stackrel{n}{N} \stackrel{\circ}{N}$ & & & & & & & & & \\
\hline Nirg & 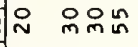 & $\ln _{n}^{n} \operatorname{sn}^{n}$ & & & & & & & & \\
\hline $\begin{array}{l}8170 N \\
\times 173 \mathrm{H}\end{array}$ & 㒸芯道 & & & & & & & & & \\
\hline $\begin{array}{c}51701 \\
70811103\end{array}=$ & 요 웅ㅇ & & in & 品足 & & & $\stackrel{\circ}{\check{I}}$ & :̊ & & in \\
\hline 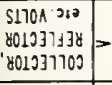 & & 吕 & $\stackrel{\sim}{\sim} \underset{0}{n}$ & 움 & 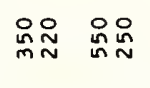 & & $\stackrel{\circ}{\circ}$ & 品昆品 & $\stackrel{\circ}{\stackrel{\circ}{~}}$ & \\
\hline$\Omega^{\circ}$ & -OO & 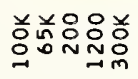 & 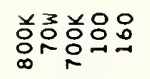 & 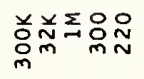 & 虽㕵总品品 & 总兽前首总 & 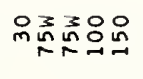 & 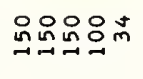 & 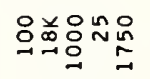 & 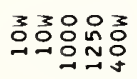 \\
\hline 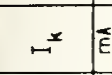 & NONO : & 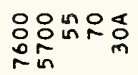 & 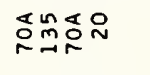 & 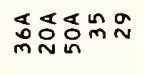 & 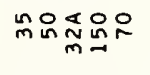 & : & 뜸유유요 & 웅ํำ & 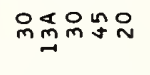 & 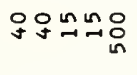 \\
\hline 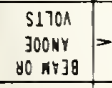 & 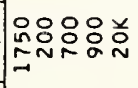 & 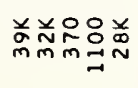 & 总品总怘怘 & 总盖总品品 & 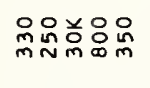 & 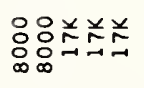 & 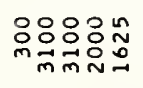 & 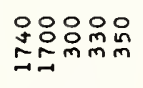 & 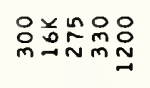 & 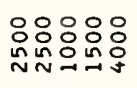 \\
\hline$F^{-} \bar{E}$ & 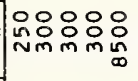 & 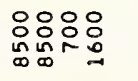 & 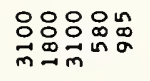 & 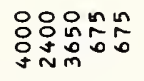 & 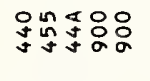 & 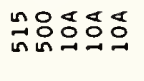 & 응융요 & 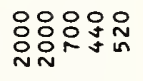 & 品预品品 & :유유 \\
\hline$\omega^{\top}$ & 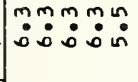 & 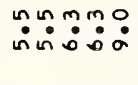 & 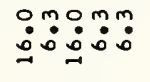 & 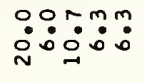 & 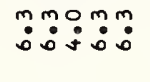 & $\begin{array}{l}M \\
\because \dot{N} \dot{N}\end{array}$ & 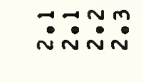 & mm:m: & $\ddot{m} \dot{m}: \ddot{m}: \dot{n}$ & 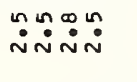 \\
\hline NOILYYJdO & |uบuvu & a auva & auauv & aavu & uvauv & $00 a 0 a$ & ưưu & טuบuv & anuu & uUu \\
\hline$y d n$ & 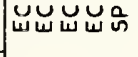 & ㅇํ욨 & $\stackrel{\mathbb{\alpha}}{\alpha} \underset{\alpha}{\alpha}$ & 岀コ岂ココ & 兰电站政 & પૅЧูড & 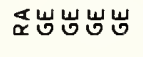 & U山心 $\sum_{w}$ & 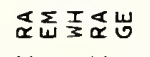 & 嵌山岕岕至 \\
\hline उNก1 & $x_{u}^{x}$ & 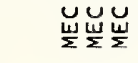 & 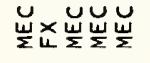 & 岀㚲岂 & 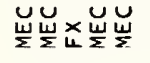 & 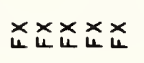 & エேレ5 & ケケ岂岂I & 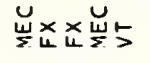 & らケらら岕 \\
\hline 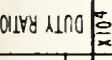 & & & $n$ in & $O 0 N$ & 욱 & $\stackrel{n}{N} \approx$ 어웡ㅇ & & & ง & \\
\hline 意 & 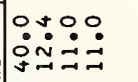 & $\stackrel{\infty}{\infty}: 0$ & $\dot{\sim} \dot{\sim} \dot{m}=\dot{N}$ & : & 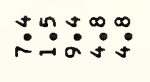 & 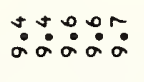 & 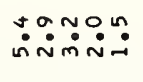 & 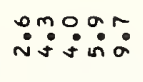 & 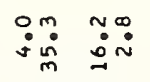 & 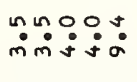 \\
\hline 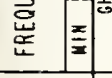 & 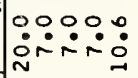 & 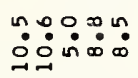 & ஸ்:0: & 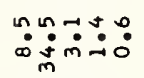 & ํำ: & 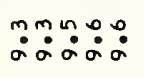 & 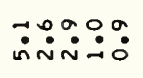 & 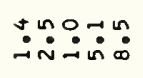 & 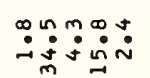 & 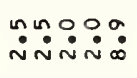 \\
\hline ONIX & 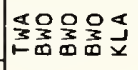 & 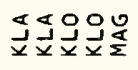 & 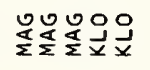 & 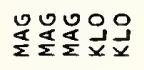 & 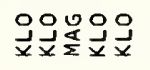 & 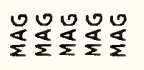 & 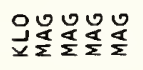 & 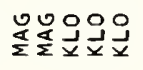 & 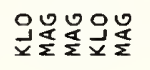 & 这造造造造 \\
\hline Tognks & " " & & $* \quad *$ & & & & & * & & \\
\hline 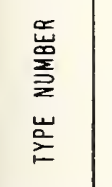 & 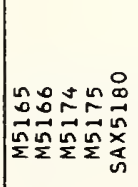 & 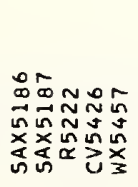 & 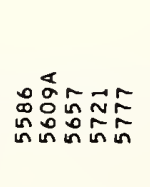 & 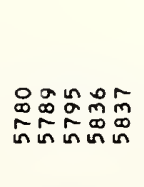 & 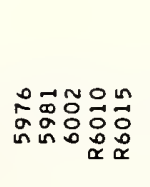 & 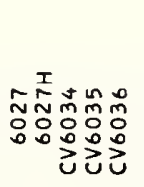 & 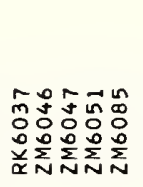 & 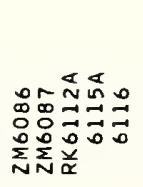 & 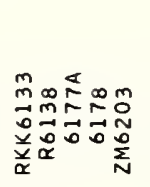 & 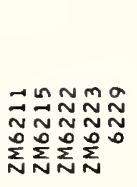 \\
\hline
\end{tabular}




\begin{tabular}{|c|c|c|c|c|c|c|c|c|c|c|}
\hline 9 911 d dח & 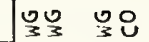 & 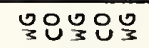 & 엉엉요 & 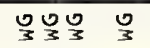 & ㅇำำ & 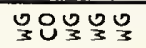 & 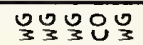 & 무꿍ㅇ & 옹ํำ & ช옹ำ \\
\hline R1AหY & 梮 & $\vec{m} \vec{m} \vec{m}$ & $\vec{m} \vec{m}$ & $\bar{\alpha} \bar{\alpha} \quad \vec{\alpha}$ & $\vec{\alpha} \vec{\alpha} \vec{\alpha} \vec{\alpha}$ & $\bar{\alpha}$ & & $\vec{\alpha} \vec{\alpha}$ & $\vec{\alpha}$ & \\
\hline ग113114 & $\sum{ }_{i n}$ & 윰욤요 & 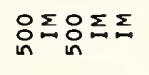 & $\Sigma$ & $\Sigma$ & $\Sigma \Sigma \Sigma$ & $\sum \Sigma \Sigma \Sigma$ & $\Sigma \Sigma \Sigma$ & 요욤유 & $\underset{\text { ñ욜 }}{ } \Sigma$ \\
\hline HLOIMOHYO & $\sim 0$ & $\circ \quad 0$ & 00 & 앙웡유웅 & $g^{n} g_{0} \sin$ & $\sim$ & $N \quad N$ & $\stackrel{n}{n} \underset{n}{n}$ & [nกNำ & in $\quad$ in \\
\hline $9: 17$ 9 & 요 & & & $\stackrel{n}{\sim}$ & $\cong$ & $\stackrel{n}{\rightarrow}$ & $m$ nn음으 & 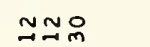 & $\cong$ & \\
\hline $\begin{array}{l}019313 \\
3510 \mathrm{H} \\
\end{array}$ & & & & & & & & & & ro \\
\hline NIYg & $\hat{n}$ & $\hat{\sim} \hat{\sim}$ & $\hat{N} \tilde{N}$ & & & & & & 윰욤 & 웅 \\
\hline $\begin{array}{l}S 1700 \\
x \rightarrow 17 y H\end{array}$ & & & & & & & & & & 웅 \\
\hline $\begin{array}{c}\text { S1701 } \\
\text { 1081100 }\end{array}=$ & & & & 응 & $\stackrel{\text { n }}{0}$ & $\stackrel{n}{\sim}$ & & & 윰요 & i \\
\hline 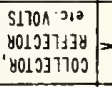 & $\begin{array}{l}n \\
\hat{m}\end{array}$ & & & 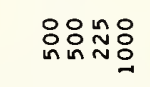 & 응 & $\stackrel{\circ}{\circ}$ & & $8 \stackrel{0}{\circ}$ & 응응영 & 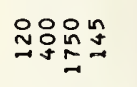 \\
\hline$a^{0}$ & $\mid \begin{array}{l}3 \\
0\end{array}$ & 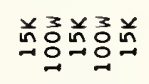 & 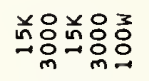 & 总呆守证只 & 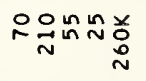 & 前品兑乲少 & 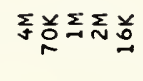 & 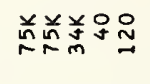 & 兑 & 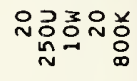 \\
\hline 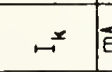 & 있 & 응윰임 & 임임윰 & $\underset{m}{\mathbb{N}} \times 0 \times \tilde{m}$ & ษ & 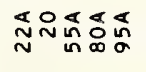 & 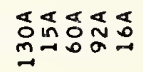 & 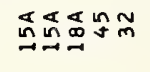 & 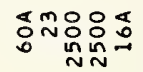 & 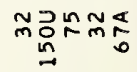 \\
\hline 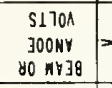 & 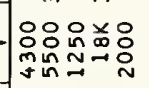 & 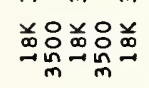 & 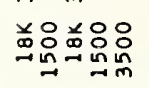 & 임윰윰요 & 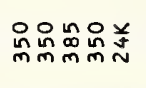 & 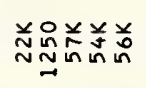 & 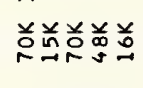 & 㒸㒸兑品品 & 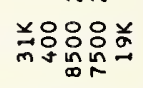 & 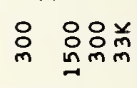 \\
\hline$\leftarrow$ & : & 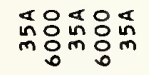 & 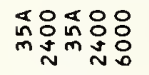 & 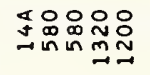 & 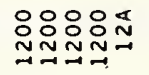 & 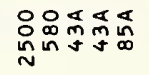 & 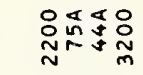 & 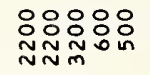 & 웅음음 & 융욤요 \\
\hline$\omega^{\leftarrow}$ & $\ddot{m} \dot{n} \dot{n} \tilde{n}$ & 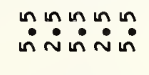 & 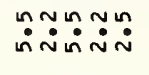 & 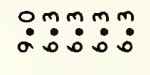 & 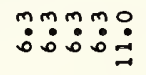 & 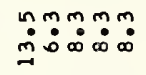 & 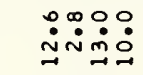 & 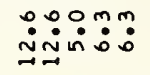 & 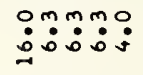 & mom: \\
\hline NOILFYjdO & aa auv & טuטu & טuטu & auvus & טưa & auaaa & aana & aacuu & auaaa & aטuט \\
\hline$\forall \pm W$ & 搱 嵌 & 岁岗岕岕 & 岁岕岕岕 & $\underset{\alpha \propto \alpha}{\alpha} \vec{\infty}>$ & $\stackrel{I}{3}>\mathbb{4}$ & $\underset{\alpha \propto \alpha}{\alpha} \underset{\alpha}{\alpha}$ & 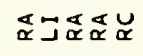 & $\underset{\propto ⿻}{\propto u}$ & 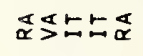 & 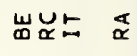 \\
\hline $3 \mathrm{~K} \cap 1$ & 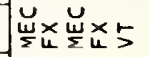 & メらメら & メ5メ5 & 岀岕山 & 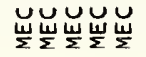 & 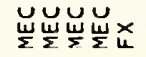 & 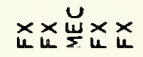 & 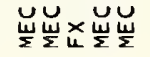 & 岕岕ら5x & 茲ら5エ \\
\hline 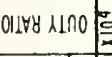 & 앙요 & & & $m$ & 2 & $\therefore$ 옹 & 음요 & $m m r$ & 으 으요음 & 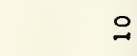 \\
\hline 意 & åa: & 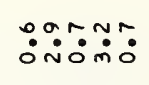 & $\ddot{0} \because \because \dot{m} \dot{m}$ & 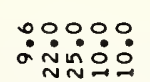 & : & 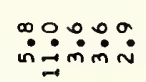 & 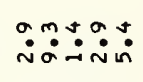 & & 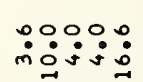 & 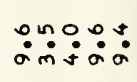 \\
\hline 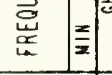 & 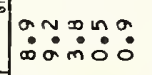 & 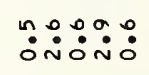 & 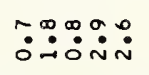 & 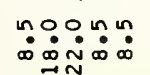 & 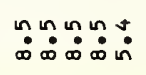 & 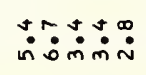 & $\begin{array}{l}\infty m \sim a \\
\dot{\sim} \\
\dot{n}\end{array}$ & ம் & ง & 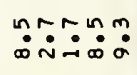 \\
\hline ONIX & 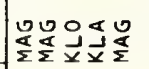 & 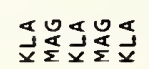 & 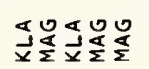 & لجُ & 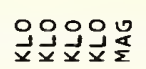 & 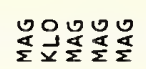 & 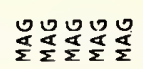 & 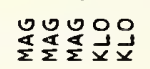 & 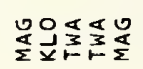 & 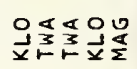 \\
\hline 70gNAS & " & " " & " $"$ & $* 11$ & * 1 & $*$ & $"$ & " & " & \\
\hline 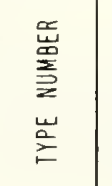 & 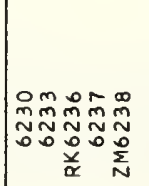 & 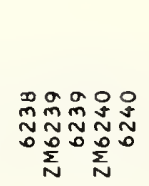 & 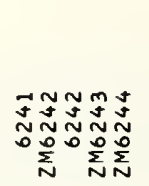 & 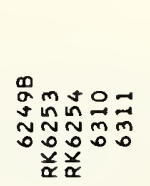 & 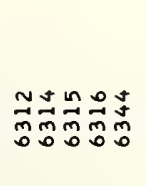 & 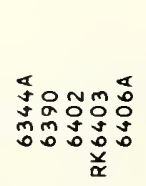 & 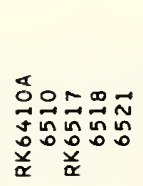 & 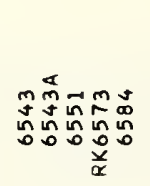 & 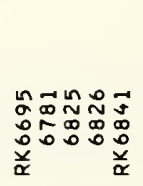 & 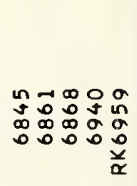 \\
\hline
\end{tabular}




\begin{tabular}{|c|c|c|c|c|c|c|c|c|c|c|}
\hline 9k17dnos & 원워눈 & 옹ㅇㅇㅇㅓ & 언언오 & ㅇㅀ ऽ엉 & 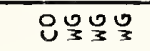 & 곤언언 & OU요 & 언연은 & 언옹요 & 온요 \\
\hline dilars & $\vec{\alpha}$ & $\underset{\propto \sim}{\vec{w}}$ & & & & 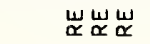 & 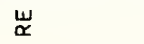 & & & \\
\hline $\begin{array}{c}01311 \\
\text { M11399YK }\end{array}$ & 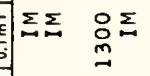 & $\Sigma \Sigma \Sigma$ & $\Sigma \Sigma \Sigma \Sigma \Sigma$ & $\sum \Sigma \Sigma \quad \stackrel{0}{\stackrel{N}{N}}$ & 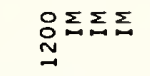 & $\Sigma \Sigma$ & $\sum \sum \sum \sum_{0}$ & $\Sigma \Sigma \Sigma \Sigma \Sigma$ & $\Sigma \Sigma \Sigma \Sigma$ & 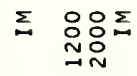 \\
\hline H101MONrg $=$ & a in & $\circ$ & m & & 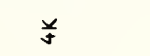 & $\circ m$ & & - & & \\
\hline $9 \times 177 n d$ & $\ln 0$ & $\simeq$ & 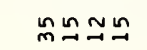 & $\infty 0$ & $\stackrel{\curvearrowleft}{\sim}$ & ナュ & & $\stackrel{n}{\sim}$ & $\checkmark$ & $\stackrel{m}{\rightarrow}$ \\
\hline $\begin{array}{l}\text { yolioys } \\
\text { 3Slow }\end{array}$ & & & & & & & $\stackrel{\circ}{m}$ & & & \\
\hline Nivg & 요 & & & ᄋ & 융요 & & m & & 음 & $\tilde{n}$ \\
\hline $\begin{array}{l}51701 \\
\times 1134 \\
\end{array}$ & & & & & 모 & & $\cong$ & & $\stackrel{N}{N}$ & \\
\hline $\begin{array}{c}51701 \\
1081 \% 03\end{array}$ & $\stackrel{\circ}{N}$ & 욤 & & 울 & $\therefore$ & 윰요 & ڤ̊ & & $:$ & \\
\hline 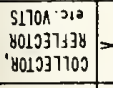 & 욤요 & 움 & & : & 임 & 암유 & $\stackrel{\circ}{\circ}$ & & : & $\stackrel{n}{{ }_{n}^{n}}$ \\
\hline $0^{\circ}$ & 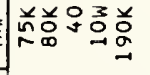 & 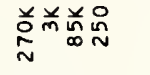 & 咨弟咨兑兑 & 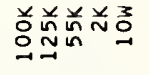 & 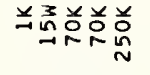 & 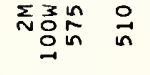 & 㭆 & 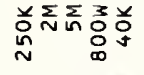 & 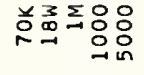 & 응으음솟 \\
\hline$\mapsto^{x}$ & 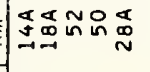 & 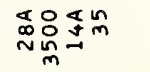 & 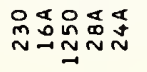 & 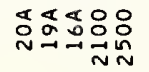 & 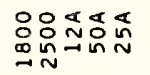 & 品品 & 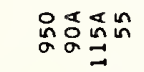 & 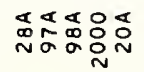 & 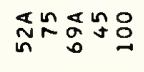 & 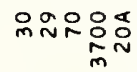 \\
\hline 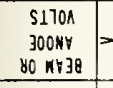 & 㒸㒸品品前 & 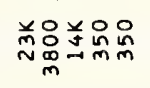 & 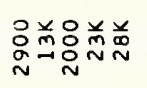 & 总㒸总品品品 & 品总恙羔 & 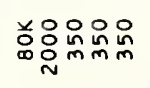 & 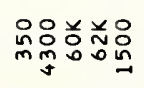 & 前并关品品前 & 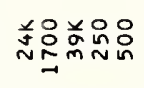 & 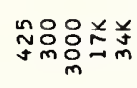 \\
\hline$\leftarrow$ & 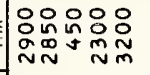 & 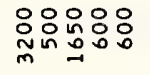 & 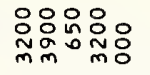 & 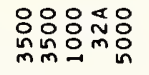 & 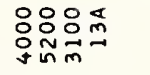 & 또융ㅇㅇㅇㅇㅇㅇ & 응융ㅇㅀㅇㅇㅛ & 总出嵒 & 积乐品品 & 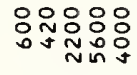 \\
\hline$\omega^{4}$ & m:mº́ & 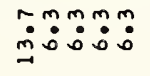 & m: & 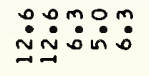 & mmo̊no & 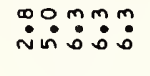 & 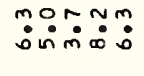 & 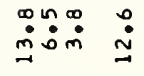 & 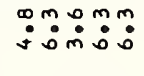 & $\ddot{m}: m: m:$ \\
\hline $\mathrm{H} 011 \mathrm{1} y \mathrm{y} \mathrm{dO}$ & aguva & a a aua & vaaa & agava & aaga & $a \subset \cup a u$ & $a a a a u$ & 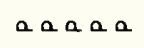 & auauu & บuบaa \\
\hline$\forall+N$ & 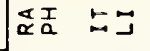 & I & $=$ & 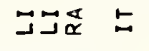 & ここコ๙ & تஃะ응 & 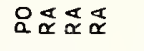 & 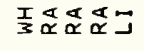 & $\underset{\alpha}{\mathbb{\alpha}} \underset{\alpha \propto \frac{T}{3}}{\mathbb{\alpha}}$ & 㚻タたヒコ \\
\hline JNח1 & 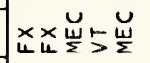 & 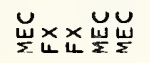 & 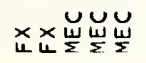 & 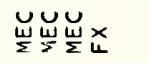 & $\times \underset{u}{x}$ & 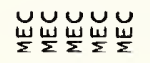 & 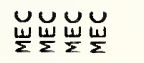 & 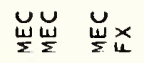 & 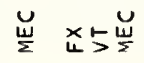 & 肴宏 岌 \\
\hline olty $\wedge$ \&nO & $\stackrel{\circ}{-} \quad m$ & $=ㅇ ㅇ \quad$ & $m \underset{N}{ }=$ & 응ㅇㅇ 응 & 음요은 & $\pm \stackrel{N}{\circ}$ & 잉요 & 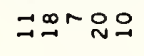 & $\approx a$ & in \\
\hline 䇋 & 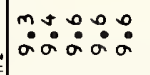 & 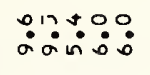 & 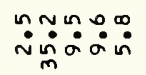 & 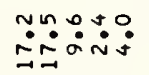 & & 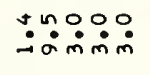 & 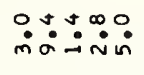 & $\therefore:$ : & 㒸: & $\begin{array}{l}m o 0 \\
\dot{y}\end{array}$ \\
\hline 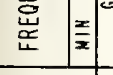 & テman & 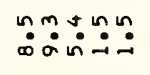 & 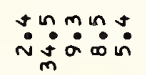 & $\begin{array}{l}\infty \\
\dot{n} \\
\end{array}$ & OO: & 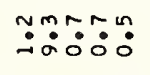 & 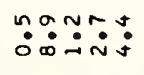 & 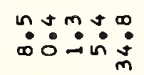 & 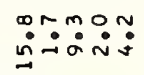 & 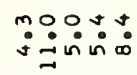 \\
\hline ONIX & 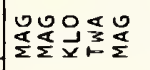 & 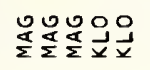 & 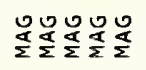 & 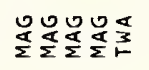 & 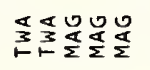 & 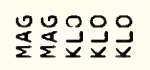 & 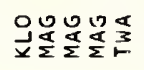 & 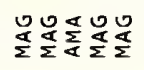 & 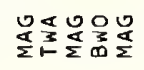 & 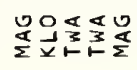 \\
\hline 108W/S & $11 \quad * \quad 1$ & & & $*$ & & & & & $"$ & " " \\
\hline 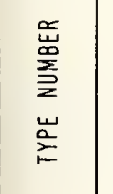 & 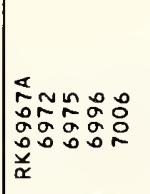 & 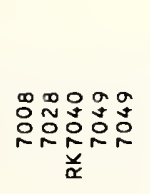 & 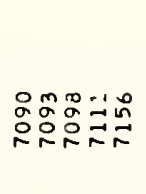 & 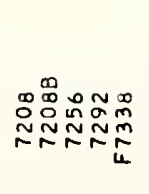 & 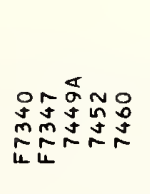 & 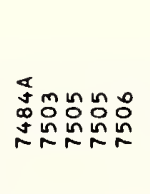 & 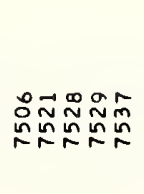 & 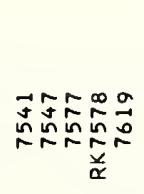 & 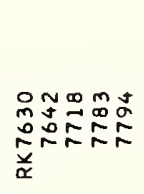 & 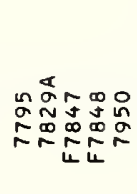 \\
\hline
\end{tabular}




\begin{tabular}{|c|c|c|c|c|c|c|c|c|c|c|}
\hline 9N17dnOS & 언요 & ৪৪০ত্ড & 뭉ㅇㅇ & ОООО유 & 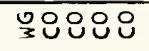 & ৪০৪ㅇㅇㄴ & O్ఞ & Оण & WO & एo \\
\hline L11Av3 & 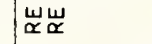 & $\vec{\alpha}$ & $\vec{\alpha}$ & $\vec{n}$ & & & $\vec{\alpha} \vec{\alpha} \vec{\alpha}$ & 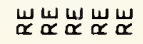 & 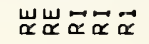 & 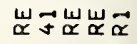 \\
\hline $\begin{array}{c}071119 \\
\text { ग119MVYK }\end{array}$ & 定 & $\Sigma \Sigma \quad \Sigma$ & $\sum \sum$ 误ミ & $\Sigma \Sigma$ & & & $\Sigma \Sigma$ & & & \\
\hline HIOIMNAY8 & $\frac{2}{2}$ & $\stackrel{\circ}{\circ} n$ & $n$ & $\star$ & & & $:::$ & 오유요 & 옹ㅇㅇㅇㅇㅛ & in \\
\hline 9N1רורס & in & $\cong$ & กq & $\stackrel{\circ}{=}$ & & & 잉요 & & & \\
\hline 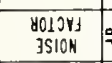 & 8 & $\stackrel{s}{m}$ & n & & & & & & & \\
\hline NiYs & i & :ீீలి & $\stackrel{\infty}{\sim} \stackrel{m}{m}$ & $\approx$ ño & m & 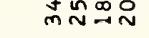 & & & & \\
\hline $\begin{array}{l}\text { S1700 } \\
\times 1173 H\end{array}$ & & 前 & : & $\stackrel{\mathscr{I}}{\Xi}$ & & & & & & \\
\hline $\begin{array}{r}51701 \\
7081000\end{array}$ & 욤 & ㅇㅇㅛ & $\stackrel{i}{N}$ & 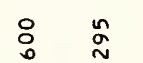 & & & & & & \\
\hline 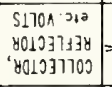 & $|=| \begin{array}{l}\circ \\
0 \\
\infty\end{array}$ & $\stackrel{n}{0} \underset{m}{m}$ & 용 & in & & & 品品品 & 음음음용 & 음음욤욤 & 음 암욤 \\
\hline$a^{\circ}$ & $\underset{n}{n}$ 弚辟总 & 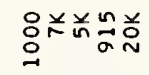 & 关兑 & 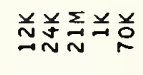 & 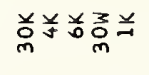 & 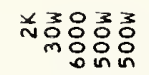 & 兑总品品 & 영ㅇㅇㅇㅇㅕ & 욤요요N & 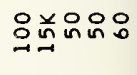 \\
\hline$m$ & ह่ & 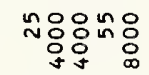 & 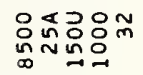 & 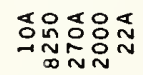 & 영윰욤욤 & 응윰웜웜 & 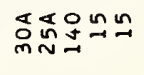 & กีก & 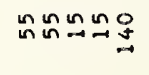 & 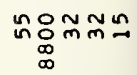 \\
\hline $\begin{array}{r}S 170 \mathrm{~A} \\
30 \mathrm{OHY} \\
\mathrm{BC} \mathrm{Ar}\} \mathrm{BO}\end{array}$ & 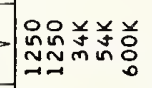 & 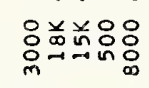 & 路品品品 & 总品嗯 & 羙爻承总品 & 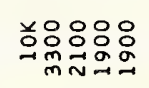 & 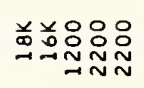 & 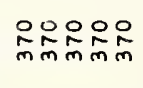 & 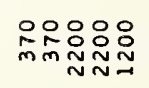 & 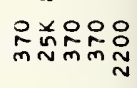 \\
\hline$\mapsto^{-}$ & 티 & 응용워용 & 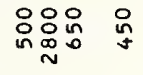 & 留照承品品 & 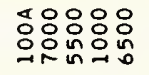 & 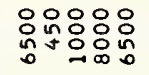 & 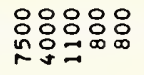 & 옴오옹옹 & 옷용요임 & 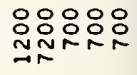 \\
\hline$\omega^{\leftarrow}$ & 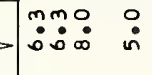 & 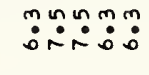 & $\ddot{m}: \ddot{0}:$ & 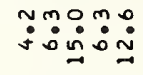 & 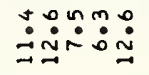 & 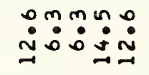 & 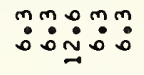 & 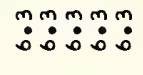 & 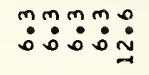 & : \\
\hline NOII $1 \forall y] d 0$ & vana & va $a$ a & $a a \cup a u$ & $0 a 0 a 0$ & Uuaa & a auuu & a auuv & טuט & טบט & vauuv \\
\hline$\forall\rfloor$ & 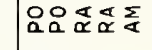 & ひハミミ出 & 山コ凯岕 & 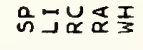 & びママママ & ZロZロロ & $\sum_{w} \sum_{w} \sum_{w} \sum_{w}$ & $\sum \sum_{w} \sum_{w} \sum_{w} \sum_{w}$ & $\sum_{w} \sum_{w} \sum_{w} \sum_{w}$ & $\sum_{w} \sum_{w} \sum_{w} \sum_{w}$ \\
\hline JNก1 & 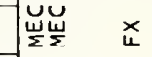 & 5 & $\begin{array}{l}x \times \\
u \underline{u}\end{array}$ & 岀xx & u & & 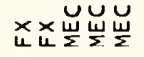 & 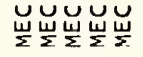 & 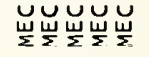 & 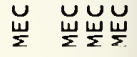 \\
\hline ontry kiso & N $_{\sim}^{\circ}$ nn nn $^{n}$ & ${ }_{m}^{N} \stackrel{n}{N}$ & $\stackrel{\circ}{N}$ & 요 음융ㅇ & m욤 & 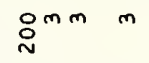 & & & & in \\
\hline 产 & 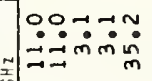 & 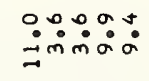 & 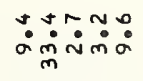 & 〜。் & 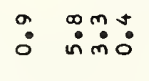 & ஸ்: & 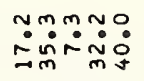 & 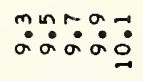 & 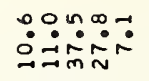 & 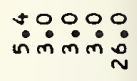 \\
\hline \begin{tabular}{l|l}
$\substack{\tilde{u} \\
4}$ & $\frac{x}{2}$ \\
\end{tabular} & & 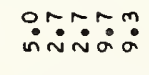 & 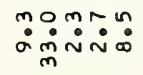 & 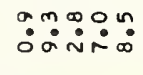 & 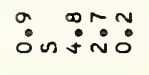 & 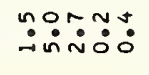 & & 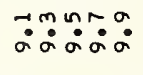 & 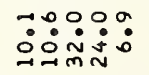 & :ペロ: \\
\hline ONIX & 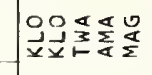 & 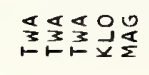 & 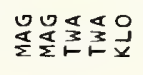 & 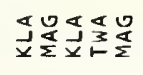 & 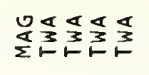 & 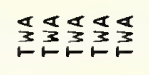 & 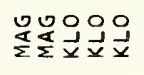 & 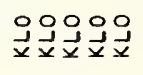 & جُ & 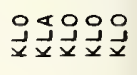 \\
\hline 7ogkNS & & & & " & & & & & & \\
\hline 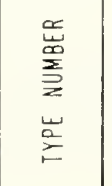 & 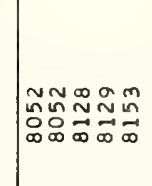 & 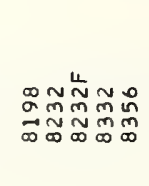 & 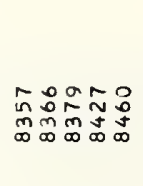 & 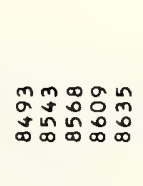 & 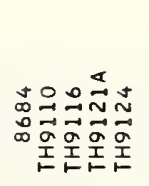 & 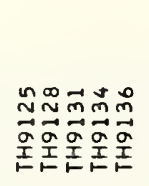 & 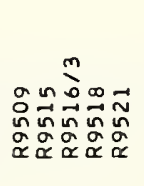 & 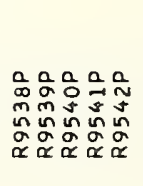 & 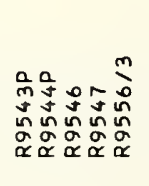 & 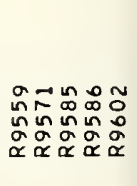 \\
\hline
\end{tabular}




\begin{tabular}{|c|c|c|c|c|c|c|c|c|c|c|}
\hline 9N17dnOS & OQYOY & OQU & 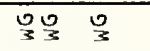 & 号号 & 号号号号员 & 吗吗号 & 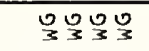 & 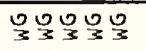 & 온언엉 & 웅웅으 \\
\hline$x 11 \operatorname{lng}$ & $\vec{\alpha} \vec{\alpha} \vec{\alpha} \vec{\alpha} \vec{\alpha}$ & $\vec{\alpha} \vec{\alpha} \vec{\alpha} \vec{\alpha} \vec{\alpha}$ & 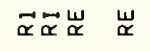 & 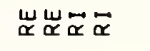 & 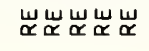 & 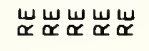 & $\underset{\propto}{\omega}$ & & & \\
\hline 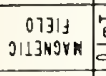 & & & $\Sigma$ & & & & $\Sigma \Sigma \Sigma \Sigma$ & $\Sigma \sum \Sigma$ & 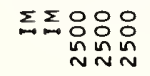 & 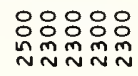 \\
\hline HLOMONYA & 용ㅇㅇㅇ & 웅웡요 & :O80 & $8^{\infty}$ & 웅유유요 & 이임요쇼 & & & & \\
\hline 9 9N177חd & & & 요 & n & & & $\infty \infty$ & $\stackrel{\infty}{\sim}$ & & \\
\hline $\begin{array}{c}8012 Y \mathrm{YJ} \\
3 \mathrm{SLON}\end{array}$ & & & & & & & & & & \\
\hline Nirg $=$ & & & & బ. & & & & & & \\
\hline $\begin{array}{l}51700 \\
\times 1734\end{array}$ & & & & & & & & & & \\
\hline $\begin{array}{c}51701 \\
1081000\end{array}$ & 윰유 & & & & & & & & & \\
\hline 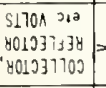 & 음음ํํํํ & 읏음욧요 & 웅요용 & 웅요요 & 음음음 & 응응으음 & 号 & & & \\
\hline $0^{\circ}$ & :음유유 & : & 员品证总品 & :윰 & 용ㅇㅇㅇㅇㅛ & ○ & 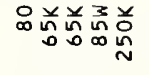 & 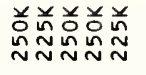 & 总总总总总 & 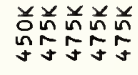 \\
\hline 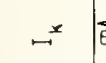 & 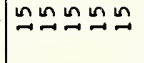 & 웍욤웜요 & 웅워묘 & 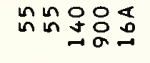 & 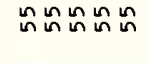 & 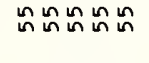 & 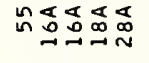 & 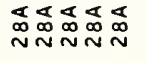 & 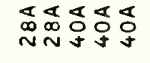 & 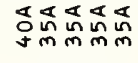 \\
\hline 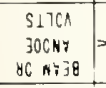 & 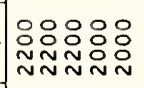 & 路品品品 & 品品品弟品 & 品品品兽弟 & 웅오울 & 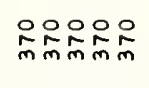 & 웅. & 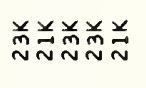 & 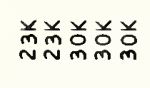 & 总总弟酋总 \\
\hline- & 음요 & 웅윰욤요 & 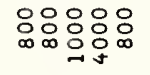 & 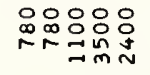 & 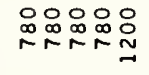 & 옦옴웜엄 & 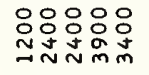 & 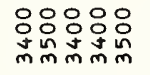 & 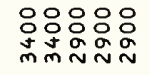 & 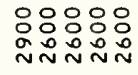 \\
\hline$\omega^{ \pm}$ & 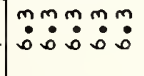 & 苂: & 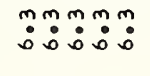 & 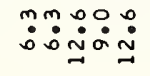 & 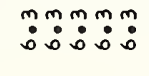 & 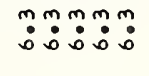 & 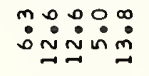 & 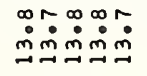 & 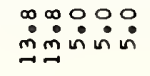 & 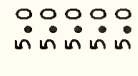 \\
\hline $\mathrm{NO} \perp \triangle \forall y] \mathrm{dO}$ & yưu & טauu & טua & uuaa & טuטu & טuטu & Ua a a & 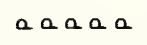 & $a a a a a$ & 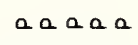 \\
\hline$\forall \pm N$ & 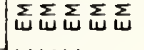 & 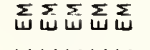 & 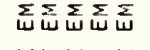 & $\sum_{w} \sum_{w} \sum_{w} \sum_{\frac{1}{3}}$ & 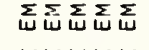 & 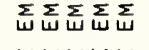 & 픈 $\frac{T}{3} \frac{T}{3}>\frac{I}{0}$ & 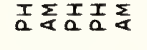 & 똠ㄱ》 & ওタ⿱丶3 \\
\hline$\frac{3 \times \cap 1}{0118 y+11 \cap 0}$ & 這岕岕 & 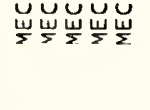 & 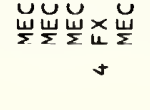 & 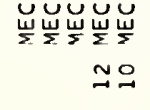 & 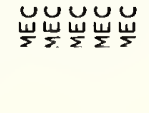 & 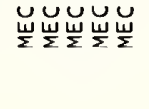 & 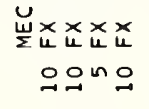 & 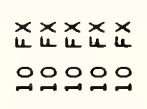 & 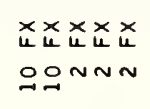 & 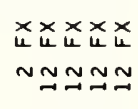 \\
\hline 总 & 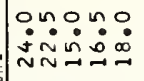 & 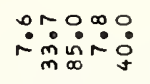 & 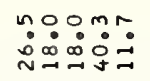 & 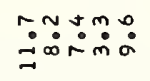 & & 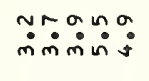 & O̊ñ & 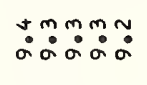 & 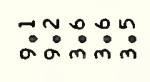 & $\ddot{m} \dot{m} \dot{m} \dot{m} \dot{m}$ \\
\hline 总 & 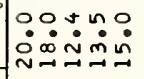 & m & 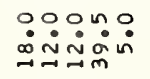 & 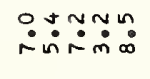 & 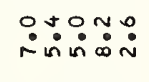 & 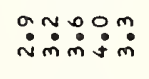 & 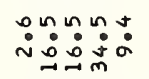 & mNmo: & 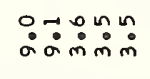 & $\dot{m} \dot{m} \dot{m} \dot{m} \dot{n} \dot{\sim}$ \\
\hline ONIX & 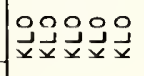 & 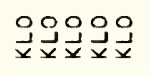 & 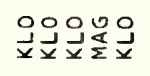 & 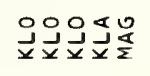 & 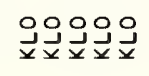 & 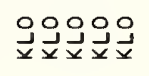 & 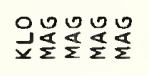 & 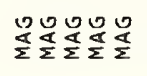 & 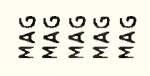 & $\frac{90}{2} \frac{1}{2} \frac{1}{2} \frac{10}{2}$ \\
\hline 70ghis & & & & & & & & & & \\
\hline 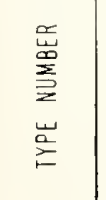 & 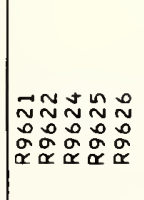 & 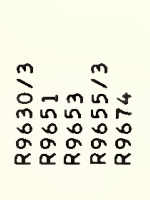 & 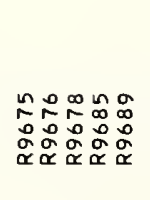 & 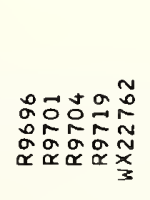 & 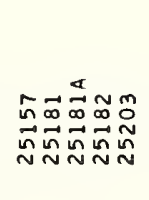 & 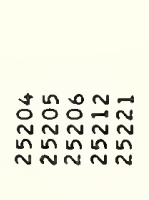 & 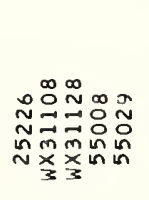 & 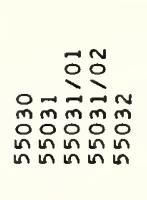 & 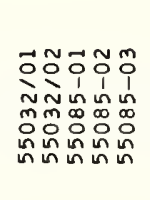 & 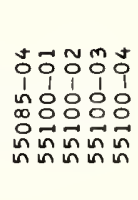 \\
\hline
\end{tabular}




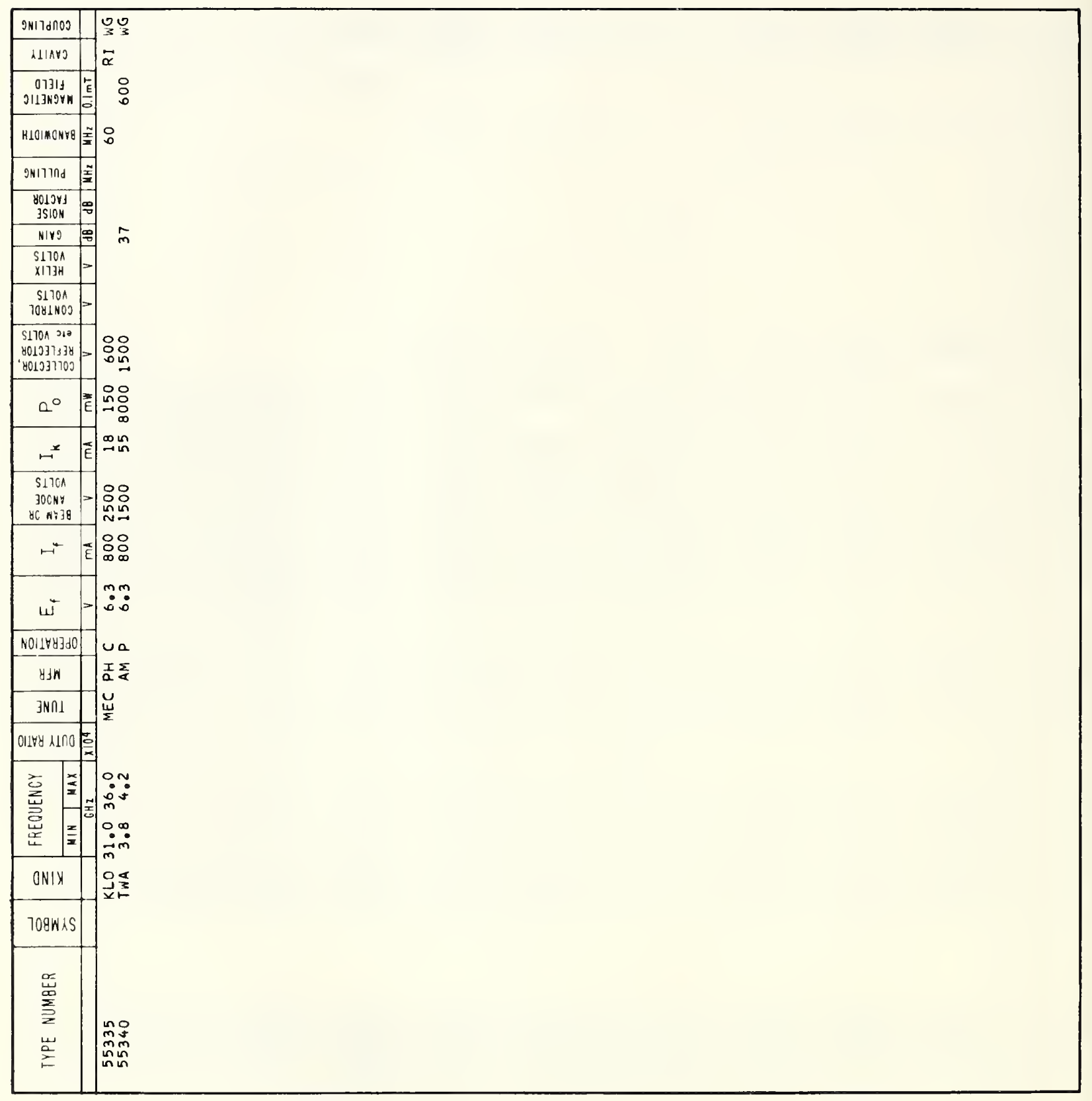




\section{Characteristic Listing of Tube Types}




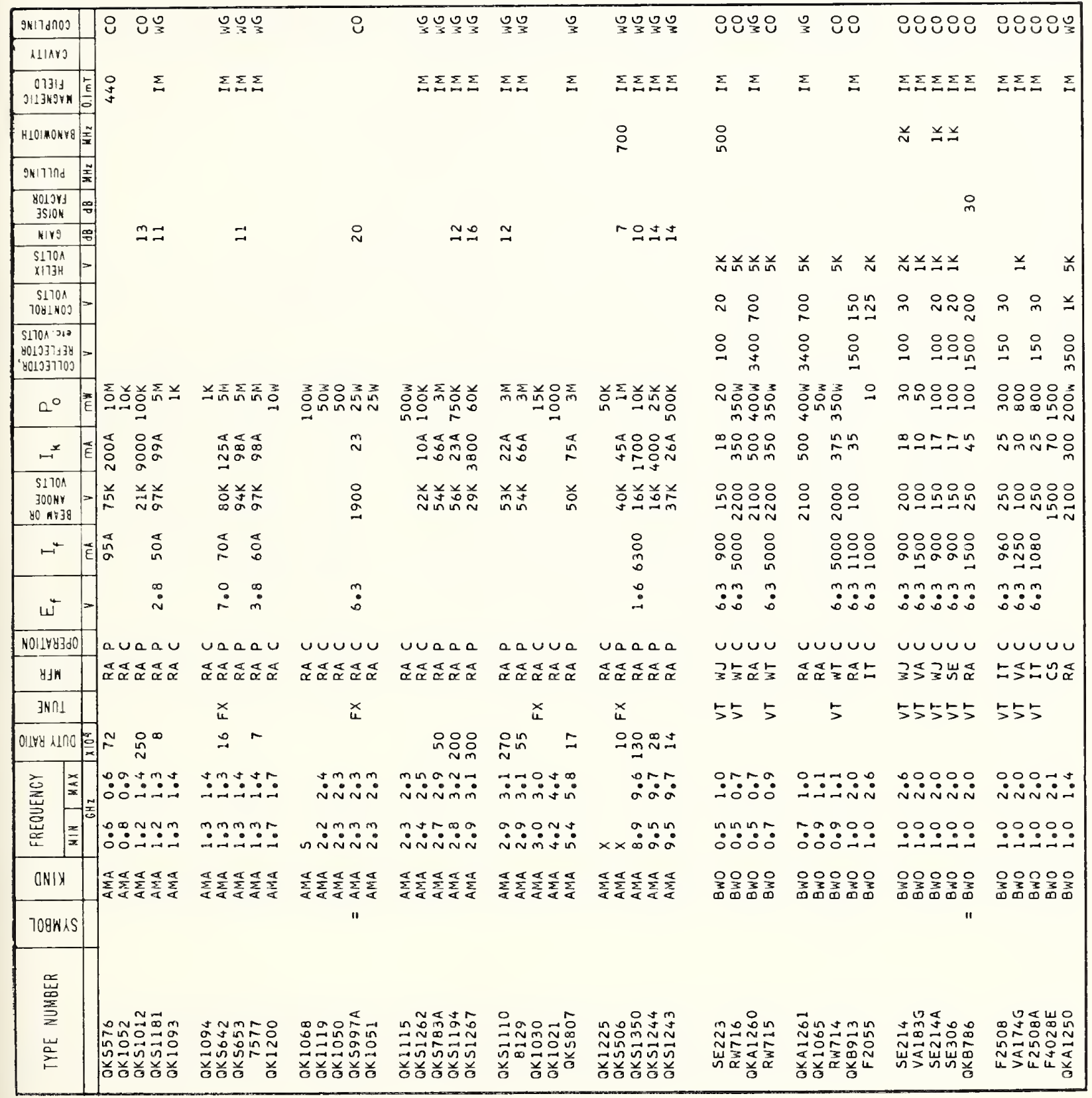




\begin{tabular}{|c|c|c|c|c|c|c|c|c|c|c|}
\hline 9M17าก0O & \multirow[t]{2}{*}{ 웅 } & \multirow[t]{2}{*}{ 용요 } & \multirow[t]{2}{*}{8808} & \multirow[t]{2}{*}{ OQ O४ } & \multirow[t]{2}{*}{ 멍요 } & \multirow[t]{2}{*}{ 엉ㅇํ } & \multirow[t]{2}{*}{8} & \multirow[t]{2}{*}{ 운원 } & \multirow[t]{2}{*}{ 웅요 } & \multirow[t]{2}{*}{888} \\
\hline Alars & & & & & & & & & & \\
\hline $\begin{array}{l}01311 \\
\text { J113н9VM }\end{array}$ & $\Sigma$ & $\sum \sum$ & $\Sigma \Sigma$ & $\sum \sum \sum \sum$ & $\sum \Sigma \sum \Sigma \Sigma$ & $\Sigma \Sigma$ & & $\Sigma \sum \Sigma$ & $\sum \Sigma$ & $\Sigma$ \\
\hline HLOMOHYB & & 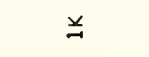 & $\stackrel{x}{N}$ & $\stackrel{2}{N}$ & & & & & & $\rightarrow$ \\
\hline 9NIITAd & 솔 & & & & & & & & & \\
\hline 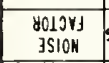 & & & 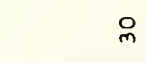 & 只 & & $\stackrel{n}{m}$ & & & & \\
\hline NiYg & & & 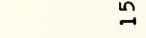 & & & & & & & \\
\hline $\begin{array}{l}S 1704 \\
\times 173 H\end{array}$ & 弚前并关 & 并兰并 & 유슴요 & $\stackrel{2}{N} \stackrel{N}{N}$ & 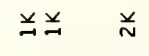 & $\ddot{\sim}$ & 前 & 兰兰 并 & 声兑并 & 前 \\
\hline $\begin{array}{c}51701 \\
1081 \mathrm{NOS}\end{array}$ & $\stackrel{Ð}{=}$ & $\underset{\sim}{\simeq}$ & 윰․ & 어 옷 & 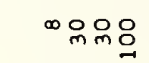 & 옥 욕옴 & $\stackrel{n}{\underset{\sim}{*}}$ & 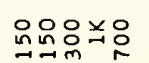 & $\stackrel{\square}{\circ}$ & 㗐 \\
\hline 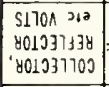 & 品 & 용ㅇ & 음욤욤 & 品品 엄 & 욤요 & \begin{tabular}{l}
0 \\
$:$ \\
\hdashline
\end{tabular} & & 品 & 窝品 & \\
\hline $0^{\circ}$ & | & 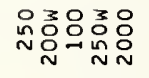 & 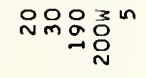 & 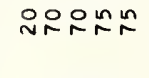 & 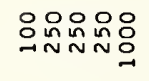 & 응응응요 & 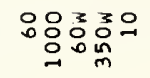 & 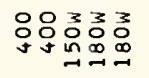 & 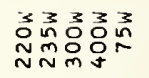 & 옹ㅇㅇㅇㅇㅇㅇ음 \\
\hline 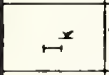 & t品品 品品 & 吃二品只 & $\stackrel{n}{n} \sim \underset{0}{0}$ & 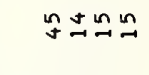 & 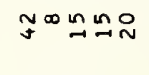 & 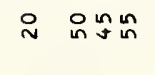 & 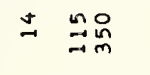 & 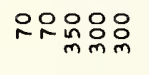 & 윰윰이 & 只员 \\
\hline 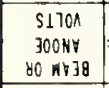 & 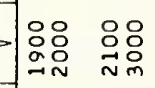 & 总员各品 & 絮员是虽 & 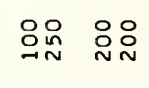 & 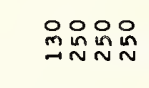 & 品 品怘品 & 总品 & 음윰유. & 음음 & 윰ำ \\
\hline$\mapsto^{+}$ & E. & 응이 & 용요 & 읽 용 & 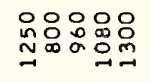 & 品 & 음음 & $\begin{array}{l}\text { OO요 } \\
\text { 요N } \underset{N}{N}\end{array}$ & : & 영 \\
\hline wॅ & $\ddot{m} \quad \stackrel{\dot{0}}{\stackrel{m}{\sim}}$ & $\stackrel{m}{:}:$ & $\dot{m}: \stackrel{m}{:}:$ & $\ddot{m} \stackrel{m}{m}: m$ & $\ddot{m} \ddot{m}: \ddot{m}: m$ & $\ddot{m} \ddot{0}: m$ & $\ddot{m}: \stackrel{m}{0}$ & $\ddot{m} \ddot{m}: 0$. & $\ddot{m}:$ & $\stackrel{m}{m} \stackrel{m}{:}$ \\
\hline NO11Yy\}dO & auvue & ưuบu & טuטu & טuטu & טuบuv & uบuบu & טuטu & บบบบa & auvuv & טuטu \\
\hline$y+w$ & 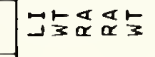 & 造亏与气 & ๔ろち๔u & 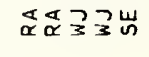 & ১アラロよひ & $\underset{\alpha \propto \alpha}{\alpha} \underset{\alpha}{\alpha} \tilde{u}$ & 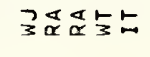 & 岀出凹凹 & 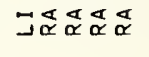 & 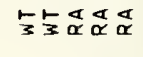 \\
\hline 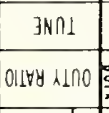 & $55 \quad 5$ & 55 & 55 & トレ トレ & 55 & 5 & 5 & 5555 & 55 & เ5 \\
\hline 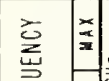 & 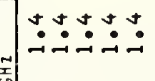 & 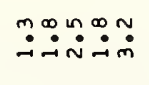 & 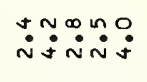 & 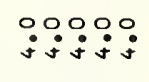 & :०००: & $\because \because \because 00$ & 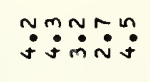 & 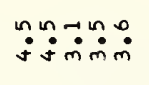 & $\ddot{m} \ddot{m} \dot{m} \dot{m} \dot{m} \dot{m}$ & $\dot{m} \dot{m} \dot{m} \dot{\sim} \dot{\sim} \dot{m} \dot{m}$ \\
\hline 总 & $\because$ & 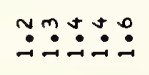 & 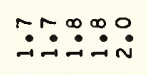 & 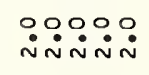 & 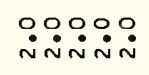 & 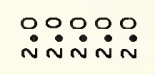 & 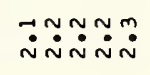 & 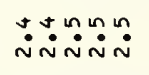 & $\dot{\sim} \dot{\sim} \dot{\sim} \sim \dot{\sim} \dot{\sim} \dot{\sim}$ & 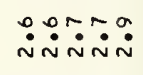 \\
\hline ONIX & 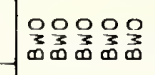 & 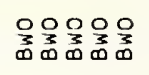 & 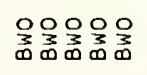 & 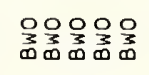 & 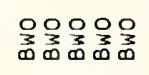 & 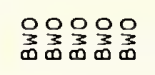 & 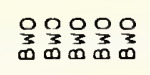 & 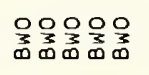 & 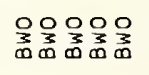 & 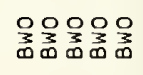 \\
\hline $709 \mathrm{~W} \times \mathrm{S}$ & 1 & & $1 "$ & " " & & " & $"$ & & & \\
\hline 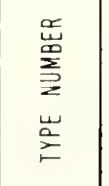 & 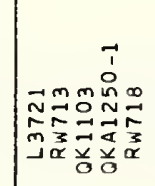 & 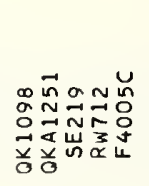 & 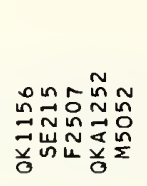 & 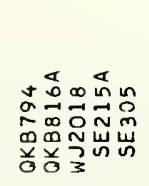 & 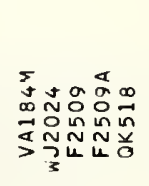 & 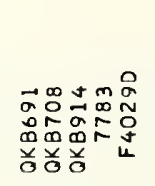 & 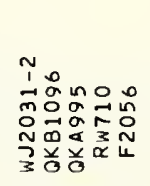 & 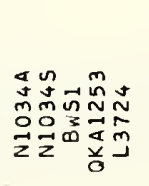 & 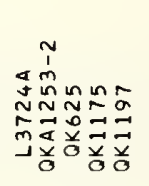 & 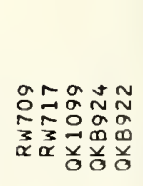 \\
\hline
\end{tabular}




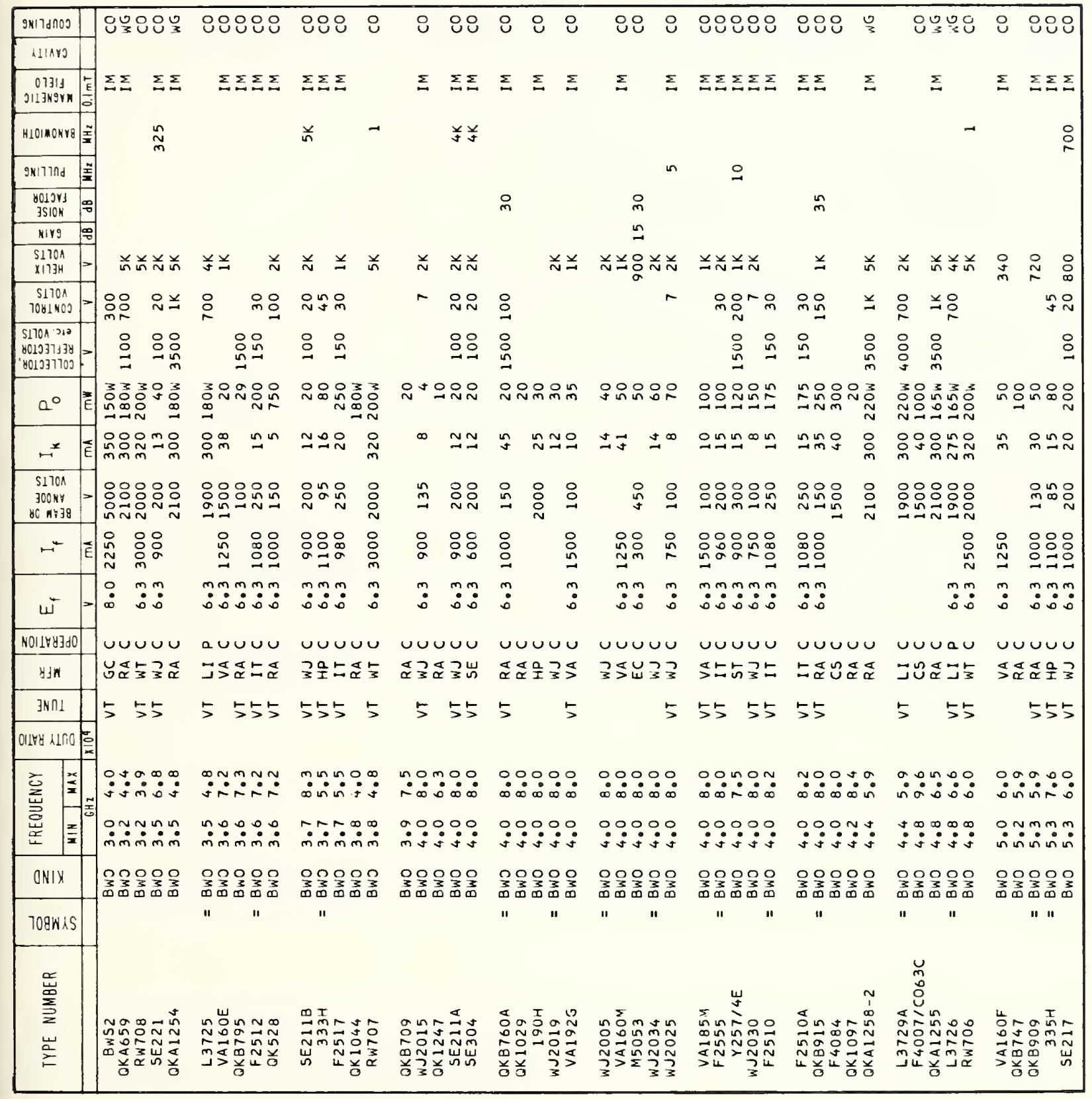




\begin{tabular}{|c|c|c|c|c|c|c|c|c|c|c|}
\hline 9kildnoo & 8 & O 원 & ๖웡요 & 88 & 운용 & 89888 & 옹요 & 언 은 & 운용 & 8O은 \\
\hline A1 ANo & & & & & & & & & & \\
\hline $\begin{array}{c}07314 \\
\text { ग113NOYK }\end{array}$ & $\underline{\Sigma}$ & $\sum \sum \sum$ & $\Sigma \Sigma \Sigma \Sigma$ & $\Sigma \Sigma \sum \Sigma$ & $\sum \Sigma \Sigma \Sigma \Sigma$ & $\Sigma \Sigma \sum \Sigma$ & $\Sigma \Sigma \Sigma$ & $\Sigma \Sigma \Sigma$ & $\Sigma \Sigma \sum \Sigma$ & $\Sigma \Sigma \Sigma \Sigma$ \\
\hline HLOMONFA & & - & & 弟 & 采 & & & $\overrightarrow{ }$ & & \\
\hline 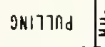 & & & & & & & & & & \\
\hline $\begin{array}{l}0019 Y 3 \\
\text { JSION }\end{array}$ & 同 & & $\stackrel{n}{m}$ & & & & 요술 & & 용 & \\
\hline Hirg & & & & & & & $\stackrel{\circ}{\circ} \stackrel{0}{m}$ & & $\stackrel{\text { n }}{\sim}$ & \\
\hline $\begin{array}{l}\text { S170A } \\
\times 1173 H\end{array}$ & $\begin{array}{l}8 \\
\infty \\
\infty\end{array}$ & 并并弚首 & 宁兰 & 说 $\underset{N}{ }$ & 弚兰兰弚兰 & 并并 & 㒸酋 & 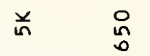 & 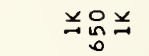 & $\underline{\sim}$ \\
\hline $\begin{array}{c}\text { S1701 } \\
7081 \mathrm{NOO}\end{array}$ & $i_{m}$ & 용 & 员品 吕怘 & $\stackrel{i}{N}$ & 융요 & 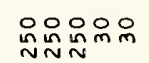 & 윰욤욤요 & on & 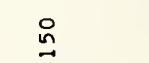 & ڤ̊ \\
\hline 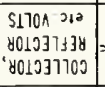 & 足 & 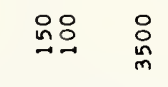 & 욤욤요 & $\stackrel{\circ}{\circ}$ & $\stackrel{\circ}{\circ}$ & 음요 & 总 & & : & \\
\hline $0^{\circ}$ & 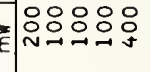 & 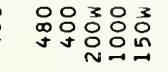 & 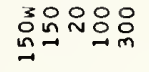 & 유윰유 & 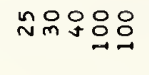 & 曽兽总品品 & :O: & 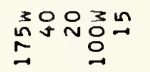 & 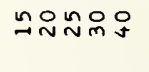 & 은요응응 \\
\hline 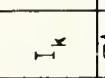 & n & $\stackrel{n}{\sim} \underset{m}{\stackrel{O}{N}} \underset{0}{0}$ & $\underset{N}{n} \approx \underset{n}{n}$ & $\simeq \stackrel{\infty}{N} \stackrel{N}{N}$ & 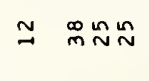 & 음요ㅁㅛㅡㅁ & $\stackrel{N}{N} \because N$ & $\underset{\sim}{\circ} \stackrel{n}{n} \underset{n}{n}$ & 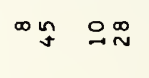 & $\stackrel{n}{\sim} \underset{N}{\infty} \stackrel{n}{m}$ \\
\hline $\begin{array}{c}5170 \mathrm{~N} \\
300 \mathrm{NY} \\
80 \mathrm{H} \rightarrow 38\end{array}$ & $>$ in & 品 $\stackrel{\circ}{\circ} \stackrel{\circ}{\sim}$ & 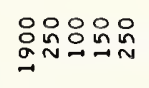 & : $\stackrel{\text { : }}{\text { 윰 }}$ & : & 음음욤요 & 음욤욤욤 & : & 总虽品品 & $\stackrel{\circ}{\stackrel{D}{N}}$ \\
\hline$\leftarrow$ & $\stackrel{\stackrel{O}{N}}{N}$ & 응응 & 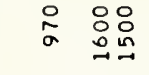 & 욤욤요 & 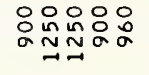 & 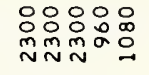 & 웅윰요 & 品: & 응용요 & 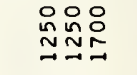 \\
\hline$\omega^{\top}$ & $\stackrel{m}{0}$ & 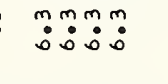 & $\ddot{m}: m m m$ & $\ddot{m}: m: m$ & 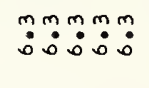 & $\ddot{n} \ddot{m} \ddot{m}: \dot{m}:$ & $\ddot{m}: m \ddot{m}^{m}: \dot{m}$ & $\ddot{m}: m: m m$ & $\ddot{m}: m \ddot{m}^{m}: \dot{m}$ & $\ddot{m}: m$ \\
\hline $\mathrm{NO1} \perp \forall y\} \mathrm{dO}$ & & 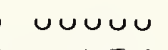 & ט & 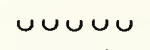 & บบบบบ & uu & טuט & บบบบท & & \\
\hline BuW & 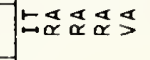 & ヒヒラ心ð & 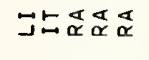 & 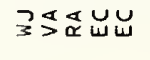 & 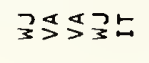 & 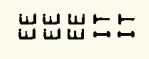 & 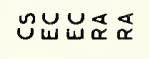 & 歺迳顿号 & 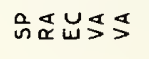 & 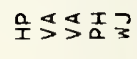 \\
\hline JNก & 5 & 55 & 55555 & 5 & 55 & 55555 & 5 & 555 & 55 & 5 \\
\hline 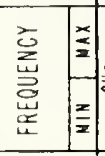 & 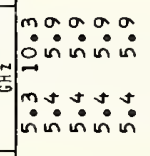 & 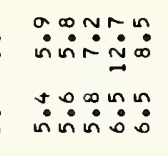 & 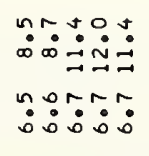 & 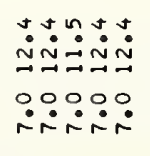 & 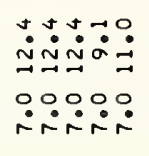 & 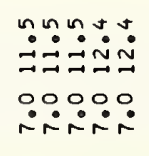 & 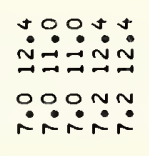 & 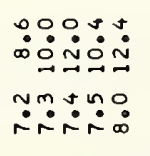 & 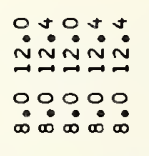 & 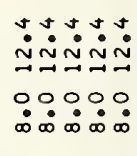 \\
\hline ONIX & 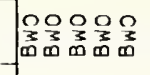 & 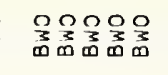 & 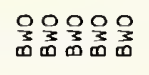 & 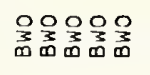 & 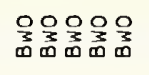 & 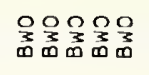 & 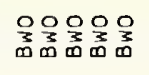 & 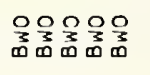 & 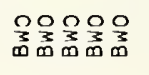 & 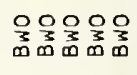 \\
\hline $708 \mathrm{~g} / \mathrm{S}$ & ]" & & & " " & $"$ & & " " " & $"$ & $"$ & \\
\hline 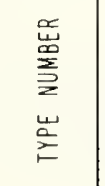 & 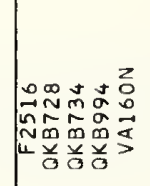 & 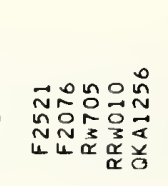 & 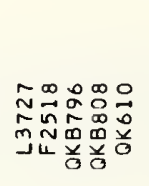 & 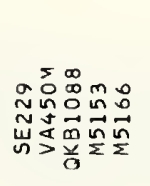 & 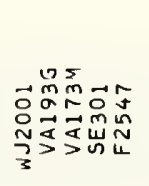 & 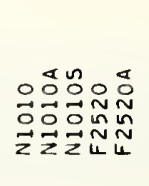 & 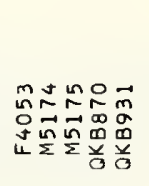 & 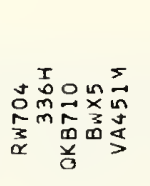 & 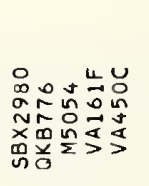 & 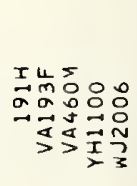 \\
\hline
\end{tabular}




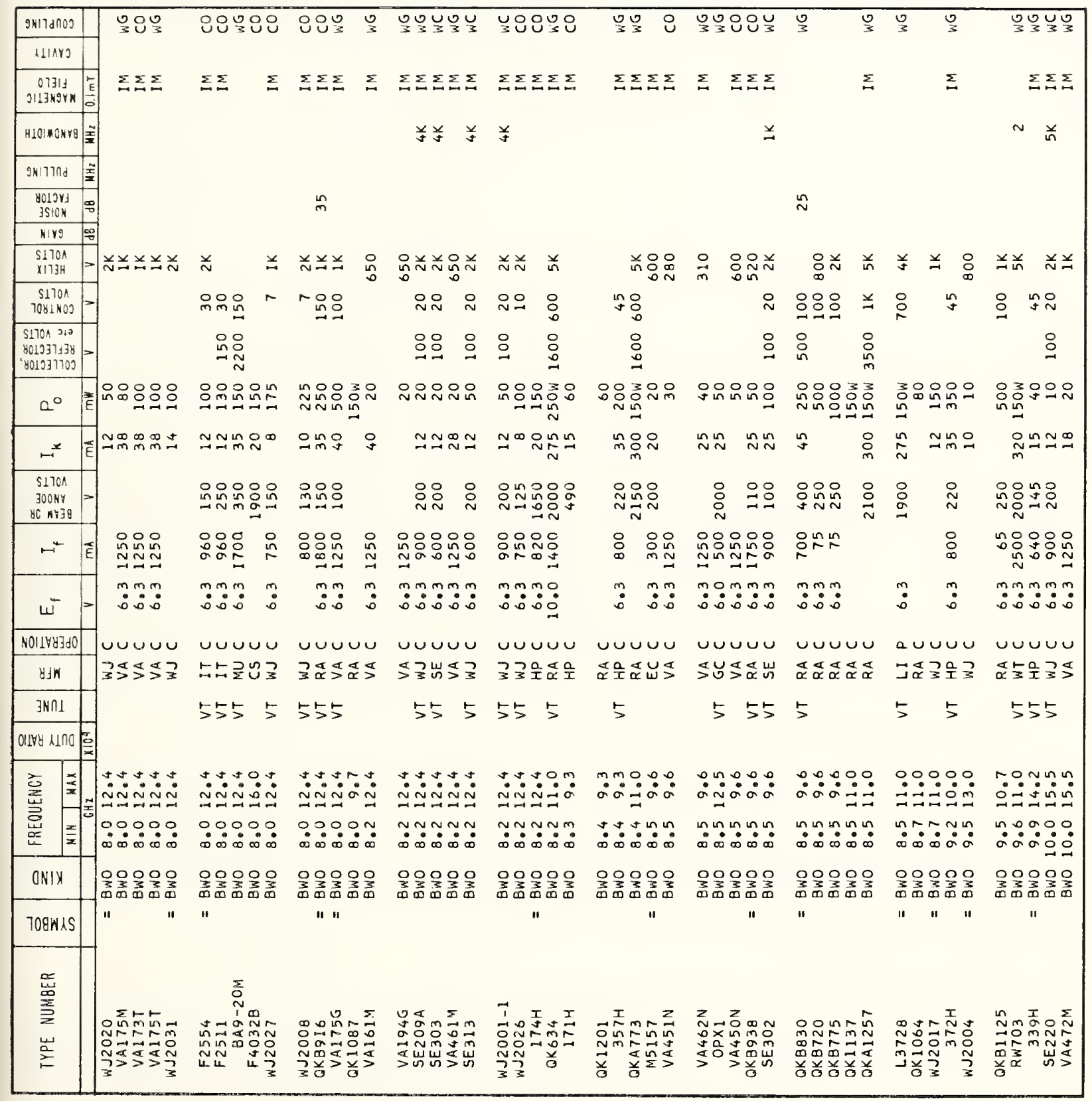




\begin{tabular}{|c|c|c|c|c|c|c|c|c|c|c|c|}
\hline 9Hוาd 103 & पणO & WO & OO $8_{3}^{\circ}$ & 원욕 & 웡요 & 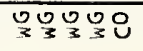 & OOßY & \multirow{2}{*}{\multicolumn{2}{|c|}{$\frac{0}{3}$}} & \multicolumn{2}{|c|}{ 号 } \\
\hline AlarJ & & & & & & & & & & & \\
\hline $\begin{array}{l}07319 \\
\text { 3119M9VK }\end{array}$ & 国 $\sum \sum \sum \Sigma$ & $\Sigma$ & $\sum_{n=1} \sum_{n=1}$ & $\sum \sum \sum \sum$ & $\sum \sum \sum \sum \sum$ & $\Sigma \Sigma \Sigma \Sigma$ & $\sum \Sigma \Sigma \Sigma$ & $\Sigma$ & & $\Sigma \Sigma$ & $\sum \sum \sum \Sigma$ \\
\hline HLOMOHYH & & $\sim$ & 居首 & $\because$ & & $\sim$ 并 & 并 & & & & 首 \\
\hline 9k17רก & & & & & & & & & & & \\
\hline $\begin{array}{c}8019 \mathrm{~s} \\
\text { JS10N }\end{array}$ & & 음 & & & & & & & & & \\
\hline hirg & & 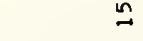 & & & & & & & & & \\
\hline $\begin{array}{l}51700 \\
\times 173 \mathrm{H}\end{array}$ & 首兰兰兰兰 & $\stackrel{\Perp}{N}$ & $\stackrel{2}{N}$ & $\cong \quad \cong \rightleftharpoons \check{N}$ & $\ddot{\sim}$ & 前 总品 & 응요 는 & & $\cong$ & $\cong \stackrel{\sim}{\sim} \cong \cong$ & $\stackrel{x}{N}$ \\
\hline $\begin{array}{c}\text { S1701 } \\
\text { 7081 1003 }\end{array}$ & $\stackrel{\circ}{\circ}$ & $\stackrel{i}{\sim}$ & iN & 요 & $\stackrel{\circ}{-}$ & 으으 & & & $\stackrel{0}{0}$ & $\stackrel{-}{\circ}$ & 욤욤 \\
\hline 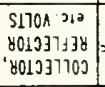 & & $\stackrel{\circ}{\stackrel{2}{N}}$ & 음 & $\stackrel{1}{\circ}$ & 品 & $\stackrel{\circ}{\circ}$ & 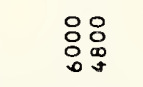 & & & & 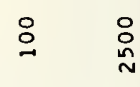 \\
\hline$a^{\circ}$ & Ho요요 & 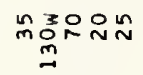 & 으으뮤유 & 옹웡ㅇㅇㅇ & 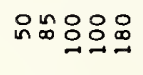 & 옹잉으요 & 윰응영응 & 눅웅웅 & & 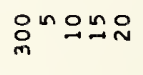 & 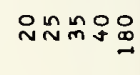 \\
\hline$\rightarrow$ & $\vec{E} \sim$ & 윰요 & $\simeq \cong \simeq n$ & $\stackrel{\simeq \simeq}{\simeq}$ & $\mathfrak{N}^{n \infty n} \sim \sim \sim$ & $=\underset{m}{O}: 00 r$ & ronging & nog & & $\simeq \stackrel{\circ}{N}$ & $\infty \simeq \cong n$ \\
\hline 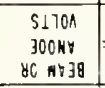 & $\Rightarrow \stackrel{\circ}{\circ}$ & 웅웅 & 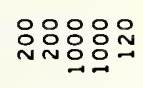 & 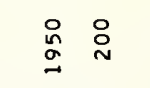 & 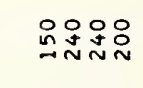 & 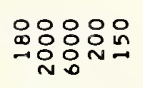 & 염윰유 & 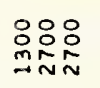 & $\stackrel{\circ}{N}$ & $\stackrel{\circ}{N}$ & 음유 \\
\hline$\mapsto^{4}$ & E & 음욤요 & 용요 & 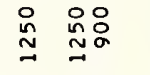 & 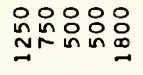 & 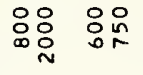 & 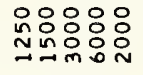 & 品 & $\stackrel{n}{m}$ & 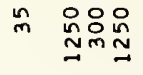 & 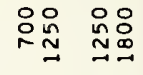 \\
\hline$w^{5}$ & 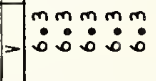 & $\ddot{0}: \ddot{m}: \dot{m}$ & $\ddot{m}:$ & $\dot{m}: m m$ & $\ddot{: m m m}: \dot{m}:$ & $\ddot{:}: m: m$ & 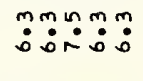 & $\ddot{m}$ & $\ddot{:}$ & $\ddot{0}: \ddot{m}: m$ & $\ddot{m}: \ddot{m}:$ \\
\hline N011 & נuuvu & טuบuv & ưưv & uบuบu & טuטu & טuטu & טuบu & yuus & & טuטu & טuטu \\
\hline 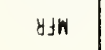 & 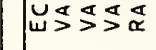 & 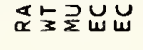 & 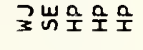 & ×ำง马? & 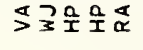 & 솛ํํำ & 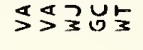 & ถูบษ & & 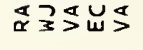 & 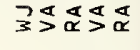 \\
\hline 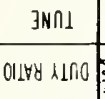 & 55 & 55 & ら5 & 5 & 5555 & 55 5ร & 5555 & & & & 55 \\
\hline 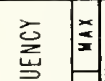 & 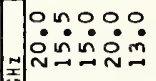 & $\because \because 0000$ & $\because \because: 000$ & 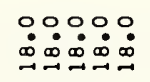 & 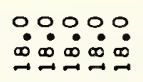 & 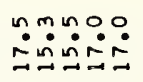 & 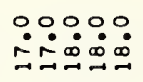 & & & Oñ & 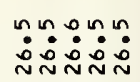 \\
\hline 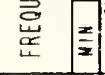 & $\because: 000:$ & $\because \stackrel{0}{0} \dot{0} \dot{0} \dot{\sim}$ & 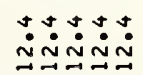 & 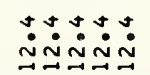 & $\dot{\sim} \dot{\sim} \dot{\sim} \dot{\simeq} \dot{\simeq}$ & 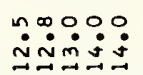 & $\begin{array}{l}00000 \\
0 \\
0\end{array}$ & $\begin{array}{l}n=0 \\
\dot{n} \operatorname{nn}: 0\end{array}$ & & 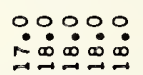 & $\begin{array}{l}000: 0 \\
0 \\
0 \\
0\end{array}$ \\
\hline ONIX & 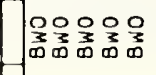 & 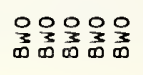 & 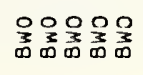 & 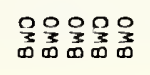 & 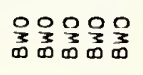 & 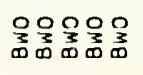 & 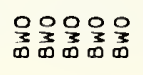 & 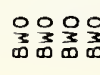 & & 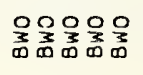 & 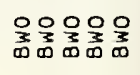 \\
\hline $700 \mathrm{~W} / \mathrm{S}$ & & & & & " " " & & & & & $"$ & " " \\
\hline 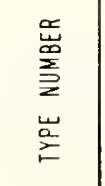 & 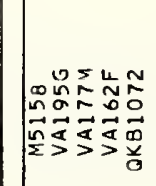 & 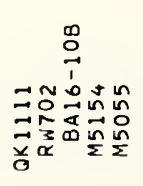 & 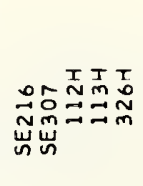 & 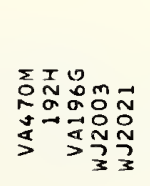 & 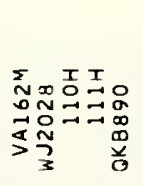 & 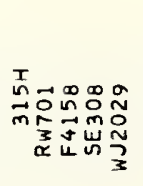 & 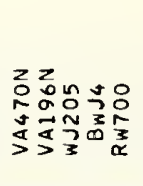 & 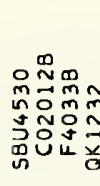 & & 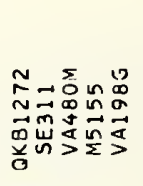 & 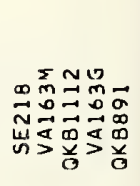 \\
\hline
\end{tabular}




\begin{tabular}{|c|c|c|c|c|c|c|c|c|c|c|}
\hline 9NIר & \multirow[t]{2}{*}{$\begin{array}{ll}O \\
\end{array}$} & जू & 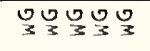 & $\frac{0}{3}$ & OU్ & पूO & पO & 인 & 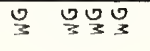 & $\stackrel{5}{3}$ \\
\hline AHANS & & & & & & & & & & \\
\hline 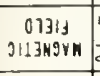 & 鳥 & $\Sigma \Sigma$ & $\Sigma 0_{0} \Sigma \Sigma \Sigma \Sigma$ & $\Sigma$ & $\Sigma \sum \Sigma \Sigma$ & $\Sigma \Sigma \Sigma \Sigma \Sigma$ & $\Sigma \Sigma \Sigma \Sigma \Sigma$ & $\sum \Sigma$ & $\Sigma \Sigma \Sigma$ & \\
\hline H1O MONFA & & & $\stackrel{\sim}{ \pm}$ & & & & & & & \\
\hline 9mוาInd & 푶옹 & & o & & & $\ln \ln i n$ & in in & & & \\
\hline $\begin{array}{r}8019 \mathrm{ys} \\
-1510 \mathrm{H} \\
\end{array}$ & 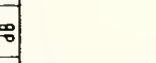 & & & & & & & & & \\
\hline N1199 & 娄 & & & & & & & & & \\
\hline $\begin{array}{l}3701 \\
\times 1173 H \\
x\end{array}$ & 前 & 兰兰兰兰 & 并弟 & $\stackrel{D}{\sim} \underset{\sim}{\stackrel{x}{*}}$ & 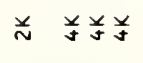 & 対爻导 & 声羊 & & & \\
\hline $\begin{array}{r}51701 \\
7081403\end{array}$ & $\Rightarrow$ in & & 负员号 & $\stackrel{\circ}{\sim}$ & 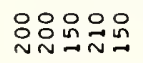 & 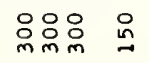 & 品品 品 & & & \\
\hline 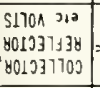 & $>>\begin{array}{l}0 \\
0 \\
\infty \\
\sim\end{array}$ & & 品 & & & & & & & \\
\hline$a^{\circ}$ & E) & $\stackrel{\infty}{N}^{\ln \ln } O=$ & 음ㅇㅇㅇㅇㅇㅇㅇㅇㅇ & 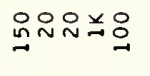 & 옹욤욤요 & 영용요 & 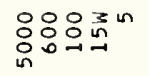 & 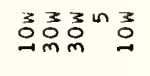 & : & 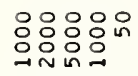 \\
\hline$\rightarrow$ & $\widehat{E}$ & $\stackrel{a}{=}$ & "뜸유요요 & 요 욤요 & 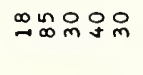 & :OOOON & 눅잉요요 & 웃 웃우 & $\stackrel{n}{m} \approx m \sim n m \tilde{m}$ & 요 \\
\hline 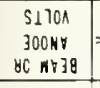 & $=$ = & & 只品 总兰 & 总羊品 & 영유 & 品品品品品 & 美虽品总 & 응 :윰용 & 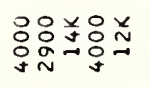 & :웅 \\
\hline$\mapsto^{-}$ & Ê & 足恕怘 & 욤음 & 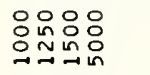 & $\begin{array}{l}\text { 응음 } \\
\text { 임윰ㅇㅁㅇ }\end{array}$ & 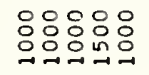 & 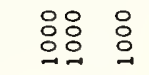 & 品 & 品 & \\
\hline$\omega^{\leftarrow}$ & $\ddot{n}$ & $\ddot{m}: m$ & $\ddot{m} \ddot{m}: \dot{m}$ & 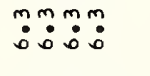 & 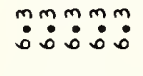 & 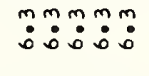 & $\stackrel{m}{m}: \stackrel{m}{:}$ & $\ddot{m}$ & $\ddot{m}$ & \\
\hline NO11甘YG $\mathrm{dO}$ & טuvuv & 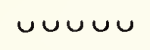 & טuบuบ & บบบบบ & 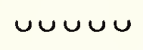 & טuטu & 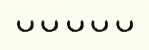 & טuטu & טuטu & uus \\
\hline$y \mathrm{yN}$ & 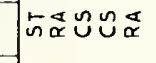 & 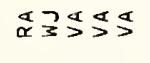 & 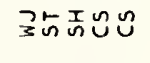 & 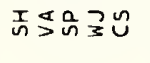 & エコさ゚元 & 石吕路吕 & 㞷并并足台 & ทังัง & クํำ吴こ呈 & 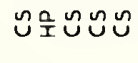 \\
\hline 3Nn1 & & & 55 & & 555 & レ5レ5 & ト5 & & & \\
\hline olyy AIro & & & & & & & & & & \\
\hline 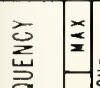 & 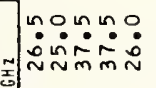 & 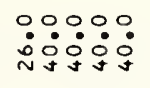 & 过品: & 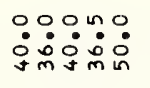 & 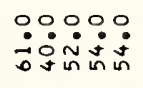 & 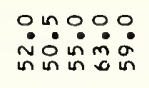 & 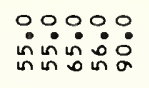 & 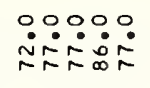 & 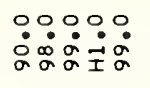 & 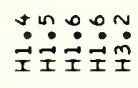 \\
\hline 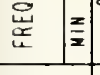 & 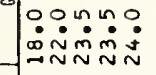 & 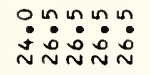 & 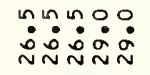 & 遇品: & 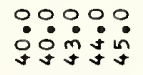 & $\begin{array}{l}\because \\
\dot{n} \\
\forall\end{array}$ & 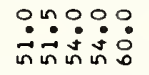 & 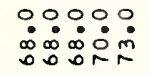 & $\because \because \because: 0: 0$ & 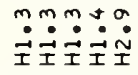 \\
\hline ONIX & 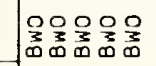 & 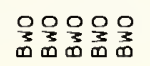 & 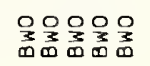 & 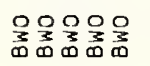 & 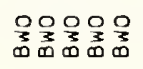 & 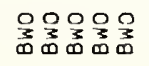 & 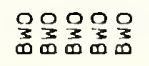 & 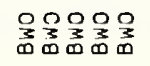 & 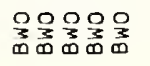 & 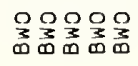 \\
\hline $708 \mathrm{~W} / \mathrm{S}$ & & & & & " " " & " " " " & " " & & & \\
\hline 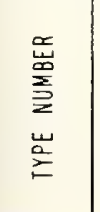 & 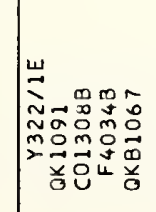 & 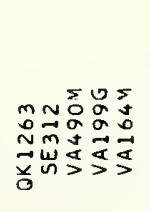 & 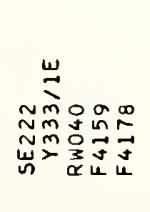 & 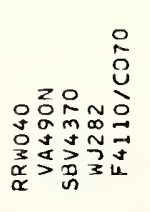 & 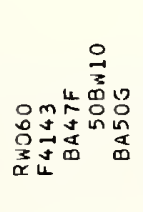 & 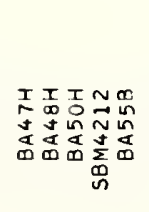 & 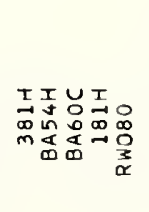 & 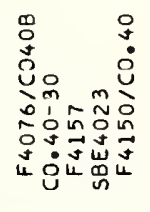 & 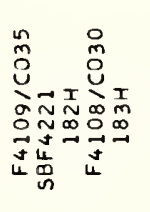 & 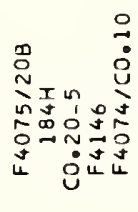 \\
\hline
\end{tabular}




\begin{tabular}{|c|c|c|c|c|c|c|c|c|c|c|c|}
\hline 9NI ld NOO & \multirow{10}{*}{ ग } & \multirow{2}{*}{$\bigcup_{3}$} & \multirow{2}{*}{ ऽ७ } & \multirow{2}{*}{ 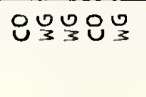 } & \multirow{2}{*}{ 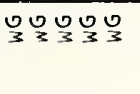 } & \multirow{2}{*}{ 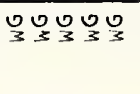 } & \multirow{2}{*}{$\begin{array}{l}0 \\
3\end{array}$} & ৪ั๐๐ & \multirow{2}{*}{ 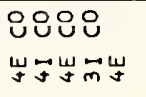 } & \multirow{2}{*}{ 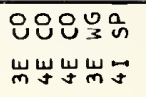 } & \multirow{2}{*}{ 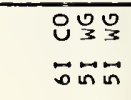 } \\
\hline 111100 & & & & & & & & 岗的崫岁 & & & \\
\hline 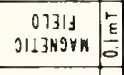 & & $\Sigma$ & & $\Sigma$ & & & $\sum \Sigma$ & $\Sigma$ & & & \\
\hline HLOIMOHYY $\frac{x}{x}$ & & & & $\stackrel{\circ}{=}$ & & & 윰 & $m$ & $n+\infty$ & $m$ & 응요 \\
\hline 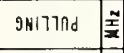 & & & & & & & & & & & \\
\hline \begin{tabular}{c|c}
$8019 \mathrm{YJ}$ \\
$3 \mathrm{SIOH}$
\end{tabular} & & & & & & & & & & & \\
\hline AIV $\$$ & & & $ㅇ ㅡ$ & $\operatorname{man}_{\sim}$ & $a r r a$ & $\exists \cong r \underset{N}{N}$ & 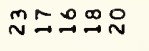 & $\stackrel{m}{m}$ & 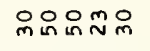 & 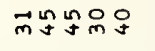 & 的出m的品 \\
\hline $\begin{array}{l}S 110 \mathrm{~A} \\
\times 117 \mathrm{HH}\end{array}$ & & & & & & & & & & & \\
\hline $\begin{array}{c}51701 \\
7041909\end{array}=$ & & & & & & $\cong$ & 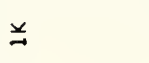 & 음 & 움윰요 & 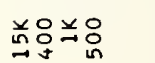 & \\
\hline 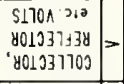 & & & & & & 首兽 & 煎 & & $\stackrel{\check{0}}{\rightarrow}$ & & \\
\hline $0^{\circ}$ & $\operatorname{lon}_{0}$ & $\ln n$ & $\sum \sum$ & 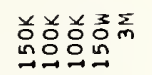 & 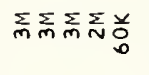 & 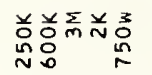 & 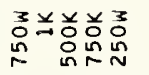 & 羊兑并弟 & 㒸兰兰兰壬 & 总首总漹 & $\sum \sum \sum_{\text {N }} \sum_{0} \sum_{-1}$ \\
\hline$\stackrel{x}{\leftarrow} \bar{\varepsilon}$ & in & in & 品 & 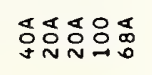 & 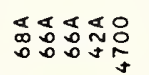 & 点 呫员员 & in 䟡 & 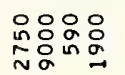 & 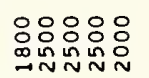 & 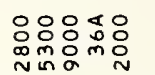 & 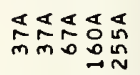 \\
\hline 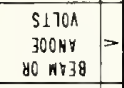 & $\begin{array}{l}: \\
:\end{array}$ & 兰 & $\stackrel{\circ}{\circ}$ & 吕兰总品兑 & 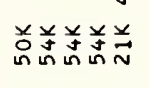 & 兑 & 吕 弟前 & 尊总酋兰 & 兰总兑兑弟 & 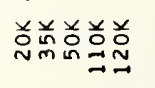 & 总总总兑兑 \\
\hline$F \bar{E}$ & & & & & & & & 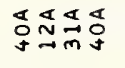 & 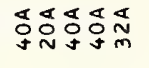 & 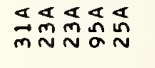 & 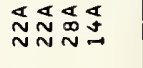 \\
\hline " & & & & & & $\dot{0}:$ & $\stackrel{m}{\circ}$ & ¿̊: & 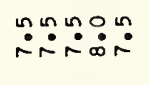 & $\begin{array}{l}\because: 0 \text { un } \\
\text { n̈: }\end{array}$ & 迸品: \\
\hline N01_ $1 \forall \mathrm{y} \mathrm{dO}$ & טuטu| & u & $a v$ & a a a va & 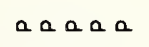 & $a, a, a$ & uvau & טuטu & טบบบ & $a \cup a a a$ & $\operatorname{araaa}$ \\
\hline 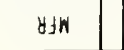 & 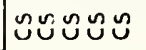 & ) & $\underset{\propto x}{\mathbb{\alpha}}$ & 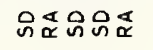 & 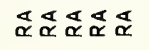 & 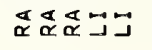 & ココถิํㅗ & 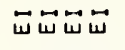 & 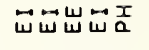 & ேேேே゙ை & コゴが \\
\hline 3Nก! & & & & & & & & 岀岕岕㟧 & 岁 & 岕岕 岁岕 & 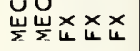 \\
\hline Oolfy lino & & & & $\therefore$ & & & $\stackrel{n}{n}$ & & & 용용 & 응 앙ㅇ \\
\hline 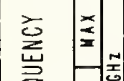 & 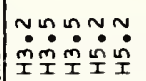 & 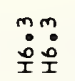 & $\ddot{0}:$ & $\dot{\because} \therefore \dot{\sim}$ & 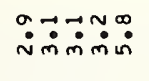 & ம் & 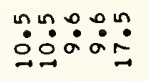 & 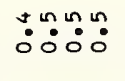 & $\ddot{0}: 0: 00$ & 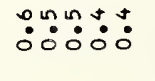 & 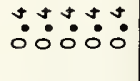 \\
\hline 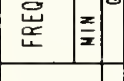 & 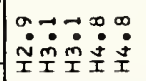 & ํํำ & $\stackrel{n}{0}$ & 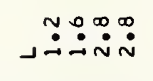 & 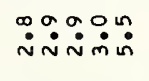 & 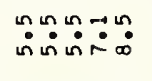 & 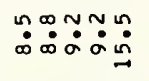 & $\ddot{n} \ddot{0}: \stackrel{*}{0}$ & $\ddot{0}: \Delta: \Delta:$ & $\dot{0}: \Delta: \Delta:$ & $\stackrel{+}{0}: \Delta: \Delta$ \\
\hline ONIX & 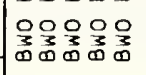 & 永号 & 选出 & 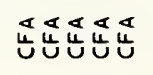 & 造芯芯出 & 巡芯芯芯选 & 巡芯話出 & 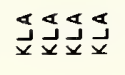 & 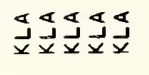 & 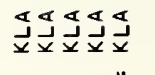 & 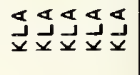 \\
\hline $708 \mathrm{~W} / \mathrm{S}$ & & & & & & & & & & & \\
\hline 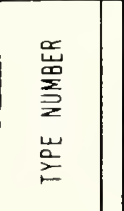 & 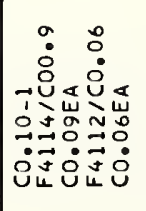 & 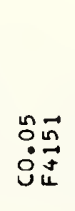 & 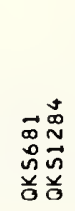 & 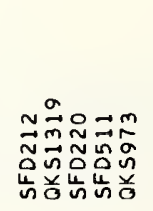 & 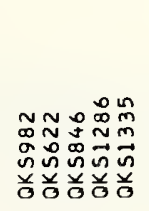 & 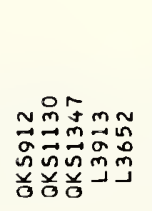 & 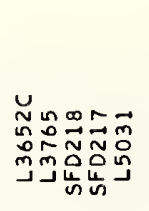 & 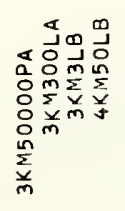 & 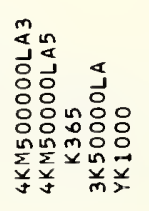 & 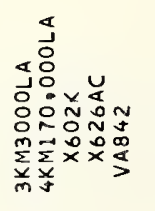 & 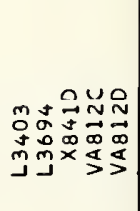 \\
\hline
\end{tabular}




\begin{tabular}{|c|c|c|c|c|c|c|c|c|c|c|}
\hline 9xild Rnos & 888 & $\frac{0}{3}$ & 언은 & 88 & 엉ㅇㅇ & 엉ㅇㅇㅇㅇ & 옹요 & 옹ㅇㅇㅇ & 엉웡으 & 8\%888 \\
\hline L110Y3 & 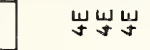 & 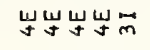 & 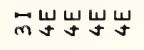 & 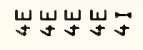 & $\vec{\sigma} \quad \underset{\mho}{\mho 山}$ & 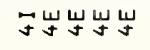 & 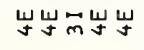 & 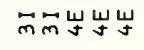 & テテす山せ & 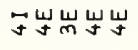 \\
\hline 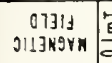 & & 品 & 总员品 & & & $\stackrel{i}{\sim}$ & & 品品 & & in \\
\hline H101monry $=$ & 10 & o & $0 \infty$ & $\infty \infty$ & $\infty$ & $\infty$ & $\operatorname{rn}$ & ס & $\infty \infty \infty$ & $\infty 0$ \\
\hline 9kורา & & & & & o & & & 웅 & ? & \\
\hline $\begin{array}{l}y 019 \mathrm{~W} \\
3 \mathrm{SIOK} \\
\end{array}$ & & & & & & & & & & \\
\hline 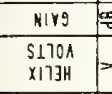 & 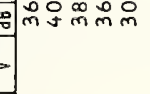 & 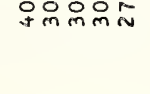 & $\hat{\sim} \sim \mathcal{F} \sim \mathcal{F} \sim$ & 品品品乎出 & 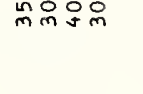 & 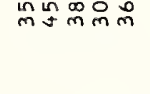 & 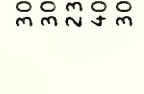 & $\tilde{N} \tilde{N}$ & 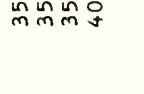 & 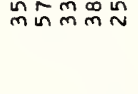 \\
\hline $\begin{array}{c}51701 \\
7001503 \\
\end{array}$ & & & 总吕 & & & 品 & 응 & & & \\
\hline 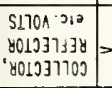 & : & 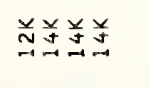 & 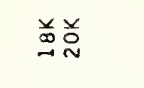 & 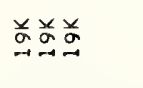 & & $\stackrel{ }{\stackrel{D}{N}}$ & 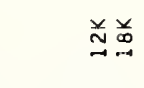 & & & \\
\hline$a^{\circ}$ & | & 总莳尌姜芯 & 吕吕并芯芯 & 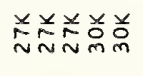 & 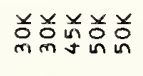 & 总兑品㒸 & 弟弟总总姜 & 前前总芯芯 & 兰㒸兑首总 & 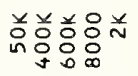 \\
\hline$\mapsto^{*}$ & 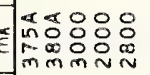 & \begin{tabular}{l}
80 \\
\hdashline 008 \\
0 \\
0
\end{tabular} & \begin{tabular}{l}
0. \\
\hdashline \\
0
\end{tabular} & 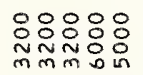 & \begin{tabular}{l}
$\circ:$ \\
\hdashline 0 \\
0
\end{tabular} & 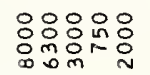 & 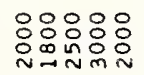 & ㅇ::ㅇㅇㅛ & 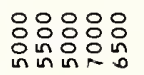 & 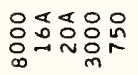 \\
\hline 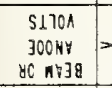 & 总总总总 & 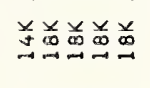 & 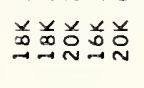 & 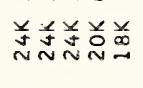 & 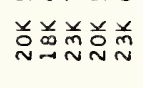 & 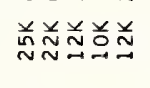 & 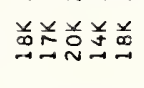 & 曾㒸㒸兑兑 & 曽总弟总 & 总芯吕高 \\
\hline$\mapsto \bar{E}$ & E E & 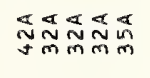 & 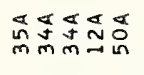 & 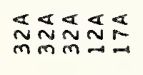 & 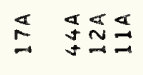 & 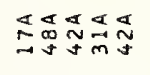 & 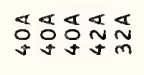 & 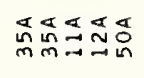 & 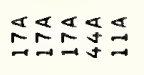 & 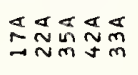 \\
\hline wั & 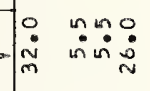 & 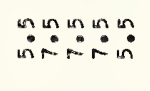 & 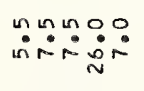 & 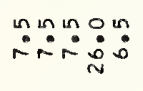 & 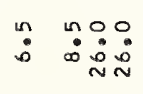 & 近 & 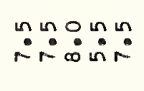 & 约 in & 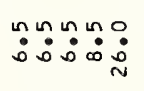 & $\because:$ \\
\hline NOIIYY马J0 & a.auuu & vuuus & vunus & unuvo & vauuv & & & yuvur & yuบu & Ua a \\
\hline$y \mathrm{dw}$ & ココ岕出 & 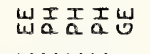 & 씸은요년 & 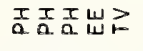 & 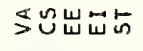 & S연 & 虬比出高 & 岁岁氙出出 & Zঙ゙出ら & 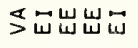 \\
\hline$\exists \mathrm{N} \cap \mathrm{\perp}$ & 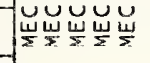 & 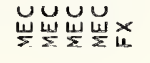 & 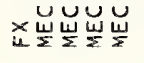 & 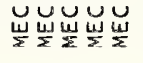 & 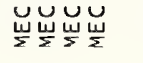 & 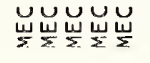 & 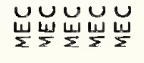 & 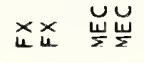 & 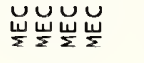 & 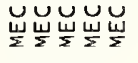 \\
\hline 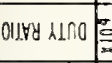 & & & & & & & & & $\stackrel{n}{n}$ & Nㅗ \\
\hline 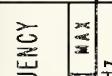 & 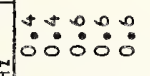 & $\because 090:$ & \begin{tabular}{l}
0.000 \\
\hdashline 0000 \\
000
\end{tabular} & $\because 9000$ & $\because \because 00: 00$ & 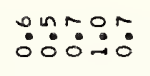 & $\ddot{\because} \because \because \because \because 0$ & $\because \because \because \because 0: 0$ & $\ddot{0}: \because \because: 0$ & $\because \because \ddot{\circ}: 0$ \\
\hline 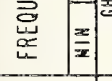 & 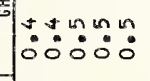 & 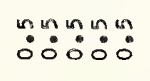 & 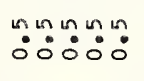 & 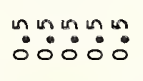 & 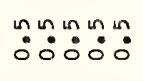 & $\ddot{0}$ & $\ddot{0}: \because 0: 00$ & $\because \because 0: 00$ & $\because \because 00: 00$ & $\ddot{0}: \ddot{0}: 0$ \\
\hline ONIX & 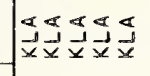 & 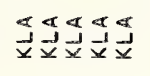 & 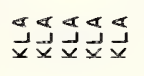 & 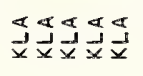 & 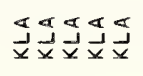 & 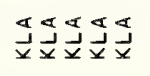 & 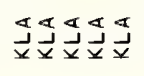 & 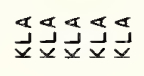 & 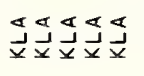 & 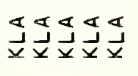 \\
\hline 70gh/S & & & $"$ & & & & & " " & & \\
\hline 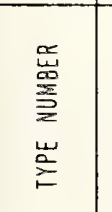 & 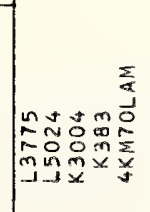 & 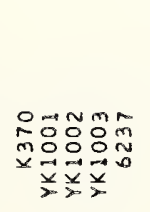 & 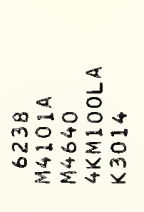 & 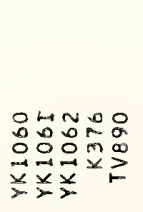 & 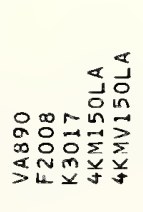 & 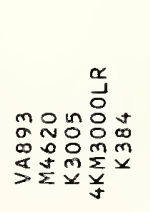 & 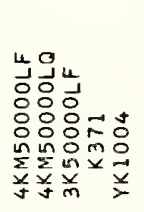 & 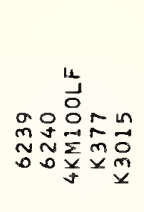 & 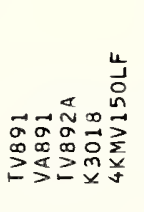 & 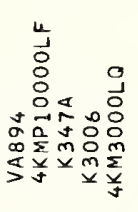 \\
\hline
\end{tabular}




\begin{tabular}{|c|c|c|c|c|c|c|c|c|c|c|}
\hline 9MITdnOS & 운운운 & 언언 & 언원오 & 800 & 논원 & 88 & Wणु & \begin{tabular}{ll}
0 \\
\multirow{3}{*}{}
\end{tabular} & पू & $\stackrel{0}{3}$ \\
\hline Allavs & $\vec{m} 山 山 心 ّ$ & 可ずす & 山テす岁す & 岁再岕ら & $\vec{m} \ddot{m} \vec{m} \vec{m}$ & $\vec{v}$ & $\vec{F} \bar{m}$ & $\vec{n} \vec{m}$ & $\vec{n}$ & $\vec{n}$ \\
\hline 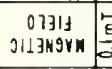 & & 品 & & 吕 & : & & $\Sigma$ & in & & \\
\hline HLOMONYG & & $\infty \quad \infty$ & oro & 000 & $+\quad \circ$ & & & 웅요 & 음으으 옹 & 우 \\
\hline 9MITTRd & E) & 웅 & 옹 & & & & & & & \\
\hline 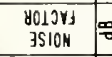 & & & & & & & & & & \\
\hline $\begin{array}{l}\text { NIY9 } \\
\text { S170A } \\
\times 173 H\end{array}=$ & : & No $\underset{\sim}{*}$ m & 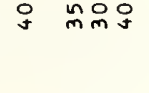 & iñs & $\hat{\sim} \stackrel{\infty}{\sim} m \bar{m} \bar{N}$ & 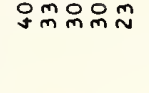 & $\stackrel{\infty}{m} m \dot{m} m \tilde{m} m$ & 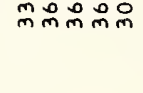 & 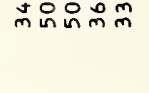 & 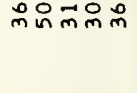 \\
\hline $\begin{array}{c}\$ 1701 \\
1001 \mathrm{NO3}\end{array}$ & 品 & & 品 & & ํํ요 & in & & & & \\
\hline 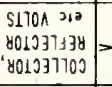 & 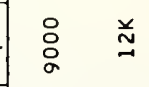 & & & $\stackrel{\stackrel{\Perp}{N}}{\sim}$ & 总 & & & & & \\
\hline $0^{\circ}$ & 㒸弟兰恙 & 芯兰芯芯首 & 弟兑兑兑并 & 兰弟总总并 & 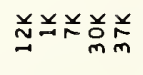 & 兰弟员品羊 & 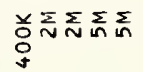 & 污 & 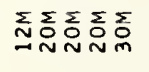 & 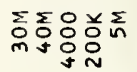 \\
\hline 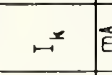 & 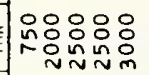 & 응요 & 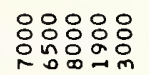 & \begin{tabular}{l}
0 \\
$\vdots$ \\
\hdashline \\
\end{tabular} & 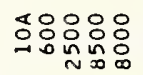 & 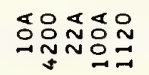 & 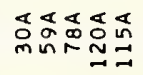 & 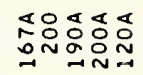 & 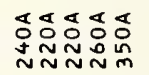 & 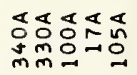 \\
\hline 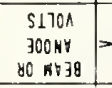 & 品总总总 & 弟弟弟兑 & 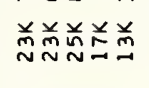 & 羊兽总皿 & 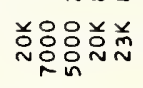 & 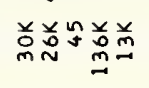 & 鸢㒸并总总 & 兑总总总兑 & 品总总㒸总 & 总总酋总总 \\
\hline 5 & 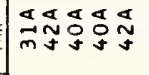 & 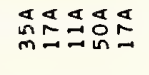 & 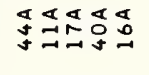 & 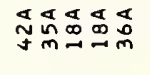 & 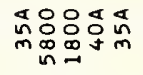 & 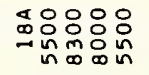 & 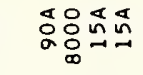 & 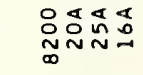 & 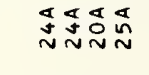 & 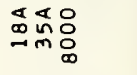 \\
\hline 山ૅ & 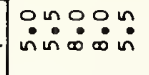 & 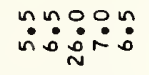 & 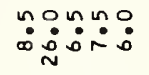 & 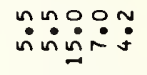 & 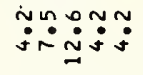 & & 遇草 & 品:움 & 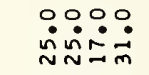 & :울 \\
\hline 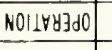 & טuטu & טบบบบ & טレンu & vuvaa & auvaa & 00000 & vasaa & Uasa & $\operatorname{aga} a$ & 00000 \\
\hline$\forall j W$ & ே岀で岀 & 岕\ら出く & 岀らさேる & 岀岕凹\品 & 跨的的的 & アロコゴ & コココこ & Шコココ山 & ペスココ & コュコココ \\
\hline \begin{tabular}{|l|l} 
3Nก। \\
\end{tabular} & 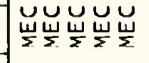 & 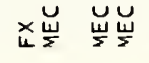 & 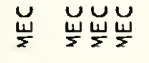 & 岀岾荘 & 岀岀 岀岀 & 岀ヶ5 & 岀岀岀岀 & 岀 岀 & 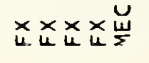 & 岁宏 始 \\
\hline $0118 y$ ansio & & & & $\stackrel{\circ}{\sim}$ & $\stackrel{\circ}{\sim}: \stackrel{0}{\sim} \sim \underset{\sim}{n}$ & 잉 : & 앙앙용 & $\cong \stackrel{\sim}{N}$ & $\stackrel{\infty}{9} \cong$ & \\
\hline 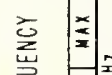 & $\because \because \because \because 0$ & $\ddot{\square}: \sigma: \sigma$ & $\because \because a: 0$ & $\because \because 0: \because$ & ヘ & 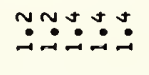 & 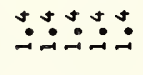 & 萿: & 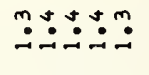 & 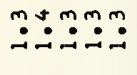 \\
\hline \begin{tabular}{l|l}
$\stackrel{\overrightarrow{3}}{u}$ \\
$\underline{w}$ \\
\end{tabular} & $\because \because 0 \therefore:$ & 范范范 & $\because \because 0^{\circ}: 0^{\infty}$ & $\begin{array}{l}\infty \\
\dot{0}: \dot{0}: \infty\end{array}$ & $\because: \because \because:$ & エִ & 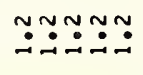 & 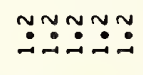 & 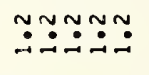 & 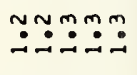 \\
\hline ONIX & 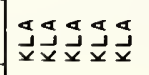 & 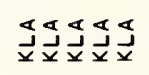 & 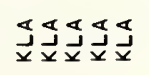 & 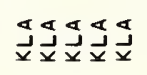 & 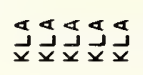 & 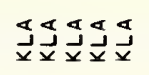 & 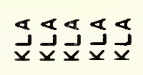 & 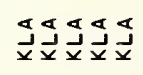 & 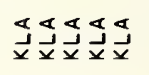 & 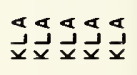 \\
\hline 70ew/S & & & & & & & & & & \\
\hline 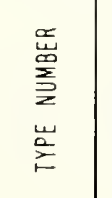 & 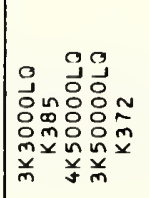 & 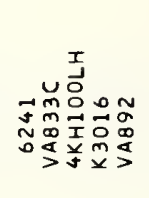 & 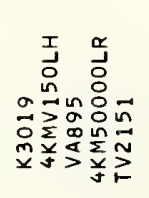 & 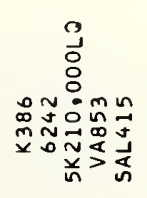 & 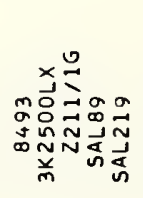 & 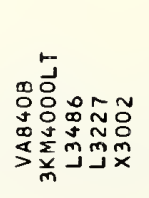 & 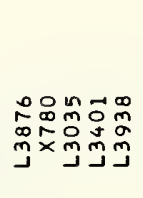 & 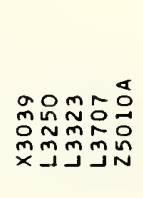 & 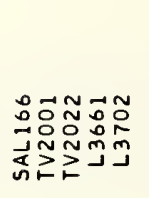 & 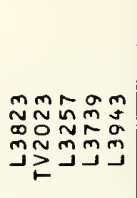 \\
\hline
\end{tabular}




\begin{tabular}{|c|c|c|c|c|c|c|c|c|c|c|}
\hline 9N17dnos & \multirow{3}{*}{$\begin{array}{r}\text { 웅엉 } \\
\text { অ岁的 }\end{array}$} & บํํำ & अणुउणु & 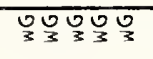 & 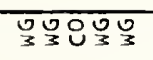 & 38 & \multicolumn{4}{|c|}{ 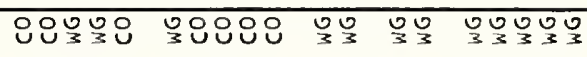 } \\
\hline Alans & & $\overrightarrow{2} \overrightarrow{2} \vec{z}$ & 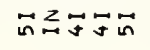 & 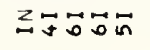 & こேすすデ゙ & $\ddot{n} z$ & $\vec{\jmath} \vec{\jmath} \vec{v} \vec{J} \vec{m}$ & $\vec{s} \vec{m} \vec{m} \vec{\sigma} \vec{m}$ & $\vec{\jmath} \vec{\jmath} \vec{\jmath}$ & $\vec{n} \overrightarrow{5} \quad \vec{n}$ \\
\hline \begin{tabular}{c|c}
071314 \\
J11399Vh
\end{tabular} & & $\sum \Sigma \sum \sum$ & & & & & $\sum_{\rightarrow=}$ & & & $\Sigma$ \\
\hline HLOMONYr $\mid \frac{\tilde{x}}{\underline{x}}$ & \pm & 몽 & 오ำ & $\infty \stackrel{\sim}{\sim}$ & 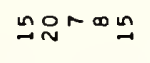 & $\cong ナ+\mathrm{p}^{\mathrm{Ln}}$ & $r \stackrel{\infty}{=}$ & $\Rightarrow \infty r \pi$ & 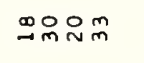 & $\cong \approx \underbrace{}_{\infty} \cong 0$ \\
\hline gNitind & & & & & & & & & & \\
\hline $\begin{array}{l}013 \mathrm{YH} \\
\mathrm{JSION} \\
\end{array}$ & & & & & & & & & & \\
\hline 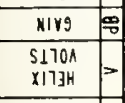 & $m$ mon & 商 & 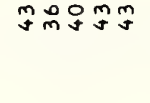 & 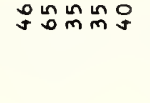 & 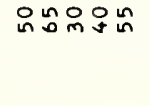 & 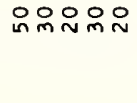 & 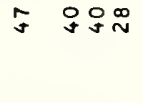 & 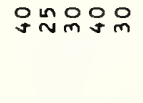 & d nn & 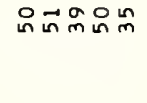 \\
\hline $\begin{array}{r}51701 \\
70811100\end{array}=$ & & & & in & 웃 & & 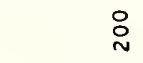 & 음 & & \\
\hline 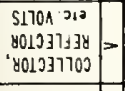 & & & & & & & & & & \\
\hline$a^{\circ}$ & 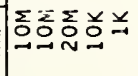 & 记兰芯弟 & 弟弟兰兑兑 & 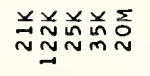 & 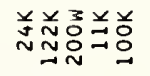 & 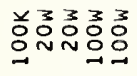 & 兰酋兑总 & 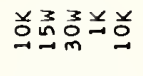 & 酋兑总导导 & $\sum_{\sin } \sum_{n=1} \sum_{n} \sum_{n}$ \\
\hline$-\infty$ & 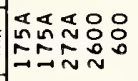 & 운 & 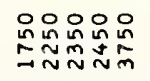 & 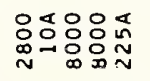 & 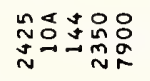 & 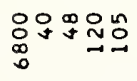 & 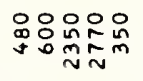 & 品品品品 & 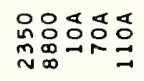 & 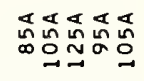 \\
\hline 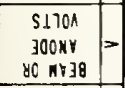 & 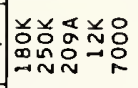 & 응요요 & 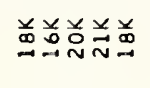 & 㒸总总总总 & 总酋总总酋 & 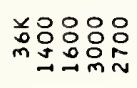 & 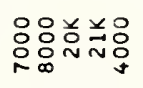 & 弟哭品品兑 & 总芯总总总 & 总总总总总 \\
\hline$\leftarrow \bar{E}$ & 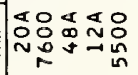 & 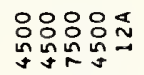 & 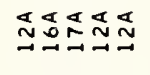 & 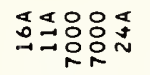 & 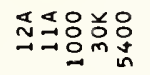 & 음 & 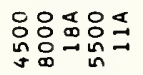 & 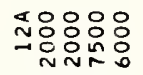 & 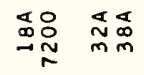 & 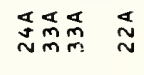 \\
\hline$\omega^{5}$ & 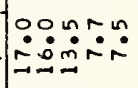 & :ํ: & 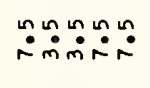 & 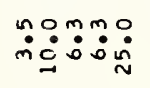 & ஜ̊m:o: & $\dot{m} \dot{m}:$ & 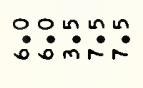 & ก:m: & $\ddot{n} \dot{0} \ddot{0} \ddot{\sim}$ & 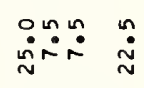 \\
\hline NOII $17 \mathrm{YJ} \mathrm{dO}$ & laaau & 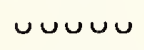 & 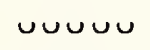 & $\cup \cup a a$ & טن & u $u$ & บบบau & บบava & $a a a a a$ & $a, a, a$ \\
\hline 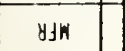 & $\exists ニ$ ถ & 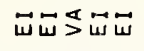 & 山岂Sび & 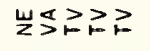 & $\vec{w}>\vec{w} \ \vec{w}$ & ルココで & 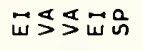 & 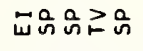 & 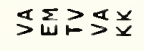 & マタSママ \\
\hline 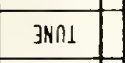 & 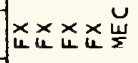 & 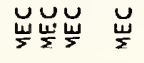 & 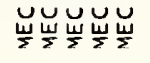 & 䢠㟧 & 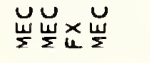 & 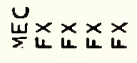 & 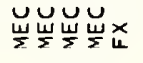 & U⿺辶巛ّ) & 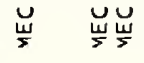 & 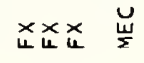 \\
\hline olyy 1100 & $N \stackrel{N}{N}$ & & & & & & 옹 & N & 온 응ㅇ & 온 \\
\hline 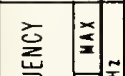 & 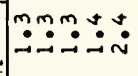 & $\dot{\sim} \dot{\sim} \dot{\sim} \dot{\sim}$ & 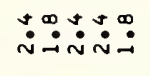 & $\begin{array}{l}\infty \\
\dot{i}\end{array}$ & $\vec{\sim} \dot{\sim} \dot{\sim} \dot{N}$ & 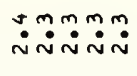 & ن் $\dot{\sim} \dot{\sim} \dot{\sim}$ & $\ddot{\sim} \dot{m} \dot{\sim} \dot{m} \dot{\sim}$ & $\dot{m} \dot{m} \dot{m} \dot{m} \dot{\sim} \dot{\sim}$ & $\ddot{m} \dot{\sim} \dot{\sim} \dot{m} \dot{m}$ \\
\hline 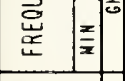 & : & 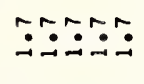 & 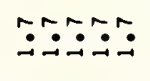 & $\because \therefore$ ¿nun & $\dot{\sim} \dot{\sim} \dot{\sim} \dot{\sim} \vec{\sim}$ & 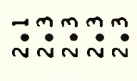 & $\dot{\sim} \dot{\sim} \dot{\sim} \dot{\sim} \dot{N}$ & 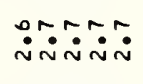 & 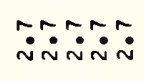 & 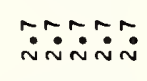 \\
\hline aNIX & | & 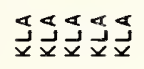 & 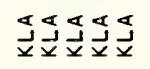 & 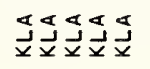 & 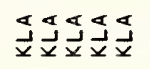 & 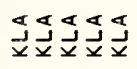 & 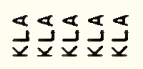 & 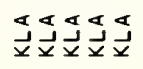 & 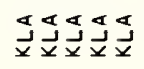 & 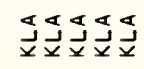 \\
\hline 708WLS & & " " " " & & & & & & & & " 1 \\
\hline 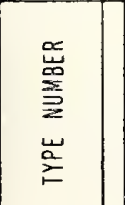 & 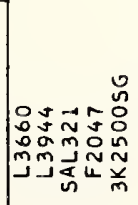 & 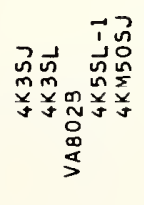 & 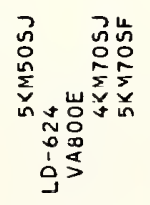 & 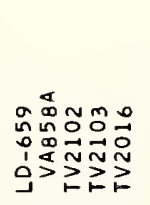 & 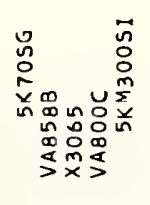 & 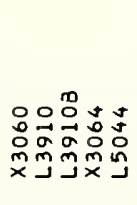 & 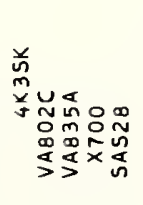 & 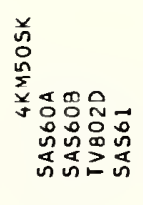 & 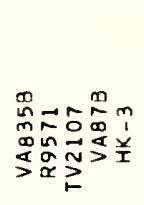 & 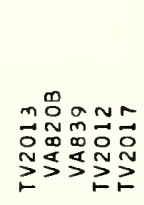 \\
\hline
\end{tabular}




\begin{tabular}{|c|c|c|c|c|c|c|c|c|c|c|}
\hline 9NITdnOS & $\stackrel{0}{3}$ & 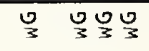 & 용요 & पूण & 오 & 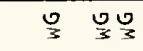 & 욱으 & एँ & $\stackrel{\square}{\Xi}$ & Wִ \\
\hline L11198s & in & 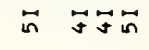 & 岗 & 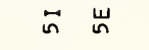 & $\vec{n} \quad$ 出 & $\vec{m} \vec{m}$ & 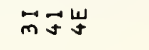 & $\vec{\alpha} \vec{n} \vec{m}$ & in & 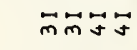 \\
\hline 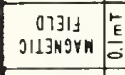 & & & $\sum \sum_{n}$ & $\Sigma$ & & $\Sigma$ & $\Sigma$ & & & $\sum \quad \Sigma$ \\
\hline HLOIMONG9 & 品品ニュ & 뜨ำㅇㅇㅇ응 & $\stackrel{\circ}{N}$ & & $\stackrel{\circ}{\sim}$ & $\stackrel{ }{\beth}$ & & $0^{\infty}$ & 윰유 & $\stackrel{\infty}{m}_{m}^{n} r \infty$ \\
\hline 9MI ITnd & & & & & & & & & & \\
\hline 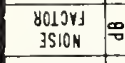 & & & & & & & & & & \\
\hline \begin{tabular}{l|l} 
NiY & \pm \\
\end{tabular} & 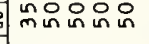 & 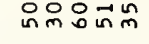 & 影 & 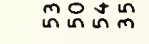 & 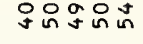 & กิm & N & 잍n $\sim$ N & 옹요 & 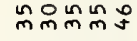 \\
\hline $\begin{array}{l}5.1701 \\
\times 173 \mathrm{H} \\
\end{array}$ & & & & & & & & & & \\
\hline $\begin{array}{c}51701 \\
1041100\end{array}=$ & & & & & & & & & & 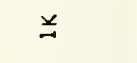 \\
\hline 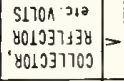 & & & & & & 㒸首 & $\begin{array}{l}\text { 兑弟 } \\
\text { N }\end{array}$ & & & \\
\hline $0^{\circ}$ & 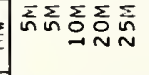 & 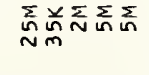 & $\sum_{n} \sum_{0} \sum_{0} \sum_{0} \sum_{N} \sum_{N}$ & $\sum \sum \sum_{N} \sum_{N} \sum_{0} \sum_{n}$ & $\sum_{n} \sum_{0} \sum_{N} \sum_{N} \sum_{\substack{n \\
0}}$ & 兑 $\sum \sum \sum \sum_{n} \sum_{0}$ & $\sum_{\sim}^{\sum} \sum_{\infty} \sum_{N} \sum_{N} \sum_{N}$ & 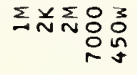 & 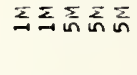 & 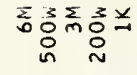 \\
\hline 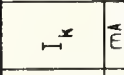 & 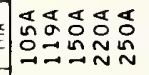 & 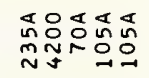 & 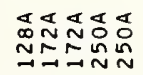 & 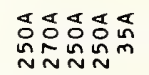 & 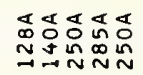 & 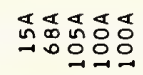 & 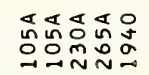 & ๔윰ㅇㅇㅛ & 雚歪出 气 & 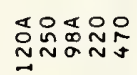 \\
\hline 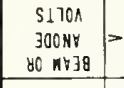 & 总总总总总 & 单总总总首 & 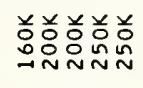 & 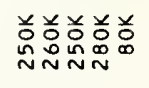 & 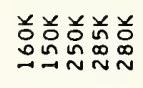 & 总㒸并兑兰 & 总㒸总总酋 & 怠总芯吠品 & 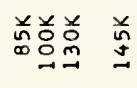 & 总品总总员品 \\
\hline$\leftarrow$ & 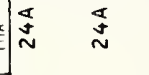 & $\stackrel{\mathbb{N}}{\mathbb{N}} \underset{\mathrm{N}}{\mathbb{S}} \underset{\mathrm{m}}{\mathbb{N}} \mathbb{N}$ & 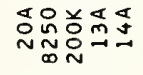 & 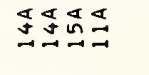 & 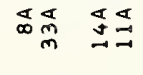 & 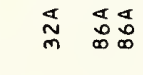 & 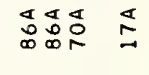 & 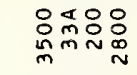 & 范 & 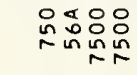 \\
\hline w" & 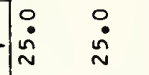 & 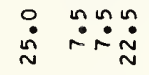 & 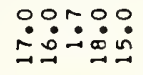 & $\begin{array}{l}0 \\
\dot{n} \\
\end{array}$ & 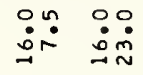 & $\stackrel{n}{\sim} \ddot{m} \dot{m}$ & $\ddot{m} \dot{0}: \dot{0}$ & 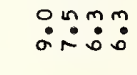 & 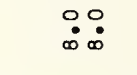 & 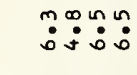 \\
\hline NOIIY8Jd0 & laoga & $a \quad 00 a$ & $a, a, a$ & $a a a a a$ & $a a a a a$ & $a a a a a$ & $a, a a a$ & $a, \cup a$ & $a, a \infty a$ & a auvu \\
\hline HJW & |マコミマる & マコミミ゙ & コココ品さ & 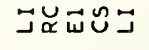 & コォロコさ & 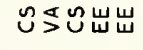 & ய山ய & コ地方品 & 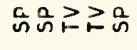 & 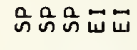 \\
\hline \begin{tabular}{l|l}
$\mathrm{N} \cap \mathrm{L}$ & \\
\end{tabular} & ×㟧 & 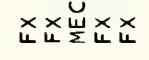 & 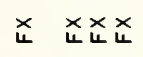 & $\begin{array}{l}\times x \times x \times \\
4 \times 4 \times\end{array}$ & 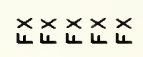 & 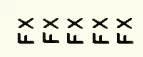 & xxx & 岀岀ヶ岀 & $\underset{u}{x \times u} \times x$ & 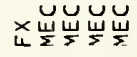 \\
\hline 0 ol18y unno & ำก유 & 웅 & 웃으. & 옾 & 윰욱 & o & $\stackrel{n}{\sim} \quad \stackrel{0}{\sim}$ & $\simeq$ 웅 & mo & 욤 \\
\hline 总 & $\vec{m} \dot{\sim} \dot{m} \vec{m} \dot{m}$ & $\ddot{m} \dot{\sim} \dot{\sim} \dot{\sim} \dot{m}$ & 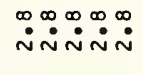 & $\dot{\sim}_{\dot{\sim}}^{\infty} \dot{\sim} \dot{\sim} \dot{\sim} \dot{\sim} \dot{m}$ & $\dot{\sim} \dot{\sim} \quad \dot{\sim} \dot{\sim}$ & $\ddot{m} \dot{\operatorname{minm}}$ & $\ddot{m} \dot{m} \dot{m} \dot{m} \dot{m}$ & $\ddot{m} \dot{m} \dot{q}$ & & 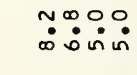 \\
\hline \begin{tabular}{|l|l|}
\multirow{x}{*}{} & $\frac{x}{x}$ \\
\end{tabular} & 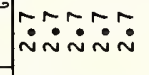 & ن் & $\dot{\sim} \stackrel{\infty}{\infty} \dot{\sim}_{\sim}^{\infty} \stackrel{\infty}{\sim} \dot{\sim}$ & 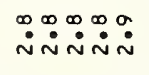 & $\begin{array}{l}\sigma \sigma \sigma \sigma \sigma \\
\dot{\sim} \dot{\sim} \dot{\sim} \dot{\sim} \dot{\sim}\end{array}$ & $\ddot{m} \dot{m} \dot{m} \dot{m} \dot{m}$ & $\because \because \ddot{n}$ & 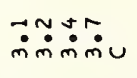 & & ט: \\
\hline ONIX & 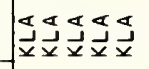 & 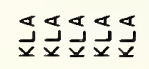 & 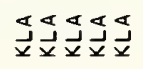 & 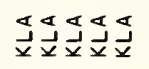 & 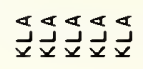 & 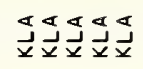 & 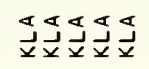 & 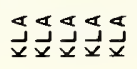 & 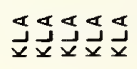 & 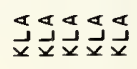 \\
\hline 708 NS & " " " & & & & & & " & " & & " " " \\
\hline 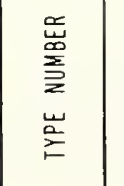 & 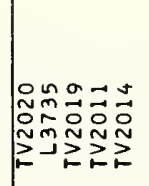 & 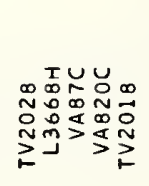 & 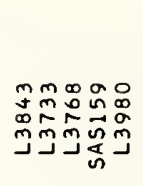 & 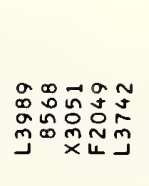 & 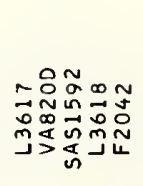 & 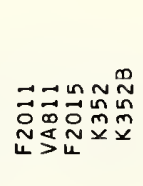 & 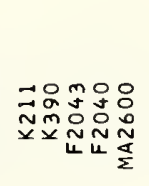 & 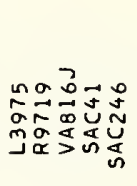 & 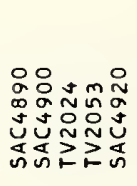 & 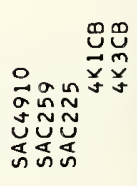 \\
\hline
\end{tabular}




\begin{tabular}{|c|c|c|c|c|c|c|c|c|c|c|}
\hline 9N1 ר dח03 & 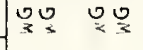 & Wִ & W & 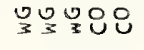 & 足芆 & 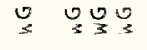 & 范 & ५७३ & 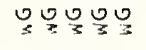 & $\bigcup_{3}{ }_{\Im}$ \\
\hline RIIAYJ & 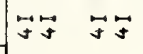 & ถูกร & デすコす & 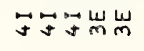 & $\vec{J} \vec{J} \quad \vec{m}$ & 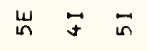 & 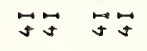 & シேேே゙て & ガすすすす & テす \\
\hline 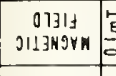 & $\sum \Sigma$ & & & $\Sigma$ & $\Sigma$ & $\begin{array}{l}\circ \\
\stackrel{0}{\infty} \\
\infty\end{array}$ & $\sum$ & $\Sigma$ & 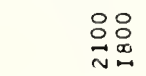 & $\Sigma \Sigma$ \\
\hline HLOMONYQ $>$ & $\simeq \simeq \operatorname{nn} \infty$ & $\stackrel{\sim}{N} \stackrel{t}{m} \stackrel{n}{N}$ & $\stackrel{\sim}{N}^{\infty \infty \infty \infty}$ & $\infty \infty m \underset{n}{n} \frac{n}{n} \underset{n}{n}$ & $\stackrel{ \pm}{\sim} \underset{\sim}{\stackrel{n}{n}}$ & $\stackrel{\circ}{\circ} \sim \infty \stackrel{0}{\sim}$ & $\infty \infty \stackrel{\infty}{\sim} \approx \stackrel{0}{=}$ & 요 뜸ㅇㅇㅇ & 品品亦 & 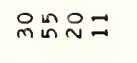 \\
\hline גחרווראי & & & & & & & & & & \\
\hline \begin{tabular}{c|c} 
yoljoy \\
בSIOH
\end{tabular}$\approx$ & & & & & & & & & & \\
\hline $\begin{array}{l}\text { NIYS } \\
S 1701 \\
X 173 H\end{array}=$ & 능ㅇㅇㅇㅇㅛ & 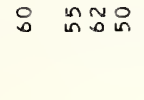 & 只会织识品 & 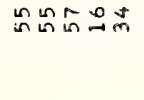 & 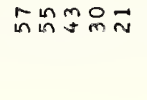 & 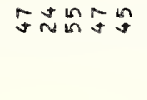 & 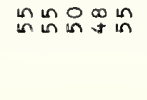 & 只品的识品 & 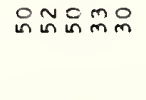 & 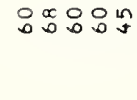 \\
\hline $\begin{array}{c}51701 \\
7081 \mathrm{NOS}\end{array}$ & $\stackrel{\circ}{n}$ & & & & & & & & 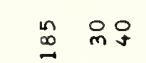 & \\
\hline 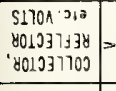 & & & & & & & & & $\underset{\sim}{\stackrel{0}{0}}$ & \\
\hline $0^{\circ}$ & $\because \underline{\sim}$ & 兰吕关弟首 & 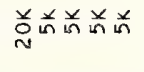 & 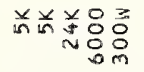 & 说兑总总 & 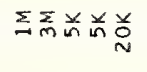 & 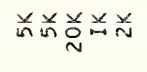 & 爫弟兰兑总 & 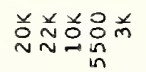 & 兑总兰并 \\
\hline$-x$ & 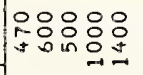 & $\begin{array}{l}\circ: \\
\circ \\
0\end{array}$ & 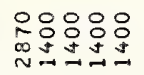 & 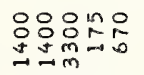 & 品号品品 & 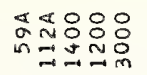 & 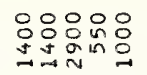 & 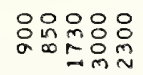 & 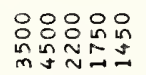 & 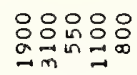 \\
\hline 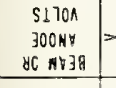 & 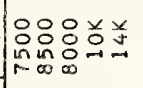 & 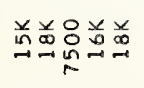 & 总总弟姜弟 & 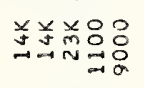 & 总盖总㒸 & 总总壬羔兑 & 姜羊咲员员员 & 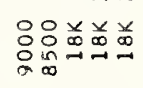 & 总总总点 & 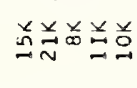 \\
\hline$\leftarrow$ & $\mid$\begin{tabular}{ll}
$O$ \\
\hdashline
\end{tabular} & 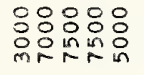 & 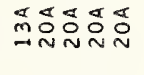 & 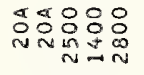 & 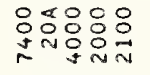 & 点 总品品 & 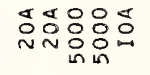 & 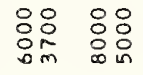 & 욤욤요 & 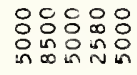 \\
\hline w" & 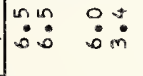 & $\begin{array}{l}0 \\
\dot{0} \\
0\end{array}$ & 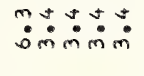 & $\dot{m} \dot{m} \ddot{m}: \dot{m}:$ & $\ddot{\circ}$ & 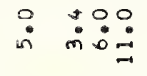 & 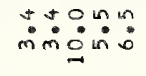 & 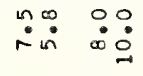 & 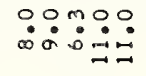 & 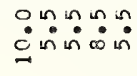 \\
\hline N011 $14 y 3 \mathrm{dO}$ & yu ves & טuบu & טuטu & unaub & vuaua & a auvo & טưu & veuve & טuטu & vauuu \\
\hline HIN & 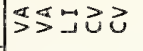 & $\vec{w} \ Z \leq$ in & 胠さ己己 & 己己่ง路 & 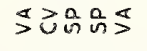 & 颌己氐洁 & 己己゙ & 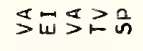 & 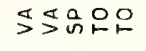 & 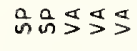 \\
\hline 3Nก1 & 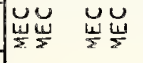 & 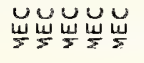 & 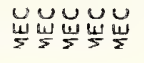 & Wu & 嵌 岀岕 & 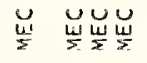 & 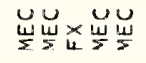 & 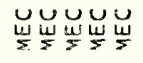 & 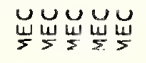 & 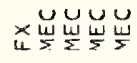 \\
\hline $0114 y$ ALro & & & & 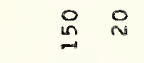 & $\simeq$ & 늠요 & & & & \\
\hline 离 & $\because \ln : 09:$ & 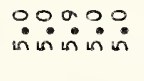 & 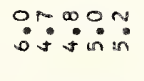 & 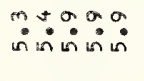 & $\begin{array}{l}\infty \\
\dot{n} \\
\dot{n}\end{array}$ & 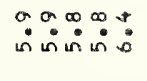 & $\because \because: \because$ & $\dot{0}: 4: 0$ & 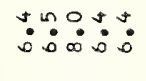 & 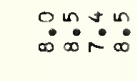 \\
\hline 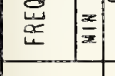 & 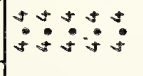 & $\begin{array}{l}* \\
\dot{q}\end{array}$ & 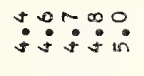 & 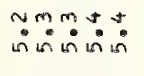 & 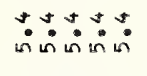 & 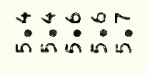 & 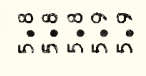 & 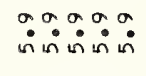 & 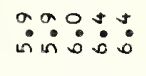 & 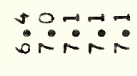 \\
\hline ONIX & 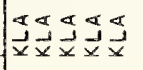 & 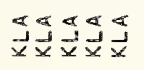 & 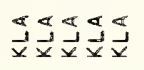 & 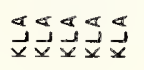 & 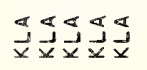 & 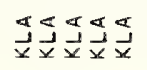 & 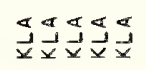 & 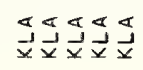 & 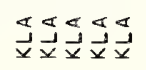 & 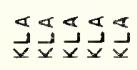 \\
\hline $708 \mathrm{~N} / \mathrm{S}$ & $"$ & & " " " & " " " & " & " & " " & & & 11 \\
\hline 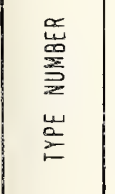 & 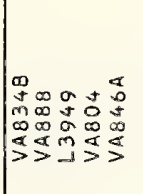 & 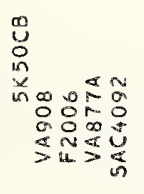 & 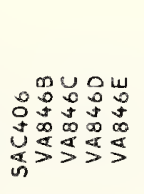 & 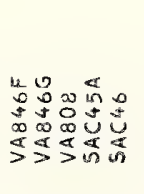 & 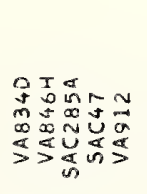 & 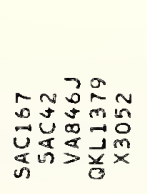 & 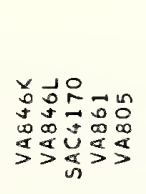 & 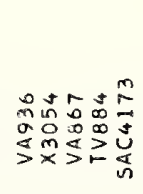 & $\begin{array}{l}m \\
m \\
0 \\
0\end{array}$ & 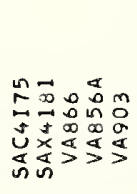 \\
\hline
\end{tabular}




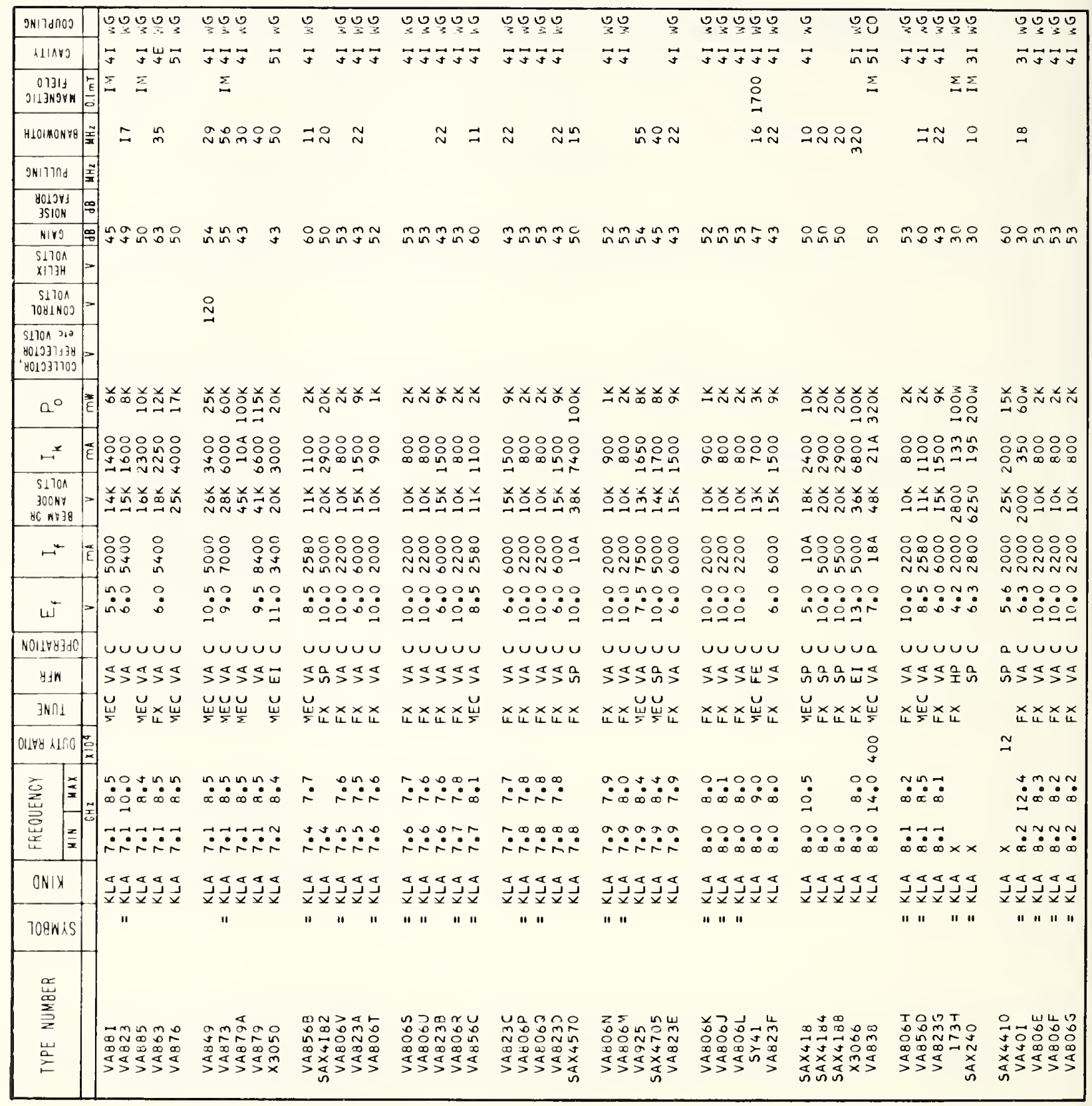




\begin{tabular}{|c|c|c|c|c|c|c|c|c|c|c|}
\hline 9N/IdnOO & OY & जO & OOSOS & OOSOY & OOOO & $\begin{array}{lll}00 \\
3\end{array}$ & 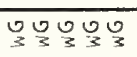 & ৩৩ৃও & 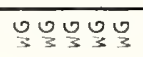 & י \\
\hline a11Ars & $\vec{J} \vec{\jmath} \vec{J}$ & コすコず & ヨ゙ずテ & テテデ & $\vec{n} \cong \backsim \overrightarrow{5}$ & $\vec{す} \overrightarrow{5}$ & 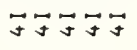 & テすずす & $\overrightarrow{\sim \vec{J}} \vec{F} \vec{J} \vec{J}$ & $\vec{m} \vec{j}$ \\
\hline \begin{tabular}{c|c}
01311 \\
J13N9YK
\end{tabular} & & & & $\sum \Sigma \quad \Sigma$ & $\Sigma \Sigma$ & $\Sigma \Sigma$ & $\sum \sum \sum \sum$ & $\Sigma \Sigma$ & $\sum \sum \Sigma$ & $\Sigma$ \\
\hline H10 MONFA & $\approx \sim \approx$ & $\approx \approx \infty$ & $\approx \sim \sim \underset{m}{\sim} \sim$ & 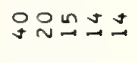 & 욨ñ & $\simeq$ 疋 & 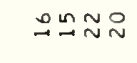 & $\approx D \quad \approx$ & $\stackrel{\sim}{\sim} \stackrel{\sim}{\sim}$ & $\stackrel{\infty}{\infty}$ \\
\hline 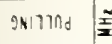 & & & & & & & & & & \\
\hline 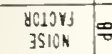 & & & & & & & & & & \\
\hline hirg & 궁ํ의 & 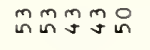 & 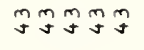 & ษกํํํํํํำ & 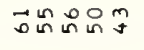 & 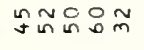 & 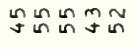 & 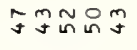 & $x \sim \tilde{n}$ & Nํํํำกn \\
\hline $\begin{array}{l}\text { S1701 } \\
\times 173 H\end{array}$ & & & & & & & & & & \\
\hline $\begin{array}{c}51701 \\
100,400\end{array}=$ & & & & & & & & & & \\
\hline 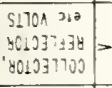 & & & & & & & & & & \\
\hline$a^{\circ}$ & 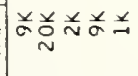 & 芯吕吕兰 & 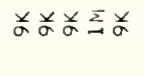 & $\stackrel{Z}{0}_{0} \cong \underline{\sim}$ & 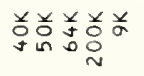 & 古兰兰兰 & 礼兑首兰 & 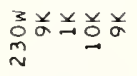 & 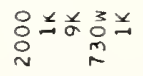 & 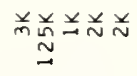 \\
\hline$-x$ & 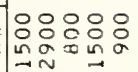 & \begin{tabular}{l}
$\circ$ \\
\hdashline \\
0 \\
$\infty$ \\
$\infty$
\end{tabular} & $\begin{array}{l}0 \\
0 \\
0\end{array}$ & 웃욤요 & 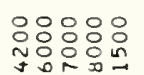 & 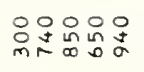 & 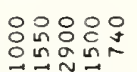 & 을 & 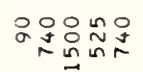 & 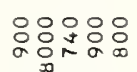 \\
\hline 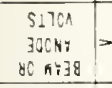 & 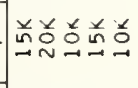 & 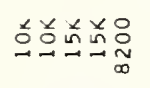 & 点芯芯怘品 & 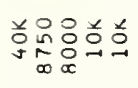 & 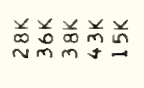 & 总品学品兑 & 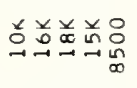 & 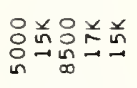 & 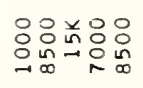 & 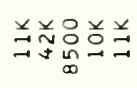 \\
\hline$\leftarrow$ & 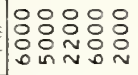 & 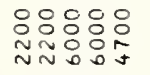 & 응영 & 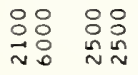 & 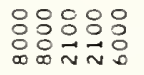 & 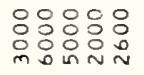 & 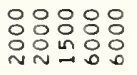 & 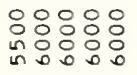 & 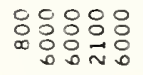 & $\begin{array}{l}\text { OOㅇㅇ } \\
\text { OOOOODO } \\
\text { NR }\end{array}$ \\
\hline w" & $\because \because \because 000$ & $\because \because 00: 0$ in & $\ddot{\bullet}: 0: 00$ & 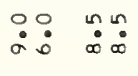 & 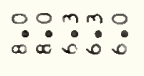 & 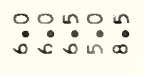 & 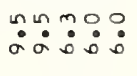 & $\because \because \because: 0:$ & $\ddot{0}: \because: 00$ & 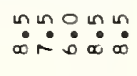 \\
\hline $1011 \% 4920$ & 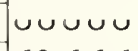 & ט৩৩ & uUuau & auטuu & $a a a b u$ & $a \cup a a u$ & $a a a u v$ & טレン & ט & 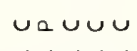 \\
\hline yin & 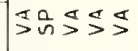 & 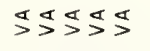 & 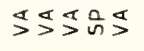 & \タタマタ & 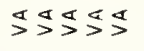 & 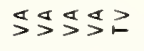 & 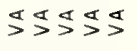 & 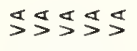 & 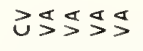 & 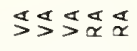 \\
\hline उNก1 & 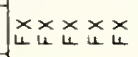 & 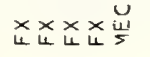 & 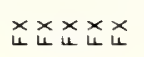 & 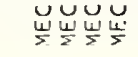 & U⿺辶ّ) & 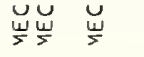 & 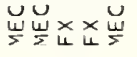 & 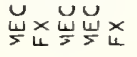 & 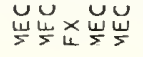 & 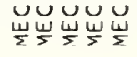 \\
\hline $015 y$ kisio & & & $\tilde{n}$ & g & No & : & $\simeq \stackrel{\text { N }}{\sim}$ & & 方 & \\
\hline \begin{tabular}{|l|l|}
$\grave{2}$ & $x$ \\
\end{tabular} & $\because \begin{array}{ccc} & +m \\
\infty & \infty & \infty\end{array}$ & 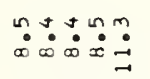 & 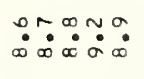 & 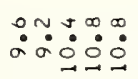 & 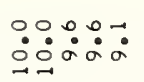 & $\dot{0} \circ \dot{0} \dot{0}: 0$ & 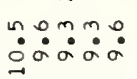 & 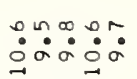 & $\begin{array}{l}\infty \circ \sigma \sim: \\
\dot{0} 0 \dot{0} 0\end{array}$ & 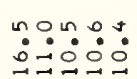 \\
\hline \begin{tabular}{|l|l|}
$\underset{\underline{w}}{\vec{d}}$ & $\frac{x}{x}$ \\
\end{tabular} & 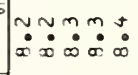 & 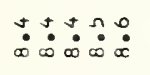 & $\dot{0}: \dot{\infty} \dot{\infty} \dot{\infty} \dot{\infty} \dot{\infty}$ & $\ddot{\sigma} \dot{\sigma} \dot{\sigma} \dot{\sigma} \dot{\alpha}$ & $\dot{\sigma} \dot{\sigma} \dot{\alpha} \dot{\sigma} \dot{\sigma}$ & 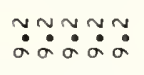 & $\ddot{\alpha} \dot{\alpha} \dot{\alpha} \dot{\alpha} \dot{\alpha} \dot{\alpha}$ & 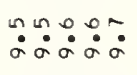 & : & $\because \because \because 0:$ \\
\hline ONIX & 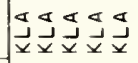 & 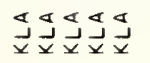 & 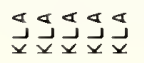 & 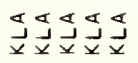 & 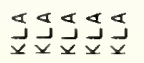 & 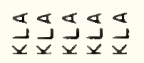 & 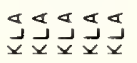 & 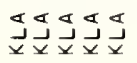 & 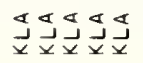 & 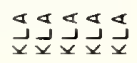 \\
\hline 709kis & $" 1$ & " " & & $"$ & & $"$ & " " " " & " & " " " " & " \\
\hline 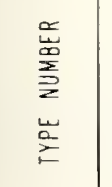 & 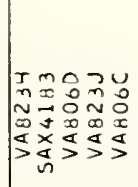 & 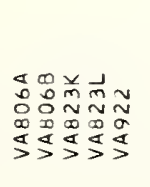 & 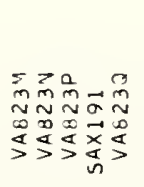 & 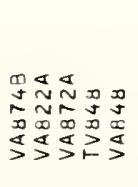 & 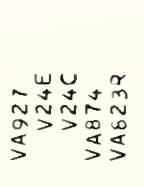 & 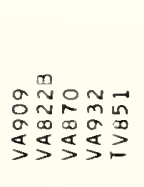 & 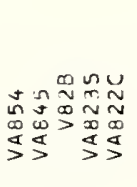 & 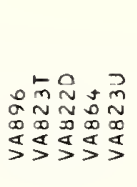 & 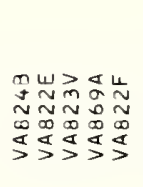 & 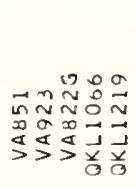 \\
\hline
\end{tabular}




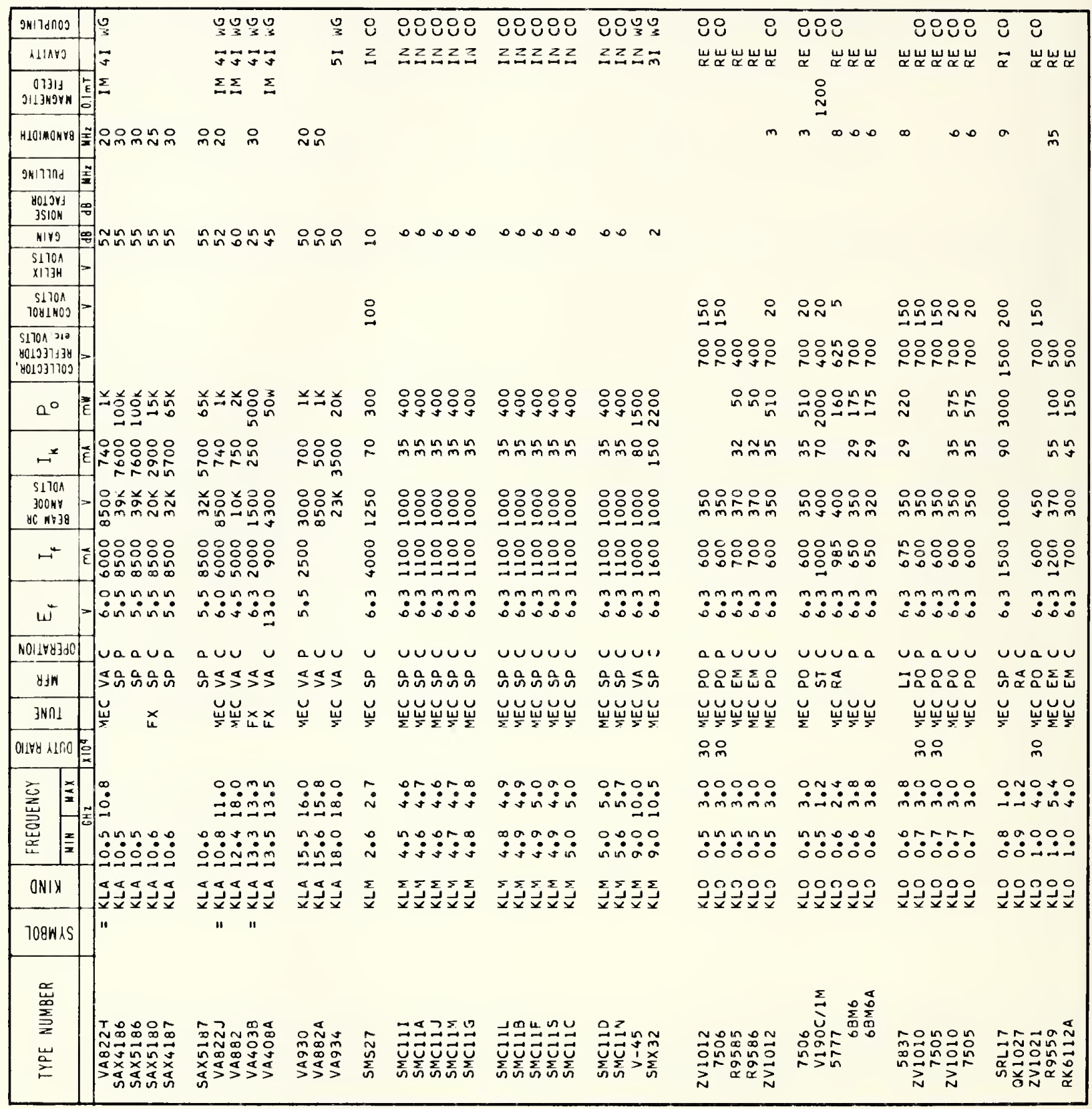




\begin{tabular}{|c|c|c|c|c|c|c|c|c|c|c|}
\hline 9MII TnOOS & 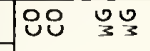 & 8888 & 888 & 옹요 & 엉 언 & 언언옳 & 88 & 원 & ॐ워용 & \\
\hline Al1AY3 & $\underset{\alpha}{\alpha} \vec{\alpha}$ & $\underset{\boldsymbol{\alpha}}{\boldsymbol{w}} \underset{\boldsymbol{\alpha}}{\mathbf{w}} \underset{\boldsymbol{\alpha}}{\boldsymbol{w}}$ & 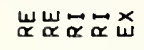 & $\underset{w}{\alpha} \vec{\alpha} \vec{\alpha} \vec{\alpha}$ & $\vec{\alpha} \vec{\alpha} \quad \vec{\alpha} \vec{\alpha}$ & $\vec{\alpha} \quad \vec{\alpha} \underset{\alpha}{w}$ & 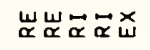 & $\underset{w}{\vec{\alpha}} \vec{\alpha} \vec{\alpha} \vec{\alpha}$ & $\vec{\alpha} \vec{\alpha} \vec{\alpha} \vec{\alpha} \underset{\alpha}{u}$ & \\
\hline $\begin{array}{c}01314 \\
\text { ग113W9Yn }\end{array}$ & $\Sigma$ & & & & & & $\stackrel{\circ}{\stackrel{\circ}{ }}$ & $\stackrel{\circ}{\stackrel{ }{*}}$ & & \\
\hline HLOMONY8 & $\begin{array}{c}x \\
x\end{array}$ & $\infty$ & $0 \infty \stackrel{0}{0}$ & 온유 & $\stackrel{\sim}{\sim}$ & $\stackrel{\sim}{\sim} \underset{\sim}{n} \sim$ & $i^{N}$ & 잉임요 & 오우으오 & 우옹 \\
\hline ג & & & & & & & & & & \\
\hline $\begin{array}{l}\text { yoljow } \\
3510 \mathrm{~N}\end{array}$ & & & & & & & & & & \\
\hline$\frac{\text { NiYg }}{\text { S1700 }}$ & & & & & & & & m & & \\
\hline $\begin{array}{l}1173 \mathrm{H} \\
\mathrm{x}\end{array}$ & & & & & & & & & & \\
\hline $\begin{array}{c}S 1701 \\
1081 \mathrm{NOO} \\
\end{array}$ & -2 & 욤욤요 & $\stackrel{\circ}{n}$ & in & in & & ? & 9 & $\stackrel{\circ}{n}$ & ํํํำ \\
\hline 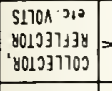 & 유 & 암윰유 & 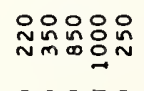 & 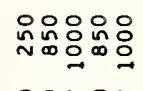 & 용요 & 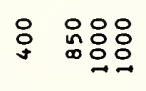 & 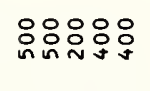 & 웅 웅욤요 & 있임임잉요 & 응용요 \\
\hline $0^{\circ}$ & 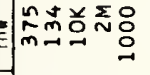 & 요요 & 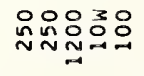 & 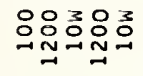 & 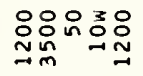 & 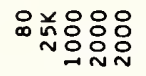 & 용ㅇㅁ임이 & 品監品品品 & 윰유유 & 总船品 \\
\hline$-x$ & zon & $m \tilde{m}$ & 虽 & 윺윺유 & 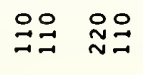 & 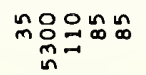 & 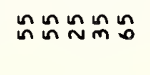 & 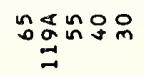 & 있일욤요 & $\stackrel{n}{\infty} \stackrel{\infty}{\sim} \stackrel{n}{\sim}$ \\
\hline $\begin{array}{r}5170 \mathrm{~N} \\
300 \mathrm{Nr} \\
80 \mathrm{nt} 3 \mathrm{~B} \\
\end{array}$ & 品总 㒸品 & 品品品品品 & 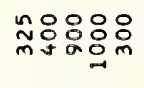 & 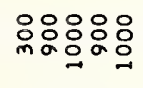 & 용 :응 & 있 & 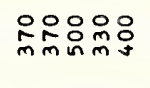 & 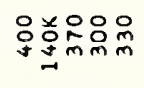 & 品品品品怘 & 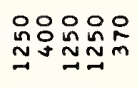 \\
\hline- & 品 & 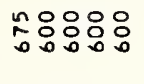 & 우숫용요 & 용융ㅇㅇ & 요 & 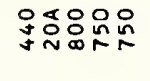 & 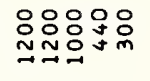 & 品 总品守 & 응음ㅇㅁㅇㅁㅇ & 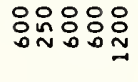 \\
\hline$\omega^{5}$ & $\ddot{0}: \stackrel{0}{0}$ & 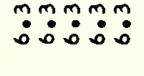 & :ח: & : & 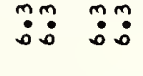 & :m: & & $\ddot{:}: \ddot{0}:$ & 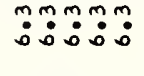 & :ח: \\
\hline NOII $\$ 43 \mathrm{dO})$ & ưuav & va auv & & & טuט & טuטu & טuטu & vauuv & a & \\
\hline Bum & 路 & ㅍㅇㅇㅇㅇㅇㅇㅇㅇ & 战옳 & ๔옹요 的 & 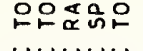 & 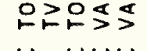 & 픈ำ & らコ恕出 & 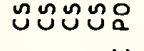 & 吃员品出 \\
\hline $3 \mathrm{~N} \cap 1$ & wü & 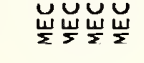 & 岀 岀岕岕 & 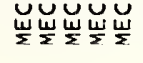 & 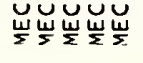 & 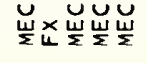 & 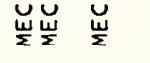 & 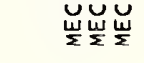 & $\stackrel{\breve{w}}{\Sigma}$ & ü \\
\hline 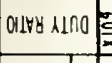 & $\stackrel{\circ}{m}$ & 윰요 & & & & & & $\stackrel{\circ}{\circ}$ & $\stackrel{\circ}{N}$ & $\stackrel{\circ}{\sim}$ \\
\hline 离 & & $\ddot{4}: \because 000$ & 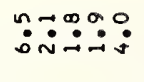 & $\ddot{\dot{N}} \dot{\sim} \dot{N} \dot{N}$ & $\dot{\sim} \dot{N} \dot{N} \dot{N} \dot{N} \dot{N}$ & $\dot{\sim} \dot{\sim} \dot{\sim} \dot{\sim} \dot{\infty}$ & $\dot{a}$ & & 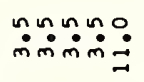 & \\
\hline 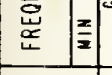 & 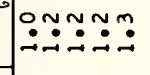 & 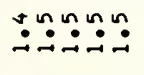 & & 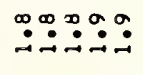 & 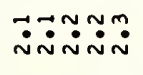 & $\dot{\sim} \dot{\sim} \dot{\sim} \dot{\sim} \dot{\sim}$ & 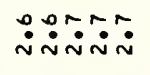 & 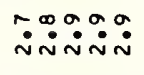 & 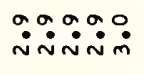 & 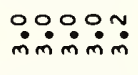 \\
\hline ONIX & 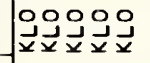 & جُ & لئ日x & & 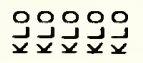 & 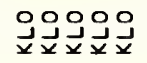 & 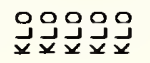 & 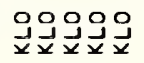 & 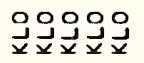 & 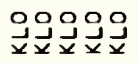 \\
\hline TogWhS & & $*$ & & & & & & & & \\
\hline 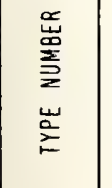 & 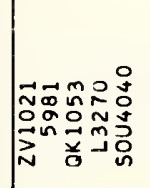 & 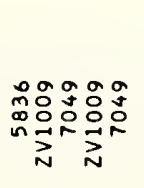 & 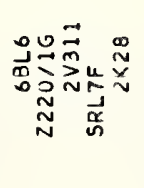 & 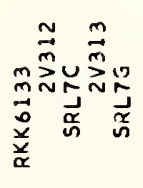 & 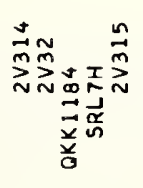 & 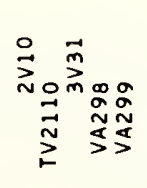 & 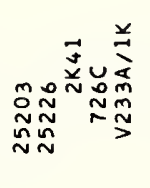 & 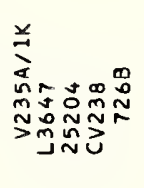 & 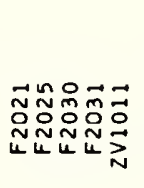 & 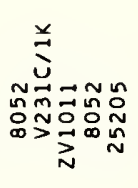 \\
\hline
\end{tabular}




\begin{tabular}{|c|c|c|c|c|c|c|c|c|c|c|}
\hline 9k17dnos & $\mid \begin{array}{ll}0 & 0 \\
3 & 3 \\
3\end{array}$ & 원ㅇㅇㅇ & $\mathrm{O}_{3}^{\circ} \cup_{3}{ }_{3}$ & 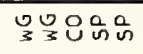 & ৪ & उon in & $\begin{array}{l}0 \\
3\end{array}$ & O & 엉요 & В8ㄴㅇㅡ \\
\hline RLIAVY & $\vec{\alpha} \vec{\alpha} \vec{\alpha} \vec{\alpha} \vec{\alpha}$ & $\vec{\alpha} \vec{\alpha} \underset{\alpha}{ } \vec{\alpha} \vec{\alpha}$ & $\underset{w}{x} \underset{\sim}{w}$ & 땀무 & $\vec{\alpha} \vec{\alpha}$ & $\vec{\alpha} \vec{\alpha} \vec{\alpha} \underset{w}{w}$ & $\underset{w}{\alpha} \vec{\alpha} \vec{\alpha} \vec{\alpha}$ & $\vec{\alpha} \underset{\alpha \underset{w}{u}}{\vec{w}}$ & $\vec{\alpha} \underset{w}{x} \vec{\alpha}$ & $\underset{w}{ } z \vec{\alpha} \vec{\alpha} \vec{\alpha}$ \\
\hline 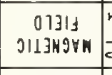 & & $\stackrel{\circ}{\stackrel{ }{~}}$ & $\stackrel{\circ}{\stackrel{\circ}{\sim}}$ & 总号 & & $\stackrel{\circ}{\stackrel{\sim}{\sim}}$ & $\stackrel{ }{\stackrel{ }{\sim}}$ & $\stackrel{\circ}{\cong}$ & 只 & \\
\hline H101MONHA & 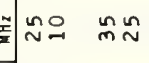 & $\stackrel{n}{N}: 0$ & No & 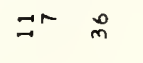 & $\underset{\sigma}{\sigma} \approx \sim$ & $\stackrel{n}{\sim} \%$ & $r \stackrel{n}{\sim} \underset{\sim}{\sim} N$ & $\stackrel{\stackrel{n}{\sim}}{\sim}=$ & $-\simeq \stackrel{N}{\sim}$ & $\stackrel{n}{m} \stackrel{n}{m}$ \\
\hline 9או17רח & & & & & & & & & & \\
\hline 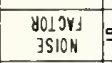 & & & & & & & & & & \\
\hline HIY & & & & & & & & & & \\
\hline $\begin{array}{l}51700 \\
\times 173 H\end{array}$ & & & & & & & & & & \\
\hline $\begin{array}{c}51701 \\
7081403\end{array}$ & & g & q & 웅 & in & q & $\stackrel{9}{q}$ & q & or & q \\
\hline 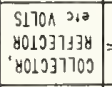 & 总品品品等 & 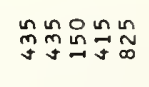 & 요 $\begin{array}{l}\text { 응 } \\
\text { 용 }\end{array}$ & 음요욤 & 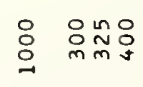 & 总 & 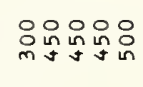 & 옷 옷유 & 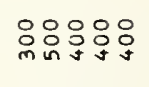 & 吕 \\
\hline$Q^{\circ}$ & ] & \begin{tabular}{l}
$\circ$ \\
\hdashline \\
0
\end{tabular} & :용요 & 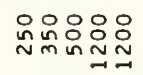 & 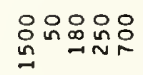 & 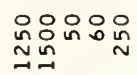 & 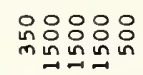 & 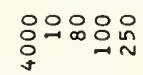 & 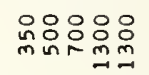 & 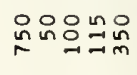 \\
\hline$-*$ & 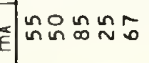 & 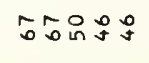 & in ${ }^{n} N$ n & 은요용ㅇㅇ & $\stackrel{\infty}{=} \stackrel{\infty}{\sim}:$ & 움 & ำเธ็กำ & 옥 등요 & ํำำธก & 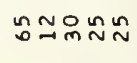 \\
\hline $\begin{array}{c}\text { S170N } \\
300 \mathrm{HY} \\
80 \mathrm{Nizg}\end{array}$ & - & 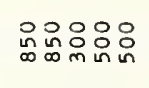 & 踣 $\underset{m}{n} \stackrel{n}{n}$ & 임윰욱을 & 응 总品品 & 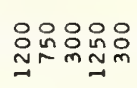 & 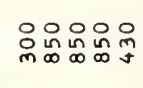 & 옹 웅용요 & 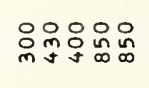 & 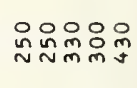 \\
\hline$\curvearrowleft$ & 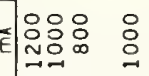 & 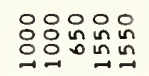 & 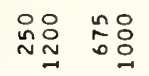 & 웅요 & 엉 웅요 & 品 & 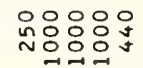 & 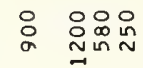 & 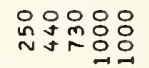 & 品品昌 \\
\hline ш & $m m m m$ & 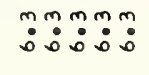 & $\stackrel{m m}{m}: m$ & mmm & 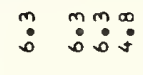 & : & mºn: & 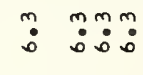 & m: & 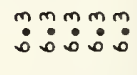 \\
\hline NOL $\angle \forall \forall \exists d O$ & טưu & טuטu & ưưu & บบưu & טuטu & ưuau & טuטu & ưư & טuטu & טưu \\
\hline yjW & $\sum_{\omega} a \underline{x} \underset{\alpha}{\alpha} \tilde{u}$ & びだேே & 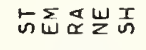 & らら立於 & 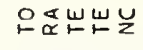 & $\vec{w}_{\alpha \propto \alpha}^{\alpha}$ 号 & レこさざ & $\sum_{w \alpha \Sigma}^{\alpha} \vdots$ & 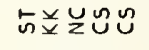 & 战宸希文 \\
\hline $3 \mathrm{~N} \cap \perp$ & $\breve{w}$ & 岀ّ & 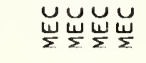 & $\frac{\breve{w}}{\Sigma}$ & U⿺乚ّ山ّ & 岕岕岕岕 & $\underset{\mathrm{w}}{\mathrm{u}}$ & 岀 & 岀岁 & 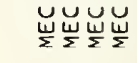 \\
\hline $0118 y$ < $1 \cap 0$ & & & & & & & & & & \\
\hline 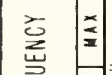 & 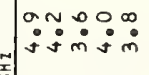 & 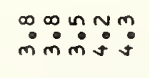 & magmin & 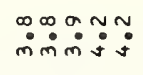 & 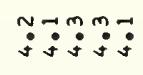 & 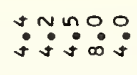 & $\because \because 8:$ & 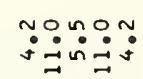 & 艧: & 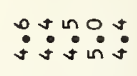 \\
\hline 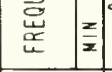 & mmmmiñ & 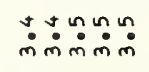 & $\ddot{m} \dot{m} \dot{m} \dot{m}: \dot{m}:$ & $\because \because \ddot{m i n} \dot{m}$ & $\ddot{m} \dot{m} \dot{m} \dot{m} \dot{m}$ & $\ddot{m} \dot{m} \dot{\infty} \dot{\infty} \dot{\infty} \dot{m}$ & $\dot{m} \dot{m} \dot{m} \dot{m} \dot{m} \dot{m} \dot{m} \dot{m}$ & 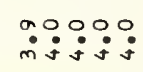 & : : : : : & 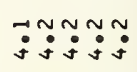 \\
\hline ONIX & 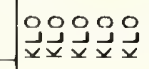 & 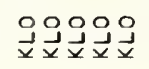 & 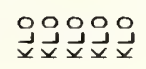 & جُ & & 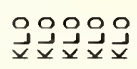 & 金金金金 & جُ & جُ & 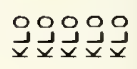 \\
\hline 708W & & & & & & $"$ & & & & \\
\hline 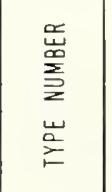 & 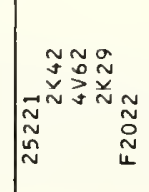 & 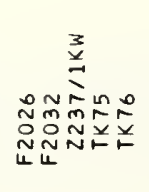 & 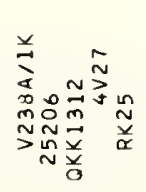 & 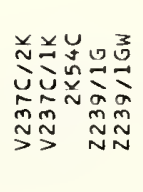 & 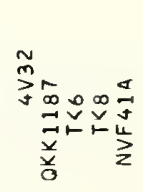 & 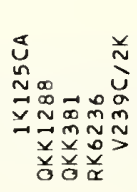 & 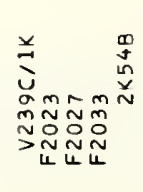 & 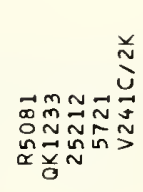 & 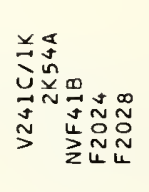 & 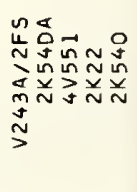 \\
\hline
\end{tabular}




\begin{tabular}{|c|c|c|c|c|c|c|c|c|c|c|}
\hline 9k17dnOS & 잃 & 언언요 & 야웡 & 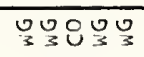 & पूण & 은물 & 엉요 & पू & 언 언 & 명요 \\
\hline telavo & $\vec{\alpha} \vec{\alpha} \vec{\alpha} \ddot{\alpha} \underset{\alpha}{w}$ & $\vec{\alpha} \vec{\alpha} \underset{w}{x}$ & $\vec{\alpha} \vec{\alpha} \vec{\alpha} \vec{\alpha} \vec{\alpha}$ & 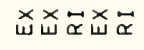 & $\vec{\alpha} \vec{\alpha} \underset{\propto}{\vec{w}} \underset{\widetilde{w}}{\vec{w}}$ & $\vec{\sim} \vec{m} \vec{\alpha} \vec{\alpha} \vec{\alpha}$ & $\vec{\alpha} \vec{\alpha} \vec{\alpha} \vec{\alpha} \vec{\alpha}$ & $\vec{\alpha} \ddot{\alpha} \quad \vec{\sim} \vec{\alpha}$ & 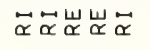 & $\vec{\alpha} \vec{\alpha} \vec{\alpha} \vec{\alpha} \vec{\alpha}$ \\
\hline $\begin{array}{c}0131 \mathrm{~J} \\
\text { ग113M }\end{array}$ & & 品 & & 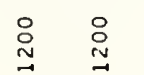 & & & & & & \\
\hline HLOMONY8 & 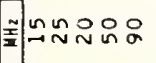 & 융요 & Jo용 & $\infty \cong \infty$ t & $\stackrel{\circ}{N}$ & tog n & ing to & $\tilde{m}$ & in & $\stackrel{\infty}{N} \stackrel{n}{m} m_{m}^{n} n$ \\
\hline 9xוาก & $\simeq$ & & & & & & & & & \\
\hline $\begin{array}{l}019 \mathrm{JJ} \\
\text { 3SIOK }\end{array}$ & 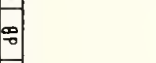 & & & & & & & & & \\
\hline $\begin{array}{l}\text { RiYg } \\
\text { S170N } \\
\times 113 H\end{array}$ & : & & & & & & & & nn & \\
\hline $\begin{array}{r}51701 \\
1001400\end{array}=$ & & of & & ơ & $\underset{j}{0}$ & $:$ & & & & \\
\hline 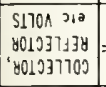 & 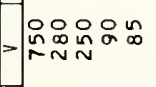 & 음욤욨요 & 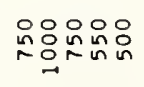 & 踪品的品 & 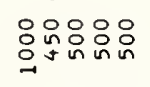 & 요욤요 & $\begin{array}{l}\text { 品气 } \\
\text { In }\end{array}$ & 음 & 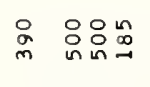 & 品 \\
\hline$a^{\circ}$ & 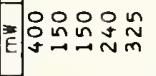 & 응용요욤요 & 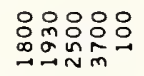 & 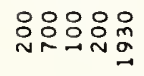 & 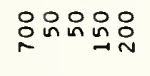 & 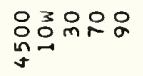 & 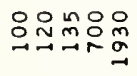 & 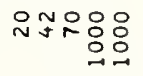 & $\begin{array}{l}\circ \\
\therefore\end{array}$ & 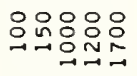 \\
\hline$\mapsto$ & 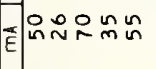 & 욤용용 & 웄음욤요 & ñ & 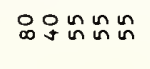 & 이욤ำ & $\tilde{m} \sim{ }_{\infty}{ }_{\infty}{ }_{\infty}$ & inn & 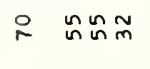 & 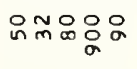 \\
\hline 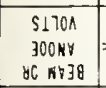 & $=$ = & 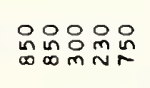 & 임임이 & 윰었윰요 & 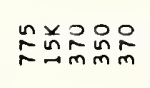 & 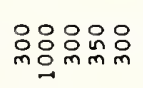 & 있윰융요 & 있m $\underset{m}{\circ} \stackrel{\circ}{\circ}$ & 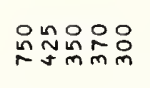 & 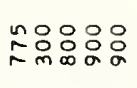 \\
\hline- & 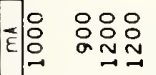 & 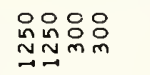 & 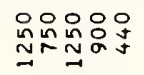 & 을웜웜요 & 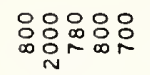 & 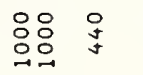 & 守道 & 品品 & 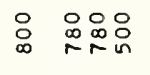 & 응융요 \\
\hline$\omega^{\longleftarrow}$ & 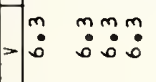 & 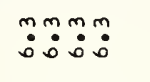 & 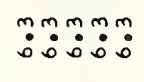 & $\ddot{m}: \ddot{m}: m m$ & $\ddot{m}: \ddot{m}^{m}:{ }^{m}: \ddot{m}^{m}:$ & $\ddot{m}: \stackrel{m}{m}:$ & $\ddot{n}: \stackrel{m}{m}: \stackrel{m}{:}$ & $\ddot{m}: m: m$ & 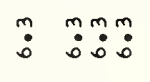 & $\stackrel{m}{m}: m{ }^{m}:{ }^{m}: \stackrel{m}{:}$ \\
\hline No1 14863d0 & טuטu & טUu & טuטuu & טuטuu & טuטuu & טu & 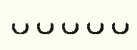 & uUu & טบט & uบuUu \\
\hline$y+\mathrm{H}$ & 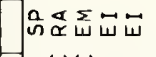 & 岀ららぬ & 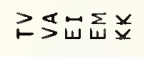 & らと泣らす & 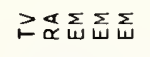 & 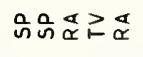 & 赔岗で & マタ乐品コ & コ岗泣勏岗 & 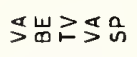 \\
\hline $3 \times \cap 1$ & 岀岀区 & 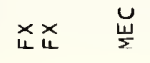 & 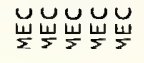 & 岀岕 岁 & 岀岀岀岀岕 & ×㐅ェ & 岕岀岀岀岀 & 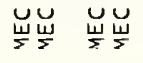 & 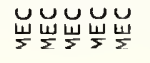 & 岀佂岀岀 \\
\hline clety $<1 \cap 0$ & & & & & & & & & & \\
\hline 离 & 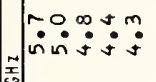 & 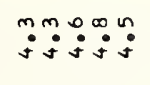 & 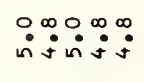 & 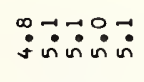 & 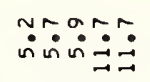 & 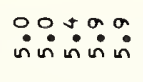 & 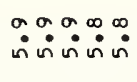 & 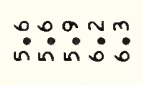 & $\because \because N N a$ : & 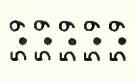 \\
\hline \begin{tabular}{l|l|}
$\substack{\bar{w} \\
w}$ & $\frac{x}{x}$ \\
\end{tabular} & 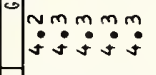 & 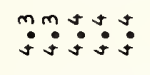 & 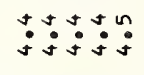 & 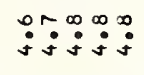 & 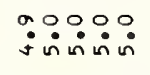 & 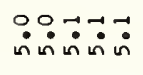 & 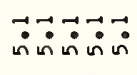 & 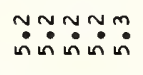 & 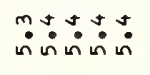 & 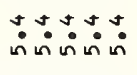 \\
\hline ONIX & لجُ & 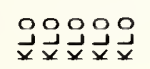 & & & 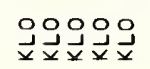 & 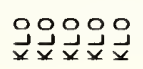 & & 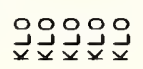 & 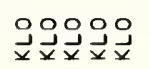 & 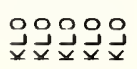 \\
\hline $708 \mathrm{w}$ <S & & & $"$ & & & " & & $"$ & & \\
\hline 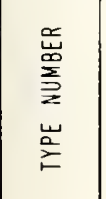 & 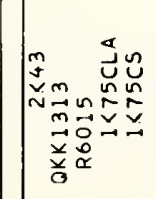 & 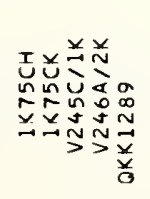 & 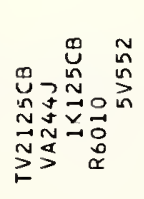 & 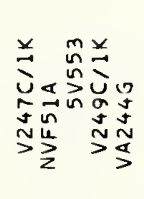 & 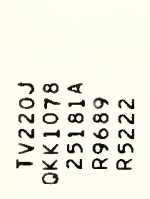 & 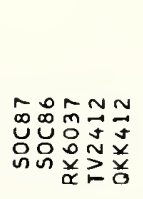 & 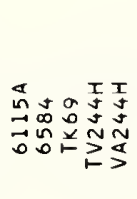 & 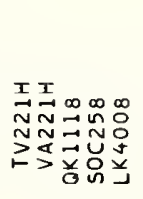 & 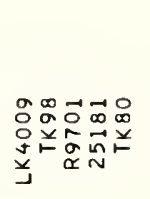 & 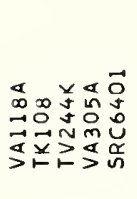 \\
\hline
\end{tabular}




\begin{tabular}{|c|c|c|c|c|c|c|c|c|c|c|}
\hline 9M17dnos & \begin{tabular}{|ll}
$W_{3}$ & 5 \\
3
\end{tabular} & ₹O & 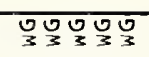 & 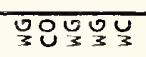 & पO & पOW\$3 & WOWW & 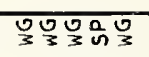 & 뭉웡 & Oত \\
\hline 111AY3 & $\vec{\alpha} \vec{\sim} \vec{\alpha} \vec{\alpha} \vec{\alpha}$ & $\underset{\alpha}{\ddot{\alpha}} \quad \underset{w}{w}$ & $\vec{\alpha} w \vec{\alpha} \vec{\alpha} z$ & $\vec{\sim} \underset{\alpha}{\alpha} \vec{\alpha} \vec{\alpha}$ & $\vec{\alpha} \vec{\alpha} \underset{\alpha}{\alpha} \vec{\alpha} \vec{\alpha}$ & $\vec{\alpha} \vec{\alpha} \vec{\alpha} \vec{\alpha} \vec{\alpha}$ & $\vec{\alpha} \vec{\alpha} \vec{\alpha} \vec{\alpha} \vec{\alpha}$ & $z z z \vec{\alpha} \vec{\alpha}$ & $\vec{\alpha} \vec{\alpha} \quad z z$ & $z \vec{\alpha} \vec{\alpha} \vec{\alpha}$ \\
\hline \begin{tabular}{l|l}
01711 \\
O1173NOVM
\end{tabular} & & $\Sigma$ & $\Sigma$ & & & & & & & \\
\hline HLOMONYG & : 15 & $:$ & 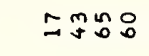 & 옹 in & $\stackrel{n}{\sim}$ & 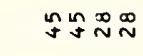 & $\stackrel{\infty}{N}: 0$ & 的的向的品 & 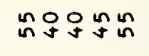 & $\stackrel{n}{m}$ \\
\hline 9 Filרnd & & & & & & & $\therefore$ & & & \\
\hline $\begin{array}{l}010 \mathrm{rJ} \\
3510 \mathrm{~N} \\
\end{array}$ & & & & & & & & & & \\
\hline Nivg & & 品 & & & & & & & & \\
\hline $\begin{array}{l}51700 \\
\times 11734 \\
\end{array}$ & & $\stackrel{D}{N}$ & & & & & & & & \\
\hline $\begin{array}{c}51701 \\
10011403\end{array}=$ & 品 & in & in & & & & & & & \\
\hline 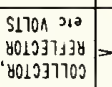 & |용 & 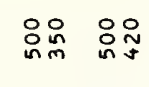 & 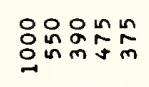 & 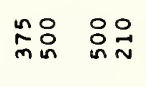 & 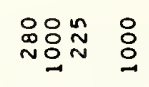 & 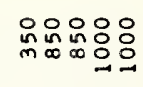 & 总 : & 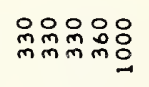 & 웄웅욤엄 & 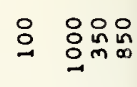 \\
\hline $\mathrm{Q}^{\circ}$ & 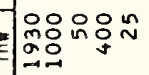 & 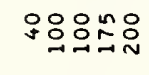 & 웃욤윰유 & 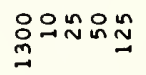 & 육윰ㅇㅇㅁ움 & :০০০০০০০ & 응응응음요 & 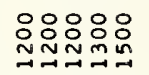 & 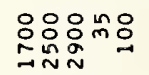 & 움웃윰영 \\
\hline$\rightarrow$ & 일옹요 & in & 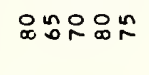 & ำ & 융용요 & 음음ㅇㅇㅛ & 중요 & 웃요욤음 & $O_{\infty}^{\infty} \vec{\infty} \stackrel{\sim}{\sim} \cong$ & 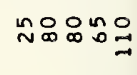 \\
\hline 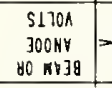 & | & 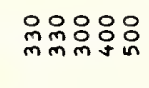 & 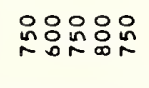 & 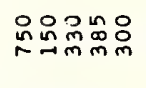 & 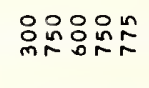 & 읏응용도 & 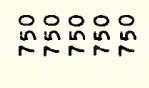 & 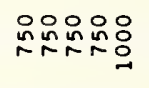 & 옳욤욤임 & 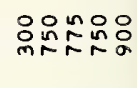 \\
\hline$F^{-}$ & 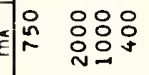 & 品品 & 윰욤욤요 & 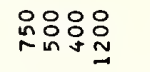 & 융요 & 잉이 & :০:০:০০ & ㅇㅇㅇ & in & 융요 \\
\hline$\omega^{4}$ & 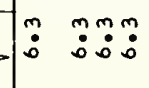 & $\ddot{m}: \stackrel{m}{m}:$ & & $\ddot{m}: \ddot{m}: \stackrel{m}{:}$ & : & 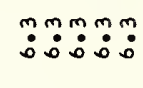 & : & $\ddot{m}: \stackrel{m}{m}: \stackrel{m}{:}$ & 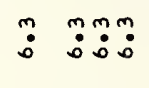 & : \\
\hline NOLIFYZdO & טuטuv & טuטu & טuט & 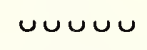 & טuטu & טuטu & טuטu & บuบuบ & ưưu & บuบu \\
\hline$\forall\} W$ & 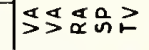 & 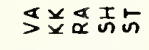 & そらコさ岂 & 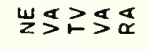 & 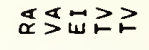 & ว゚ロォ & マママコココ & 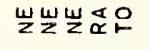 & 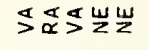 & 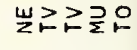 \\
\hline כאח। & 柊岕岕 岁 & 岕岕岕岕 & 岀 岀岀 & 岕岕岕区岀 & 岕谂岕 & U⿺辶巛پ & 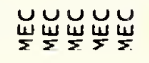 & 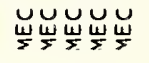 & 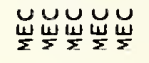 & 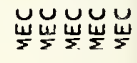 \\
\hline Ollty $\lambda \perp \cap 0$ & & & & & & & & & & \\
\hline 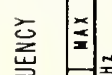 & 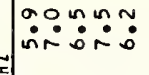 & 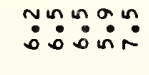 & $\ddot{\square}: \overrightarrow{0}:$ & $\because \ddot{0} \because \ddot{0}$ & :̊ำ & 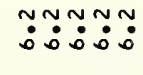 & 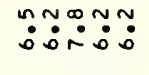 & $\because \because \because: 5$ & 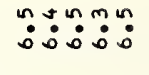 & 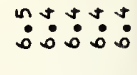 \\
\hline 言 & 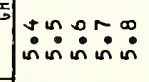 & 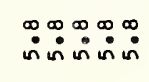 & 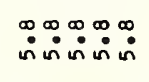 & 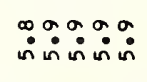 & 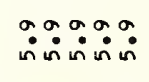 & $g a g \sigma a$ & 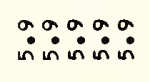 & 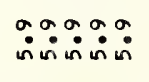 & 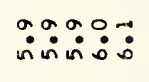 & 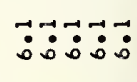 \\
\hline ONIK & 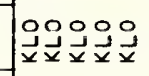 & 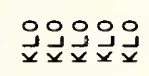 & 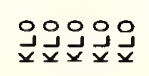 & 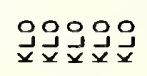 & 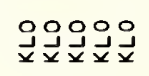 & 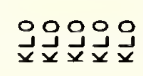 & 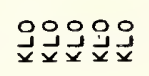 & & 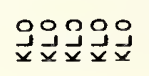 & 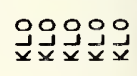 \\
\hline 108W $1 \mathrm{~S}$ & & " & & & & & & & & \\
\hline 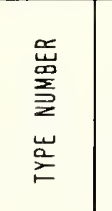 & 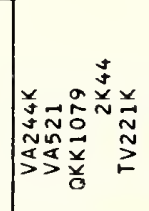 & 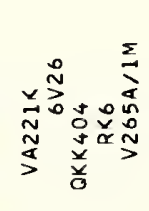 & 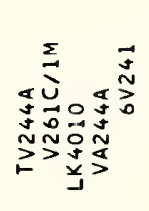 & 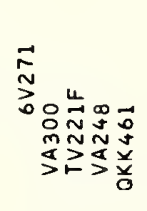 & 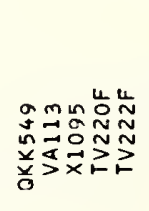 & 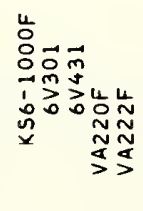 & 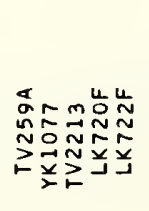 & 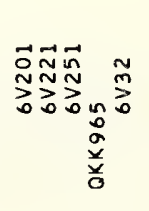 & 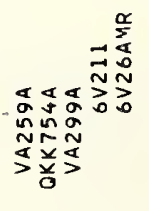 & 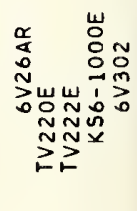 \\
\hline
\end{tabular}




\begin{tabular}{|c|c|c|c|c|c|c|c|c|c|c|}
\hline 9N17dnos & & 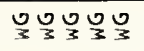 & 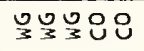 & 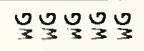 & 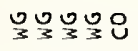 & 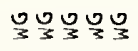 & OUß & OYOY & 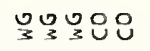 & OU: \\
\hline HIIAYJ & $\vec{\alpha} \vec{\alpha} \vec{\alpha} \vec{\alpha} \vec{x}$ & $\vec{\alpha} \geq z z \vec{x}$ & $\vec{\alpha} \vec{\alpha} \vec{\alpha} \vec{\alpha} \vec{\alpha}$ & $z Z \vec{\alpha} \vec{\alpha} z$ & $\vec{\alpha} \vec{\alpha} \vec{\alpha} \vec{\alpha} \vec{\alpha}$ & 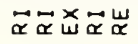 & $\vec{\alpha} \vec{\alpha} \vec{\alpha} \vec{\alpha} z$ & $\geq \vec{\alpha} \vec{\alpha} \vec{\alpha} \vec{\alpha}$ & $\vec{\sim} \vec{x} \vec{\alpha} \vec{\alpha}$ & $\vec{\alpha} \vec{\alpha} \vec{\alpha} \vec{\alpha} \vec{\alpha}$ \\
\hline 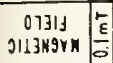 & & & & & & $\Sigma$ & & & & \\
\hline H10MONY8 & ก๊ & O & 둉요요 & 요용 & $\ln \frac{9}{4}$ & $\infty \quad \stackrel{\infty}{\sim}$ & 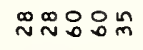 & 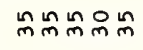 & 의 & $g$ \\
\hline \begin{tabular}{l|l} 
9k17ากd & 站 \\
\end{tabular} & $\therefore$ & & & & & & $\stackrel{-}{-}$ & & & \\
\hline \begin{tabular}{l|l}
$0019 y$, \\
$3510 \%$
\end{tabular}$=$ & & & & & & & & & & \\
\hline \begin{tabular}{l|l} 
NIY9 & $\$ 0$ \\
\end{tabular} & & & & & & & & & & \\
\hline $\begin{array}{l}S 110 \AA \\
x 113 \mathrm{H}\end{array}$ & & & & & & & & & & \\
\hline $\begin{aligned} 517101 \\
10811003\end{aligned}$ & & & & & : & in & & & & \\
\hline 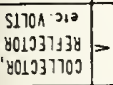 & 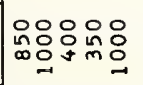 & 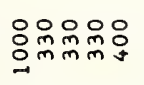 & 吠品品品品 & 육윰음 엄 & 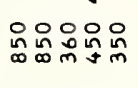 & 웅용요 & 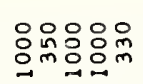 & 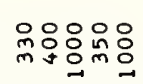 & 公 & 品品品 : \\
\hline$\Omega^{\circ}$ & 응ㅇㅇㅇㅁ & 엄웜웜 & 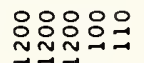 & 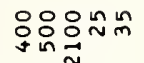 & 응응욤ㅇㅁ & ㅇㅇㅁ임융 & ㅇ:ㅇㅇㅁㅇ & : & 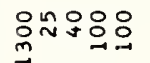 & 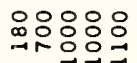 \\
\hline 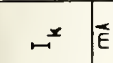 & 음ㅇㅇㅇㅇㅇㅇㅇ & 임유뭉 & 임요ㅇㅛㅛn & 웃요욤N & 을응ㅇㅇㅇㅛ & 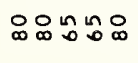 & 음잉임ㅇ & 웅임요 & $\stackrel{n}{\sim} \underset{m}{n} \underset{\sim}{\sim} \stackrel{\infty}{\sim}$ & ஸ્ง \\
\hline 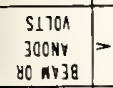 & 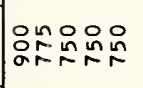 & 읏읏요솟읏 & 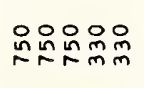 & 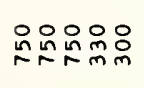 & 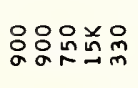 & 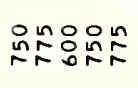 & 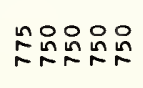 & 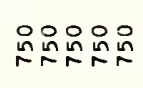 & 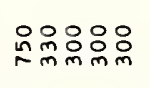 & 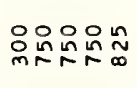 \\
\hline- & OOO০ & 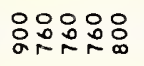 & 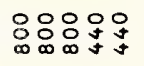 & 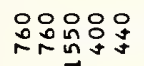 & 임용워 & 응윰용요 & ㅇㅇㅇㅇㅇㅇㅇㅇㅇ & 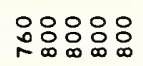 & 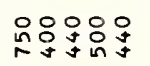 & ㅇㅇㅇㅇㅇㅇㅇㅇㅇㅁㅇㅛ \\
\hline$\omega^{5}$ & 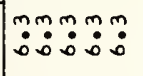 & 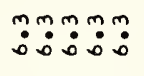 & :M:m: & : & 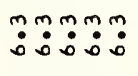 & $\ddot{0}: \dot{m}: \dot{m}: \dot{m}$ & $\ddot{0} \ddot{0}: \dot{m}: \dot{m}: \dot{0}$ & 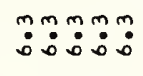 & m:mm & 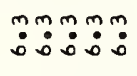 \\
\hline NOI LYy $3 \mathrm{dO}$ & טuuu & טuטu & vưue & vunuv & טuטus & ưư & טuטu & ưư & טuטu & טuט \\
\hline $\mathrm{yJN}$ & 오コココ & 岃崖崖出 & 岀山岕岕立 & 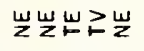 & 은요コ๔ & ママレ゙ゴ & 咲ココ岂 & 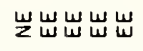 & 岂れ岂つ号 & 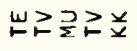 \\
\hline JNח & UuUuUu & $\begin{array}{l}u \\
\text { wu } \\
\Sigma\end{array}$ & U⿺辶ّ) & 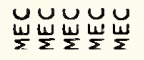 & 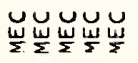 & 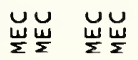 & 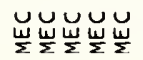 & 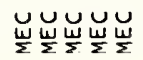 & 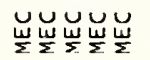 & 过Uư⿺ \\
\hline Oalyy xinO & & & & & & & & & & \\
\hline$\sum_{\underline{z}}^{\bar{c}}$ & $\ddot{\bullet}: \Delta:$ & 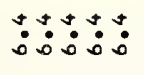 & $\ddot{0}: \dot{0} \ddot{\sim} \dot{\sim}$ & 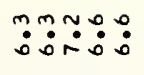 & 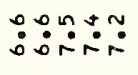 & $\ddot{0}: \ddot{0}: \dot{0}:$ & $\because \because: 0: 0$ & $\ddot{0}: \ddot{0}: 00$ & 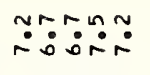 & 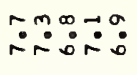 \\
\hline 总 & $\overrightarrow{0} \dot{0} \overrightarrow{0} \dot{0}$ & $\overrightarrow{0} \ddot{0} \overrightarrow{0} \dot{0}$ & $\overrightarrow{0} \ddot{0} \dot{0}:$ & : & 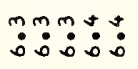 & $\dot{0}: \Delta: \Delta$ & $\ddot{0} \dot{0}: \Delta:$ & $\dot{0}: \dot{0}: \dot{0}$ & 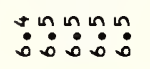 & 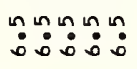 \\
\hline ONIX & & 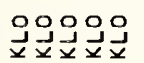 & 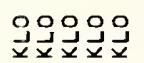 & 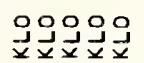 & 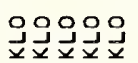 & جُ & 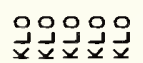 & 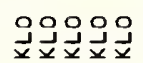 & 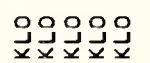 & 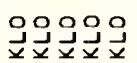 \\
\hline 70owis & & & & & & & & & & \\
\hline 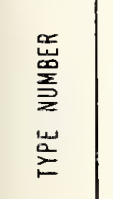 & 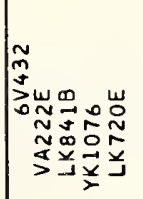 & 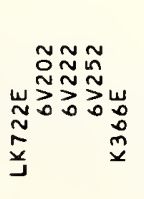 & 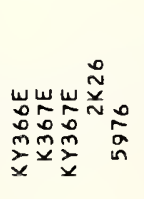 & 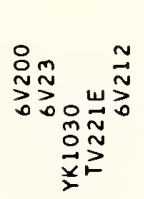 & 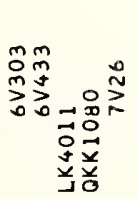 & 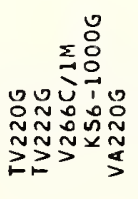 & 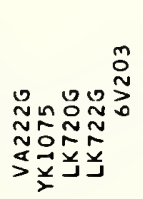 & 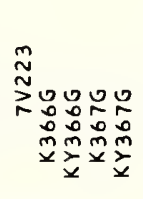 & 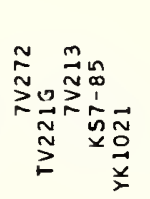 & 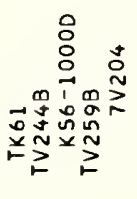 \\
\hline
\end{tabular}




\begin{tabular}{|c|c|c|c|c|c|c|c|c|c|c|}
\hline 9NITdחOS & & 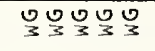 & O్ర & 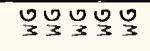 & ญॐำ路 & 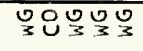 & 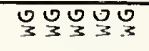 & 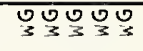 & WO & 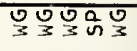 \\
\hline RLIAYS & $z_{\| \vec{\alpha}} \vec{\alpha} \quad \vec{\alpha}$ & $\ddot{\alpha} \vec{\alpha} \vec{\alpha} \vec{\alpha}$ & $\vec{\alpha} \vec{\alpha} \vec{\alpha} \vec{\alpha} \vec{\alpha}$ & $z \geq z \vec{\alpha} \tilde{\alpha}$ & $\vec{\alpha} \vec{\alpha} \vec{\alpha} \vec{\alpha} \vec{\alpha}$ & $z \vec{\alpha} \times \vec{\alpha} \vec{\alpha}$ & $\vec{\alpha} \vec{\alpha} \vec{\alpha} \vec{\alpha}$ & $\vec{\alpha} \vec{\alpha} \vec{\alpha} \vec{\alpha} \vec{\alpha}$ & $\vec{\alpha} z z z \vec{\alpha}$ & $\vec{\alpha} \vec{\alpha} \vec{\alpha} \vec{\alpha} \vec{\alpha}$ \\
\hline $\begin{array}{c}0131 \pm \\
\text { ग113\%9\% }\end{array}$ & & & & & & & & & & \\
\hline HLOMONY8 & 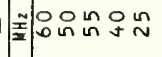 & in & $\stackrel{\infty}{\sim} \underset{\sim}{*} \underset{\sim}{\infty}: 0:$ & 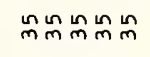 & 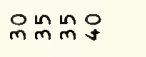 & $\stackrel{q}{\infty} \stackrel{n}{\sim}$ & in & $\stackrel{\infty}{\sim} \underset{\sim}{\sim} \stackrel{\infty}{\sim}$ & 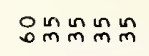 & ñ m \\
\hline 9MIITIA & $\hat{\mathbf{x}}$ & & 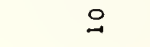 & & & & & $\stackrel{ }{\sim}$ & & \\
\hline 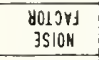 & $\infty$ & & & & & & & & & \\
\hline Hirg & 果 & & & & & & & & & \\
\hline $\begin{array}{l}51700 \\
\times 173 H\end{array}$ & & & & & & & & & & \\
\hline $\begin{array}{c}\text { S1701 } \\
1081403\end{array}$ & & & & & & N & & & & \\
\hline 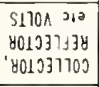 & 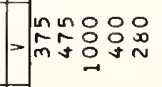 & 응 용요 & 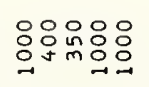 & 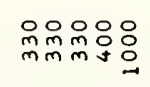 & 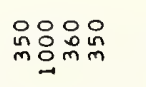 & 응요 & 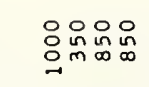 & 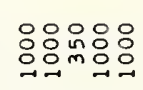 & 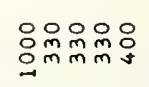 & 遂: \\
\hline$a^{\circ}$ & | & 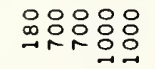 & 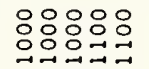 & 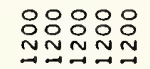 & 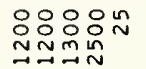 & O요ㅇㅛㅛ & 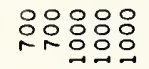 & 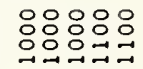 & 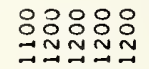 & 웄웜웜유 \\
\hline$\leftarrow$ & $\vec{E} \mid \sim ⿴ 囗 十)$ & 잉이욤으 & 임암잉ㅇ & 웃웅요 & 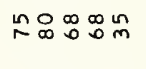 & 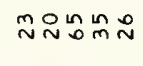 & 있욤을 & 임요 & 임우숭요 & 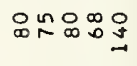 \\
\hline 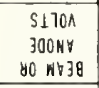 & $=\mid \begin{array}{ll}\circ \\
\end{array}$ & 웃욛ㅇㅇㅇㅇㅁㅇ & 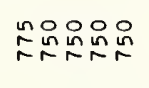 & 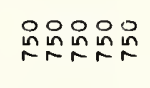 & 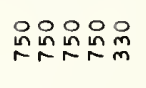 & 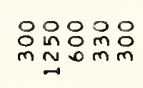 & 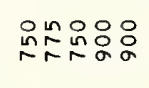 & 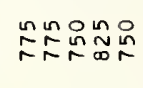 & 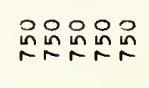 & 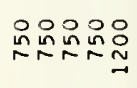 \\
\hline$\leftarrow^{-}$ & | & $\begin{array}{l}: \\
\infty \\
\infty\end{array}$ & 둥용ㅇㅇㅇ & 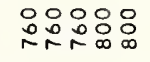 & \begin{tabular}{ll}
$\circ$ & $\circ$ \\
\hdashline & $\circ$ \\
$\infty$ & 0
\end{tabular} & 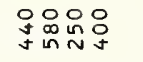 & O:০০:০ & 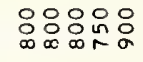 & 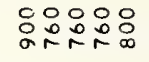 & $\begin{array}{l}\circ: 0 \\
0 \\
\infty\end{array}$ \\
\hline$\omega^{5}$ & 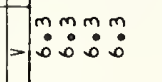 & $\ddot{m} \dot{m}: m m m$ & $\ddot{m} \dot{m}: m m m$ & $m m m m m$ & $\ddot{m} m \quad \stackrel{m}{0}$ & $\begin{array}{l}m m m \\
\dot{0} \\
\dot{0}\end{array}$ & 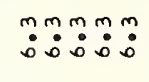 & 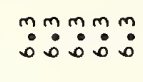 & 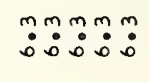 & $\stackrel{m}{m} \stackrel{m}{m} \stackrel{m}{\dot{0}} \stackrel{0}{\sim}$ \\
\hline NOII $\forall y\} d 0$ & טuטuט & טบบบ & บบบบบ & טuטu & טuטu & טบบบ & טบט & טיטיט & טuטu & טuטu \\
\hline$y j k$ & 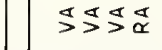 & 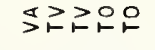 & タコマココ & 岂岂岂岀 & 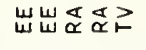 & 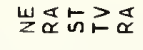 & 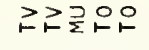 & 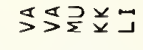 & コ岂岂岀出 & 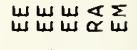 \\
\hline $3 \mathrm{~N} \cap 1$ & 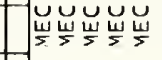 & 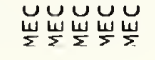 & 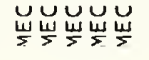 & 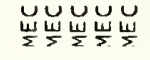 & 岀岕岕岕崖 & 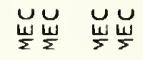 & 岀岀岕岀岀 & 崖岀岕岕 & 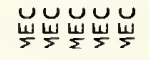 & 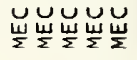 \\
\hline 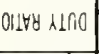 & & & & & & & & & & \\
\hline 总 & $\ddot{m} \ddot{\sim} \ddot{\sim} \dot{0}$ & 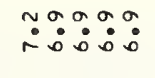 & $\because \because a^{\circ}: \sigma^{\circ}: \dot{0}$ & $\because \sigma \sigma a \sigma:$ & $\ddot{a} \dot{\sigma}: \sigma: 0$ & $\ddot{r} \because \ddot{r} \dot{r}$ & $\ddot{\because ヘ ヘ ゚ ロ ~}$ & 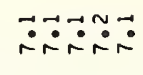 & $\ddot{\sim} \ddot{\sim} \dot{\sim}$ & 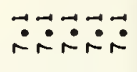 \\
\hline 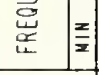 & 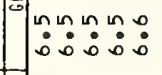 & 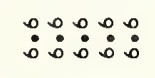 & $\because \because: \because: \bullet:$ & 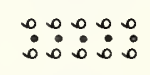 & $\ddot{0}: \ddot{0}: \ddot{0}:$ & $\because \because \dot{0}: \sigma: \dot{0}$ & $\dot{\square}: \sigma a \sigma$ & $\ddot{\circ}: \sigma a:$ & $\ddot{0}: a \circ: a$ & $\because \because a \because \because \dot{0}$ \\
\hline ONIY & | & 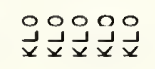 & 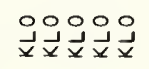 & 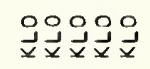 & 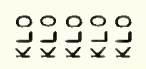 & لُجِ & 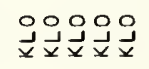 & 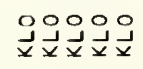 & & لُx \\
\hline $708 \mathrm{~W} / \mathrm{S}$ & & & & & & & & & & \\
\hline 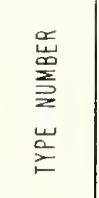 & 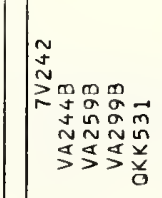 & 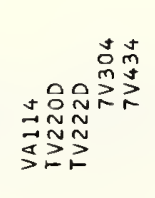 & 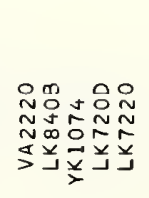 & 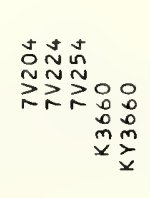 & 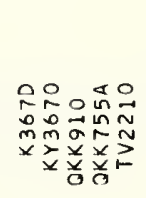 & 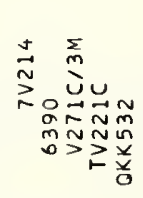 & 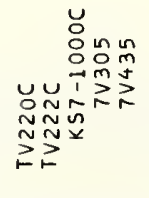 & 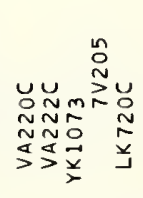 & 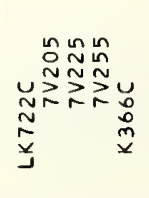 & 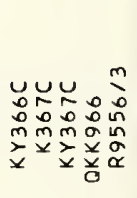 \\
\hline
\end{tabular}




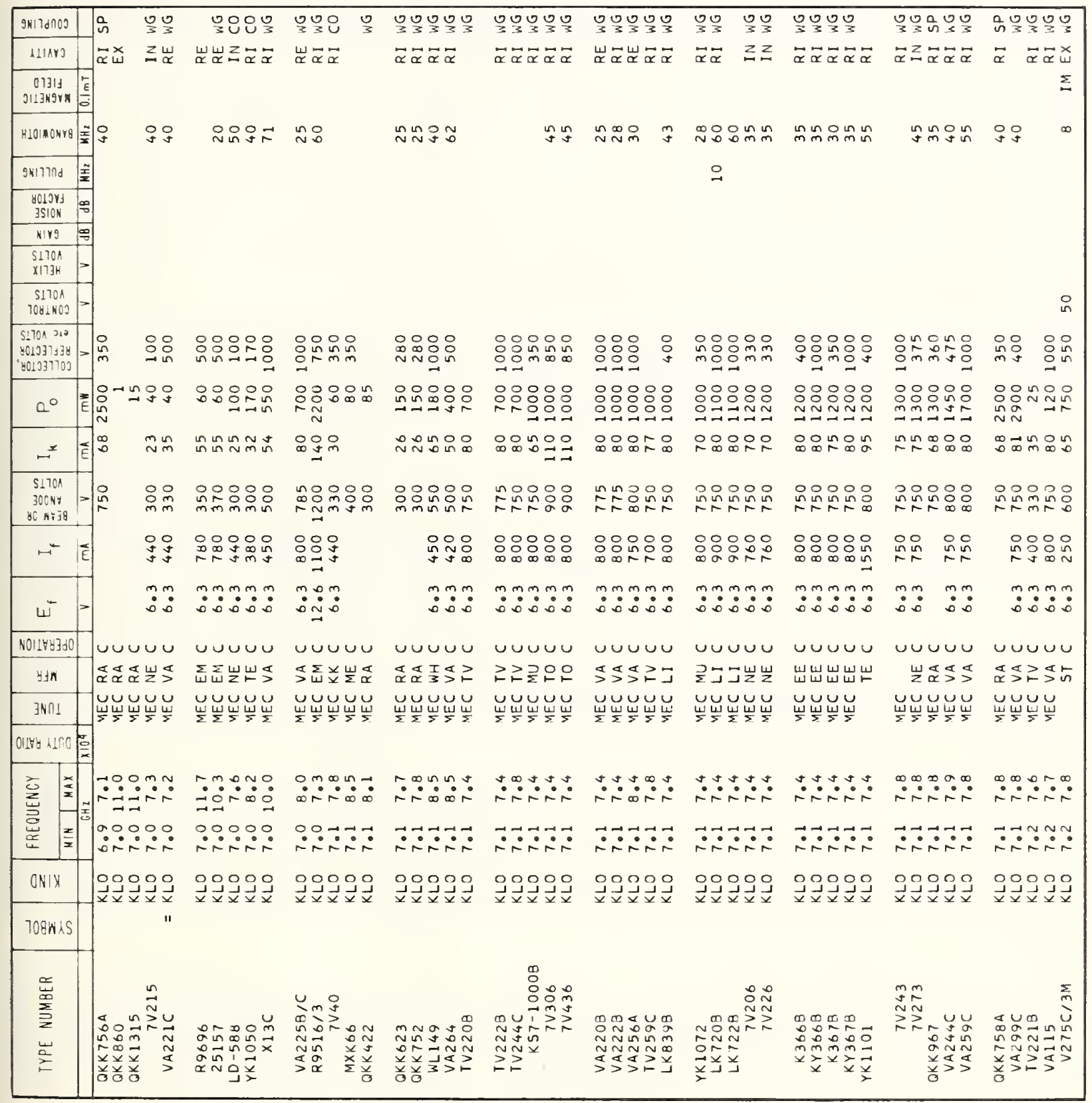




\begin{tabular}{|c|c|c|c|c|c|c|c|c|c|c|}
\hline 9אוTd InOS & 웅요 & 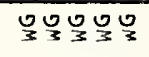 & & 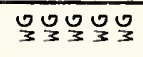 & 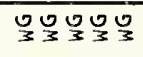 & 帚 品 & ڤ్ & 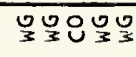 & 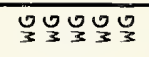 & 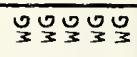 \\
\hline Alans & $\vec{\alpha} \approx \vec{\alpha} \vec{\alpha}$ & $\underset{\alpha}{\alpha} \vec{\alpha} \vec{\alpha} \vec{\alpha}$ & $\vec{\alpha} \vec{\alpha} \vec{\alpha} z z$ & $z \vec{\alpha} \bar{\alpha} \vec{\alpha} \bar{\alpha}$ & $\vec{\alpha} \vec{\alpha} \vec{\alpha} \vec{\alpha} \vec{\alpha}$ & $\vec{\alpha} \vec{\alpha} \vec{\alpha} \underset{\alpha}{\alpha} \underset{\alpha}{u}$ & $\vec{\alpha} \vec{\alpha} \geq z \vec{\alpha}$ & $\vec{\alpha} \vec{\alpha} \vec{\alpha} \vec{\alpha} \vec{\alpha}$ & $\vec{\alpha} \vec{\alpha} \geqq \vec{\alpha} \vec{\alpha}$ & $\vec{\alpha} \ddot{\alpha} \vec{\alpha} \vec{\alpha} \vec{\alpha}$ \\
\hline $\begin{array}{c}01311 \\
\text { ग113\%9rk }\end{array}$ & & & & & & & & & & \\
\hline HLOInONY & 푚ㅇㅇㅇㅇํㅇㅇㅇㅇㅇ & $\stackrel{n}{\sim}$ & 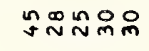 & 잉요료 & 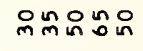 & 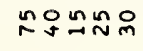 & 응 운요 & N: & 织品品 & $\tilde{N} \vec{N} \quad \infty \vec{N}$ \\
\hline גחורוואי & : & & & 이 & & & & & & \\
\hline $\begin{array}{c}\text { Holori } \\
3510 \mathrm{~N} \\
\end{array}$ & 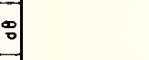 & & & & & & & & & \\
\hline Mirg & 희 & & & & & & & & & \\
\hline $\begin{array}{l}5170 n \\
\times 1173 H\end{array}$ & & & & & & & & & & \\
\hline $\begin{array}{c}51701 \\
1081 \mathrm{NOS} \\
\end{array}$ & & & & & & $\therefore$ & & & & \\
\hline 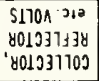 & 음임욤요 & 路品品品 & 品品品 首 & 잉윰융유 & 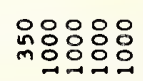 & 영 용ㅇㅇㅇㅇㅛ & : & 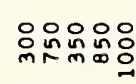 & 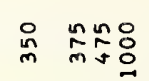 & 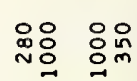 \\
\hline $0^{\circ}$ & $\mid$ & :ঃ০০০০০ & 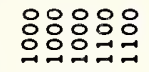 & 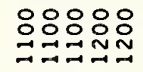 & 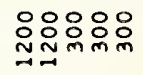 & 웅용요 & 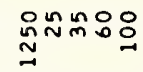 & 욪유 & 음ㅇㅇㅛ & 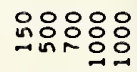 \\
\hline$\sim$ & E) & 임유요 & 움옹우우 & 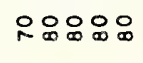 & กำ & 出经识品品 & 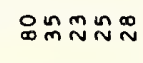 & 웅엄윰요 & 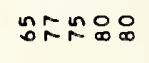 & 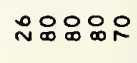 \\
\hline $\begin{array}{r}5170 \mathrm{~A} \\
30 \mathrm{NY} \\
80 \mathrm{Kr} 3 \mathrm{~g} \\
\end{array}$ & - & 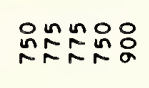 & 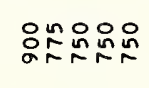 & 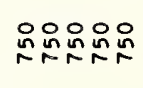 & 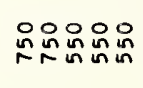 & 最莡品品 & 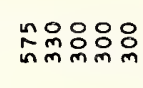 & 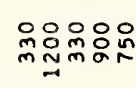 & 옷욧용요 & 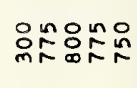 \\
\hline$H^{*}$ & 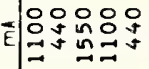 & 응용요 & 응음ㅇ요유 & 응응용요 & 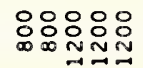 & 인융요 & 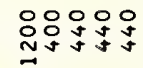 & 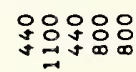 & 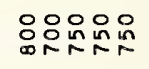 & 윰용요 \\
\hline$\omega^{ \pm}$ & & חִ & :m: & 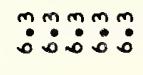 & 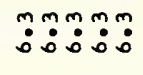 & 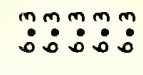 & 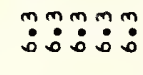 & 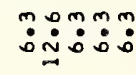 & 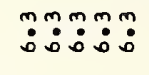 & : \\
\hline $\mathrm{NOOL} \quad 1 \forall \forall 3 \mathrm{dO}$ & |ưư & טบט & บบบบบ & voưu & บบบบบ & บบบบบ & บบบบบ & טuบu & บบบบบ & uu \\
\hline$y J W$ & 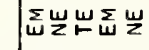 & 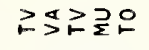 & 요골 & 岂さコ岕山 & 岀U $\frac{T}{3} \frac{T}{3} \frac{T}{3}$ & 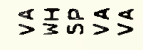 & \マ岂岩 & 뜨늠으 & 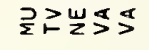 & 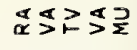 \\
\hline $3 \mathrm{~N} \cap 1$ & Uü & 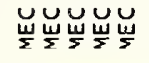 & 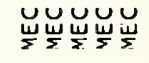 & 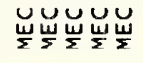 & 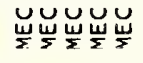 & 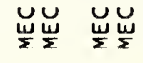 & 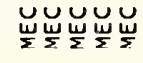 & 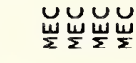 & 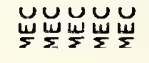 & 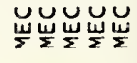 \\
\hline Ollyy $k 1 \cap 0$ & & & & & & & & & & \\
\hline 曾 & 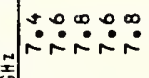 & 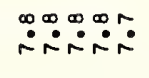 & 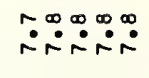 & 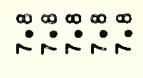 & 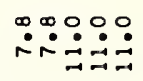 & 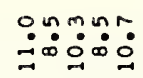 & 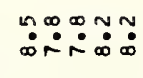 & 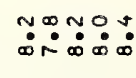 & 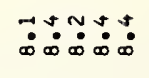 & 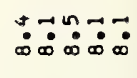 \\
\hline 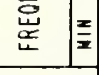 & & 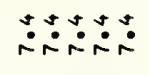 & 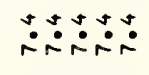 & 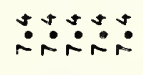 & 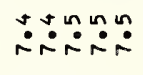 & 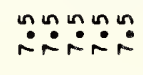 & $\ddot{i}: \ddot{i}$ & 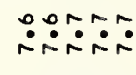 & 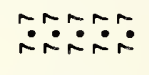 & 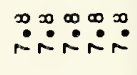 \\
\hline ONIX & لج & 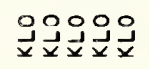 & 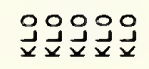 & & 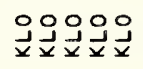 & 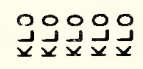 & 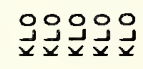 & 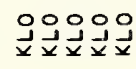 & 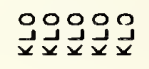 & 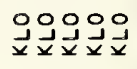 \\
\hline 70gW $\mathrm{AS}$ & & & & & & & & & & \\
\hline 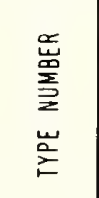 & 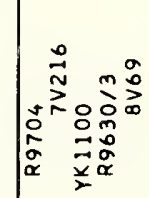 & 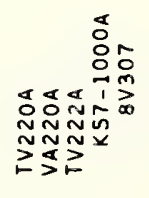 & 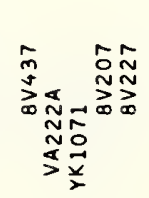 & 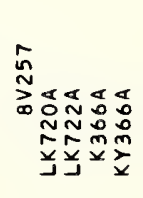 & 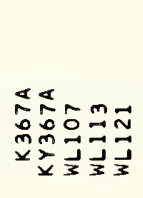 & 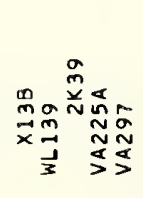 & 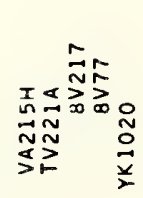 & 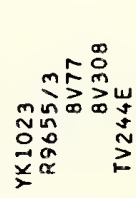 & 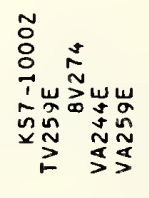 & 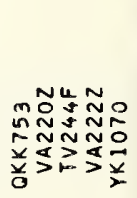 \\
\hline
\end{tabular}




\begin{tabular}{|c|c|c|c|c|c|c|c|c|c|c|}
\hline 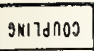 & 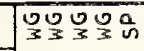 & $a_{3} 00 \%$ & 잃 & OU్ & W & 次 & 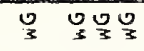 & $\stackrel{\frac{0}{3}}{\frac{0}{3}}$ & SUO & 980 \\
\hline LI1Aro & $\leq z \vec{\alpha} \vec{\alpha} \vec{\alpha}$ & $\vec{\alpha} \vec{\alpha} \vec{\alpha} \vec{\sim} \vec{\alpha}$ & $\vec{\alpha} \vec{\alpha} \quad \vec{N}$ & $\vec{\alpha} \ddot{\alpha} \vec{\alpha} \vec{\alpha} z$ & $\vec{\alpha} \vec{\alpha} \vec{\sim} \vec{\sim} \tilde{\alpha}$ & $\ddot{\alpha} \geq \vec{\alpha}$ & $\vec{\alpha} \vec{\alpha} \quad \vec{\alpha} \alpha$ & $\vec{\sim}$ & $\vec{\alpha} \vec{\alpha} \vec{\alpha} \vec{\alpha} \vec{\alpha}$ & $\vec{\alpha} \vec{\alpha} \vec{\alpha}$ \\
\hline 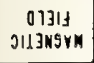 & & & & & & & & & & \\
\hline HIOLNONY8 & | & 옹요 & 용 & 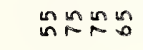 & 品 & 용ㅇㅇㅇ & 总 & 웅요요 & in & 孚角话品 \\
\hline גחרוואפ & $\therefore$ & & & & & & & & & \\
\hline 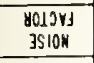 & & & & & & & & & & \\
\hline Nirg & & & & & & & & & & \\
\hline $\begin{array}{l}S 170 \Omega \\
\times 1173 H\end{array}$ & & & & & & & & & & \\
\hline $\begin{array}{c}\text { S170R } \\
30811003\end{array}$ & & & & & & & & & & \\
\hline 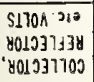 & mor:o: & 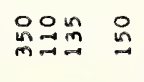 & 웅요 & 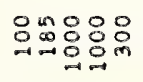 & 品品 & 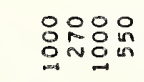 & ㅇoㅇ윰욤 & $\begin{array}{l}n \\
\stackrel{0}{0} \\
\stackrel{0}{0}\end{array}$ & 芭品品品品 & 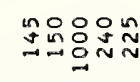 \\
\hline$a^{\circ}$ & {$\left[\begin{array}{ll}88880 \\
080 \\
7\end{array}\right.$} & 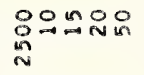 & \begin{tabular}{l}
80 \\
8908 \\
\hdashline
\end{tabular} & 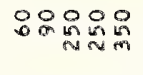 & $\begin{array}{l}08080 \\
\text { miñ }\end{array}$ & $\begin{array}{l}\text { DO } \\
\text { Mn } \\
\text { N }\end{array}$ & 응욤요 & 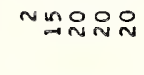 & 유유요 & 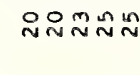 \\
\hline- & 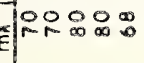 & ロッñ & 음욤점 & 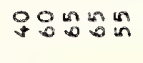 & 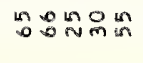 & ำ요요 & $\stackrel{n n}{n}$ in $N$ & 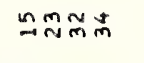 & 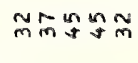 & $\approx N \tilde{N}$ \\
\hline 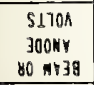 & 100000 & $\begin{array}{l}\text { 요요요 } \\
\text { nN }\end{array}$ & 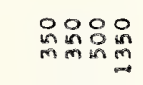 & 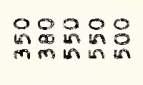 & 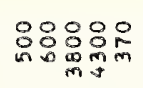 & 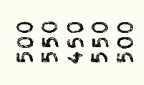 & 응용ㅇㅇㅇ & 영융요 & 용요요요 & 영융요 \\
\hline+ & 10:8: & 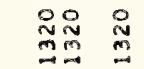 & 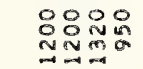 & 요욤요 & 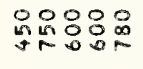 & 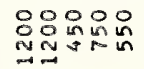 & 品品 总品 & 용요 & 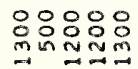 & 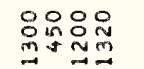 \\
\hline w" & 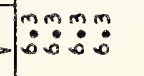 & $\ddot{3}:$ & 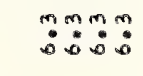 & $\ddot{0}: \ddot{0}:$ & 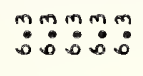 & 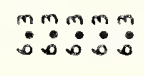 & 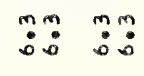 & $\ddot{0}: \dot{m}:$ & 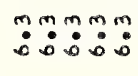 & 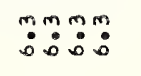 \\
\hline HollyGtdo & |ưues & vusur & unusu & yuuse & yuuve & ưuuv & ưưu & vauvu & uטuu & yuuve \\
\hline 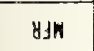 & 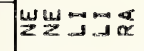 & 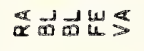 & xum & 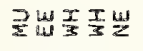 & Zsosin & 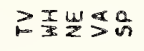 & 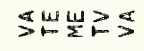 & 出古的总崖 & 㤎びマ出 & 㟧 \\
\hline J*ก! & $\mid \begin{array}{l}4 \\
\end{array}$ & 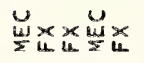 & 湠崖出宏 & 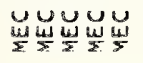 & 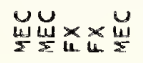 & 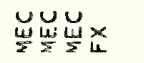 & 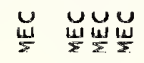 & 宏 㟧5 & I & エ吉崖岀 \\
\hline olry alnio & & & & & & & & & & \\
\hline 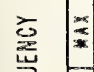 & 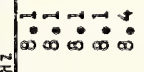 & × & 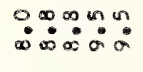 & $\because \infty$\begin{tabular}{c}
0 \\
\hdashline
\end{tabular} & $\dot{\vdots}=$ & 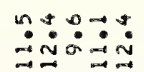 & $\begin{array}{l}n=00 \\
=0000\end{array}$ & $\because 0 \stackrel{0}{0}: 00$ & 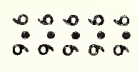 & $\because: 00$ ino \\
\hline 总 & & $\begin{array}{l}\because: 0 \% \\
\because \infty 800\end{array}$ & $\begin{array}{l}\because \because: 0 \\
\infty \\
\infty\end{array}$ & 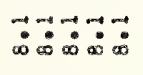 & 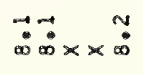 & 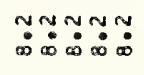 & 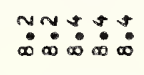 & 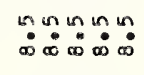 & 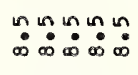 & 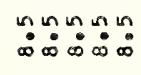 \\
\hline ONIX & $\mid \begin{array}{l}90900 \\
x \times x x x\end{array}$ & $\begin{array}{l}90900 \\
x \times x \\
x \\
x\end{array}$ & 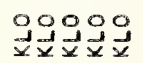 & 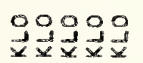 & 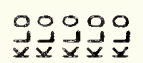 & 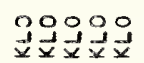 & 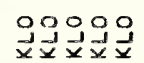 & $\begin{array}{l}00900 \\
\dot{\vec{x}} \overrightarrow{\mathbf{x}} \mathbf{x} \overrightarrow{\mathbf{x}} \mathbf{x}\end{array}$ & 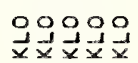 & 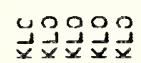 \\
\hline 708 ws & & " " " & " " & & & & & & & \\
\hline 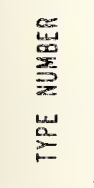 & 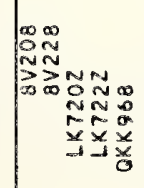 & 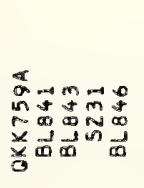 & 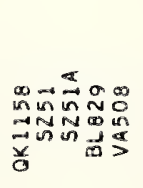 & 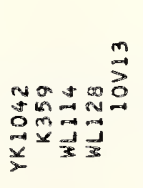 & 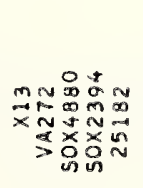 & 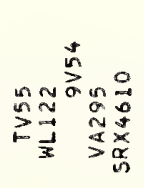 & 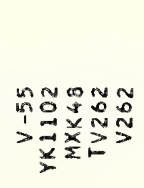 & 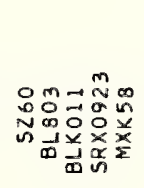 & 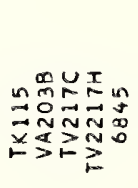 & 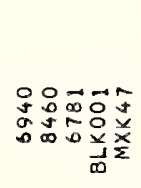 \\
\hline
\end{tabular}




\begin{tabular}{|c|c|c|c|c|c|c|c|c|c|c|}
\hline 9NiTdnOS & $\begin{array}{ll}0 & 9 \\
3 & \end{array}$ & 원 & 옹어 & O $\quad \stackrel{3}{3}$ & 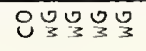 & 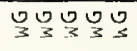 & & 원 & एँ్ & $\frac{0}{3} \frac{0}{3}{ }_{3}$ \\
\hline RLARY & $\vec{\alpha} \vec{\alpha} \vec{x} \vec{x}$ & $\vec{\alpha} \vec{\propto} \vec{\propto} \vec{\alpha} \vec{\propto}$ & $\vec{\alpha} \vec{\alpha} \vec{\alpha} \vec{\alpha} \vec{\alpha}$ & $\vec{\alpha} \vec{\alpha}$ & $\vec{\alpha} \vec{\alpha} \ddot{\alpha} \vec{\alpha} \vec{\alpha}$ & $\vec{\alpha} \vec{\alpha} \vec{\alpha} \vec{\alpha} \vec{\alpha}$ & $\vec{\alpha} \vec{\alpha} \vec{\alpha} \vec{\alpha} \vec{\alpha}$ & $\vec{\alpha} \vec{\alpha} \quad \vec{\alpha} \vec{\alpha}$ & \multirow[t]{2}{*}{$\vec{x} \ddot{x}$} & $\vec{\alpha} \vec{\alpha} \vec{\alpha} \vec{\alpha} \vec{\alpha}$ \\
\hline 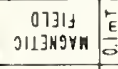 & & & & & & & & & & \\
\hline HLOMONA & unon & ㄱㅇㅇㅇ요요 & 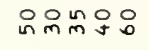 & 웡용요 & 옹ํำ & 았앙ㅇㅇㅇㅇㅇ & 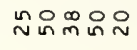 & ơ in $^{\text {in }}$ & \multirow[t]{5}{*}{$\stackrel{?}{q}$} & 유ํㅇํํํํ요요 \\
\hline 9אורורח & & & & & & & & & & \\
\hline 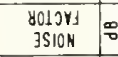 & & & & & & & & & & \\
\hline \begin{tabular}{l|l}
$N \mid Y 9$ & To \\
\end{tabular} & & & & & & & & & & \\
\hline $\begin{array}{l}51700 \\
\times 173 H\end{array}$ & & & & & & & & & & \\
\hline $\begin{array}{c}51701 \\
1081003\end{array}$ & $\frac{n}{m}$ & & & in & & & $\underset{m}{\stackrel{n}{n}}$ & 路 & 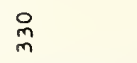 & $\begin{array}{l}\text { OO } \\
\text { No }\end{array}$ \\
\hline 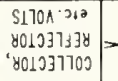 & 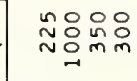 & $\stackrel{n}{ \pm} \stackrel{\circ}{\stackrel{0}{N}}$ & 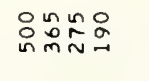 & $\stackrel{n \sim}{\sim} \underset{\sim}{N}:$ & 응요응 & 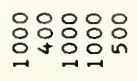 & 옹 & 오ํ & 음음 & 응음 \\
\hline$a^{0}$ & $\stackrel{\sim}{\sim} \sim \tilde{N} \cong \tilde{N} \sim \tilde{m}$ & 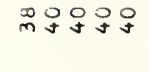 & 字隹出品品 & 只品品品 兄 & ํํำำก & 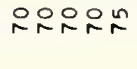 & 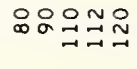 & 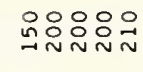 & 施品品品品 & 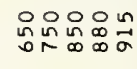 \\
\hline 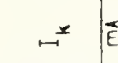 & 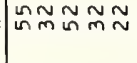 & $\stackrel{n}{\sim} \stackrel{n}{m}$ & ํㅡ으요 요 & 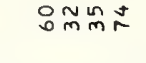 & 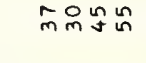 & $\mathcal{N}$ in $\mathcal{N} \underset{J}{N}$ in & 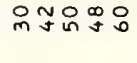 & in ${ }_{\mathrm{m}}^{\circ} \mathrm{f}$ & in & 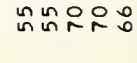 \\
\hline 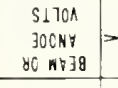 & 음ㅇㅛㅛㅇㅛ & 임임욤ㅇ & 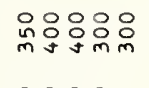 & 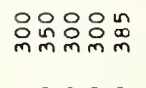 & 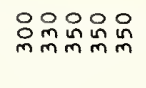 & 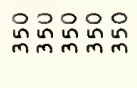 & 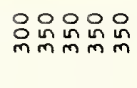 & 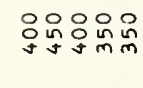 & 응응응 & 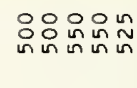 \\
\hline$\mapsto$ & 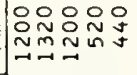 & : & 융용용 & 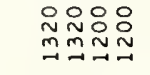 & 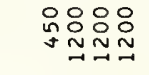 & 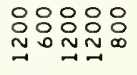 & 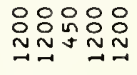 & 品 : & 옴어 & 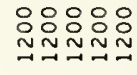 \\
\hline шँ & 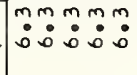 & $\ddot{\dot{0}} \quad \stackrel{m}{\dot{0}}$ & 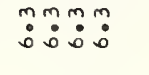 & 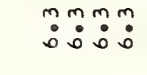 & 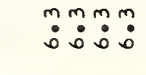 & mºmmm & & $\stackrel{m}{m}: \stackrel{m}{:}$ & $\stackrel{m}{m}: \stackrel{m}{0}$ & 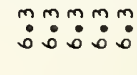 \\
\hline 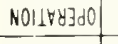 & yuuv & yuบu & uUuUu & טuטu & טuט & טuטu & טuטu & טuטu & บบบư & vous \\
\hline HuW & $\frac{1}{3} \cos ^{\alpha}$ & 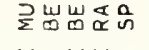 & 岀出灵岗 & 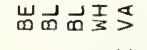 & 岗怘ミさ岀 & 음⿱口凵 & 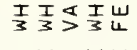 & 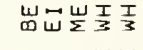 & 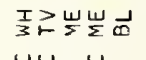 & $\frac{T}{3} \frac{T}{3} \ \stackrel{\Phi}{>}$ \\
\hline JNก! & 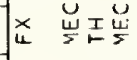 & 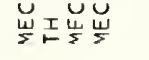 & 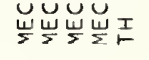 & I $\quad \times \quad \breve{u}$ & 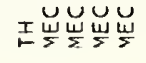 & U⿺乚ّ山ّ & 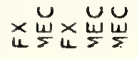 & 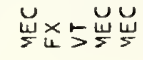 & 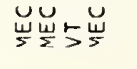 & 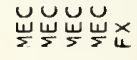 \\
\hline $0114 y$ a 150$]$ & & & & & & & & & & \\
\hline 竞 & $\begin{array}{l}\because \because 0 r: \text { : } \\
\because \because 000\end{array}$ & 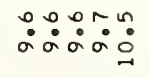 & 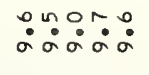 & 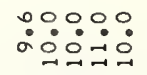 & 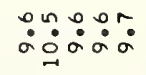 & & $\because \because \because 00$ & & \multirow{2}{*}{ 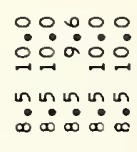 } & 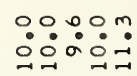 \\
\hline 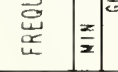 & 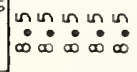 & 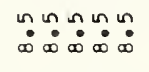 & 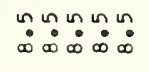 & 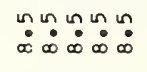 & 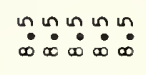 & 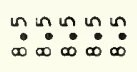 & 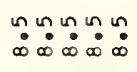 & 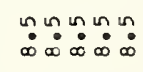 & & 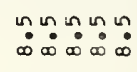 \\
\hline ONIX & $\begin{array}{l}0 \\
\overrightarrow{\vec{A}} \\
\vec{x}\end{array}$ & 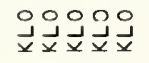 & 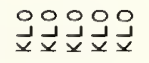 & 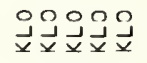 & 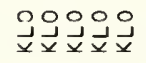 & 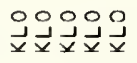 & 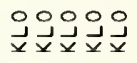 & 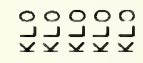 & \multirow[t]{2}{*}{$\begin{array}{l}0 \\
\vec{x} \\
\vec{z}\end{array}$} & 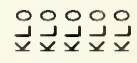 \\
\hline $708 \mathrm{w} / \mathrm{S}$ & * * & & * " & $"$ & $" 1 "$ & $" * \|$ & $"$ & & & \\
\hline 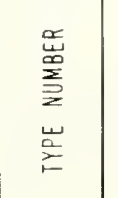 & 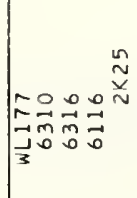 & 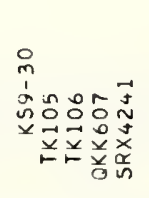 & 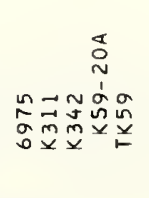 & 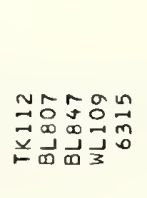 & 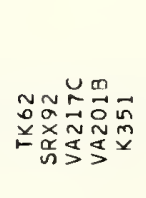 & 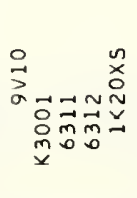 & 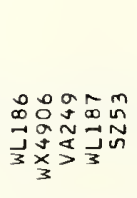 & 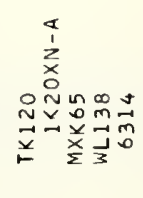 & 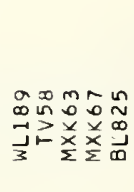 & 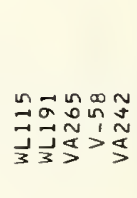 \\
\hline
\end{tabular}




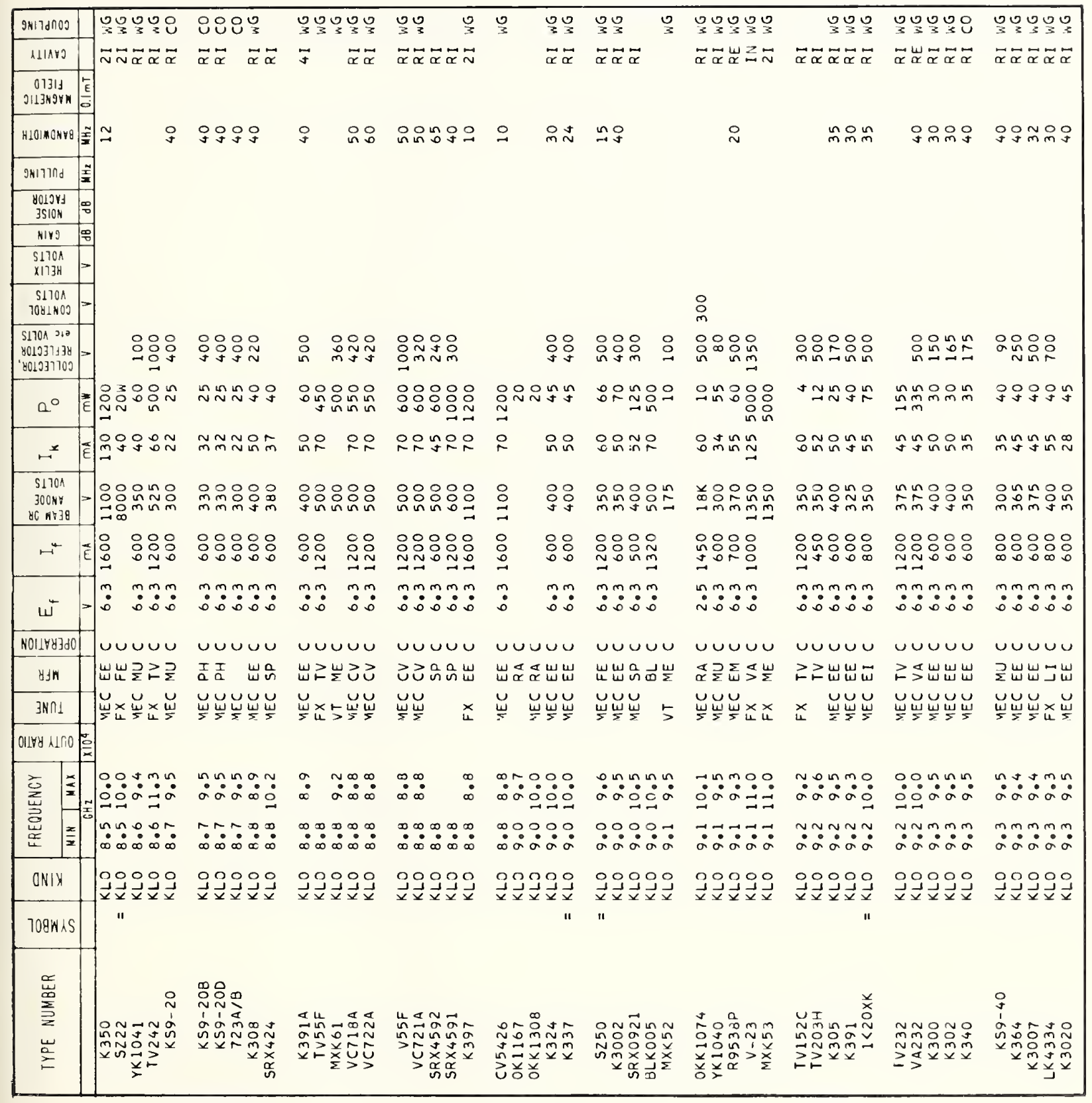




\begin{tabular}{|c|c|c|c|c|c|c|c|c|c|c|}
\hline 9M17dnOS & 号号号号 & Wु & 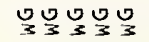 & 可) & ३ญूँ३ & కW & 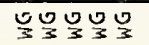 & 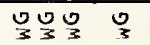 & Uতু & गु \\
\hline AlaYs & $\vec{\alpha} \underset{\alpha}{\alpha} \quad \vec{\alpha}$ & $\vec{\alpha} \ddot{\alpha} z$ & $\vec{\alpha} \vec{\alpha} \vec{\alpha} \vec{\alpha} \vec{\sim}$ & $\vec{N} \vec{\alpha} \vec{\alpha} \vec{\sim} \vec{N}$ & $\vec{\sim} \vec{\sim} \vec{\sim} \vec{N}$ & $\vec{\sim} \tilde{\sim} \tilde{\sim} \tilde{\sim}$ & $\vec{\sim} \vec{x} \quad \vec{\alpha} u$ & $\vec{\alpha} \widetilde{\alpha}$ & $\vec{\alpha} \vec{\alpha} \vec{\alpha} \widetilde{\alpha} \widetilde{\alpha}$ & $\vec{\alpha} \vec{\alpha} \quad \underset{\alpha}{\alpha} \vec{\alpha}$ \\
\hline $\begin{array}{c}01311 \\
\text { ग11369YK }\end{array}$ & & & & & & & & & & \\
\hline H1OIMONYP & 요 & 웅유요 & $\stackrel{\circ}{N}$ & mo & & $\stackrel{\circ}{\sim}$ & 용 9 \& & 요 & 용ㅇ & 응 \\
\hline Sחורואי & 줄 & & & & & & & & & \\
\hline $\begin{array}{l}\text { yolisy } \\
\text { 3S10N }\end{array}$ & & & & & & & & & & \\
\hline nivg & & & & & & & & & & \\
\hline $\begin{array}{l}\text { S1700 } \\
\times 173 \mathrm{H}\end{array}$ & & & & & & & & & & \\
\hline $\begin{array}{c}51701 \\
1001003\end{array}$ & & & $\stackrel{0}{\simeq}$ & & & & & & : & \\
\hline 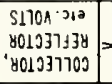 & 승요 & 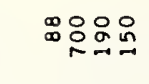 & : & 음 & & & $\stackrel{\circ}{\$}$ & 总些 & 웕 & 응 웅요 \\
\hline $0^{\circ}$ & lino:움요 & 跑的号品 & 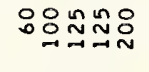 & 品品: & :O:O: & 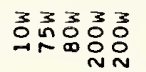 & 芯 & 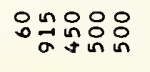 & 品品瓶距 & 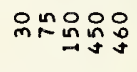 \\
\hline 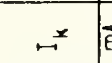 & $\ln _{N}$ o in $n$ & 우으묘요 & 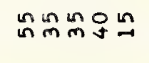 & 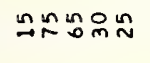 & 있임윰요 & 운음웜워 & 䠋品 典 & 배브욤요 & 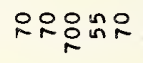 & 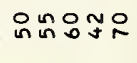 \\
\hline $\begin{array}{c}5170 \mathrm{~A} \\
300 \mathrm{HY} \\
\mathrm{HC} \mathrm{Nr39}\end{array}$ & 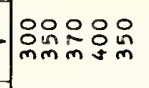 & 응용요욤 & 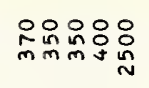 & 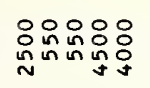 & 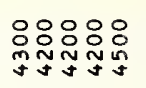 & $\underset{\mathrm{g}}{\mathrm{g}} \mathrm{g}$ & 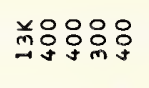 & 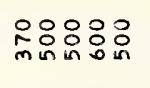 & 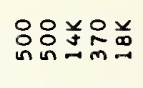 & 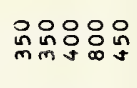 \\
\hline 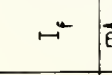 & 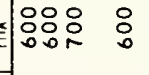 & 울윰요 & 응요 욤요 & 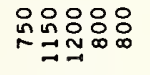 & 옷옷윳윳용 & 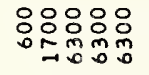 & :OOO & 임요=요 & 옹융ㅇㅇㅇㅛ & : \\
\hline w" & 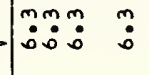 & $\dot{m}: m=m$ & 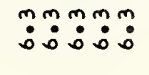 & 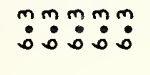 & 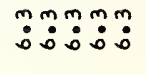 & $\dot{m} \dot{0} \dot{m} \dot{m} \dot{m}$ & $\ddot{m} \dot{m}: \dot{m}:$ & $m \ddot{m}: m: m$ & 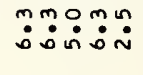 & $\ddot{m}:$ \\
\hline $\mathrm{NOI} / \mathrm{YH} \mathrm{H} \mathrm{dO}$ & טuטu & טuטu & טuטu & טuטu & עטuט & טuטu & טuטu & vưuv & טuטus & טuטus \\
\hline YJW & 玹㐫崖出 & Фวコさ岂岂 & 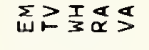 & ハムざタ & 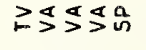 & 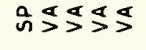 & 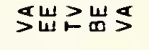 & 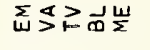 & 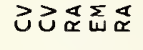 & 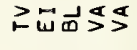 \\
\hline $3 N \cap 1$ & 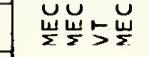 & 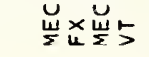 & 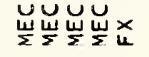 & 荘状嵌 $x$ & 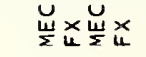 & 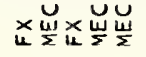 & 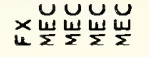 & 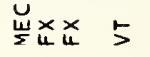 & 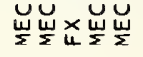 & 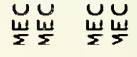 \\
\hline $011 \forall y<1 \cap 0$ & & & & & & & & & & \\
\hline 总 & 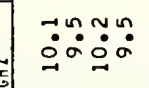 & 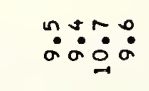 & 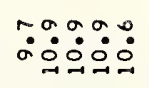 & 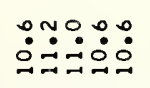 & $\because \because 0: 00$ & 遇: & 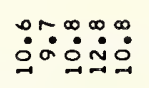 & 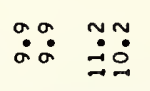 & 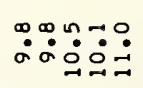 & $\ddot{m}: 0$ \\
\hline 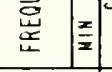 & 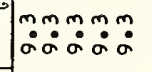 & 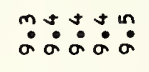 & 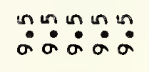 & 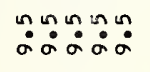 & 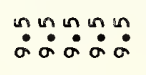 & 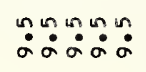 & 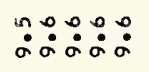 & 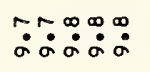 & 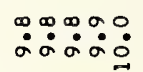 & :웅워 \\
\hline ONIX & 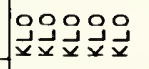 & & 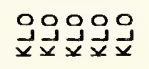 & 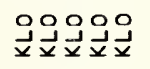 & 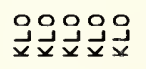 & 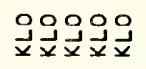 & 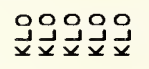 & 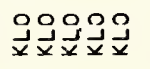 & 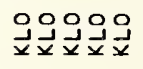 & \\
\hline 708W 25 & & & & & & & & $"$ & & \\
\hline 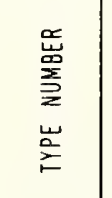 & 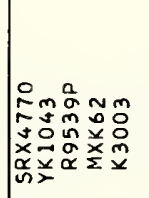 & 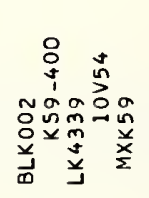 & 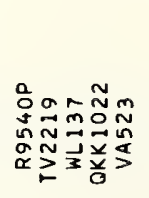 & 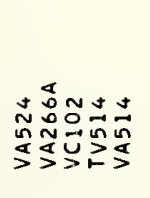 & 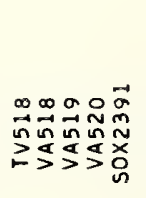 & 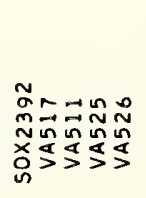 & 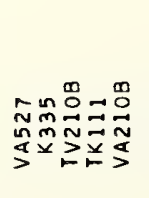 & 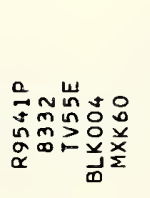 & 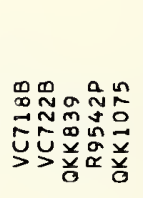 & 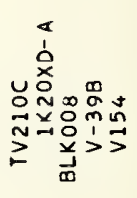 \\
\hline
\end{tabular}




\begin{tabular}{|c|c|c|c|c|c|c|c|c|c|c|}
\hline 9M17dnOS & $\frac{0}{3}$ & 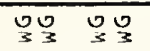 & उु్ & ৩তু३ & 응 & Yত३ & 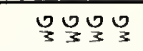 & WW & 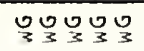 & 员员 \\
\hline ALIAYJ & $\vec{\alpha} \vec{N}$ & $\underset{\alpha}{\tilde{N}}$ & 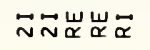 & $\vec{\alpha} \vec{\sim} \quad \vec{\sim}$ & $z$ & $\overrightarrow{\alpha \vec{\alpha}} \quad \vec{\alpha} \vec{\alpha}$ & $\sum \ddot{\alpha \vec{\alpha} \vec{\alpha}}$ & $\vec{\alpha} \vec{\alpha} \vec{\alpha} \vec{\alpha} \vec{\alpha}$ & $\vec{\alpha} \vec{\alpha} \vec{\alpha} \vec{\alpha} \ddot{\alpha}$ & $\vec{\alpha} \vec{\alpha} \vec{\alpha} \quad \vec{\alpha}$ \\
\hline ת) & & & & & & & & & & \\
\hline HLOMONYA & gn $^{2}$ & 只 & 웅워 & $\stackrel{N}{N}$ & $\stackrel{\circ}{\sim}$ & in & $\tilde{m} N$ & m요용ㅇㅇㅇ & $\stackrel{\circ}{\sim}$ & 요요 \\
\hline 9M17רח & 촌 & & & & & & & & & \\
\hline $\begin{array}{c}\text { yolows } \\
\text { 3SION }\end{array}$ & & 운 & & 으오 & & & & & & \\
\hline Mirg & & & & & & & & & & \\
\hline $\begin{array}{l}51700 \\
\times 11334\end{array}$ & & & & & & & & & & \\
\hline $\begin{array}{c}\text { S1701 } \\
\text { 10811400 }\end{array}$ & $\stackrel{\circ}{\circ}$ & & & & & & & & & $\therefore$ \\
\hline 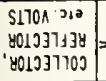 & 品 & 임요 & $\stackrel{\circ}{\Xi}$ & 品 & 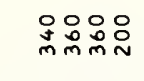 & 임음 品 & 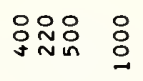 & 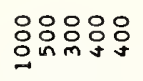 & 임임요 & 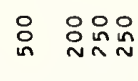 \\
\hline $0^{\circ}$ & E & 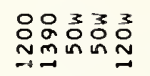 & 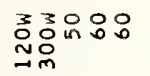 & 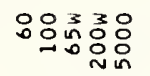 & 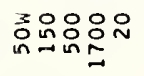 & 오ำํำ & 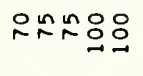 & 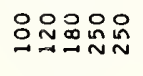 & 울웅ํำ & ํํㄱ윰ํำ \\
\hline$-x$ & 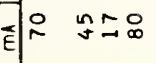 & 융이 싱응 & 웜워 & 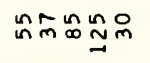 & 요 & 织告品品品 & 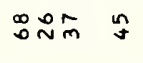 & 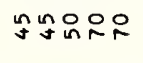 & :요ㅇㅛㅛ & 욱昌品 \\
\hline $\begin{array}{r}51701 \\
300 \mathrm{HF} \\
80 \mathrm{HY} 39\end{array}=$ & - & 品 & 兰兰品品品 & 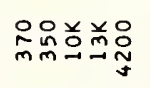 & 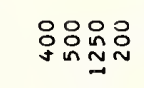 & 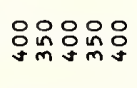 & 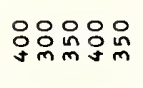 & 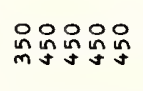 & 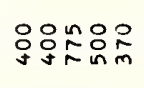 & 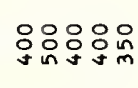 \\
\hline- & 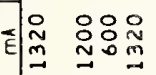 & 品 品怘 & 总号品 & 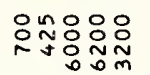 & 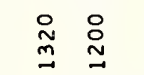 & 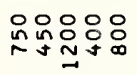 & 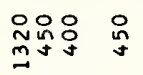 & 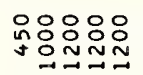 & 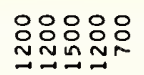 & 유: \\
\hline$\omega^{\star}$ & 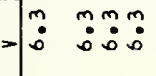 & 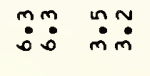 & $\because \dot{m} \dot{m}: \dot{m}$ & 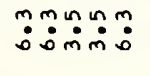 & $\stackrel{m}{m}:$ & 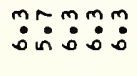 & $\ddot{2} \ddot{0}: \stackrel{m}{:}$ & :m:m: & $\ddot{m}: m \ddot{m}: m$ & $\ddot{m} \ddot{m} \dot{m}: m$ \\
\hline NO1 $17 y 3 \mathrm{dO}$ & טיטטי| & טטuט & טטuט & טบบบ & บบบบบ & טטט & טบט & บบบบy & טuบu & טִט \\
\hline$y \mathrm{Jn}$ & هు & 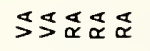 & 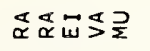 & 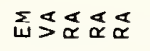 & 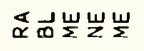 & コ孚レムさ & هు & 甹岕出出 & ZZ忘 & \兵踥崫 \\
\hline JNก1 & 訔岕崖 & 岁 & 岀×獶岕岕 & 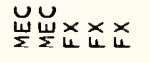 & 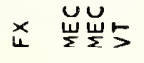 & 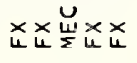 & 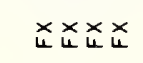 & ×岕岕岕岕 & 岀岀 $\times$ & 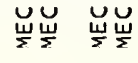 \\
\hline olity anno & & & & & & & & & & \\
\hline 䇋 & 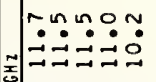 & 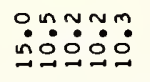 & mற் & $\stackrel{0}{0} \quad \stackrel{0}{0}$ & 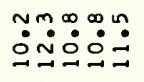 & 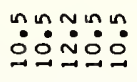 & 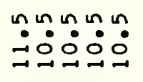 & 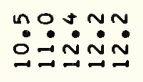 & 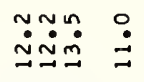 & 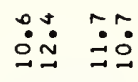 \\
\hline \begin{tabular}{l|l|}
$\stackrel{\vec{Z}}{\tilde{x}}$ & $\frac{x}{x}$ \\
\end{tabular} & $\because 00: 00$ & 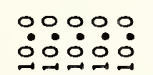 & 웅무웅 & $\ddot{0}: \because 0$ & 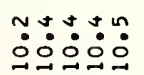 & ñ. & 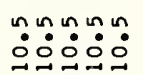 & نٌ & ن & :웅우웅웅 \\
\hline ONIX & | & 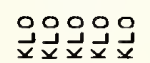 & 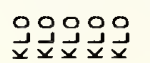 & 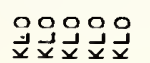 & & ئ日) & 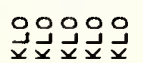 & 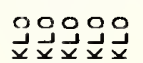 & 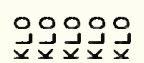 & لُو \\
\hline 708W & & $"$ " & & & & & & " " " & " & \\
\hline 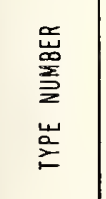 & 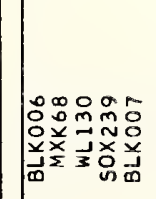 & 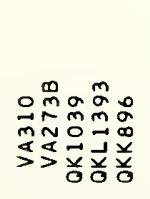 & 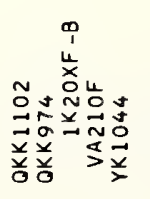 & 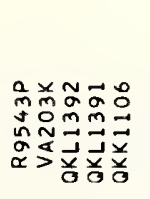 & 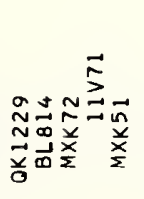 & 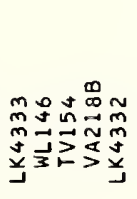 & 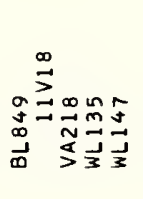 & 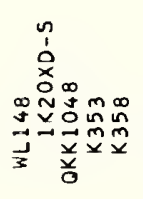 & 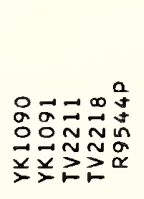 & 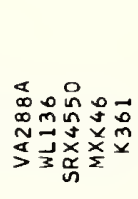 \\
\hline
\end{tabular}




\begin{tabular}{|c|c|c|c|c|c|c|c|c|c|c|}
\hline 9N17dnOO & 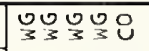 & 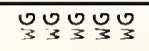 & 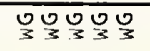 & 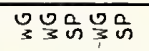 & OOY & OOSO & OOY & OOS & 엉요 & 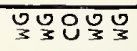 \\
\hline $111 \mathrm{AV}$ & 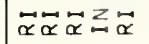 & 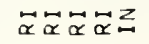 & $\underset{\sim}{\sim} \underset{\alpha \breve{\alpha}}{u}$ & 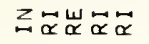 & 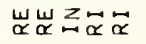 & $\ddot{\alpha} \ddot{\alpha} \ddot{\alpha}$ & $\vec{\alpha} \widetilde{\alpha} \widetilde{\alpha} \widetilde{\alpha} \vec{\alpha}$ & $\vec{\alpha} \mathfrak{\sim} \ddot{\alpha} \quad \vec{\alpha}$ & 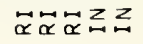 & $\vec{\alpha} \vec{\alpha} \tilde{\alpha} \ddot{\alpha}$ \\
\hline \begin{tabular}{c|c|c|}
01311 \\
ग113W9YK
\end{tabular} & & & & & & & & & & \\
\hline HLOIMOHYO & 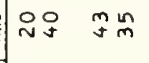 & 옹요 우쇼 & f & nㅡㅁํ앙ㅇㅇ & t & $\stackrel{\circ}{\stackrel{N}{0}}$ & 드웅요 & ofing in & 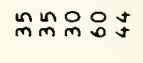 & ming \\
\hline 9w/7רח & & & & & & & & & & \\
\hline 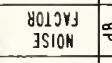 & & & & & & & & & & \\
\hline HIVI $\frac{\alpha}{\tau}$ & & & & & & & & & & \\
\hline $\begin{array}{l}\text { S1701 } \\
X \backslash 73 H\end{array}$ & & & & & & & & & & \\
\hline $\begin{array}{c}S 170 \mathrm{~A} \\
70 \mathrm{~B} 1 \mathrm{HOS}\end{array}=$ & & & & $\stackrel{\circ}{N}$ & $\stackrel{\circ}{\circ}$ & in & $\stackrel{n}{\sim}$ & & & q \\
\hline 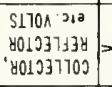 & 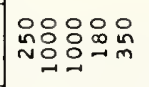 & 오ํํํํํำ & 샛ㅇㅇㅇㅇㅇㅇㅇㅇ & 윳음용요 & 응용요 & 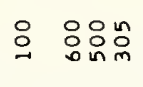 & 앙용ㅇㅁ & 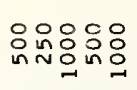 & 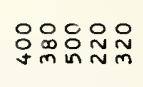 & 웅ㅇㅇㅁㅇㅇㅁ \\
\hline$a^{\circ}$ & 충용ㅇㅇㅁㅇ & 웅엄욤요 & 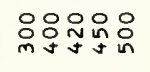 & 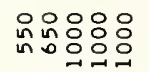 & 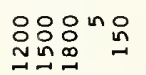 & 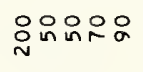 & 品品肙品品 & 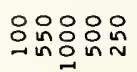 & 옥음욤욤 & 옥옥윰어 \\
\hline- & 웝 $\sim \underset{\sim}{\sim} \sim$ & 요요묘요 & 品 射出员 & 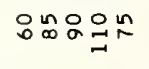 & 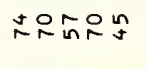 & no & 요요ำ & 옹음 & in & 웅으 \\
\hline 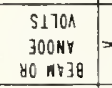 & 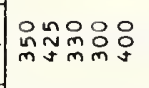 & 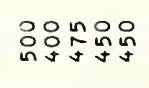 & 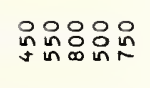 & 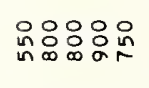 & 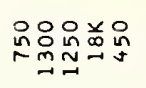 & 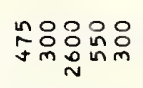 & 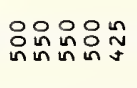 & 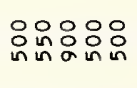 & $\underset{\sim}{n} \underset{\sim}{n}$ 品 & 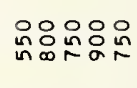 \\
\hline$H^{-}$ & 응ㅇㅇㅇㅝ & 음옹요 & 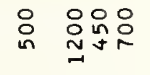 & 品 总品 & 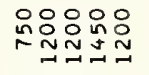 & 옹요 & 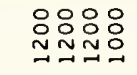 & 品品品 & 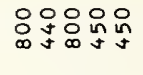 & 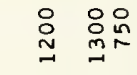 \\
\hline "َ & mºmm & 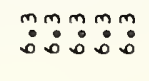 & 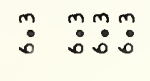 & $\ddot{m} \dot{m}: \dot{m}: \dot{m}$ & mamun & $\ddot{m} \ddot{m} \ddot{0} \dot{m}: \dot{m}$ & $\ddot{m} \dot{m}: \dot{m}: \dot{m}$ & $\ddot{m}: m: m:$ & 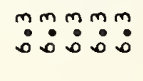 & $\ddot{:}: \ddot{m}$ \\
\hline Nol $14 y\} \mathrm{dO}$ & טuטu & טuטu & טuטu & טuטu & טuט & טuטu & טuט & טuט & טuטu & uu \\
\hline 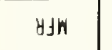 & 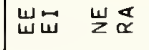 & யே\ 岀岂 & 岂崖 Z & 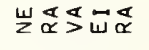 & 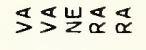 & 的更敉崖 & 山゙ミア & ே岂ே岂。 & 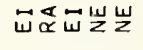 & 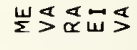 \\
\hline 3Nก1 & 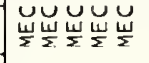 & 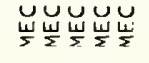 & 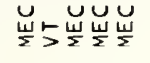 & $\begin{array}{l}\breve{U} \\
\\
\end{array}$ & 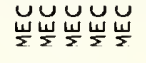 & 岁岁区 & 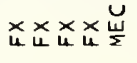 & 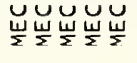 & 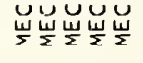 & 5岀 \\
\hline O1日8y $11 \cap 0$ & & & & & & & & & & \\
\hline 离 & 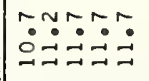 & シュュュ゚ & $\because \because \because \because \dot{\tilde{N}}$ & & 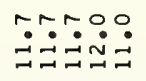 & 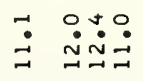 & 莳莳: & 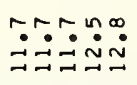 & 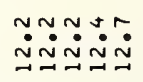 & 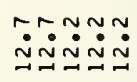 \\
\hline 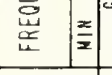 & 뭉ㅇㅇㅇㅇㅇ & $\because \because \because \therefore \circ$ & $\because \because \because 0$ & $\because \because \dot{0} \dot{0} \dot{0}$ & $\because \because \because a: 0$ & 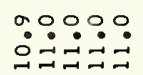 & $\because \because \because \because シ$ & 〜 N & そュュペ & シュュュュ \\
\hline ONIX & & جُ & طُ & 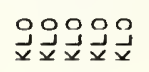 & 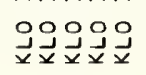 & 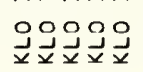 & 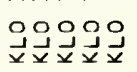 & 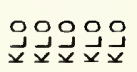 & 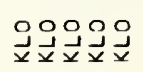 & 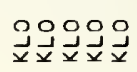 \\
\hline $709 \mathrm{~W} / \mathrm{S}$ & & $"$ & & & $"$ & " & " " & $" \quad "$ & & \\
\hline 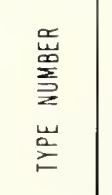 & 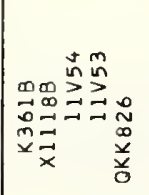 & 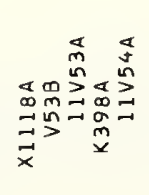 & 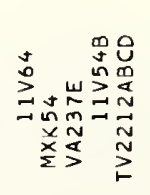 & 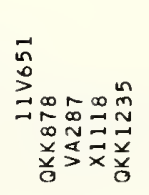 & 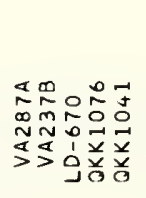 & 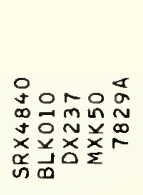 & 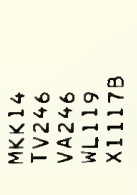 & 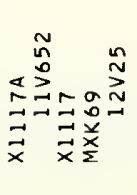 & 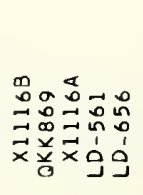 & 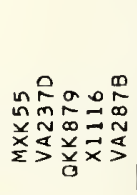 \\
\hline
\end{tabular}




\begin{tabular}{|c|c|c|c|c|c|c|c|c|c|c|}
\hline 9N17dnos & Uঙ & Зיర & 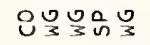 & ড & ॠण్ & 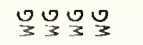 & OYOSO & U్ & 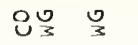 & Sू \\
\hline A11Av2 & $\vec{\alpha} \vec{\alpha} \ddot{\alpha} \vec{\alpha} \vec{\alpha}$ & $\stackrel{u}{\alpha} \vec{\alpha} \vec{\alpha} \vec{\alpha}$ & $\vec{\alpha} \vec{\alpha} \ddot{\alpha} \vec{\alpha} \vec{N}$ & $\vec{\sim} \vec{\alpha} \vec{\alpha} \vec{\alpha}$ & $\vec{\alpha} \vec{\alpha} \vec{\alpha} \vec{\alpha} \vec{\alpha}$ & $\vec{\alpha} \vec{\alpha} \quad z \ddot{\alpha}$ & $\vec{\alpha} \underset{\alpha}{\alpha} \underset{\alpha}{u}$ & $\vec{\alpha} \vec{\alpha} \quad \vec{\alpha} \vec{\alpha}$ & $\vec{\alpha} \vec{\alpha} \vec{\alpha} \vec{\alpha} \vec{\alpha}$ & $\vec{\alpha} \vec{\alpha} \tilde{N}$ \\
\hline 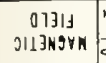 & & & & & & & & & & \\
\hline HLOMONG8 & 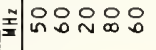 & 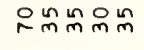 & 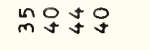 & 뇨 & 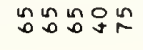 & 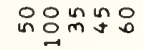 & 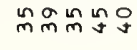 & 일앙 욤 & 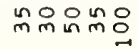 & nno \\
\hline גחרוואפ & $\frac{\tilde{x}}{2}$ & & & & & & & & & \\
\hline $\begin{array}{l}8013 \gamma] \\
\text { JS1ON }\end{array}$ & : & & & & & & & & & \\
\hline Nirg & & & & & & & & & & \\
\hline $\begin{array}{l}51701 \\
\times 11734 \\
\end{array}$ & & & & & & & & & & \\
\hline $\begin{array}{c}5170 \wedge \\
7061 \mathrm{NOS}\end{array}=$ & & & q & & & & q & & o & \\
\hline 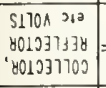 & | & 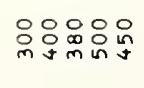 & 응요 & 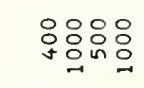 & 응ㅇㅇㅇ & 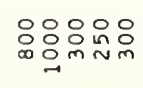 & 응응응 & 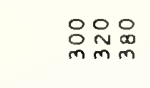 & :O:০: & iñ \\
\hline $0^{\circ}$ & $\mathbb{E}$ & 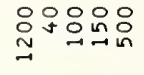 & 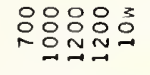 & 으뭄윰유 & 웅요요요 & 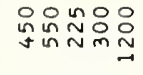 & 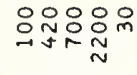 & 욤욤욤요 & 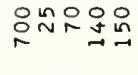 & 윰용요 \\
\hline$\rightarrow$ & 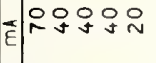 & 응ำㄴำㅇํㅇำ & 웃온 & 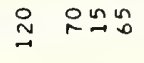 & 욱: & 군 & 앙앙유 & inํ의 & 요쇼요 & 듯유요 \\
\hline $\begin{array}{c}\text { S170N } \\
300 \mathrm{NY} \\
\forall C \mathrm{Hr} 3 \mathrm{~B}\end{array}$ & 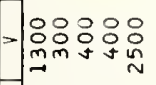 & $\begin{array}{l}\text { Oñ } \\
\text { ONO } \\
\infty\end{array}$ & 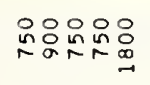 & 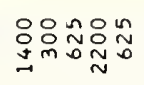 & 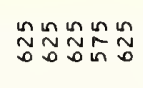 & 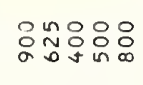 & 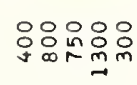 & 응용ํ & 은음음 & 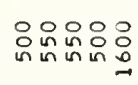 \\
\hline$\mapsto$ & $\mid$\begin{tabular}{llll}
0 & 0 & 0 & 0 \\
0 & 8 & 0 & 0 \\
\hdashline & 0 & 0 & 0
\end{tabular} & 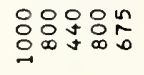 & 总员 & : & 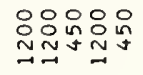 & 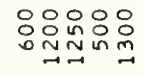 & 足 & 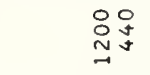 & :०: & 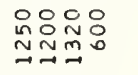 \\
\hline 山ँ & 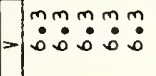 & 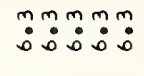 & $\stackrel{m}{m} \stackrel{m}{\dot{0}} \stackrel{m}{:}$ & $\ddot{m} \quad \stackrel{m}{m} \dot{m}: m$ & $\dot{m} \ddot{m} \dot{m}: m m$ & 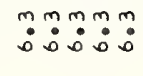 & $\ddot{m} \dot{m}: \dot{m}$ & $\ddot{m}:$ & $\ddot{m} \stackrel{m}{m}:$ & $\ddot{m} \dot{m} \dot{m}: m$ \\
\hline NOIIFy $\mathrm{dO}$ & |งบบบ & บuบuv & บัuบa & บuบuv & vounu & צuטu & yuบuv & ưuบu & บบบบบ & บuบuv \\
\hline 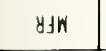 & 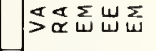 & 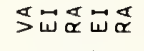 & 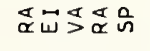 & 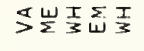 & 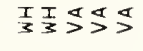 & 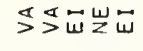 & 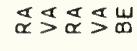 & 岕岗\さひ & व & 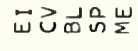 \\
\hline $3 \mathrm{~N} \cap 1$ & $\mid$ & 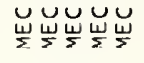 & 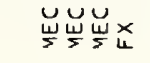 & 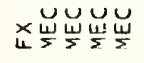 & 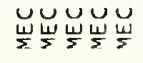 & U్Wّ & 岀岁 & U⿺辶巛ّ & × & $\underset{4}{\breve{w}}$ \\
\hline $0118 y \times 1 \cap 0$ & & & & & & & & & & \\
\hline 䇋 & 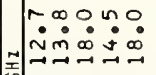 & 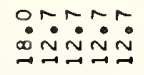 & 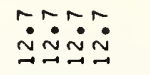 & : & $\because$ & 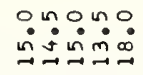 & 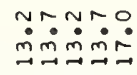 & 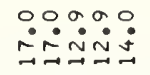 & 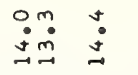 & $\dot{m} \dot{m} \dot{m}$ \\
\hline 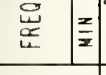 & 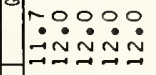 & $\underset{\sim}{\sim} \underset{\sim}{\sim} \underset{\sim}{\sim} \underset{\sim}{\sim} \sim$ & 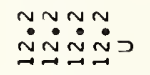 & 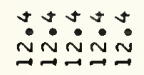 & 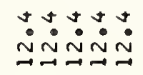 & 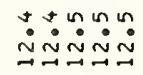 & 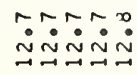 & 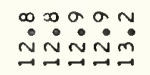 & 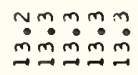 & 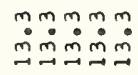 \\
\hline ONIX & 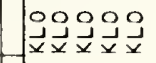 & 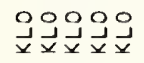 & 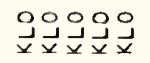 & 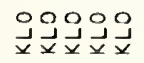 & 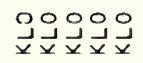 & 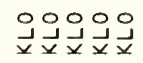 & 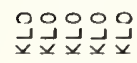 & 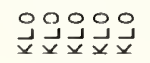 & 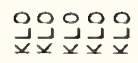 & 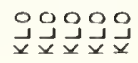 \\
\hline $708 \mathrm{~W} / \mathrm{S}$ & & & & & & " & & & & $"$ \\
\hline 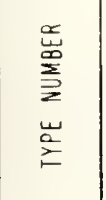 & 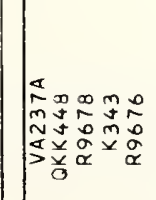 & 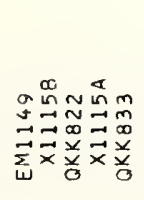 & 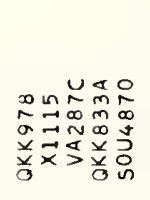 & 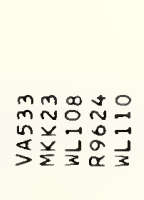 & 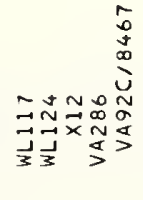 & 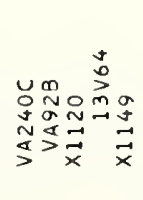 & 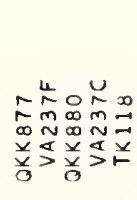 & 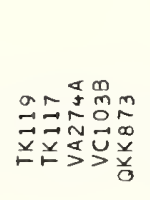 & 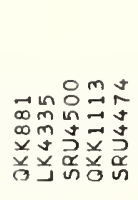 & 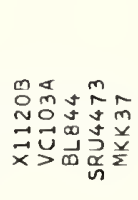 \\
\hline
\end{tabular}




\begin{tabular}{|c|c|c|c|c|c|c|c|c|c|c|}
\hline 9אוI d 103 & in & گ్ & 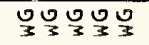 & 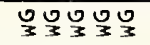 & 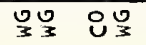 & בֶּ & OUß & O్ & 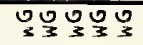 & $\left.{ }_{3}^{0}\right)_{3}$ \\
\hline Alaro & 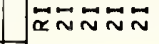 & $\ddot{\sim} \tilde{N} \tilde{N}$ & $\vec{\sim} \vec{\alpha} \vec{\sim} \vec{\alpha} \vec{\alpha}$ & $\ddot{\alpha \widetilde{\sim}} \tilde{\sim} \tilde{N}$ & $\vec{\alpha} \vec{\alpha} \quad \vec{\alpha} \vec{\alpha}$ & $\ddot{\propto} \ddot{\propto} \ddot{\alpha} \underset{\propto}{\widetilde{u}}$ & $\ddot{\alpha} \underset{\alpha}{\alpha} \ddot{\alpha} \quad z$ & $\underset{\sim}{\boldsymbol{\alpha}} \boldsymbol{\sim} \widetilde{\alpha} \widetilde{\alpha}$ & $\vec{\alpha} \ddot{\alpha} \underset{\alpha}{\boldsymbol{\alpha}} \vec{\alpha}$ & $\vec{\alpha} \vec{\alpha} \tilde{\alpha}$ \\
\hline $\begin{array}{c}01311 \\
\text { ग113N9YK } \\
\end{array}$ & 息 & & & & & & & & & \\
\hline HLOMONY8 & 푼 & & 용 in & ㅇ & No & กูน & 趴品 & 은요옹 & mo o in & 䒽品 量 \\
\hline פMIIITnd & $\underline{\mathbf{y}}$ & & & & & & & & & \\
\hline $\begin{array}{r}801319 \mathrm{H} \\
3510 \mathrm{~N} \\
\end{array}$ & $\infty$ & & & & & & & & & \\
\hline NIYO & : & & & & & & & & & \\
\hline $\begin{array}{l}{ }_{51700} \\
\times 1133 \mathrm{H}\end{array}$ & $\Rightarrow$ & & & & & & & & & \\
\hline $\begin{array}{c}51701 \\
10811103\end{array}$ & & & 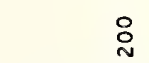 & & & & ? & & 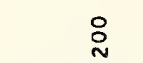 & \\
\hline 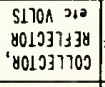 & $=10$ & & 운 웅 & 웅음 & 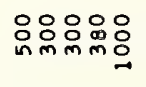 & 응윰욤욤 & 움욨 & 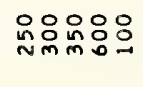 & 음음욧용 & 융요 \\
\hline $0^{\circ}$ & = & 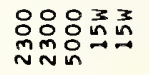 & 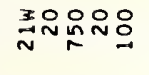 & 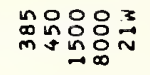 & 임임욤음 & 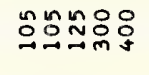 & 옷용요요 & 음ㅇㅇㅇㅇㅝ & 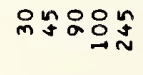 & 员员욨요 \\
\hline$\mapsto$ & Elog & $\stackrel{ \pm}{\sim}$ & F墭品 & 믐으 & 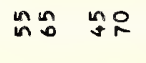 & 움욤요 & 우요 in & 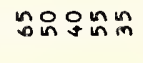 & $\stackrel{n}{\xi} \vec{m} \underset{\sim}{N} \sim \underset{N}{\infty}$ & $\tilde{ก} \tilde{m} \tilde{N}$ \\
\hline $\begin{array}{r}\text { S170 } \\
300 \mathrm{HF} \\
80 \mathrm{AB} 3 \mathrm{Bg}\end{array}$ & 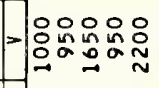 & 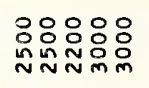 & 을 & 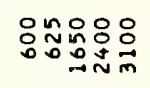 & 品品品昌告 & 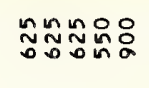 & 윳용ㅇㅇㅇㅇㅇㅇㅇㅇ & 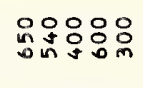 & 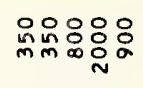 & 옴욤요 \\
\hline$\omega^{-}$ & 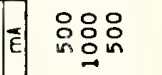 & ㅇㅇㅇ & 응 융요 & 욱융융 & 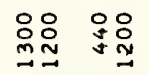 & 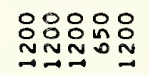 & 号 & 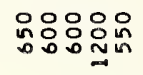 & 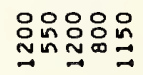 & 융용 용응 \\
\hline L" & $\stackrel{m}{m}: m$ & $\stackrel{m}{m}:$ & 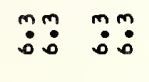 & 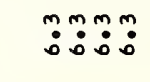 & 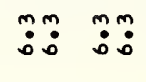 & : & $\stackrel{m}{:}:$ & $\ddot{m}: m: m$ & 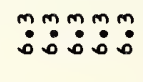 & 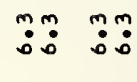 \\
\hline NOIIVy $3 d 0$ & บuบuบ & טบบบ & טuטu & טuטu & טuט ט & טنטיט & טטuט & טuטu & טuטu & טบบบ \\
\hline$\forall J W$ & 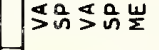 & $\unlhd \ \frac{\omega}{\Sigma}$ & 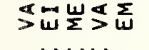 & 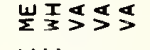 & 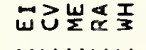 & $\frac{1}{3} \frac{1}{3} \ \$ & 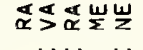 & 岂心品嵅品 & 顷品品】 & 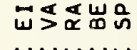 \\
\hline JNก1 & 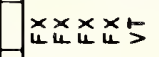 & 雄 & 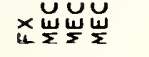 & 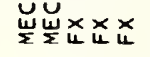 & 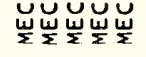 & 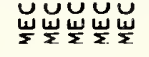 & 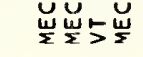 & 岀 & xüّ & 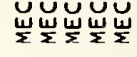 \\
\hline $0: 1 \forall y, k i \cap 0$ & & & & & & & & & & \\
\hline 总 & $\ddot{m} \dot{m} \ddot{m} \dot{m} \dot{m}$ & $\ddot{m} \dot{m} \dot{m} m \dot{m}$ & m: & 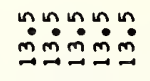 & åñ & 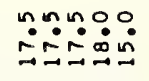 & 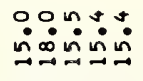 & 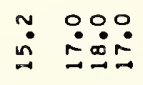 & 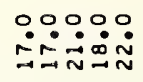 & $\begin{array}{l}0 \\
0 \\
0\end{array}$ \\
\hline 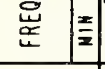 & $\dot{m}_{\dot{m} \dot{m} \dot{m} \dot{m} \dot{m} \dot{m}}^{m}$ & $\dot{m}_{\dot{m}}^{m} \dot{m} \dot{m} \dot{m} \dot{m} \dot{m}$ & mand & 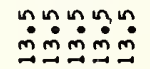 & 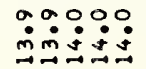 & \begin{tabular}{l}
$0: 000$ \\
0 \\
\hdashline \\
\end{tabular} & 㝵莳莳 & 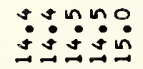 & & 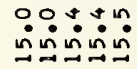 \\
\hline ONIX & 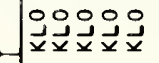 & 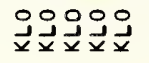 & 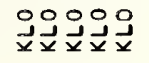 & 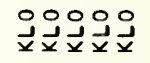 & 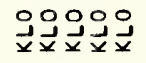 & 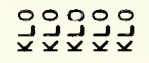 & $\begin{array}{l}0 \\
\vec{x} \\
\vec{x} \\
\end{array}$ & 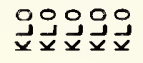 & 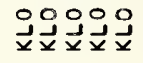 & 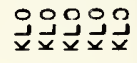 \\
\hline 700W/S & & & & " " & $"$ & " & " & & & $"$ \\
\hline 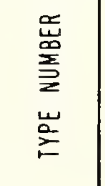 & 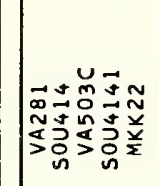 & 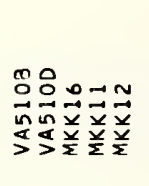 & 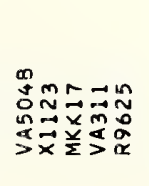 & 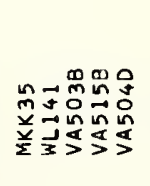 & 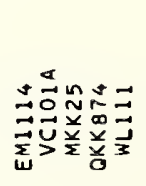 & 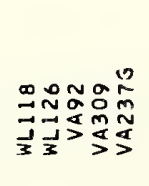 & 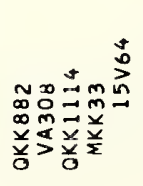 & 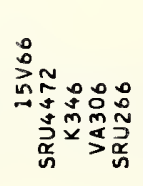 & 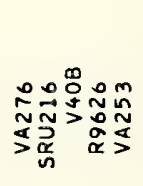 & 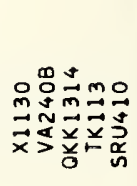 \\
\hline
\end{tabular}




\begin{tabular}{|c|c|c|c|c|c|c|c|c|c|c|}
\hline $9 \times 17 \mathrm{dnO0}$ & 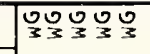 & ७ुषु & 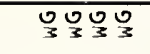 & WO & 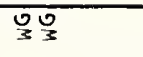 & $\begin{array}{ll}0 \\
\end{array}$ & OO० & 잎이 & 야요 & 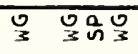 \\
\hline Alsars & $\vec{\alpha} \quad \vec{\alpha} \vec{\alpha} \vec{\alpha}$ & $\vec{\sim} \vec{\alpha} \quad \vec{\alpha} \vec{\alpha}$ & $\vec{\alpha} \vec{\alpha}$ & $\vec{\alpha} \ddot{\alpha} \vec{\alpha} \vec{\alpha} \vec{\sim}$ & $\vec{\alpha} 山 \vec{\alpha} \vec{\alpha} \vec{\sim}$ & $\vec{\alpha} \vec{\alpha}$ & $\ddot{\alpha} \boldsymbol{\alpha} \ddot{\sim} \tilde{N} \vec{\sim}$ & $\vec{\sim} \vec{\alpha} \vec{\alpha} \quad \vec{\alpha}$ & $\vec{\alpha} \vec{\alpha} \ddot{\alpha} \vec{\alpha} \vec{\alpha}$ & $\vec{\alpha} \vec{\sim} \underset{\alpha}{\sim}$ \\
\hline $\begin{array}{c}0131 \mathrm{I} \\
\text { g113kgr }\end{array}$ & & & & & & & & & & \\
\hline HLOINOWY & 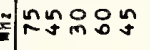 & 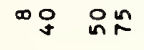 & ก & 용ํㅇำ & 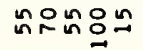 & o요 & 용ํㅛ $\cong \simeq$ & no웡ㅇㅇ & 용용요 & 유ํ용 \\
\hline dחרורוא9 & 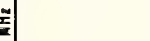 & & & & & & & & & \\
\hline 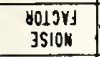 & : & & & & & & & & & \\
\hline Mirg & & & & & & & & & & \\
\hline 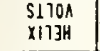 & & & & & & & & & & \\
\hline $\begin{array}{c}\text { S170A } \\
10811603\end{array}$ & $\stackrel{\circ}{\sim}$ & & & & & & & 음유 & & \\
\hline 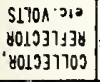 & 임ㅇㅇㅇㅇㅇㅇ & 율윰ㅇㅇㅛ & 익品品品品 & 움욤욤 & 윰용으 & 品品品: & : & 윰욤욤요 & 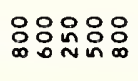 & : \\
\hline $0^{\circ}$ & 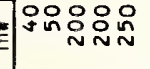 & 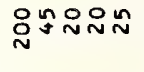 & 입워윰요 & 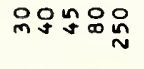 & 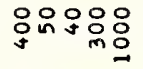 & 움윰윰유 & 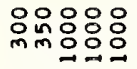 & 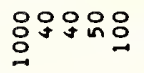 & 品虽虽怘是 & 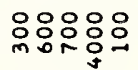 \\
\hline$\approx$ & $\left\{\begin{array}{l}n \\
+\end{array}\right.$ & $: \vec{m} \quad \tilde{m} \tilde{s}$ & in $n$ & $\tilde{N}=$ m & 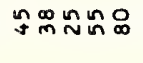 & nn $\quad \stackrel{ \pm}{N}$ & 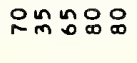 & 品o & 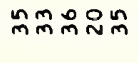 & ำกษ๋ \\
\hline $\begin{array}{r}5170 \mathrm{~A} \\
300 \mathrm{NY} \\
80 \mathrm{ny3} \\
\end{array}$ & 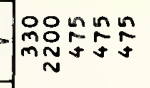 & 영욤욜 & 品品䠌品 & 암윰임윰 & 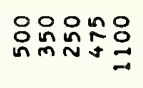 & 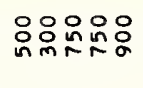 & 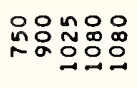 & 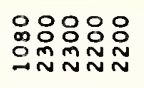 & 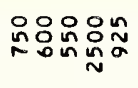 & 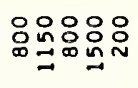 \\
\hline 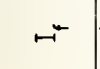 & 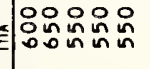 & 品品 品品 & 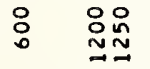 & 品品品品品品 & 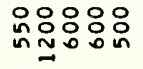 & 品: & 品员品品品 & 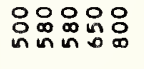 & 品品员员品怘 & 总品员员昆 \\
\hline " & 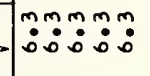 & 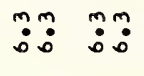 & $\ddot{m} \quad \stackrel{m}{:}:$ & : & 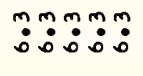 & $\ddot{m} \stackrel{m}{:} \quad \stackrel{m}{0}$ & & :m: & : & m:mm: \\
\hline N011YY3d0 & uUu & บบบบบ & บบบบบ & טuטu & טuטu & 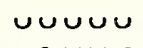 & טuบu & yuuvu & טบטบ & טטיט \\
\hline 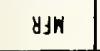 & 欧羊的战的 & 的的岂触 & 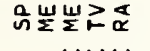 & 衫路品 & 新的的的 & 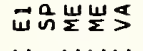 & ๔马经的 & 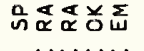 & 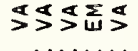 & 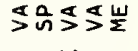 \\
\hline उNกI & 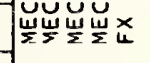 & 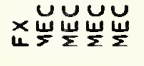 & 岀岀岁 & 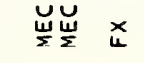 & $\begin{array}{ll}\breve{w} & x\end{array}$ & 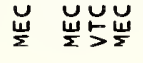 & 岀岁始始 & 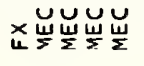 & 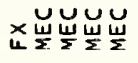 & 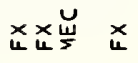 \\
\hline alve inno & & & & & & & & & & \\
\hline 总 & 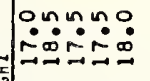 & 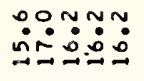 & $\because \because 0: 0$ & $\because 0: 0: 0$ & 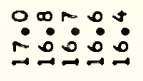 & $\because \cong 0:$ & 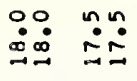 & 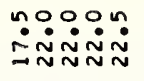 & 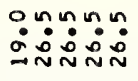 & 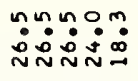 \\
\hline \begin{tabular}{|l|l|}
$\substack{\tilde{u} \\
w}$ & $\frac{x}{x}$ \\
\end{tabular} & 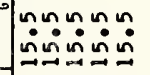 & $\ddot{n}$ & ம் & 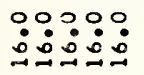 & 車: & 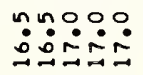 & 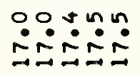 & تص: & : & : \\
\hline ONIX & 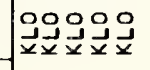 & جو & 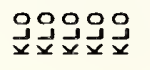 & 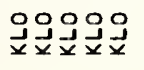 & 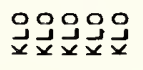 & 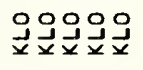 & جو & 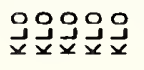 & 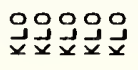 & \\
\hline $708 \% / S$ & & & & $"$ & & $"$ & " " & & " " & " \\
\hline 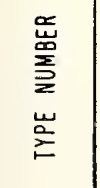 & 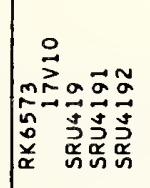 & 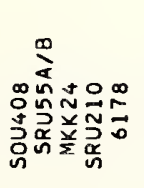 & 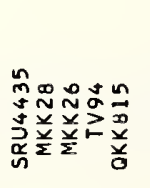 & 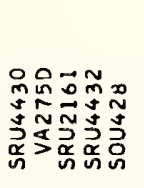 & 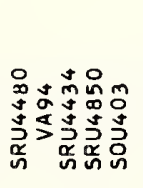 & 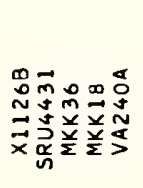 & 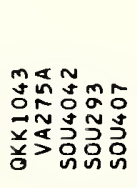 & 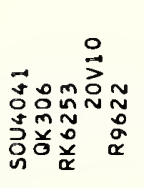 & 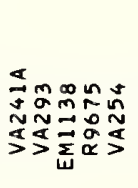 & 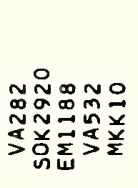 \\
\hline
\end{tabular}




\begin{tabular}{|c|c|c|c|c|c|c|c|c|c|c|c|}
\hline 9Mi Td DOS & SOS & $000 \%$ & З & 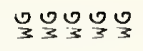 & 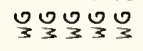 & \%० & O० & & 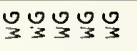 & 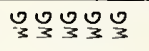 & 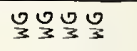 \\
\hline dians & $\vec{\alpha} \quad \vec{\alpha} \vec{\alpha} \vec{\alpha}$ & $\vec{\alpha} \vec{\sim} \vec{\sim} \vec{\alpha} \vec{\alpha}$ & $\vec{\alpha} \ddot{\alpha} \quad \vec{\alpha}$ & $z \vec{\alpha} \vec{\alpha}$ & $\vec{\alpha} \vec{\alpha} \quad \vec{\alpha} \vec{\alpha}$ & $\vec{\propto} \vec{\propto} \widetilde{\propto} \widetilde{\sim} \underset{\sim}{u}$ & $\vec{\alpha} \vec{\alpha} \vec{\alpha}$ & 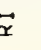 & $\vec{\sim} \vec{\alpha} \vec{\alpha} \vec{\alpha} \vec{\alpha}$ & $\vec{\sim} \vec{\sim} \widetilde{\propto} \ddot{\propto} \underset{\sim}{u}$ & $\vec{\alpha} \vec{\alpha} \vec{\alpha} \vec{\alpha} \vec{N}$ \\
\hline $\begin{array}{c}0131 \mathrm{~d} \\
\text { ग113k9rk }\end{array}$ & & & & & & & & & & & \\
\hline HLOINONYA & 풀ㅇㅇㅇㅇㅇㅇㅇㅇㅇㅇ & 용워 & 영응요요 & 음요 & $\stackrel{n}{\sim}:$ 음 & 응웅ㅇㅇㅇ & 둥요 & : & 웅용 & 용 & 응용ㅇㅇㅇㅛ \\
\hline 9N/רחnd & & & & & & & & & & & \\
\hline 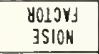 & & & & & & & & & & & \\
\hline NIYO & & & & & & & & & & & \\
\hline $\begin{array}{l}5170 \AA \\
\times 177 H \\
\end{array}$ & & & & & & & & & & & \\
\hline $\begin{array}{c}51701 \\
1001 \mathrm{NOS}\end{array}$ & $\stackrel{\circ}{\sim}$ & 음 & 음 윰엄 & 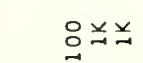 & & & & & & & \\
\hline 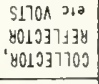 & 응융요 & 욤 & 음윰윳요 & 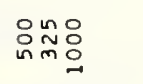 & 임염윰요 & 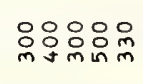 & 음욤 & 8 & 웅욤요 & ㅇ: & :O: \\
\hline$a^{\circ}$ & 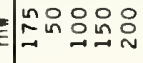 & 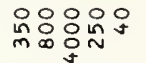 & 잉욤요 & 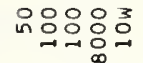 & 엄요요 & 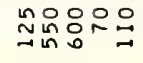 & $\stackrel{n}{\cong}$ & : & 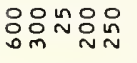 & 응ㅇㅇㅇ욤요 & 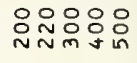 \\
\hline$\leftrightarrow$ & 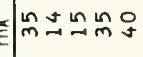 & 암은 in & $\alpha$ añ & 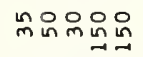 & 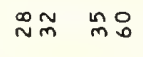 & 옹웅ㅇㅇ & o놉료 & nn & 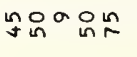 & 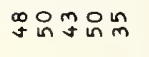 & 윴용요 \\
\hline $\begin{array}{r}51701 \\
300 \mathrm{Nr} \\
y 0 \mathrm{NB} 3 \mathrm{Bg}\end{array}$ & 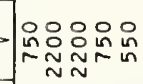 & 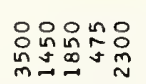 & 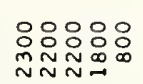 & 응응응응 & 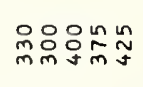 & 육용요 & 응ㅇㅁ & 品 & 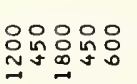 & 克跑品品品 & 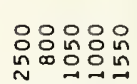 \\
\hline$\mapsto$ & E & 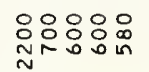 & 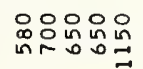 & : & 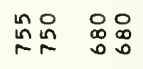 & 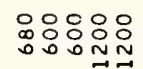 & 욱웡요 & : & 응 & 융ㅇㅇㅇㅇㅛ & 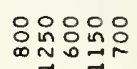 \\
\hline шँ & : & 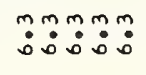 & m: & 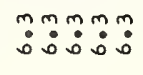 & $\ddot{m}: m: ?$ & 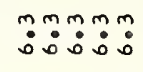 & $\ddot{m} \ddot{0}:$ & ?: & m:m & 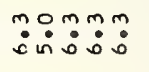 & m:mm \\
\hline NOII $\forall y 3 \mathrm{dO}$ & טuטu| & טuטu & טuטu & טuטu & טuטu & טuטu & voune & & טuטu & טบטu & uue \\
\hline$\forall \mathrm{JWN}$ & 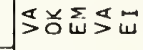 & 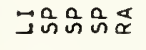 & 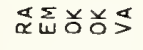 & 炭匹ココ & 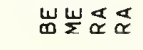 & ๔ํํำ己 & 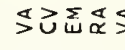 & $\$$ & 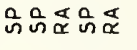 & 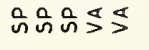 & $\sum \stackrel{5}{>}$ \\
\hline JNก1 & 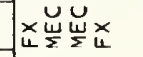 & 岀xx & 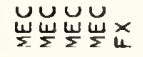 & 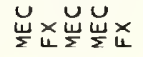 & 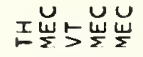 & 荘 $\quad \stackrel{u}{\Sigma} \frac{u}{\Sigma}$ & 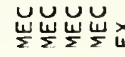 & & × & 质 & 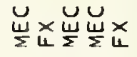 \\
\hline Olity $x$ x nO & & & & & & & & & & & \\
\hline 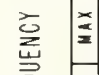 & 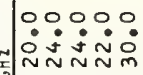 & 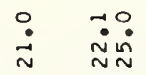 & ڤ̊뮤: & 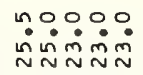 & 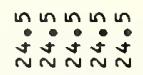 & 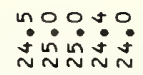 & $\begin{array}{l}+a \infty \\
\dot{\sim} \dot{N} \dot{N} \\
\sim\end{array}$ & & 迎 & 品 & 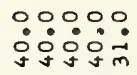 \\
\hline 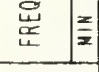 & $\because: \because: 00:$ & $\begin{array}{l}0: \because 0: \\
\dot{N} \dot{N} \dot{N} \dot{N}\end{array}$ & $\begin{array}{l}0: 0: 00 \\
\dot{N} \mathbf{N} \dot{N} \dot{N} \dot{N}\end{array}$ & 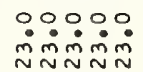 & 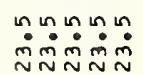 & 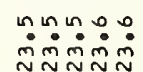 & 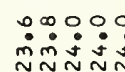 & & ominn & o:on: & 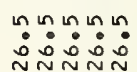 \\
\hline ON!X & 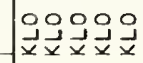 & 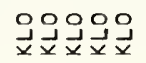 & 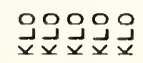 & 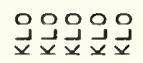 & 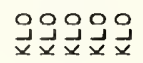 & 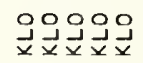 & 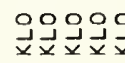 & 䱛 & 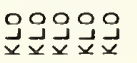 & 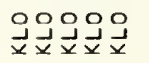 & 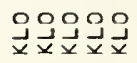 \\
\hline $709 \mathrm{~W} / \mathrm{S}$ & & $"$ & & " " " & * " " & " " " " & $"$ & & $"$ & $"$ & $"$ \\
\hline 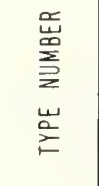 & 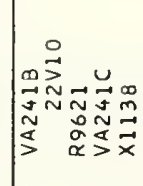 & 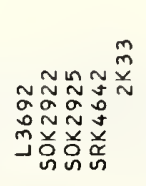 & 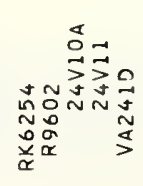 & 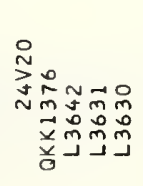 & 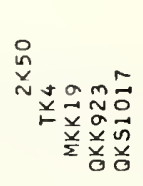 & 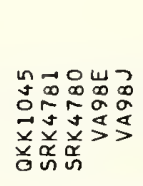 & 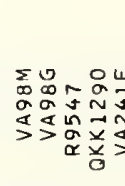 & & 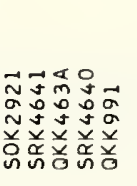 & 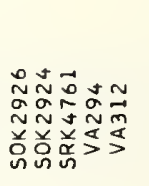 & 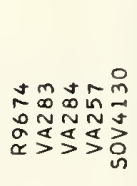 \\
\hline
\end{tabular}




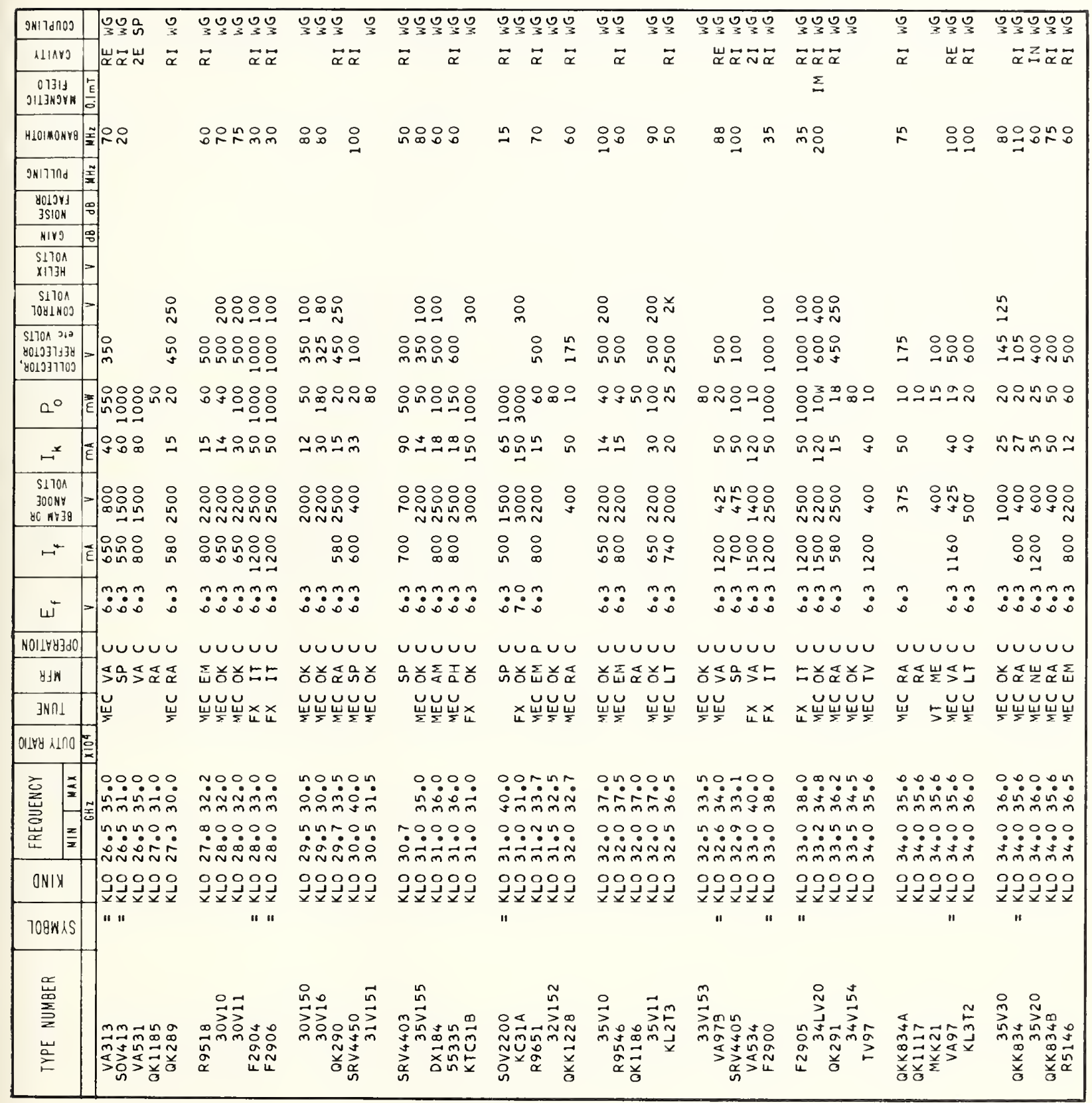




\begin{tabular}{|c|c|c|c|c|c|c|c|c|c|c|c|c|}
\hline 9אוTd ח0J & 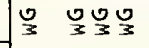 & OU & OU३ & OO3 & 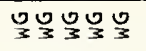 & 30 & گ్ & گँ & 우 & 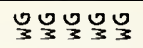 & ॠण & ॠण \\
\hline d11avs & $\bar{\alpha} \tilde{N}$ & $\vec{\alpha} \vec{\alpha}$ & $\vec{\alpha}$ & $\vec{\alpha} \vec{u} \vec{\alpha}$ & $\vec{\alpha} \vec{\alpha} \vec{\sim} \vec{\alpha} \vec{\alpha}$ & $\tilde{\alpha} \tilde{N}$ & $\vec{\alpha}$ & & $\vec{\alpha}$ & 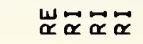 & $\vec{\alpha} \vec{\alpha} \vec{\alpha} \vec{\alpha}$ & \\
\hline 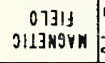 & & & & & & & & & & & & \\
\hline H1O:monv8 & in $\cong$ & $: 0$ & & № & 요요 & in & 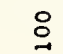 & 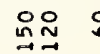 & $:$ & 우요 & 융ㅇำ & 웅요 \\
\hline dחרוואפ & & & & & & & & & & & & \\
\hline $\begin{array}{l}\text { 8019yt } \\
\text { 3Sion } \\
\end{array}$ & & & & & & & & & & & & \\
\hline MIY9 & & & & & & & & & & & & \\
\hline $\begin{array}{l}\text { S1701 } \\
X I 173 H\end{array}$ & & & & & & & & & & & & \\
\hline $\begin{array}{c}\text { S170n } \\
\text { 70y1 } 103\end{array}$ & : & : & 吕兰并兰 & : & $\stackrel{n}{\approx}$ & & 윰유 & 욤 & $8 \stackrel{0}{0}$ & ํㅠำ: & : & : 윰유 \\
\hline 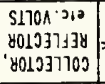 & 임융 & 욤 & & 品 总品品 & 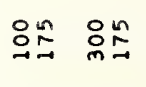 & $\stackrel{\text { D }}{N}$ & 요요 & 음 & 움 & 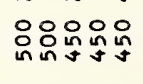 & 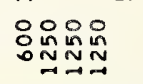 & 品品 \\
\hline$Q^{\circ}$ & 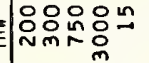 & 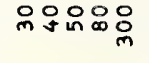 & 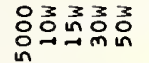 & ng욤 & 影怘 & 윳요 & $n$ & 용요용 & 品 & No: ${ }^{n} \sim \stackrel{n}{N}$ & 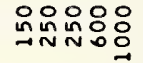 & 응응흥유문 \\
\hline 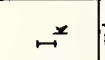 & lo & $\cong n$ 品定 & 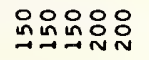 & ㄱ & $\mathcal{O}_{n \rightarrow \infty}^{n} \underset{\infty}{\infty} \stackrel{\infty}{\sim}$ & $\therefore:$ & 웅 & 융 & $\stackrel{n}{\infty} \underset{-\infty}{-\infty}$ & 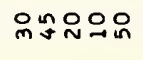 & กำกำกำ & 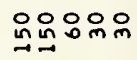 \\
\hline 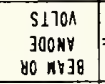 & - & 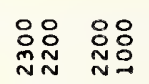 & 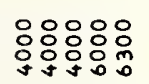 & 을 웅요 & 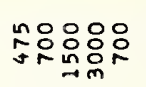 & 욤 & $\begin{array}{l}\text { :0 } \\
\text { :o: } \\
\text { min }\end{array}$ & 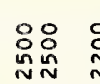 & 융 & 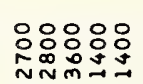 & 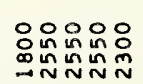 & 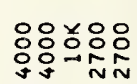 \\
\hline$H^{-}$ & $\mid \begin{array}{ll}0 \\
0\end{array}$ & : & :ㅇ: & 용 & 움융ㅇㅇㅇ & 品 & 웅 & 只品 & 品 & 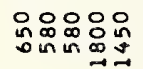 & 음요 & 品 品品 \\
\hline$\omega^{\leftarrow}$ & : & 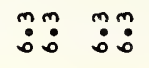 & 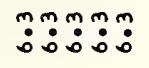 & 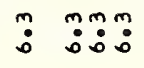 & : & $\ddot{m}: \stackrel{m}{:}$ & $\ddot{m}:$ & & $\ddot{m}:$ & 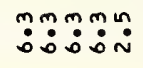 & : & 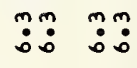 \\
\hline NOIIYGJdO & טענ & טuบu & טuטu & טuטu & טuטu & טu & טu & uUu & טU & uטu & uบuvy & טuטu \\
\hline$\forall J W$ & 岀 & コふ※ðる & コココゴ & 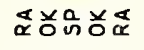 & 的的品品 & $\dot{a} ₫ \propto$ & 送 & 吕吕凹 & : & 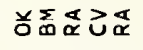 & 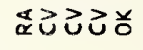 & ココ圣芫。 \\
\hline 3Nก1 & 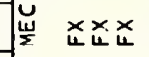 & 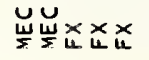 & 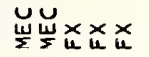 & 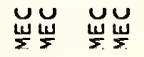 & xuuㅍ & $\underset{4}{x}$ & 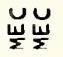 & wu & xu & 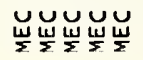 & ưưu & 品 \\
\hline OLEY AIno & & & & & & & & & & & & \\
\hline 曾 & 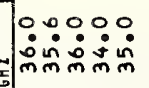 & 品品品品 & 㝵品品品品 & 㶽: & $\begin{array}{l}: \\
:\end{array}$ & 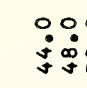 & 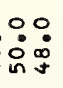 & 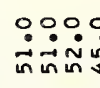 & 品品 & 过落品品 & 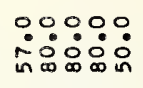 & $\begin{array}{l}0 \\
\dot{0} \\
\dot{0}\end{array}$ \\
\hline 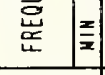 & :0:0: & 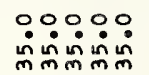 & 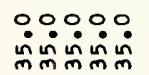 & 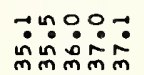 & †무요 & a: & $\therefore \frac{7}{9}$ & $0: 000$ & $\left\{\begin{array}{l}0 \\
0 \\
0\end{array}\right.$ & $\because: 00:$ & 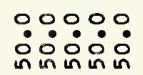 & 㝵㷎 \\
\hline ONIY & 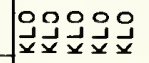 & 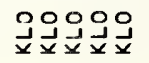 & 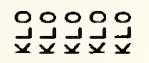 & 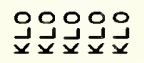 & 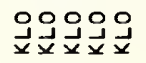 & 舟金金 & جُ & 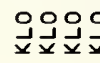 & 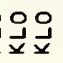 & 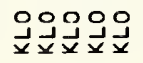 & 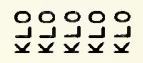 & 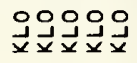 \\
\hline T08WAS & & $" \quad "$ & " " " " " & & " & & & & $"$ & & " " & " " \\
\hline 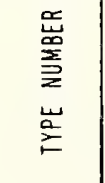 & 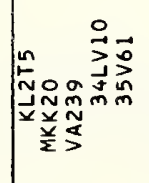 & 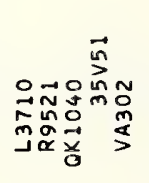 & 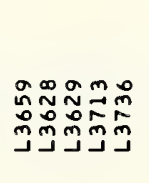 & 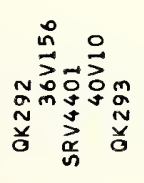 & 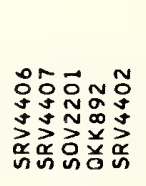 & 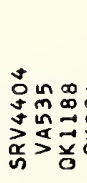 & 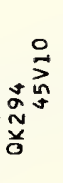 & 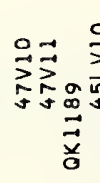 & 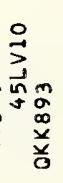 & 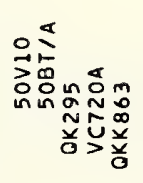 & 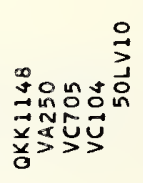 & 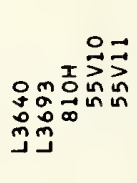 \\
\hline
\end{tabular}




\begin{tabular}{|c|c|c|c|c|c|c|c|c|c|c|}
\hline 9N17dnOS & О్య & \O & 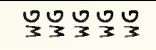 & 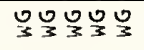 & 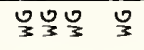 & 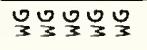 & 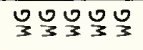 & 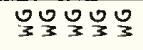 & צ' & 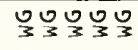 \\
\hline Al11K3 & $\vec{\alpha} \vec{\alpha} \bar{\alpha}$ & $\vec{\alpha} \vec{\alpha} \vec{\alpha} \quad \vec{\alpha}$ & $\vec{\alpha} \underset{\alpha}{w}$ & $\vec{\alpha} \vec{\alpha} \vec{\alpha} \vec{\alpha} \vec{\alpha}$ & $\vec{\alpha} \vec{\alpha} \quad \vec{\alpha}$ & & $\vec{\alpha} \vec{\alpha} \vec{\alpha} \vec{\alpha}$ & $\vec{\alpha} \vec{\alpha}$ & $\vec{\alpha} \vec{x}$ & $\vec{\alpha} \vec{\alpha} \vec{\alpha} \vec{\alpha}$ \\
\hline 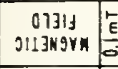 & & & & & $\stackrel{\circ}{\sim}$ & & & & & \\
\hline H.OIMONY & in & $\stackrel{\circ}{\leftrightarrow}$ & 웅용ㅇ욤ㅇ & nㅗ욤욤요 & $\stackrel{\circ}{\circ} \stackrel{\circ}{\stackrel{n}{n}}$ & & 욤요 & 옹임욤유 & $\therefore \stackrel{\circ}{\sim} \stackrel{\circ}{\stackrel{n}{n}}$ & 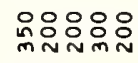 \\
\hline 9ו17רd & & & & & & & & & & \\
\hline $\begin{array}{l}\text { yoljys } \\
\text { JSION }\end{array}$ & & & & & 。 & & & & & \\
\hline Nirg & & & & & & & & & & \\
\hline $\begin{array}{l}51700 \\
\times 113 \mathrm{H}\end{array}$ & & & & & & & & & & \\
\hline $\begin{array}{c}51701 \\
7041100\end{array}$ & :임윰유 & 号品 & : & : & 品品 & 兰兰兰兰 & 品品 & :임윰요 & 於品品 & $\stackrel{N}{N}$ \\
\hline 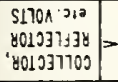 & 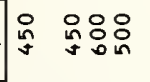 & 识品品 品 & 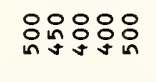 & 웁윱윰융ㅇ & 육융요 & & 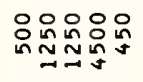 & 음음ㅇㅇㅁ & 응 品品品 & 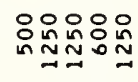 \\
\hline $0^{\circ}$ & No윰요 & $\stackrel{n}{\sim} \stackrel{n}{\sim} \cong$. & 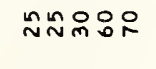 & $\stackrel{n}{\sim} \cong \cong$ & 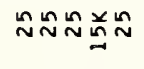 & 응응요요 & 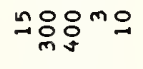 & Nㅇ유수요 & 음응ㅇㅇㅛ & " 윰요 \\
\hline$\omega^{x}$ & 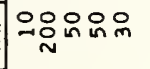 & 으용요 & 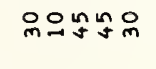 & 우유워 & 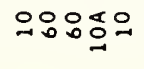 & 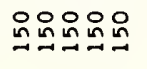 & 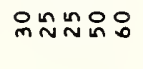 & 유숌요 & : $\underset{\sim}{0} \underset{N}{N} \stackrel{N}{N}$ & $\stackrel{n}{\sim} \tilde{N} \tilde{N} \cong \stackrel{n}{\sim}$ \\
\hline $\begin{array}{r}51701 \\
300 \mathrm{NY} \\
80 \mathrm{KY3g}\end{array}$ & | & 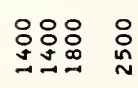 & ㅇㅇㅇㅇㅛ & 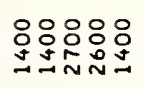 & 웅요 & 음웅웡워 & 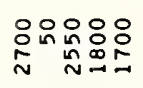 & 용요 & g: & 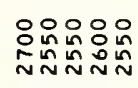 \\
\hline$\mapsto$ & 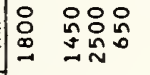 & 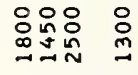 & 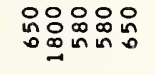 & 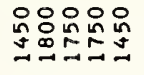 & 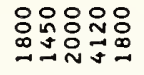 & :용유윰유유 & 융ㅇㅇ요요 & 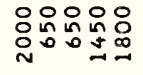 & 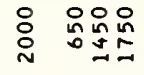 & 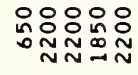 \\
\hline$\omega^{\prime \prime}$ & mon:m & 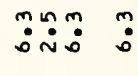 & : & $\dot{\sim} \dot{n} \dot{m} \dot{m} \dot{n} \tilde{n}$ & 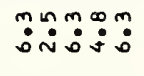 & : & 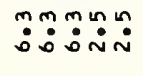 & 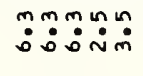 & $\ddot{m}: \ddot{n} \dot{\sim}: 0$ & 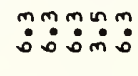 \\
\hline NOIIYYJdO & טuטu & טบט & טบט & טuטu & uบua & טuטu & טuטu & טuטu & บaUuU & טบט \\
\hline$y \mathrm{IJn}$ & 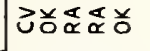 & 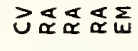 & 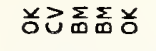 & 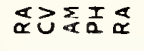 & 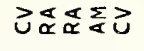 & コココココ & ๖つさぬ๕ & 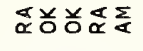 & 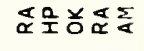 & るひさエさ \\
\hline 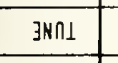 & 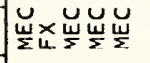 & WU & 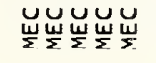 & 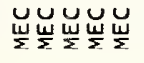 & U⿺乚一匕 & xưw & U⿺乚ّ山心 & 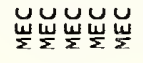 & 㞭 & 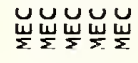 \\
\hline outro sino & & & & & 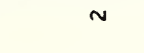 & & & & $=$ & \\
\hline 惫 & ํำ & 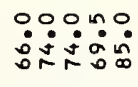 & 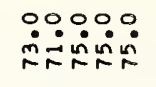 & 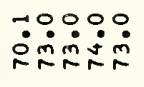 & :웅우 & & 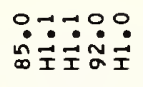 & 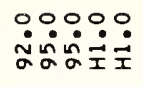 & 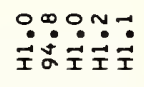 & 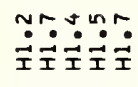 \\
\hline \begin{tabular}{l|l|}
$\stackrel{a}{\tilde{x}}$ & $\frac{x}{x}$ \\
\end{tabular} & 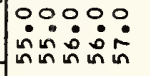 & 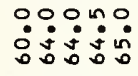 & 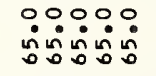 & 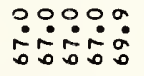 & 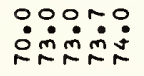 & 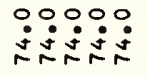 & :O: & : : : : & 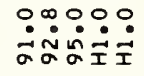 & 至主主主主 \\
\hline ONIX & 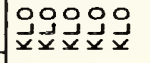 & 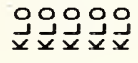 & 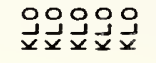 & 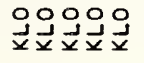 & 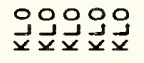 & 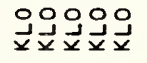 & 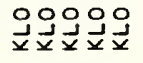 & 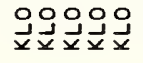 & 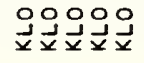 & 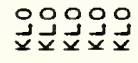 \\
\hline 7ogkis & $"$ & $"$ & & & & " " " " " & & & & " " \\
\hline 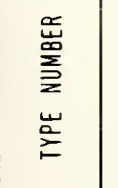 & 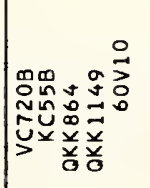 & 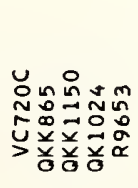 & 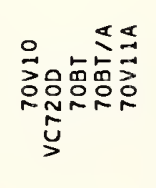 & 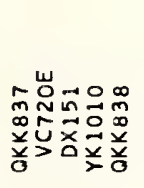 & 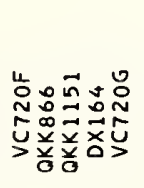 & 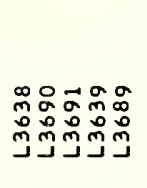 & 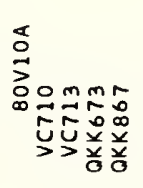 & 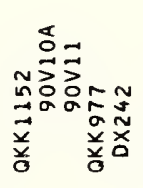 & 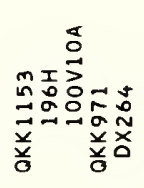 & 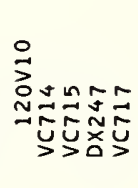 \\
\hline
\end{tabular}




\begin{tabular}{|c|c|c|c|c|c|c|c|c|c|c|}
\hline 9N17dnos & \multirow[t]{3}{*}{ 8ㅇ } & 웅요웅 & 옹옹 & 옹오옹 & 옹워 & ㅇ 888 & 웅옹 & 웅옹 & 엉 옹 & 옹오온 \\
\hline AL1AY3 & & & & & & & & & & \\
\hline 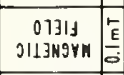 & & & $\Sigma$ & & $\Sigma$ & $\sum \sum \sum$ & & & $\Sigma \Sigma$ & $\Sigma$ \\
\hline HLOIMONY & & & & & & & & & $N$ & \\
\hline 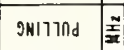 & & & & & & & & & m & \\
\hline $\begin{array}{l}\text { yolivis } \\
\text { 3sion }\end{array}$ & & & & & & & & & & \\
\hline \begin{tabular}{l|l} 
NIY9 & : \\
\end{tabular} & & & & & & & & & & \\
\hline $\begin{array}{l}\text { S1770 } \\
\times 1734\end{array}$ & & & & & & & & & & \\
\hline $\begin{array}{r}51700 \\
1041402\end{array}$ & $\stackrel{\circ}{\circ}$ & 욕 욧 & $\stackrel{0}{\circ}$ & : & 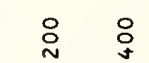 & & : & 品品 : & : & 号 \\
\hline 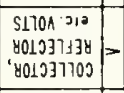 & 음 & 응 & $\stackrel{\circ}{N}$ & $\stackrel{\circ}{:}:$ & 움 & : & 은 & 잉 & & : \\
\hline$\alpha^{\circ} \bar{E}$ & 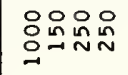 & 음임용음 & 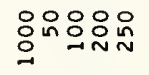 & 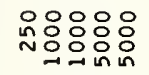 & 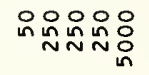 & 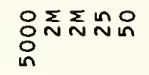 & 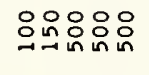 & 음윰융ㅇํ & 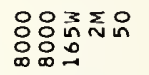 & 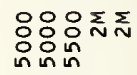 \\
\hline \begin{tabular}{l|l}
$\mapsto^{x}$ & $\bar{E}$ \\
\end{tabular} & 웅의 in & mammin & no mon & $n \infty \infty 00$ & 으뇨묘 & 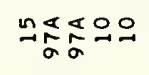 & 엉으으으 & $\infty 0 \infty \div 0$ & 느융요 & ผnన N \\
\hline 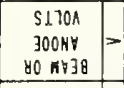 & 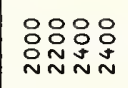 & 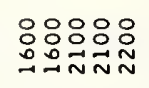 & 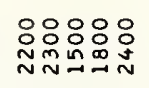 & 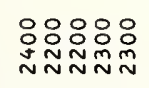 & 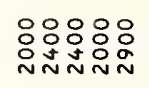 & 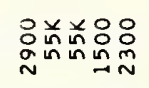 & 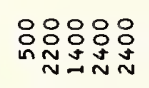 & 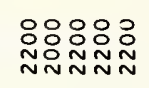 & 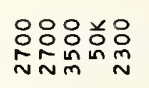 & 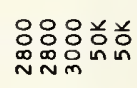 \\
\hline$F \vec{E}$ & 음유 & 음윰욤유 & : & 㗊: & 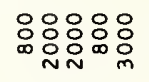 & 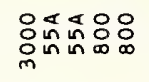 & 음ㅇㅇㅇㅇㅇㅇㅁ & 응융ㅇํㅇㅇํ & 음을 & 음윰 \\
\hline wั & 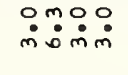 & $\ddot{m} \dot{m} \dot{m} \dot{m} \dot{m}$ & 艊品品品 & $\ddot{m} \dot{m} \dot{m} \dot{m} \dot{m}$ & $\ddot{\dot{m}} \dot{\dot{m}} \dot{m} \dot{0} \dot{m}$ & 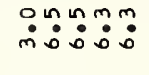 & 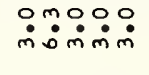 & 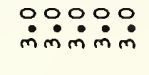 & $\dot{m} \dot{m} \dot{m} \dot{n}$ & $\ddot{n} \dot{m} \dot{0}$ \\
\hline NOILYGJdO & vuvu & vouve & vưur & ưuu & vưuu & uaauv & ưưu & uuvuu & uvuau & uvua \\
\hline$\forall J w$ & $\frac{\alpha}{\Sigma}>\frac{\alpha}{\Sigma} \frac{\alpha}{\Sigma}$ & $\frac{\alpha}{\Sigma} \frac{\alpha}{\Sigma} \frac{\alpha}{\Sigma} \frac{\alpha}{\Sigma} \frac{\alpha}{\Sigma}$ & $\frac{\alpha}{\Sigma} \tilde{w} \frac{\alpha}{\Sigma} \vec{w} \frac{\alpha}{\Sigma}$ & $\frac{\alpha}{\Sigma} \frac{\alpha}{\Sigma} \frac{\alpha}{\Sigma} \frac{\alpha}{\Sigma} \frac{\alpha}{\Sigma}$ & $\vec{w} \frac{\alpha}{\Sigma} \frac{\alpha}{\Sigma} \vec{w} \frac{\alpha}{\Sigma}$ & 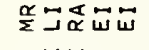 & $\frac{\alpha}{\Sigma} \vec{w} \frac{\alpha}{\Sigma} \frac{\alpha}{\Sigma} \frac{\alpha}{\Sigma}$ & $\frac{\alpha}{\Sigma} \frac{\alpha}{\Sigma} \frac{\alpha}{\Sigma} \frac{\alpha}{\Sigma} \frac{\alpha}{\Sigma}$ & $\frac{\alpha}{\Sigma} \frac{\alpha}{\Sigma} \beth \propto \widetilde{\varpi}$ & $\frac{\alpha}{\Sigma} \frac{\alpha}{\Sigma} \vec{w} \alpha \widetilde{\alpha} \alpha$ \\
\hline $3 N \cap 1$ & ら555 & ら5ケ5 & ら55ケ5 & ら55ケケ & ケら5ケ5 & ๖岀岕ヶレ & ら5ケらら & ケ5ケ5ケ & ケร岀メ & ケケケメ岕 \\
\hline $0.118 y$ anno & & & & & & $\stackrel{\infty}{N}$ & & & $\stackrel{\infty}{-}$ & \\
\hline 曾 & 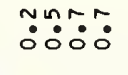 & 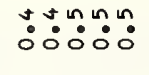 & \begin{tabular}{l}
$n \sim 0$ \\
\hdashline \\
\hdashline
\end{tabular} & $\because \because \because \because 0^{n}$ & 决 & $\stackrel{\infty}{0}: \Delta \circ: \circ:$ & & :゚:゚゚ & 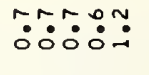 & 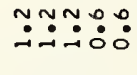 \\
\hline 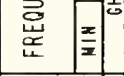 & 뭄ำ & N : : : N & 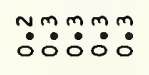 & $\ddot{0}: \ddot{m}: m$ : & $\dot{0}: \stackrel{+}{0}: \dot{0}:$ & 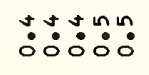 & 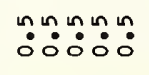 & 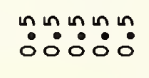 & 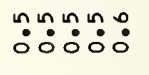 & 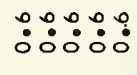 \\
\hline ONIX & 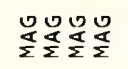 & 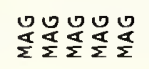 & 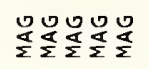 & 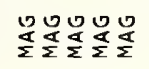 & 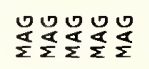 & 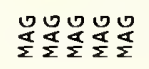 & 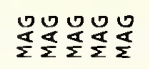 & 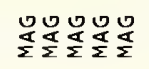 & 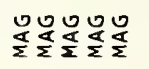 & 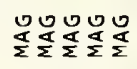 \\
\hline $708 \mathrm{~W} / \mathrm{S}$ & & & " " & & & " " " " & & & " & $"$ \\
\hline 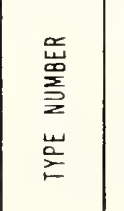 & 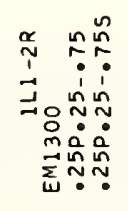 & 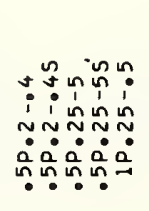 & 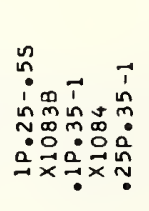 & 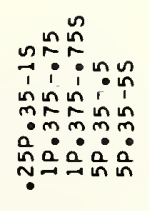 & 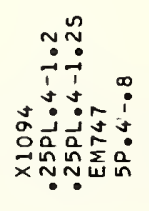 & 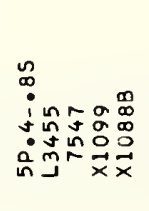 & 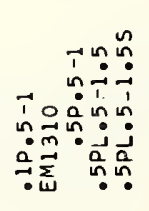 & 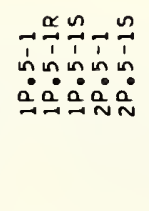 & 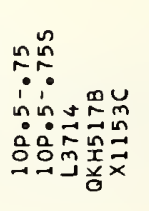 & 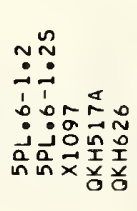 \\
\hline
\end{tabular}




\begin{tabular}{|c|c|c|c|c|c|c|c|c|c|c|}
\hline 9N17dnOS & 웅요 & יㅜㅇㅇㅛ & יㅜㅇㅇㅛ & 언언어 & 운웡어 & 언운요 & 엉ㅇㅇㅇ & ㅇ 우 & 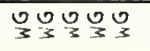 & $\frac{00}{3}$ \\
\hline RIAAY & & & & & & & & & & \\
\hline 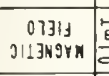 & & $\sum \sum$ & $\Sigma \Sigma \Sigma$ & $\Sigma$ & & $\Sigma$ & $\sum \Sigma$ & 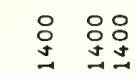 & 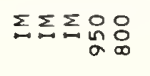 & $\Sigma \Sigma$ \\
\hline HOOLOHYG $\div$ & & & & & & & N & & & \\
\hline 9אורוาח & 필 & & & & & & 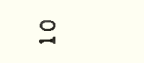 & ง & $\operatorname{lng} a$ & \\
\hline 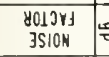 & 5. & & & & & & & & & \\
\hline \begin{tabular}{l|l|l|} 
NIYg & $\frac{\pi}{2}$ \\
\end{tabular} & & & & & & & & & & \\
\hline $\begin{array}{l}5170 \AA \\
\times 173 \mathrm{H}\end{array}$ & & & & & & & & & & \\
\hline $\begin{array}{c}\text { S170A } \\
\text { 70y } 1 \text { NO3 }\end{array}$ & 임웜워 & $\stackrel{0}{\simeq}$ & & 뭉ํํ & 吕 & 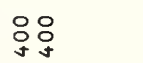 & $\stackrel{\circ}{\sim}$ & & & \\
\hline 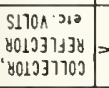 & 品 & : & & & $\stackrel{\circ}{\circ}:$ & & $\stackrel{\circ}{N}$ & & & \\
\hline$a^{\circ}=$ & E & 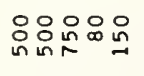 & 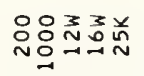 & 酋品品品品 & 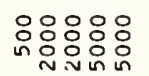 & 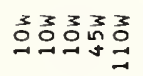 & 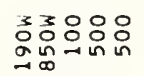 & 差兑兑兑总总 & $\sum \sum_{N} \sum_{N} \sum_{n} \sum_{n}$ & 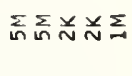 \\
\hline$\mapsto$ & $\bar{E}$ & 으으능유 & Nㅗㅇㅛㅛ & 응으으음 & 음뜸요 & $\stackrel{N}{N}$ 임 & 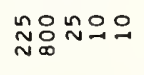 & 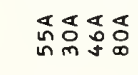 & 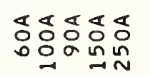 & 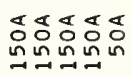 \\
\hline 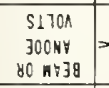 & 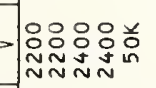 & 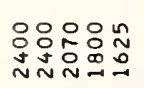 & 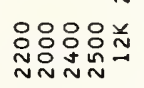 & 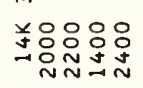 & 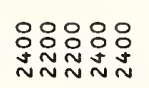 & 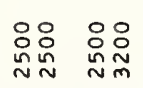 & 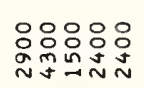 & 弟鬲总羔 & 居啰兑总总 & 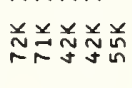 \\
\hline$\mapsto$ & E| $\mid$\begin{tabular}{l}
$O$ \\
\hdashline \\
0 \\
D:
\end{tabular} & 응ㅇㅁㅇㅇㅁㅇㅁㅇ & 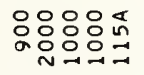 & 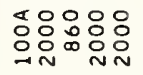 & 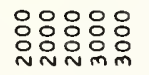 & 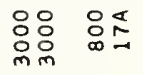 & 代品品品 & 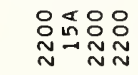 & 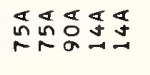 & 总 局 \\
\hline w' & $\ddot{m} \dot{m} \dot{0} \dot{0}$ & $\ddot{m} \dot{m} \dot{m}: \dot{m}: \dot{m}$ & 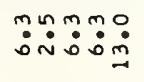 & 这范品品 & 向品品品 & $\ddot{m} \dot{m} \dot{m} \ddot{n}$ & $\ddot{0} \ddot{0} \dot{0} \dot{m} \dot{m}$ & 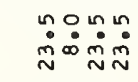 & 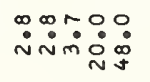 & $\stackrel{\circ}{\circ} \dot{\sim} \dot{0}$ \\
\hline N01 \&Yy $\mathrm{dO}$ & uuvua & טuטu & טuטu & טuטu & טuטu & טuטu & טuטu & vaaaa & a a a a a & $a a a a a$ \\
\hline HJW & $\frac{\alpha}{\Sigma} \frac{\alpha}{\Sigma} \frac{\alpha}{\Sigma} \frac{\alpha}{\Sigma} \alpha$ & $\frac{\alpha}{\Sigma} \frac{\alpha}{\Sigma}$ Ш゙யU & யே岕でむ゙ & 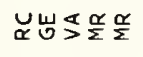 & $\frac{\alpha}{\Sigma} \frac{\alpha}{\Sigma} \frac{\alpha}{\Sigma} \frac{\alpha}{\Sigma} \frac{\alpha}{\Sigma}$ & $\frac{\alpha}{\Sigma} \frac{\alpha}{\Sigma} \propto \varpi \varpi$ & 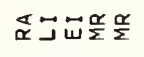 & ๔I & ๔ & 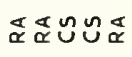 \\
\hline 3Nก1 & $5555 x$ & 55555 & 5555x & 圠5 555 & 55555 & ケレメே岕 & 岂岁っレレ & 岀 岀岕 & 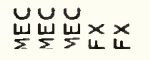 & 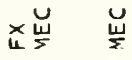 \\
\hline $0116 y$ גIno & $\stackrel{\infty}{*}$ & & & & & & & 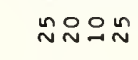 & $\mathfrak{Z} \simeq \simeq \backsim \mathfrak{N}$ & $\stackrel{\infty}{\sim} \leadsto \curvearrowleft \curvearrowleft$ \\
\hline 䇋 & Un: & 过さ゚ロ & 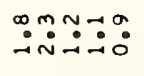 & 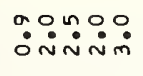 & 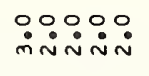 & 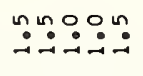 & 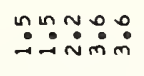 & 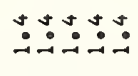 & シささささ & 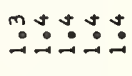 \\
\hline 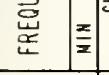 & $\ddot{\circ} \ddot{\circ} \because \ddot{\circ}$ & $\ddot{0} \dot{0} \dot{0} \dot{0} \dot{0}: \dot{0}$ & $\ddot{\circ} \dot{0} \dot{0} \dot{0} \dot{0}$ & 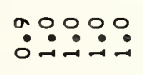 & $\because \because \because: \circ$ & $\because \because \because \because:$ & 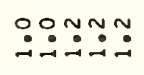 & 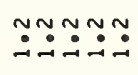 & 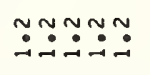 & 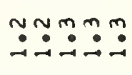 \\
\hline ONIX & $\mid$ & 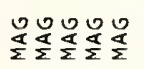 & 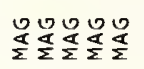 & 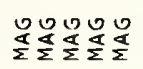 & ソั0 & 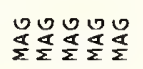 & 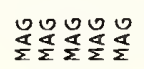 & 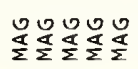 & 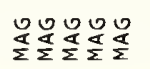 & 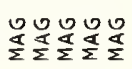 \\
\hline 708WAS & & & " " " & & & " & & & & \\
\hline 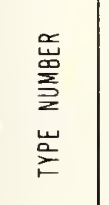 & 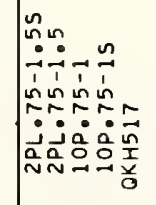 & 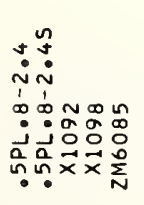 & 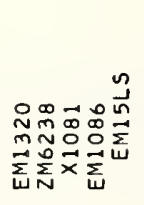 & 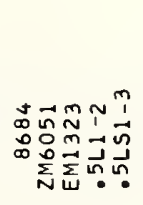 & 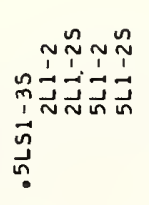 & 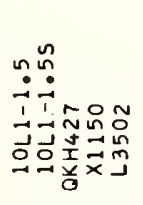 & 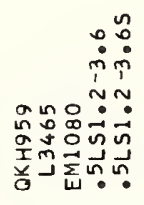 & 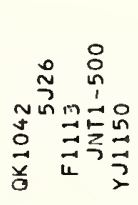 & 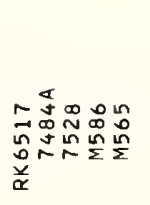 & 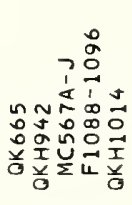 \\
\hline
\end{tabular}




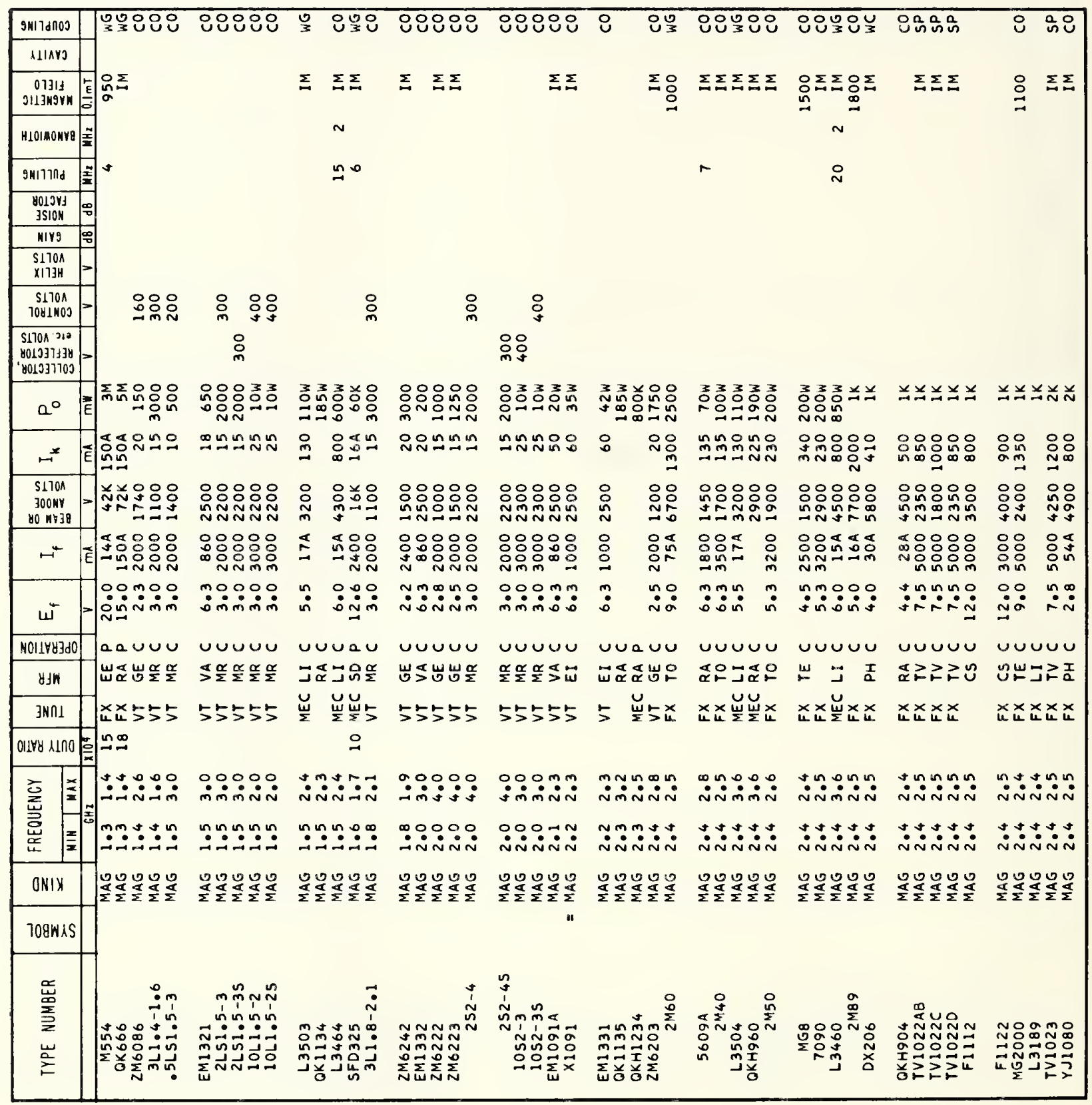




\begin{tabular}{|c|c|c|c|c|c|c|c|c|c|c|}
\hline 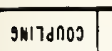 & ¿ צü & 눙엉어 & 언언무 & 엉ㅇㅇㅇㅇ & O & 8808 & 엎 푼 & जOOO & 엉ㅇㅇ & 원 \\
\hline A11AY & & & & & & & & & & \\
\hline $\begin{array}{c}01311 \\
\text { ग11399\% }\end{array}$ & $\Sigma \Sigma$ & $\Xi$ & $\Sigma$ & $\Sigma \Sigma \Sigma$ & $\stackrel{\circ}{\vdots}$ & 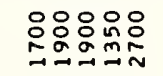 & 요 & $\sum$ 乏足足 & 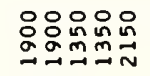 & 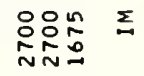 \\
\hline HLOMONYW9 & & & & : & & 웅 & & & 우우 & - \\
\hline 9Milา & & $\simeq$ & & & & $\cong n$ & $\cong \mathrm{rn}$ & & $\cong$ & 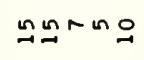 \\
\hline 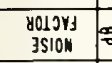 & & & & & & & & & & \\
\hline Mirg & & & & & & & & & & \\
\hline $\begin{array}{l}S 110 \Lambda \\
\times 1173 H \\
\end{array}$ & & & & & & & & & & \\
\hline $\begin{array}{c}51701 \\
1041103\end{array}=$ & & 용 & 品品 & 号 & 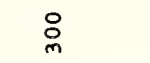 & & & $\stackrel{\circ}{\circ}$ & & \\
\hline 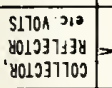 & & ঃ & & - & & & & & & \\
\hline$a^{\circ}=$ & 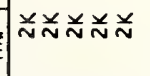 & 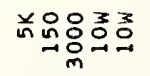 & 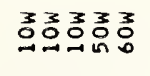 & 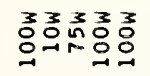 & 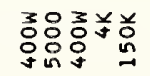 & 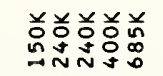 & 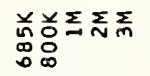 & 朔路总总 & 曾曾兑兑总总 & 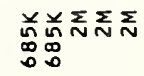 \\
\hline$=$ & 잉요 & 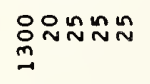 & 엉워용 & 옴암요 & $\stackrel{n}{\sim}$ & 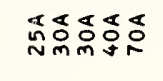 & 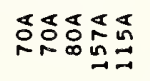 & 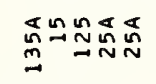 & 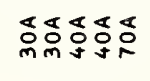 & 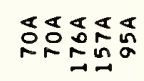 \\
\hline $\begin{array}{c}5170 \mathrm{~A} \\
300 \mathrm{wr} \\
80 \mathrm{Wr} 39\end{array}$ & 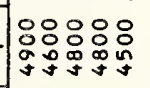 & 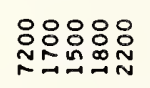 & 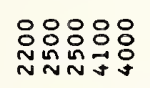 & 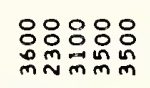 & $\stackrel{\circ}{\circ}$ & 次잉ㅁㅇㅁㅁ & 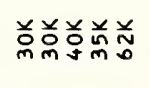 & 쏫웜웜웅 & 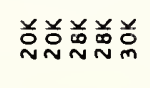 & 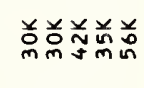 \\
\hline$\rightarrow \vec{E}$ & 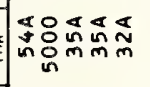 & 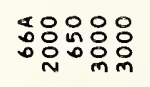 & 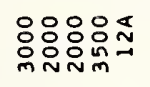 & 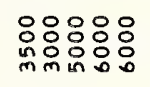 & $\stackrel{\circ}{\circ}$ & 옹요 & 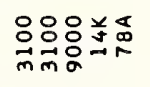 & 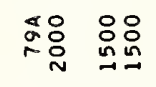 & 움윰윰웅 & 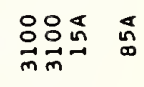 \\
\hline$\omega^{ \pm}$ & ن̊ñ & 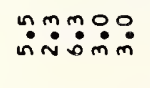 & 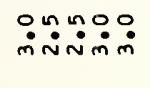 & 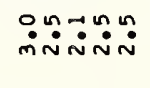 & $\stackrel{m}{\dot{m}} \quad \stackrel{m}{0}$ & m: & : : & 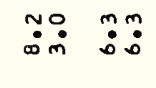 & $m m: \because: 00$ & $\begin{array}{l}: \\
\dot{0} \\
\dot{0}\end{array}$ \\
\hline MOIIYYJdO & טuטuט & บบบบ & ưu & ט & บบบบa & 00000 & 0,000 & auva & $0 \circ 000$ & 00000 \\
\hline 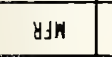 & I & エ & $\frac{\alpha}{\Sigma} \omega_{\circlearrowleft} \omega \frac{\alpha}{\Sigma} \alpha$ & 受古岁岁岕 & $\underset{\alpha}{\alpha} \frac{\alpha}{\alpha} \underset{\alpha}{\alpha} \frac{\alpha}{\alpha}$ & 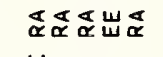 & 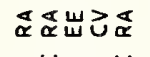 & $\circ \frac{\alpha}{\Sigma} \propto \varangle \propto$ & 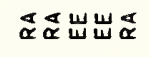 & 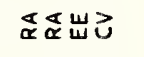 \\
\hline JKח1 & ]$_{u}^{x} \quad x \times x$ & ×ே5555 & ラケらケメ & ららららら & $5 \quad \stackrel{u}{\Sigma}$ & 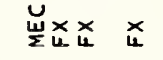 & 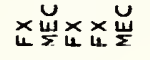 & 荘々 㟧㟧 & $\begin{array}{ll}x & x \\
4 & x\end{array}$ & $x_{4}^{x} \times x_{u}^{x}$ \\
\hline Oury AnO & & & & & $\stackrel{i}{i}$ & 오오응 & $O^{n} \cong$ & 요 & 오요 & 오요 \\
\hline 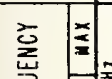 & 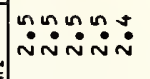 & 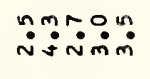 & 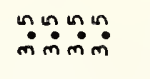 & 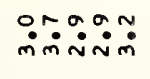 & $\stackrel{\infty}{\infty} \stackrel{\infty}{\sim} \stackrel{\infty}{\sim} \stackrel{\infty}{\sim} \dot{\sim}$ & $\begin{array}{l}\infty \infty \sim: \infty \\
\dot{\sim}\end{array}$ & 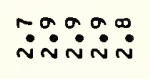 & $\ddot{\sim} \dot{m} \dot{n} \dot{\sim} \dot{\sim}$ & 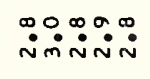 & 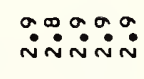 \\
\hline 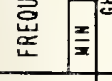 & ஸे山゙் & 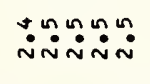 & 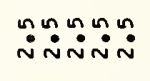 & ஸ̊ñ⿻ & 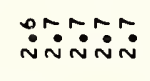 & 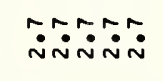 & 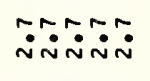 & ஸें & $\begin{array}{l}\infty \infty \infty \infty \infty \infty \infty \\
\dot{N} \dot{N} \dot{N} \dot{N}\end{array}$ & $\dot{\sim}_{\dot{N}}^{\infty} \dot{\sim}^{\infty} \dot{\sim}^{\infty} \dot{\sim}$ \\
\hline ONIX & 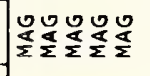 & 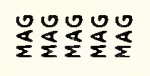 & 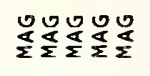 & 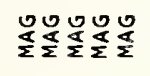 & 造造造造是 & 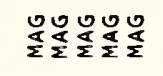 & 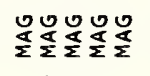 & 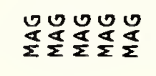 & 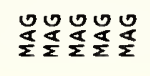 & 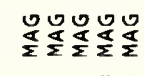 \\
\hline 7ognks & & & & & & & & & & \\
\hline 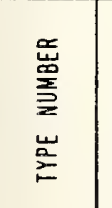 & 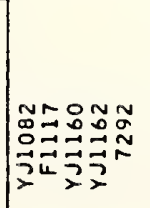 & 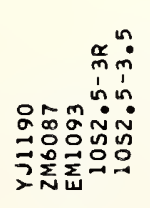 & 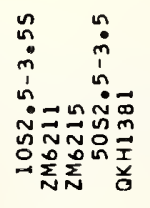 & 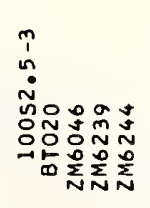 & 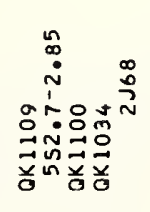 & 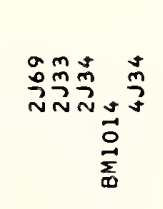 & 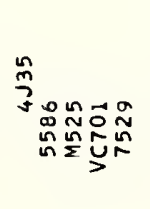 & 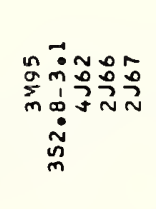 & 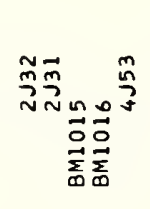 & 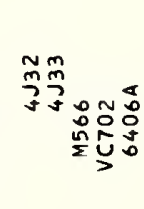 \\
\hline
\end{tabular}




\begin{tabular}{|c|c|c|c|c|c|c|c|c|c|c|}
\hline 9k17dnOS & \О & 잉 & 8 & 엉요 & סु) & 8웅으 & 웅오웅 & ㅇㅇㅇㅛ & 욱원어 & 운웡어 \\
\hline ALAVY & & & & & & & & & & \\
\hline 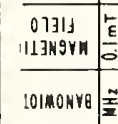 & $\sum \sum \sum \sum$ & $\sum \Sigma \sum \Sigma$ & \begin{tabular}{l}
$\stackrel{8}{\circ}$ \\
\hdashline \\
$\vdots$
\end{tabular} & 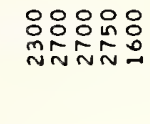 & 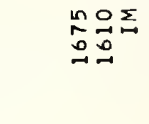 & $\Sigma \Sigma$ & 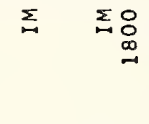 & 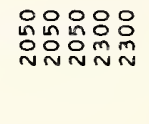 & 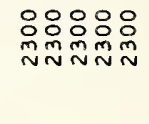 & 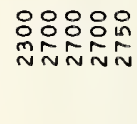 \\
\hline 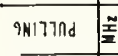 & & & $\stackrel{n}{\rightarrow}$ & $\stackrel{n}{\sim}$ & rr & $\stackrel{\circ}{\sim}$ & $m \infty$ & rrr & & $\stackrel{\sim}{\sim} \approx$ \\
\hline 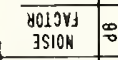 & & & & & & & & & & \\
\hline \begin{tabular}{l|l} 
HIY9 & I \\
\end{tabular} & & & & & & & & & & \\
\hline $\begin{array}{l}5170 \AA \\
\times 17 y H\end{array}$ & & & & & & & & & & \\
\hline $\begin{array}{c}51701 \\
70411003\end{array}=$ & & & & & & : & $\cong \cong$ & & & \\
\hline 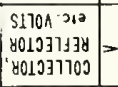 & & & & & & ষ্ণ & & & & \\
\hline$a^{\circ}$ & 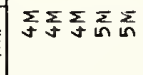 & 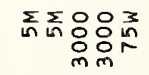 & 预兰㒸总兑 & 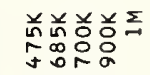 & $\sum \sum \sum \sum_{N} \sum_{N}$ & 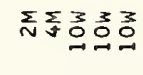 & 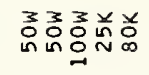 & 兑兑兑总兑 & 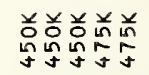 & 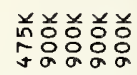 \\
\hline$r$ & 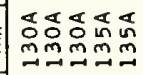 & 芯芯 울움 & 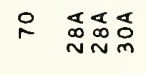 & 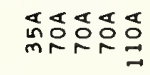 & 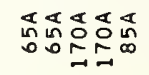 & 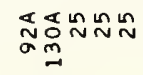 & 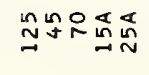 & 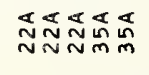 & 芯芯芯芯倠 & 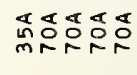 \\
\hline $\begin{array}{c}5170 \AA \\
300 \mathrm{HY} \\
\text { yo } \mathrm{HB} 3 \mathrm{Bg}\end{array} \mid>$ & 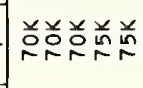 & 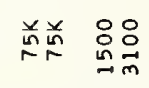 & 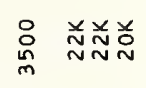 & 总总樼希芯 & 总总兽总总 & × & 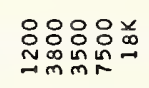 & 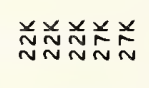 & 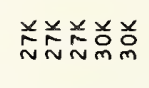 & 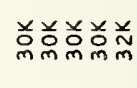 \\
\hline+ & 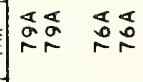 & 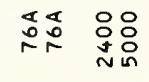 & 음 & 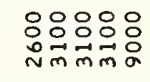 & 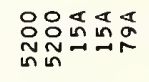 & 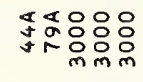 & 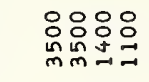 & 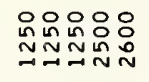 & $\begin{array}{l}\text { :ㅇㅇㅇㅇㅇ } \\
\text { 윤융 }\end{array}$ & 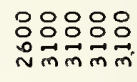 \\
\hline$\omega^{\leftarrow}$ & 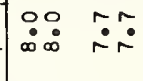 & $\ddot{\sim} \stackrel{\sim}{\sim}$ & 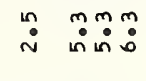 & 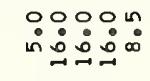 & 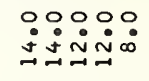 & 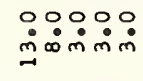 & $\dot{m} \dot{m} \dot{m} \dot{0} 0$ & $\because \because: 000$ & 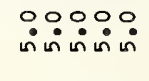 & 웅요 \\
\hline 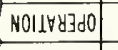 & anaog & anuvu & Uuaa & a.aua & $000 a 0$ & a auvu & บบบaa & 0,000 & $00 a 0 a$ & a a a a \\
\hline$\forall \leq W$ & 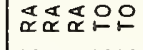 & 요๙岕山 & 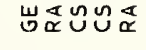 & 〉৫氐出 & 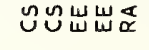 & $\underset{\alpha}{\alpha} \underset{\alpha}{\alpha} \frac{\alpha}{\Sigma} \frac{\alpha}{\Sigma} \frac{\alpha}{\Sigma}$ & $\underset{\alpha}{\varangle} \frac{\alpha}{\Sigma} \frac{\alpha}{\Sigma} \underset{\alpha}{\alpha}$ & 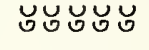 & U్Uㅓऽ & 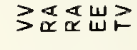 \\
\hline 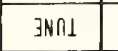 & ưx & 訔岕 & $\stackrel{5}{>}$ & 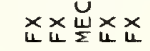 & $x_{L}^{x} \times \underset{u}{x}$ & メேே゙55 & 5รメ爻 & $x_{u}^{x} \times x_{u} \times x$ & 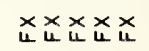 & $x_{\mathbb{L}}^{x} \times x_{\mathbb{L}} \times x_{u}$ \\
\hline $01184 \wedge 1 \cap 0=\frac{F}{2}$ & $\quad$ 엉ㅇㅇ & 으우 & $\ln \backsim 0$ & $\simeq \operatorname{On}^{n} \stackrel{n}{\sim}$ & 웅요듀 & $r \infty$ & 옹 & 응ㅇㅇㅇㅇㅇㅇ & 웅우워 & 주우응ㅇㅁㅇ \\
\hline 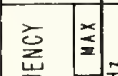 & 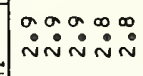 & $\ddot{\sim} \dot{\sim} \dot{\sim} \dot{m} \dot{m}$ & 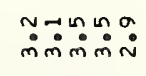 & 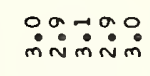 & m்n் & 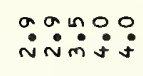 & $\dot{m} \dot{\sim} \dot{m} \dot{m} \dot{m}$ & $\ddot{m} \dot{0} \dot{m} \dot{m} \dot{m}$ & 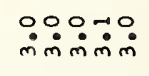 & $\dot{m} \dot{m} \dot{m} \dot{m} \dot{m} \dot{m}$ \\
\hline 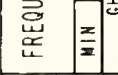 & 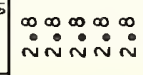 & $\begin{array}{l}\infty \infty a \sigma a \\
\dot{\sim} \sim \dot{\sim} \sim \dot{\sim}\end{array}$ & $\begin{array}{l}a \sigma a \sigma o \\
\dot{\sim} \dot{\sim} \dot{\sim}\end{array}$ & $\begin{array}{l}a \sigma a \sigma a \\
\dot{\sim} \dot{\sim} \dot{\sim} \dot{\sim}\end{array}$ & $\dot{\sim} \dot{\sim} \dot{\alpha} \dot{\alpha} \dot{\sim}$ & 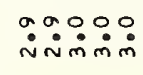 & 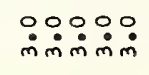 & $\ddot{m} \dot{m} \dot{m} \dot{m} \dot{m}$ & mंn்min & 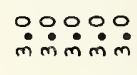 \\
\hline ONIX & 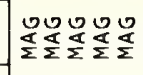 & 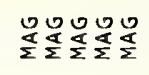 & 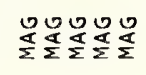 & 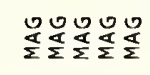 & 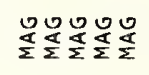 & 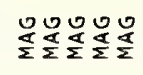 & 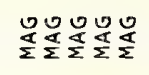 & 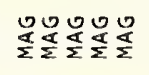 & 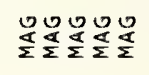 & 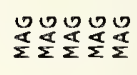 \\
\hline $709 \mathrm{k} \times \mathrm{S}$ & & & & & & & & & & \\
\hline 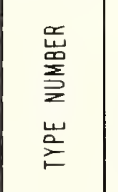 & 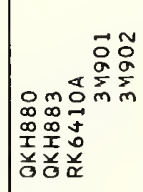 & 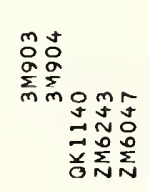 & 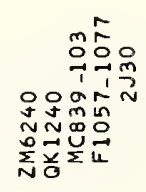 & 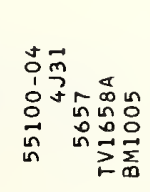 & 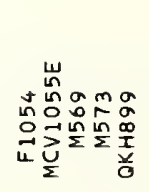 & 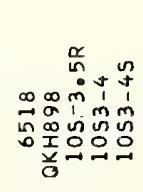 & 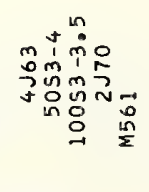 & 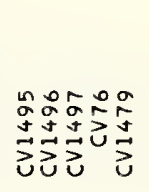 & 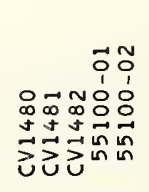 & 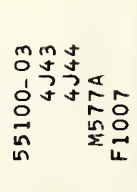 \\
\hline
\end{tabular}




\begin{tabular}{|c|c|c|c|c|c|c|c|c|c|c|}
\hline 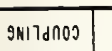 & \multirow{4}{*}{ 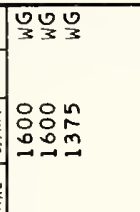 } & \multirow{3}{*}{ 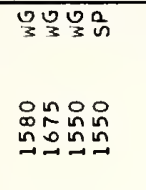 } & \multirow{4}{*}{ 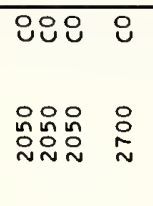 } & \multirow{4}{*}{ 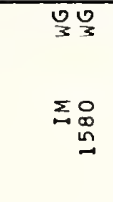 } & \multirow{4}{*}{ 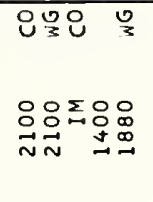 } & \multirow{4}{*}{ 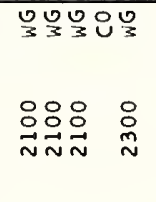 } & \multirow{4}{*}{ 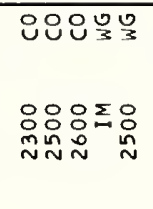 } & \multirow[t]{2}{*}{ 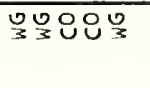 } & \multirow[t]{2}{*}{ 웅ํ } & \multirow[t]{2}{*}{ 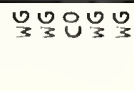 } \\
\hline ALAYS & & & & & & & & & & \\
\hline \begin{tabular}{|c|c|}
01311 & $\frac{E}{0}$ \\
J11399VK
\end{tabular} & & & & & & & & 吕 & 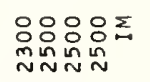 & 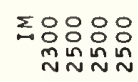 \\
\hline HLOMOHYG & & & & & & & & & & \\
\hline 9N1717nd & hr & RAr & rrNñ & $\stackrel{n}{\simeq}$ & $r$ & & 으 & 욱 & 웅 & 으우 \\
\hline 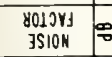 & & & & & & & & & & \\
\hline NIYY & & & & & & & & & & \\
\hline $\begin{array}{l}S 1101 \\
\times 173 H\end{array}$ & & & & & & & & & & \\
\hline $\begin{array}{c}51701 \\
10411003 \mid\end{array}$ & & & & & & : & & 号 & & \\
\hline 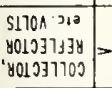 & & & & & & & & 号 & & \\
\hline$\alpha^{\circ}$ & $\sum \sum \sum \sum \sum \sum$ & 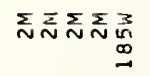 & 중잉ㅇㅁㅇㅇㅇㅇ & 兑 & 弟兑强兑兑 & 关㒸总路兑 & 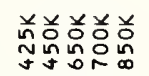 & 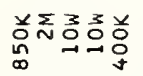 & 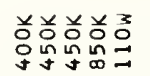 & 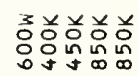 \\
\hline- & 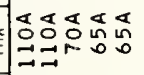 & 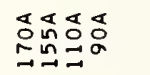 & 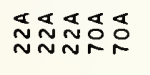 & 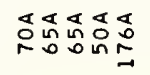 & 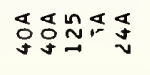 & 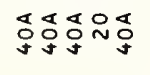 & 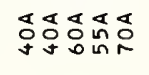 & 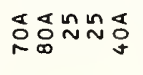 & 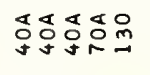 & 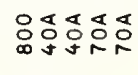 \\
\hline 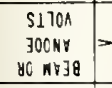 & 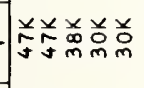 & 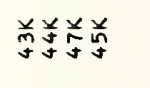 & 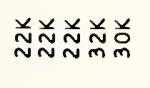 & 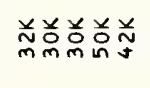 & 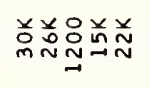 & 酋酋兑怘总 & 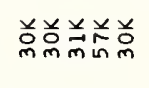 & 㒸羊总品怘 & 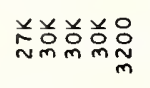 & 总总总总总 \\
\hline 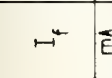 & $\mid$\begin{tabular}{l}
$\circ$ \\
\hdashline \\
\hdashline
\end{tabular} & 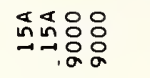 & 员员品品品品 & 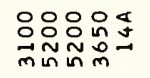 & 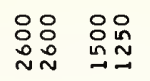 & 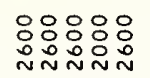 & 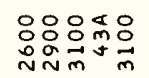 & 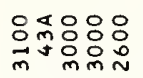 & 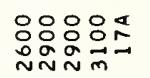 & 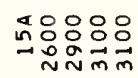 \\
\hline "َّ & 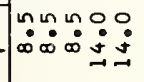 & 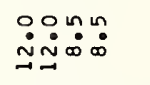 & 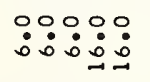 & :우요 & $\ddot{n} \dot{0}: \dot{0}:$ & $\because \because 0: 0: 0$ & மீம் & 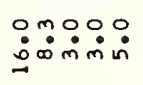 & பำ & 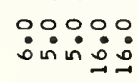 \\
\hline NOIIFYJdO & $0 a 0 a 0$ & $a a a v u$ & $a a a a a$ & $a a a a a$ & anua & $a, a \cup a$ & $a, a n a$ & a auva & $0 a 0 a U$ & UaOa \\
\hline$y \mathrm{~J} N$ & แய山コ & 岀山岀さ凹 & 次号‡出 & そこコ岂ய & 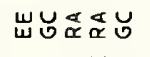 & 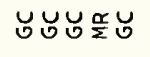 & ư & 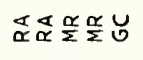 & 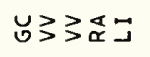 & JU \\
\hline 3Nก1 & 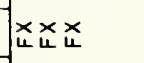 & 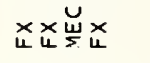 & 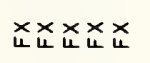 & $\underset{u}{x} \quad \breve{y}_{z}$ & 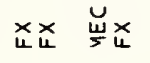 & 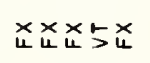 & 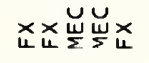 & メ岁ららメ & 전ㅈuㅍ & 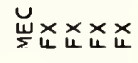 \\
\hline Ollyy $\times$ ino $=$ & 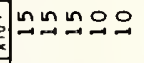 & 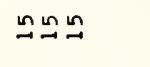 & 으으우음으 & 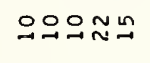 & $\operatorname{O}^{N} \stackrel{N}{0}^{\circ}$ & $N N N \quad N$ & 우어우으 & 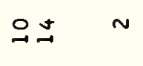 & NNNO & Nㅜㅇㅇㅡ \\
\hline \begin{tabular}{l|l}
$\bar{z}$ & $=$ \\
& $=$
\end{tabular} & $\because \dot{m} \dot{m} \dot{m} \dot{m}$ & $\ddot{m} \dot{m} \dot{m} \dot{m}$ & $\vec{m} \vec{m} \dot{m} \dot{m} \vec{m}$ & 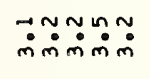 & mimiñmin & $\dot{m} \dot{m} \dot{m} \dot{m} \dot{m} \dot{m}$ & 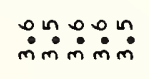 & 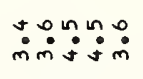 & 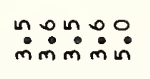 & 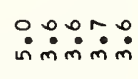 \\
\hline 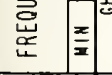 & $\ddot{m} \dot{m} \dot{m} \dot{m} \dot{m}$ & $\ddot{m} \dot{m} \dot{\dot{m}} \dot{\dot{m}} \dot{\mathrm{m}}$ & $\overrightarrow{\dot{m}} \overrightarrow{\dot{m}} \dot{m} \dot{m} \overrightarrow{\dot{m}} \overrightarrow{\dot{m}}$ & $\overrightarrow{\dot{m}} \overrightarrow{\dot{m}} \overrightarrow{\dot{m}} \overrightarrow{\dot{m}} \overrightarrow{\dot{m}}$ & $\ddot{m} \ddot{m} \dot{m} \dot{m} \dot{m}$ & $\dot{m} \dot{m} \dot{m} \dot{m} \dot{m} \dot{m} \dot{m}$ & $\dot{m} \dot{m} \dot{m} \dot{m} \dot{m} \dot{m}$ & $\dot{m} \dot{m} \dot{m} \dot{m} \dot{m} \dot{n} \dot{m}$ & $\dot{m} \dot{n} \dot{n} \dot{m} \dot{n} \dot{n} \dot{m}$ & $\dot{m} \dot{m} \dot{m} \dot{m} \dot{m} \dot{m}$ \\
\hline ONIX & $\mid \frac{\pi}{2} \frac{\pi}{2} \frac{\pi}{2} \frac{\pi}{2} \frac{0}{2}$ & 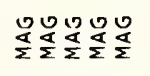 & $\frac{0}{2} \frac{\pi}{2} \frac{0}{2} \frac{\pi}{2} \frac{\pi}{2}$ & 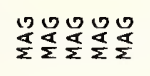 & 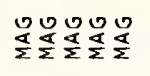 & 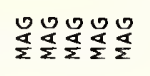 & 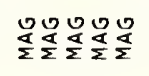 & 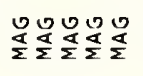 & 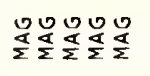 & 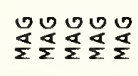 \\
\hline $708 \mathrm{~W} / \mathrm{S}$ & & & & & & & & & & \\
\hline 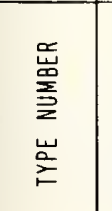 & 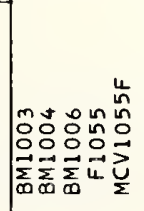 & 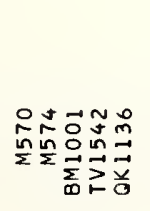 & 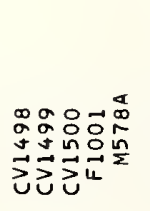 & 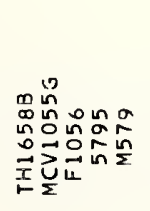 & 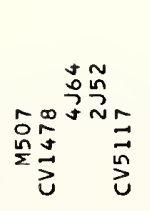 & 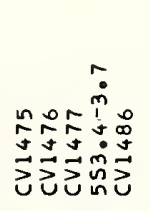 & 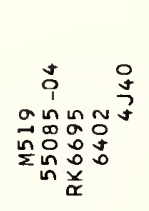 & 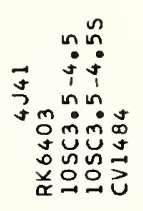 & 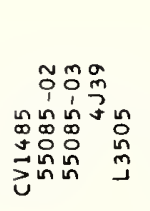 & 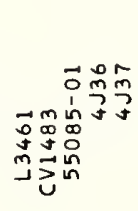 \\
\hline
\end{tabular}




\begin{tabular}{|c|c|c|c|c|c|c|c|c|c|c|c|}
\hline 9MITdnos & 엉ㅇㅇ & $8 \% 8$ & 언 인 & QOWण & 뭉으 & $\frac{5}{3}$ & 웅요 & ํㅗ 88 & 운옹 & 옹응 & 은 응 \\
\hline Rlars & & & & & & & & & & & \\
\hline 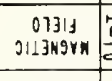 & 基 & $\Sigma \Sigma \sum \Sigma$ & $\Sigma \Sigma \Sigma \Sigma$ & 总 & $\Sigma$ & $\Sigma$ & $\Sigma$ & $\Sigma \Sigma$ & & $\Sigma$ & $\Sigma$ \\
\hline 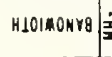 & & & N & - & & & & & & & \\
\hline 9w:177ח & 푼요 & 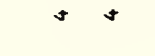 & $\stackrel{n}{N}$ & 우 & $\sigma \simeq$ & $\cong$ & $\simeq 0$ & & $\cong$ & $\stackrel{n}{\simeq}$ & $\stackrel{\sim}{\sim}$ \\
\hline \begin{tabular}{c|c}
$0015 r 3$ \\
3sion
\end{tabular} & & & & & & & & & & & \\
\hline Mirg & & & & & & & & & & & \\
\hline $\begin{array}{l}51704 \\
\times 1173 H\end{array}$ & & & & & & & & & & & \\
\hline $\begin{array}{c}\text { S1701 } \\
1081 \mathrm{NOO}\end{array}=$ & 号 & & & & & & & & & & \\
\hline 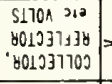 & : & & & & & & & & & & \\
\hline$a^{\circ}$ & E & 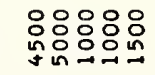 & 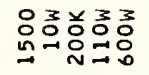 & 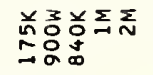 & 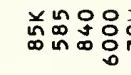 & & 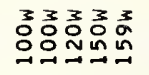 & 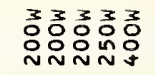 & 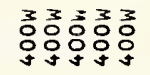 & 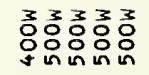 & 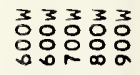 \\
\hline 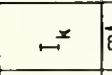 & Eㅇํㅇㅇㅛ & 융요 윳ำ & ఓ & 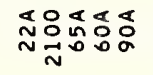 & : & $\stackrel{n}{N}$ & :০:০:০০: & 品 & 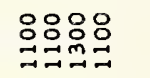 & 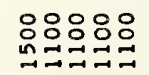 & 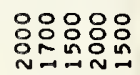 \\
\hline $\begin{array}{c}\text { S170R } \\
300 \mathrm{HY} \\
80 \mathrm{~K} \forall \mathrm{Gg}\end{array}=$ & 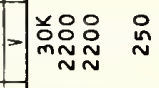 & 影吕 令怘 & 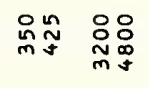 & 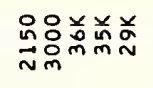 & 弟 & $\stackrel{\circ}{\sim}$ & 总品品品品品 & 品 & 品员: & 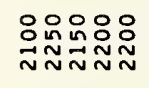 & :융유 \\
\hline$F^{-}$ & Eี & 落 路 & :요욛 & 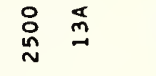 & 윰욤요 & $\stackrel{\text { O }}{\text { I }}$ & 용요 & & $\stackrel{\circ}{\circ}$ & :̊ & 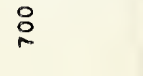 \\
\hline$\omega^{\leftarrow}$ & $=\ddot{0}$ & $\ddot{m}$ & 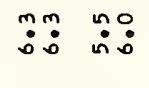 & $\stackrel{n}{\stackrel{n}{m}} \stackrel{m}{0}$ & $\therefore \circ$ & $\ddot{:}$ & $\because \because$ & & $\ddot{\circ}$ & 落 & in \\
\hline NOI IYYJdO & מטuטu & טuטu & טuau & $00 a 0 a$ & a a auc & & $a \circ a \circ a$ & a a va a & $a a a a a$ & $a a a a a$ & a.a.a \\
\hline$y J N$ & $\alpha \frac{\alpha}{\Sigma} \frac{\alpha}{\Sigma} \propto \frac{T}{3}$ & $\frac{T}{3} \frac{T}{3} \propto \frac{T}{3} \frac{T}{3}$ & 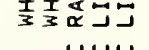 & コ岂岀匹㟧 & 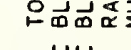 & & 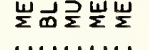 & 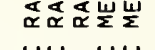 & 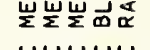 & ※而臬崖崖 & の岂崖崖 \\
\hline $3 N \cap 1$ & X55 & 岕㟧 $x \underline{w}$ & 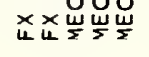 & 岕始始希 & 如岕岕 & 宏 & 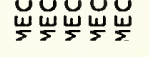 & 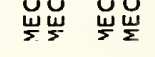 & 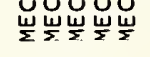 & 岕岕 岕岕 & 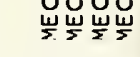 \\
\hline OLIFY $\wedge \cap \cap 0=$ & 은 & & & 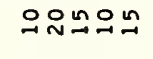 & 요윳 & 운 & 유옹유요 & 윰유 웃우 & 융ํำ & 유ํํㅠำ & ํํํํํํำ \\
\hline 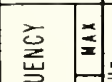 & 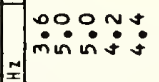 & s:s: & Mำ & 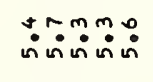 & 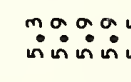 & & 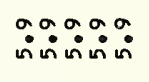 & 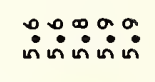 & 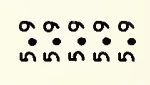 & 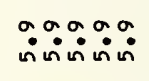 & 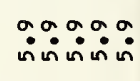 \\
\hline 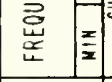 & 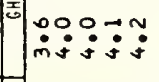 & 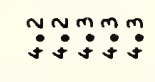 & mago: & 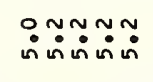 & 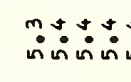 & & 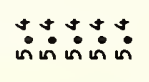 & 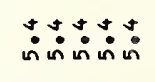 & 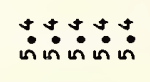 & 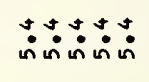 & 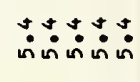 \\
\hline ONIY & 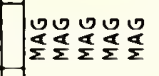 & 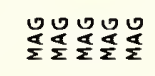 & $\frac{0}{2} \frac{0}{2} \frac{0}{2} \frac{\pi}{2} \frac{0}{2}$ & 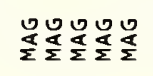 & 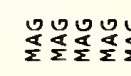 & & 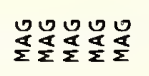 & 这造造造造 & 安造造造曹 & 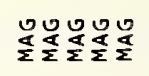 & 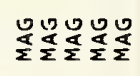 \\
\hline $708 \mathrm{~W} / \mathrm{S}$ & $"$ & " " & " & & & & & & & & \\
\hline 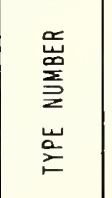 & 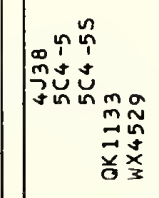 & 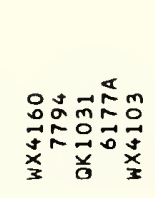 & 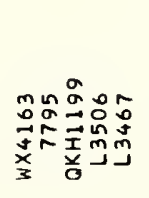 & 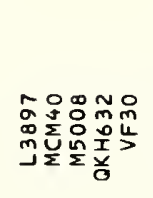 & 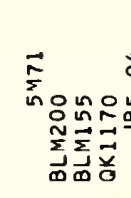 & & 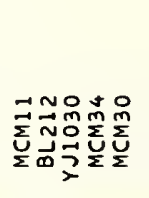 & 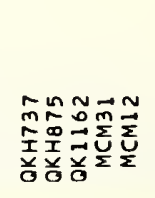 & 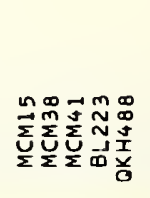 & 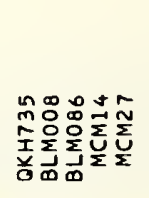 & 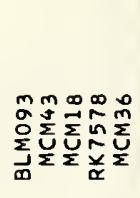 \\
\hline
\end{tabular}




\begin{tabular}{|c|c|c|c|c|c|c|c|c|c|c|c|}
\hline 9N17dROS & \multirow[t]{4}{*}{80} & \multirow{4}{*}{ 웡 운 } & \multirow{4}{*}{$\begin{array}{c}80 \text { 옹 } \\
\Sigma\end{array}$} & \multirow{3}{*}{ 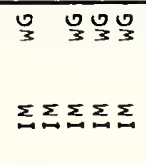 } & \multirow{4}{*}{ 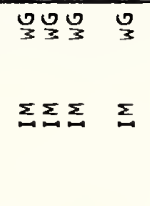 } & \multirow{3}{*}{ 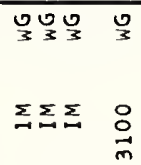 } & \multirow{2}{*}{\multicolumn{2}{|c|}{ 윅 }} & \multirow[t]{2}{*}{ 욱요 } & \multirow[t]{2}{*}{ 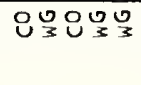 } & \multirow[t]{2}{*}{ 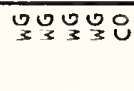 } \\
\hline A11AYS & & & & & & & & & & & \\
\hline $\begin{array}{l}071311 \\
\text { ग11399VM }\end{array}$ & & & & & & & $\sum \Sigma$ & $\Sigma$ & & 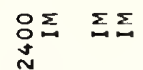 & $\Sigma \Sigma \Sigma \Sigma$ \\
\hline H10IMONYB & & & & & & $\rightarrow$ & & & N & N & \\
\hline פחורוואי & 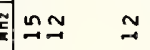 & $\cong \simeq \simeq$ & $\simeq$ & $m \backsim$ & mmnñ & 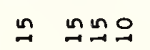 & sm & & $n m N$ & $\cong \stackrel{n}{\sim} n$ & 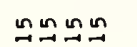 \\
\hline 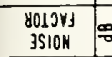 & & & & & & & & & & & \\
\hline HIY9 & & & & & & & & & & & \\
\hline $\begin{array}{l}S 1700 \\
\times 113 \mathrm{H} \\
\end{array}$ & & & & & & & & & & & \\
\hline $\begin{array}{c}51701 \\
1081103\end{array}=$ & & & & & & & & & & & 응 \\
\hline 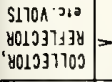 & & & & & & & & & & & \\
\hline $0^{\circ}$ & 客兰兰兰兰 & 兰兰兰兰 & 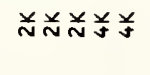 & 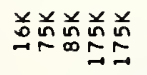 & 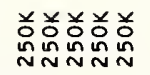 & 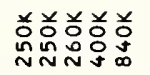 & $\sum \sum \sum_{N} \sum_{\text {in }}$ & & $\sum_{i} \sum_{0}^{\Sigma}$ & 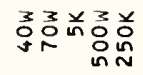 & 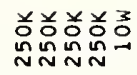 \\
\hline- & 음옴옴 & 옹요 & 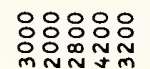 & 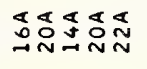 & 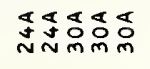 & 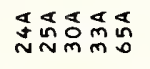 & 항ㅇㅇㅇㅇㅇㅇ & & 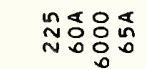 & 억제 & 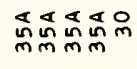 \\
\hline 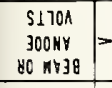 & 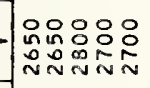 & 융을 & 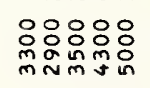 & 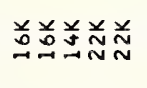 & 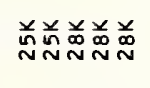 & 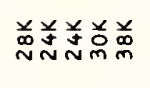 & 总兑品前 & & 总前品总 & 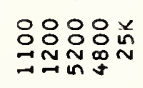 & 芯芯芯芯怘 \\
\hline$\mapsto$ & E. & 옹윰유 & $\stackrel{\circ}{\cong}$ & 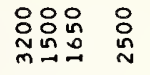 & 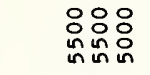 & 용 品 & $\stackrel{a}{a}$ & i & $\stackrel{\circ}{\stackrel{\circ}{\cong}}$ & 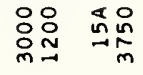 & 总员员昂品 \\
\hline " & $=\left[\begin{array}{ll}\because & 0 \\
\text { in }\end{array}\right.$ & 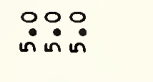 & $\stackrel{\circ}{\circ}$ & 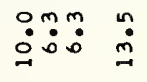 & 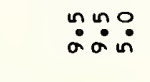 & 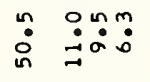 & & $\dot{0}$ & $\dot{m}: \dot{0}$ & 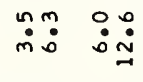 & 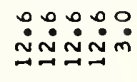 \\
\hline Nol17yyjdO & acaa & aava & aana & aaca & aaga & aoaa & 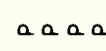 & a a & a a a a & vacua & a.aou \\
\hline$y J W$ & 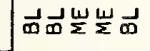 & 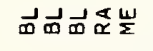 & 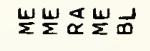 & 巡迎品 & 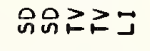 & そ岀 & ดิดั山् & 요 & 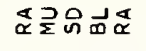 & 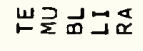 & 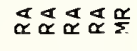 \\
\hline $3 \mathrm{~N} \cap 1$ & 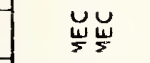 & $\stackrel{\breve{u}}{\Sigma}$ & 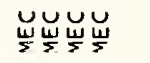 & 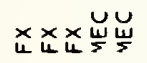 & 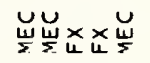 & 岁岕岕区x & 岁х & $x \breve{u}$ & ×xxư & 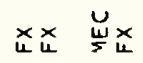 & $\begin{array}{l}\times \times \times \times 5 \\
u k u k s\end{array}$ \\
\hline $00117 y+1 n 0$ & 유유유 & 유유 욤 & 윙유어어 & 우응으용ㅇ & 욱워 $\simeq$ & $\simeq m g n n$ & 우융ㅇㅇㅇ & 우우 & 우웅ㅇㅇㅇㅡ & 욤요 & 으우우으 \\
\hline 产 & 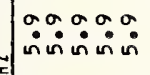 & 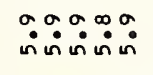 & 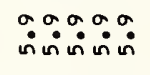 & 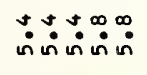 & 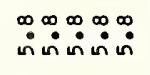 & 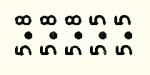 & 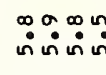 & & 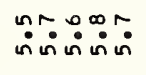 & 番 & : \\
\hline 总 & பn் & $\dot{n} \cos$ & 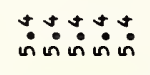 & 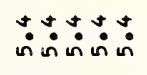 & 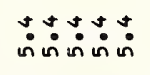 & 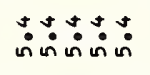 & 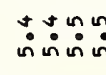 & & 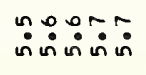 & மீ & 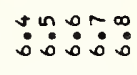 \\
\hline ONIX & 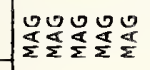 & 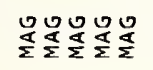 & 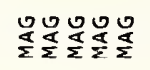 & 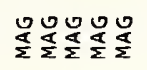 & 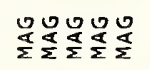 & 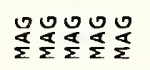 & 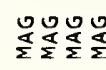 & $\frac{0}{2} \frac{0}{2}$ & 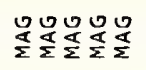 & 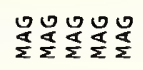 & 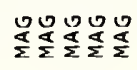 \\
\hline 708W/S & & " & & $"$ & " & $"$ & & & & " & " " " " \\
\hline 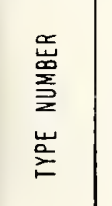 & 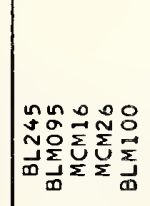 & 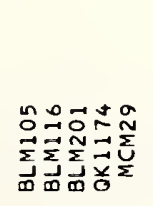 & 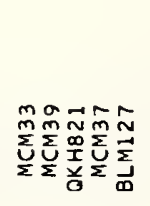 & 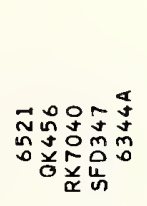 & 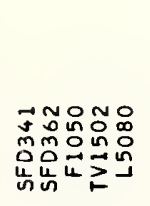 & 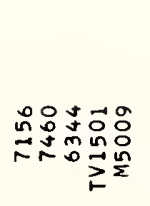 & 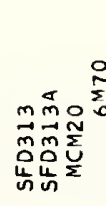 & 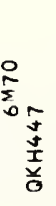 & 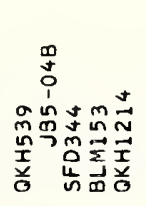 & 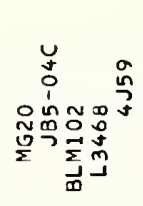 & 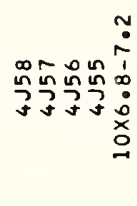 \\
\hline
\end{tabular}




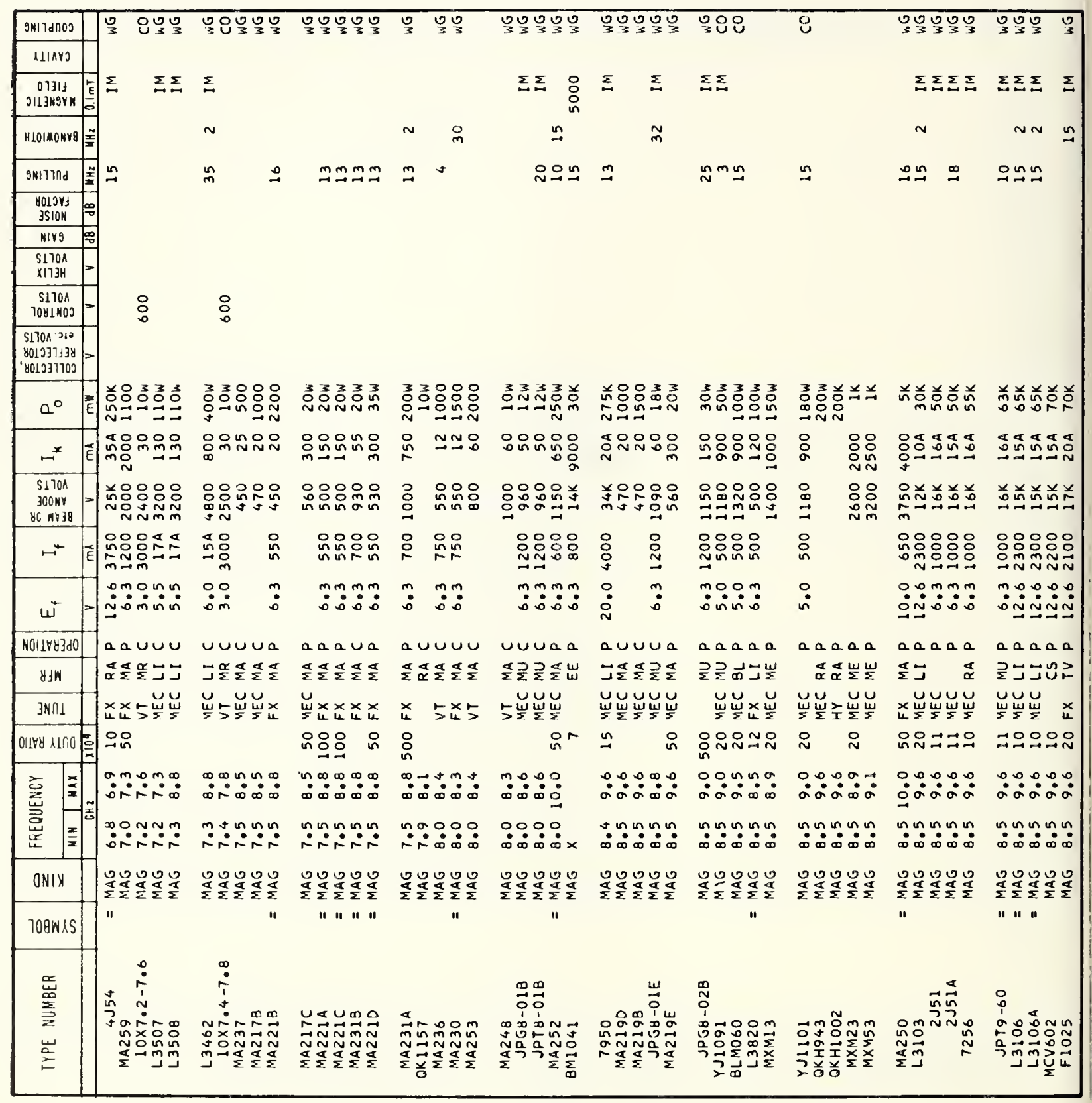




\begin{tabular}{|c|c|c|c|c|c|c|c|c|c|c|}
\hline 9HITdnOS & Оण्ड & OOY & OOY & 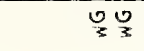 & צ'צ & צ' & & 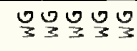 & O్ర & Oֵ \\
\hline RIAAYJ & & & & & & & & & & \\
\hline $\begin{array}{c}11311 \\
\text { ग11399YK }\end{array}$ & $\Sigma \Sigma$ & $\Sigma \Sigma \Sigma \Sigma \Sigma(n$ & $\sum \sum \sum \sum$ & $\Sigma$ & $\sum_{n=1} \sum_{n=1} \sum_{n=1}$ & $\sum_{n=1} \sum_{n=-} \sum_{n}$ & $\sum_{n} \sum \sum \sum \sum$ & $\sum \Sigma \sum \Sigma \Sigma$ & $\sum \Sigma$ & $\sum \sum$ \\
\hline HLOMOANG & & & & & $N N N$ & $\sim$ & & & $\infty$ & \\
\hline 9N177nd & $\operatorname{tin} \cong \approx$ & 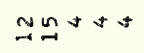 & $\stackrel{n}{\sim} \cong$ & $m \underline{n}$ & $\cong$ & 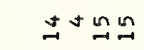 & $\underset{-1}{\sim} \simeq \simeq \simeq \sim$ & $\underset{\sim}{\operatorname{nn}}$ 오N & $\cong \simeq$ & 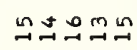 \\
\hline 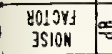 & & & & & & & & & & \\
\hline HIY & & & & & & & & & & \\
\hline $\begin{array}{l}\text { SI170A } \\
X 113 H\end{array}$ & & & & & & & & & & \\
\hline $\begin{array}{c}51701 \\
7001 N 03\end{array}$ & & & & & & & & & & \\
\hline 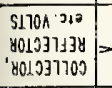 & & & & & & & & & & \\
\hline $0^{\circ}$ & 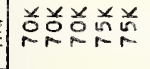 & 并关总总兑 & 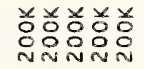 & 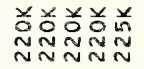 & 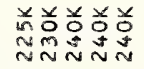 & 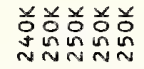 & 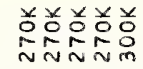 & 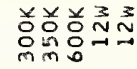 & 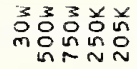 & 恙总品怘怘 \\
\hline$r^{*}$ & 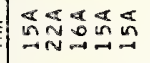 & 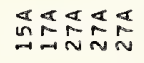 & 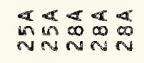 & 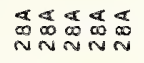 & 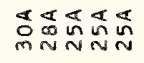 & 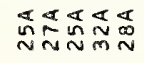 & 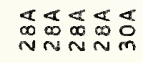 & 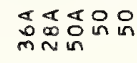 & 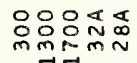 & 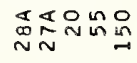 \\
\hline $\begin{array}{r}\$ 170 \mathrm{~N} \\
300 \mathrm{KY} \\
80 \mathrm{KH}=\mathrm{B}\end{array}$ & 奇畐芯总总 & 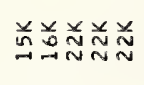 & 总恙总芯总 & 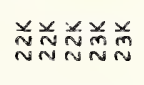 & 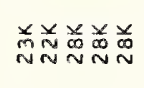 & 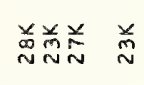 & 恙前前前 & 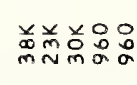 & 品品品兑总总 & 前胥品品怘 \\
\hline- & $\begin{array}{l}88880 \\
08080 \\
N N+N N N \\
N N N N\end{array}$ & $\begin{array}{l}\text { : } \\
\text { N: } \\
\text { N }\end{array}$ & 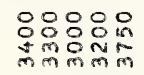 & 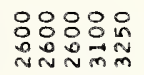 & 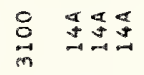 & 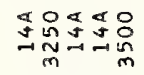 & 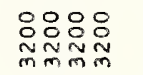 & 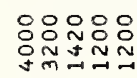 & 品 萧 & 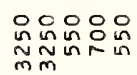 \\
\hline$\omega^{*}$ & 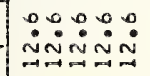 & 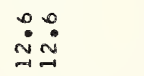 & $\ddot{n}_{n \rightarrow \infty}^{\infty} \dot{m}_{n \rightarrow-\infty}^{\infty} \dot{m}^{\infty}$ & $\therefore: 00 \dot{m}_{-1}^{\infty}$ & $\min _{n \rightarrow-1}^{\infty} \dot{0} 00$ & 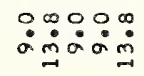 & 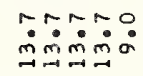 & 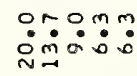 & $\stackrel{\circ}{\infty}$ & 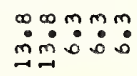 \\
\hline NOIIYUJdO & aacao & $a, a, a$ & avaaa & araan & a a a a a & aabao & a a a a & a a $a \cup u$ & $a a a a$ & a a uua \\
\hline$y \pm n$ & 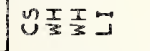 & ำ & 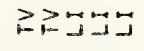 & 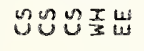 & \begin{tabular}{l}
$I \frac{T}{a} \alpha \alpha \alpha$ \\
\hdashline$\alpha \alpha$
\end{tabular} & 这立名 & $\because コ \quad \frac{r}{3}$ & 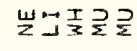 & コ崖崖甹采 & 山己 \\
\hline $3 N \cap 1$ & 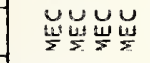 & 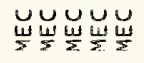 & 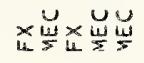 & 容 & 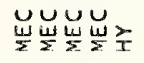 & 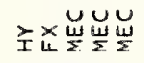 & 岁文崲嵌 & 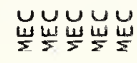 & 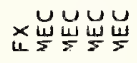 & 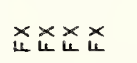 \\
\hline OIIEY $\lambda I n O=$ & 웃ㅇㅁㅁㅛ & 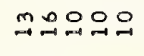 & $\stackrel{\circ}{\circ} \stackrel{2}{a}=$ & 음음어윰에 & $=m m n$ & $m$ m우요 $m=$ & $m=m$ & 움요 & 옷옥 & 음요 웅 \\
\hline 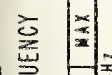 & 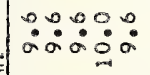 & 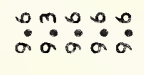 & $\because \because \because: 0$ & $\because \because 002$ & 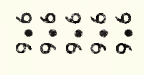 & 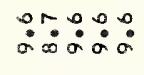 & 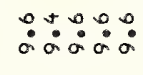 & 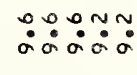 & $\because \because 0 \%$ 品 & $\dot{\infty}: \because \because: 0$ \\
\hline $\begin{array}{l}\vec{z} \\
\tilde{z} \\
u\end{array}$ & 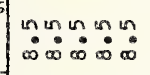 & 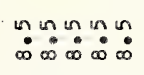 & 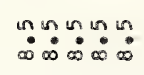 & 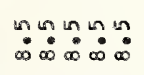 & 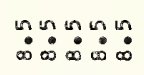 & 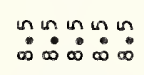 & 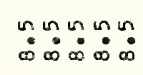 & 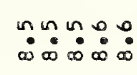 & $\because \because \because \because:$ & 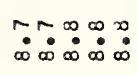 \\
\hline ONIX & 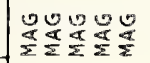 & 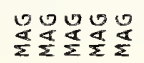 & $\begin{array}{l}00000 \\
\frac{5}{2} \frac{0}{2} \frac{\pi}{2} \frac{x}{2}\end{array}$ & $\begin{array}{l}0000 \\
\frac{3}{2} \frac{4}{2} \frac{4}{2} \frac{4}{2}\end{array}$ & 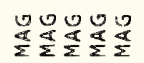 & 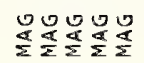 & 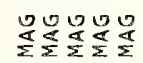 & 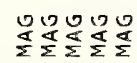 & 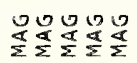 & 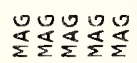 \\
\hline 70gNAS & & & "1 & & " & " " & & * & $"$ & " " \\
\hline 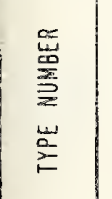 & 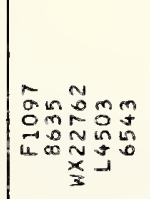 & 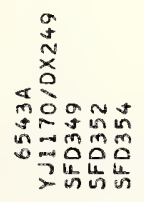 & 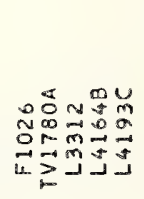 & 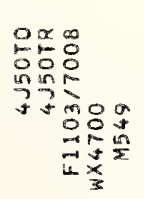 & 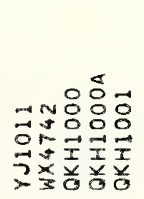 & 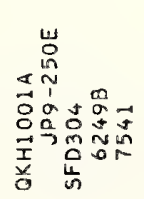 & 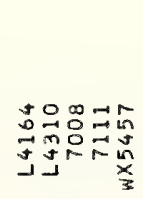 & 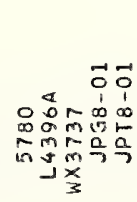 & 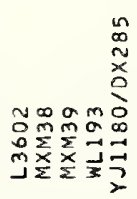 & 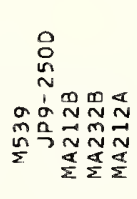 \\
\hline
\end{tabular}




\begin{tabular}{|c|c|c|c|c|c|c|c|c|c|c|}
\hline 9พוาd & O०ण & प0 & 38 & 엉요 & एण & 어ㅇㅠㅜ & 뮤요 & 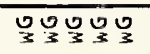 & पू & ดु३ \\
\hline LLIAVS & & & & & & & & & & \\
\hline $\begin{array}{c}01311 \\
\text { J113M9VK }\end{array}$ & $\sum \sum \Sigma$ & $\Sigma$ & $\Sigma$ & 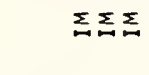 & 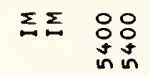 & 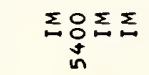 & $\sum_{n} \sum_{n=1}$ & $\Sigma$ & & $\sum \sum \sum$ \\
\hline H101m0nY9 & n & $\sim$ & & $\sim N N$ & $N-$ & N & $\sim$ & & & $N N$ \\
\hline 9 9ורד & $\underline{\mathbf{I}} \underset{\sim}{\sim} \sim \sim \sim \sim \sim$ & $m$ & $\stackrel{\sim}{\sim}$ & $\because$ 유요 & 올 $\cong \Perp$ & $\cong \cong \bumpeq$ & 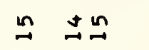 & $\cong$ & & 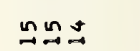 \\
\hline $\begin{array}{l}8019 y\} \\
3510 \mathrm{~N}\end{array}$ & & & & & & & & & & \\
\hline HIV9 & & & & & & & & & & \\
\hline $\begin{array}{l}51701 \\
\times 11734\end{array}$ & & & & & & & & & & \\
\hline $\begin{array}{c}51700 \\
10 y 1403\end{array}$ & & & & & & & & & & \\
\hline 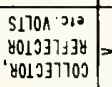 & & & & & & & & & & \\
\hline$a^{\circ}$ & 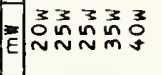 & 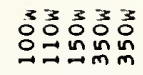 & 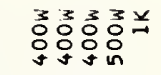 & 兰兰兰并 & 当吕兑首并 & 弚弚总总总 & 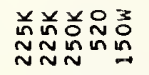 & 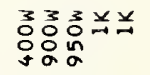 & 壬弚弚羊 & 总兑关足足 \\
\hline 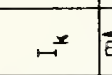 & 品品品品京 & 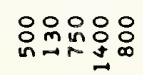 & 음윰윰요 & 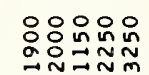 & 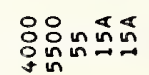 & 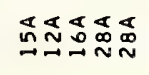 & 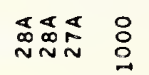 & 음용요 & 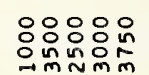 & 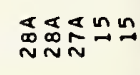 \\
\hline $\begin{array}{c}5170 \mathrm{~A} \\
300 \mathrm{NY} \\
80 \mathrm{ABg}\end{array}$ & 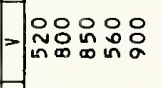 & 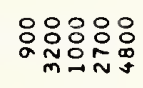 & 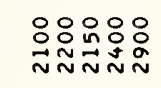 & 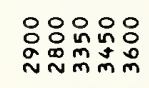 & 路品品㒸㒸 & 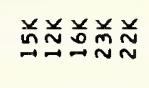 & 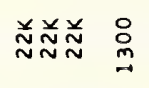 & 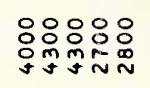 & 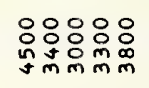 & 恙胥永品只 \\
\hline 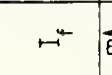 & 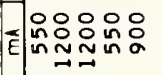 & 옹 & 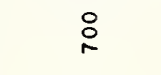 & 음응ㅇㅇㅇㅇㅇㅇ & 응 용유 & 음용욤요 & 突品㶽足 & in & 응 & 일요 \\
\hline$\omega^{\omega}$ & 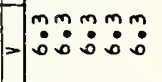 & $\ddot{n} \dot{m}:$ & : & $\dot{m}: m: m$ & $\ddot{m}: m: m$ & 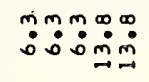 & 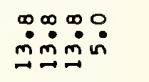 & $\stackrel{\circ}{\circ} \stackrel{\circ}{n}$ & $\dot{m}_{0}^{m}$ & $\dot{m} \dot{m} \dot{m} \dot{m}$ \\
\hline NO11Yy3d0 & aaca & auaau & $a a a a a$ & $a a a a a$ & a aua & auaa a & $a, a a b$ & 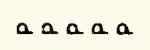 & $a, a a a$ & $a a, u a$ \\
\hline$y J W$ & 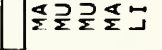 & 乔コ希岂コ & 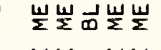 & 岂めコココ & コ山岂そマ & そマ 出コ & 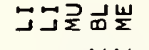 & 这这《崖あ & ๔岂岂岸 & 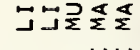 \\
\hline 3Nก1 & $\begin{array}{ll}x \times & x \\
k\end{array}$ & 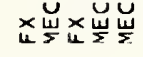 & 岕岕 岕岕 & 岀 $\times x \times x$ & 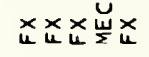 & 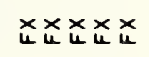 & 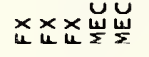 & 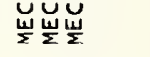 & 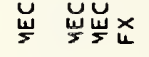 & 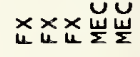 \\
\hline OLIFY xino & D N N 읏 & 응 & ต & 오슴요음 & 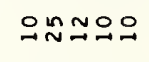 & 으웜으 & 음웅음 & 픔어유요 & 음윰유 & 으으으 \\
\hline 产 & 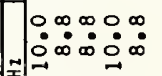 & 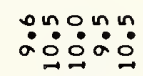 & 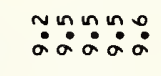 & 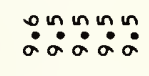 & 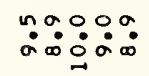 & 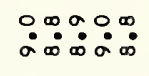 & 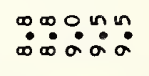 & 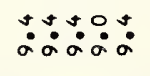 & பீ人。 & 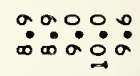 \\
\hline 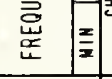 & 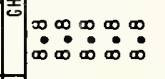 & 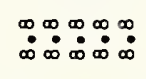 & 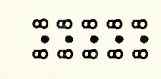 & 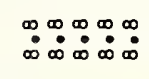 & 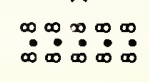 & 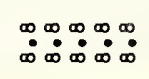 & 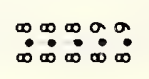 & 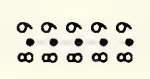 & $\begin{array}{l}\sigma: \sigma: \sigma: \sigma . \\
\dot{\infty} \dot{\infty} \dot{\infty} \dot{\infty} \dot{\infty}\end{array}$ & 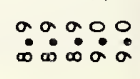 \\
\hline ONIX & 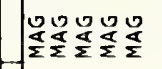 & 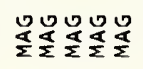 & 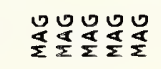 & 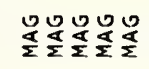 & 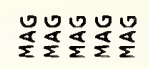 & 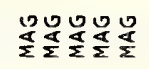 & 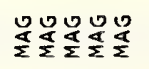 & 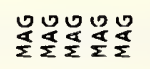 & 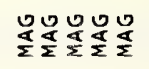 & 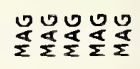 \\
\hline 708WLS & & $"$ & & & & & & & & \\
\hline 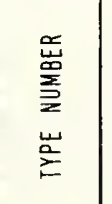 & 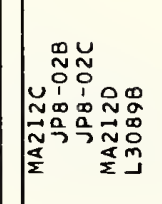 & 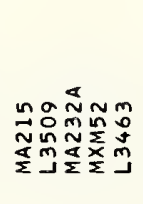 & 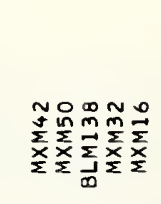 & 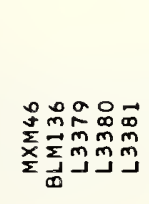 & 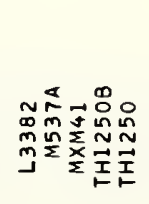 & 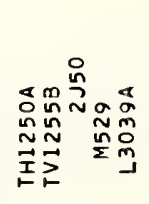 & 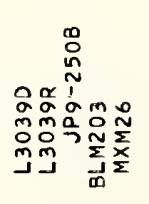 & 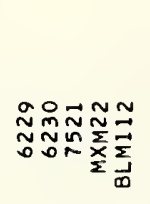 & 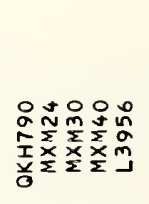 & 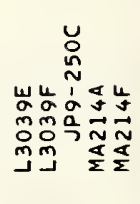 \\
\hline
\end{tabular}




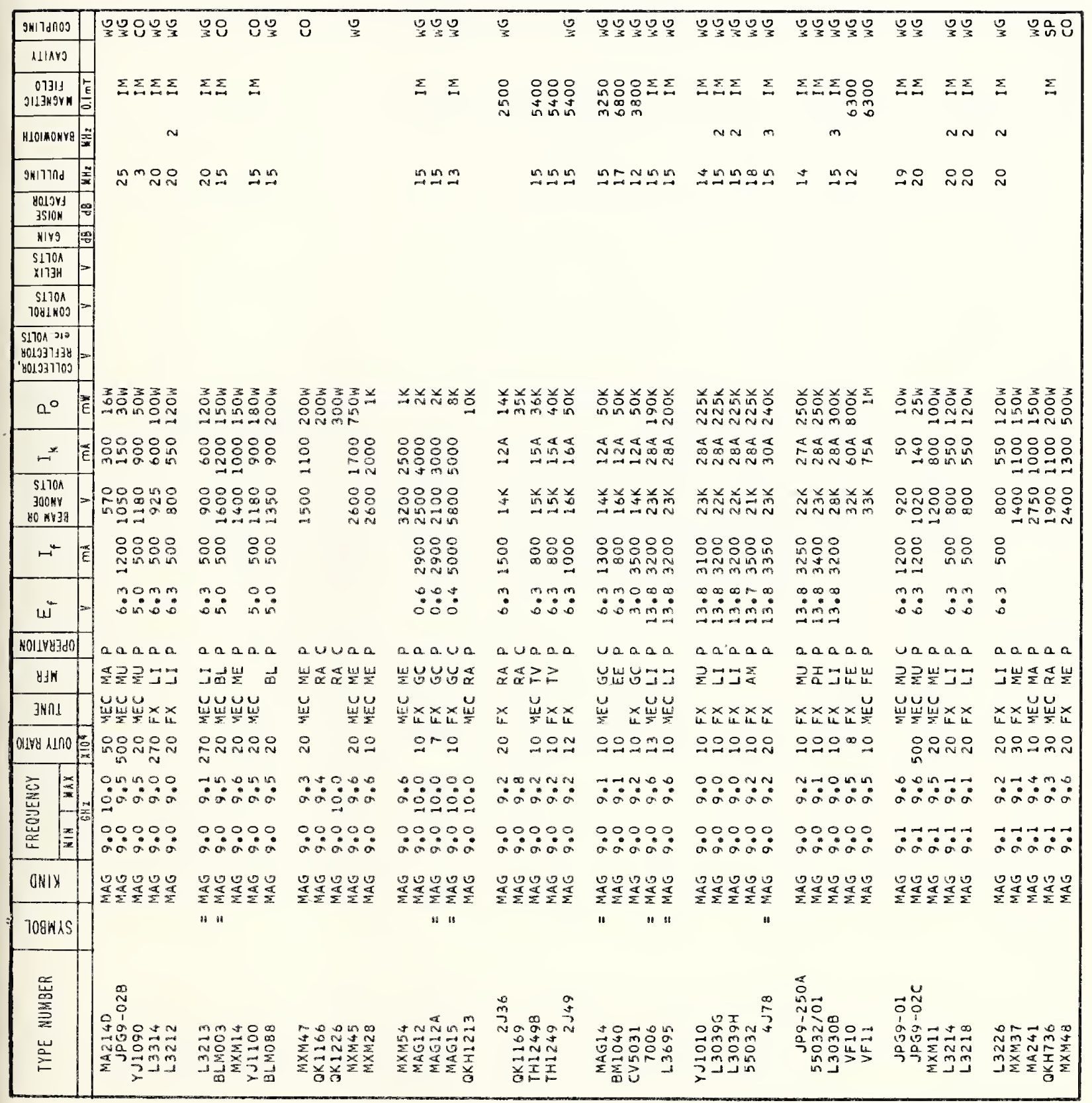




\begin{tabular}{|c|c|c|c|c|c|c|c|c|c|c|}
\hline 9NITdกOS & \multirow[t]{2}{*}{ W్ } & \multirow[t]{2}{*}{ 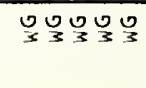 } & \multirow[t]{2}{*}{ पू } & \multirow[t]{2}{*}{ \ָ } & \multirow[t]{2}{*}{ Oुß } & \multirow[t]{2}{*}{ 잉 } & \multirow[t]{2}{*}{ ॐО } & \multirow[t]{2}{*}{ Wुßुण } & \multirow[t]{2}{*}{ 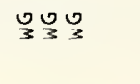 } & \multirow[t]{2}{*}{ 잉요 } \\
\hline ILIArJ & & & & & & & & & & \\
\hline 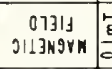 & 舁 & $\Sigma \sum \sum \Sigma$ & $\sum \sum \sum$ & $\Sigma \sum \Sigma \Sigma$ & $\Sigma \Sigma \Sigma \Sigma \Sigma$ & $\Sigma \Sigma 0 \Sigma 0$ & $\sum \Sigma \Sigma \Sigma \Sigma$ & $\sum \Sigma \Sigma \sum$ & $\sum \sum \Sigma$ & $\sum \sum \sum \sum$ \\
\hline HLIMONFG & $N$ & N & mNNm & m & NNNNN & & no & $N N$ & m & $\underset{m m m}{m m}$ \\
\hline 2אורנים & 垶 & a 유N유 & 윰ํำ & 욤 $\stackrel{\infty}{\sim}$ & 유유유 & 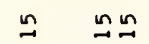 & 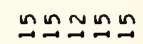 & ヘュュュ゚ロ & $n=$ & $\stackrel{\sim}{\sim}$ 요 \\
\hline 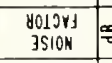 & & & & & & & & & & \\
\hline Hir? & : & & & & & & & & & \\
\hline $\begin{array}{l}5170 \mathrm{~A} \\
\times 17 \mathrm{H}\end{array}$ & & & & & & & & & & \\
\hline $\begin{array}{c}\text { s1701 } \\
70811009\end{array}$ & & & & & & & & & & \\
\hline 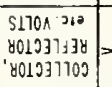 & & & : & & & & & & & \\
\hline $0^{\circ}$ & 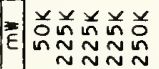 & 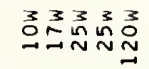 & 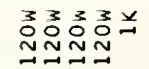 & 兰并前并 & 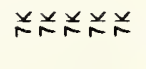 & 弟弚弚兑兑 & 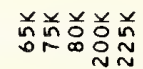 & 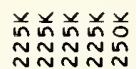 & 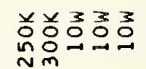 & 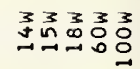 \\
\hline 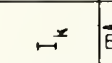 & 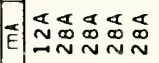 & 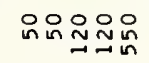 & 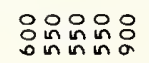 & 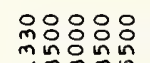 & 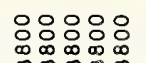 & : & 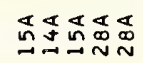 & 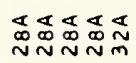 & 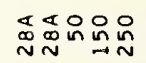 & 옴윳요 \\
\hline 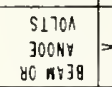 & 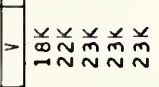 & 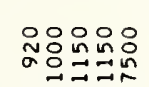 & 음요 & 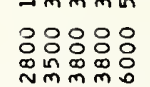 & 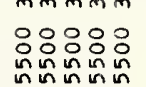 & 品总兰兰弟 & 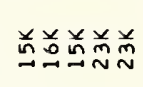 & 胥胥弟弟总 & 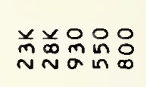 & 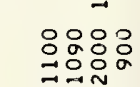 \\
\hline$\leftarrow$ & ㅌ| & : & 웅움욤용 & 음 㒸: & ㅇ:ㅇㅇㅁ & 음ㅇㅁㅇㅇㅇㅇ & 욤윰유 & 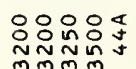 & 욤엄 & 음요요 \\
\hline$\omega^{4}$ & 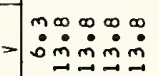 & 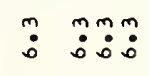 & $\ddot{n}$ & $\ddot{0} \ddot{0} \ddot{0}: \ddot{0}$ & 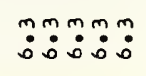 & 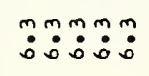 & $\dot{\sim} \dot{\sim} \dot{\sim} \dot{\sim} \dot{\sim} \dot{m}$ & $\ddot{n} \dot{m} \dot{m} \dot{m} \dot{m} \dot{\sim}$ & 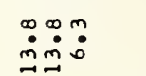 & 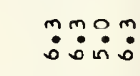 \\
\hline NOII $1 \forall y 3 d 0$ & 00000 & ưaa & $a a a a v$ & $a a n a 0$ & 00000 & a a ua a & a a a a & ana a & anua & vouaa \\
\hline$\forall J W$ & 岀コ岕出 & 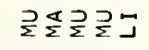 & コココゴ & 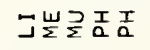 & ココココ & 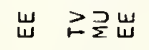 & 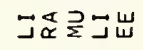 & ココ岕交希 & エコマ崖崖 & 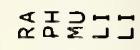 \\
\hline JNกL & 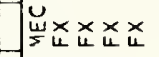 & 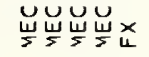 & $\underset{u}{\times} \times x \times \underline{u}$ & 嵌xxx & 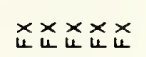 & 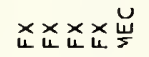 & 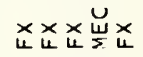 & 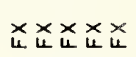 & 진 & 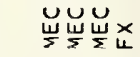 \\
\hline $011+\forall y$ aLrio & 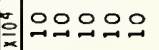 & $\operatorname{nin} \mathcal{N}^{\circ}$ & 우ํㅇํ & 유N요 $\underset{N}{N}$ & 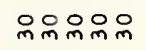 & 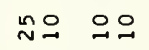 & 으으으고으 & 으으으으으 & $\stackrel{m \simeq}{ } \cong$ & $\approx$ 웅 \\
\hline 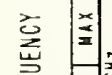 & 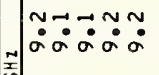 & 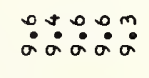 & 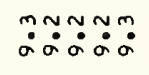 & 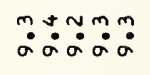 & 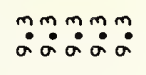 & 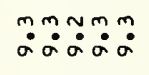 & 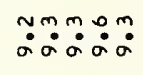 & 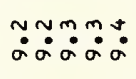 & 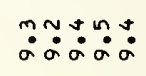 & 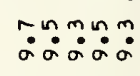 \\
\hline 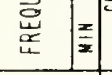 & $\vec{\sigma} \dot{\circ} \dot{a} \dot{a} \dot{a}$ & 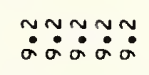 & 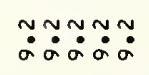 & 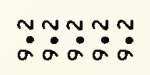 & 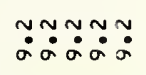 & 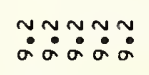 & 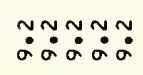 & 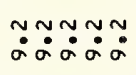 & 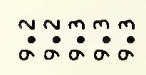 & 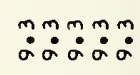 \\
\hline ONIX & 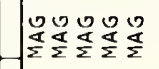 & 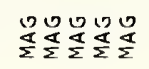 & 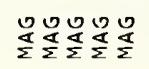 & 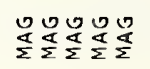 & 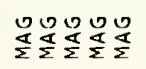 & 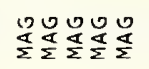 & 更 & 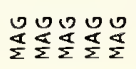 & 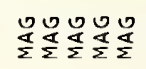 & 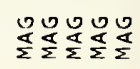 \\
\hline $709 w \times S$ & & & & & & & " " " & & & \\
\hline 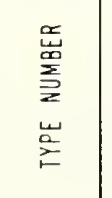 & 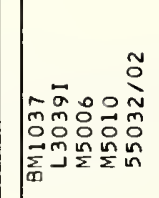 & 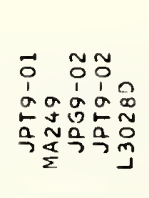 & 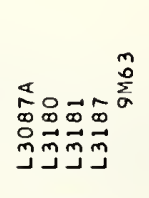 & 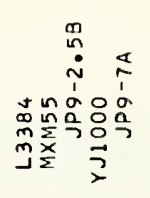 & 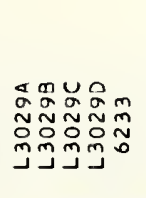 & 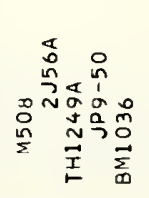 & 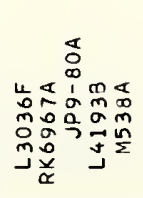 & 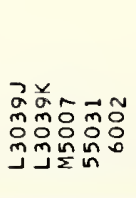 & 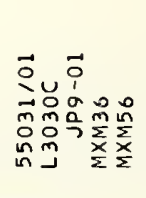 & 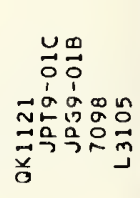 \\
\hline
\end{tabular}




\begin{tabular}{|c|c|c|c|c|c|c|c|c|c|c|c|}
\hline 9N17dnos & 80000 & $\stackrel{5}{3}$ & & טָ & 号号 & 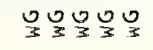 & OUß & & & & פ \\
\hline h11AY & & & & & & & & & & & \\
\hline \begin{tabular}{c|c}
07311 \\
ग119MVYK
\end{tabular} & $\sum \sum \Sigma \Sigma \Sigma$ & $\Sigma$ & $\Sigma$ & $\sum \sum \sum \sum$ & $\Sigma \Sigma \Sigma$ & $\sum \sum \sum \Sigma$ & $\sum \Sigma \sum \sum \Sigma$ & $\sum \Sigma$ & $\sum \sum \sum \sum \sum \sum$ & $\sum \sum \sum \sum \Sigma$ & $\sum \circ 8: 8$ \\
\hline HLOMONYI: & $m \sim$ & $\sim$ & m & $\min N$ & m & m & & in & & in & \\
\hline 9M17าnd & ПNN유 & $\stackrel{\circ}{N}$ & $\stackrel{i}{N}$ & ํㅠํํํำ & 옥 운 & 무요묘 & $\stackrel{n}{n} \cong$ & 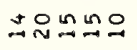 & $\stackrel{n}{\sim} \pm \stackrel{n}{n}$ & 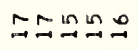 & 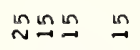 \\
\hline \begin{tabular}{c|c|}
$80115 \% 1$ \\
$3510 n$
\end{tabular}$=$ & & & & & & & & & & & \\
\hline nirg $\approx$ & & & & & & & & & & & \\
\hline 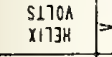 & & & & & & & & & & & \\
\hline $\begin{array}{c}51701 \\
\text { cor11003 }\end{array}=$ & & & & & & & & & & & \\
\hline 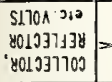 & & & & & & & & & & & \\
\hline $0^{\circ}$ & 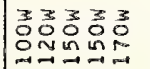 & 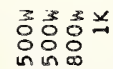 & & 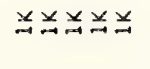 & 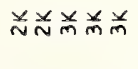 & 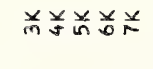 & 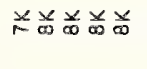 & 弟弟㒸羔总 & 弟兑兑兑兑 & $\underset{N}{\mathbb{N}} \underset{N}{\mathbb{N}} \underset{\sim}{\mathbb{N}} \underset{N}{*}$ & 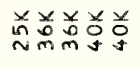 \\
\hline$\stackrel{\infty}{-}$ & 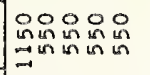 & 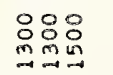 & 总 & 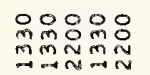 & 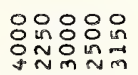 & 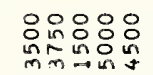 & 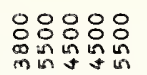 & 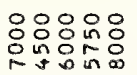 & 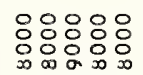 & 응요요 & 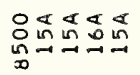 \\
\hline 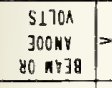 & 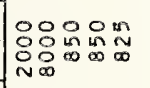 & 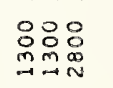 & $\begin{array}{c}8 \\
\substack{0 \\
N \\
\sim}\end{array}$ & 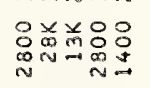 & 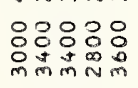 & 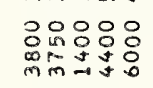 & 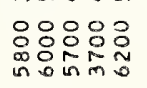 & 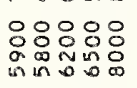 & 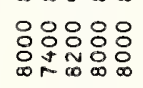 & 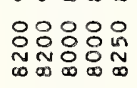 & 总酋总总 \\
\hline-4 & 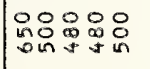 & :웅 & 品 & 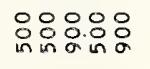 & 只品 只 & 용요요요요 & 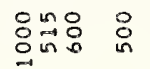 & 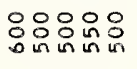 & 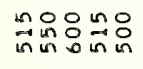 & 品品总品品 & 용요 \\
\hline$\omega^{5}$ & 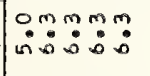 & $\ddot{m}:$ & 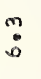 & $\ddot{0}$ & $\stackrel{m}{m}: m$ & 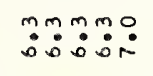 & $\ddot{0}: \stackrel{m}{0}:$ & $\ddot{n}$ & 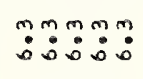 & : & 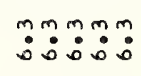 \\
\hline NOIIYYG & acaca & $\cos u$ & & a a.a.a & aacas & a a o a a & aana & aaca & $a a a a$ & a a a a & aaaaa \\
\hline Yjo & ココ弪圭先 & ココ岂晨 & & コココゴゴ & 山むコ崖引 & 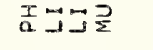 & 马 预堊山 & 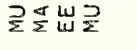 & & 出出 & 山己ZZ Z \\
\hline 3 XNก1 & 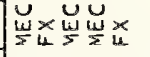 & 宏肴希 & & $\underset{u}{x} \times x \underset{u}{u}$ & 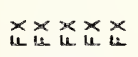 & $\begin{array}{l}x \times x \times x \\
u k u k u\end{array}$ & 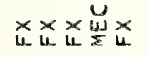 & 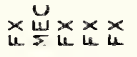 & 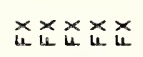 & 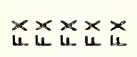 & 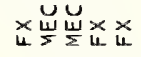 \\
\hline 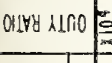 & 온유N유 & 요요 & $\therefore$ & 있유욤요 & 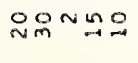 & 응ㅇㅁㅇㅇㅛ & 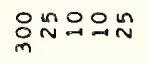 & NON $\underset{N}{ }$ 요 & $\stackrel{n}{\sim} \mathcal{N} \underset{\sim}{n} \stackrel{n}{N}$ & $\cong \curvearrowleft \underset{\sim}{n} \underset{\sim}{n}$ & 응워음 \\
\hline 总 & nmm & mát: & & mmmm & 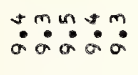 & 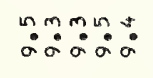 & ma & 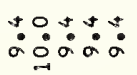 & $a a^{+}+\Delta$ & 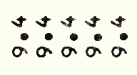 & 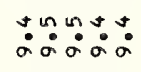 \\
\hline 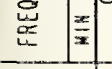 & $\begin{array}{l}m m m \\
\dot{\alpha} \dot{0} \sigma \dot{0}\end{array}$ & ๓ேm & & 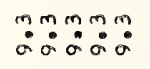 & 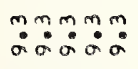 & mmmm & måna & 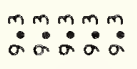 & mamm: & 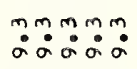 & 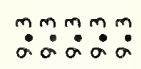 \\
\hline ONIX & 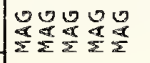 & 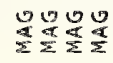 & & 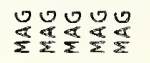 & $\begin{array}{ll}0 \\
\frac{8}{2}\end{array}$ & 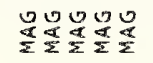 & 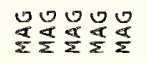 & $\begin{array}{ll}0 \\
2 \\
2\end{array}$ & 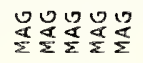 & $\begin{array}{l}10000 \\
\frac{\pi}{2} \frac{\pi}{2} \frac{\pi}{2} \frac{\pi}{2} \frac{\pi}{2}\end{array}$ & 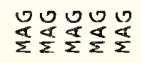 \\
\hline $709 \mathrm{~N}$.S & " " & " & & & & " " & $"$ & & * & & \\
\hline 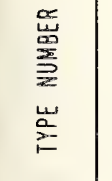 & 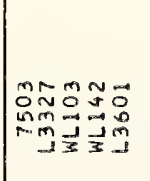 & 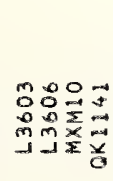 & & 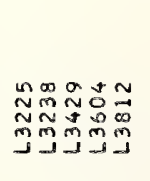 & 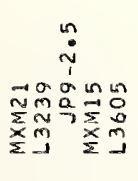 & 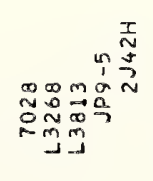 & 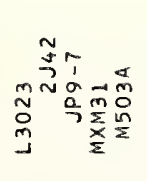 & 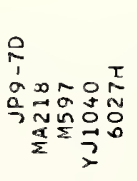 & 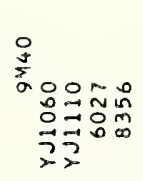 & 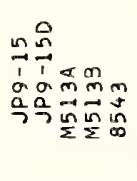 & 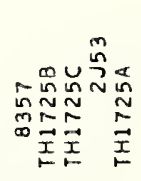 \\
\hline
\end{tabular}




\begin{tabular}{|c|c|c|c|c|c|c|c|c|c|c|}
\hline 9HITdnos & \multirow[t]{2}{*}{ OOO } & \multirow[t]{2}{*}{ O్ర } & \multirow[t]{2}{*}{ WO } & \multirow[t]{2}{*}{ 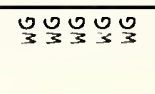 } & \multirow[t]{2}{*}{ 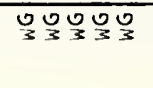 } & \multirow[t]{2}{*}{ 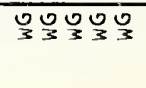 } & \multirow[t]{2}{*}{ प } & \multirow[t]{2}{*}{ 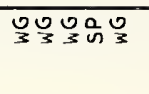 } & \multirow[t]{2}{*}{ प } & \multirow[t]{2}{*}{ 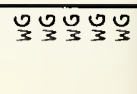 } \\
\hline RLIAvJ & & & & & & & & & & \\
\hline $\begin{array}{c}01714 \\
\text { ग113M9VK }\end{array}$ & 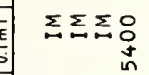 & 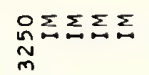 & $\sum \Sigma \sum \Sigma \Sigma$ & $\Sigma \Sigma \Sigma \Sigma \Sigma$ & $\sum \Sigma \Sigma \Sigma \Sigma$ & $\sum \Sigma \Sigma \Sigma \Sigma($ & $\Sigma \Sigma \Sigma$ & $\Sigma \Sigma \Sigma \sum$ & $\Sigma \sum \Sigma \Sigma$ & $\Sigma \Sigma \Sigma \Sigma \Sigma$ \\
\hline HLOMOHYA & $\tilde{\varepsilon}$ & $\sim N \sim$ & $\stackrel{n}{\sim}$ & $N N$ & 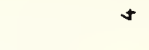 & & - & & & 오 \\
\hline פחי & $m \stackrel{m}{a} \simeq$ & 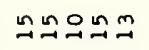 & 우묨요 & 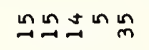 & $\simeq \approx$ & $\circ$ & $\stackrel{n}{\sim}$ 유묘 & $\stackrel{\infty}{\sim} \underset{\sim}{-\infty}$ & $\stackrel{\infty}{\sim}$ & $\stackrel{\infty}{\sim} \quad$ 는 \\
\hline \begin{tabular}{c|c}
$\begin{array}{c}\text { 8019YJ } \\
\text { 3SIOH }\end{array}$ & $\approx$ \\
\end{tabular} & & & & & & & & & & \\
\hline Mirg & & & & & & & & & & \\
\hline 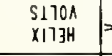 & & & & & & & & & & \\
\hline $\begin{array}{c}5170 \mathrm{~N} \\
1081000\end{array}$ & & & & & & & & & & \\
\hline 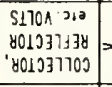 & & & & & & & & & & \\
\hline $0^{\circ}$ & 总总兑兑总 & 弟曾兑兑兑 & 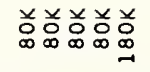 & 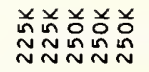 & 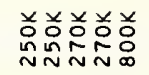 & 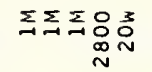 & 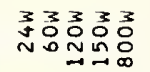 & 㒸并并并兑 & 弚总㒸芯兑 & 酋并兑总胥 \\
\hline$\mapsto$ & 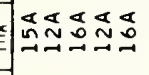 & 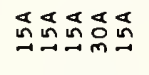 & 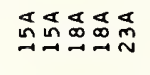 & 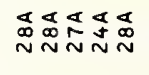 & 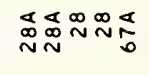 & 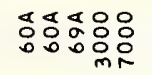 & 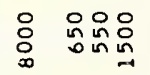 & 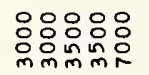 & 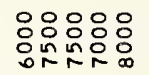 & 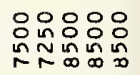 \\
\hline $\begin{array}{r}5170 \mathrm{~N} \\
300 \mathrm{NH} \\
80 \mathrm{KBg}\end{array}$ & 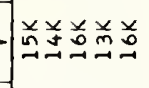 & 弟关关关弟 & 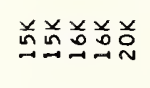 & 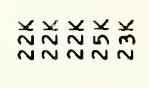 & 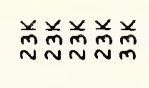 & 前侖总品品品 & 을 总怘怘 & 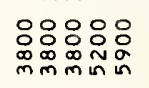 & :음요 & 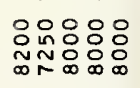 \\
\hline$\mapsto$ & $\mid$\begin{tabular}{l}
0 \\
\hdashline \\
$\infty$
\end{tabular} & 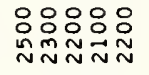 & 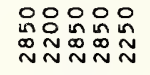 & 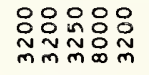 & 용요 & 总品总品品 & 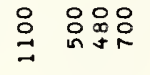 & 음용ํํ in & 品品品品品 & 숫음음용ํํ \\
\hline$w^{t}$ & : & 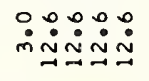 & 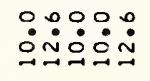 & 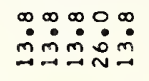 & 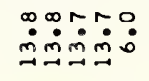 & $\ddot{\sim} \dot{\sim} \dot{\sim} \dot{m} \dot{0}:$ & 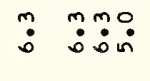 & $\ddot{0}: \ddot{m}:$ & 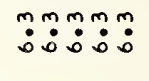 & 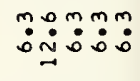 \\
\hline NOIIY $\forall \mathrm{ZdO}$ & $0 a 00 a$ & 00020 & $a \circ a a a$ & aacao & $a a a c a$ & aoaa & aUa@a & 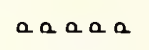 & aacas & $0 \Omega 000$ \\
\hline$\forall \mathrm{JW}$ & 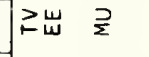 & Чב $\geq \Xi$ & ว $\frac{\partial}{\Sigma} \frac{\partial}{\Sigma} \frac{T}{2} \frac{\partial}{\Sigma}$ & ココミ゚ัコ & 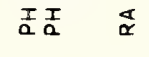 & 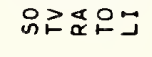 & 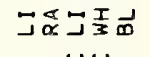 & 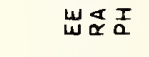 & ココ コト & 旁ココ山山山岕 \\
\hline JNAL & $\mid \begin{array}{l}x \times x \\
\mathbf{L} u[u\end{array}$ & 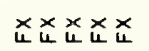 & $x_{u}^{x} x_{u}^{x} x_{u}^{x}$ & 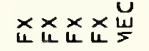 & 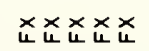 & 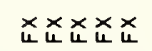 & 希 & 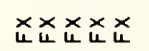 & $x_{u}^{x} \times x_{u}^{x}$ & $x_{u \in u}^{x \times x}$ \\
\hline $0117 y$ A1:0 & 으으으으으 & 욱으몽ㅇㅁㅇㅡ & 으윰ำ & 으어웅ㅇㅁㅗ & 으오우으오 & 으움으음 & $\therefore$ 오슈 & NNOO' & 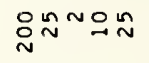 & ำ \\
\hline 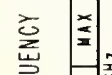 & 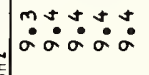 & 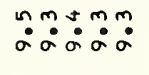 & 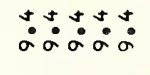 & 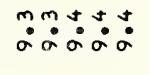 & 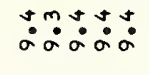 & 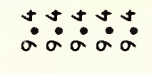 & 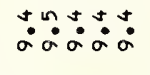 & 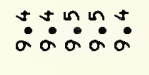 & 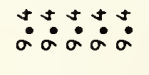 & 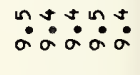 \\
\hline 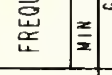 & 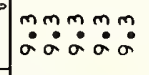 & 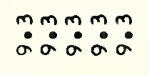 & 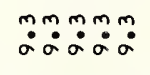 & 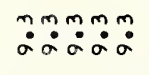 & 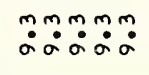 & 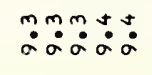 & $\dot{\alpha} \dot{\alpha} \dot{\alpha} \dot{\alpha} \dot{\alpha}$ & 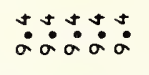 & $\dot{\sigma} \dot{\alpha} \dot{\alpha} \dot{\alpha} \dot{\alpha}$ & $\dot{\sigma} \dot{\alpha} \dot{\alpha} \dot{\alpha} \dot{\alpha}$ \\
\hline ONIX & 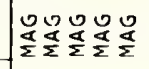 & 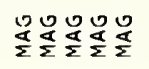 & 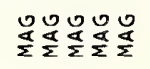 & 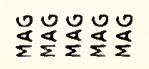 & 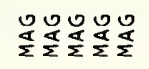 & 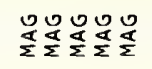 & 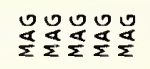 & 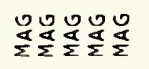 & 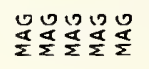 & 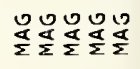 \\
\hline 700W/S & & & & & & & " " & & & \\
\hline 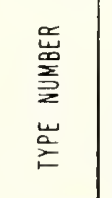 & 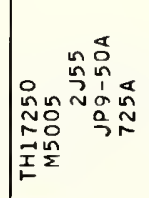 & 空 & 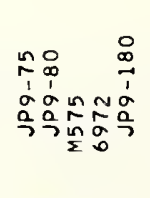 & 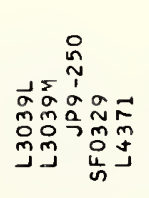 & 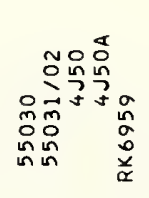 & 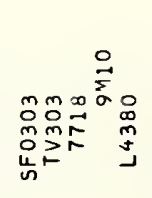 & 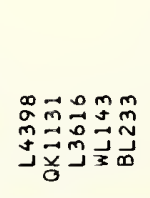 & 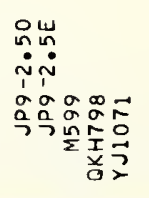 & 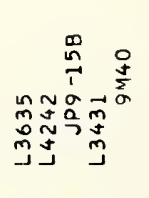 & 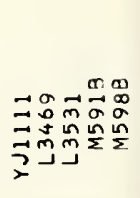 \\
\hline
\end{tabular}




\begin{tabular}{|c|c|c|c|c|c|c|c|c|c|c|}
\hline 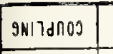 & \multirow[t]{2}{*}{ उ్య } & \multirow[t]{2}{*}{ 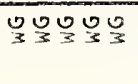 } & \multirow[t]{2}{*}{ WO } & \multirow[t]{2}{*}{$0 \% \frac{1}{3}$} & \multirow[t]{2}{*}{ \ } & \multirow[t]{2}{*}{ U్ } & \multirow[t]{2}{*}{ WO } & \multirow[t]{2}{*}{ 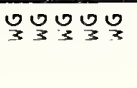 } & \multirow[t]{2}{*}{ 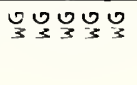 } & \multirow[t]{2}{*}{ Y̧ } \\
\hline alars & & & & & & & & & & \\
\hline 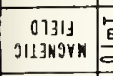 & $\sum \sum \sum \sum \sum \Sigma \Sigma_{n}$ & 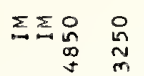 & 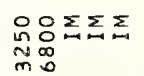 & $\sum \sum \sum \sum \sum$ & $\sum \sum \sum \sum$ & 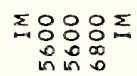 & $\Sigma \Sigma \sum \Sigma \Sigma$ & 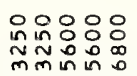 & 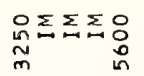 & 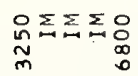 \\
\hline H101makpg $=$ & 욤 & $N$ & N & ONN & $\mathrm{m}$ & & $N m$ & & & \\
\hline 9w/7nd & 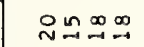 & 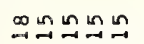 & 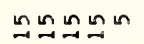 & $\ln _{n \rightarrow-1} \min _{n=-1}$ & $i n$ & ๓ேேュே & แOำ & 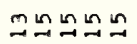 & 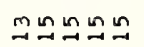 & mッュェ \\
\hline 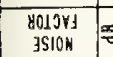 & & & & & & & & & & \\
\hline Rirg & & & & & & & & & & \\
\hline $\begin{array}{l}\$ \$ 1701 \\
\times 1173 \mathrm{H}\end{array}$ & & & & & & & & & & \\
\hline $\begin{array}{c}170 \mathrm{R} \\
1081003\end{array}$ & & & & & & & & & & \\
\hline 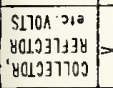 & & & & & & & & & & \\
\hline$a^{\circ}$ & 羊姜点芯总 & 总总总总㮍 & 关关总总䓛 & 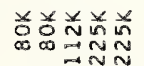 & 总兑兑总兑 & 居兑总总 & 惢号前弟 & 弟首兑兑兑 & 兑总总总总 & 兑出兑总兑 \\
\hline- & 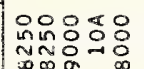 & 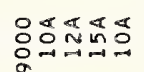 & 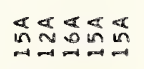 & 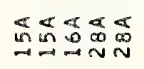 & 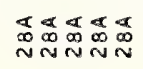 & 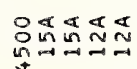 & 赵品: & 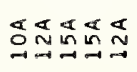 & 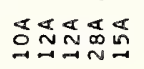 & 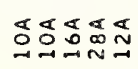 \\
\hline $\begin{array}{c}5170 \mathrm{~N} \\
300 \mathrm{NY} \\
80 \mathrm{BE} 3 \mathrm{BR}\end{array}$ & 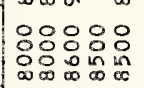 & 品并弟总点 & 兑希希总点 & 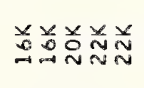 & 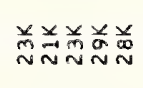 & 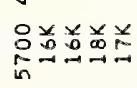 & 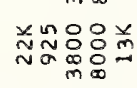 & 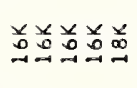 & 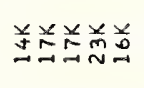 & 弟总兰首酋 \\
\hline$\mapsto$ & 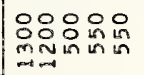 & $\begin{array}{l}\circ 8 \\
8 \\
0\end{array}$ & $\begin{array}{l}\circ 8 \\
\circ 808 \\
\infty \\
m\end{array}$ & 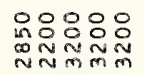 & 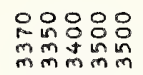 & 응ㅇㅇㅇ용요 & 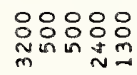 & 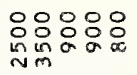 & 品喕品员员 & 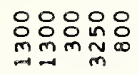 \\
\hline ш" & 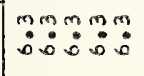 & 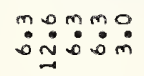 & 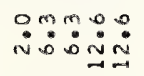 & 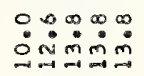 & 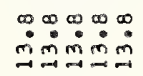 & $\begin{array}{l}m m m \\
\dot{0}: \dot{0}: \dot{0}\end{array}$ & 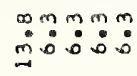 & 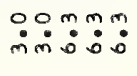 & 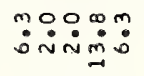 & : \\
\hline No11YY3dO & aca $a$ & $a \operatorname{aga}$ & $a, a c a$ & casaa & aabaa & a a a a & a a a a & a a & $\operatorname{araa} a$ & $a a a a$ \\
\hline$\forall נ W$ & تコّ & 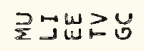 & 次出出 & 叉岂ココさ & コゴコロ & 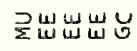 & ココ灵コU & سّ سّ سّ سئ & 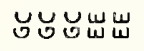 & \\
\hline ]Nก1 & $\mid \begin{array}{l}x \times x \\
4\end{array}$ & 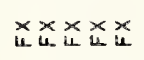 & 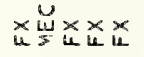 & 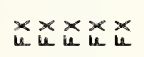 & 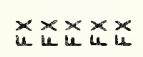 & 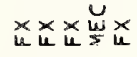 & 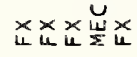 & 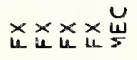 & 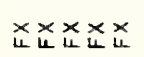 & xxuuu \\
\hline 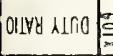 & 욱우요 & "n & 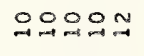 & 움웃ㅇㅇㅇㅇㅁ & 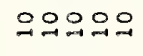 & 엄응ㅇㅁ우으 & 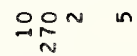 & in엉ㅇㅇㅇㅇㅡ & in어우웅ㅇ & $n \backsim \simeq 0$ \\
\hline 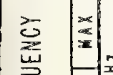 & 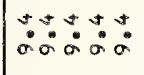 & 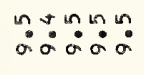 & 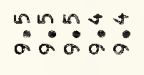 & åa & 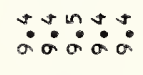 & $\because \stackrel{\circ}{\circ}$ & 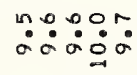 & 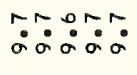 & 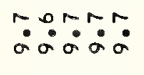 & 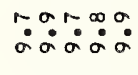 \\
\hline 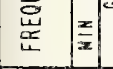 & $\dot{\sigma} \dot{0} \sigma \dot{0}$ & 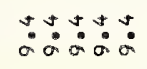 & $\dot{\sigma} \dot{\alpha} \dot{0} \dot{0}$ & 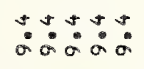 & $\dot{a} \dot{0} \dot{0} \dot{0}$ & 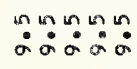 & ஸீ: & 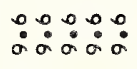 & 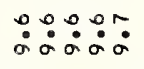 & 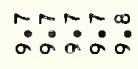 \\
\hline ONIX & 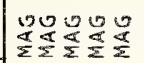 & 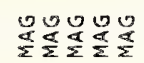 & 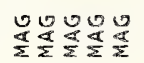 & 00000 & $\begin{array}{l}00100 \\
\frac{5}{2} \frac{4}{2} \frac{x}{2} \frac{x}{2}\end{array}$ & 包0 & 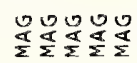 & 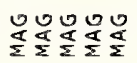 & 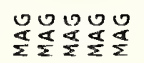 & 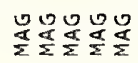 \\
\hline 108WKS & " " & & $"$ & & $"$ & & $" 1$ & & & \\
\hline 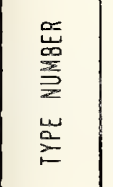 & 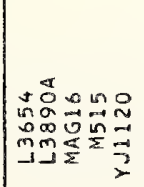 & 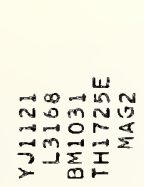 & 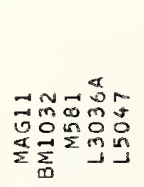 & 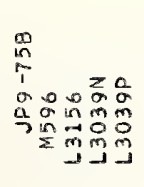 & 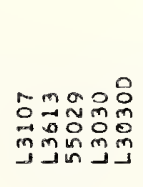 & 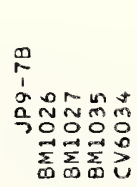 & 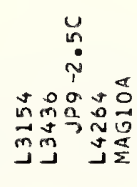 & 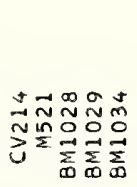 & 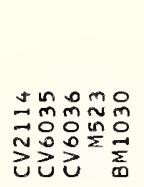 & 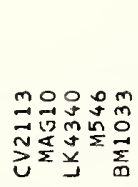 \\
\hline
\end{tabular}




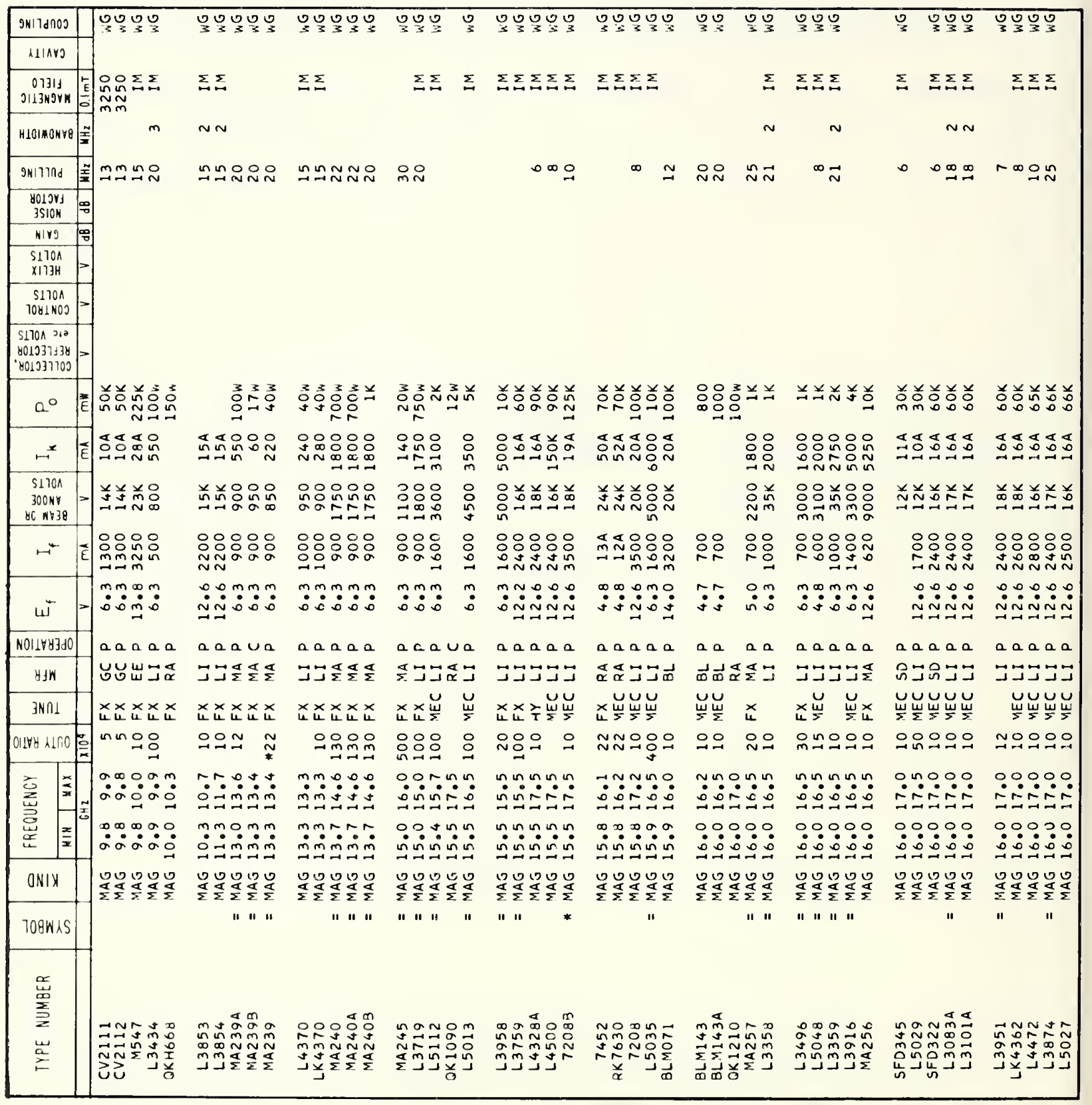




\begin{tabular}{|c|c|c|c|c|c|c|c|c|c|c|}
\hline 9xildno3 & \multirow{3}{*}{ 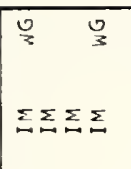 } & \multirow[t]{2}{*}{0000} & \multirow[t]{2}{*}{ 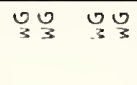 } & \multirow[t]{2}{*}{ OOS } & \multirow[t]{2}{*}{ OO } & \multirow[t]{2}{*}{ 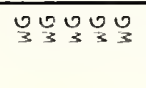 } & \multirow[t]{2}{*}{ טצ } & \multirow[t]{2}{*}{ 워 } & \multirow[t]{2}{*}{$\mathscr{Z}_{3} U_{3} U_{3}$} & \multirow[t]{2}{*}{$\bigcup_{3} \cup \cup \cup$} \\
\hline Al:nvo & & & & & & & & & & \\
\hline $\begin{array}{c}01314 \\
\text { 3113\%9rk }\end{array}$ & & 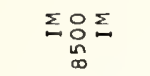 & $\Sigma \Sigma \sum \Sigma$ & $\Sigma \Sigma \Sigma \Sigma$ & $\Sigma$ & $\sum \sum \Sigma$ & $\sum \Sigma \Sigma \Sigma$ & $\Sigma \Sigma$ & $\sum \sum \sum$ & $\sum \Sigma \sum \sum \sum$ \\
\hline H1OMONY $x_{2}^{2}$ & $\stackrel{D}{\sim}$ & & $\sim$ & & & & & $\stackrel{n}{\sim}$ & & \\
\hline 9אורח & $\stackrel{\sim}{\sim}$ & $\stackrel{\sim}{\sim} \sim \infty \simeq$ & $\stackrel{\circ}{\sim}$ & $\simeq$ & $\stackrel{\sim}{\sim}$ & $\circ$ & $\simeq \infty \infty$ & 윰요 & $\stackrel{D^{\circ}}{\sim} \sim$ & 알웡의의 \\
\hline $\begin{array}{c}\text { yolors } \\
\text { JSION }\end{array}=$ & & & & & & & & & & \\
\hline Airg $=$ & & & & & & & & & & \\
\hline $\begin{array}{l}51700 \\
\times 1174\end{array}$ & & & & & & & & & & \\
\hline $\begin{array}{c}51701 \\
10 y 1403\end{array}=$ & & & & & & & & & & \\
\hline 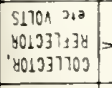 & & & & & & & & & & \\
\hline $0^{\circ}$ & 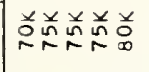 & 莌总总总点 & 兰并兑弚兑 & 勇并总兑总 & 莌品总总兑 & 居兑兑品点 & 关占酋关兑 & 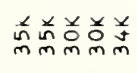 & 差并兑兑品 & 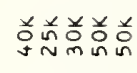 \\
\hline 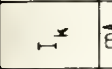 & 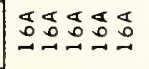 & 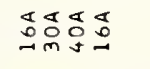 & 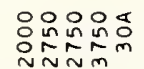 & 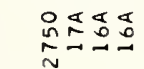 & 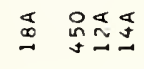 & 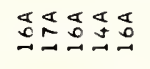 & 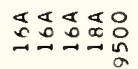 & 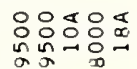 & 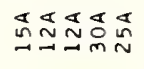 & 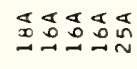 \\
\hline 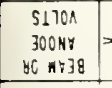 & 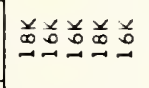 & 总总总总 & 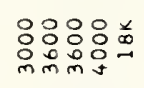 & 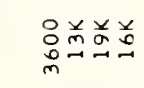 & 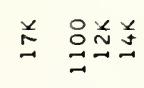 & 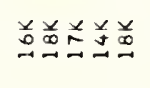 & 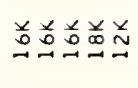 & 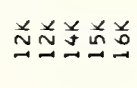 & 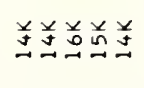 & 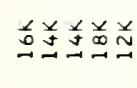 \\
\hline$\leftarrow^{*}$ & 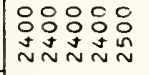 & $\begin{array}{l}\circ \\
\stackrel{\circ}{\circ} \\
\stackrel{\circ}{N}\end{array}$ & 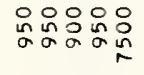 & 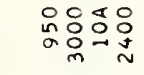 & 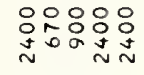 & 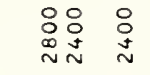 & 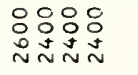 & 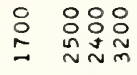 & 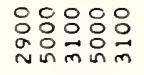 & 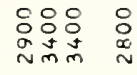 \\
\hline$\omega^{ \pm}$ & 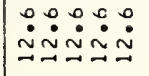 & $\stackrel{\dot{\sim}}{\cong} \quad \stackrel{\check{\sim}}{\cong}$ & 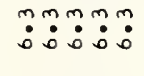 & 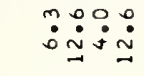 & 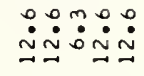 & 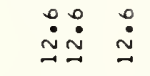 & 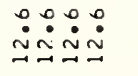 & 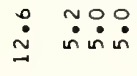 & 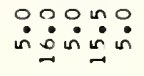 & 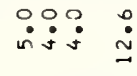 \\
\hline 101184380 & $a, a, a$ & 0,000 & a ava & Ua a a a & $a, a, a$ & a a a a & a a a a & $0, a, a$ & $0 a 0 a$ & aoga \\
\hline$y\rfloor n$ & コュマココ & コ出山コす & コココゴ & 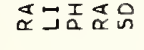 & そ古更ココ & ペ๕コ๔コ & コ汸汸コه & 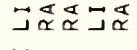 & 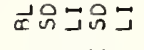 & 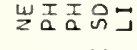 \\
\hline उNก1 & 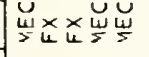 & 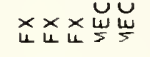 & $\begin{array}{ll}x \times & \times x \\
u\end{array}$ & 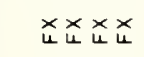 & 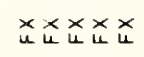 & $x_{u}^{\times x \times x}$ & $\times \times \times \times \breve{u}$ & 岀x $\times x$ & 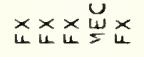 & $\times \times \times \breve{u} \times$ \\
\hline outy alro & $\therefore \quad \simeq 0$ & 음ㄷㅇㅇㅁㅇㅢ & 임윰임 & 윰으으오 & 으욤요 & MNON & 으으으으 & omor & $\operatorname{rin} 0$ ino & $\operatorname{rmm\infty }$ \\
\hline 总 & 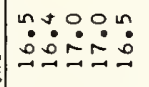 & 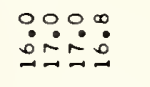 & 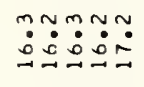 & \begin{tabular}{l}
0 \\
$\dot{0}$ \\
\hdashline
\end{tabular} & 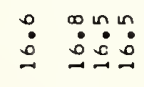 & 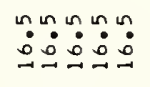 & 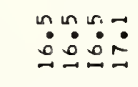 & 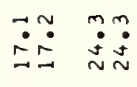 & 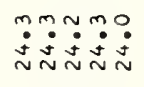 & 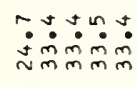 \\
\hline \begin{tabular}{l|l|}
$\stackrel{\vec{B}}{\vec{x}}$ & $\frac{x}{x}$ \\
\end{tabular} & :0욤요 & ن & ن & ن & 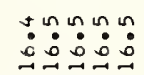 & 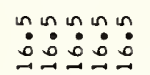 & ن & $\stackrel{\sim}{\dot{\sim}} \times \stackrel{\infty}{\sim} \stackrel{\infty}{\sim} \dot{\sim}$ & 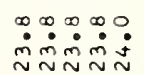 & $\ddot{\dot{\sim}} \dot{\sim} \dot{\sim} \dot{m} \dot{m} \dot{m}$ \\
\hline ONIX & 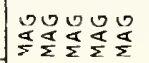 & $\begin{array}{ll}0 \\
\frac{1}{2}\end{array}$ & 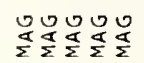 & 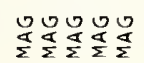 & 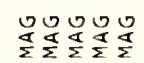 & 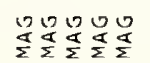 & 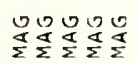 & 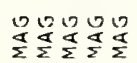 & 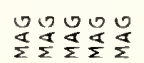 & 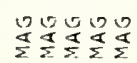 \\
\hline $708 \mathrm{k} \times \mathrm{S}$ & $" 11$ & & " " " " " & " " & & " " " & & " " & & \\
\hline 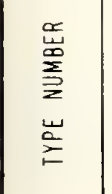 & 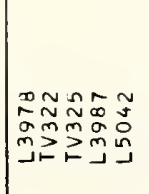 & 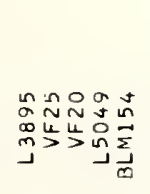 & 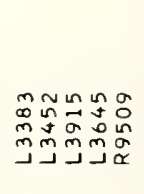 & 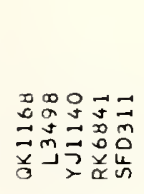 & 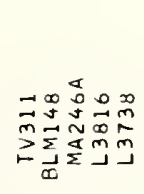 & 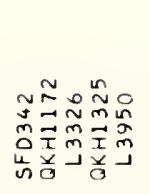 & 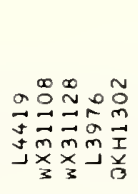 & 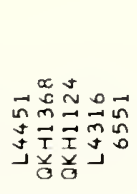 & 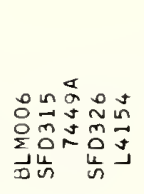 & 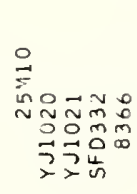 \\
\hline
\end{tabular}




\begin{tabular}{|c|c|c|c|c|c|c|c|c|c|c|}
\hline 9N17dnOO & 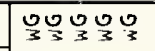 & O० & 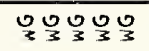 & O००ऽ & OUß & उOS & 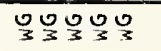 & 온 & 운운 & 엉엉ㅇㅇ \\
\hline Alars & & & & & & & & & & \\
\hline \begin{tabular}{c|c}
01311 \\
$113996 \mathrm{~F}$
\end{tabular} & $\sum \sum \sum \sum$ & $\sum \sum \sum \sum \Sigma \Sigma$ & $\Sigma \Sigma \Sigma \Sigma \Sigma$ & $\Sigma \sum \Sigma \Sigma \Sigma$ & 兰 $\sum \Sigma \Sigma$ & $\sum$ & & $\Sigma \Sigma$ & $\Sigma$ & 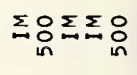 \\
\hline 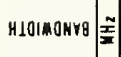 & $\therefore$ & $\stackrel{n}{\sim}$ & & in & & & & & & \\
\hline 9אורור & 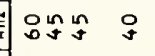 & 욱느용으음 & 응ㅇㅁㅇㅛ & 윰ㅇ ${ }^{\infty} \infty$ & 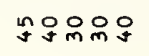 & 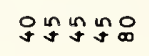 & 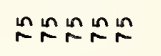 & & & \\
\hline \begin{tabular}{l|l} 
Y01391 \\
3510N
\end{tabular} & & & & & & & & q & & \\
\hline \begin{tabular}{l|l} 
NIY & 0 \\
\end{tabular} & & & & & & & & กNก๊ก & 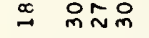 & qmengin \\
\hline $\begin{array}{l}\text { S1700 } \\
\times 173 H\end{array}$ & & & & & & & & $\stackrel{\circ}{\sim}$ & 弚 并 & 芯 弟 \\
\hline $\begin{array}{c}51701 \\
7081 \mathrm{NOO}\end{array}$ & & & & & & & & in & & \\
\hline 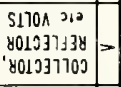 & & & & & & & & 육윰 & $\begin{array}{l}\text { OO } \\
\text { O⿱口口 } \\
\text { N }\end{array}$ & $\stackrel{\circ}{\stackrel{0}{m}}$ \\
\hline $0^{\circ}$ & 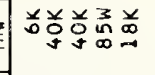 & 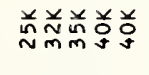 & 弟并并弟兑 & 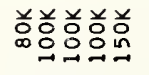 & 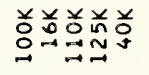 & 关并弟兑总 & 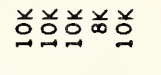 & 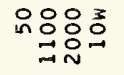 & 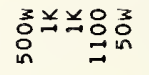 & 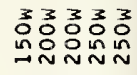 \\
\hline 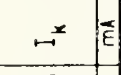 & 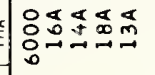 & 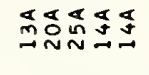 & 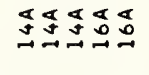 & 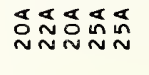 & 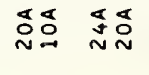 & 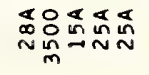 & :ㅇㅇㅇㅇㅇ & $0_{0}^{\circ} \underset{\infty}{\infty} \stackrel{n}{\infty}$ & 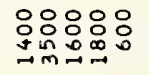 & 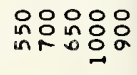 \\
\hline $\begin{array}{r}5170 \mathrm{~A} \\
30 \mathrm{NY} \\
80 \mathrm{M} \rightarrow 3 \mathrm{Bg} \\
\end{array}$ & 㒸酋并关弟 & 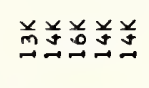 & 并品兑总酋 & 品前并并前 & 慈前兑兰慈 & 关品品兰并 & 总总首 & 있웅요 & 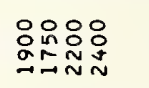 & 品 : \\
\hline$\leftarrow$ & $\mid \begin{array}{l}O O \\
080 \\
0\end{array}$ & 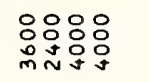 & 임 & 品品 五品 & 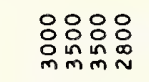 & 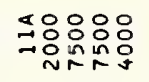 & 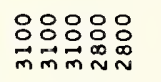 & 은 & 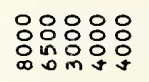 & 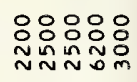 \\
\hline$\omega^{ \pm}$ & 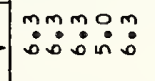 & m:m: & $\stackrel{\circ}{\circ}$ & 我 & 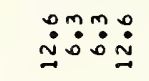 & $\ddot{m}: m m m m:$ & 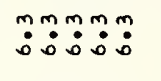 & 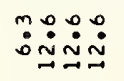 & 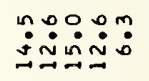 & 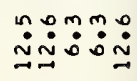 \\
\hline 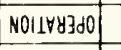 & $a a a n a$ & $a 0 a 0 a$ & 00000 & 00000 & $a a 0 a$ & 0.000 & $a 0 a a n$ & טuטu & vauvu & טuบu \\
\hline$\forall \pm N$ & コココろシ & ココ立品品 & ติธี丶万 요 & ะ品昌ニ只 & 岀造コココ & ェコココ & 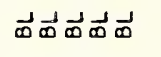 & 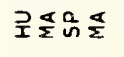 & 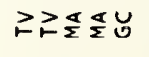 & 强的系品 \\
\hline \begin{tabular}{l|}
$3 \cap \perp$ \\
\end{tabular} & xxur & 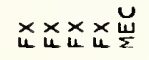 & 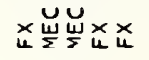 & 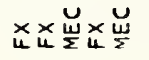 & 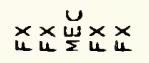 & $x_{4}^{x} \times x_{u}^{x} \times$ & $\begin{array}{l}x \times \times \times x \\
4\end{array}$ & & & \\
\hline $0148 \mathrm{x} \times 10^{0}=\frac{0}{2}$ & $0 \sin$ & Dosin & $\ln i n$ in $m$ in & 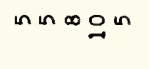 & $\stackrel{n n \infty \infty O}{\longrightarrow}$ & 음음워 & $\simeq \simeq \simeq$ in $\mathrm{n}$ & & $\stackrel{\circ}{\sim}$ & \\
\hline 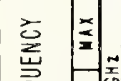 & 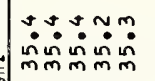 & ONmNn & №ñ & №ñ & to:웅 & 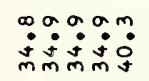 & 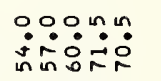 & $\ddot{0} \ddot{0} \dot{0}: \dot{0}$ & 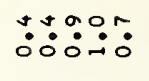 & 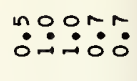 \\
\hline 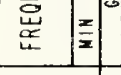 & 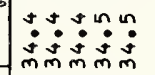 & 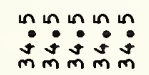 & 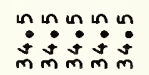 & 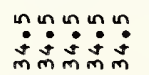 & 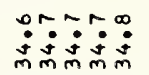 & $\begin{array}{l}\infty a \sigma a n \\
\dot{m} \\
\dot{m}\end{array}$ & 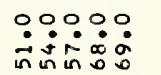 & 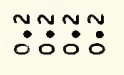 & 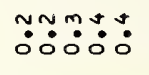 & $\ddot{\circ} \dot{0} 0: \Delta$ \\
\hline ONIK & $\frac{912000}{2} \frac{0}{2} \frac{\alpha}{2} \frac{\alpha}{2} \frac{\alpha}{2}$ & 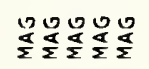 & 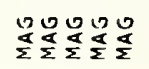 & 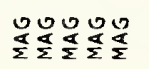 & 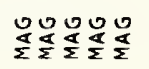 & $\begin{array}{ll}0 \\
\frac{8}{2} \\
\frac{\alpha}{2}\end{array}$ & 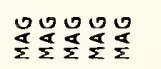 & 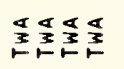 & 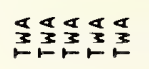 & 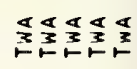 \\
\hline 708WAS & & & & & " " & " " " & & & $"$ & \\
\hline 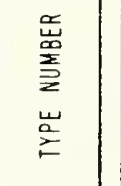 & 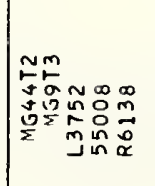 & 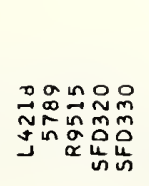 & 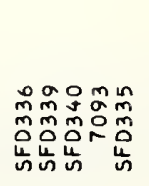 & 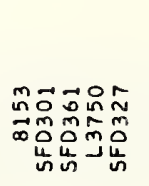 & 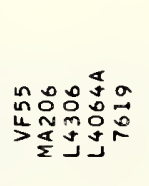 & 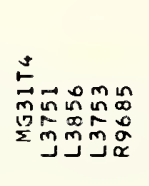 & 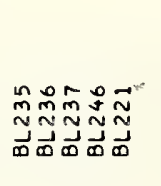 & 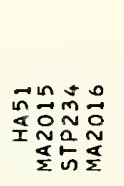 & 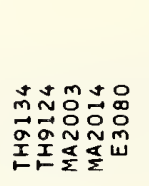 & 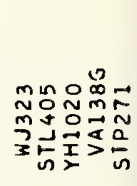 \\
\hline
\end{tabular}




\begin{tabular}{|c|c|c|c|c|c|c|c|c|c|c|}
\hline SNITd TnOS & 언언 & 웅웅으 & ㅇบำ & ะบํำ & 옹 & 농요 & 운온 & 돈요 & 8088 & ¿ㅇㅇ \\
\hline Alars & & & & & & & & & & \\
\hline 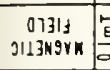 & $\sum \Sigma$ & $\sum \sum_{-\infty} \underset{\infty}{0}$ & 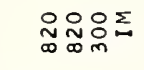 & $\sum D_{i n} \sum \sum \Sigma$ & $\Sigma \Sigma \Sigma \Sigma \Sigma$ & $\sum \sum$ - & $\Sigma$ & $\Sigma \Sigma \Sigma$ & $\sum \sum \Sigma \sum \Sigma$ & 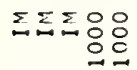 \\
\hline H101MONR8 & in & & & & 品 & 吕 & 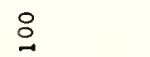 & & $\stackrel{\circ}{\rightarrow}$ & \\
\hline 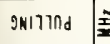 & & & & & & & & & & \\
\hline $\begin{array}{l}\text { Yoljors } \\
3510 \mathrm{~W}\end{array}$ & & nos & 음우요 & $=\stackrel{\sim}{N} \underset{N}{ }$ & mo & $\stackrel{s}{m}$ & & $m$ & $\stackrel{m}{m} \stackrel{n}{m}$ & $0 \sin 0$ 品 \\
\hline Nivg & 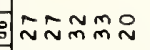 & $\tilde{m} \tilde{m} \underset{N}{ } \sim \sim$ & 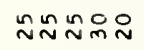 & 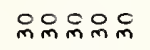 & 요툐 & 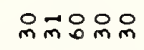 & OÑON & 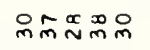 & 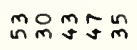 & 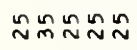 \\
\hline $\begin{array}{l}\text { S170A } \\
\times 173 H\end{array}$ & N & $\stackrel{O}{\simeq}$ & 옳용유 & 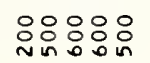 & 并 $\quad \stackrel{x}{N}$ & $\stackrel{x}{N}$ & 兰 & 并 & & : \\
\hline $\begin{array}{c}51701 \\
7081403\end{array}=$ & 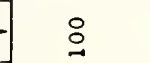 & & in & $\stackrel{\circ}{\circ}$ & 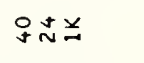 & & 品 & o & $\stackrel{\circ}{\sim}$ & \\
\hline 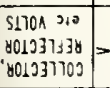 & : & 庤 & $\begin{array}{l}\text { 응유 } \\
\text { 웅유 }\end{array}$ & 동용요 & $\stackrel{\circ}{\circ}$ & 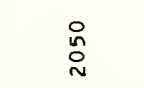 & 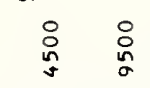 & $\begin{array}{l}: \\
:\end{array}$ & $\begin{array}{l}0 \\
\stackrel{\leftrightarrow}{n} \\
\end{array}$ & 용 \\
\hline$a^{\circ}$ & $\mid \begin{array}{ll}3 \\
0 \\
0 \\
0\end{array}$ & 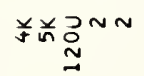 & NMmO & in: & 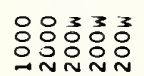 & 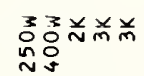 & 品㒸总兑兑 & 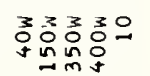 & 윳유. & $\stackrel{2}{\simeq}^{N N}$ \\
\hline$\mapsto$ & $\left.\mid \begin{array}{ll}\circ \\
0 \\
\infty\end{array}\right)$ & \begin{tabular}{l}
$\circ$ \\
\hdashline \\
0 \\
\end{tabular} & $N N+\infty$ & 웅ㅇㅇㅇㅇㅇ & 임욤ㅇㅇㅇ & 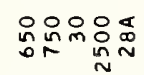 & 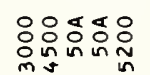 & 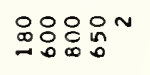 & nn in & \\
\hline 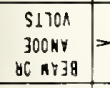 & 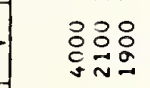 & 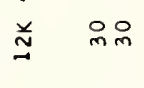 & 으웅요 & 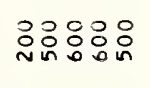 & 萹 总 & 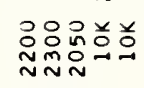 & 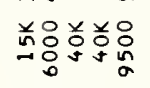 & 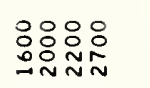 & 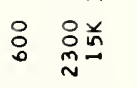 & $\stackrel{ }{\simeq}$ \\
\hline$\rightarrow \quad \tilde{E}$ & 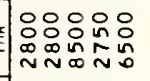 & $\begin{array}{l}: \\
\stackrel{D}{0} \\
\sim\end{array}$ & 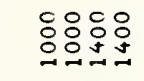 & 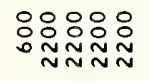 & : & 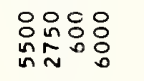 & $\begin{array}{l}\text { :O } \\
\text { : } \\
\text { O }\end{array}$ & 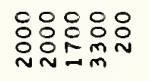 & 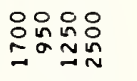 & $\stackrel{\circ}{\circ}$ \\
\hline$\omega^{\leftarrow}$ & 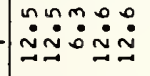 & $\stackrel{0}{\ddot{0}} \quad \ddot{0}$ & 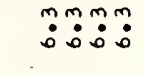 & $\ddot{\because}: \dot{0}: \dot{R}$ & $\ddot{0} \ddot{0}: \dot{0}$ & : & $\stackrel{0}{\stackrel{\dot{I}}{\cong}}$ & 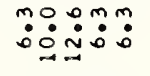 & : & $\ddot{m}:$ \\
\hline N01178] & טuvur & a auuv & טuט & טuט & 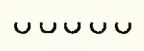 & vuvaa & avaa & uบuบu & ưuau & טuטu \\
\hline$\forall J W$ & 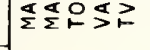 & ココアㄱㅗㅗㅗㅗ & 구ㄱㅗㅗ고고고 & 곺고고고ㄱㅗㅗ & ய路ミs & 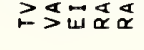 & 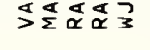 & 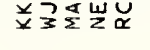 & 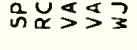 & 굮고고 \\
\hline JMn1 & & & & & & 5 & & u & & \\
\hline 01164 a 1150 & m & 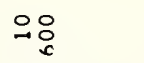 & & & & 웅응 & @ & & $\stackrel{\circ}{\circ}$ & \\
\hline 衣 & $\ddot{0} \because \because 00$ & $\because \because \because \because \because$ & ப் & $\because \because \because:$ & $\because \because \because ゚ 。$ & $\because \because \because \because 0$ & $\because \because: 00:$ & 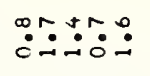 & 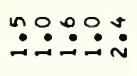 & $\ddot{\sim} \dot{N} \dot{\sim} \dot{\sim} \dot{N}$ \\
\hline 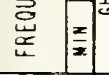 & $\ddot{0}: \stackrel{t}{0}: \Delta \stackrel{0}{0}$ & $\because::_{0}^{+n}::_{0}^{n}:$ & 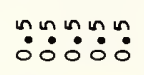 & $\stackrel{n}{n}: \stackrel{n}{n^{n}}::^{n}:{ }^{n}:$ & 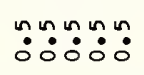 & 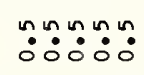 & $\ddot{n}: \stackrel{n}{n}: \dot{n}: 0:$ & $\ddot{0}: \because: 0_{0}^{\infty}$ & $\ddot{\infty}: 00: 0$ & $\because \because \because ̊$ \\
\hline ONIX & 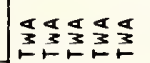 & 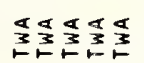 & 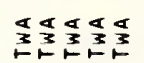 & 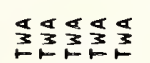 & 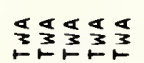 & 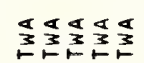 & 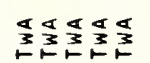 & 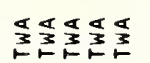 & 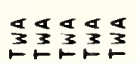 & 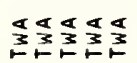 \\
\hline 708WAS & " " " " & & " & & $"$ & " & & & " & \\
\hline 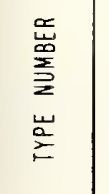 & 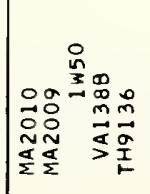 & 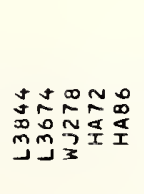 & 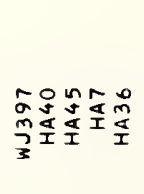 & 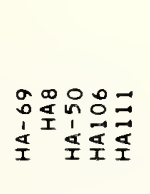 & 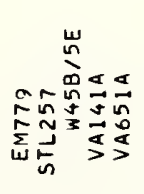 & 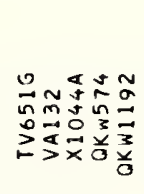 & 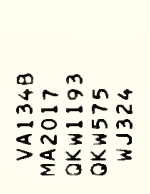 & 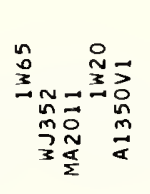 & 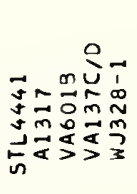 & 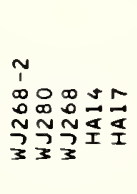 \\
\hline
\end{tabular}




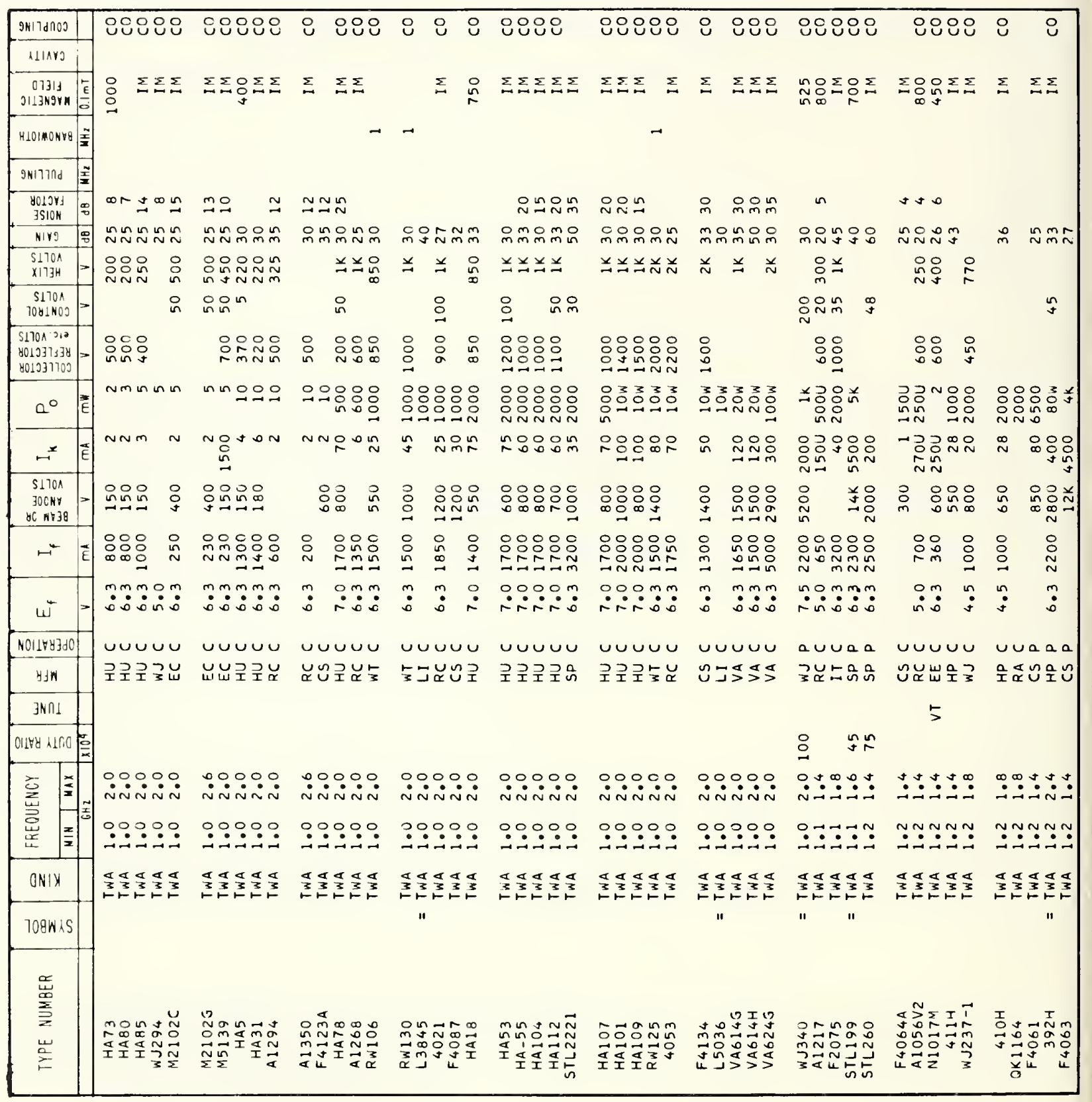




\begin{tabular}{|c|c|c|c|c|c|c|c|c|c|c|}
\hline 9NII InOO & \multirow{3}{*}{ 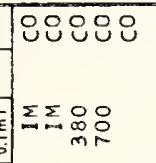 } & \multirow[t]{2}{*}{ 언옹 } & \multirow[t]{2}{*}{ ธ8908 } & \multirow[t]{2}{*}{ Оㅇㅇㅇ } & \multirow[t]{2}{*}{ O O } & \multirow[t]{2}{*}{ Тับ098 } & \multirow[t]{2}{*}{88080} & \multirow[t]{2}{*}{8808} & \multirow[t]{2}{*}{ 웅 } & \multirow[t]{2}{*}{8858} \\
\hline Alikrs & & & & & & & & & & \\
\hline \begin{tabular}{c|c}
07311 \\
ग113N9VK
\end{tabular} & & $\sum \Sigma \sum \Sigma$ & 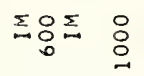 & 음욤요 & $\Sigma \stackrel{\Sigma}{0}$ & $\sum \sum \sum \sum_{n}$ & $\Sigma \Sigma$ & $\overbrace{0}^{\circ}$ & $\Sigma \Sigma \sum \Sigma$ & $\Sigma$ \\
\hline HLOMOAre & in & 品 & $\stackrel{P}{m}$ & & $\stackrel{P}{N}$ & $\stackrel{i}{N}$ & $\rightarrow$ & $\stackrel{\text { n }}{m}$ & & \\
\hline 9wורחกd & 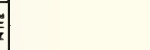 & & & & & & & & & \\
\hline 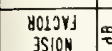 & & 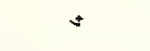 & $\stackrel{n}{=}$ & 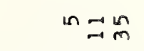 & $\stackrel{\circ}{m}$ & & $\stackrel{i}{N} \dot{m}$ & $\stackrel{\sim}{\sim}{ }_{m}$ & & \\
\hline Mirg & 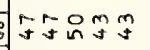 & mo욤요 & $m \stackrel{m}{m} \stackrel{n}{\sim}$ & 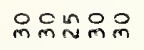 & $m \sim \mathcal{m}$ & qoxo品? & 옴ำ & 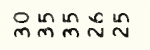 & 윰요요 & $\tilde{N} \stackrel{n}{n}$ \\
\hline $\begin{array}{l}51704 \\
\times 1134\end{array}$ & & $\underset{\sim}{\infty} \underset{\xi}{\sim}$ & $\because \quad \stackrel{\circ}{\sim}$ & 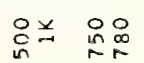 & $\stackrel{x}{\sim}$ & 次 & 并弚并前前 & 娄前 & $\ddot{\sim}$ & 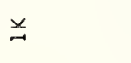 \\
\hline $\begin{array}{c}51701 \\
1081403\end{array}$ & 尊 & 品 & & 웅 & $\stackrel{\circ}{\sim}$ & nro & in & q & & \\
\hline 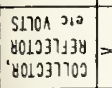 & & 品 & 옹욤요 & 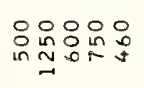 & 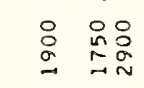 & 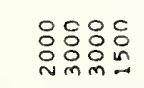 & 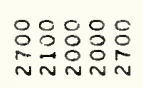 & 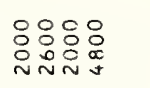 & 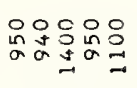 & $\stackrel{\circ}{\cong}$ \\
\hline $0^{\circ}$ & 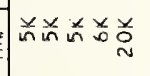 & 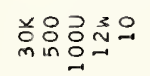 & 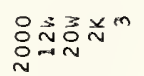 & 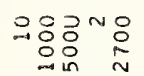 & 욤요 & 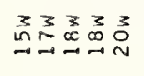 & 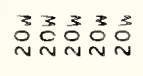 & 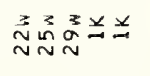 & 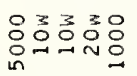 & 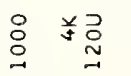 \\
\hline$\varpi^{x}$ & 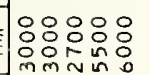 & 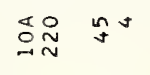 & 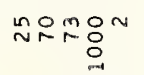 & 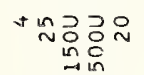 & 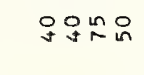 & 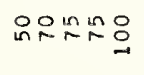 & 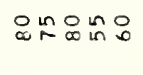 & 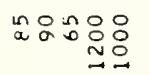 & 우순 & 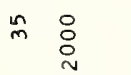 \\
\hline 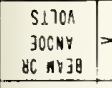 & 总品弟羔弟 & 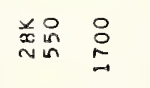 & 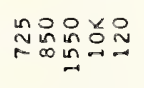 & 踏 品品 & 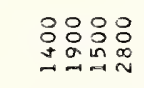 & 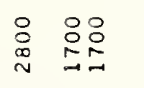 & 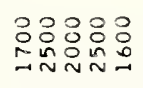 & $\begin{array}{l}\text { 品品 } \\
\text { 品品 }\end{array}$ & 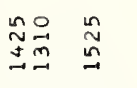 & $\stackrel{D}{\cong}$ \\
\hline$\varpi^{-}$ & 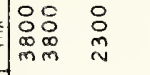 & 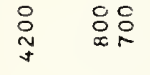 & 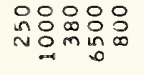 & 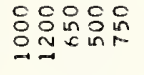 & 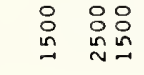 & 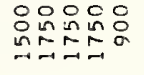 & 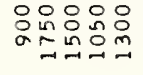 & 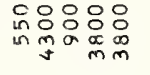 & 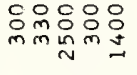 & $\stackrel{\circ}{\circ}$ \\
\hline$\omega^{\star}$ & 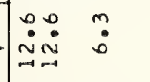 & $\stackrel{0}{\vdots} \quad \dot{n}$ & 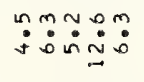 & 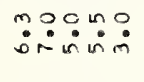 & $\ddot{m}: \ddot{m}$ & 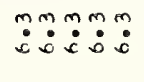 & m: & 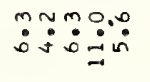 & 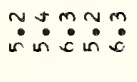 & $\dot{m}: \dot{0}$ \\
\hline N011Yyzdo & acaco & auuvu & uvau & 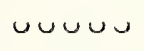 & 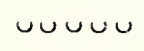 & טuט & טuטu & טuบu & טưu & טuט \\
\hline Yin & 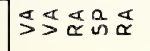 & 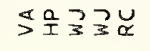 & 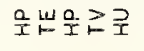 & 主王U⿺乚一匕 & 此こひ & $Z_{\propto} u_{0} \quad I$ & 보는 & 山岁岂山ே & 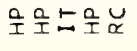 & $\left.\breve{x}_{\propto} \geq \mathfrak{z}\right\}$ \\
\hline 3Nก1 & & & & & 5 & & & 5 & & \\
\hline $0014 y+41,0=$ & $\mid \begin{array}{ll}80 \\
08000\end{array}$ & q & $\stackrel{\circ}{\text { i }}$ & & & & & & & \\
\hline 咅 & 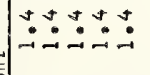 & $\ddot{\sim} \dot{\sim} \dot{\sim} \dot{\sim}$ & $\dot{\sim} \dot{\sim} \dot{\sim} \dot{\sim} \dot{\sim} \dot{\sim}:$ & $\ddot{\sim} \dot{\sim} \dot{\sim} \dot{\sim} \dot{\sim}$ & $\ddot{\sim} \dot{\sim} \dot{\sim} \dot{\sim} \dot{\sim}$ & $\dot{\sim} \dot{\sim} \dot{\sim} \dot{\sim} \dot{\sim} \dot{\sim}$ & $\ddot{\sim} \dot{\sim} \dot{\sim} \dot{\sim} \dot{\sim}$ & $\dot{m} \dot{\sim} \dot{\sim} \dot{\sim} \dot{\sim}$ & $\ddot{\sim} \dot{\sim} \dot{\sim} \dot{\sim} \dot{j}$ & \\
\hline 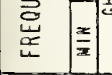 & 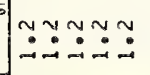 & 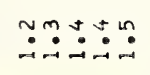 & 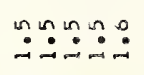 & $\because \because \therefore$ & $\therefore \therefore \therefore$ & $\because \because \because \therefore$ & $\because \therefore \therefore \therefore$ & $\because \because \therefore \therefore$ & 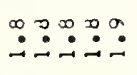 & $\dot{A} \backsim \dot{\sim} \dot{\sim}$ \\
\hline ONIX & $\mid \begin{array}{l}a \\
z \\
F\end{array}$ & 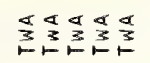 & 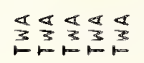 & 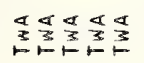 & 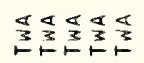 & 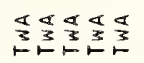 & 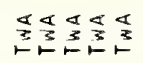 & $\begin{array}{l}4 \\
3 \\
5 \\
1\end{array}$ & 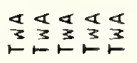 & 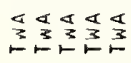 \\
\hline 708kis & & " " & " " & & & & & & $" 1$ & \\
\hline 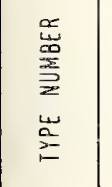 & 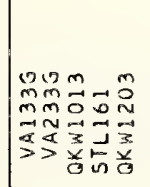 & 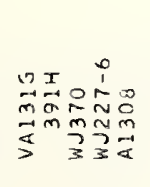 & 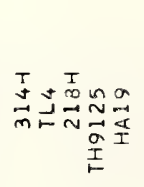 & 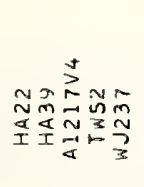 & 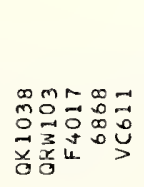 & 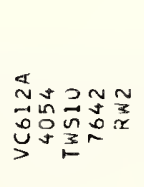 & 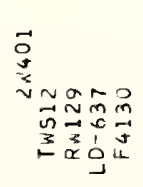 & 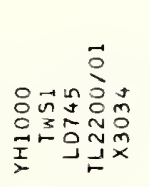 & 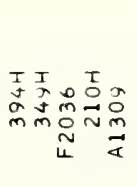 & 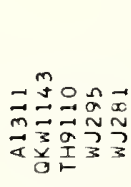 \\
\hline
\end{tabular}




\begin{tabular}{|c|c|c|c|c|c|c|c|c|c|c|}
\hline 9HIIdกOS & 오 옹 & י৪י৪৪י & 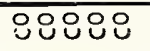 & ㅇ 웅으 & 운 운 & 웅ㅇㅇㅇ & 우 80 & ৪웡ㅇㅇ & 옹워 & 웅웅오 \\
\hline AlAYs & & & & & & & & & & \\
\hline \begin{tabular}{|l|l}
01311 & $\frac{E}{0}$ \\
ग11399VK & $\frac{E}{0}$
\end{tabular} & $\Sigma$ & $\sum \Sigma \Sigma_{0}$ & $\sum \sum \sum \sum \Sigma$ & $\Sigma \Sigma \Sigma \Sigma$ & $\sum \sum D_{0}$ & $\sum \sum \sum_{-1}$ & $\sum_{\sim} \sum_{\sim} \sum_{i}$ & $\sum \sum \sum \sum \sum \sum$ & $\sum \sum \sum \sum \sum \sum$ & $\sum \Sigma \Sigma$ \\
\hline HLOIMOHYg $\mid \tilde{x}$ & & $\stackrel{\sim}{N}$ & & & & $\sim$ & & & & N \\
\hline 9w17nd & & & & & & & & & & \\
\hline 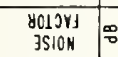 & $\infty \infty \sim \curvearrowleft \infty$ & $\cong ㅡ$ & $\stackrel{2}{=}=$ & $\cong \simeq$ & 임 & $m$ D & $\stackrel{m}{\sim} \stackrel{\sim}{\sim}$ & $\stackrel{\sim}{\sim}$ & 웅 & 음 \\
\hline \begin{tabular}{l|l} 
nivg & क \\
\end{tabular} & 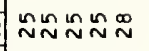 & 픔임요 & 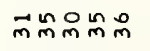 & 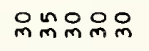 & m $\sim N$ NOP & 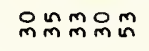 & 이묘 & mmmpmm & 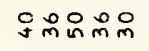 & 임요mmm \\
\hline $\begin{array}{l}S 170 \mathrm{~A} \\
\times 1133 \mathrm{H} \\
\end{array}$ & $\stackrel{0}{i n} \underset{v}{\stackrel{n}{f}}$ & 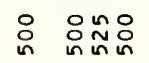 & $\underset{\sim}{\stackrel{n}{\sim} \underset{\sim}{\sim}}$ & 용욤요 & $\stackrel{\sim}{\sim} \cong \quad \underline{\sim}$ & $\underline{\underline{Z}} \underline{\sim} \underline{\underline{Z}}$ & $\cong \quad \cong$ & $\underline{\underline{x}} \quad \underline{\underline{ }}$ & $\stackrel{\circ}{\sim}$ & 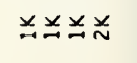 \\
\hline $\begin{array}{c}\text { S1701 } \\
1081403\end{array}=$ & & 요 & & $\therefore \quad$ in & : & inm & in & 요 & $\stackrel{8}{8}$ & \\
\hline 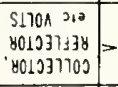 & $\begin{array}{ll}0 & n \\
j & f\end{array}$ & 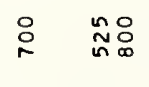 & 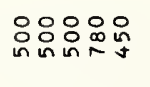 & 음 & N요 & 응응유 & 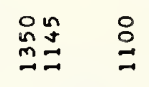 & 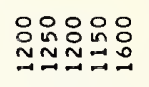 & : & 응욤윰유 \\
\hline$a^{\circ}$ & $\stackrel{D}{N}^{D-n m+~}$ & 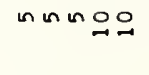 & 음으우으음 & 음엉ㅇㅇ어어 & 응융ㅇㅇㅇㅇㅇㅇ & 응ㅇㅇㅇㅇㅇㅇㅇㅇ & 응응응ㅇㅇㅇㅇ & 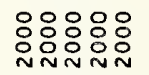 & 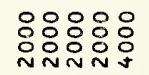 & $\begin{array}{l}03 \\
830 \\
0\end{array}$ \\
\hline$\cong \vec{\varepsilon}$ & $\sim N$ & NNNTN & 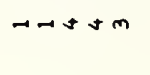 & $\rightarrow N M N N$ & + N $_{\infty} \mathrm{D}_{\mathbb{N}}^{\text {N }}$ & 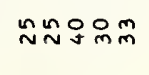 & 임잉요 & 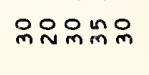 & 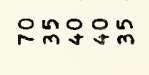 & 있용ㅇㅇㅇㅇㅛ \\
\hline 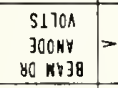 & 욤 & 음욤옥욤요 & & 앳ㅇㅇㅇㅇㅇㅇㅇㅇ & 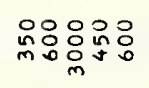 & 육응응용 & 品 总员员品 & 웅 & 용요 & 음음욤 \\
\hline$\mapsto^{4}$ & $\stackrel{\circ}{\equiv}$ & 윰욤음음 & 음요 & : & 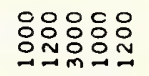 & 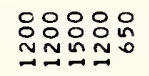 & 品 임 & 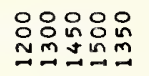 & 응용요 & 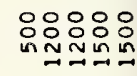 \\
\hline w" & $\stackrel{\infty}{\infty}: \stackrel{m}{0}$ & $\ddot{m}: \ddot{m}: \dot{m}: \dot{m}$ & $\ddot{m}: m: m: m$ & $\ddot{:}: m:_{0}^{m}:$ & m: & 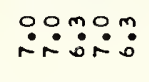 & $\ddot{m}: \dot{m}: \dot{m}$ & $\ddot{m}:{ }^{m}:{ }^{m}: a^{m}:$ & $\ddot{m}: \mathfrak{m}^{m}:{ }^{m}$ & 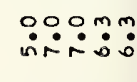 \\
\hline N0118y3do & uบuบ & uบuบu & 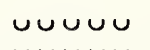 & 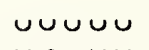 & บบบบa & บบบบบ & ソンuטu & บบบบบ & บบบuบ & 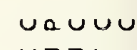 \\
\hline$y \mathrm{ym}$ & 굻고고곡 & 곱uㅗ로고 & 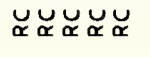 & びさ导岙 & 고고뚤로고 & 고곤고용 & 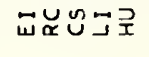 & 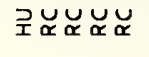 & ヒコココ呈 & 议고고 \\
\hline $3 \mathrm{~N} \cap 1$ & & $\underset{u}{x}$ & & & & & & & & \\
\hline 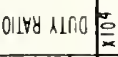 & & & & & $\simeq$ & & & & & $\simeq$ \\
\hline 衣 & 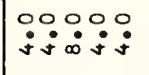 & :00:0 & 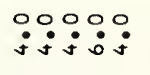 & 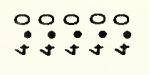 & $\because \because: 00$ & $\because \because \because \because:$ & 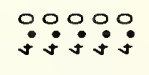 & $\because \because \because 8:$ & $\ddot{0}: 00: 0$ & 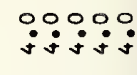 \\
\hline 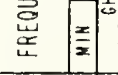 & ஸ்: & 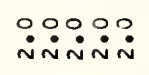 & 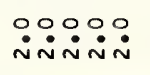 & 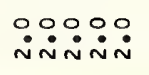 & 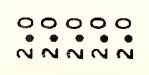 & 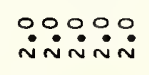 & 華流兑 & 产满芒 & 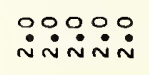 & 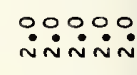 \\
\hline ONIX & 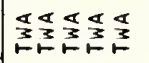 & 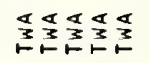 & 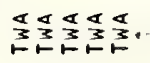 & 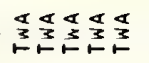 & 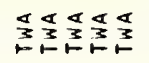 & 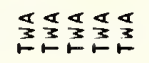 & 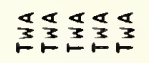 & 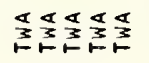 & 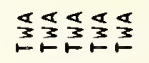 & 乐乐乐舟 \\
\hline TogW/S & " " & & & & & & $"$ & & " " " & \\
\hline 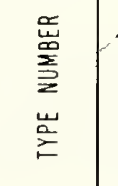 & 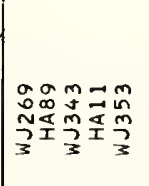 & 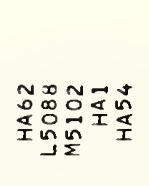 & 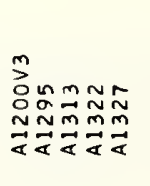 & 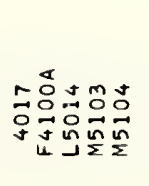 & 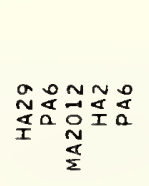 & 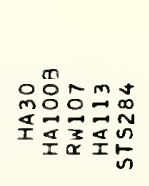 & 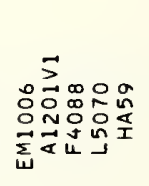 & 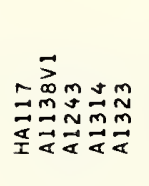 & 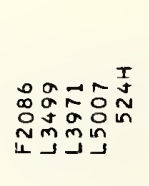 & 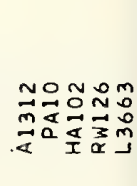 \\
\hline
\end{tabular}




\begin{tabular}{|c|c|c|c|c|c|c|c|c|c|c|}
\hline 9.17 dח & \multirow{2}{*}{\multicolumn{2}{|c|}{ 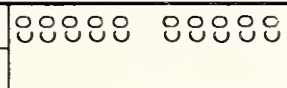 }} & \multirow[t]{2}{*}{ ソேல৪ } & \multirow[t]{2}{*}{ ৪৪๐י๐: } & \multirow[t]{2}{*}{ 웅ㅇํ웅 } & \multirow[t]{2}{*}{ יㅜㅇㅇㅇ } & \multirow[t]{2}{*}{ 도요 } & \multirow[t]{2}{*}{ 운웅 } & \multirow[t]{2}{*}{ ๐ㅜㅇํำ } & \multirow[t]{2}{*}{ 웅ํํ } \\
\hline L1/Ars & & & & & & & & & & \\
\hline 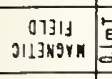 & 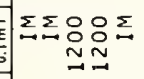 & $\underset{N}{\stackrel{O}{0} \Sigma \sum \sum \Sigma}$ & 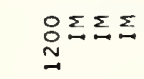 & $\sum \sum \sum \sum \sum$ & $\sum \sum \sum-\sum_{\infty}$ & $\sum O_{\infty} \sum_{-\infty}$ & $\sum \sum \sum \sum \Sigma$ & $\sum \Sigma \underbrace{}_{0} \sum_{0}$ & $\sum \Sigma \Sigma$ & 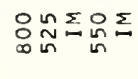 \\
\hline HLOMONYA & $\stackrel{x}{N}$ & $\stackrel{\sim}{\exists}$ & $\stackrel{\square}{N}$ & & & & & & & \\
\hline Sחדרואי & & & & & & & & & & \\
\hline $\begin{array}{l}\text { ycisys } \\
\text { ySION }\end{array}$ & io & 윰윰 & $\stackrel{n}{m}$ & & $\infty$ & $\sin \cong$ & $\stackrel{n}{m}$ & 요요으요 & $\stackrel{n}{\sim} \stackrel{N}{n}_{m}$ & $\infty \infty \stackrel{m}{*}$ \\
\hline Mirg & :mmón & 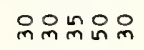 & ㅍmㅁ윰욤요 & 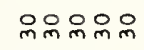 & 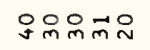 & $\stackrel{\sim}{\sim} \underset{\sim}{\sim}$ & 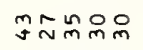 & 윰 $\sim$ N & 있욨 盀 & 온 $\underset{\sim}{*}$ 品 \\
\hline $\begin{array}{l}517010 \\
\times 1173 H \\
\end{array}$ & $=\underset{N}{N}$ & 믄 & $\underset{m}{\mathrm{~m}} \mathrm{~m}$ & $\underset{\infty}{\square}$ & 品 & no & 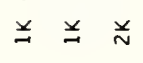 & 웅ํํำ & 品品并并前 & 品品㶽前 \\
\hline $\begin{array}{c}51701 \\
1081403\end{array}=$ & 웅유 & : & $\stackrel{\circ}{\circ}$ & ㅇ & $\stackrel{\circ}{-}$ & & : & $\stackrel{\sim}{\sim}$ in & 응요요 & :웜 \\
\hline 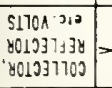 & . & $\stackrel{\circ}{\circ}$ & : & & 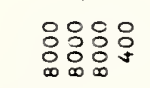 & 용 & 옹염 & 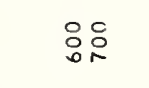 & $\begin{array}{r}: 80 \\
\therefore: \\
\sim \\
\sim\end{array}$ & 웅욤욤욤 \\
\hline $0^{\circ}$ & 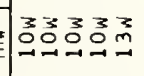 & 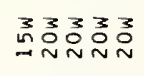 & 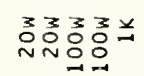 & 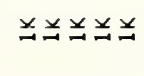 & $\underline{\sim}$ & 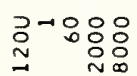 & 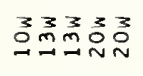 & $\underset{N}{3} \rightarrow m n$ & 우 & no: \\
\hline$\rightarrow$ & 纱品品品 & 总웅요 & 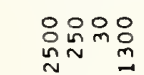 & $\begin{array}{l}\text { O:O: } \\
\text { ODN }\end{array}$ & 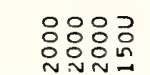 & 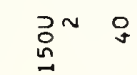 & 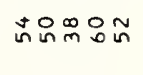 & $\stackrel{D}{n}_{n} \sim N$ & $\sim \sim O_{\infty} \cong \tilde{N}$ & 强品跑 \\
\hline 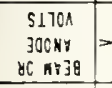 & 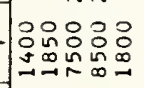 & $\begin{array}{l}: \\
:\end{array}$ & 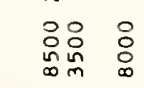 & 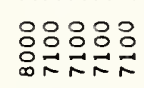 & 要 & : 음 & 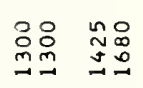 & 움웅 & 斿号贺品品 & 号 :윰 \\
\hline$\mapsto$ & 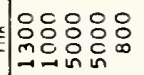 & 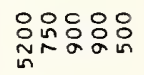 & 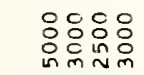 & 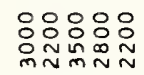 & 음윰윰 & in & 옹요 & 융요요 & 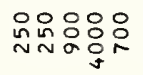 & 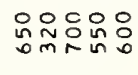 \\
\hline 山' & mammån & 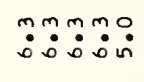 & $\ddot{m}: \ddot{m}: \ddot{m}$ & 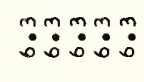 & 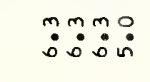 & $\ddot{0}$ & 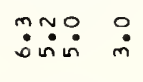 & $\ddot{n} \dot{0} \dot{0}$ & $\ddot{m} \ddot{m}: \dot{m}: \dot{m} \dot{m}: \dot{m}$ & $\dot{n}$ \\
\hline NOIIFy3dO & Uuagu & auvuu & Uava & 00000 & $00 a 0 u$ & טuטu & טuบu & טuטu & uบuvu & \\
\hline$y \cdot m$ & ひコこニ? & 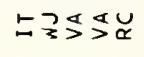 & コニアび & Ш소솓소오 & そヒこヒと & 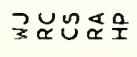 & 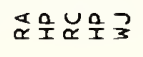 & 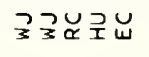 & 넜눙엉요 & 岗出亗ら \\
\hline ] $\mathrm{N} \cap 1$ & 5 & & 5 & & & & & & & \\
\hline $0118 y+1500$ & in i⿱ 口 & in & in $\simeq$ N & 웃ㅇㅇㅇ음 & 용ㅇㅁ & & & & & \\
\hline 曾 & 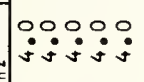 & 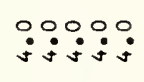 & 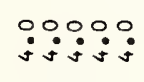 & 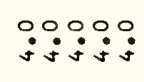 & 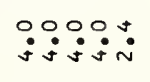 & $\dot{\sim} \dot{\sim} \dot{\sim} \dot{\sim} \dot{\sim}$ & 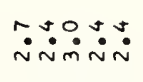 & $\dot{\sim} \dot{v} \dot{\sim}: \dot{s}$ & 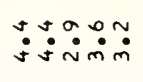 & $\because \because \dot{\square}$ \\
\hline 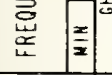 & :O: & $\dot{\sim} \dot{\sim} \dot{\sim} \dot{\sim} \dot{\sim}$ & 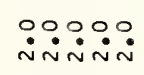 & ஸ்: & $\dot{\sim} \dot{\sim} \dot{\sim} \dot{\sim} \dot{\sim}$ & 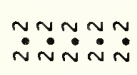 & $\because \cong \sim \sim ⿻ 上 丨$ & 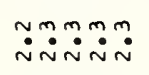 & 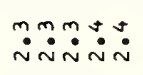 & $\stackrel{n}{\sim} \dot{\sim} \dot{\sim} \dot{\sim} \dot{\sim}$ \\
\hline ONIX & 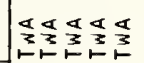 & 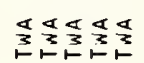 & 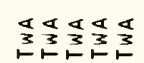 & 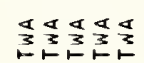 & 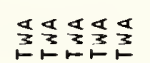 & 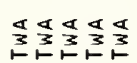 & 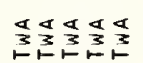 & 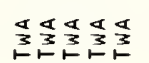 & 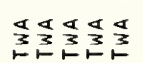 & 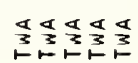 \\
\hline Togwis & & " & $"$ & " " " & & " " & " " " & & & " \\
\hline 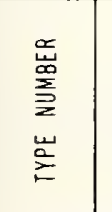 & 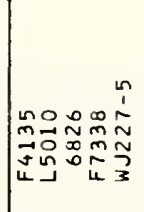 & 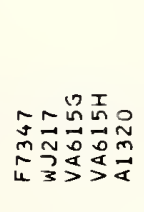 & 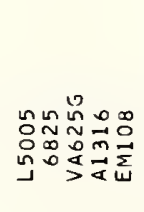 & 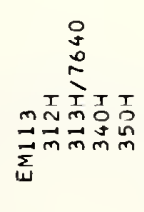 & 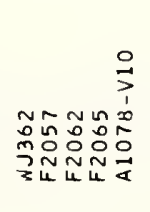 & 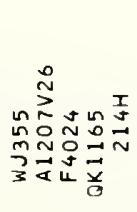 & 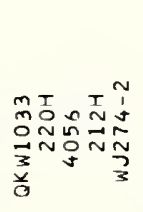 & 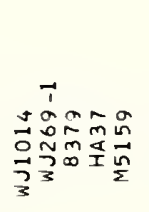 & 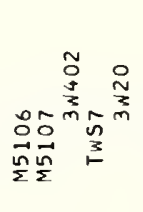 & 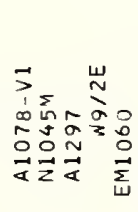 \\
\hline
\end{tabular}




\begin{tabular}{|c|c|c|c|c|c|c|c|c|c|c|}
\hline $9 \times 17$ TnO3 & 엉엉ㅇㅇ & 웅요 & 엉엉 & ত্ডYSOO & 80088 & 엉요 & 8388 & O্তOOO & 웅ㅇㅇ & SOSOS \\
\hline LIAAY & & & & & & & & & & \\
\hline \begin{tabular}{|c|} 
O1311 \\
J113K9YM \\
\end{tabular} & 鳥 & $\sum_{-1} \sum_{n} \sum_{n}$ & 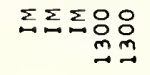 & 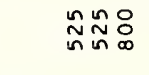 & 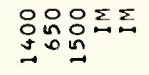 & $\Sigma$ & $\sum \sum \Sigma$ & $\Sigma \Sigma \Sigma$ & $\Sigma \Sigma \Sigma$ & $\sum_{n} \sum_{n} \sum_{n} \sum_{n=1}$ \\
\hline HLOMOMNY & & & & 总 & & & & 응 & 争 & \\
\hline גחרוואפ & & & & & & & & & & \\
\hline $\begin{array}{c}\text { BOIJW } \\
\text { 3SION }\end{array}$ & $=0$ & $\stackrel{9}{-}$ & $\stackrel{a}{\sim}$ & roin & soro & $\stackrel{n}{m}$ & & & & \\
\hline$x(y)$ & 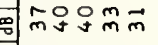 & $\stackrel{\infty}{N} \stackrel{m}{\sim} \sim{ }^{\circ}: 0$ & $\stackrel{n}{n} \tilde{m} \sim \tilde{m} \tilde{m}$ & 융우요요 & $\stackrel{\sim}{\sim} \stackrel{\sim}{\sim} \vec{m}$ 品 & 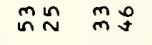 & 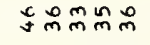 & 무ำm & 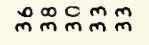 & 묠욜 \\
\hline $\begin{array}{l}51701 \\
\times 113 \mathrm{H} \\
\end{array}$ & $\simeq \stackrel{x}{\sim}$ & & 首酋首 & 总员管 & 윰욤용 & 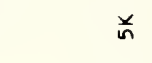 & 㒸 & & & \\
\hline $\begin{array}{c}\text { S1701 } \\
1081103\end{array}$ & 있 : & 욤요 证 & 弚 & 우수 & 용 & $\stackrel{\circ}{\sim}$ & $\stackrel{n}{m}$ & : & $\stackrel{i}{N}$ & $\stackrel{i}{n}$ \\
\hline $\begin{array}{l}51704212 \\
801937134 \\
801237700\end{array}$ & 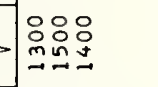 & 吕 & 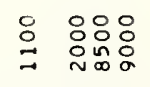 & 응요 & 品 움 & & $\begin{array}{l}\circ \\
\text { O } \\
\text { o }\end{array}$ & 엉 & & 음 \\
\hline $0^{\circ}$ & E & 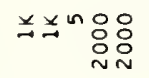 & 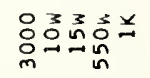 & 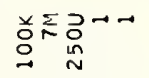 & Nmmmo & 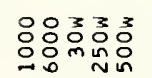 & 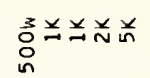 & 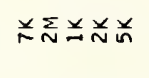 & 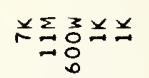 & 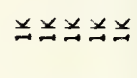 \\
\hline$-x$ & E & 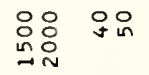 & 었임용응 & 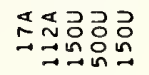 & $\begin{array}{l}\text { 응응ㅇㅇ } \\
\text { 잉 }\end{array}$ & 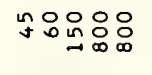 & 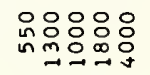 & 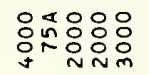 & 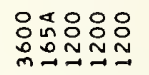 & 용육: \\
\hline 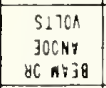 & ${ }_{m} \quad \frac{O}{0}$ & 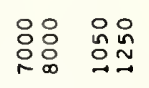 & $\stackrel{\substack{n \\
\infty}}{\rightarrow}$ & 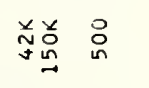 & $\stackrel{2}{\sim} \stackrel{0}{\mathfrak{j}}$ & 음유 & 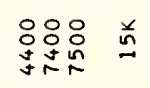 & 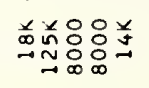 & 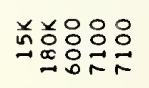 & 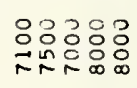 \\
\hline 5 & Eิ & 응 & 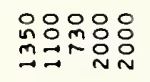 & 岱西㶽品品 & 品品品品 & 욤음윰유 & 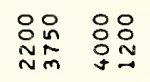 & 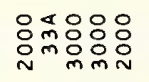 & 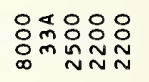 & :요요 \\
\hline$\omega^{ \pm}$ & $\ddot{\dot{0}} \dot{0} \dot{0}$ & $\ddot{\dot{0}} \dot{0} \ddot{0} \dot{0}$ & 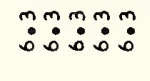 & 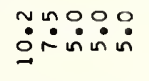 & 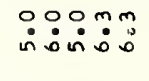 & 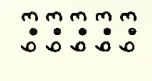 & 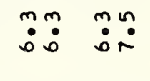 & 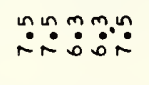 & 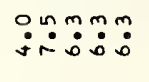 & $\ddot{m} \ddot{0}: \dot{m}$ \\
\hline NOLI $\forall Y\}]$ IO & Uบบa & a auu & Uuvaa & a auu & ưuuv & Uuava & acao & $00 a 0 a$ & aaca & 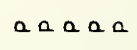 \\
\hline$y \leq N$ & 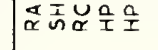 & 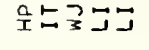 & 는요요 & 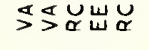 & 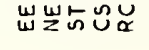 & 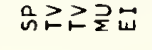 & 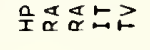 & ハムロロロ゙ & 秝蛙呈呈 & 呈呈呈にニ \\
\hline 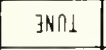 & & & & 55 & & 5 & 5 & & & \\
\hline Oleyt $\times 1$ rio & 옴 & 욤 & 응 & 吕 & & mo & 品品品 N & ㅁำ & 슛ㅇㅇㅇ & 윰유유 \\
\hline \begin{tabular}{l|l|}
$\bar{z}$ & $=$ \\
\end{tabular} & 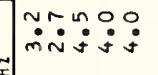 & 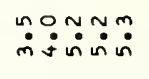 & 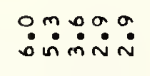 & 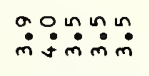 & $\ddot{m} \dot{\sim} \dot{m} \dot{m} \dot{m}$ & imminmin & 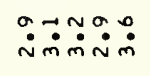 & 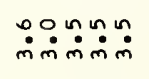 & 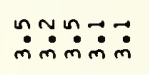 & 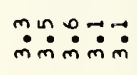 \\
\hline 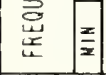 & 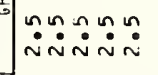 & $\dot{\sim} \dot{\sim} \dot{\sim}: \dot{\sim} \dot{\sim}$ & $\dot{\sim} \dot{\sim} \dot{\sim} \dot{\sim} \dot{\sim}$ & $\dot{\sim} \dot{\sim} \dot{\sim} \dot{\sim}$ & 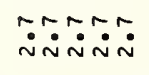 & 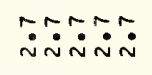 & 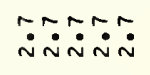 & $\dot{\sim} \dot{\sim} \dot{\sim} \dot{\sim}_{\dot{\sim}}^{\infty} \dot{\sim}$ & $\dot{\sim} \dot{\sim} \dot{\sim} \dot{\sim} \dot{\sim} \dot{\sim}$ & $\dot{\sim} \dot{\sim} \dot{\alpha} \dot{\sim} \dot{\alpha}$ \\
\hline ONIX & 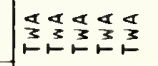 & 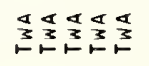 & 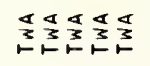 & 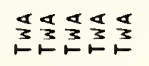 & 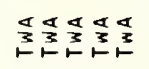 & 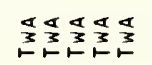 & 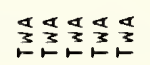 & 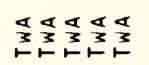 & 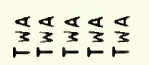 & 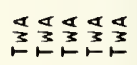 \\
\hline $708 W / S$ & $"$ & " " & & & & & $"$ & $" 1 "$ & $"$ & \\
\hline 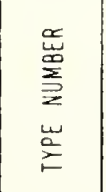 & 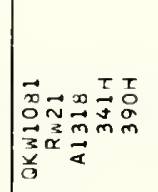 & 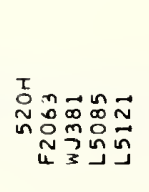 & 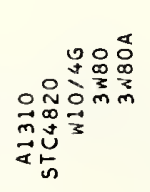 & 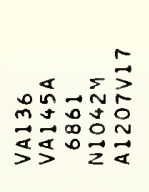 & 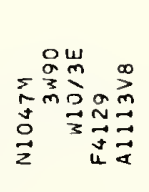 & 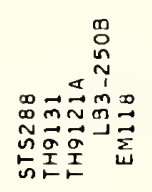 & 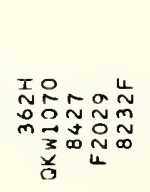 & 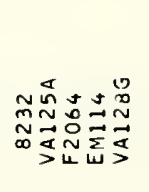 & 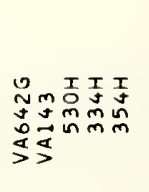 & 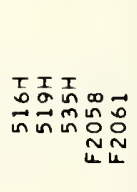 \\
\hline
\end{tabular}




\begin{tabular}{|c|c|c|c|c|c|c|c|c|c|c|}
\hline 9x: 7dnos & \multirow{2}{*}{\multicolumn{2}{|c|}{ บ8 웡 }} & \multirow[t]{2}{*}{ 웅요 } & \multirow[t]{2}{*}{ ওOSOY } & 은요 & \multirow[t]{2}{*}{ 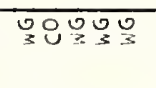 } & \multirow[t]{2}{*}{ 언몽요 } & \multirow[t]{2}{*}{ 옹요 } & \multirow[t]{2}{*}{ 요 } & \multirow[t]{2}{*}{ 80808 } \\
\hline RLAKI & & & & & & & & & & \\
\hline 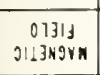 & 自 $\leq \sum \sum \sum 8$ & $\sum \sum \sum \sum 0$ & $\sum \Sigma$ & 距 & $\sum 0_{\infty} \sum$ & 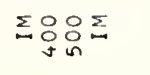 & $\sum \sum \sum \sum$ & $\sum \sum$ & 品品 & $\Sigma \sum \Sigma \Sigma$ \\
\hline H10MONY & & & $\stackrel{\circ}{\sim}$ & & & & & & & \\
\hline פומו & $\hat{x}$ & & & & & & & & & \\
\hline $\begin{array}{ll}3019 Y_{j} \\
3 S 10 \mathrm{~K}\end{array}$ & 量 & & 을 & $\stackrel{n}{\sim} \approx m$ & & $\vec{\sim} \quad \hat{v}$ & $\stackrel{n}{\sim}$ & $\rho_{m}^{\circ \infty}$ & & $m_{m}^{\infty} \simeq$ \\
\hline Hirg & : & Nining & 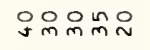 & $\stackrel{a}{m} \sim \mathcal{n} \sim \sim m$ & 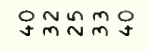 & 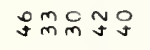 & mmmmon & 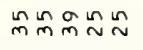 & $\stackrel{n}{\sim} \tilde{m} \tilde{m} \cong$ & 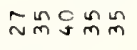 \\
\hline $\begin{array}{l}S 1701 \\
\times 1173 \mathrm{H}\end{array}$ & 요 & $\stackrel{x}{\sim}$ & $\underline{\sim} \underset{\sim}{\sim} \underset{\sim}{ }$ & $\stackrel{\sim}{\sim} \cong$ & $\stackrel{x}{\sim} \quad \stackrel{x}{m}$ & 苾品前前前 & 㒸前南吕 & 은용ㅇㅇ & $\stackrel{x}{N}$ & $\stackrel{x}{m}$ \\
\hline $\begin{array}{c}51701 \\
10411003\end{array}$ & & in & & 요 & $\stackrel{\circ}{\sim}$ & 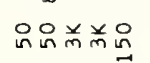 & 음요요 & $\stackrel{\circ}{\stackrel{n}{*}}$ & & $:$ \\
\hline 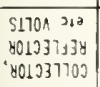 & 曽品 & 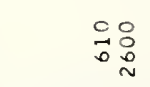 & 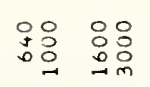 & 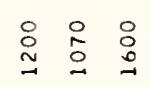 & 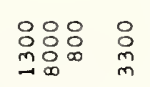 & 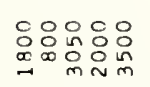 & $\begin{array}{l}\text { 응요 } \\
\text { 음윰요 }\end{array}$ & 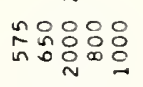 & 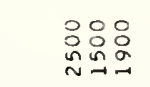 & \begin{tabular}{l}
$\circ 0$ \\
$\therefore 0$ \\
$\vdots$ \\
\hdashline
\end{tabular} \\
\hline$\alpha^{\circ}$ & E. & 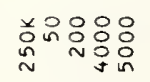 & 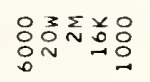 & 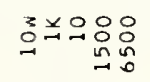 & 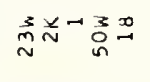 & 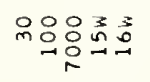 & 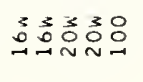 & 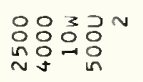 & 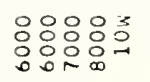 & 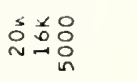 \\
\hline$-x$ & E. & $\underset{\sim}{\infty} \sim \sim \underset{\sim}{\sim} \sim$ & 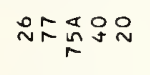 & 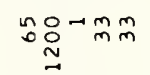 & 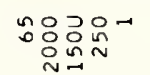 & 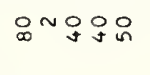 & 요의의 & 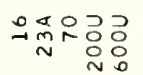 & 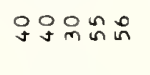 & 웅요 \\
\hline 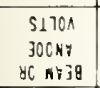 & $=\mid$\begin{tabular}{ll}
0 \\
\hdashline \\
0
\end{tabular} & 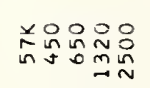 & 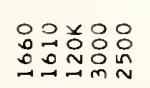 & $\stackrel{\circ}{\circ} \stackrel{\circ}{\circ}$ & $\begin{array}{l}\circ \\
: \\
\vdots \\
\infty\end{array}$ & 요 & 总 & 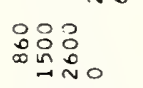 & 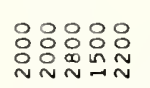 & 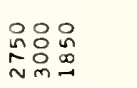 \\
\hline 5 & E| & : & 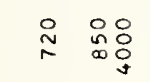 & 음욤요 & 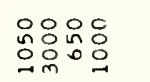 & 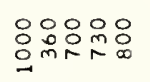 & 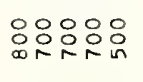 & 임 & 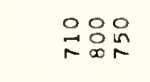 & 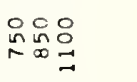 \\
\hline$\omega^{\top}$ & $\Rightarrow: \dot{m}: \dot{m}: 0$. & & $\ddot{i} \ddot{i} \dot{m} \dot{m} \ddot{m} \dot{m}$ & $\ddot{x}: \ddot{m}: \ddot{m}: \dot{m}$ & 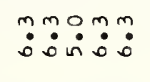 & 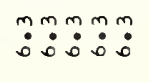 & $\ddot{m}:$ & $\dot{m i n} \dot{0} \dot{0} \because \ddot{0}$ & 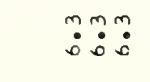 & $\ddot{m}: \stackrel{m}{0}$ \\
\hline NOILYYIJO & $a 0 \Omega 2 a$ & טuטu & טuav & vauvu & vausu & & & & Uua & uve \\
\hline HiN & 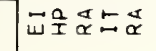 & 오오ㅁㅗㅗ오포 & 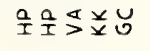 & 甹呈议娟 & IேUココ & ら岀らら岀 & I岂岂岂? & そฺ̧出出 & 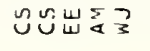 & 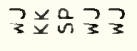 \\
\hline $3 \mathrm{~N} \cap 1$ & & & $\underset{u}{x}$ & & & 5 & & 55 & 5 & $\underset{u}{x}$ \\
\hline $011 y 4$. LI, & 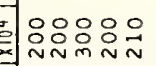 & $:$ & & in & $\therefore \cong$ & & & & & \\
\hline 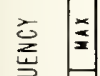 & $\because \ddot{m} \dot{m} \dot{m}$ & 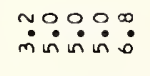 & $\ddot{n} \because \ddot{m} \dot{m}$ & 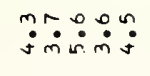 & 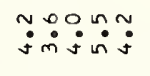 & & & 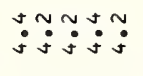 & & $\ddot{j} \because \dot{0}: 00$ \\
\hline 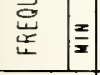 & |0 & $\ddot{\sim} \dot{m} \dot{m} \dot{m} \dot{m}$ & 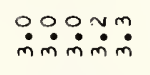 & $\ddot{m} \dot{m} \dot{m} \dot{m} \dot{m}$ & $\dot{m} \dot{m} \dot{m} \dot{m} \dot{m} \dot{m}$ & $\dot{m} \dot{m} \dot{m} \dot{m} \dot{m}$ & & $\ddot{m} \dot{m} \dot{m} \dot{m} \dot{m}$ & $\ddot{m} \dot{m} \dot{m} \dot{m} \dot{m} \dot{m}$ & $\dot{m} \dot{m} \dot{m} \dot{0}=$ \\
\hline ONIY & 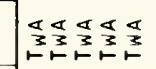 & 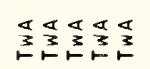 & 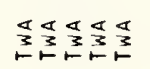 & 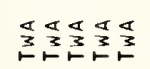 & 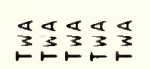 & 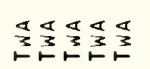 & 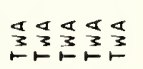 & 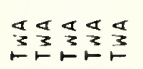 & 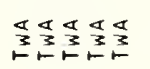 & 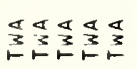 \\
\hline $700 \mathrm{~W} / \mathrm{S}$ & & " & & $"$ & & & & & & \\
\hline 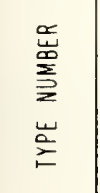 & 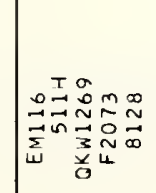 & 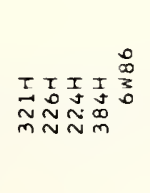 & 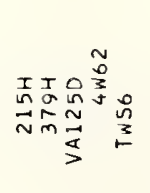 & 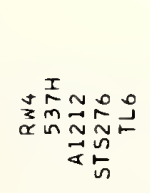 & 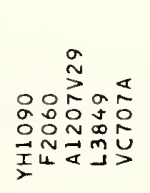 & 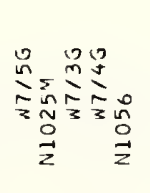 & 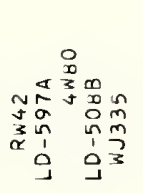 & 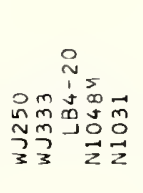 & 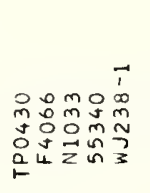 & 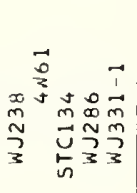 \\
\hline
\end{tabular}




\begin{tabular}{|c|c|c|c|c|c|c|c|c|c|c|}
\hline 9Hו Td InOS & \multirow[t]{2}{*}{ ४8080 } & \multirow[t]{2}{*}{ 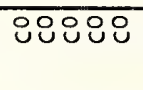 } & \multirow[t]{2}{*}{ 운용 } & \multirow[t]{2}{*}{ 원 웅어 } & \multirow[t]{2}{*}{ 엉ㅇㅇㅇㅇ } & \multirow[t]{2}{*}{ 웅ㅇㅇㅇ } & 889 & \multirow[t]{2}{*}{ י্ব০ণ্ডি } & \multirow[t]{2}{*}{ 운엉 } & \multirow[t]{2}{*}{ ০্তিত্তি } \\
\hline LEIAVS & & & & & & & & & & \\
\hline $\begin{array}{l}01311 \\
\text { ग11399VM }\end{array}$ & $\sum_{0}$ & $D_{j} \sum \sum \sum$ & 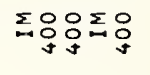 & $\sum \sum \sum \Sigma$ & $\Sigma \Sigma \Sigma \Sigma$ & $\sum \sum \Sigma \Sigma \Sigma$ & $\sum \sum \sum \sum_{\infty} \sum_{\infty}$ & $\sum \Sigma \sum \Sigma$ & $\Sigma \sum \Sigma \Sigma$ & 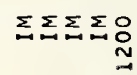 \\
\hline HLOMONYA & & & $\stackrel{\sim}{N}$ & & 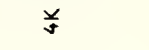 & & & & & \\
\hline \multicolumn{11}{|l|}{ 9אורורח } \\
\hline \begin{tabular}{l|l}
801951 & \\
35104 & 0
\end{tabular} & $\circ$ 응 & $\pm \approx$ & 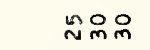 & $\infty m \cong$ & 을 & $\stackrel{\sim}{\sim} \stackrel{\sim}{\sim}$ & 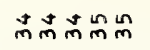 & 望盀 & $\stackrel{n}{\sim} \underset{m}{n}$ & 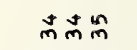 \\
\hline Mirg & 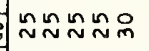 & 음욤음유 & 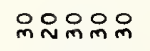 & 冓品品品品 & 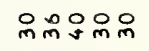 & 品前兹m & 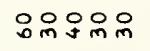 & 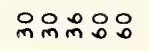 & 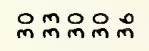 & 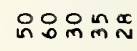 \\
\hline $\begin{array}{l}51701 \\
\times 113 H \\
\end{array}$ & 음윳 & 응용요 & 용용요 & 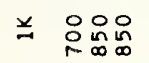 & $\stackrel{\circ}{\circ} \underset{\infty}{\circ} \stackrel{\circ}{\circ}$ & 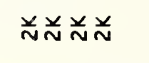 & 并前 弚 & $\stackrel{N}{N}$ & 㓅 & 并前前 \\
\hline $\begin{array}{r}51701 \\
70011000\end{array}=$ & 옥 & in & & 品 㶽品 & 兰 & in in: & 웡ㅇㅇㅇㅇㅛ & 只品 褋 & in in & 은요ㅇㅛㅛ \\
\hline 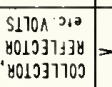 & 융요 & 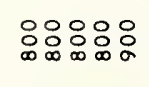 & 응요 & : & 总品品 & 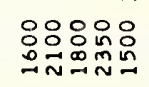 & 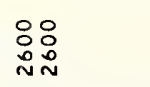 & & 鬲品 & 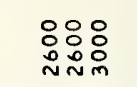 \\
\hline$a^{\circ}$ & 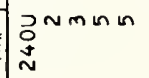 & 오으오으으 & 으으음으으 & 으음유으으 & 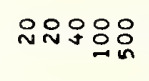 & 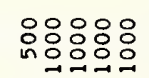 & 응ㅇㅇㅇ융ㅇㅁㅇㅁㅇ & 양ㅇㅇㅇㅇㅁ & 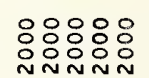 & 욜요 \\
\hline- & $N N \sim$ & NNNMN & Nmmmm & NHง NN & $m m-\simeq O$ & 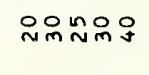 & 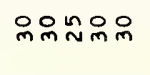 & 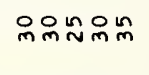 & 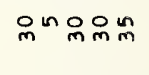 & 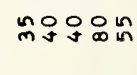 \\
\hline 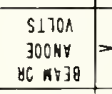 & 응 & 总员呫号 & 总品品品 & 윰용요 & 음ㅇㅇㅇㅇ & 옹용요 & 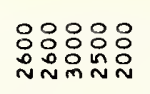 & 욤요 & 怘 : & 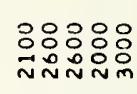 \\
\hline+5 & 品 움 & 음음윰요 & 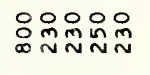 & 음 N & 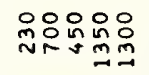 & 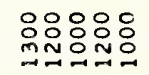 & 응용요 & 웅응ㅇㅇㅇㅇㅁㅇ & 总品品品品 & 응ㅇㅇㅇㅇㅛ \\
\hline шॅ & $\ddot{\text { mo }}:$ & 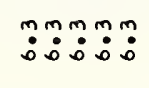 & 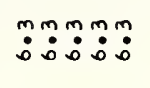 & $\ddot{m}: \ddot{m}: \stackrel{m}{0}$ & 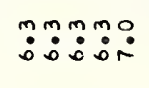 & 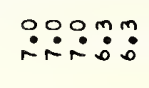 & :m: & 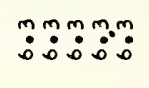 & 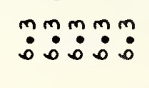 & $\ddot{0}: 00$ \\
\hline NOLIVY\}dO & טuטu & טuuu & บบบบy & ưuuu & uuuuv & טuuu & טuטu & טuטu & uauu & טuט \\
\hline YנA & ३고곯ㅂㅂ & 고고로로무 & 通㞻怘品 & 慈コ㞻㞻 & 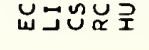 & 고고ㄱㅗㅗㄹㅗ & 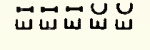 & 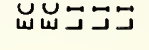 & 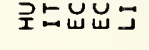 & 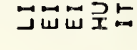 \\
\hline 3Nก1 & & & & & & & & & & \\
\hline $0186 y<1 n 0 \div$ & & & & & & & & & 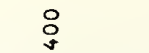 & \\
\hline 离 & 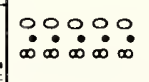 & $\because \because \because \frac{0}{\infty} \dot{\infty} \dot{\infty}$ & 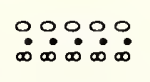 & 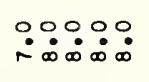 & 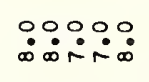 & $\ddot{\infty} \dot{\infty} \dot{\infty} \dot{\infty} \dot{\infty} \dot{\infty} \dot{\infty}$ & :0: & 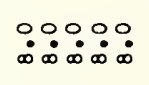 & 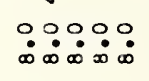 & $\ddot{\infty} \dot{\infty} \dot{\infty} \dot{\infty} \dot{\infty} \dot{\infty} \dot{\infty}$ \\
\hline 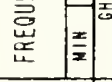 & 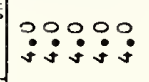 & :0:0:0 & 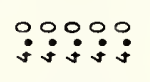 & $\because: 0: 0$ & :゚: : : & 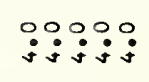 & 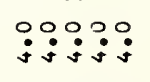 & 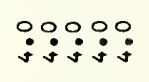 & $\because: 9: 0$ & 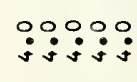 \\
\hline ONIX & 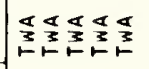 & 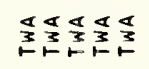 & 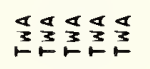 & 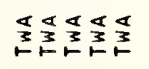 & 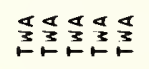 & 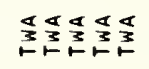 & 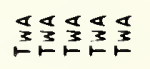 & 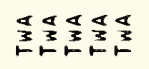 & 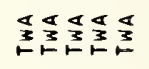 & 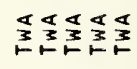 \\
\hline $708 \mathrm{~W} \times \mathrm{S}$ & & & & & $"$ & & " " " & $"$ & & " " " \\
\hline 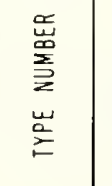 & 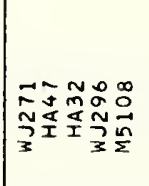 & 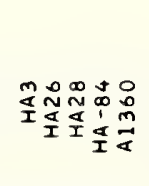 & 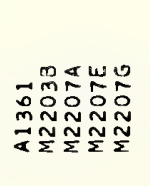 & 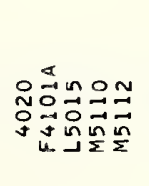 & 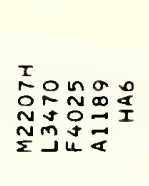 & 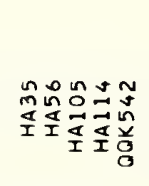 & 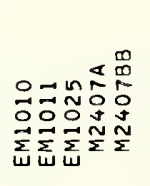 & 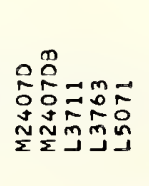 & 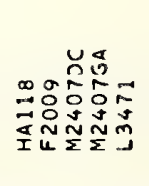 & 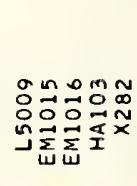 \\
\hline
\end{tabular}




\begin{tabular}{|c|c|c|c|c|c|c|c|c|c|c|}
\hline 9NIIdnOS & \multirow{2}{*}{\multicolumn{2}{|c|}{ 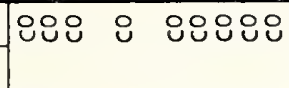 }} & \multirow[t]{2}{*}{ 웅요 } & \multirow[t]{2}{*}{ 운 운 } & \multirow[t]{2}{*}{ บบษำ } & \multirow[t]{2}{*}{ ㅇํํㅇํำ } & \multirow[t]{2}{*}{ ㅇบㅇํํㅇ } & \multirow[t]{2}{*}{ ㅇํ 운 } & \multirow[t]{2}{*}{ ๐๐ำ } & \multirow[t]{2}{*}{ 동으 } \\
\hline Alars & & & & & & & & & & \\
\hline 07114 & $\underline{E}$ & $\sum \sum \sum \sum \sum$ & $\sum \sum \sum \sum$ & $\sum_{n=} \sum_{n} \sum \sum$ & $\begin{array}{ll}\sum \Sigma \Sigma & 0 \\
0\end{array}$ & $\sum \Sigma \Sigma \Sigma$ & $\sum \sum \sum \Sigma \Sigma$ & $\Sigma: 0$ & $\sum \sum \sum \sum$ & ミ요 \\
\hline HLOMOHYP & $\tilde{\tau}$ & $\stackrel{\sim}{*}$ & & & & & & & & \\
\hline 9אי & $\underline{\mathbf{x}}$ & n & & & & & & & & \\
\hline $\begin{array}{l}8013 \times 3 \\
3510 \mathrm{~N}\end{array}$ & in & 융요 & $\stackrel{n}{m}$ & 눙 & $\stackrel{\circ}{\sim}$ & $n+\underset{m}{n}$ & 웅 & $\exists$ & $\stackrel{m}{m} \stackrel{n}{m}$ & $\stackrel{n}{m}$ \\
\hline Nivg & 엽웅요 & 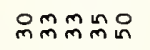 & 욱유윰요 & 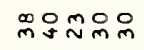 & 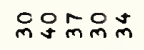 & 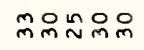 & $\stackrel{m}{\sim} m \sim n \sim ⿻ 上 丨$ & 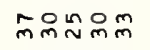 & :0:0:0 & 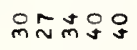 \\
\hline $\begin{array}{l}5170 A \\
\times 173 H \\
\end{array}$ & 学 & 弟 & 前 $\stackrel{\square}{5}$ & 㗖怘 品怘 & 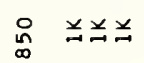 & $\stackrel{D}{\sim}$ & 总兰并 & : & 并芯䛔 & $\stackrel{\check{N}}{\sim}$ \\
\hline $\begin{array}{c}51701 \\
7081 \text { NOS }\end{array}$ & 융요 & 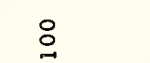 & & $\cong \quad$ in & 요 & in & : & & 옹옹ํ요 & in \\
\hline 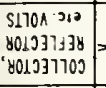 & $\stackrel{\circ}{\stackrel{n}{\sim}} \stackrel{n}{\Xi}$ & & $\stackrel{\circ}{\circ}$ & 용 & $\begin{array}{l}\text { :OO } \\
\text { :욤 }\end{array}$ & 总 & 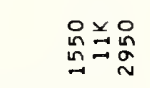 & 을 & : & $\stackrel{:}{:}$ \\
\hline $0^{\circ}$ & 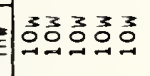 & 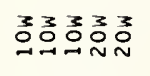 & 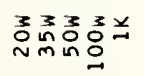 & 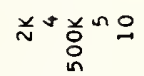 & 옥융ㅁㅇㅇㅁㅇㅇㅁ & 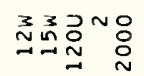 & 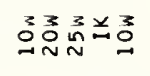 & ㄴ 잉으 & 응ㅇㅁㅇㅇㅁㅇㅁㅇ & 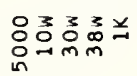 \\
\hline$\mapsto$ & foon & 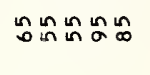 & : & 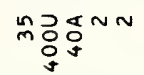 & N ถูก N N & 设早 & 느요 & :mamo & 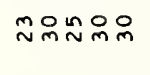 & 윰움요 \\
\hline 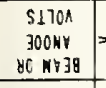 & 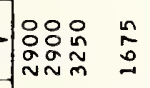 & 음윰윰윰 & $\begin{array}{l}\text { 品品 } \\
\text { 品 }\end{array}$ & 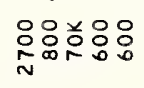 & 品品 吕吕 & 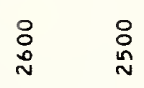 & 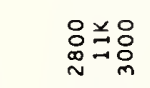 & 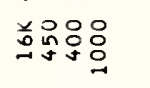 & 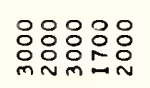 & 品品品 \\
\hline- & Ex & 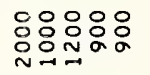 & $\underset{\text { O }}{\stackrel{\text { }}{ }}$ & 品 总品 & 品 总品品 & 䠐 & 品 & 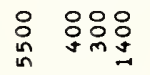 & 응:용요 & 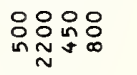 \\
\hline$\omega^{5}$ & 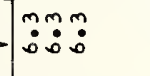 & $\ddot{m}: m \cdot m: m$ & $\ddot{0}$ & 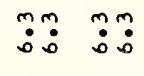 & $\stackrel{m}{:} \stackrel{m m}{a}: \stackrel{m}{:}$ & $\because \dot{0} \quad \dot{0}$ & $\ddot{m}:$ & $\stackrel{n}{\stackrel{n}{r}}: \stackrel{m}{: 0 m}:$ & $\ddot{0}: m: m: m$ & 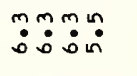 \\
\hline NOLIFy] & טư & טบטบ & טuט & auauu & טuบuท & טuטu & vuvau & auuvu & טuטu & บบaUa \\
\hline$\forall+M$ & 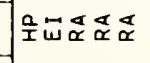 & 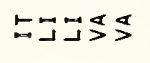 & 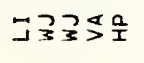 & 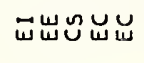 & 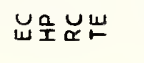 & 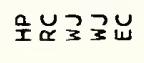 & 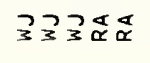 & 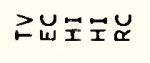 & ேコロゴ & 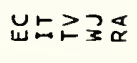 \\
\hline JNก1 & & & & $\stackrel{5}{5}$ & 5 & & & & & \\
\hline $0168 \times 1 \times 10$ & $\stackrel{\circ}{N}$ & & $\stackrel{\circ}{\circ}$ & $\stackrel{\circ}{\circ}$ & & & $\stackrel{\circ}{n}$ & m & & $m \stackrel{\sim}{\sim}$ \\
\hline 竧 & 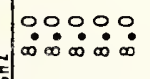 & 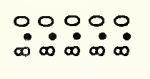 & 舟: & 㝵華芒 & 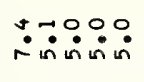 & 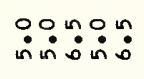 & 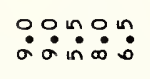 & 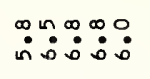 & 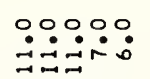 & 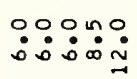 \\
\hline $\begin{array}{ll}\stackrel{\overrightarrow{3}}{\underline{x}} & \frac{x}{2} \\
\end{array}$ & 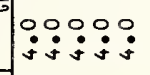 & 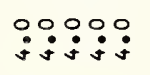 & 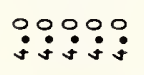 & 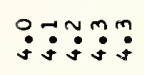 & $m q_{j}+t: j$ & 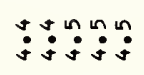 & 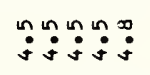 & 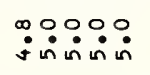 & 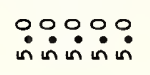 & 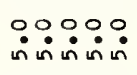 \\
\hline ONIX & 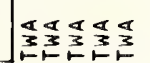 & 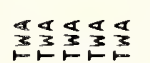 & 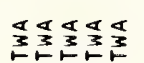 & 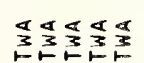 & 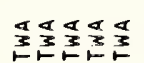 & 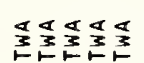 & 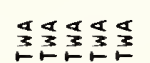 & 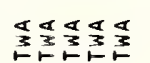 & 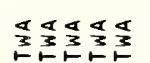 & 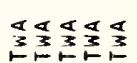 \\
\hline 708WRS & $"$ & & $"$ & & $"$ & $"$ & $"$ & & $"$ " & $"$ \\
\hline 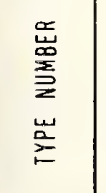 & 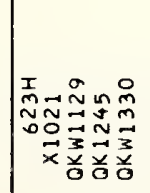 & 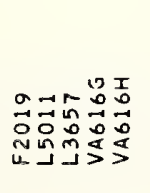 & 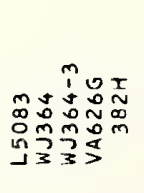 & 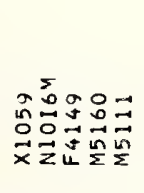 & 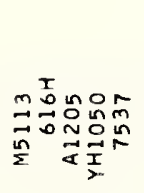 & 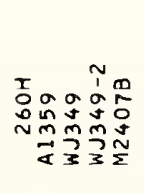 & 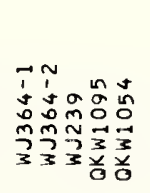 & 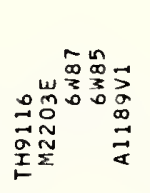 & 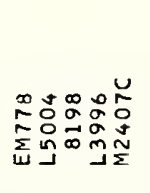 & 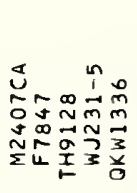 \\
\hline
\end{tabular}




\begin{tabular}{|c|c|c|c|c|c|c|c|c|c|c|}
\hline 9N17dnOS & 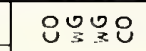 & 옹 & 언언 & 언 옥 & 언언어 & 옳원 & $\mathrm{O}_{3}{ }^{0}{ }^{\circ}$ & 언요 & 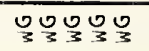 & OU్ \\
\hline Alang & & & & & & & & & & \\
\hline \begin{tabular}{|c|c}
01314 \\
ग113994
\end{tabular} & 음음 & $\sum \Sigma \sum \Sigma$ & $\sum \sum \sum \sum$ 品 & 品 $\sum_{n=n} \sum_{n}$ & $\sum \sum \sum$ & $\sum \sum \sum \sum \sum_{n} \sum_{n}$ & $\sum \Sigma \sum$ & 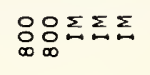 & $\sum \sum \sum \sum \Sigma$ & $\Sigma$ \\
\hline HLOMOHYP $\tilde{\underline{\Sigma}}$ & & & & & 品 & & & & & \\
\hline 9MII7רnd & & & & & & & $\cong$ & & & \\
\hline \begin{tabular}{l|l}
$8019 Y\}$ \\
$3510 \mathrm{H}$
\end{tabular} & ง & $\min _{m}^{n}$ & 용 & & 궁요 & $\checkmark$ & 욤 & $\stackrel{N}{N} \stackrel{\infty}{\sim}$ & $\stackrel{\sim}{\sim}$ & 요ㄹㅛㅛ \\
\hline $\begin{array}{ll}\text { Nirg } & 0 \\
\end{array}$ & 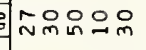 & 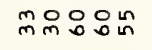 & ํํํํํำ & $\tilde{\sim} \stackrel{\infty}{N} \underset{v}{m} \stackrel{m}{+}$ & 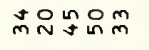 & 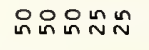 & 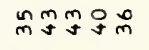 & 영영ㅇㅇ & 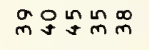 & 品 \\
\hline $\begin{array}{l}S 170 N \\
\times 173 \mathrm{H} \\
\end{array}$ & $\stackrel{-}{\exists}$ & 弚 & 并希品 & $\underset{\sim}{\stackrel{m}{N}}$ & 并 $\quad \stackrel{⿱}{\sim}$ & $\stackrel{\sim}{\sim}$ & 并并并前 & 并并并前 & 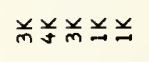 & 并并并前 \\
\hline $\begin{array}{c}51701 \\
7081403\end{array}$ & & 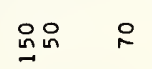 & 용 & 올 & in & 욱 & 品前 & 우용ㅇㅇ & 品品 品 & 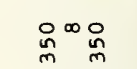 \\
\hline 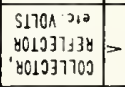 & 羊 & & $\stackrel{\infty}{\circ}$ & $\stackrel{\stackrel{ }{\leftrightarrows}}{\sim}$ & 号 & 品 & 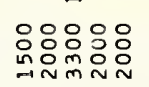 & 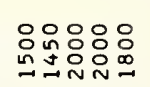 & 路品 品 & 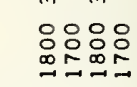 \\
\hline$a^{\circ}$ & 决单品 & 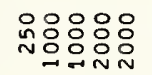 & 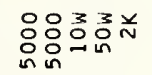 & 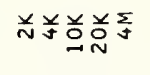 & 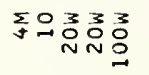 & 항망요 & 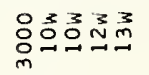 & 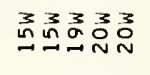 & 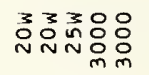 & 응응융유 \\
\hline$-x$ & 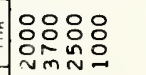 & ro ${ }_{m}^{n} n$ & 용ㅇㅇㅇㅇㅁ & 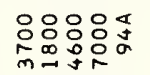 & 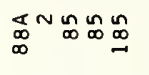 & 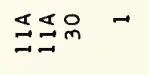 & No워 & 只员乐肙尔 & 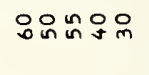 & Nํ워워 \\
\hline 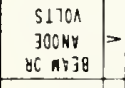 & 兰盖总 & 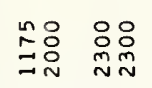 & 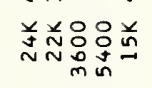 & 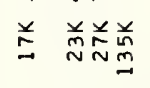 & 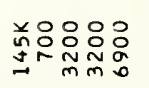 & 兰兰品 只 & 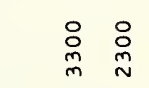 & $\begin{array}{l}\text { 옹 } \\
\text { 옷 } \\
\text { N }\end{array}$ & 品怘 & 음음요 \\
\hline$\leftarrow$ & 응을 & 응 : & 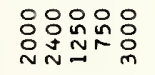 & 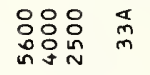 & 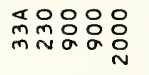 & 응요 & 율울옳윰 & 응용용요 & 응용욤 & 응융ㅇㅇㅇ \\
\hline$\omega^{\prime \prime}$ & $\ddot{0}: 00$ & 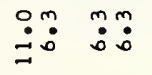 & :OM: & $m m i n$ & 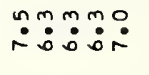 & 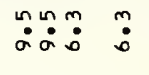 & $\because \stackrel{m}{m} \stackrel{m}{m}: m$ & 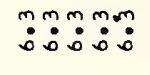 & 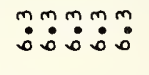 & $\ddot{m}: m m$ \\
\hline $\mathrm{NOI} 1 \mathrm{Fy} \mathrm{dOC}$ & oaguu & טuบu & $a a \cup a a$ & $0 a 0 a 0$ & auvuu & a auvu & uบuvu & uv & uu & uu \\
\hline 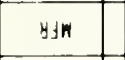 & ヒロஃマ? & タัuさコさ & ३근요 & ヒヒア号な & \Uঙホ? & 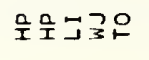 & 爻出らア & エル山上岂岂 & ラ山岕稂 & 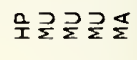 \\
\hline $3 N \cap 1$ & $x_{u}^{x}$ & & & & & & $\underset{\amalg}{\longleftarrow}$ & $\underset{\breve{u}}{\breve{u}}$ & $\underset{\Sigma}{\breve{w}}$ & レレ5 \\
\hline $0118 y$ a ario & 유윰요 & & ㅇN 옥 & 윰ำ & $\stackrel{P}{\sim}$ & 욤 & & & & \\
\hline 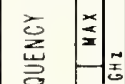 & 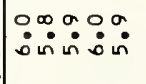 & 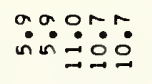 & 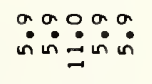 & $\begin{array}{l}\because 090.9 \\
\dot{n} \sin \end{array}$ & 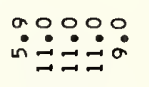 & 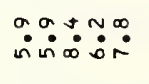 & 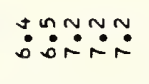 & 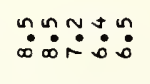 & 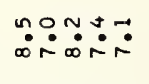 & 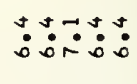 \\
\hline 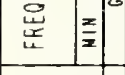 & 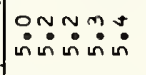 & 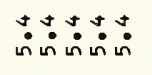 & 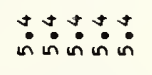 & 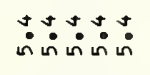 & 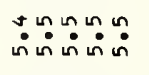 & 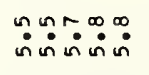 & 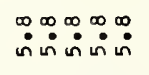 & 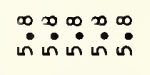 & 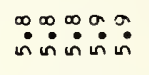 & 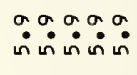 \\
\hline ONIX & 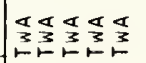 & 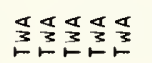 & 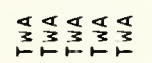 & 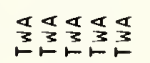 & 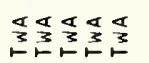 & 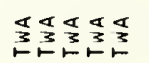 & 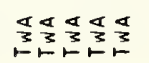 & 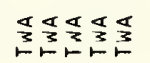 & 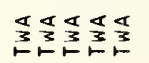 & 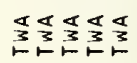 \\
\hline $709 \mathrm{~W} / \mathrm{S}$ & $"$ & " & $"$ & 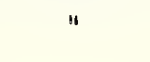 & & & & & & \\
\hline 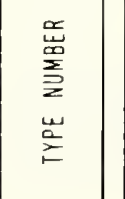 & 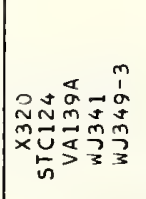 & 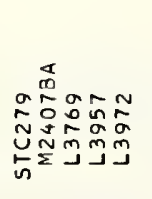 & 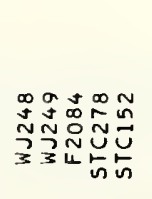 & 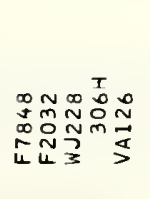 & 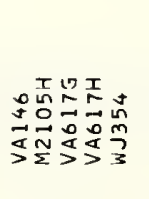 & 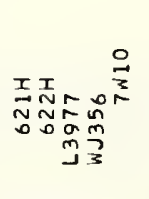 & 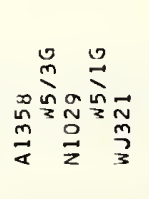 & 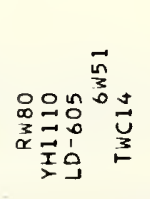 & 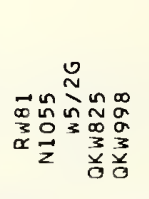 & 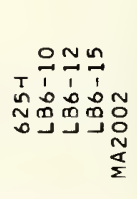 \\
\hline
\end{tabular}




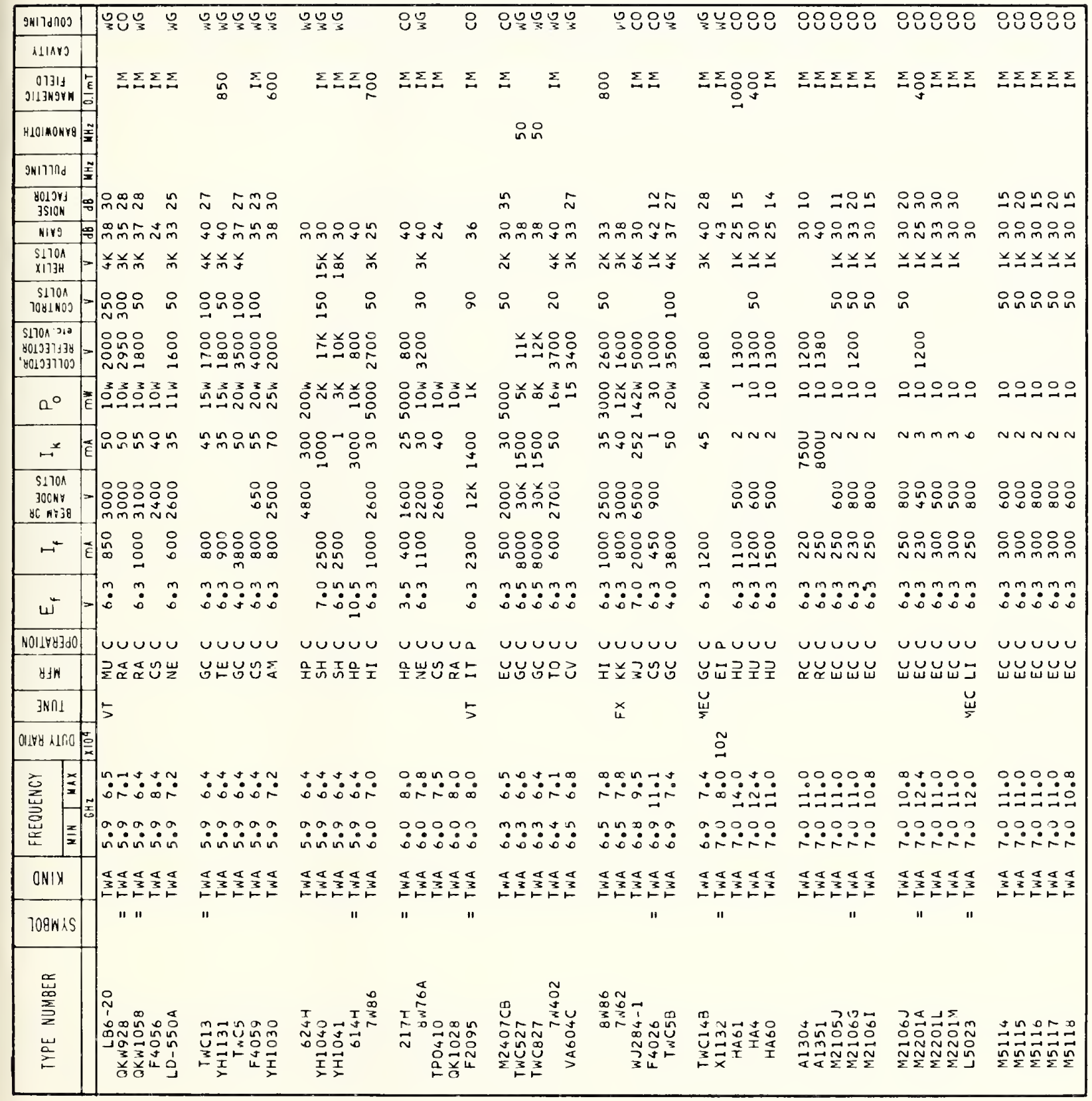




\begin{tabular}{|c|c|c|c|c|c|c|c|c|c|c|}
\hline 9k17dnOO & _운옹으 & 온원은 & 운웅은 & 몽요 & 언언언 & 운언옹 & 웅요 & 언언원언 & 언엉오 & 엉ㅇㅇㅇ \\
\hline RLARS & & & & & & & & & & \\
\hline $\begin{array}{c}01314 \\
\text { ग113HOYK }\end{array}$ & E & 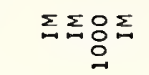 & $\sum \Sigma \Sigma \Sigma \Sigma$ & $\sum \Sigma \Sigma \Sigma$ & $\sum \sum \sum \Sigma$ & 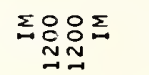 & 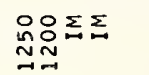 & $\sum \sum \sum \sum \sum$ & $\sum \sum \sum \sum$ & $\sum \Sigma$ \\
\hline HLOMONY8 & $\stackrel{F}{f}$ & & & 羊 & & & & & & \\
\hline 9 9xו7רח & & & & & & & & & & \\
\hline $\begin{array}{l}\text { yolows } \\
\text { 3SIOK } \\
\end{array}$ & 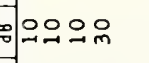 & 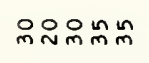 & 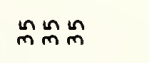 & 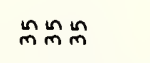 & 品 & 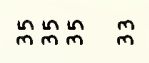 & minm & $\stackrel{\circ}{m}$ & 융ㅇ & \\
\hline Hirg & 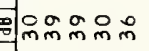 & 앝임욤요 & 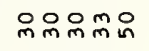 & 임욤ำ & 品出出㗊 & 윰있웅 & 앝임욤요 & 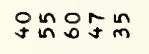 & 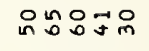 & $g \circ \quad m m$ \\
\hline $\begin{array}{l}51700 \\
\times 1173 H\end{array}$ & 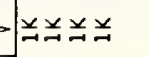 & 并芯弚并 & 弚弚 & 弚 & 并并并首 & 弚羊羊前 & 弚弚羊羊 & 并 & $\stackrel{\circ}{\stackrel{\circ}{n}}$ & 兰兰兰并 \\
\hline $\begin{array}{c}51701 \\
7001 \mathrm{HOS}\end{array}$ & $=$ in & 品 品品品 & 只品品品品 & 응요욤 & 品 ơ & 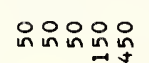 & 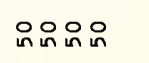 & $\stackrel{\infty}{\circ}$ & : & $\therefore$ \\
\hline 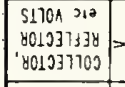 & : & 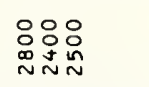 & & 吕 & 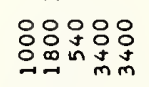 & 品 & & 品 & 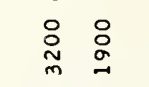 & 兰兰兰弟 \\
\hline $0^{\circ}$ & "ำ & 응응ㅇㅁㅁㅁㅇ & 응ㅇㅁㅇㅁㅁㅇㅁ & :옹융ㅇㅁㅇ & 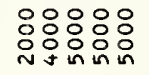 & 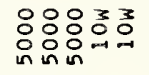 & 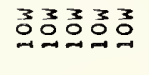 & 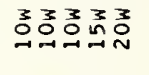 & 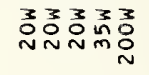 & 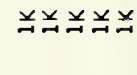 \\
\hline$\mapsto$ & Ez & 음주임요 & 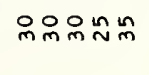 & 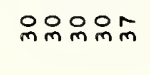 & 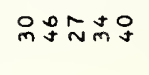 & 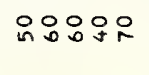 & 영요 & 웅용요 & 胫足 ño & :ㅇ: \\
\hline $\begin{array}{r}\text { S170 } \\
300 \mathrm{HY} \\
80 \mathrm{n} \rightarrow 3 \mathrm{~B} \\
\end{array}$ & - & 욤음 & 응ㅇㅇㅇㅇㅇㅁㅇㅁ & 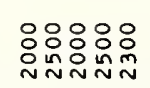 & 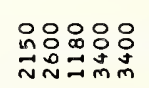 & 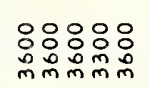 & $\begin{array}{ll}0 & 0 \\
0 & 0 \\
0 & 0 \\
0 & 0 \\
m & 0 \\
m\end{array}$ & 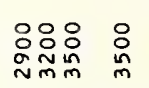 & $\begin{array}{l}\text { OO } \\
\text { 요 } \\
\text { No } \\
\end{array}$ & $\underline{\Xi} \Xi$ \\
\hline$\leftarrow$ & 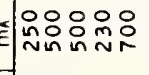 & 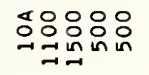 & 음윰ㅇㅇㅇㅇㅁ & 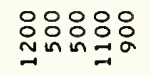 & 윰양ㅇㅇㅇ용 & 웅윰요 & 응요음요 & $\begin{array}{l}\text { 응응음 } \\
\text { 엄엉 }\end{array}$ & 욤요 & 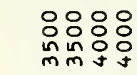 \\
\hline ш" & 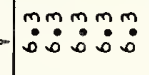 & 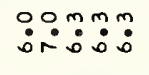 & 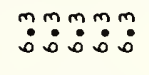 & 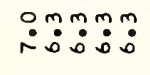 & $\ddot{m}: \because \dot{m}: m:$ & $\ddot{0}: m: m: m$ & $\ddot{m}: m$ & mmmmm: & 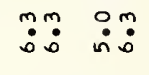 & $\ddot{m}: \stackrel{m}{:}: m$ \\
\hline N011YYZJd0 & טuטu & טuטu & uบuบu & טuטuv & טUטuン & טUטu & & טưu & ưuva & $a a a a a$ \\
\hline$\forall \pm n$ & 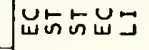 & 브ㅇㅗㅗㄱㅗㅃㅆّ & ב怘楳コ & ว品㞻ココ & コミ゚づル & 岀岂出 & ב岕出品 & コココらか & アロコアす & 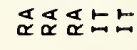 \\
\hline JNח1 & & & 岁 & & 5 & & & $\stackrel{\breve{~}}{\Sigma}$ & $\stackrel{\breve{U}}{\Sigma}$ & \\
\hline $018 \forall 4 \times 150=$ & & & & & & & & & 욱 & 음웅융ㅇㅁㅇㅜ \\
\hline 离 & 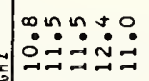 & 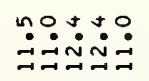 & 芯芯芯 & さ゚シ゚ & 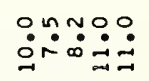 & 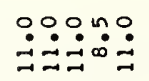 & $\because \because 00:$ & :゚요 & 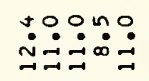 & ○०О: \\
\hline 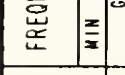 & 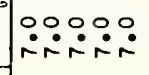 & 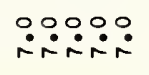 & $\ddot{r} \ddot{i} \ddot{i} \dot{r}$ & 兵官落 & 華芒芒芒 & $\ddot{r} \dot{r} \dot{r} \dot{r}$ & 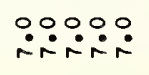 & 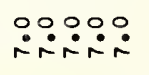 & 品芒芒 & 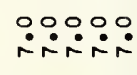 \\
\hline ONIX & 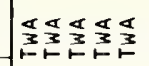 & 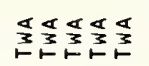 & 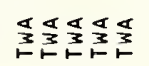 & 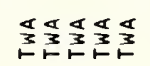 & 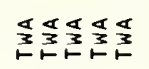 & 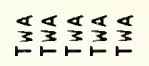 & 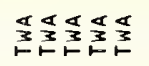 & 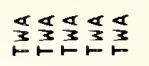 & 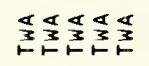 & 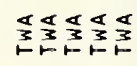 \\
\hline $700 \mathrm{w}$. & & & & & & $"$ & & $"$ & " & \\
\hline 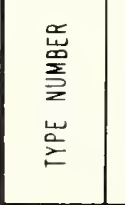 & 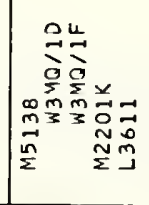 & 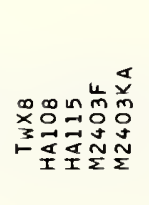 & 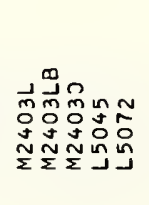 & 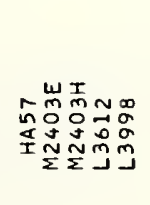 & 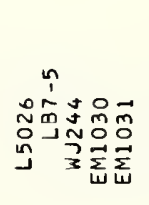 & 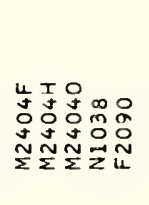 & 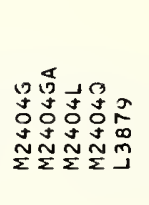 & 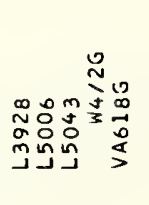 & 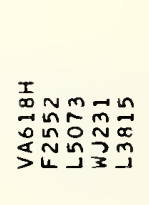 & 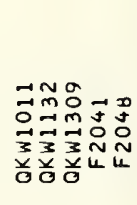 \\
\hline
\end{tabular}




\begin{tabular}{|c|c|c|c|c|c|c|c|c|c|c|}
\hline 9NIId InOS & \multirow{2}{*}{\multicolumn{2}{|c|}{ 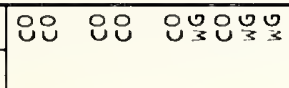 }} & \multirow[t]{2}{*}{ 웅ㅁำ } & \multirow[t]{2}{*}{ 웅요 } & \multirow[t]{2}{*}{ 뫂ㅇㅇㅇ } & \multirow[t]{2}{*}{ 어웡어 } & \multirow{2}{*}{\multicolumn{2}{|c|}{ 엉 웡 }} & \multirow[t]{2}{*}{ 웅ํㅇ } & \multirow[t]{2}{*}{ 언옹 } \\
\hline hIANS & & & & & & & & & & \\
\hline ग113139\% & $\sum \sum \sum \Sigma$ & $\sum \sum \sum_{-\infty} \sum_{\infty} \sum_{\infty}$ & $\sum \sum \sum \Sigma$ & $\sum g_{-} \sum_{-1}$ & $\Sigma \Sigma \Sigma \Sigma$ & $\sum \sum \sum \sum \Sigma$ & $\sum \underset{\sim}{g} g_{-}$ & $\Sigma \stackrel{g}{g}$ & $\Sigma \Sigma \Sigma 0$ & $\Sigma \Sigma \Sigma$ \\
\hline HLOMONYA & & & & & & & & & & \\
\hline 9אורור & & & & & & & & & & \\
\hline $\begin{array}{l}8019 \mathrm{YS} \\
3 \mathrm{SOON}\end{array}$ & $\stackrel{\infty}{\sim}$ & $\stackrel{n}{m} \stackrel{\infty}{\sim}$ & $\stackrel{\infty}{\sim}$ & $\tilde{\sim} \stackrel{\infty}{\sim}$ & oñ & $\stackrel{1}{\circ} \div$ & 임요 & 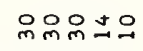 & $a=0$ & $\stackrel{t}{m} \stackrel{m}{m}$ \\
\hline nivg & 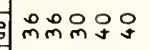 & minmmigo & ํํํํํํํำ & 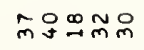 & 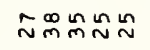 & 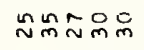 & mo욤 & 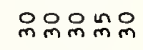 & $\stackrel{\sim \infty}{\sim} \underset{\sim}{\sim} \sim m$ & 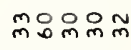 \\
\hline 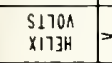 & 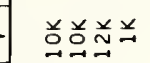 & 弟㒸并岢 & 芯前羊 & 声并并 & 羊 & 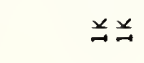 & 并兰 & 兰兰兰 & 决 & 并鬲并 \\
\hline $\begin{array}{c}51700 \\
1081 N 03\end{array}$ & - & 음윰요 & o & $\stackrel{-}{\circ}$ & 兑 & 品 & & & & 웅요 용 \\
\hline 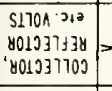 & 응ํำ & 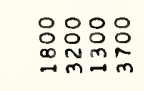 & 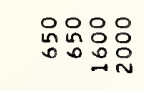 & 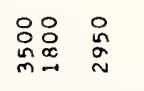 & $\begin{array}{l}\text { 웅 } \\
\text { 品克 }\end{array}$ & $\stackrel{\circ}{\stackrel{8}{7}}$ & 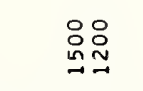 & $\stackrel{ }{\stackrel{D}{\sim}}$ & 品啰 & 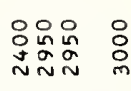 \\
\hline $0^{\circ}$ & $\underline{A} \underline{x} \underline{\underline{a}}$ & 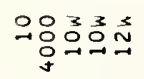 & 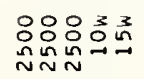 & 茎产 : & 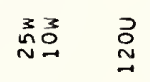 & 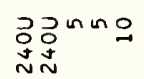 & 으으으으으 & 응ㅇㅇㅇㅇㅇㅁ & 엄옹ㅇㅁㅇㅇㅛ & 음음음ㅇㅁㅇ \\
\hline$-x$ & 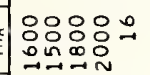 & 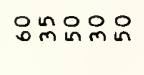 & JAmi & 品先m $\cong$ P & o 品 & $N$ & 吾m & $m m m-$ & $m \infty \stackrel{N}{N}$ & 오ำำ \\
\hline $\begin{array}{c}\text { S170n } \\
300 \mathrm{Ny} \\
80 \mathrm{NBz}\end{array}$ & 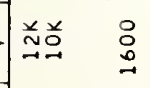 & 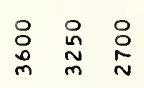 & 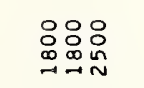 & 品 & : & 总品 & 品等品 & 음웁윰음 & 윰용요 & 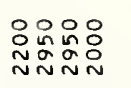 \\
\hline$H^{*}$ & 总品品品品 & 品品 & 있있용ㅇㅁ & 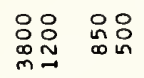 & 品足 & 怘克 & 윰유욤 & :율묘 & 욤유 & 总品吕品品 \\
\hline$\omega^{\leftarrow}$ & mmmm: & $\ddot{m}: \ddot{m}: m$ & $\dot{m} \dot{m}: \dot{m}:$ & $\ddot{q}: \stackrel{m}{:}:$ & $\ddot{m} \ddot{0} 0$ & $\ddot{n}: \dot{0}:$ & $\ddot{0}: \dot{0}$ & $\ddot{m}: \dot{m}:$ & $\ddot{0}: \stackrel{m}{0}$ & $\ddot{i}: \dot{m}: \dot{m}:$ \\
\hline NOIIYyJdO & aagau & บuบuv & טuטuט & טuบuv & auvuu & uuvur & vưue & ưuuv & uuvuv & u \\
\hline$y+n$ & こコこ㐫ロ & 岀忈乐山。 & 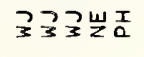 & 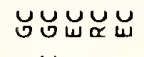 & 诘马马马 & 국구ㅆㅗㅗ & 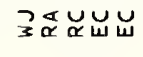 & 过跑? & 굻ㅂㅃ노로 & 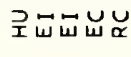 \\
\hline JNก1 & & & & 岁 & 5 & & 5 & & & \\
\hline $0116 y$ unO & 음음음 & & & & in & & & & & \\
\hline 咅 & 挰: & 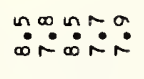 & 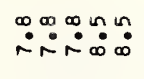 & 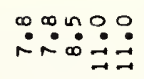 & 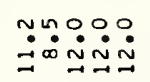 & 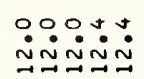 & 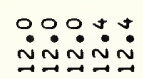 & 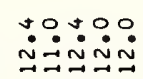 & 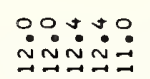 & 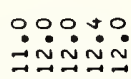 \\
\hline 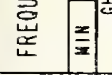 & 華芒茫 & $\ddot{\sim} \because \because \dot{\therefore}$ & 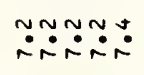 & $\dot{\sim} \dot{\sim} \dot{\sim} \dot{\sim} \dot{\sim}$ & 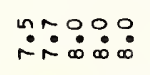 & $\because \because 0: 0$ & $\because \because \because \because: 0$. & $\ddot{\infty} \dot{\infty}: \dot{\infty}: \dot{0}$ & 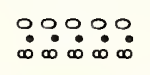 & 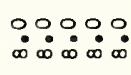 \\
\hline ONIX & 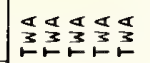 & 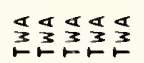 & 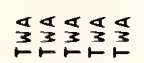 & 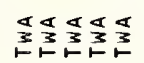 & 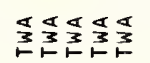 & 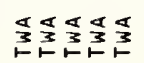 & 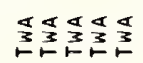 & 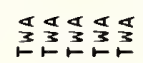 & 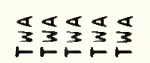 & 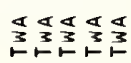 \\
\hline $70 \mathrm{WW}$ AS & $"$ & $"$ & & & & & & " & $"$ " & " " \\
\hline 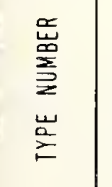 & 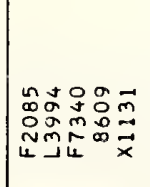 & 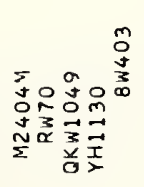 & 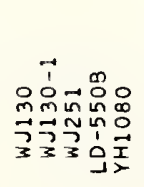 & 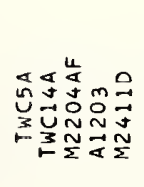 & 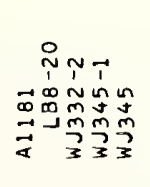 & 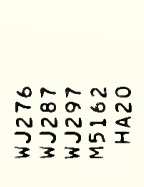 & 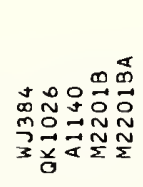 & 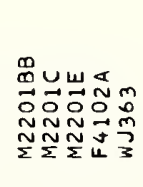 & 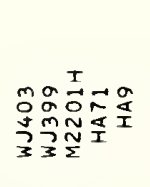 & 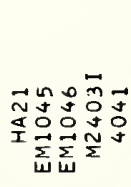 \\
\hline
\end{tabular}




\begin{tabular}{|c|c|c|c|c|c|c|c|c|c|c|}
\hline 9N17dnOS & \multirow[t]{2}{*}{ 온옹으 } & \multirow[t]{2}{*}{ บ용 } & \multirow[t]{2}{*}{ บํㅇ } & \multirow[t]{2}{*}{ 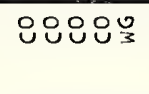 } & \multirow[t]{2}{*}{ ৪ ৪:8 } & \multirow[t]{2}{*}{ 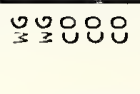 } & \multirow[t]{2}{*}{ 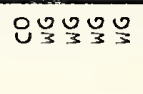 } & \multirow[t]{2}{*}{ З०\% } & \multirow[t]{2}{*}{ ソ゚ั } & \multirow[t]{2}{*}{ Q } \\
\hline LHavo & & & & & & & & & & \\
\hline 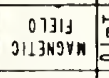 & 苜 & $\sum \sum \sum \sum$ & 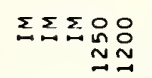 & 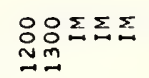 & $\sum \sum \sum \sum$ & ㅇㅇㅇ음 & $\sum_{-\infty}$ & $\sum \sum_{-1} \sum_{\infty} \sum_{-\infty}$ & $\sum \sum \sum$ & $\sum \sum \Sigma \sum$ \\
\hline H101MONG9: & & & & & 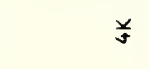 & 品 & & $\stackrel{\sim}{a}$ & & \\
\hline \multicolumn{11}{|l|}{ 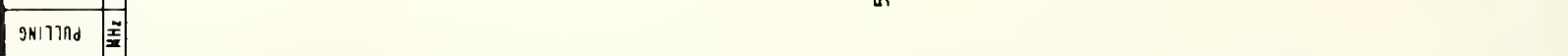 } \\
\hline $\begin{array}{c}\text { Holows } \\
3510 \mathrm{~N}\end{array}$ & D & $\dot{m} \vec{m}$ & $\stackrel{n}{n} \quad n n$ & $\stackrel{n}{m} \stackrel{n}{m}$ & & $\simeq$ & s & ๑ం o & g & \\
\hline nirg $=$ & 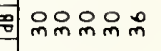 & 옹있요 & 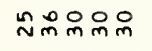 & 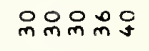 & 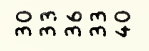 & Go 虽 NNN & ongngog & 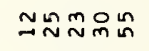 & $\approx$ 象品肙 & 영영ㅇㅇㅇ \\
\hline $\begin{array}{l}5170 \mathrm{~A} \\
\times 173 \mathrm{H}\end{array}=$ & $\rightarrow \underset{N}{ } \rightarrow \underset{y}{*}$ & 当前 & $\frac{a}{3} \quad \frac{3}{3}$ & 羊 弚 & 흐믐 & $\underset{\sim}{\sim} \stackrel{\sim}{\sim} \cong \ddot{N}$ & 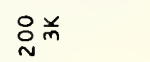 & ? & $\stackrel{\circ}{\sim}$ & $\stackrel{\Xi}{\Xi}$ \\
\hline $\begin{array}{c}51701 \\
104: \text { NOJ }\end{array}=$ & 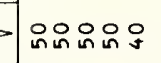 & in in & 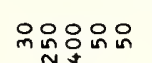 & 品品 品 & 움욤웅 & & 웅 & in: & : & N \\
\hline 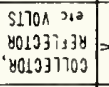 & $=\stackrel{\circ}{\stackrel{D}{n}}$ & 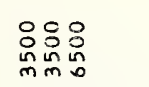 & $\stackrel{\square}{\circ}$ & 品 品 & & 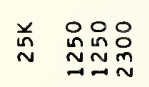 & 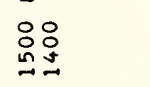 & $\stackrel{\circ}{\cong}$ & $\stackrel{\circ}{\sim}$ & $\cong$ \\
\hline $0^{\circ}$ & E) & 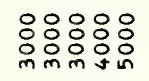 & 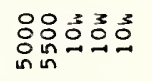 & 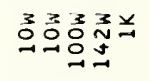 & 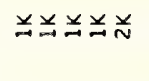 & 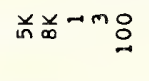 & 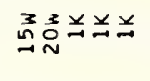 & 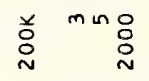 & 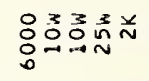 & 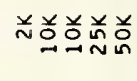 \\
\hline$\rightarrow \bar{E}$ & E & 욜요 & 웅옹요 & 으욪유 & 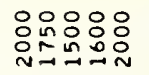 & in: $\underset{N}{N} \sim N \infty$ & 웅 总品 & 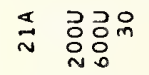 & 웅융 & 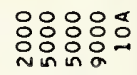 \\
\hline $\begin{array}{r}5170 \AA \\
300 \mathrm{KH} \\
8 \mathrm{KY} ; \mathrm{ig}\end{array} \mid=$ & 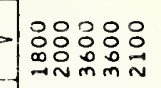 & 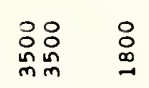 & 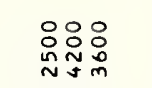 & 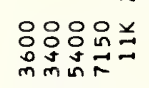 & 兰兰兰兰 & 웅윰요 & 吕 兰兰 & 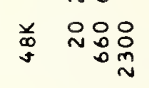 & 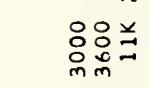 & 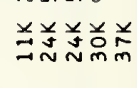 \\
\hline$m^{-}$ & E & 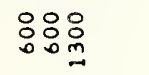 & 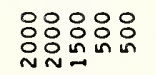 & 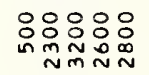 & 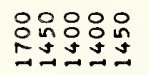 & 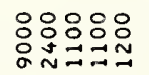 & 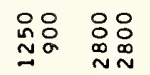 & 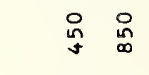 & $\underset{n}{\stackrel{8}{0}} \stackrel{0}{N}$ & 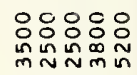 \\
\hline w" & $\ddot{m} \ddot{m} \dot{m}: \dot{m}: \dot{m}$ & $\ddot{0} \ddot{m}: \ddot{m}$ & $\ddot{m}:{ }^{m}:{ }^{m}:{ }^{m}: \dot{0}$ & mammin & 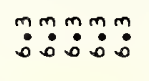 & 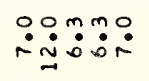 & $\ddot{m}_{\dot{0}}^{m} \stackrel{n}{i} \stackrel{n}{n}$ & $\stackrel{0}{:}$ & $\ddot{m}:$ & mo:00 \\
\hline N011 $18 \mathrm{y} \mathrm{dO}$ & & ưau & טuטu & 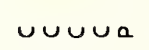 & $a \operatorname{aga} a$ & auuuv & ưava & aบuบu & บบบบa & $a a a a a$ \\
\hline मJW & 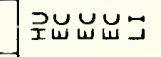 & 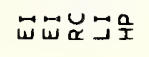 & 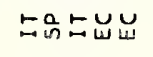 & 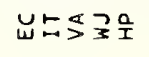 & コココココ & 버ㅁㅜㅗ고로고 & Е山的侱呈 & 呈预嵌コ & コ๙゙コロ呈 & 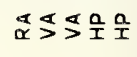 \\
\hline $3 \mathrm{~N} \cap 1$ & & & 5 & 5 & u & & & $u_{u}^{x}$ & & \\
\hline oldy a drio & & in & & $\stackrel{\text { O }}{2}$ & 융유 & $\circ$ & 요 N & in & 品 & 임엄욤요 \\
\hline 总 & 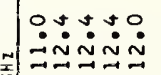 & $\begin{array}{l}\because \because 000 \\
\cong 0\end{array}$ & 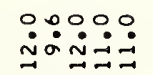 & 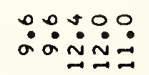 & $\because \because \because 00$. & $\ddot{\sigma} \dot{\sim} \underset{\sim}{\tilde{N}}$ & 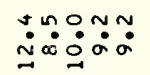 & 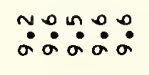 & 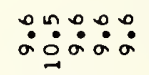 & 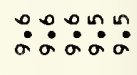 \\
\hline 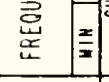 & 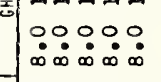 & $\because \because \therefore: 0$. & 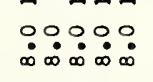 & $\because \because \dot{\infty} \infty \dot{0}$ & $\because \because \therefore: 0$. & $\dot{\infty} \times \underset{\infty}{\sim} \sim \tilde{\infty}$ & 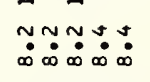 & 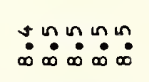 & 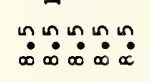 & 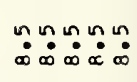 \\
\hline ONIX & 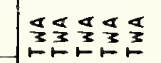 & 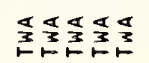 & 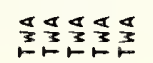 & 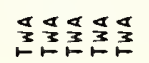 & 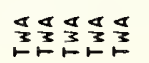 & 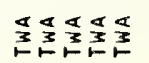 & 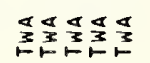 & 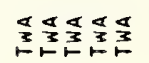 & 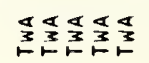 & 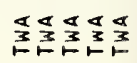 \\
\hline $708 \mathrm{w} \times \mathrm{S}$ & & " " " & $"$ & & & & " " " & & $"$ & " " \\
\hline 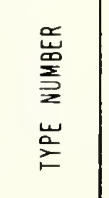 & 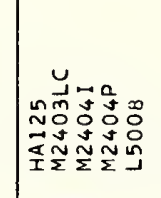 & 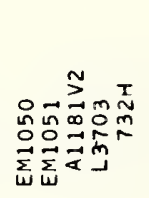 & 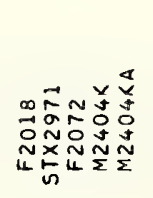 & 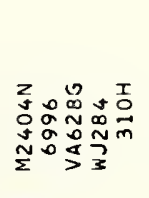 & 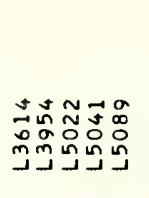 & 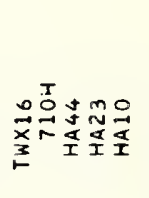 & 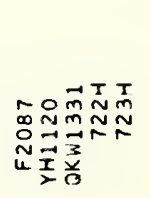 & 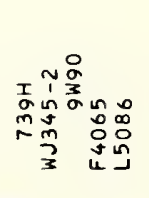 & 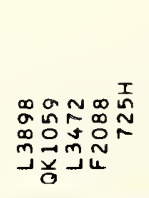 & 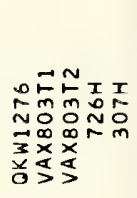 \\
\hline
\end{tabular}




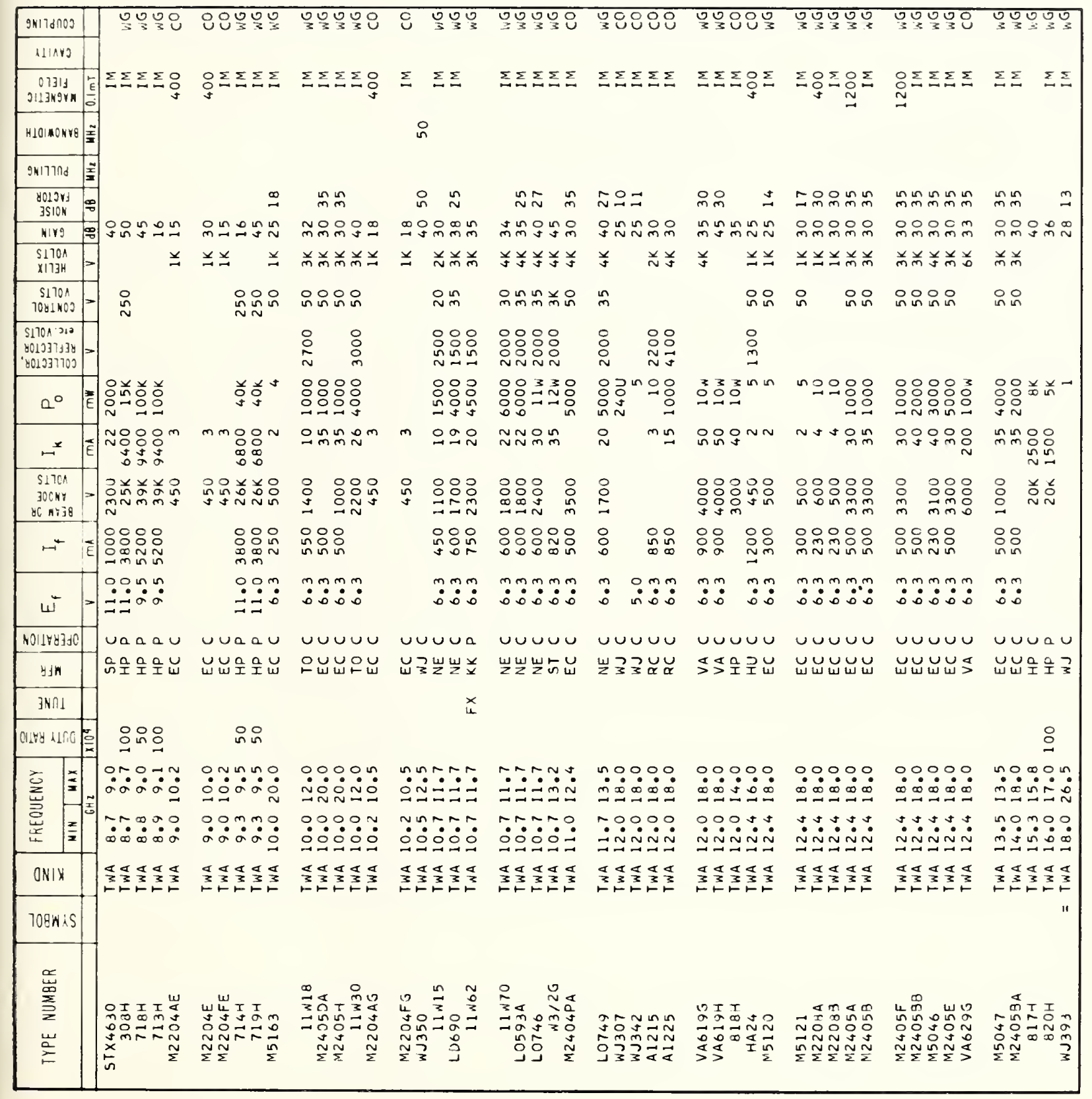




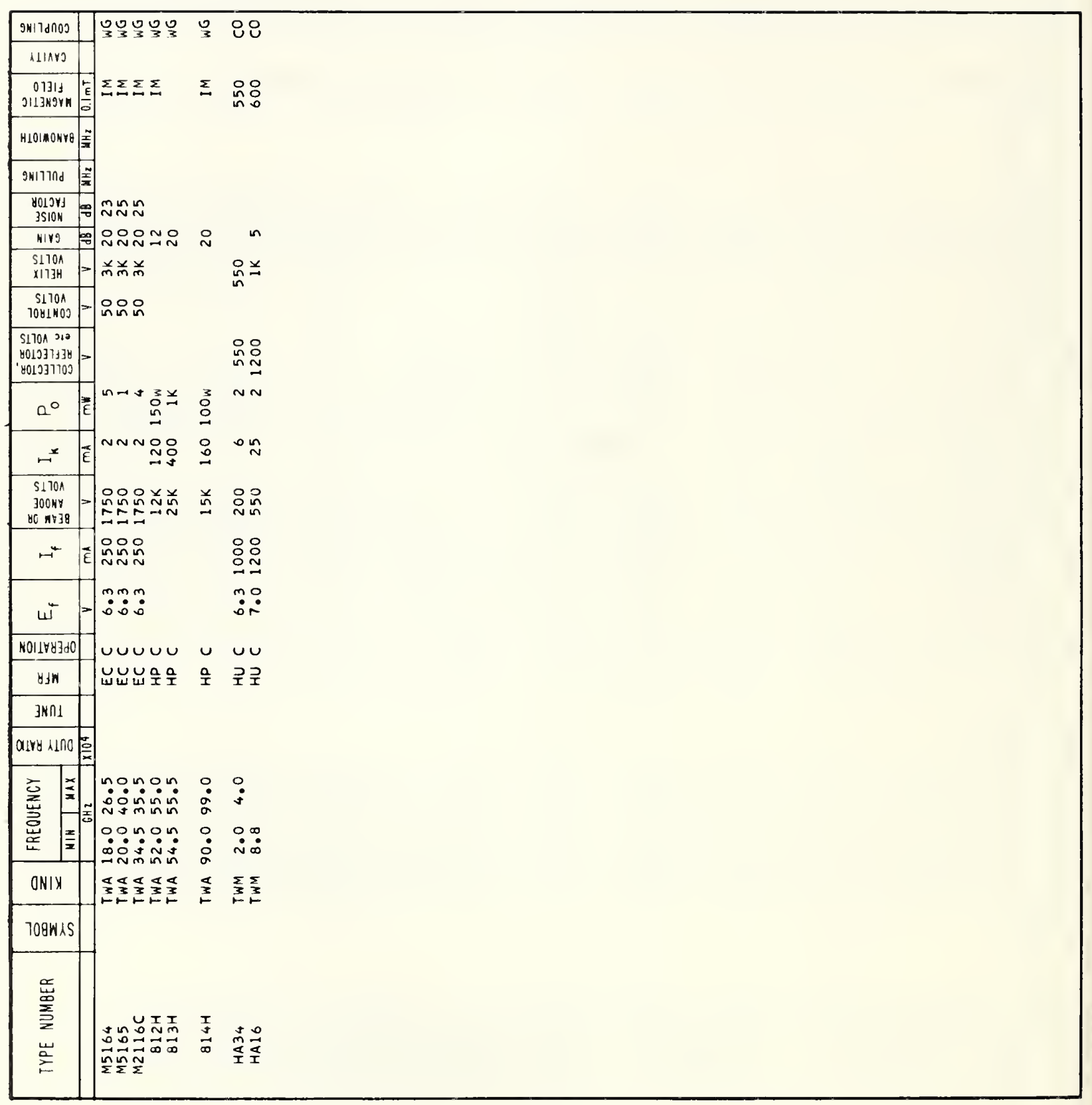




\section{THE NATIONAL BUREAU OF STANDARDS}

The National Bureau of Standards ${ }^{1}$ provides measurement and technical information services essential to the efficiency and effectiveness of the work of the Nation's scientists and engineers. The Bureau serves also as a focal point in the Federal Government for assuring maximum application of the physical and engineering sciences to the advancement of technology in industry and commerce. To accomplish this mission, the Bureau is organized into three institutes covering broad program areas of research and services:

THE INSTITUTE FOR BASIC STANDARDS . . . provides the central basis within the United States for a complete and consistent system of physical measurements. coordinates that system with the measurement systems of other nations, and furnishes essential services leading to accurate and uniform physical measurements throughout the Nation's scientific community, industry, and commerce. This Institute comprises a series of divisions, each serving a classical subject matter area:

- Applied Mathematics-Electricity-Metrology-Mechanics-Heat-Atomic Physics-Physical Chemistry-Radiation Physics- Laboratory Astrophysics ${ }^{2}$ - Radio Standards Laboratory, ${ }^{2}$ which includes Radio Standards Physics and Radio Standards Engineering-Office of Standard Reference Data.

THE INSTITUTE FOR MATERIALS RESEARCH . . . conducts materials research and provides associated materials services including mainly reference materials and data on the properties of materials. Beyond its direct interest to the Nation's scientists and engineers, this Institute yields services which are essential to the advancement of technology in industry and commerce. This Institute is organized primarily by technical fields:

-Analytical Chemistry-Metallurgy-Reactor Radiations-Polymers-Inorganic Materials-Cryogenics $^{2}$-Office of Standard Reference Materials.

THE INSTITUTE FOR APPLIED TECHNOLOGY ... provides technical services to promote the use of available technology and to facilitate technological innovation in industry and government. The principal elements of this Institute are:

- Building Research-Electronic Instrumentation-Technical Analysis-Center for Computer Sciences and Technology-Textile and Apparel Technology Center-Office of Weights and Measures -Office of Engineering Standards Services-Office of Invention and Innovation-Office of Vehicle Systems Research-Clearinghouse for Federal Scientific and Technical Information ${ }^{3}$-Materials Evaluation Laboratory-NBS/GSA Testing Laboratory.

\footnotetext{
${ }^{1}$ Headquarters and Laboratories at Gaithersburg, Maryland, unless otherwise noted; mailing address Washington, D. C., 20234.

2 Located at Boulder, Colorado, 80302.

${ }^{3}$ Located at 5285 Port Royal Road, Springfield, Virginia 22151.
} 


\section{PERIODICALS}

JOURNAL OF RESEARCH reports $\mathrm{Na}$ tional Bureau of Standards research and development in physics, mathematics, chemistry, and engineering. Comprehensive scientific papers give complete details of the work, including laboratory data, experimental procedures, and theoretical and mathematical analyses. Illustrated with photographs, drawings, and charts.

Published in three sections, available separately:

\section{- Physics and Chemistry}

Papers of interest primarily to scientists working in these fields. This section covers a broad range of physical and chemical research, with major emphasis on standards of physical measurement, fundamental constants, and properties of matter. Issued six times a year. Annual subscription: Domestic, \$5.00; foreign, $\$ 6.00^{*}$.

\section{- Mathematics and Mathematical Physics}

Studies and compilations designed mainly for the mathematician and theoretical physicist. Topics in mathematical statistics, theory of experiment design, numerical analysis, theoretical physics and chemistry, logical design and programming of computers and computer systems. Short numerical tables. Issued quarterly. Annual subscription: Domestic, $\$ 2.25$; foreign, $\$ 2.75^{*}$.

\section{- Engineering and Instrumentation}

Reporting results of interest chiefly to the engineer and the applied scientist. This section includes many of the new developments in instrumentation resulting from the Bureau's work in physical measurement, data processing, and development of test methods. It will also cover some of the work in acoustics, applied mechanics, building research, and cryogenic engineering. Issued quarterly. Annual subscription: Domestic, $\$ 2.75$; foreign, $\$ 3.50^{*}$.

\section{TEGHNICAL NEWS BULLETIN}

The best single source of information concerning the Bureau's research, developmental, cooperative and publication activities, this monthly publication is designed for the industry-oriented individual whose daily work involves intimate contact with science and technology-for engineers, chemists, physicists, research managers, product-development managers, and company executives. Annual subscription: Domestic, \$1.50; foreign, \$2.25*.

*Difference in price is due tc extra cost of foreign mailing.

\section{NONPERIODICALS}

Applied Mathematics Series.

tables, manuals, and studies.

Building Science Series. Research results, test methods, and performance criteria of building materials, components, systems, and structures.

Handbooks. Recommended codes of engineering and industrial practice (including safety codes) developed in cooperation with interested industries, professional organizations, and regulatory bodies.

Miscellaneous Publications. Charts, administrative pamphlets, Annual reports of the Bureau, conference reports, bibliographies, etc.

Monographs. Major contributions to the technical literature on various subjects related to the Bureau's scientific and technical activities.

National Standard Reference Data Series. NSRDS provides quantitative data on the physical and chemical properties of materials, compiled from the world's literature and critically evaluated.

Product Standards. Provide requirements for sizes, types, quality and methods for testing various industrial products. These standards are developed cooperatively with interested Government and industry groups and provide the basis for common understanding of product characteristics for both buyers and sellers. Their use is voluntary.

Technical Notes. This series consists of communications and reports (covering both other agency and NBS-sponsored work) of limited or transitory interest.

\section{CLEARINGHOUSE}

The Clearinghouse for Federal Scientific and Technical Information, operated by NBS, supplies unclassified information related to Governmentgenerated science and technology in defense, space, atomic energy, and other national programs. For further information on Clearinghouse services, write:

Clearinghouse

U.S. Department of Commerce Springfield, Virginia 22151

Order NBS publications from:

Superintendent of Documents

Government Printing Office

Washington, D.C. 20402 




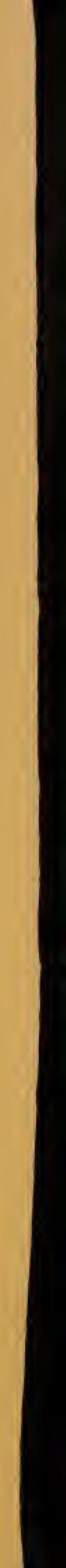

